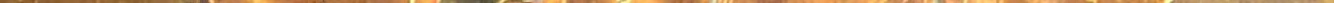




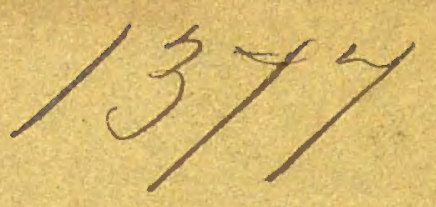




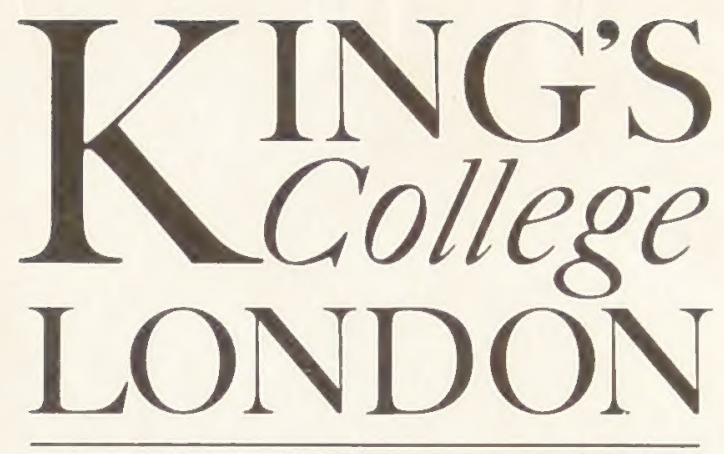

GLMPIS QLI37 SER

Library

SERRGS, E. RIA.

AnATOMRE COMPAREE

DU CERVEAV

1827

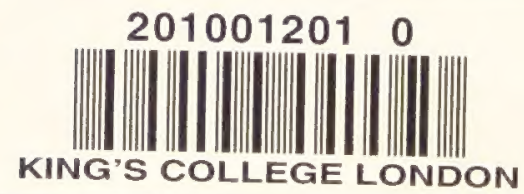


\&

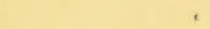


Digitized by the Internet Archive in 2015

https://archive.org/details/b21300197_0002 


\title{
AN TOMTE
}

COMPARÉ

\section{DU CET $\mathbb{V} \mathbf{E} \mathbb{U}$}

\section{DANS IES QUATRE CLASSES}

\section{DES ANIMAUX VERTÉBRÉS,}

\author{
APPLIQUÉE \\ A IA PHYSIOLOGIE ET A LA PATHOLOGIE DU SYSTÈMe NERVEUX;
}

\section{PAR E. R. A. SERRES,}

Chevalier de l'Ordre royal de la Légion-d'Honneur, Médecin ordinaire de l'Hopital de la Pitié, Professeur agrégé de la Faculté de Médecine de Paris, Chef des Travaux anatomiques de l'Amphithéâtre des Hopitaux, Professeur d'Anatomie et de Physiologie du même établissement, Membre de l'Académie royale de Médecine de Paris, de la Société Philomatique, de la Société de médecine Démocrite, Anaxagoras disséquaient dejà le cerveau il y a près
de trois mille ans; Haller, Vicq-d'Azyr et vingt anatomistes
vivans l'ont disséquéd vivans lont disséqué de nos jours; mais, chose admirable! il n'en est aucun qui n'ait encore laissé des découvertes a faire à ses successeurs.
Covieg.

OUVRAGE QUI A REMPORTÉ LE GRAND PRIX A L'INSTITUT ROYAL DE FRANCE.

\section{TOME SECOND.}

Arec un Atlas de seize Planches grand in- $4^{\circ}$, représentant trois cents sujets, dessinces ef lithographiées par Fraxez, sous les yeux de l'auteur, et accompagnées d'une Esplication.

$$
1826 .
$$

\section{A PAIS,}

CHEZ GABON ET COMPAGNIE, LIBRAIRES, RUE DE L'ÉCOLE-DE-MÉDECINE;

A MONTPELLIER, GHEZ LES MÊMES LIDRATRES.

\footnotetext{
A BRUXELLES, AU DÉPÔT GB́NÉRAL DE LIBRAIRIE MÉDICA

Marché aux Poulets, $\mathrm{n}^{\circ}, 1213$, au coin de a
} 


$$
562882
$$

IMPRIMERIE DE GUEFFIER 


\section{TABLE}

DES MATIÈRES

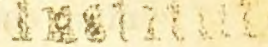

$2+7$

CONTENUES DANS LE SECOND VOfUNG I ? ?

\section{TROISIÈME PARTIE.}

Des formes permanentes du système nerveux chez les vertébrés et les invertébrés. - Rapports, fonctions et maladies de l'axe cérébro-spinal des vertébrés.

\section{GHAPITRE PREMIER.}

Concordance du système nerveux dans le règne animal. - Explication du système nerveux des invertébrés. 5

\section{CHAPITRE II.}

Apercu sur le siége de l'instinct. - De l'action des nerfs trijumeaux des vertébrés, comparée à celle des ganglions céphaliques des articulés. ....... 50

Histoire d'une altération organique du nerf trijumeau, suivie de la perte de la vue, de l'odorat, de l'ouie et du goat du même côté. .......... 6 7

CHAPITRE III.

Anatomie comparative de la moelle épinière, dans les quatre classes d'animaux vertébrés. . . . . . 9 9 
Tableau comparatif des dimensions de la moelle épinière chez les mammifères.

Tableau comparatif des dimensions de la moelle, épinière chez les oiseaux. ......... 175

Tableau comparatif des dimensions de la moelle épinière chez les reptiles. ........... 17g

Tableau comparatif des dimensions de la moelle épinière chez les poissons............. 178

\section{CHAPITRE IV.}

Anatomie comparative de la moelle allongée dans les quatre classes d'animaux vertébrés.

Corollaires physiologiques et pathologiques sur la moelle épinière et la moelle allongée...... 219

Histoire d'une maladie organique de la moelle épinière. 234

Tableau comparatif des dimensions du bulbe rachidien chez les mammiféres. .

Tableau comparatif des dimensions de la moelle allongée chez les oiseaux. ...................... 49

Tableau comparatif des dimensions de la moelke allongée chez les reptiles. ............ 251

Tableau comparatif des dimensiors de la moelle allongée chez les poissons. ............. 253

\section{CHAPITRE V.}

Des tubercules quadrijumeaux, et de leurs analogues, les lobes optiques, dans les quatre classes des vertébrés 253 Tabieau comparatif des dimensions des tubercules quadrijumeaux chez les mammifères. . . . . . 356

Tableau comparatif des dimensions des tubercules quadrijumeaux chez les oiseaux. ........ 359 
'Tableau comparatil des dimensions des tubercules quadrijumeaux chez les reptiles. . . . . . . . 340 Tableau comparatif des dimensions des tubercules quadrijumeaux chez les poissons. ......... 341

\section{CHAPITRE VI.}

Anatomie comparée du cervelet, dans les quatre classes des animaux vertébrés. . . . . . . . 34\%

Tableau comparatif des dimensions du cervelet chez les mammifères. ..................... 423

Tablenu comparatif des dimensions du cervelet chez les oiseaux.......................... 425

Tableau comparatif des dimensions du cervelet chez les reptiles. .................... 426

Tableau comparatif des dimensions du cervelet chez

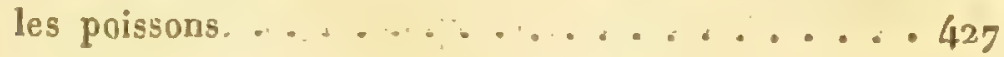

\section{CHAPITRE VII.}

Anatomie comparative des hémisphères cérébraux et du lobe olfactif dans les quatre classes des vertẻbrés. . . 428 De la couche optique et du corps strié. ...... 45 I Système des commissures des hémisphères cérébraux. 448 Commissure antérieure et postérieure. . . . . 450 De la commissure molle. ........... 458

De la bandelette demi-circulaire. . . . . . . 459 Du corps calleux................. 480

De l'épiphyse et de l'hypophyse cérébrales. (Glande pinéale et glande pituitaire.). ......... 482 Du lobule optique. ................... $49^{8}$ De l'éminence mamillaire. .......... 5 5 2 
Dès lobes cérébraux. . . . . . . . . . $516^{\circ}$

Du lobe olfactif. . . . . . . . . . . 565

Résumé des caractères propres à l'encéphale des quatre classes d'animaux vertébrés. ........ . 579

Tableau comparatif des dimensions des lobes cérébraux chez les mammifères. : . . . . . . . . 588

Tableau comparatif des dimensions des lobes cérébraux chez les oiseaux. ................. 590

Tableau comparatif des dimensions des lobes cérébraux chez les reptiles. ............... 591

Tableau comparatif des dimensions des lobes cérébraux chez les poissonis. . ............... 592

\section{CHAPITRE VIII.}

Corollaires physiologiques et pathologiques sur le cervelet, les tubercules quadrijumeaux et les hémisphères cérébraux. .......... . 595

De l'action du cervelet sur les organes génitaux. . . . 60 r Action du cervelet sur les mouvemens volontaires.. . . 613 Action et maladies de la protubérance annulaire. . . . 632 Action et maladies des tubercules quadrijumeaux. . . . 642 Action et maladies des hémisphères cérébraux. . . . . 653 


\section{ANATOMIE \\ COMPARÉE \\ DU CERVEA}

D A N S

LES QUATRE CLASSES DES ANIMAUX VERTÉBRES.

\section{TROISIÈME PARTIE.}

DES FORMES PERMANENTES DU SYSTEME NERVEUX CHEZ LES VERTÉBRÉS ET LES INVERTÉBRÉS. - RAPPORTS, FONCTIONS ET MALADIES DE L'AXE GÉRÉBRO-SPINAL DES VERTÉBRÉS.

Les sciences exactes se composent de deux orAres de faits, des faits particuliers, qui en sont les élémens, et des faits généraux, qui sont une abstraction des premiers. Les premiers servent de base aux seconds, les scconds cxpliquent les premiers. Toute science positive doit tendre à présenter ce double enchaînement des vérités qui li constituent.

Dans la Première l'arlie de cet ouvrage j'ai exposé le développement de l'axe cérébro-spinal chez les embryons; jen ai déterminé les diverses parties.

II. 
Dans la Seconde, j’ai présentéles lois du système. nerveux; j'en ai fait l'application à la névrogénie; j’ai donné les rapports comparatifs des nerfs encéphaliques dans les quatre classes d'animaux vertébrés.

Je vais essayer, dans cette Troisième Partie, de présenter le tableau général du système nerveux dans le règne animal. Je dirai d'abord ce qu'il est chez les invertébrés; je chercherai à ramener à un type commun les modifications nombreuses qu'il offre daus leś diverses classes de ce grand embranchement. Après avoir déterminé ses rapports avec celui des vertébrés, je m’occuperai de l'axe cérébro-spinal de ces derniers; j'exposerai les lois de son déreloppement et ses formes permanentes; je montrerai les rapports de ses diverses parties dans les quatre classes, ct je dirai un mot de leurs fonctions et de leurs maladies chez l'homme.

Ces rapports, ces fonctions et ces maladies ont beaucoup occupé les anatomistes, les physiologistes et les médecins.

Les rapports ont été méconnus, parce que, itune part, on comparait des ternes dont la valeur était inconnuc et changeait à tout moment; de l'autre, parce que n'ayant pas décomposé l'encéphale, on appréciait les rapports en masse, au lieu de les étudier de partie à partie.

En troisième lieu, l'ilée que tout embryon était primitivement ce que devait ctre l'animal qui en provenait, avait fait négliger les rapports de l'axe 
cérébro-spinal avec les diverses régions du corps. On laissait échapper les conditions d'existence et de forme qui lient les centres du systeme nerveux aux variations nombreuses de la périphérie des ètres; on mettait ainsi hors de la question les données les plus importantes de ce systène, puisqu'clles se ratlachent aux nodifications diverses que le corps des animaux présente dans les quatre classes, et à leur manière de vivro, qui résulte de ces modifications. On avait vaguement dit que l'axe cérébro-spinal du système nerveax répétait en petit tout l'aninal; il fallail le prouver.

I.es fonctions et les maladies n'étaient guère mieux précisées que les rapports. On étudiait en masse les effots de l'axe cérébro-spinal du système nerveux; on rapportait des phénoménes analogues à des parties que l'on croyait dissimilaires, et des phénomènes opposés étaient attribués à d'autres parties que l'anatomie déclarait analogues.

La physiologie expérimentale n'avait pas encore imaginé d'agir isolément sur les diverses partics du système nerveux, pour en apprécier les divers usages; et la pathologie n'avait tiré aucun parti de cet isolement d'altération que les maladies nous présentent si fréquemment, et nous présentent dégagées des complications diverses que l'expériinentation introduit nécessairement dans le problème.

Ainsi la ploysiologie tâlonnail, privée des bases quo devait lui fournir l'anatomic comparative: la 
pathologie éclairait bien de loin en loin les routes ténébreuses de cette étude; mais ses lueurs étaient perdues au milieu du vague des hypothèses et des suppositions de toute nature que faisaient naître les difficultés du sujet.

On observait, on expérimentait beaucoup; les faits semblaient contredire les faits, les expériences étaient détruites par d'autres expériences, les explications s'accumulaient pour concilier ce qui était inconciliable. Aussi la science restait-elle stationnaire au milieu de ces longs et pénibles efforts.

L'anatomic, la physiologie et la pathologic du système nerveux s'étant arrêtées par les mêmes causes, devaient s'éclairer par les mêmes moyens.

Déterminer rigoureusement les élémens divers dont se composent l'encéphale et la moelle épinière ;

Comparer les parties similaires pour saisir leurs analogics et leurs différences, ou en d'autres termes, exprimer leurs rapports;

Expérimenter sur les parties analogues pour en découvrir les usages;

Observer leurs altérations et les symptômes qu'elles produisent, pour en éclairer les maladies:

Telle est la marche que suivent les anatomistes, les physiologistes el les pathologistes de nos jours. Telle est la cause des progrès rapides que font depuis quelque temps ces trois branches importantes de nos connaissances. 


\section{CHAPITRE PREMIER.}

Concordance du système nerveux dans le règne animal. - Explication du syslème nerveux des invertébrés.

L'Explication des êtres organisés est le but définitif de l'anatomie comparative; ses moyens sont la détermination et les rapports des organes qui. les composent.

Les rapports sont des abstractions résultant de la comparaison des parties similaires dans tous les animaux.

Quand on jette un coup-d'oeil sur l'ensemble du règne animal, l'esprit est frappé d'abord de la dissemblance des êtres qui le composent; mais quand on étudie leur organisation intérieure, ces dissemblances s'effacent peu-à-peu, les ressemblances s'établisseut, et l'observateur voit sc dérouler le plan vaste, mais uniforme, del'organisation animale.

Tout paraît différent, tout paraît analogue, selon le point de vue d'après lequel on considère le règne animal. Si l'on n'en mesure quela surface, les dissemblances frappent les yeux d'une classe à l'autre, d'une famille à la famille qui l'avoisine, d'un genre à un autre genre, et quelquefois même rlune fspècr à unc espòce. Ces dissemblances, or- 
données avec méthode et d'après certaines règles, forment les cadres de la zoologie; la zoologie n'est donc autre que les dissemblances extérieures des animaux systématisées.

Mais à mesure que l'anatomiste pénètre de l'extérieur à l'intérieur des animaux, à mesure qu'il s'avance vers les grands appareils organiques qui en occupent le centre, il voit se grouper les analogies, il voit toutes les parties rentrer les unes dans les autres. Il tombe cufin sur les analogies d'une manière aussi inévitable qu'il était d'abord tombé sur les différences, en ne considérant que l'extérieur.

Les ressemblances organiques sont donc assujéties à la même loi que ícur dúveloppement, elles procèdent de la circonférence au centre. Plus un système organique est extéricur, plus il cst différent, considéré dans l'ensemble des êtres; plus il est central, plus il est analogue. De là les variétés infinies que nous offre l'enveloppe extérieure des animaux ; ces varićtés sont déjà moins grandes dans les muscles sous-cutanés, moins sensibles encore dans les muscles profonds; les os sont plus analogues que les muscles; et les viscères centraux, les poumons, le cœur, le canal intestinal, etc., plus ressemblans encore que les muscles et les os.

Analogie d'organisation dans les régions profondes des animaux, dissemblance dans les régions superficielles, tel est donc le secret à l'aide duquel la nature a varié de tant de manières le spectacle que nous offrent les êtres organisés. 
Telle est aussi la source des deux grandes méthodes qui ont divisé et divisent encore les anatomistes.

Ceux qui n'ont considéré que l'anatomie extérieure des animaux, ont dû nécessairement puiser dans la nature le principe des diversités organiques.

Ceux, au contraire, qui n'ont considéré que les régions profondes, ont dû aussi inévitablement tomber sur le principe de leur identité.

Pour juger quel est et quel a dû être l'esprit de cet ouvrage, il n'est besoin que de réfléchir à la position de l'axe cérébro-spinal des vertébrés. Sa situation au centre des régions des animaux nous a commandé, pour ainsi dire, de nous maintenir constamment sur la ligne des analogies. En vain aurions-nous cherché dans la moelle épinière et le cerveau des mammifères l'empreinte des caractères anatomiques d'après lesquels la zoologie les a si bien classés. Quoique la classe des oiscaux, si fixe dans son organisation, ne représente en quelque sórte qu'une grande famille, les différences extérieures sont toutefois assez marquées pour y établir des coupes distinctes : or c'est encore en vain que nous en aurions cherché les caractères dans leur encéphale.

Les embryons reproduisent en petit ce que nous présentent sur un plan plus large les animaux parfaits. I.es formes primitives de la moelle épinière et de l'encéphale sont identiques dans toutes les classes, quoique par leur circonférence ces pe- 
tits êtres diffèrent infiniment les uns des autres. Ainsi l'embryon du reptile ne répète pas le poisson à l'époque où son encéphale est icthyomorphe. L'embryon de l'oiseau n'est ni poisson ni reptile, aux époques où son cerveau reproduit le type de celui de ces classes. Enfin un embryon de mammifère est toujours mammifère, quoique son axe cérébro-spinal revête fugitivement et successivement les formes encéphaliques des trois classes inférieures. Les différences sont à l'extérieur, et les ressemblances à l'intérieur.

Si c'était ici le lieu de développer ces grands principes de lanatomie comparative, je montrerais que c'est à l'extrémité des membres que se trouvent les plus grandes variations des os, des muscles, des artères et des nerfs des animaux; je ferais voir que dans chaque système en particulier les dissemblances vont toujours en diminuant de la circonférence au centre, et que c'est toujours au centre que les anatomistes retrourent leur point de ralliement. Je n'en citerai qu'un exemple. Parcourez les travaux des anatomistes illustres de nos jours sur la détermination des pièces osseuses du crâne des poissons : une idée générale domine dans la nature et dans ces recherches, c'est que les plus grandes dissemblances portent sur les pièces les plus extérieures, ct que les analogies se groupent à mesure que l'on se rapproche du cestre. Aussi n'est-ce que sur les pièces du centre que les Cuvier, les Geoffroy-Saint-Hilaire, les Oken, les Bo- 
janus, les Spix, les Rosenthal et les Backer offrent quelqu'accord; leur discordance est frappante sur les pièces de la circonférence, parce que c'est en effet à la circonférence que la nature a placé les différences les plus tranchées.

Rentrant maintenant dans le sujet spécial de cet ouvrage, cherchons à apprécier les différences de l'encéphale le plus compliqué des vertébrés , celui des mammifères; c'est toujours à la circonférence que nous rencontrerons les dissemblances dans la forme, l'étendue et les rapports des différens lobes, dans les rapports, l'étendue et la forme des circonvolutions quand elles existent; le corps calleux, la voûte, la protubérance annulaire et le corps trapézoïde, qui occupent le centre, ne varient que dans les proportions; ces dernières parties offrent toujours la même forme. la même position et les mêmes rapports.

Celte fixité des parties centrales tient donc à une loi primordiale; elle est d'autant plus remarquable, qu'en descendant l'échelle des développemens, ce sont précisément ces mêmes parties qui disparaissent dans les classes inférieures.

A quoi tient cette nouvelle source des dissemblances encéphaliques? Toujours à la même cause, à la loi du développement cxcentrique des animaux.

Si, en effet, l'encépbale se fût développé du centre a la circonférence, quelles eussent été les parties fondamentales de cet organe dans toutes les classes? Néressairement re serait le corps cal- 
leux, la voùte, la protubérance annulaire, le corps trapézoïde, et les commissures antéricures et postérieures: or, ce sont précisément celles qui disparaissent.

Le corps calleux manque d'abord chez les oistaux; la voûte disjointe existe encore; elle disparaît chez les reptiles, et ne se reproduit plus chez les poissons.

Le pont de varole a cessé d'exister chez les oiseaux, les reptiles et les poissons.

Les commissures des couches optiques persistent chez les oiseaux : l'antérieure disparaît chez les reptiles, et toutes les deux cessent de se montrer chez la plupart des poissons.

Si l'observation directe n'avait pas établi le développement excentrique des organes, ce grand fait d'encéphalotomie comparative eût suffi pour le démontrer; car l'encéphale se développant d'arrière en avant, on voit que la commissure postérieure doit d'abord se développer, puis la commissure antérieure et postérieure, puis la voûte, comme chez les oiseaux, puis enfin le pont devarole et le corps calleux chez les mamnifères.

Les divers développemens de l'encéphale des quatre classes correspondent donc aux développemens divers de l'embryon des classes supérieures. De cette vérité en dérive une autre très-importante, c'est que les animaux parfaits, considérés dans l'ensemble des quatre classes, reproduisent les mêmes rapports que nous avons signalés chez. 
los embryons. Cette double concordance devait être constatée avec rigueur, pour entreprendre avec quelque succès la détermination et l'explication du système nerveux des animaux invertébrés.

Car dans l'état présent de la science, on ne peut guère traiter les hautes questions de l'anatomie comparative, sans embrasser dans leur ensemble la généralité des êtres dont se compose le règne animal. Nos considérations seraient nécessairement imparfaites, et nous serions exposés à nous voir démentir chaque jour par la nature, si nous bornions nos explications à une famille, à une classe, ou même à l'une des grandes coupes qui ont été faites dans l'immeuse série des êtres organisés. Des mollusques à l'homme, l'anatomic comparative doit les expliquer tous; de même que la zoologie, qui n'est au fond qu'une sorte d'anatomie comparéc des surfaces, les a tous classés. Ces deux sciences doivent assigner à chaque être, à chaque groupe, sa valeur réelle dans le grand problème dont se compose le règne animal.

L'Académie Royale des Sciences n'ayant mis au concours que le système nerveux des animaux vertébrés, jai dû m’altacher principalement aux êtres qui cn composent les quatre classes, et puiser dans les notions qu'ils m'ont fournies, les bases de la détermination et des rapports de cet imporlant système. Mais cres rapporls et ces déterminations laisseraicnt encore quelque chose à désirer, si nous laissions en arrière le système nerveux des 
animaux invertébrés, et si nous ne montrious dans quel rapporl il se trouve avec celui des vertébrés.

On a ćchoué, jusqu'à ce jour, dans ce rapprochemcnt. La diversité des ètres dont se compose la grande série des invertébrés, en est, je pense, la causé. Au premier aperçu, tous les vertébrés. ont un air de famille, qui rappelle, mêne aux. yeux les moins cxercés, une organisation identique sous beaucoup de rapports. Chez les invertébrés, tout concourt au contraire i repousser cette identité : un mollusque ne rappelle en aucune manière un ver, un ver ne saurait donner à l'esprit l'idce d'un insecte, ni un insecte nous faire soup. conner l'organisation d'un crustacé. La nature semble ne marcher que par des dissemblances.

Mais, comme nous l'avons déjà dit, aux yeux de l'anatomiste, ces dissemblances ne sont qu'extérieures : à mesure qu'il pénètre dans l'intérieur de l'animal, à mesure qu'il s'avance de la circonférence au centre, il voit les dissemblances s'effacer graducllement, t les ressemblances organiques se dessiner de plus en plus; enfin, parvenu au centre de l'animal, il retrouve a tous une physionomie commune.

On voit, d'après ce principe, que, chez les articulés de:mème que chez les vertíbrés, le système nerreux', le plus central de tous les systènes organiques, doit offrir, plus que tout autre, des traits de resscinblance; il doit rapprocher et presque confondro drs ctres que, d'aprèsleur extéricur, la 
zoologie tient avec juste raison à des distances considérables les uns des autres.

Des vers aux insectes $t$ t aux crustacés, les dissemblances extérieures sont si nombreuses, si variées, si discordantes quelquefois, qu'il a fallu toute la sévérité des principes zoologiques de nos jours, pour échelonner ces êtres avec ordre et dans une méthode régulière. Or, voyez leur système nerveux, vous le trouvez construit sur le même plan : c'est chez tous un cordon longitudinal, renflé de distance en distance, s'ouvrant pour donner passage à l'oesophage, et formant constamment en avanl et en arrière de cette entréc du canal nutritif, un double groupe de ganglions.

Au premier aperçu, les mollusques s'éloignent beaucoup de cetle homogénéilé du système nerveux: le cordon nerveux central disparaît, ou plutôt se disjoint sur une partie de sa longueur; il ne reste plus sur le centre que les ganglions pro et méta-œsophagiens, encore ccux-ci sont-ils quelquefois désassemblés. Cette particularité d'organisation a fait dire que ces animaux étaient privés de moelle épinière, dans le sens où on l'accordait aux vers, aux insectes." et aux crustacés. Souvent mème, comne dans? l'aplysie et le bulla aperta, on n'a pu dislinguer les ganglions cérébraux proprement dits; d'autres fois enfin, le système nerveux s'est contourné en cercls: comme dans l'astérie (asteria auriantiaca) et daus la mulette des peintres; dans ces diverses mélamor. 
phoses on n'a plus reconnu l'analogue du cordon central des invertébrés supérieurs; lominé toujours par l'idée que le système nerveux se développe du centre à la circonférence, on n'a pu ni concevoir, ni expliquer, ni ramener à son véritable type un système nerveux disjoint dans sa partie moyenne, et interrompu dans l'axe qui était censé lui donner naissance.

On a procédé chez les invertébrés de la même manière que chez les vertébrés : on est parti du syslème nerveux tout formé dies ètres les plus élevés de celte grande série, et on est descendu de proche en proche vers ceux qui occupent le bas de l'échelle; on a voulu expliquer le simple par le composé : on a échoué de mênse que chez les aninaux vertébrés, et comme on le voit, toujours par les mèmes raisons, louj̣ours par les mêmes causes.

Or, nous avons déjà démontré que chez les animaux vertébrés, les classes supérieures étaient expliquées par les inférieures; nous avons démontré que l's embryons des vertébrćs supérieurs trasersaicnt successivement les phases rlu système nericux des inférieurs : nous avons vu enfin quc ioutes les dissemblances nerveuses ćlaient produite's chez ces animaux, par quelques métamorphoses de plus ou de moins.

En serait-il de méme de la grande série des animaux invertébrés? Les inférieurs scraient-ils ves embryons permanens des supéricurs? Le syslime nerveux d'en haut pourriti-il itre ramené 
à celuid'en bas, et celui d'en bas pourrail-il nous offrir les premiers traits de celui d'en haut? On sent toute l'importance et toute la difficulté de ces questions, dans l'étal présent de la science. Jo vais ncianmoins en tenter la solution.

J'ai déjà dit, dans la Deuxiène Parlie de cet ouvrage, et dans les lois du systeme'nerreux, que, chez les jeunes larves des insccies; le système nerveux procédait dans sa marche de la circonféreuce au centre ; que primitivement il existait deux cordons au centre de la larve, desquels provenait le cordon unique qui, plus tard, se manifestait dans la série des métamorphoses de ces êtres. Cette fusion des deux cordons en un seul ne se fait pas brusquement dans toute la longueur, il commence toujours par les ganglions, qui sont immédiatement en avant et en arrière de l'œesophage: ces ganglions, en marchant à la rencontre les uns des autres, se touchent d'ahord; puis ils s'unissent en conservant sur leur milieu un raphé peu profond; enfin, ce raphé disparaît souvent, el-les deux ganglions paraissent alors n'en faire plus qu'un seul.

Après ces ganglions antérieurs, les inférieurs, ou ceux qui termincut la prétendue moclle épinière, sont ceux qui s'adossent et se confondent de la même manière que les précédens; puis viennent successivement les ganglions interposćs entre la tête cl la queue de la larve. En mêmo: tcups que les deux ganglions sr rounissent en ü 
seul, situé alors sur l'axe central de l'animal, les cordons intermédiaires qui les unissent de haut en bas suivent la même marche; ils s'adossent, adhèrent d'abord ensemble, et puis se confondent si intimement, qu'il n'y en a réell ement plus qu'un. Sont constamment exceptés de ce mécanisme les deux cordons qui, à droite et à gauche, sont situés sur les côtés de l'osophage. L'œesophage s'interposant, en cet endroit, entre les deux cordons nerveux, les maintient écartés, et donne lieu, de cette manière, à l'anneau osophagien signalé par tous les anatomistes. L'anneau œesophagien n'est donc, de cette manière, qu'un trou de conjugaison formé par l'adossement en avant et en arrière des deux cordons primitifs de l'axe nerveux des invertébrés ( 1 ).

Il est rare que les traces de ce double développement soient effacées dans toute la longueur du cordon; indépendamment de l'anneau œesophagien, il arrive souvent qu'un ou plusieurs groupes de ganglions en conservent les traces; il arrive plus souvent encore que les filets intermédiaires sont désunis d'un ganglion à unautre, ainsi que nous allons bientôt le faire remarquer.

Auparavant, nous devons observer que parmi

(1) Le unécanisme de sa formation est absolument le même que celui qui donne naissance aux ouvertures du diaphragme et aux trous dont est si fréquemment perforé le système osseux. 


\section{DANS LE RLEGE ANIMAL.}

les vers, l'ascaride lombricoide de l'homme, de mème que celui du cheval, conservent pendant toute leur existence l'état primitit et embryonnaire des larves des insectes. Chez eux, on trouve constamment les deux cordons désunis dans toute la longueur de l'animal. Il y a un cordon à droite et un cordon à gauche.

Chez l'écrevisse de rivière, la moitié du cordon nerveux est désuni daus sa partic antérieure; les ganglions, quoiqu'adossés les uns aux autres, conservent évidemment les traces de leur jonction primitive. Il en est de même de la nymphe du ver à soie. Les filets inter-ganglionnaires sont doubles dans presque toute leur longueur, et les ganglions eux-mêmes paraissent résulter de la réunion des deux ganglions primilifs. La chenille épineuse de l'ortie, la nymphe de l'abeille, et l'abcille elle-même, retracent, en totalití ou cn partie, ce mode primilif de développement. Dc là résultent les deux grandes variétés que présente, chez les vers, les insectes et les crustacés, l'ensemble de leurs ganglions; tantôt, en effet, los ganglions paraissent uniques, c'est le cas oú la fusion des deux ganglions a été complète; tantôt, au contraire, les ganglions ne sont pas entièrement confondus, un raphé median les divise on deux parties, l'unc droite, l'aulre garche; quelquefois ce raphé est trés-profond, conmme chez l'aphrodite herissée; lo ganglion paraît échancré ct prend la forme d'un cocur. Dans ce cas, la fusion des II. 
deux ganglions est imparfaite, et conserve des traces plus marquées de sa double origine. En général les formes variées de l'axe nerveux de ces invertébrés dépendent du plus ou moins de rapprochement de ses deux cordons élémentaires.

Cela posé, descendons à l'explication du système nerveux des mollusques. Si nous voulions trouver cette explication dans l'insecte parfait, nous serions arrêtés par des difficultés insurmontables; mais si, de même que chez les vertébrés, nous cherchons à expliquer le système nerveux des invertébrés inférieurs, par celui des embryons des invertébrés supérieurs, une partie de ces difficultés disparaîtront, et nous serons sur une route plus rapprochée des analogies. En effet, lá plus grande, je dirai même l'uniquuc difficulté, qui se présente dans ce rapprochement, c'est de ramener le cordon unique et serré des invertébrés supérieurs, au double cordon, désuni en grande partic, de la plupart des mollusques. Mais si, cessant de mettre en rapport les nollusques avec les insectes parfaits, vous les comparez à l'état primitif de leurs larves, vous voyez, chez les uns et chez les autres, un cordon à droite et un cordon à gauche, ou un système nerveux pour chaquc côté de l'animal. Chez les mollusques ce double système sera tantôt réuni en haut seulement, autour de l'osophage, de même que chez les insectes et les crustacés ; tantôt il sera réuni en haut et en bas, comme cela arrive dans le premier développe- 
ment de la larve, et chez l'ascariáe lombricü̈de du cheval et de l'hommi.

Mais la désunion plus ou moins compléle des deux systèmes nerveux primitifs est le caractère constant du système nerveux des mollusques; un autre caractère moins général sc lire de l'absence des ganglions sur les cordons, à partir de l'oesophage jusqu'à l'anus. Justifiors ces caractères par des exemples.

Si vous considérez le système nerveux bien peu développé de l'hyale, vous trouvez qu'il consiste en quatre ganglions méta-œsophagiens, séparés et écartés les uns des autres. L'anneau osophagien est complété en devant par un cordon transversal. Chez l'aplysie (aplysia fasciata), les ganglions pro-œesophagiens sont réunis; mais les méta-œsophagiens sont écartés l'un de l'autre; un cordon transversal, qui du ganglion droit se porte au gauche, complète l'anneau oesophagien. Des ganglions méta-oesophagiens partent des faisceaux nerveux qui descendent le long de l'animal, sans préseuter de ganglions, et sans communiquer entre eux à droite et à gauche. Le systèmc nerveux de la dolabelle répète la même disposition.

Chez le Bulla aperta, on trouve sur les côtés de l'oesophage dcux ganglions uniques, très-écartés l'un de l'autre. De la partic supéricure de chaque ganglion s'élève un cordon qui, se réunissant ì son congénère, forme l'arc nesophagicu antérieur; de même; en bas, l'arc aesophagieu postérieur 
est furmes par deux cordons semblables; enfin, de la partie inférieure du ganglion partent les cordons primitifs, qui, très-éloignés l'un de l'autre, parcourent les flancs de l'animal sans communiquer entre eux. Le pleuro-branche offre une disposition analogue, excepté que chez ce dernier, les deux ganglions sont adossés par leur partie interne.

La Tritonic ( Tritonia hombergii) est plus simple encore. Le système nerveux de droite est tout-à-fait distinct de celui de gauche: des deux ganglions prooesophagiens qui se remarquent des deux côtés, les antérieurs sculs se touchent légèrement par la partie convexe; du reste, tout le système nerveux est désuni.

Les Doris, dont le systeme nerveux paraît plus compliqué, sont exactement la répétition de celui de la tritonie, avec cette différence, que chez certaines espèces les ganglions pro-cesophagiens ne sont pas très-distincts; chcz ceux où ils existent, comme chez le doris solea, ces ganglions sont adossés de la même manièrc que chez la tritonie.

Ces différens systèmes nerveux représentent donc pius ou moins l'état primitif de celui des larves des insectes; mais une analogie plus frappante cncore nous esi offerte par la clie boréale. parmi les gastéropodes, ei par la mulette des peintres (unio pictorum).

Le système nerveux de la clie boréale est com- 
posé de deux cordous longitudinaux présentant chacun cinq renflemens ganglionnaires de chaque côté. De ces ganglious, les antérieurs sont seuls rapprochés l'un de l'autre; les deux suivans communiquent entre eux par deux filets transverses, qui forment un double anneau autour de l'osophage; les deux inférieurs sont tout-à-fait disjoints, écartés et entièrement isolés. Cet état représente exactement celui de la larve à l'époque où les ganglions pro et méta-ocsophagiens, encore écartés l'un de l'autre, ne communiquent entre eux que par des filets transverses, qui disparaissent à mesure que les ganglions marchent à la rencontre les uns des autres. $\mathrm{Si}$, en effet, vous prenez une larve à cette époque, et si vous écartez sa partie moyenne, vous voyez les ganglions antérieurs communiquant entre eux, comme ceux de la clie boréale; tandis que les ganglions inférieurs sont, de même que chez ce dernier animal, tout-à-fait écartés; vous faites d'unc larve une clie boréale, et il faudrait peu de chose pour faire de cette dernière une nymphe dinsecte. Il suffirait pour cela d'imiter artificiellement le procédé de la nature; de rapprocher les ganglions écartés, de les faire tous rémir sur la ligne noćdiane; vous auriez alors, comme chez toutes les nymphes, un cordon central unique, rentré dr distance ca distance, et ouvert sculement pon' fonner passage it l'oesophage.

Cela arrive en parlie chez la mulede des peinurs: 
ici les ganglions pro-øesophagiens, qui étaient réunis chez la clie boréale, sont très-écartés chez la mulette; les méta-œesophagiens écartés, au contraire, chez la clie, sont adossés chezla mulette; mais, chez cette dernière, les cordons qui longent le corps de l'animal sont renflés à leurs extrémités : ces deux ganglions inférieurs sont adossés l'un à l'autre, comme cela arrive chez les larves des insectes, à la deuxième période de leur formation.

La clie boréale correspond ainsi à l'époque de la larve où les ganglions oesophagiens sont écartés les uns des autres et joints ensemble par les filets transverses, et la mulette à l'époque plus avancée à laquelle les ganglions oesophagiens et les ganglions de l'anus sont encore les seuls adossés; tandis que, dans le reste de leur étendue, les cordons sont encore disjoints.

Le système nerveux de la mulette des peintres représente un long anneau réuni en avant et en arrière par l'adossement des ganglions ‘qui le terminent; celui d'une espèce d'astérie (asteria aurantiaca) répèłe jusqu'à un certain point cette disposition, avec la seule différence que tous les ganglions sont écartés, que l'anneau est plus ouvert, et qu'il représente un véritable hexagône, dont les six angles sont occupés par un ganglion, et dont les côtés sont formés par les filets de réuriọ. Rapprochez ces ganglions qu'un alegré dc développement inférieur tient écartés; et chez 
l'astérie, de même que chez la clie boreale, vous reproduirez le système nerveux des nymphes et des insectes ( 1 ).

On a placé le système nerveux des mollusques céphalopodes bien au-dessus de celui des insectes et des crustacés; tout paraît se réunir au contraire pour le mettre plus bas. Il se rapproche tellement de celui de la tritonie et des doris, qu'on est surpris de voir que cette analogie n'ait pas frappé les zootomistes. Si nous prenons la sèche pour exemple, nous voyons les ganglions céphaliques réunis de même que chez les doris; il part ensuite de la partie inférieure et externe de chaque ganglion un filet nerveux considérable qui diverge en dehors de devant en arrière, et qui se termine par un ganglion volumineux, duquel rayonnent les nerfs en tous les sens, excepté par le côté interne du ganglion. Il résulte de cette disposition divergente des cordons, que ces ganglions sont très-écartés inférieurement.

Rapprochez ces ganglions ainsi écartés, ramenez-les au point de contact, et vous aurez le système nerveux de la mulette des peintres; ou mieux encore, vous formerez celui de certains

(1) Chez cette astérie, de même que chez la clie boréale. tnut le système nerveux est formé de matière blanche, les ganglions eux-mêmes sont d'un blanc laiteux comme les filets. Ces faits sont à ajouter à tous ceux que j'ai déjà donnés sur la formation primitive de la malière blanche avant la matière grise. 
mollusques gastéropodes, tels que l'hélice vigneronne.

Prenant pour terme de comparaison les larves des insectes, et suivant la marche excentrique de leur système nerveux, on voit que les deux cordons primitifs convergent l'un vers l'autre, et tendent à s'unir sur la ligne médiane, excepté à la partic qui correspond à l'oesophage, leur jonction étant arrêtée par cet organe. En descendant ensuite vers les mollusques, on trouve que la marche des cordons est plus ou moins interrompue, que conséquemment leur systène nerveux est plus isolé ou plus disjoint que chez les nymphes et les insectes parfaits. D'où il résulte que les mollusques sont, quant à leur système nerveux, des larves, ou des embryons, plus ou moins avancés, des insectes.

Cet écartement des cordons chez certains invertébrés, et leur réunion chez d'autres, tient à la même loi et à la même cause que ceux de l'axe cérébro-spinal des vertébrés. N'avons-nous pas vu, chez ces derniers, que chez les oiseaux, les cordons postérieurs de la moelle épinière ne se réunissent pas dansleur renflement inféricur? N'avonsnous pas vu que les lames latérales du cerveau, si étroitement confondues chez la plus grande partic des vertébrés, étaient disjointes et déjctées sur les côtés du quatrieme ventricule, sur li plupar sles poissons cartilagineux? N'avons-nous pas ru, cnfin, que Ins hemisplowes cóbraux, si intime- 
ment unis chez les mammifères, étaient tout-àfait disjoints et isolés chez tous les poissons osseux? La nature suit donc une marche invariable dans ses développemens; elle réunit et confond à son gré, ou elle tient à distance les élémens primitifs du système nerveux; et, chose qui ne saurait trop être admirée, c'est que des causes si simples produisent toutes ces variétés de formes que nous présente ce systèmc dans tout le règne animal.

Parmi les métanorphoses singulières que nous ont offertes les embryons des vertébrés, une de colles qui ont le plus frappé les anatomistes est l'ascension de la moelle épinière dans son étui. Je fus très-frappé de ce phénomène la première fois que je l'observai; mon étonnement cessa quand j'eus remarqué ce qui se passe dans le systène nerveux des insectes pendant la métamorphose. Jc vis se manifester en grand, chez les insectes, un phénomène, qui ne se montre qu'en petil, et pour ainsi dire, qu'en miniature, chez certains vertébrés.

Le speclacle de la métanorphose des insectes a dans tous les temps frappé les philosophes et les naturalistes: les preniers y puisérent les idées de la métempsycose, relatives à la perfectibilité des êtres organisés; les seconds, plus philosophes sans doute, soccupèrent à étudier le mécanismo:

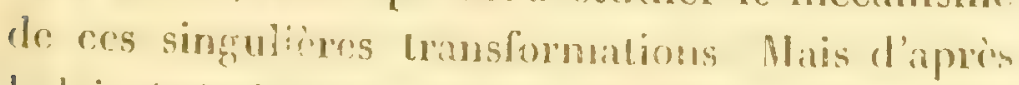

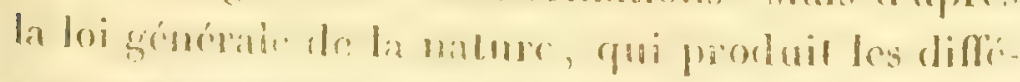

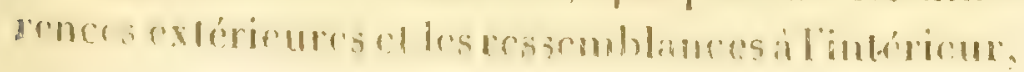


on voit de suite que les métamorphoses des organes doivent être d'autant plus générales et d'autant plus sensibles, qu'elles s'opèrent sur les organes les plus extérieurs : la métamorphose marche de la circonférence au centre; plus les organes sont extérieurs, plus ils sont métamorphosés; plus ils sont intérieurs, plus ils restent stationnaires. Cela est, et cela devait être, par des raisons puisées dars l'organisation même, mais qu'il serait trop long et peut-être hors de propos de développer ici. Je ne rappelle cette loi que pour expliquer pourquoi les entomologistes qui ont suivi avec tant de persévérance et avec un si rare bonheur tousles déguisemens des larves et des nymphes, n'ont presque rien dit des changemens que subit le système nerveux, le plus central de tous les organes.

Un des effets les, plus frappans de la métamorphose, celui qui tombe sous les yeux des observateurs les moins exercés, est la longueur du ver ou de la chenille, comparće à la brièveté de l'insecte qui en provient. L'axe nerveux du ver et de la chenille s'étendant sur toute sa longueur, que se passe-t-il pendant cette métamorphose? Le système nerveux se raccourcit-il à mesure que l'insecle approche de son état parfait, ou bien disparaît-il en partie? Le système nerveux de la chenille étant le même qui doit servir à l'insecte, on voit qu'il doit nécessairement se produire l'un on l'autre de ces effets. Quel est celui des deux qui se: produit? c'est ce qu'on va voir. 
Si on observe une jeure larve à l'époque où les deux cordons se sont adossés ou réunis sur la ligne, médiane, on trouve que les ganglions sont rapprochés les uns des autres; mais à mesure que la chenille s'étend en longueur, à mesure aussi l'axe nerveux s'étend. Cette extension est due à l'étendue que prennent les filets inter-ganglionnaires. Cet accroissement en longueur étant arrèté, et la chenille observée au moment où va commencer la métamorphose, la longueur totale de l'axe nerveux est portée à son maximum.

Représentons cette longueur par le nombre cent, et suivons la nymphe.daus ses périodes de transformation. A la première période on observe un raccourcissement de l'axc nerveux, qui peut être évalué à cinq; la longueur totale n'est plus que de quatre-vingt-quinze; à la deuxième période, survient un nouveau raccourcissement qui, à raison de son étendue constante, peut être évalué à vingt; la longueur lotale est alors représentée par soixante-quinze; à la troisième, se manifeste un troisième raccourcissement, plus considérable encore, et qui égale vingt-cinq; l'axe nerveux est réduit à cinquante, juste la moitié de sa longueur à l'époque où a commencé la métamorphose. Cies dimensions sont l'expression exacte de ce que j'ai observé sur la cheville épineuse.

C.r raccourcissement considérable n'a pu s'opirer que par des changemens survenus sur toute. la longueur de l'axe nurveux. Ces changemens 
doivent être observés dans les cordons in ter-ganglionnaires, et dans la position des ganglions eux-mêmes.

En ce qui concerne les cordons, on remarque que leur abréviation n'est d'abord qu'apparente, car ces cordons étaient chez la chenille tout-àfait droits et tendus. A la première période ils se fléchissent légèrement et forment un petit arc du ganglion au ganglion voisin; à la seconde période la flexion est plus prononcée; à la troisième, ils sont tout-à-fait flexucux. Cependant cette flexion ne représente pas la longueur perdue; car, en étendant le cordon de la troisième période, on ne lui rend jamais sa longueur primitive. Constamment, pendant cette période, l'anneau œesophagien s'ouvre de plus en plus; à mesure que les angles de la boutonnière qu'il représente se rapprochent, les côtés s'écartent nécessairement l'un de l'autre.

Enfin la nymphe coutinuant son déreloppement, la longueur totale du cordon reste stationnaire; mais les cordons, qui étaient flexueux d'un ganglion à l'autre, deviennent tout-à-fait droits et tendus, comme ils étaient chez la chenille, de manière qu'ils ont réellement perdu la moitié de leur longueur dans le cours de la métamorphose.

L'axe nerveux a éprouvé pendian celie concentration des changemens remurquables; si la diminution de la longueur des cordons arait ete 
égale sur tous, la disposition générale serait restée la mème, l'axe nerveux de l'insecte serait en raccourci le même que celui de la chenille ou du ver; mais il n'en est pas ainsi, la concentration est très-différente dans les cordons intcrnes ganglionnaires. Chez les uns elle cst si forte, que les cordons disparaissent tout-à-fait; il en résulte que des ganglions, qui chez la chenille ou chez le ver étaient à une longue distance l'un de l'autre, se trouvent amenés chez l'insecte parfait au point de contact, et souvent même sont confondus les uns avec les autres. Dans d'autres, la concentration étant plus faible, les ganglions que ces cordons séparent se trouvent simplenıent plus rapprỏchés chez l'insecte quc chez la larve. De la diversité de concentration des cordons résulte donc la diversité de posilion relative que pré-sentent les ganglious de l'axe nerveux des animaux articulés.

En général, les cordons qui se contractent le, plus sont ceux qui, chez la larve, séparent les uns des autres les ganglions pro-oesophagiens et méta-oesophagiens. Chez les larves, ces ganglions sont maintenus très-écartés les uns des autres, à cause de la longueur des cordons qui les unissent. Chez l'insccte parfait, les corlons avant disparu presque complètement, ces ganglions se trouvent groupés antour de l'ouverture oesophagienne.

Chez la chenille du chou ct chez la chenilio épineuse, par exemple, les deux ganglions pro-oeso pha- 
30 CONCORDANCE DE SYSTEME NERVEUX

giens, de chaque côté, sont à une distauce de cinq millimètres l'un de l'autre; les quatre ganglions méta-œesophagiens sont séparés par une distance à-peu-près semblable. A mesure que la nymphe se métamorphose, cette distance diminue : elle est d'abord de quatre millimètres, puis de trois, puis de deux, et enfin chez l'insecte parfait ces ganglions sont tombés les uns sur les autres; ils sont réunis et même tellement confondus, qu'il n'y a plus qu'un ganglion en avant de l'osophage et un autre en arrière. On ne croirait jamais que chacun de ces ganglions provient de la fusion des quatre ganglions de la larve, si l'on n'avait suivi leur marche pas à pas.

A mesure que ces ganglions tombent les uns sur les autres, l'anneau osophagien éprouve des modifications proportionnées au degré de leur - rapprochemcnt. D'abord l'anneau est très-long, puis il s'élargit beaucoup, comme une boutonnière dont on rapprocherait les deux angles; puis les cordons se raccourcissant, il se rétrécit de nouveau, et souvent, comme chez le papillon provenant de la chenille du chou, ce n'est plus qu'un simple trou très-étroit.

Les cordons qui unissent les aritres ganglions éprouvent une concentration proportionnelle, de telle sorte qu'ils sont maintenus chez la chenille, la nymphe et l'insecte parfait, à une distance relalive au raccourcissement total de l'axe nerveux.

Mais chez plusieurs insectes, quelque chose 
de plus remarquable encore s'opère dans un ou dansdeux des ganglions qui suivent immédiatement les méta-œsophagiens. Chez les chenilles, ces ganglions ont le mêne volume que ceux du reste de l'animal; comme chez ces dorniers il part quatre filets nerveux de sa surface externe. Dans la première métamorphose de la nymphe, on voit encore ces ganglions dans le même état, ainsi que les filets nerveux qui s'y rendent. A la seconde période de la métamorphose, les nerfs disparaissent : les ganglions existent encore, leur volume est un peu diminué; c'est un simple renflement du cordon central, il ne s'y rend ou il n'en part aucun des filets qu'on y remarquait précédemment. Enfin, à la troisième et dernière période dela nymphe, ces ganglions disparaissent complètement; le cordon nerveux sur lequel ils formaient un renilement, devient uni comme les cordons inter-ganglionnaires. C'est sans doute une chose fort singulière que de voir ainsi disparaître une partie du système nerveux de la chenille et de la nymphe dans le cours des métamorphoses de cette dernière. Chez la chenille du chou, quatre des ganglions qui suivent les méta-œesophagiens disparaissent, deux de chaque côté; chez la chenille épineuse, il n'y en a qu'un à droite et un à gauche. Ce fait me parut trop important pour la névrogénie en général, pour ne pas chercher à apprécier avec soiu dans quel ordre, dans quelle succession s'opérait cet anéantissement d'un système nerveux déjà tout 
formé. On conçoit, en effet, que la disparition du système nerveux devait suivre la mème marche que son apparition. Si le système nerveux se développait du centre à la circonférence, le centre devait d'abord disparaître; puis, de proche en proche, la circonférence devait cesser d'exister. Si les ganglions sont la matrice des nerfs, le ganglion devait précéder la disparition des filets nerveux; si, au contraire, les lois de la névrogénie que j'ai exposécs, sont exactes, l'inverse devait avoir lieu, la perte de ce système nerveux devait s'opérer de la circonférence au centre; ses irradiations excentriques devaicnt d'abord cesser d'ètre, puis l'atrophie et l'anéantissemeat devaient gagner graduellement l'insertion des nerfs aux ganglions, puis enfin ces ganglions cux-mêmes. Or, c'est exactement ce qui arrive. Les nerfs disparaissent d'abord à la périphérie dans le commencement de la métamorphose; puis à la fin de la première période on n'en trouve plus que des lambeaux flottans. Au commencement de la seconde, les nerfs excentriques ont tout-à-fait disparu, le ganglion qui occupe le centre de l'axe reste encore seul isolé, ne recevant plus de nerf. Enfin il disparaît lui-mème le dernicr, lorsqu'il n'y a plus de vestige des radiatious nerveuses qui venaient s'y implanter.

Que de questions sont renfermées dans l'explication de ce fait! Où se rendaient ces nerfs? à quels organes était destiné cet appareil nervcux chez la 
chenille et chez la nymphe, dans le premicr temps de sa métamorphose? Jo crois mètre assurć sur plusiours nymphes, quil se distribuail daus lorgane destiué a la sécrétion du lissu i l'aile duquel la nymphe fabri fue sa prisun moncutané. Celte cnveloppe lemince, l'organe devient inutile, il a rempli son but; c'est un appareil désormais inulil", il disparnît, et avec lui l'appareil nerveux qui lui élail destiné. Si cela est, on voit que cé phénomène est analngue à cenx qui se passent sur certaines parlics los mammiferes; sur le thynus, qui, d'abord tris-volumineux chrz l'enoloryon, est réluit à ricu chez l'animal adulle; sur l's curps sur-rénaux qui, si imporlans et si diviloppis chez les jeuncs foctus, devienuent iontà-fitit inutiles apres la naissance. Enfin il correspourl au fait si remarquabl.', el si pu remarqué, parce quil se passe journcllement suus nos yeux, de la disparili.n des premicres dents des mammifères. lci, comme chez les ny:nphes, ın apparcil disparaît tout entier, et en mène temps ses artères, ses veines, ses nurfs cesscut d'existur. La nature se répète parlout. Une circouslance que notis ne devons pas omettre, c'est que la meme loi priside au déseloppoment el à l'anéantisscment du systeme nerveux, il se forme et se détruit de la circonférence au centre.

Telle est, en général, la narche dusystèmenerveux dins la inctamorphose des insectes; mais la sonuthe asile offre sous ce rapport une exception II. 
bien remarquable et bien digne de l'attention des anatomistes et des entomologistes.

Chez le ver de cette mouche, les ganglions, au nombre de onze, sont primitivement adossés les uns aux autres; ils sont ramenés au point de contact, et sanscordons intermédiaires qui les séparent, comme chez les autres vers. De plus, au lieu d'être étendus perpendiculairement les uns au-dessous des autres, comme dans les larves en général, ils sont roulés sur eux-mêmes de manière à former un anncau ganglionnaire, dont les deux extrémités sont cependant interrompues.

On conçoit bien, d'après cette disposition primitive, que la métamorphose ne peut s'opérer comme dans les larves ordinaires. Il ne saurait y avoir ni concentration, ni raccourcissement d'un système nerveux qui est tout ramassé sur luimême, et qui occtape le plus petit espace possible. Les cordons ne sauraient se raccourcir, puisqu'il n'y en a pas. Que se passe-t-il donc dans la métamorphose de ce ver? L'inverse, justement l'inverse de ce qui se remarque dans la métamorphose ordinaire. Ordinairement, comme nous venons dc le voir, les ganglions, éloignés chez la larve, se rapprochent chez la nymphc, les cordons de séparation se raccourcissent, disparaissent mème quelquefois entièrement, ce qui fait que plusieurs ganglions, primitivement séparés, tombent les uns sur les autres. L'effet ordinaire de la métamorphose est donc de rapprocher les ganglions 
qui composent l'axe nerveux des articulés; mais ici les ganglions sont tous agglomérés les uns contre les autres, leur rapprochement est donc impossible. Le ver de l'asile représente l'insecte. parfait sous ce rapport; aussi l'effct de la métamorphose chez ce ver est-il de le ramener à l'état de la larve des insectes ordinaires.

En effet, si l'on suit la mélamorphose du ver de l'asile, on observe d'abord que, de circulaire qu'il était, son système nerveux central devient droit et se replace longitudinalement sur l'axe de l'animal, comme cela a lieu chez les autres nymphes. Mais ce redressement de l'anneau n'eût point produit la longueur bécessaire pour s'étendre tout le long de la nymphe, si les ganglions étaient restés adossés les uns contre les autres; il a fallu que les ganglions s'écartâssent, et, du moment qu'ils se sont écartés, il a dû se produire des cordons qui d'un ganglion se portâssent au ganğlion voisin: c'est exactement ce qui a lieu. A mesure que l'anneau circulaire du ver de l'asile se redresse, on voit se disjoindre les ganglions, et lorsquils cessent de se correspondre, on observe cntre cux un ou deux petits filets intermédiaires. Ces filets s'allongent a mesure que la nymphe se développe: plus la nymphe grandit, plus les ganglions s'écarlent, plus les cordons intermédiaires s'allongent. Au lieu d'une concentration des cordons inter-ganglionnaires, il y a donc ici une veritable cxtensina: la mélamorphose du système nerveux 
s'opère tout-d-fait en sens inverse de la marche commune. Ce phénomène est sans doute trèsremarquable; et ce qui contribue encore à le rendre plus singulier, c'est que la longueur totale du ver de l'asile est pour le moins le double de celle de la nymphe et de la mouche qui en proviennent; d'où il résulte que, lorsque l'auimal est le plus long, son système nerveux central est le plus court; et lors. que l'animal est le plus court, son système nerveux cst le plus long. Une opposition si manifeste aux règles ordinaires a ses raisons dans l'organisation de la larve de cette mouche; mais je serais trop écarté du but de cet ouvrage, si je m'arrêtais « les développer.

Voilà donc deux effets très-opposés dans la métamorphose : dans l'un, le système nerveux se raccourcit; dans l'autre, il s'allonge. Quelle en est la cause? c'est une question bien digne de l'attention des anatomistes. On sait que chez les vertébrés, le. système nerveux n'est point élastique; on sait que, Jorsqu'on coupe un serf sur un animal vivant, les deux bouts restent en contact et ne s'écartent point. Je nc connais d'exception a ce principe que le fait que nous a lait connaître M. Desmoulins, en ce qui concerne la moelle épinière de la lamproie: mais, d'un autre côté, on sait aussi que le système nerveux est très-élastique chez les animaux articulés. Chez un ver, une chenille et un insecte, on peut doubler la longueur d'un filet nerveux en le tirant "n sens opposé; et si on cesse la trac- 
tion, le filet reprend sa longueur primitive. Peut-on expliquer par l'élasticité le raccourcissement et l'allongement qui se produisent dans le cours de la métamorphose? Je ne le pense pas; et voici les faits sur lesquels je fonde mon opinion.

Dans le passage de la première à la dcuxième période de la métamorphose de la nymphe, le système nerveux central se raccourcit de la moitié ; mais nous avons remarqué que céte abréviation de l'axe nerveux avait lieu, parce que les cordons, de droits qu'ils étaient, devenaient flexueux : or, si l'élasticité était l'unique cause de ce phénomène, cette flexion ne devrait point aroir lieu, les cordons devraient toujours rester tendus, comme ils le sont chez la chenille, et comme ils le deviennent de nouveau dans la do ruière période de la métamorphose. L'extensibilité ne saurait expliquer non plus l'allongement des filets nerreux de la nymphe de l'asile. Si on cherche à redresser l'anneau ganglionnaire de sou ver, on peut lui donner un tiers de plus quesa longueur primilive; et si on cesse la traction, il reprend a’ssitôt sa longueur et sa forme circulaire. Dans cette expérience les ganglions paraissents'allonger: nn naperçoit pas de filet d'un ganglion à l'autre. Chez la nymphe, au moment où l'anneau se redresse, les filets sont toujours droits et tendus; ils ne sont point roules, ni autour, ni en avant. ni en arriere de chaque ganglion. leur allonge. 
ment est une aclion vitale qui se produit dans le cours du développement. Je ne connais rien chez les vertébrés qui se puisse rapprocher de ce qui se passe chez la nymphe de l'asile; mais, quant à la contraclion du système nerveux dans la métamorphose ordinaire, nous en avons vu plusieurs exemples chez les mammiféres, les reptiles ct les oiseaux.

Chez les oiscaux, nous avons remarqué que dans les premiers temps del'incubation, l'axe longitudinal de l'encéphale était beaucoup plus prononcé que le transversal; nous avons observé plus tard que, dans la marche de l'incubation, l'encéphale se concentrait; que le cervelet et les hémisphères cérébraux, si éloignés primilivement, étaient ramenés au point de contact, et que pendant ce temps les lobes optiques éprouvaient une demi-rotalion qui, de la face supérieure de l'encéphale, les ramenait vers sa base.

Nous arons vu que, chez certains mammifères, la concentralion de la moelle épinière produisait l'ascension de sa tclminaison, effet surtout remarquable dans la métamorphose du tétard des batraciens. Peut-être est-ce une cause semblable qui fait remonter la moelle épinière de la lamproic et de l'esturgeon. L'examen des embryons de ces poissons pourrait seul éclairer cette conjecture.

Le système nerreux du scarabée monocéros offre dans ses transformations quelque chose d'a. 
valogue à celuide l'asile. L'axé nerveux esl composé chez cet insecte de quatorze pelits ganglions tous réunis les uns aux autres, et en ligne droite sur l'axe du corps. Chaque ganglion paraît destiné aux anneaux dont se compose le ver, au nombre de quatorze également. Mais, au lieu de s'étendre sur toute la longueur de l'animal, ils ne ront guères au-delà du quatrième ou du cinquième anneau; et lęs nerfs inférieurs qui en partent, sont d'une longueur considérable pour pouvoir rejoindre les anneaux inférieurs et leurs dépendances. C'est une espèce de queue de cheval, mais beaucoup plus divergente que celle de la lamproie, de l'esturgeon, de la grenouille, des chauve-souris sans queue et de l'homme. Cet axe nerveux est le mêne que celui du ver de l'asile, excepté qu'il est droit au lieu' d'être en forme d'anneau. Dans la nymphe du scarabée monocéros, les ganglions s'écartent commedanscedcrnier. Chaqueganglion. vient se placer vis-à-vis de la division annulaire à laquelle il correspond; ce mécanisme s'effectue de la même manière par le développement de filets inter-ganglionnaircs quin'existaient pas chezlever. Cette métaimorphose change tout-à-fait la disposi. tion du système nerveux chez la nymphe. Les filets nerveux, quiavaient une direction parallèle ou oblique pour se rendre a leur destiuation inféricure, deviennent horizontaux et transverses, à mesure que les ganglious descendent lc long du corps de. l'auimal. En nème temps, ils se raccourcissent 
beaucoup, l'rspace qui sćpare l'anncau de son gang'ion dimiluant par l'eff l de celte marche des ganglions. Ainsi, dans le mêne trmps, pendant que les filds nerveux inter-ginglionnaires se forment et s'allong'nt, les radialions nerveuses se raccourcissent. L'effet est inverse dans li's filets qui unissent les ganglions les uns aux autses, ct dans ceux qui parient des ganglions moyens el inlérieurs pour se rendre aux parlies excentriques de l'animal.

En définilif clonc, le système nerveux des mollusifues cst l'itit ambryonnaire plus ou moins avancé des larves des insectes.

Dans la premicre période de ces larves, le systéme nerveux est double et désuai daus toute sa longueur : caractère du syslime nervenx que nous royons constiluer l'état permanent de quelques vers, notamment du lombricoïde du cheval et de l'homme.

En second licu, le système nerveux se réunil en avant aux environs de l'ouvirture oesophasienne, et se maintient écarlé ct à distance dans le resie de son álendue. Cette seconde périosle de la larve est la plus commune dans l'ćlat permanent du système nerveux des mollusques : c'est la disposition permanente du systemc nerveux de lihyale, du pneumoderme, lu pleuro branche, de l'onchidie (c-sch. Peronii), de la phyllitie (phyllidia trilincala), de laplysic, de la dolinbelle, du bulla aperta, des doris, de la tritonie 
(tritonia hombergii), et de l'hélice vigncronne.

En troisicme lieu, le système nerveux de la larve se réunit par sa terminaison inférieure; ses deux cordons longitudinaux sont entrecoupés par des ruflemens ganglionnaires : c'eșt l'élat qui correspond à la mulette des peintres et à la clie boréale.

En quatrième lieu, enfin, les deux cordons primitil's reuflés de dis'ance en distance, se réunissent, s'adossent ou se confondent complètement sur la ligne médiane. Cette dernière mćtamorphose, à laquelle ne s'élèveut jamais les mullusques, constitue l'axe nerveux que l'on remarque sur la plupart des vers, sur les chenilles, chez tous les insectes et chez tous les crustacés.

Le caractère général du système nerveux des invertébrésest donc d'être symétrique: plus ou moins séparé, chrz lesiufíricur: en deux faisceaux longitudinaux, il est réuni en un seul chrz les supérieurs, commechez les insectes et chez les crustacés.

Constamment celte jonclion des parties droite et gaucho s'opére par l'intermèle des ganglions, soit qu'ils se porient à la rencoutre les uns des autres, quils s'adossent, qu'ils s'unissent, se confordunt, soit qu'ils s'envoicnt réciproquement des filets de communication.

Constamanen? une double communication s'iSablit antour de l'ouverture nesophagienne. Celte: sommunication sefrectur regalemeni do dewx ma- 
nières, ou par des filets, ou par l'adossement des ganglions pro et méta-œesophagiens.

Les communications de la partic droite avec la partie gauche du système nerveux sont toujours directes, commeles faisceaux de réunion des deux lames de la moelle épinière, ou comme les principales commissures des hémisphères cérébraux.

Il en résulte que tous les organes reçoivent leur nerf de la partie du système nerveux qui correspond à leur côté.

Le croisement des deux systèmes nerveux qu'on remarque dans l'axe cérébro-spinal des vertébrés n'existe donc pas chez les invertébrés.

Le peu d'expériences physiologiques qui ont été faites sur le système nerveux prouvent que sun action est directe dans toute son érendue, en avant comme en arrière de l'ouverture œsophagienne.

Voilà donc le système nerveux des mollusques et des articulés ramené à un même type; mais dans quels rapports se trouve ce système avec celui des vertébrés? Ou, dans d'autres termes, a quelle partie clu sysieme nerveux des vertébrés correspond celui des invertébrés? Cctle question importante a beaucoup occupé les anatomistes. et elle a étć résolucbien difléremment, sclon les divers points de vac d'a près lesquels elle a été traitée.

Les uns , comme Ackermann, ont pris la névrogénie pour base de leurs rapports; ils ont suppose que l'ivolution de ce système commençant 
par le grand sympathique, l'axe nerveux des invertébrés devait correspondre au nerf intercostal des vertébrós; mais les travaux des anatomistes modernes ont établi un ordre de développement inverse à celui que supposait Ackermann. Ils ont vu que la moelle épinière devançait toutes les autres parties centrales du système nerveux, au lieu de se former la dernière : cette preuve et le rapport qu'on en déduisait ne sont donc pas admissibles.

D'autres ont pris pour guide la position de l'axe du système nerveux; considérant que chez les vertébrés la moelle épinière occupait l'axe central des animaux, ils ont pensé que l'axe nerveux de la plupart des articulés, occupant la même position, et recevant, comme la moelle épinièrc, les radiations nerveuses de toutes les partics de l'animal, n'était autre que la moelle épinière des vertébrés. Mais indépendamment des objections par lesquelles nous avons déjà réfuté cette analogie (1), il en est une qui se présente à la simple inspection de ces parlies; car tandis que la moelle épinière des vertébrés est un corps à-pcu-près cylindrique, l'axe nerveux des articulés qu'on lui comparait, est, au contraire, interrompu dans toute sa longueur par une séric de ganglions juxta-posés en quelque sorte sur les deux cordons qui le consti-

(1) Tom. T**, pug. 25,, 255 . 
tuent. Daprès cette dissemblance dans les formes, auxquelles les anatomistes se sont toujours assujélis dans leurs déterminations, l'axe nerveux des articulés ne pouvait donc être ramené à la moelle épinière des vertébrés.

Désespérant d'y arriver par cette voie, MM. Gall et Spurzheim ont pris une autre marche. L'axe nerveux des invertébrés ne pouvant être expliqué par la moclle épinière des vertébrés, ils ont imaginé d'expliquer au contraire cette dernière partie par la structure anatomique de la première. Ils ont supposé en conséquence que primitivement la moclle épinière des vertébrés était un cordon renflé de distance en distance, coinme l'axe nerveux des articulés: mais cette supposi-. tion, de même que celle d'Ackermann, s'est trouvée démeutic par la névrogénic, et la question a été remise en doute.

Par sa posilion, l'axe nerveux des articulés se rapprochait donc de la moelle épinière, dont l'écartait sa structure, qui le ramenait à celle du grand nerf intercostal. II est arrivé alors ce qui uanque rarement de survenir dans les questions difficiles, et sur lesquelles les sentimens sont opprosés : des anatomistes ont pensé que l'axe nerveux des articulés était à-la-fois un composé de la moelle épinière des vertébrés et du grand neri sympathique. Cette opinion de Weber et de Ilechal ne contenta ni ceux qui regardaientl'axe ner- 
reux des articulés comme la moclle épinierc des vertébrés, ni ccux quil'assimilaient au grand nerf intercostal.

La science en était là, quand je préscntai mon ouvrage à l'Institut: on y vit que je n'adoptais ni l'une ni l'autre de ces déterminations, et que j'en proposais une nouvile, fondée sur l'ordre des développeinens que suit le système nerreux dans tout le règne animal. Aussi long-temps qu'on a cru que le système nerveux se développait du centre à la circonférence, on a dû chercher à rallier uniquement l'axe nerveux des invertébrés aux parlies centrales des vertébrés; mais du mo. ment où japerçus au contraire que toutr's les parties de ce système se formaient de la circonférence au centre, je dus nécessairement, et par les mêmes raisons, être ramené sur une autre base de détermination; celte base fut celle des ganglions intervertébraux (1).

En ramenant le système nerveux des invertébrés à sa véritable expression, cetle déterminalion fait cesser les contradictions que présentent l'une ou l'autre des opinions précédemment émises.

Si, d'une part, ce que l'on aja pas remarqué jusqu'à ce jour, la division du système nerveux des mollusques favorisait son rapprochement avec le grand intercostal, de l'autre, sa conti-

(1) Voyes le I" volume, pay. 254.255 . Voyes le Rappurt de M. le haron Cuvier, 1821 . 
unité chez les articulés repoussait cette analogie.

Si cette continuité chez les articulés rapprochait leur axe nerveux de la moelle épinière, de l'autre sa disjonction chez les mollusques répugnait à cette détermination.

Dans l'une et dans l'autre supposition, les ganglions encéphaliques que l'on comparait au cerveau ne pouvaient être ni conçus ni expliqués. Dansl'hypothèse du nerf intercostal, comment un cerveau se trouvait-il sans moelle épinière, et comment les ganglions du grand sympathique faisaient-ils saite au cerveau? Aujourd'hui qu'il est bien reconnu que la moelle épinière ne dérive pas du cerveau et le précède dans son développement, cette opinion devenait inconciliable avec les faits.

Dans l'hypotlè̀se de la moelle épinière, ce que l'on regardait comme le cerveau, faisait bien suite à ce qu'on nommait la moelle épinière chez les articulés; mais chez les mollusques, l'axe nerveux était interrompu. On avait dit qu'ils étaient privés de moelle épinière; on rctombait donc dans la même invraisemblance d'un cerveau sans moelle épinière. Cette invraișemblance conduisait à une autre: chez certains mollusques, les ganglions oesophagiens sont d'autant plus développés, que les cordons du tronc le sont moins; alors on disait que ces mollusques avaient un cerveau beaucoup plus développé que les animaux articulés. Ils se trouvaient, sous ce rípport, placés à la tête des invertébrés, de même que l'homme 
occupe le sommel des vertébrés. 'Tel était le cercle de contradictions dans lequel on se trouvait enfermé, et dont on ne pouvait sortir, parce que l'état de la science n'en offrait pas encore les moyens.

Dans notre détermination, ces contradictions disparaissent; on voit la nature s'élever du simple au composé par une gradation insensible, comme le degré de complication des êtres dont on considère le système nerveux. Que les cordons intervertébraux et les ganglions encéphaliques des invertébrés soient entièrcment isolés, entièrement confondus, ou coufondus ou isolés en partic seulement, c'est toujours le même systeme organique, dont les états divers correspondent à des divers degrés de développement.

En résumé donc, le système nerveux du grand embranchement des invertébrés correspond aux cordons intervertébraux ct aux ganglions de la cinquiène paire des animaux vertébrés. Chez ces dernicrs, l'axe cérébro-spinal du système nerveux est un nouvel ordre d'organes sur-ajoutés à ceux des animaux invertébrés. Tous les rapporis que nous avons à faire connaître sur ce dernier axe ne peuvent donc être appliqués qu'aux quatre classes composant l'embranchement los vertébrés.

I a noureauté des rapports que je viens d'exposer me fera pardonner sans doute les détails dans Insquels jai étr obligŕ de descendre pour 
montrercombien le système nerveux des larves différait de celuilles insectes parfaits(1). A vant mes travaux, les anatomistes avaicut adopté une opiuion qui sopposait invinciblinent aux progrès uliérieurs de la science. On avail dit, ct on araitcru, que les embryons étaient la miniature des animaux parfails. Dans celle supposition, l'élude de l'embryogénic, sans êlre entièremı́nt dilaiss'ce, ne présentait aux anatomistes qu'un méliocre intérèt : elle prometlait de montrer en petit ce que présentait en grand l'anatomie des adultes; elle élait donc sans utilité comme sans but. Mais me livrant à cette étude sans idée préconçue, cherchant ce qui était, et non ce qui devait être d'après dles vues à priori, je rlécourris un ordre de faits inconnus, dans lesquels jeutrevis la solution d'une multitude de problèmes qui avaient inutilement fatigué le génie investigateur des anatomistes et des physiologistes : appliquant présen-

(1) Je suis entré dans ces développemens relatifs au sys tème nerveux des invertébrés, et dans ceux que je vais présenter dans le chapitre suivant, d la'sollicitation de MI. Latreille, Geoffroy-Saint-Hilaire et Aınpère, auxquels je les arais communiqués. Ces illustres professeurs les ont jugés néces jaires pour éclairer les principes que j’avais exposés dans mon premier volume. Ils m'avaient conseillé aussi d'y joindre quelques-uns des dessins que j'ai fait exécuter à ce sujet, et qui leur ont paru d'un grand intérêt pour cette partie de la science; je regrette beaucoup d'avoir été dans l'impossibilité de Je faire. 
DANS LE REGTE ANIMAL.

tement une partie de ces faits à l'explicaliou du système nerveux dans tout le règne animal, on a vu que les embryons rendent raison del'état permanent des animaux parfaits; on verra par la suite que l'état permanent répète, chez les vertébrés, de même que nous venons de l'établir pour les invertébrés, la série des formes transitoires que nous ont offertes leurs emhryons. 


\section{CHAPITRE II.}

Apercu sur le siége de l'instinct. - De l'Action des ner/s trijumeaux des vertébrés, comparée à celle des ganglions céphaliques des articulés.

$O_{\mathrm{N}}$ ne peut parler du système nerveux des invertébrés, sạis qu'aussilôt l'admirable industrie de certains d'entre eux ne se présente à l'esprit.

Ces êtres ont des impressions, des sens qui les leur transmettent, et des déterminations qui paraissent réglées sur ces impressions. Or, comme chez les vertébrés les impressions aboutissent à l'encéphale, et queles déterminations en partent, on n'a pu croire que des êtres en qui ces facultés se montrent, fussent dépourvus de l'organe qui en est l'instrument. On a donc cherchè un encéphale et une moelle épinière chez les invertébrés, parce que les vertébrés ont une moelle épinière et un encéphale. Pour en venir à ce résultat, on a négligé toutes les considérations ánatomiques qui pouvaient en éloigner. On a fait abstraction des formes, de la position, de la structure et des rapports des deux organes que l'on assimilait l'un à l'autre.

Mais, on mêne temps que l'on a imellait cher 
les invertébrés un cerveau, les psychologisles étaient loin de confondre leurs actes avec ceux des animaux vertébrés. La nécessité qui les dirige, et la répétition exacte qui les caractérise, les a fait distinguer avec soin des déterminations qui paraissent être le résultat t'une combinaison prémćditée. On a nommé instinct la faculté d'où dérivent ces actes.

La zoologie des invertébrés, fondée sur l'examen attentif des caraclères extérieurs, est très. avancée; l'anatomie, surtout celle des régions profondes, l'est beaucoup moins; la physiologic laisse presque tout à désirer. Nos connaissances sont toutà-fait nulles en ce qui concerne les fonctions diu système nerveux: au momentoù les physiologistes se disposent à entrer dans ce champ nouveau de recherches, il était donc bien nécessaire que l'anatomie comparative déterminât à quellc partie du système nerveux des vertébrés pourraient être rapportés les phénomèncs observés.

J'ai déjà touché ce point difficile de la science dans lá Seconde Partie de cet ouvrage; jai cherché à faire ressortir, chez les animaux vertébrés, les rapports anatomiques du nerl trijumeau avec tous les sens; j'ai montré que chacun d'eux avait deux cordons nerveux : le nerf' spécial de la sensation, et un nerf accessoire provenant de la cinquième paire. J'ai dit ensuite que les articulés ćtant privés de l'organe encéphalique, manquaient nécessaircment des nerfs propres des sens, qui sc mettent 
en relation avec l'encéphale; il ne leur restait, et il ne devait leur rester que les branches analogues à celles du nerf trijumeau chez les vertébrés. A l'appui de ces vues nouvelles, j'ai fait connaître avec détail l'organisation de la taupe, du rat-taupe du Cap, de la chrysochlore et du protée: chez tous ces animaux, le nerf oplique manque, et une branche de la cinquième paire le remplace. Ils scrvent, sous ce rapport, d'intermédiaires entre les articulés et les vertébrés. C'est pour l'anatomiste un anneau qui joint ces deux grands cmbranchemens du règne animal.

Des faits de cette nature heurtaient trop directement les hypotheses adoptées, pour ne point éprouver une vive opposition; pour s'en débarrasser, on a dit: la taupe a un nerf optique, ainsi le veulent les analogies, et il est absurde de croire, a-t-on ajoulé, que le nerf trijumeau exerce une influcnce quelconque sur l'action d'un ou de plusieurs sens. Prouvons par de nouveaux faits et. de nouvelles expériences, que cette action est réelle, et montrous que la taupe est privée du uerf optique. Ramenons ainsi aux faits, des auteurs qui, pour s'en être malheureusement écartés, ont consumé lcurs veilles et leur génie à étayer des systèmes qui s'écroulent de toule part.

Ecarto:s de cette discussion les dissidences des divers auteurs; car dans les sciences d'observation, un fait est, ou n'est pas ; s'il cst, qu'importent ces 
autorités qui l'ont rejeté? et s'il n'est pas , qu'importent aussi celles qui l'ont admis? La taupe a-t-clle un nerf optique? J'ai dit qu'elle n'en avait pas, et chacun peut s'en assurer en examinant la base de l'encéphale de ce singulier animal. J'ai cru inutile, et il eût été peut-être déplacé alors de mettre en avant les principes de la science, pour prouver ce fait, qui, je dois le dire, me paraissait tres-simple: aujourd'hui, il n'en est pas de mêne, il faut l'étayer par le raisonnement, en partant des bases sur lesquelles sont établies en anatomie les individualités organiques.

Depuis que l'anatornie est devenue une science; depuis qu'elle ne nomme pas nerf tout ce qui est blanc; muscle, tout ce qui est rouge; artère , tout ce qui est creux, les anatomistes se sont imposé la loi d'assigner à chaque organc des caractères fixes, et un nom qui rappelle à l'esprit ces principaux caractères. Plus tard, la zoologie, ( qui, comme nous l'arons dit, n'est que l'anatomie des surfaces, ) a adopté cette logique anatomique, et lui a dû ses plus brillans succès. On a purgé les genres de ces espèces, dites anomales, qui rompaient les affinités naturelles (Cuvicr, Geoffroy-Saint-Hilaire), et on n'a pas hésité à faire un genre nouvcau pour une seule espéce, lorsque cette especce ne pouvait prendre son rang dans les genres qui l'avoisinaicnt.

T.es espèces anatomiques se déduisent de leur posilion, de leur forme, de Inurs rapports, du point 
de leur départ eł de leur terminaison, après que leur structure a déterminé le genre auquel elles appartiennent. C'est d'après cette méthode que tous les anatomistes, sans aucune exception, ont donné le nom de nerf optique à un nerf qui de l'oil se rend aux tubercules quadrijumeaux, ou à leurs analogues, les lobes optiques, et qui, dans sa marche, s'adosse et s'entrecroise avec son congénère. Existe-t-il un nerf optique chez la taupe? C'est, en d'autres termes, demander s'il y a chez cet animal un nerf qui des tubercules quadrijumeaux se porte à l'oeil, ou qui de l'oil se dirige vers les tubercules quadrijumeaux, en s'adossant à celui du côté opposé.

S'il manquait à un nerf l'une de ces trois conditions, des doutes şéleveraient avant de l'assimiler au nerf optique; s'il en manquait deux, on rejeterait cette analogie; et si aucun de ces trois caractères n'existait, quel serait l'anatomiste qui prendrait sur lui re confondre des parties si fondamentalement opposées l'une à l'autre? Or, y a-t-il un nerf chez la taupe qui, du fond du globe de l'oil, se dirige, comme le nerf optique, vers les apophyses d'Ingrassias? Non. Y a-t-il un nerf qui des tubercules quadrijumeaux se porte vers la mème partie du sphénoïdc? Non. Y a-t-il nu nerf sur la base de l'encéphale de la taupe qui se joigne et sc croise, commc le nerf optique, ave: cului du côté opposé? Non, certainement non; rien de semblable ne se remarque ni chez la 
musaraigne, nichezle rat-taupe du Cap, ni cliezle protée, ni chez la taupe. Ce n'est pas toutcfois qu'on n'ait prétendu le contraire dans ces derniers temps. M. Durondeau dit avoir vu un nerf optique suivre chez la taupe la marche ordinaire, de l'œil à la base de l'encéphale; M. Gall a avancé la même opinion; mais à l'examen, toutes ces suppositions se sont évanouies. Il n'est plus resté, pour soutenir cette assertion, que le petit filet sus-sphénoïdal, que Carus et Treviranus ont regardé comme un nerf optique rudimentaire; encore ces anatomistes illustres n'ont-ils hasardé cette conjecture que dans l'idée où ils étaient que re petit filet se joignait à la branche du nerf trijuneau pour aller s'épanouir dans le globe de l'oil: supposition qui ne s'est point réalisée dans les nombreuses recherches que MM. Cuvier, GeoffroySaint-Hilaire, Lisfranc, Bailly et moi avons tentées à ce sujet.

Toute la discussion portant désormais sur cc petit filet, ou sur ces petits filets, car il y en a deux de chaque côté; c'est à leur détermination que doivent s'attacher les anatomistes pour éclairer ce point curieux de li science. Exposons d'abord ce que montre la dissection délicate et difficilc de cette partie, nous jugerons mieux ensuite les conjectures qu'elle a fait naîtrc.

Si l'on soulève l'encéphale de la taupe d'avant en arrière, on apercoit, vers le milieu de la base Jo sel organe, quatre petils filels grisâtres, Irans- 
parens, adhérens d'une part à l'encéphale, et se dirigeant ensuite vers la base du crâne : les filets externes sont les plus gros et les plus longs; ils se rendent des cellules etmoïdales sur les côtés de la base du cerveau, entre le tuber cinereum et la base du lobe, côtoyant dans ce trajet le côté internedu nerf trijumeau auquel ils adhèrent par ún petit repli de l'arachnoïde. Un millimètre environ plus en arrière, on distingue deux autres filets plus petits, égalant à peine le tiers d'un cheveu pour le volume; ces filets s'élèvent de la partie moyenne et antérieure du corps du sphénoïde: séparés l'un de l'autre par l'intervalle d'une ligne ou d'une ligue et demic, ils se dirigent parallèlement l'un à l'autre vers la base du cerveau, à laquelle ils adhèrent vers la partie moyenne du tuber cine. rcum. Leur position est plus interne que celle des premiers; ils n'ont conséquemment aucun rapport avec le tronc des nerfs trijumeaux.

Arrivés à la base de l'encéphale, ces pelits filets du centre convergent plus l'un vers l'autre; Ic plus souvent ils se continuent par arcade, et, adhìrent tantôt à la matière grise du tuber cinereum, tantôt à la strie médullaire blanchâtre que Carus considère comme la commissure inféricure de la division moyenne de l'encéphale, tåntôt en avaut, et tantôt en arrière de cette même commissure. Quelque soit le point de leur adhérence, si on dissèque dans l'eau distillée, et qu'on soulève l'encéphale avec beaucoup de précaution, on les 
voit abandonner la base du cerveau, et s'eu écarterd'un millimètre environ en déplissant une lame a rachnoïdale ext rêmement mince, qui du cerveau, se réfléchit sur ces deux petits filets, ainsi que sur les deux précédens situés un peu plus en dehors.

Raisonnons dans la supposition que ces filets sont nerveux. Nous trouvons que Carus a pris-les externes pour les rudimens du nerf optique, parce qu'il les voyait s'adosser au nerf trijumeau, et qu'il pensait qu'ils se joignaient à la branche supérieure de ce nerf qui se porte dans l'œil. Trériranus, au contraire, le regarde comme le nerf de la troisième paire, et il réserve le nom de nerf optique aux filets internes; mais il a tort alors de sétayer de la supposition de Carus, puisque ces derniers filets sont sans communication avec le nerf de la cinquième paire. M. Gall a adopté l'idée de Tréviranus, ot M. Bailly, anatomiste distingué, qui a soutenu l'opiuion de ces auteurs, n'a porté son attention que sur les filets moyens.

A près avoir montré que ces filets ue se rendent pas à l'ocil, après avoir vu qu'en prolongeant leur ligne d'insertion sur le sphénoïde, ils tomberaient à un pouce au moins au-dessous de l'oeil de la laupe; apres avoir fiit remarquer que ces filets ne s'adossaicut pas sur le sphénoïde, mais divergeaient ylutôt qle de se rapprocher, je fis remir"guer que, par lour adhérence a l'oncephalr, ils 
n'avaient ni ne pouvaient avoir aucune connesion ni arec les tubercules quadri-jumeaux . ni avec la partie postérieure des couches optiques. Je fis voir ainsi que ces filets étaient dépourvus de tous les caractères propres au nerf optique. $\mathrm{O}_{4} \mathrm{n}$ insista beaucoup alors sur leur rapport avec la petite commissure inférieure de l'encéphale, en attribuant à cette commissure une influence trèsgrande sur le nerf optique en général : influence que jignore, parce qu'elle n'a pas été développée. Mais en admettant encore cette nouvelle supposition, elle ne prouverait rien en faveur de cè prétendu nerf optique de lá laupe: car, $1^{\circ}$. Carus el Tréviranus ont toujours vu ce filet s'insérer sur la matière grise du tuber cinereum; $2^{\circ}$. dans le grand nombre de taupes que jai disséquées dans cette vuc, je l'ai vu le plus souvent s'adosser au point désigné par ces célèbres anatomistes ; quelquefois je l'ai vu adhérer ḋ la petite commissure, plus rarement je l'ai rencontré en arrière d'elle; plus rarement encore j’ai observé un des filets s'insérant en avant, et l'autre en arrière: dans toutes ces rariélés, en disséquant avec le soin que nécessite une préparation si minutieuse, j’ai vu ces deux filets se réunir par arcude, en se défachant de la base de l'encéphale, et déplissant par une légèrc traction un repli de l'arachnoïde. qui semble les y assujétir. La ténuité extrême de ces filets et leur ćlasticité ne mont jamais permis. ni aux autres anatomistes, de les suiver dans la 
profondeur du tuber cinereum, ou de la petite commissure, quand ils y adhèrent (1).

Ainsi, $1^{\circ}$. ces filets ne se rendent pas à l'oeil ; $2^{\circ}$. ils ne se rendent pas aux tubercules quadrijumeaux ni à la couche optique; $5^{\circ}$. ils ne s'adossent pas sur le sphénoïde; $4^{\circ}$. ils n'adhèrent pas le plus souvent à la petite commissure inférieure de l'encéphale. Dans toutes les suppositions possibles, ces filets ne peuvent donc être regardés comme le nerf optique rudimentaire.

J'ai supposé jusqu'ici que ces filets étaient nerveux; mais le sont-ils réellement? je ne le pense pas. A la vérité, ils blanchissent par l'action de l'alcohol; mais les filets externes, que Tréviranus considère comme le nerf de la troisième paire, blanchissent aussi; or, nous allons prouver que ces derniers n'appartiennent pas au système nerveux.

Leur couleur grise, leur transparence parfaite,

(1) Au moment où je corrige cette page, un de nues éléves, M. Joseph Martin, mapporte une musaraigne. commune. Le rierf optique manque comme chez la taupe et la musaraigne muselle; les filets sus-sphénoïdaux sont doubles; les internes offrent une disposition un peu différenle de ceux de la taupe. Ils se touchent presque sur le sphénoïde, nu ils s'arrêtent, et ils adhèrent ì liı base de l'encéphale en avant de la strie médullaice moyenne. IIs sont d'un gris cendre; les cxterues sont rougeâtres; la troisième. la quatrieme el la sixieme paires manquent Une branche do l'ophthal uique de la cinqueme paire se remil a l'neil. ol sy omporte de minme que rhey la taupe. 
leur élasticité très-prononcée, me paraissent les réunir au système vasculaire, et voici les raisons sur lesquelles je m'appuie.

Premièrement, ce petit réseau vasculaire existe chez la plupart des mammifères doués de nerfs optiques; après avoir incisé ces nerfs à leur entrée dans le crâne, si on les soulève, on aperçoit en arrière d'eux quatre petits filets transparens, analogues, par leur position et leur marche, aux filets de la taupc. J'ui disséqué ces filets chez les carnassiers, les ruminans, los rongeurs, et chéz les oiseaux, qui ont un nerf optique si développé (1).

En second lieu, j’ai injecté constanment ces filets chez ces divers animaux, soit au vermillon, soit au mercure : assez souvent chez les petits animaux les filets internes ne s'injectent pas.

Puisque les animaux pourvus de nerf optique ont les filers que l'on considèrc comme tels chez la taupe, c'est une preuve sans réplique que ces filets ne sunt point le nerf optique rudimentaire. Mais sont-ils vasculaires? c'cst une nouvelle queslion à l'appui de laquelle je vais présenter le résultat de mes recherches.

(r) En disséquant l'encéphale de l'crnithorhynque arec M. Laurillard, prosecteur de M. Cuvier, et conservateur du Cabinct d'Anatomie comparée au Jardin du Roi, j'ai trouvé de wême ces filets, dont la disposition cst analogue it cell. de lá taupe. M. Laurillard eut même la bunté de m’en faire un dessin, que je conserve avec ceux que jaithit executer chez la taupe et rhez divers autres animanx. 
Chez plusieurs taupes strangulées, jai va les filets extéricurs remplis de sang; sur plusieurs j'ai remarqué une molécule jaunâtre occupant le centre des filet; moyens, et parcourant sa longueur, quand on abaissait et relevait l'encéphale avec précaution. J'ai injecté plusieurs de ces animaux: conslamment je suis parvenu à remplir, par le vermillon et le mercure, les filets externes; ce qui prouve que ce n'est pas la troisième paire. Une ou deux fois seulement, sur environ trente injections, je suis parvenu à rougir les filets internes; encore m'est-il resté des doutes sur leur injection; car, quand on soulevait l'encéphale pour les examiner, les molécules rouges remontaient vers l'encéphale, et se réunissaient en nappe le long du petit repli arachnoïdal qui les applique contre l'encéphale. Linjection remontait-elle par la capillarité extrême de ces filets ? c'est un fait que je n'ose assurer.

Si ces filets sont vasculaires, ils devront disparaîtṛe dans une classe chez laquelle le réseau vasculaire qui avoisine le tuber cinereum chez les mammifères et les oiseaux, disparaît aussi; ce sera, je crois, une preuve évidente qu'ils ne sont ni des filets nerveux, ni consćqucmment le nerf optique rudimentaire. Or, c'est ce qui arrive chez. les reptiles. Chez eux on ne voit plus les petits filets en arrièrc de leur nerf optique; ct chez lc protée qui, counme la taupe, est privé de ce nerf, on ne rencontre plus les filets grisâtres qui ont 
trompé les anatomistes à ce sujet. Je ne les ai pas rencontrés sur le dernier protée dont j'ai examiné le cerveau, et Tréviranus, qui a disséqué plusieurs protées dans cette intention, ne les a non plus jamais aperçus.

Il me paraît donc rigoureusement établi que la taupe est privée de nerf optique, comme aussi le rat-taupe du Cap, la chrysochlore, la musaraigne et le protée, et que si cet animal jouit de la vision, comme le prouvent les faits intéressans et les expériences curieuses, exposés dernièrement à la Société Philomatique par MM. les professeurs Geoffroy-Saint-Hilaire, Duméril et Blainville, cette fonction ne peut s'exécuter que par l'intermède du nerf de la cinquième paire.

Autre hérésie physiologique, a-t-on dit, que de supposer que la cinquième paire puisse exercer une influence quelconque sur la vue ou les organes des sens. Ne supposons rien; laissons parler les expérieuces physiologiques et les faits pathologiques, et nous verrons cette seconde objection réfutéc plus victorieusement encore que la précédente.

Ces faits et ces expériences vont nous porter sur un champ nouveau de recherches dont il est impossible de prévoir les résultats. Nous allons voir se réunir sur un même point l'anatomie comparative, la physiologie expérimentale et la pathologie, trois sciences qu'on croit si étrangères les unes aux autres, et qui tôt ou tard n'en feront qu'une. 
()uiconque eût annoncé, il y a quelques années, que la section de la cinquième paire chez les animaux, et les altérations de ce werf chez l'homme, porteraient une telle atteinte à l'action des sens, que la plupart d'entre eux seraient paralysés, eût trouvé devant lui toute la cohorte des anciennes idées, au travers de laquelle il n'eût pu se faire jour; on n'eût pas manqué de dire qu'il y avait un nerf optique qui seul pouvait voir, comme on l'a fait dans la discussion précédente : un nerf olfactif qui seul pouvait sentir; un nerf acoustique qui seul pouvait entendre : on eût ainsi, pendant quelque temps, enrayé la marche des idées; mais les progrès rapides de la science du système nerveux ont prévenu ces objections.

Parmi les êtres organisés, les animaux seuls sont doués de deux proprićtés qui les caractérisent, la sensibilité et la motilité; le système nerveux en est le siége. Mais chaque nerf ou chaque partic du système nerveux cst-elle egalement propre à développer et à transmettre l'une et l'autre de ces propriétés? ou bien existe-t-il des nerfs et des parties, dans l'axe cérébro-spinal, destinées spécialement à la sensibilité, d'autres à la motilité? Personne n'ignore combien est ancienne la division des nerfs, en nerfs du sentiment et en ceux du mouvement; chacun sait aussi combien furent iufruclueuses les recherches de nos pères. pour démontrer l'isolcment de ces deux actions, dont ils avaient un pressentiment vaguc. Ces idées, 
tour-à-tour suivies avec persévérance, délaissées ensuite, puis reprises de nouveau par l'école de Haller, et délaissées encore quand l'impulsion de ce grand homme s'éteignit, ont acquis enfin des recherches modernes, un degré de certitude qui promet à la science les plus importantes acquisitions. Les résultats déjà obtenus par Charles Bell, par le professeur Mayo, et par M. Magendie, sont connus de tout le monde; c'est en les poursuivant sans relấche que le dernier de ces physiologistes a été conduit à pratiquer sur les animaux vivans la section de la cinquième paire. Déjà le professeur Mayo l'avait tentée; mais, n'agissant sur les animaux qu'après la mort, ou ne les laissant vivre que peu de temps, il méconnut les effets remarquables que nous a dévoilés le physiologiste français (1), et dont les plus surprenans, et sans aucun doute les plus inattendus, sont relatifs à la perte de la vue, de l'odorat et de l'ouie, qui suit inmédiatement la section de la cinquième paire. Voici l'ordre de leur manifestation.

$A$. Vingt-quatre heures après la section, la cornée commence à devenir opaque; après soixante-douze heures, elle l'est beaucoup plus . l'opacité augmente graducllement, et cinq ou six jours après la section, elle est de la blancheur de l'albâtre.

(1) Journal de Phys. experim. et de Path. ; numéro d'arril 1824 , pag. 176 . 
$B$. Vers le deuxième joúr la conjonctive s'injecte; on voit aussi l'iris devenir rouge et s'enflammer comme la conjonctive. Il se développe à la surface antérieure de l'iris de fausses membranes, qui, comme l'iris, ont la forme d'un disque percé à son centre. Ces fausses membranes finissent par remplir la chambre antéricure de l'œil, et contribuent à faire paraître la cornée opaque.

C. Vers le huitième jour, le tissu de la cornée s'altère visiblement, son centre s'ulcèrc, et sa circonférence se détache de la sclérotique (1); l'œil se vide et se réduit à un petit tubercule.

$D$. La vue paraît être très-affaiblie et presque perdue dès que les deux nerfs sont coupés; l'animal se conduit comme s'il était aveugle.

$E$. La langue est insensible du côté où le nerf est coupé, ct des deux, si les nerfs le sont à droite et à gauche; dans ce dernicr cas, la mâchoire inférieure est pendante chez les animaux, la déglutition est très-gênée.

Quand un seul nerf est coupé, la moilié de la langue devient blanchâtre, les gencives quittent les dents, ou, d'après l'expression ordinaire, les dents sont déchaussées.

F. Quoique les altérations morbides de la

(1) Cette circonstance prouve la différence d'organisation de ces deux membranes, différence expliquéc par l'organogénie, comme je le prouverai dans un autre ouvrage.

II. 
membrane pituitaire soient moins sensibles physiquement que celles de l'oil et de la bouche, néanmoins sa sensibilité disparaît; aucun corps odorant ne l'affecte, soit qu'il soit placé à distance ou en contact; les corrosifs même ne l'excitent pas; circonstance que nous avons déjà vue produite par la section du nerf olfactif. Cet effet est en grande partie expliqué par l'anatomie comparative; car on sait d'une part que les divisions du nerf olfactif ne dépassent pas les cornets ethmoïdaux, et de l'autre j’ai montré que l'étendue de la chambre olfaclive était principalement due, chcz les animaux, au volume du cornet inférieur, sur la surface duquel se distribuent uniquement les filets de la cinquième paire.

G. M. Magendie, à qui j'emprunte ces faits importans, croit avoir remarqué aussi que la section de la cinquième paire entraîne la perte de l'ouie ; circonstance qui expliquerait l'audition des animaux dont le nerf acoustique paraît n'être qu'une division du trifacial ( 1 ), et qui en serait expliquée à son tour.

Des résultats si nouveaux ne pouvaient manquer d'exciter vivement l'attention des physiologistes; à une autre époque, peut-être, ils eussent été stèriles pour la médecine; les médecins n'eussent pas manqué de renouveler contre eux toutes les préveutions qu'ils ont conçues contre la phy-

(1) Tnm. Ier, pag. $331,3(1)$ 
siologie expérimentale. Mais à peine étaient-ils connus, que j'en fis à l'homme les plus heureuses et les plus utiles applications. J'avais, depuis plusieurs mois, dans ma division, à l'hôpilal de la Pitié, un jeune homme qui avait successivement perdu l'neil droit, et la vue, l'odorat et le goût du même côté. Quelle élait la cause de cette affection simultanée des sens, dont les annales de la science n'offraient point d'exemple? on va le voir dans l'observation détaillée que j'en présente ici.

Histoire d'une altération organique dunerf trijumeau, suivie de la perte de la vue, de l'odorat, de l'oviic et du goat du même côté.

Hubertin-Joseph Lainé, âgé de vingt-six ans , garcon chapelier, entra dans ma division à l'hôpital de la Pitié, le 29 septembre 1825 . Sa constitution était délicate, son tempérament lym phatique; sa yie avait été régulière, mais il s'était souvent livré à la masturbation.

Son air était hébêté, sx physionomie donnait au premier aspect l'idée d'un homme imbécille; il paraissait concevoir lentement et comprendre avec difficulté les questions qu'on lui adressait. Lorsqu'il avait compris et qu'il voulait répondre, on voyait qu'il éprourait de la difficulté à s'cxprimer. Il prononçait difficilement, et le peu qu'il disait, scmblait exiger de sa part un effort Irè- 
prononcé. Son crâne était volumineux proportionnellement au reste du corps. Quelques élèves, soupçonnant un commencement d'hydrocéphale, crurent remarquer un écartement entreles pariétaux et les temporaux; mais la saillie très-prononcée des yeux me fit rejeter cette conjecture. Les os maxillaires et les jugaux étaient un peu écartés, ce qui avait produit l'aplatissement du nez; les os de la face du côté droit étaient un peu plus volumineux que ceux du côté gauche, ce qui faisait que la face était légèrement déjetée dans ce dernier sens. Le malade avait quelque peine à mouvoir la langue: les mouremens et la sensibilité des membres n'étaient point affectés; toutefois , il agissait moins librement des extrémités inférieures que des supérieures.

Tel est l'état dans lequel il se présenta à nous; il nous apprit d'ailleurs, qu'il était affecté d'épilepsie, maladie dont il faisait remonter l'invasion à une des deux années précédentes, sans pouvoir lui assigner de cause. Il avait perdu son père et sa mère; il avait une sour sourde et muette; il n'était en relation habituclle avec personne; circonstances qui nous privèrent de plus amples détails sur sa vie antérieure, que nous ne pouvions du reste connaître par lui-même à cause de son ctat moral.

Daus les premiers temps nous ne portions uotre attention que sur les accès d'épilepsie, qui se remarquaient asser. fréquemment, et qui toujours 
commençaient par une conviulsion du côté droit ; l'oeil droit était en outre affecté d'un ophthalmie chronique, que nous jugeâmes de nature scrophuleuse, à cause de la constitution du malade. Nous combattîmes ces affections par les moyens ordinaires, et nous nous abstînmes le plus possible de lui adresser des questions à cause d'une circonstance qu'il est im portant de ne pas négliger. Il nous était mort depuis quelque temps des malades affectés d'épilepsie, dont les altérations morbides de l'encéphale avaient vivement excité notre in térêt; ce jeune homme nous présen tait quelques-uns des symptômes que nous avions précédemment observés. Nous nous arrêtâmes plusieurs jours de suiteà sonlit, afin d'apprendre de luil'ordre de leur succession, et le sentiment qu'il en éprouvait pendantl'accès, ou dont il devait conserver le souvenir après l'accès, dont la durée ne dépassait pas ordinairement un quart-d'heure. Or il nous arriva fréquemment de provoquer le retour de l'accès par l'attention qu'il metlait à nous comprendre, et les efforts qu'il était toujours obligé de faire pournous répondre. Aussitôt que nous nous fûmes aperçu du mal que lui faisaient les renseignemens que nous cherchions a obtenir, nous cessâmes nos recherches, et pendant près de deux mois nous nous contentânes de l'examiner en passant à la visite, sans lui adresser d'autres questions que celles qui élaicnt relatives à ses besoins. 
Le calme moral, le repos dans lequel il restait, firent diminuer considérablement le retour des accès; il restait souvent huit, quinze et vingt jours sans en éprouver, tandis qu'au moment de son entrée ils se répétaient tous les jours, quelquefois deux ou trois fois. L'appétit était revenu, le malarle mangeait les trois-quarts de la portion, et digérait bien; l'embonpoint revenait de jour en jour; de jour en jour aussi il paraissait plus content et plus gai.

Mais ce bien-être ne fut pas de longue durée: vers le milieu du mois de décembre, une ophthalmie aiguë se manifesta sur l'oeil droit: elle fut accompagnée d'un ocdème des paupières, et d'une opacité commençante de la cornée transparente ; I'iris de ce côté ne présenta rien de particulier. Un séton fut appliqué à la nuque : l'ophthalmie se dissipa au bout de dix à douze jours; mais pendant que l'inflammation de la conjonctive diminuait, l'opacité de la cornée augmentait de plus en plus, de telle sorte que, l'ophthahmie ayant disparu, la cornée parut épaissie et tout-à-fait upaque dans toute son étendue. La perte de la vue de ce côté en fut le résultat inévitable; du reste, les accès épileptiques devinrent plus fréquens et se manifestèrent principalement pendant ia nuit. J'administrai les anti-spasmodiques, le nitrate d'argent associé au camphre : les accidens se calmèrent de nouveau, et le malade revint à-peupries dans l'état où nous l'avons vu précédemment. 
Toutefois, nous nous étions aperçu que l'oil droit était insensible, dans le cours de janvier et en fév rier ${ }_{1} S_{24}$. Plusieurs fois les accès épileptiques avaient eu lieu sous nos yeux. Je remarquai que les convulsions dont il était affecté, se manifestaient exclusivement du côté droit, et consistaient dans une extension et une flexion alternative du bras et de la jambe de ce côté, mais principalement du bràs, qui souvent, pendant plusieurs minutes, restait dans une rigidité télanique : du reste, la face était aussi injectée, spécialement du côté droit. Ayant remarqué encore que nos questions le fatiguaient beaucoup et provoquaient le retour des accidens épileptiques, nous discontinuâmes des recherches qui lui paraissaient si nuisibles. Dans le cours des mois d'avril et de mai, nous nous aperçûmes qu'il quittait rarement son lit, ct nous apprîmes qu'il lui était resté une faiblesse très-grande du bras et de la jambe du côté droit.

I e $1^{\text {er }}$ juin $\$_{24}$, je fus invité par M. Magendie a aller voir les animaux sur lesquels il avait pratiqué la section de la cinquième paire : à l'aspect des effets que j'aperçus sur les yeux de deux ou trois lapins, je fus frappé d'un trait de lunière relativement au malade qui fait le sujet de celte observation. Jo me rappelai que l'ophthalmie de l'oeil droit, etlopacité de la connéc qui en avail été la suife, avaient offert une warche a-peu-près scmblable a celle dnut ces animanx étaient affectés. 
Est-ce d'une affection de la cinquième paire que serait atteint notre malade? telle est la conjecture qui s'offrit à mon esprit. Rentré à l'hôpital, je me rendis aussitôt auprès du malade, je m'assurai que le globe de l'oeil droit était insensible, ainsi que la partie interne de la paupière. Je constatai que la narine du même côté était insensible aux odeurs, tandis que l'œil gauche et la narine correspondante jouissaient de leur sensibilité naturelle. Je n'aperçus rien sur les gencives, ni dans l'intérieur de la bouche. C'en était assez cependant pour m'arrêter à cctte idée, après en avoir abandonné d'autres pour lesquelles j'avais répété quclques expériences importantes (1).

Craiguant d'aggraver l'état du malade en le soumettant pendant la visite aux expériences qu'il devenail indispensable de tenter pour avoir sur le diagnostic de sa maladie quelque chose de plus positif, je pris la résolution de le voir seul dans le cours dela journée le 7 et le 1 juin; je vérifiai

(1) Cette observation me préscntant des phénomènes insolites dans les maladies organiques du cerveau, j'attribuai les effets observés sur l'œeil, a la lésion du nerf intercostal, avant de connaitre les expériences de M. Magendie sur la cinquième paire.

On sait que le célébre médecin Petit, de Namur, arait fait la section du nerf intercostal, chez le chien, an niveau de la troisième el quatrième vertébre cervicale, pour prouver que ce nerf exercait une induence sur certaines parties de l'vil. (Histoire de i'Academie Royale des Sciences, année. 
de nouveau l'insensibilité du globe de l'ocil droil, la perte de l'odorat du même cêté ; je plaçai du poivre en poudre sur la moitiédroite de la langue, il ncle sentit point; je l'appliquai a gauche, il en ressentit vivement l'impression.

Du 15 au 20, je m'aperçus que les gencives s'enflammaient du côté droit, d'abord à la mâchoire supérieure, puis à l'inférieure. Le 23, j'invitai M. le professeur Lisfranc à se joindre à moi pour répéter les précédens essais. Un stylet boutonné fut promené sur tơute la surface de l'oeil droit ; celuici resta immobile, il n'y eut point de clignotement de la paupière; le malade n'en avait aucun sentiment. L'oeil gauche, au contraire, était très-sensible, le malade fermait l'oeil en voyant approcher lestylct, et quand on était parvenu à l'appliquer sur sa surface, il y portait sa main pour le frotter et détruire la démangeaison que cet attouchement avait provoquée. Nous lui fìmes prendre du tabac par la narine droite, il ne le sentit point; il le sentit vivement de la gauche, et éternua deux fois.

1707.) Parmi les phénomines observés, il avait constaté la rougeur de la conjonclive et l'opacité de lis cornće, que j'observais chez ce inalade. Je repretai celte expérience, qui ne me réussit qu'une lois, et qui determina l'aplatissement et l'opacilé pirrtielle de la corné, l'injection de la conjonctive et une alteration sensible dı bord libre des paupières. Je rapporterai ailleurs les résultats de celte expérience, qui . an ce qui concerne l'ocil, different de ceux produits par la section de la cinquième paire. 
Je lui fis flairer une potion éthérée; il reconnut la présence de l'éther de la narine gauche, et ne put jamais le distinguer de la narine droite. Je n'aperçus rien du reste sur la membrane pituitaire. II est inutile de dire que nous fermions avec soin la narine opposée à celle sur laquelle on faisait l'essai.

Nous examinions avec soin l'intérieur de la bouche, et nous reconnûmes sur les gencives droites un commencement d'affection scorbutique qui n'cxistait pas à gauche; les, gencives étaient rouges, quelques plaques blanches existaient çà ct là; elles étaient gonflées au pourtour des alvéoles, surtout à la mâchoire inférieure. La langue se mouvait toujours avec quelques difficultés; du poivre en poudre fut encore appliqué et non ressenti sur la partie droite de cet organe; sur la gauche, il excita le ptyalisme et une vive cuisson. L'ouie du côté droit ne parut nullement affectée.

Cet état restait stationnaire. Dès le 5 juillet, je fis observer aux élèves l'affection scorbutique qui se remarquait du côté droî́ et point à gauche. II survint à cette époque une gêne de la respiration que nous n'avions pas remarquée jusqu'à ce jour. Le 11 juillet, l'affection scorbutiquè avait fait des progrès; la parlic gauche en était encore exempte. L.e 16 , les gencives sont plus boursouflées à gauche, fendues vers la partic qui embrasse le collet des dents, laffection commenes a s'étemire aux 
gencives du côté gauche. Du 17 au $1^{\text {*r }}$ août, l'intérieur de la bouche devient de plus en plus malade; mais la partic droite reste toujours plus profondément affectée que la gauche; du côté droit, les racines des dents commencent à abandonner leurs alvéoles, c'est-à-dire à être déchaussées. Cet état fait des progrès jusqu'au 10 août; à cette époque les gencives étaient écartées à droite du collet des dents; il existait entre ces dernières et les gencivès des vides, où avaient pénétré du tartre et des parcelles d'alimens.

Sa santé d'ailleurs déclinait de jour en jour. Le soin que nous avions eu de ne point fatiguer le malade, avait presque fait cesser les accès épileptiques; mais une cachexie générale s'était lentement développée. Le malade était d'une faiblesse extrème. La respiration était gênée plus à droite qu'à gauche. Le pouls était concentré, petit et fréquent. La face, qui s'était conservẻe jusqu'alors, offre une altération profonde des traits. L'appétit disparaît, et le malade tombe dans une taciturnité qui jamais ne s'était manifestée.

On conçoit, d'après l'exposé de tout ce qui précede, qu'il ne me restait aucun doute sur le siége de la maladic. Trop de caractéres, trop de signes indiquaient unelésion de la cinquième paire, pour qu'il fût possiblede la méconnaître, quoique ce fît le premier fait de ce genre qui s'offrait aux regrards des médecins, ct que je ae fusse dicigé., Aans co: diagnostic, que par les induclions de la 
physiologie expérimentale et de l'anatomie comparative. Aussi, dès le 7 août, discutant devant la Sociétć philomatique sur le prétendu nerf optique de la taupe, et réfutant les objections qui s'élevaient contre l'action que j'avais attribuée à la cinquième paire sur les sens, je n'hésitai pas à m'avancer devant cette honorable assemblée, en, l'assurant que sous peu de jours je produirais un cas de pathologie qui justifierait ce que j'avais avancé. Tout, dès-lors, annonçait une fin prochaine de ce malade.

Jusques-là, je n'avais fait qu'énoncer aux élèves les conjectures que j'avais formées sur le siége de la maladie. Je m'étais abstenu, par les raisons indiquées plus haut, de faire devant eux les expériences qui m'avaient mis sur la voie du diagnostic. Je jugeai cependant qu'un fait de cette importance devait être publiquement constaté : en conséquence, le 10 août, à la visite du matin, j'invitai M. Dimbarre, interne de ma division, jeune médecin très-instruit, et excellent observateur, à répéter lui-même les cxpériences devant les autres ćlèves.

M. Dimbarre frotta l'oeil droit avec les barbes d'une plume à écrire: le malade n'en eut aucun sentiment, il n'y eut point de clignottement des paupières: la face interne de ces dernières parties était également insensible. La mème expérienco faite sur l'oeil gauche, produisit une vive sensation et un clignotement long-temps prolongé. On réi- 
téra deux ou trois fois cet essai, parce que le malade n'en parut pas fatigué, et que cette insensibilité de la conjonctive, de la cornée et de la face interne de la paupière, jointe à l'immobilité complète du globe de l'oil et de ses dépendances , excitait un vif étonnement parmi les assistans.

On passa ensuite aux fosses nasales, on introduisit la plume dans la narine droite, on l'agita dans tous les sens : le malade y fut complètement insensible. On passa à la narine gauche, la sensibilité la plus vive se manifesta dès son introduction. On présenta à la narine droite un flacon contenant de l'ammoniaque liquide : le malade en ressentit une faible impression après une forte inspiration; à gauche, l'approche du flacon ne put même être supportée.

On vint à la bouche: nous constatâmes de nouveau l'altération profonde des gencives du côté droit, beaucoup plus affeciées que celles du côté gauche. La langue ne parut pas sensiblement altérée, le malade la portait hors, de la bouche, en ligne directes Du sulfate de quinine, réduit en poudre, fut appliqué sur la partie droite de la langue, le malade ne le sentit point, ne le dégusta point; on en mit sur le côté gauche, il le cracha aussitôt. Interrogé sur la savçur qu'il lui avait trouvée, il en désigna l'amertume par le terme de chicotin, usité parmi le peuplc.

On a remarqué dans les expériences premières que nous fimes sur ce malade, que l'audition 
n'avait point éprouvé d'altération sensible. Le malade paraissait entendre également bien de l'un et de l'autre côté. On a dû remarquer aussi que dans ses expéricnces sur les animaux, M. Magendie n'était pas sûr que l'ouie fût affectée, ce dont il est du reste très-difficile de s’assurer dans les vivisections. Cette circonstance tient peut-être à ce que la partie de la cinquième paire qui communique avec l'oreille, est la dernière à ressentir l'effet de la section ou de l'altération du nerf : c'est du moins ce qu'on pourra inférer du fait extraordinaire que nous rapportons. L'ouïe se conserva du côté droit et du côté gauche jusqu'au 3 ou 4 août; mais vers le 5 et le 6 , il devint presque sourd de l'oreille droite. Informé par le malade de ce nouvel accident, je fis appliquer un vésicatoire à la nuque. Les 7,8 et 9 , la surdité diminua, et le 10 , au moment où on répétait les 'expériences précédentes, il entendait encore bien moins de loreille droite que de la gauche, la sensibilité était parfaitement conservée dans toute l'étendue du côté droit de la face et du col. Si on pinçait légèrement la peau, il en ressentait aussi.bien l'impression à droite qu'àgauche. Tel fut le résultat des expériences faites publiquement ả. la visite du 10 août.

Le malade était d'ailleurs dans une triste position; la faiblesse était extrême, la respiration était courte, précipitée; le côté droit de la poitrine était immobile, lc son qu'il rendait était mat, on 
n'entendait pas la respiration par l'applicalion du cylindre; le pouls était très-petit, trés-fréquent ct facileàdéprimer : la face, décomposéc, offrait déjà un aspect cadavérique; ces mauvais symptômes augmentèrent d'intensité le 11 . Il mourut dans la nuit du 11 au 12 août, après une agcnie de buit à neuf heures.

Aussitôt que je fus informé de sa mort, jécrivis à MM. les docteurs Magendie, Lisfranc et Georget, afin qu'ils se réunissent à moi pour faire l'ouverture du cadavre. Elle fut faite le lendemain à deux heures, en présence deces médccins, et d'un concours nombreux d'élèves, alors présens à l'amphithéâtre des hôpitaux.

Avant de procéder à la nécropsie, je fis lire l'histoire de la maladie par l'interne de ma division, j'en résumai ensuite les circonstances principales, en les rapprochant des effets qui suivent la section de la cinquième paire chez les animaux. Je fis remarquer l'aitération de la cornée de l'oil droit ; l'ophthalmie chronique et aiguë qui avait précédé cette transformation de tissu; l'insensibilité complète du globe de l'ocil, qui avait supporlé les irritations les plus vives sans que ce malade en eût la conscience, sans qu'il y eût eu lc cliguotencnt des paupieres qui a constamment licu toutes les fois que la sensation la plus légère affecte l'ocil. Je fis observer ensuite que dans toules les expéricnces la membrance pituilaire avait paru insensible, soic qu'on l'ait irritée avre un stylet ou les barbes d'une: 
plume, promenées en divers sens dans la nariné droite. Toutefois, l'odorat n'avait pas' complètement disparu, puisque le malade avait senti les polions éthérées, puisqu'il avait été affecté par l'ammoniaque liquide. Cette différence apparente de ce qu'avait éprouvé le malade et de ce qu'on avait observé chez les animaüx, me parut expliquée par la difficulté qu'il y a à s'assuner si un animal éprouve ou non une sensation, toutes les fois que celle-ci n'est pas portée à un degré qui l'affecte vivement ou désagréablement.

Eu rappelant ce qu'on venait d'entendre sur les altérations des gencives et du goût, j'observai que les effets étaient identiques chez les animaux et chez l'homme; que dans les expériences, de même que dans le cas pathologique, les gencives du côté expérimenté ou du côté malade avaient abandonné le collet des dents et avaient élé profondément alcérées; le goût avait disparu sur la moitié de la langue. Notre malade, dans ces diverses expériences, n'avait senti ni le poivre, ni le sulfate de quinine, que l'on avait placés sur la partie droite à diverses reprises et à des époques éloignées les unes des autres. Ces faits confirmaient donc ce que l'expérience avait appris; et, ce que l'expérience ne pouvait apprendre sur les animaux, ils constataient positivement que cette sensation était anéantie. Il en était de mêrne de l'ouie ; car si chez les animaux il est difficile de s'assurer si ce sens est perdu ou non d'un seul côté, il n'en était pas de 
même chez l'homme: notre malade nous indiqua lui-même l'ćpoque à laquelle la surdité du côté droit commença à se manifester. En définitif, donc, les altérations de l'œil, celles des fosses nasales, de la bouche et del'oreille, se réunissaient pour nous permettre de pronostiquer que nous allions trouver sur le cadavre une altération plus ou moins profon te de la cinquième paire, soit à son insertion snr la protubérance annulaire, soit vers son ganglion.

Désirant donner à notre pronostic le degré de cerlitude que mes recherches antécédentes sur les maladies des hémisphères cérébraux m'avaient appris, je rappelai aux élèves de ma division que les accidens épileptiques de ce jeune homme avaient suivi la même marche que ceux d'une femme décédée quelques jours auparavant, et sur le cadavre de laquelle nous avions trouvé une inflammation chronique dans l'hémisphère opposé au côté sur lequel les convulsions étaient plus prononcées. Il était donc à présumer qu'avec la lésion de la cinquieme paire, nous trouverions de plus une altération plus ou moins grande de l'hémisphère gauche du cerveau.

Cela posé, on procéda à l'ouverture du cadavre. Le crâne fut scié avec le plus grand soin pour ne point ébranler l'encéphale; celui-ci étant mis a découvert, la face interne de la dure-mère parut injectée du côté droit; du côté gauche elle était épaissie, d'un blanc sale; la tente du cervelet, II. 
plus épaisse encore, adhérait à la face supérieure de cet organe. L'encéphale fut détaché de la base du crâne avec la plus grande attention, le tronc de la cinquième paire du côté droit se détacha néanmoins de la protubérance annulaire, par le soul effet du soulèvement de cette dernière partie.

La base du crâne ainsi dégagée parut inégale; elle était plus éiroite à droite qu'à gauche : cette inégalité portait plus spécialement sur la fosse sphénoïdale.

La dure-mère étant détachée de la fosse sphénoïdale droite, nous a percûmes à son côté interne le ganglion du nerf trijumeau dans un état insolite : ce ganglion était boursouflé, d'un gris jaune, une petite quantité de sérosité en séparait les grauulations. A sa partie interne, la portion duganglion d'où se détachait le nerf ophthalmique étaît rouge, injectée; cette injection et cette rougeur étaient partagées par la dure-mère, qui la recouvrait. En arrière du ganglion, les faisceaux nerveux étaient isolés par une petite quantité de sérosité. Les faisceaux internes étaient d'un blanc plus mat que les externes, les uns et les autres étaient un peu ternes; cette disposition faisait ressortir les faisceaux musculaires du nerf trijumeau qui, parfaitement sains, occupaient le côté interne du nerf, ct qui prassaient au-dessous du ganglion, après avoir dépassé la ligne supéricure $d u$ rocher. Tout-à-fait en arrière, le tronc "du nerf qui débordait dans la fosse occipitale était 
jaune comme le ganglion lui-même ; celte couleur se remarquait dans l'étendue de deux lignes environ. Il est à remarquer encore que les filets musculaires ne partageaient point cette altération; ils étaient dans leur état ordinaire; en arrière comme en avant.

L'altération du ganglion et son hypertrophie sc prolongeaient en arant sur ses trois principales divisions; le nerf ophthalmique paraissait le plus anciennement affecté, l'e nerf maxillaire inférieur était un peu plus altéré que le supérieur. Ces trois nerfs étaient d'un jaune terne, dont la coloration contrastait avec celle des nerfs opposés, qui étaient découverts. Ils conservaient cette couleur jusqu'à leur sortie du crâne; au-delà, le nerf ophthalmiqué la perdait avant d'arriver à la fente sphénoïdale : le nerf lacrymal, le nerf frontal et le nerf nasal, nous offrirent $\mathrm{du}$ reste leur structure ordinaire. La couleur jaune du maxillaire supérieur disparaissait tout-à-fait dans la fosse sphéno-maxillaire, les rameaux orbitaires, dentaires antérieurs, postérieurs et supérieurs, et les branches du sousorbitaire, disséqués ávec soin, ne présenlèrent aucun changement dans leur texture ni dans leur organisation; la troisieme branche du nerf trijumeau, ou le nerf maxillaire inférieur, conservait son boursouflement et sa couleur jaune dans le crâne, et en partie dans son trajet dans la fosse xygomatique: dans cette fosse, il paraissait divisé cu deux parties par l'altération dont il avait été le 
siége; une partic interne conservait encore la nuance jaune du tronc, l'externe ne différait pas du nerf du côté opposé. De la première partaient les rameaux dentaire inférieur, lingual et auriculaire; de l'autre se détachaient plus spécialement les rameaux temporaux profonds, -les ptérygoïdiens, le masséterin et le buccal. En disséquant ces derniers rameaux d'avant en arrière, nous aperçûmes quils correspondaient aux faisceaux intacts qui se remarquaient au côté interne et inférieur du ganglion de Glaser. Cet isolement des branches musculaires du nerf trijumeau, produit par cette altération pathologique, est un fait d'autant plus important que l'anatomie normale est loin de pouvoir la mettre en évidence d'une manière aussi marquée.

Le nerf optique droit est, en arrière de l'œil, un peu moins volumineux que le gauche. Dans le reste de leur trajet, ces deux nerfs sont identiques.

La cornée de l'œil droit est opaque et épaissie dans toute son étendue. L'iris est adbérente à sa face postérieure, ce qui détruit l'espace désignć sous le nom de chambre antérieure. La pupille est contractée; la face antérieure de l'iris est couverte d'une fausse membrane blanchâtre, qui adhère à la face postérieure de la cornée. Sur cette dernièrc on aperçoit plusieurs petits vaisseaux formant deux demi-cercles. La choroïde est un peu rougeâtre; l'humeur vitrée paraît moins transparente que dans l'oil gauche. 
La membrane muqueuse nasale est un peu injectée à la narine droite, principalement dans la portion qui correspond au cornet nasal inférieur.

Les gencives sont noires du côté dòroit à la mấchoire supérieure et inférieure. Son tissu, mou, boursouflé, se déchire avec la plus grande facilité. Les dents sont tout-à-fait déchaussées en haut et en bas; le tissu osseux formant les alvéoles supéricures et inférieures, est comme injecté. A gauche, le lissu des gencives est brun, un peu ramolli ; mais l'altération est bien moins profonde que du côté opposé,

La langue ne présente d'abord aucune trace d'altération à sa superficie; mais disséquée avec soin, le tissu muqueux paraît un peu plus mou à droite qu'à gauche.

L'oreille droite ne présente aucune lésion sensible, soit dans son appareil osseux interne, soit dans ses nerfs. Lenerf acoustique, la portion dure de la septième paire dans l'aqueduc de Fallope, la corde du tympan, sont dans leur état normal.

L'encéphale fut examiné avec le même soin que les parties dont nous venons de présenter l'état insolite. Ce qui d'abord frappa nos regards, fut le côté droit de la protubérance annulaire, correspondant à l'insertion du nerf trijumean altéré. A la place de ce nerf qui, comme nous l'avons dit, s'était détaché en soulevant le cerveau, nous trouvâmes une matière gélatincuse, jaune, ana-- 
logue à celle qui existait à l'extrémité du nerf, restée libre au niveau du bord supérieur du rocher. Ecartant ensuite les faisceaux transverses du pont, nous suivîmes les traînées de cette matière jaune, dans l'étendue environ de deux lignes: en même temps, nous remarquâmes, au côté interne de la matière gélatineuse, deux petits faisceaux blancs, intacts, que nous mîmes à découvert jusqu'au bord supérieur du bulbe rachidien. Ces faisceaus élaient la continuation des faisceaux médullaires quiexistaient sur le cốté interne du ganglion sphénoïdal de la cinquième paire. Les filets musculaires étaient donc sains dans toute leưr étendue, ils paraissaient n'avoir point participé à l'altération profonde dont le nerf trijumeau de ce côté avait été atteint. Du côté gauche, le nérf de la cinquième paire était dans son état normal.

En outre, l'hémisphère gauche du cerveau était ramolli, légèrement jaune à sa surface supérieure, principalement en avant et en arrière. Sa face inférieure était tellement adhérente à la dure-mère, qu'une petite partie de la substance corticale resta attachée à cette membrane, au moment où on soulevait l'encéphale pour en considérer la base. Tout le lobe moyen et postérieur parut alors ramolli et jaune; cette alltération s'étendait dans la profondeur du lobe jusqu'au niveau du demicentre ovale du côté gauche. La couche optique et le corps strié du même côté étaient un peu plus moụs que dụ côté opposé. L'hémisphère 
gauche du cervelet offrait une altération analogue à celle de l'hémisphère cérébral du même côté; elle était néanmoins beaucoup moins profonde. Le ventricule latéral gauche était plus étendu que le droit ; la glande pinéale est plus volumineuse et plus dure que dans l'état normal (1).

Les deux poumons sont tuberculeux à leur sommet.

Un fait de cette nature n'a pas besoin d'un long commentaire, pour peu que l'on soit au courant de la marche sévère que présentent de nos jours la physiologie expérimentalc et la pathologie; l'accord de ces deux sciences sur un point si important de leurs recherches devait nécessairement arriver, du moment que M. Magendie avait précisé les effets résultant de la section de la cinquième paire; du moment qu'il élait descendu de la causc aux effeis, la pathologic ne pouvait manquer de remonter des effets à la cause. Mais se serait-on attendu que les effets fussent absolument les mêmes? Se serait-on attendu qu'une maladie de l'homme répétât de point en point tout ce que la physiologic expérimentale avait fait connaître? Disons-le hautement, un tel accord est encore trop rare pour ne pas être signalé à l'attention des physiologistes et des médecins; de ces

(1) Jai fait peindre ces diverses alturations; ces dessins feront partie du grand ouvrage que ie publierai apres celuici sur les maladies organiques du cerveau ct de la moelle épinière. 
derniers surtout, dont la plupart regardent comme oiseuses les recherches expérimentales sur les animaux.

Tout a concouru pour donner à cette nouvelle acquisition de la science toutes les applications que la médecine pourait désirer; car à peine avions-nous constaté par l'autopsie le siége positif de cette maladie, que deux nouveaux malades, un homme et une fernme, se présentèrent lans ma division, offrant les symptômes primitifs que nous avait offerts le jeune homme dont nous venons de tracer l'histoire : rougeur de la conjonclive, insensibilité de la cornée, dureté de l'ouïe, diminution de la sensibilité de la langue, tels élaient les préludes d'une affection qui aurait eu peut-êlre les tristes suites de la précédente, si, éclairé comme nous l'étions, nous n'en॰eussions arrêté la marche. I a femme fut guérie en cinq semaines, et l'homme, sur lequel MM. Magendie et Édwards répétèrent avec nous les expériences, lut rétabli, et sortit après vingt-six jours de séjour dans l'hôpital (1). De semblableś faits, je le répète, n'ont pas besoin de commentaires; ils prouvent que la cinquième paire exerce une influence très-puissante sur l'action des sens, chez

(1) C'est par des faits de cette nature, c'est par des résultats semblables que la physiologie expérimentale doit repousser les allaques sais cesse renouvelées contre elle. et qui portèrent timt d'amerlume daus la carrière scientifique des Harvey, des Malpighi et des Haller. W'ne malarlie it:- 
les mammifères, et même chez l'homme; ils expliquent parfaitement la vision des animaux privés du nerf optique, et pourvus d'un nerf oculair: provenant du rameau supérieur du nerf trijumeau. Ils ourrent un nouveau champ aux études de l'action du système nerveux chez les vertébrés et les invertébrés.

J'ai parlé, dans le premier volume (1), de deux enfans anencéphales chez lesquels les ganglions des nerfs trijumeaux, réunis dans le crâne, servaient d'inserlion aux différens nerts des sens. L'un d'eux vécut dix-huit heures; l'autre deux jours et une nuit; tous les deux prirent le sein de leur mière; tous les deux ouvraient et fermaient les yeux; ils exécutèrent des acies que nous sommes accoutumé à attribuer exclusivement à l'encéphale et que nous pourons expliquer aujourd'hui malgré son absence, puisque les deux ganglions des nerfśs trijumeaux étaient réunis et très-développés.

Ces actes étaicnt purement instinctifs comme ceux qu'exécutent les articulés. Nous pouvons présumer, d'après sela, quc les actes instinctifs sont sous la dépéndance de la cinquième paire, et que

connue signalée et déterminée, deux malades guéris dans l'espace de moins de six mois, et dans une seulc division de l'hôpital de la Pitié, voili les meilleurs argumens ì opposer aux détracteurs de l'analomic comparative et de la physiologie expérimentale.

(1) Page infi. 
le cerveau n'y prend que peu ou point de part. Lorsque l'encéphale disparaît complètement, et que la cinquième paire le remplace, les actes instinctifs devront donc se manifester au plus haut degré. C'est le cas des invertébrés (1).

Toutes leurs déterminations ont un caractère qui les distingue; elles sont purement instinctives : elles sc répètent si exactement, si nécessairement, lors même que les résultats nous paraissent les plus surprenans, qu'on voit manifestement que la volonté n'y est pour rien, et que, conséquemment, leurs actes sont de simples effets organiques. Tous les zoologistes et tous les psychologistes sont d'accord sur ce point; tous considèrent ces animaux comme les êtres instinctifs par excellence. Si cela est, on entrevoit sans peine la conséquence, qui en découle.

Si les crustacés et les insectes sont les êtres instinctifs par excellence, et s'ils n'ont pas d'encé-

(1) Je ne puis m'empêcher, dans l'intérêt de la science, de fini.e remarquer ici, que M. Ampère, auteur des deux articles qui ont paru dans les Annales des Sciences naturelles, sur la détermination du système solide et du système nerveux des animaux articulés, est arrivé, par des considérations psychologiques, ì des vues peu différentes de celles énoncées dans le premier volume de cet ouvrage, et de celles que je développe présentement. (Voy.Ann. sc. nat., tom. III, pag. 202.)

Une réflexion sur laquelle nous devons insister, c'est que M. Ampère et moi, nous sommes parvenus à ces résultats par des roies très-différentes. 
phale, ce dernier organe est donc élranger à l'instinct.

Si leur système nerveux céphalique représente le système ganglionnaire de la cinquième paire, la cinquième paire est donc le siége de l'instinct chez les animaux (1).

C'est dans cette direction que nous paraissent devoir être dirigées désormais les recherches physiologiques sur les facultés et les actes de cet ordre; et si l'on parcourt a'un coup-d'oeil la classe des mammifères, on verra les actes encéphaliques décroître des quadrumanes aux rongeurs, tandis que les actes instinctifs accroissent dans la même proportion. Cola est, et un instant de réflexion montre que cela devait être; car il fallait bien que la perte des facultés encéphaliques fût balancée par l'accroissement des facultés instinctives, jusqu'à ce qu'enfin les facultés encéphatiques venant à disparaître, les animaux pussent vivre sous la seule influence des actes instinctifs.

Or, ces données générales de la psychologie me paraissent en rapport avec les données de l'anatomic comparative, car nous voyonș l'encéphale décroître chez les mammifères en raison directe du développement des actes encéphaliques, et nous voyons les actes instinctifs se développer en

(1) Voy. cncorc à ce sujet les belles idées de M. Ampère, Mémoire déjic cilé, Annales des sciences naturelles, tom. II . pag. 305. 
raison directe aussi du développement de la cinquième paire. De là naît encore le rapport inverse qui s'obscrve chez les nammifères entre l'étendue de l'encéphale et le volume de la cinquième paire: plus l'encéphale diminue, plus la cinquième paire augmente; le développement de la cinquième paire compensc la diminution de l'encéphale; ce que les animaux perdent d'un côté, ils le gagnent de lautre.

Dans un.ouvrage de cette nature, je suis forcé de me restreindre a chaque instant: je ne ferai donc que rappeler l'industrie des chauve-souris, des taupes et des autres mammifères qui sont dans ces conditions organiques, et j'indiquerai seulement que les actes qui ont été attribués à une iutelligence supéricure ne sont que des effets instinctifs en rapport avec le développement que présente la cinquième paire chez tous ces animaux.

J'expliquerai de la mêmie manière l'industrie des phoques et des castors, qui les a fait placer si haut, par ceriains psychologistes, dans l'échelle de lintelligence. Les actes qui chez ces animaux scmblent justifier cotte assertion, découlent de la même source que ceux des abeilles, dont ceux-ci ne sont qu'une bien faible imitation; l'anatomie comparative peut, je crois, en fournir la preuve.

Si les actes des phoques et des castors étaient des ofrels encéphaliques, ces animaux devraient 
ètre sur la même ligae pour le développement de l'encéphale; le castor devrait même occuper un rang plus élevé que le phoque. Or, comparez lcur encéphale, et voyez quellc distance immense sépare ces animaux sous ce rapport: le phoque égale presque les quadrumanes par l'étendue de son encéphale, et le castor est descendu jusqu'aux rongeurs par les formes imparfaites du mème organe; l'un est aux degrés inférieurs de l'échelle, l'autre en occupe les marches supéricures. Deux organes si différens ne peuvent donc produire un ordre d'effets si rapprochés.

Mais si ce sont des effets instinctifs, et si la cinquième paire en est l'organc producteur, tout s'explique : ces animaux, si éloignés par leurs autres caractíres, se touchent par celui-ci. Les phoques et les castors sont remarquables par le développement du nerf trifacial; le castor surtout dépasse tellement tous les mammifères par le volume et l'éteridue de ce nerf, que nul ne peut lui être comparé sous ce rapport; nul aussi ne peut être mis sur sa ligne pour les facultés instinctives. Ils sont aux vertébrés ce que les abeilles sont aux invertébrés; et chez les uns comme chezles autres, les effets sont proportionnés à la cause, les actes sont en rapport avec l'organe qui les produit.

Si l'on se rappelle maintenant que le système nerveux se développe de la circonférence au centre, on trouvera peut-être, daus la formation primitive de la cinquiène paire, la raison pour laqualle les 
actes instinctifs se manifestent constamment chez les animaux avant les actes encéphaliques; on expliquera de cette manière aussi comment des fotus anencéphales ont pu exercer certains actes, tels que la succion du lait de la mère, quoiqu'ils fussent privés de cerveau. Il est peu d'anatómistes. qui n'aient été dans le cas d'observer ce phénomène une fois au moins dans le cours de leur vie. Or, il est inexplicable dans la théorie qui fait partir ces actes de l'encéphale; et il se trouve d'autant mieux expliqué dans nos idées, que personne n'ignore que, chez les monstres anencéphales, la cinquième paire est d'autant plus développée que l'encéphale est plus imparfait, ou que mêmo il manque complètement.

Tous les physiologistes et les psychologistes ont échoué dans leurs tentatives pour ramener les facultés instinctives aux facultés de l'encéphale. L'admirable travail des abeilles et des arachnides supposerait une intelligence à laquelle ne s'élèvent jamais les animaux les plus élevés de la classe supéricure des vertébrés, supérieure même à celle ¿̀ laquelle pourrait parvenir un homme isolé, s'il cherchait à exécuter un travail si géométriquement parfait. Or, voyez la contradiction dans laquelle on se trouvait nécessairement entraîné par ces idées! On convenait, d'une part, que les insectcs étaient millc fois plus descendus que le dernier des vertébrés, relativement à leur cerveau, et l'on attribuait en même temps à cet organe des 
effets mille fois supérieurs à ceux que produisent les vertébrés les plus favorisés par l'étendue de leur encéphale : une contradiction si manifeste ne pouvait être dans la nature; elle n'existait que dans nos idées.

J'ajouterai une dernière considération à ce sujet: c'est que l'homme, auquel nul être ne peut être comparé pour le développement de l'encéphale et de ses facultés, est le plus descendu de tous par ses facultés instinctives, parce qu'il est le plus inférieur de tous, relativement au volume proportionnel de la cinquième paire. Je remarquerai ensuite que l'abeille est à la tête des invertébrés par ses facultés instinctives, parce que ses ganglions crâniens sont les plus développés : elle occupe, sous le rapport des ganglions de la cinquième paire, le haut de l'échelle anatomique des invertébrés, comme, sous celui de l'encéphale, l'homme occupe le sommet des animaux vertébrés.

De tout cc qui précède nous pouvons donc conclure :

$1^{\circ}$. Que, chez les arimaux vertébrés et l'homme, la cinquième paire exerce une influence très-marquée sur l'action des sens;

$2^{\circ}$. Que la taupe est privée de nerf optique, comme la chrysochlore du Cap, le rat-taupe, le protée, etc., et que cette absence n'empêche pas chez elle l'cxercice de la vision;

$3^{\circ}$. Que la cinquième paire paraît être le siége de l'instinct ou des actes irréfléchis chez les animaux; 
$4^{\circ}$. Que, chez les animaux vertébrés, le déve. loppement de l'instinct paraît en rapport avec celui de la cinquième paire;

$5^{\circ}$. Que les ganglions céphaliques des animaux articulés étant les analogues de ceux de la cinquième paire, les propriétés instinctives sont les plus développées chez ces êtres;

$6^{\circ}$. Enfin, que les vues nouvelles que pourront nous dévoiler les expériences physiologiques sur le système nerveux des invertébrés, doivent être comparées à ceux de la cinquième paire et des ganglions intervertébraux chez les animaux vertébrés. 


\section{CIIPITRE III.}

Anitomic comparative de lie moelle eppiniors, dans les quatrc classes d'animaux verlébrés.

Le système nerveux des inverlébrés n'est lonc que le système nerveux latéral des vertébrés. Chez ces derniers, un appareil nouveau vient en former le centre; cet appareil est formé de la moelle épinière et de l'encéphale : l'axe cérébro-spinal, nom par lequel je l'ai désigné, est donc exclusivemunt dévolu aux animaux vertébrés (1). Ce que nous avons à dire de ses formes permanentes, de sa structure, de ses rapports, de ses fonctions et de ses maladies, n'est donc applicable qu'aux quatre classes qui composent l'embranchement supéricur du règne animal. Que d'efforts il a fallu faire avant d'arriver à ce résultat! de cơmbien d'inconnues, de combien de fausses idées il a fallu débarrasser la science, pour préciser avec quelque rigueur les termes dont devait se composer le problème de l'anatomie comparée du cerveau!

(1) M. le professeur Latreille a heurcusement cmpleyé rette idée, en désignant les animaux vertébrés sous le nom de spini-cirébraux. (Voyez Familles naturelles du rigne animal, pag. 38.) 
L'axe cérébro-spinal du système nerveux est le point de convergence et de divergence de tout l'animal; il répète en petit les caractères principaux des modifications infinies que nous offrent les êtres composant les quatre classes des veriébrés. Son anatomie comparative exigeait, en quelque sorte, un abrégé de toute l'anatomie comparée et les.traits les plus saillans de la zoologie; car touies les parties de l'organisation de l'animal se licnt les unes aux autres, sc correspondent et s'expliquent mutuellement. Comment concevoir en effet, et apprécier à leur juste valeur les modifications nombreuses de la moelle épinière, si on les sépare des modifications principales que nous offre le tronc des invertébrés? A quoi nous cût servi de dire, que tantôt le calibre de cette parlie est égal dans toute son étendue, que tantôt il est renfié çà et là, si nous n'avious signalé en même temps à quelles dispositions générales $d \mathbf{u}$ tronc correspondaient ces renflemens ou leur absence? Quel intérêt la science eût-elle retiré de nos observations diverses sur les variations de l'encéphale, si nous n'avions simultanément exposé les principales variations des organes des sens? Un animal étant donné, déterminer, par l'examen extérieur de ses formes, les formes ct les rapports de l'axe cérébro-spinal de son systéme nerveux : tel doit étre le but définitif de cette partie de la science.

Mais que de routes détournées il nous a fallu prendec pour prourer l'utilité de cette nouvelle 
manière d'envisager l'anatomie comparative! que de rapports organiques il a fallu constater, avant d'arriver aux rapports correspondans que nous répète l'axe cérébro-spinal! Ostéologic, myologie, angiologie, névrologic, il nous a fallu tout examiner, tout comparer, pour en rallier les faits principaux aux variétés de cet axe.

Depuis Démocrite, les philosophes sont à la recherche des fonctions du système nerveux; depuis Hippocrate, les médecins poursuivent sans relâche l'étude de ses maladies. Trois mille ans de veilles ont échoué devant cettc noble tentative; le génie même en avait désespéré, parce que la pathologie attendait de la physiologic les données qui lui étaient indispensables, et que la physiologie demandait à son tour à l'anatomie comparative les bases sans lesquelles elle ne pouvait avancer avec certitude. Anatomie générale, physiologie, pathologie du systèmé nervcux, tout devait donc s'éclaircr en même temps, parce que toutes ces sciences s'étaient arrêtées par les mêmes causes : telle était sans doute la penséc de l'Académie des Sciences, lorsqu'on mit celte question au concours, puisque de toutes les parlies de l'Europe les ouvrages qu'elle a fait éclore portent l'empreinte de ce triple caractère. Cict accord unanime des ana lomistes et des physiologistes prouve unegrande vérité, c'est que nous tcnons les fils de cc labyrinthe qui avait paru incxtricable à nos péres, et qu'on persévérant dans les roules tracées, nous 
pouvons espérer d'en connaître uu jour tous les détours. Lancé dans cette carrière depuis un si grand nombre d'années, je n'ambitionne d'autrc gloire que celle de coutribuer par mes efforts à atteindre ce grand résultat.

La détermination de la moelle épinière n'a été le sujet d'aucune contestation chez les animaux vertébrés; encaissé dans le canal de conjugaison que forment les vertèbres par leur réunion, cette position fixc et l'homogénéité de ses furmr's dans toutes les classes n'ont offert aucune prise aux conjectures diverses dont certaines parties de l'encéphalc ont été l'objet. Considéréc dans l'ensemble des quatre classes, clle offre dans ses dimensions des variétés nombreuses et des rapports très-importaus, rélativement à l'appareil locomoteur des animaux : c'est donc sous ce double point de vue que l'anatomie comparative doit la considérer.

Au premier aspect, la moelle épinière paraît un organe unique ou impair : sa forme est celle d'uu cylindre plus ou moins aplati, en avant, en arrière ou sur les côtés, selon les classes où on l'observa; plus ou moins étendue dans le canal vertébral, selon les classes, les familles et les espèces; d'un calibre égal ou différent dans ses diverses régions, selon les conditions locomotrices des animaux; creuse ou solide; toujours fermée en avant dans toutes les classes sans exception; ferméc aussi en arrière le plus généralement, mais ouverte chez les oiseaux dans la partie qui corres- 
pond au renflement inférieur ( 1 ), et chez les reptiles en haut de la région cervicale (2). Ces formes permanentes dérivent de ses formes primitives, et ces dernières ront nous en fournir toute l'explication.

l'hypothèse du développement central de la moelle épinière ne pouvait se concevoir avec l'existence d'un canal daus l'étendue de son axe: un vide ne pouvait être, dans celle illée, la sourc primitive de tout le système nerreux. C'est cn vain que Charles litienne, Colombo, Piccoluomini, Bauhin, Bartholin, Valsalva, Morgagui, Haller et Portal, disaient l'avoir observé, même chez l'homme, dans toute l'étendue de la moclle épiuière, ou dans une de ses régions seulement: l'existence de ce canal a été niée jusqu'à ces derniers temps, ct elle a élé niée parce qu'elle r'était pas conçue et que sa formation ne pouvait ètre expliquée.

D'après nos lois expérimentales de l'organisation, la manifestation de ce canal dans le centre de la moelle épinière est aussi inćvitable chez lous les vertébrés, que sa formation cst impossible dans l'axe nerveux des iuvertébrés. La's lames de la moclle épinière, qui succident au liquide qui la constitue dans les preniers temps chez tous les cmbryons, réunies on avont d'ahorl.

(1) I', Ir, fir. $75,11 \times 2$

(2) V.I, fig. 1\%, 10 万. 
puis en arrière, laissent nécessairement un espace entre elles; cet espace occupe le centre des lames roulées sur elles-mêmes, et forme l'axe de ce canal. Or, on conçoit que, pour contenir ce liquide et pour favoriser la marche des lames qui lui succèdent, il était nécessaire que la dure-mère formât un étui pour les contenir; il fallait, de plus, que cet étui fût favorisé lui-même par le canal cartilagineux des vertèbres. De là, l'ordre des développemens de ers trois parties. D'abord, le canal cartilagineux se développe; puis vient celui de la dure-mère; puis, en troisième lieu, celui de la moelle épinière. Chez tous les jeunes embryons, le rachis est dès-lors composé de trois cylindres creux, emboîtés les uns dans les autres, et qui sont, en procédant de la circonférence au centre, le canal vertébral, alors cartilagincux, le canal de la dure-mère, et le canal de la moelle epinièrc. Les lames du premier cylindre sont cartilagincuses; les vertèbres doivent en provenir : celles du sccond sont fibreuses; celles du troisième sont deux membranes nerveuses. Rien de scmblable n'existant chez les invertébrés, l'abscnce du canal dans leur axe nerveux provient donc de cotte différence primilive du systèrae nerveux.

D'après le mécanisne de la formation du canal épinien, on voit que plus les cmbryons sont jeunes, plus les lames de la moelle épinière sont minces, flus conséquemment le caual doit être 
large : c'est ce qui se remarque chez l'embryon des oiseaux, les septième, huitième, neuvième et dixième jours del'incubation; chez le tétard des batraciens, jusqu'aux trentième et trente-cinquième jours de leur formation; chez les singes, le chien, le chat, le mouton, le veau et le cheval, jusqu'au milieu de leur formation; chez l'homme, du troisième au cinquième mois de la conception; chez le lapin, jusqu'aux deux tiers de son développement.

Le canal de la moelle épinière se rétrécit donc à mesure que les lames nerveuses qui en forment les parois acquièrent de l'épaisseur : or, l'épaississement de ces lames s'opérant de la circonférence au centre, le rétrécissement du canal est en raison directe de l'épaisseur des nouvelles lames qui sont déposées par les artères qui pénètrent dans l'intérieur du canal. Enfin, toutes les couches fibreuses de la moellc épinière étant sécrétées, le centre du canal est oblitéré en totalité ou cn partie par les couches de matière grise appliquées sur la face interne des lames de la moelle épinière.

Cette dernière circonstance explique l'absence ou la permanence de ce canal chez les animaux adultes; clle explique aussi les différences de sa capacité, selon les classes ou les familles : plus les couches de la matière grise sont épaisses, plus le canal est étroit, chez les animaux paŕaits. Or, la matière grise de l'intérieur de la moclle épinière allant en diminuant d'épaisseur de l'homme aux 
singes, aux cétacées, aux phoques, aux cirnassiers, aux ruminans et aux rougeurs, la capacité du canal doit augmenter dans la mème progression.

La capacité du canal de la moelle épinière est donc en raisou inverse de l'épaisseur de la conche grise quicn occupclc centre. Chezl'homme adulte colle couche étant trés-ćpaisse, le canal est complètement oblitéré; chez les singes, la couche grise ayaut un peu diminué, le canal n'est pas herméliquement fermé : on en trouve les rudimens chez lc drill, le mandrill, je papion.

Chez lo damphim, le marsouin et les phoques, if est plus large que chez les singes; son diamitre

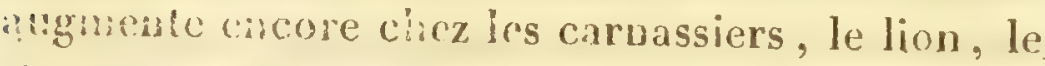
igre, la panthere, le luup, l'hyene, le reuard, le chat, le chicu, l'ours brun, l'ours noir d'Amérique, elc. parce que la couche de matière gri ic est moins apaisse cicz ces derniers animaux que chez le phoque et les cétacées.

C'est toujours i cause de la décroissance de la matière grise que les pachydermes el les ruminans. out ce canal plus dilaté encore que les carnassiers. comme je l'ai observé chcz le cheval, le chameau, le lama, le nouton, le bouf, les chèvres. Linfu, les rongeurs sont, de tous les mammifères; ceux qui ont le canal de la moelle épinière le plus large, parce que ce sont ceux chez lesquels la couche. grise est la plus mince : c'est donc clrez les rongeurs, lelis que Ios licres, les lapins, les rats, Ito 
castor, l'agouli, qu'il faut observer ce canal, pour en avoir une idée exacte dans cette classe.

Si ces principes sont justes, c'est-à-dire, si, d'une part, la capacité du canal épinien augmentè en raison inverse de l'épaisseur de la couche grise, et si, de l'autre, cette couche se dépose de la circonférence au centre, on voit que les cmbryons des mammifères supérieurs offriront, à leurs diverses périodes, les capacités différentes que cc canal noús présente dans les direrses familles. Le raisonnement indique que cela doit ètre; l'observation prouve rigoureusement que cela est.

Soit l'embryon humain : observé au troisième mois, le diamétre du canal épinien égale au moins celui des rongeurs; au quatrième, sa capacité se rapproche de celle des pachydermes et des rımiuans; au cinquième, son diamètro rappelle celui des carnassiers; au sixième, c'est celui des phoques 't des cŕtacées; au septième, le canal n'est plus que rucimentaire, comme on le remarque chez les singes : enfin, au huitième ct au neuvième, il cst complètement oblitéré; circonstance qui se remarque exclusivement chez l'homme, parce que l'homme adulte est celui de tous les nammiferes qui a le plus de matière grise dans le centre de lit moellc épinière. Les cmbryons des singes, des carnassiers et des ruminans répètent exactement certe disposition : plus ils sont jennes, phus leur canal bpinion est dilaté; plus ils se rapprochent. 
sous ce rapport, des familles qui leur sont inférieures.

J'ai remarqué, en 1820 , que chez les animaux composant les trois classes inférieures, la matière blanche de la moelle épinière prédomine tellement sur la matière grise, que celle-ci forme souvent une couche imperceptible chez certains reptiles et certains poissons (1). Quelle est la conséquence de ce fait relativement au canal de la moelle épinière? On l'entrevoit de suite : on voit que, chez les oiscaux, les reptiles et les poissons, la capacité du canal épinicn devra dépasser encore celle qu'on lui remarque chez les rongeurs. C'est, en effet, ce qui est, toute proportion gardée, ainsi que je l'ai constaté chez l'orvet, les vipères, les couleuvres, le caméléon, le tupinambis, les crocodiles, le caïman, les tortues, les crapauds et les grenonilles, parmi les reptiles; les passereaux, Is perroquets, les canards, la poule, les cigognes, la bondrée, l'aigle, l'au-

(1) Cette disposition a été découverte aussi en 1822 che les poissons par M. Desmoulins. Cet anatomiste distingué a été conduit à ce fait par des rues bien différentes de celles que j'expose ici. Je remarque cettc circonstance pour deux motifs; le premier, pour montrer que lorsque les faits sont exacts, les obscrvations y amènent par différentes voies; ct le second, pour ne rien ôter à M. Desmoulins du mérite de son observation; car je n'avais rien publić des miennes al l'époque ou elle parut. 
truche, le casoar, parmi les oiseaux; les raies, les squales, l'esturgeon, les trigles, le congre, la morue, le brochet, la carpe, le merlan, la perche, la bandroie, le turbot, l'anguille, parmi les poissons.

La moelle épinière est donc formée, chez tous les vcrtébrés, de deux cylindres enchâssés en quelque sorte l'un dans l'autre; l'extérieur est formé par la matière blanche, l'intérieur par la matière grise, brune ou rougeâtre, selon les animaux où on la considère, ou l'âge des individus dans la même espèce. Ces deux cylindres ne sont point développés en raison directe l'un de l'autre, comme on l'a dit, et comme l'ont supposé plusieurs des systèmes imaginés pour expliquer les fonctions. du systeme nerveux; ils se trouvent, au contraire, lans touies les classes, dans une proportion inverse. Plus on descend de l'homme aux rongeurs, et de ceux-ci aux oiseaux, aux reptiles et aux poissons, plus l'épaisseur du cylindre extérieur ou de la maticre blanche augmente, plus le cylindre intéricur diminue. Si on se rappelle maintenant que le volume des nerfs spinaux décroît progressivement de l'homme aux poissons, on aura une nouvelle et derniere preuve que la maticre grise n'entre pour ricn dans lcur développement, dans leur origine, dans leur nutrition ou dans lcur renforcement.

On mesure l'épaissour de ces denx cylindres et Inar disposition relative, par une section trans- 
versale dans un ou dans plusieurs des segmens dela moclie épinière, ainsi que l'ont pratiqué Blasius, Hygmore, Winslow, Monro, Huber, Lieutaud, Haller, Soemmering, Chaussier, Rolando. Cette disposition varie selon les classes, les familles et les espèces; elle varie même selon les régions où on la pratique chez le même individu. Ces variétés sont soumises aux formes de la moelle épinière: c'est pour n'avoir pas fait cette distinction que les autcurs ont tant varié les uns des autres sur la forme qu'affeclait la matière grise dans l'intéritur de la moelle épinière.

Dans les animaux chrz lesquels la moelle épinière est un cylindrc arrondi, comme chez les poissons osseux, chez les ophidiens ef les chéloniens parmi les reptiles, et chez tous les oiseaux, dans sa plus grande étendue, la malière grise offre uno forme cruciale, dont la partie moyenne correspond aux denx sillons. Cette disposition est lit même dans toutes les régions où la moclle épinière est arrondie : aiusi, on la remarque toujours tell: dans la régioñ dorsale de tous les mammiferes et de. l'homme, et duns la région caudale de tous ceux chez lesquels la moelle épinière se prolonge audola des vertèbres lombaires.

Au contraire, les animaux chez lesquels le cylindre épinien est aplati d'avant en arricre, comme chuzles poissons cartilagincux et les batraciens, la disposilion de la malite grise affecte la forme d'un croissuml ou se rapproche do la forme de l'os 
hyoüde de l'homme : celte disposition est la mene cincz tous les animaux dont les parlies ou la moelle épinière s'aplatit. Ainsi, on la retrouve c̀ la région cervicale de tous les mammifères et de l'homme; on la retrouve dans les renflemens supérieur et inférieur des mảmmifères et des reptícs, et seulement très-prononcéc dans le renflement inféricur des oiseaux, leur renflement supérieur conservant toujours une forme cylindrique. La disposition générale de la matière grise est donc assujétie aux formes diverses de la moelle épinière.

La moelle épinière est donc plus'ou moius arrondie ou plus ou moins aplatie d'avant en arrière, selon les animanx et selon les réǵions des mèmes animaux où on l'observe. Quoiqu'elle ne remplisse pas exactement le canal vertébral, néanmoins la variété de ses formes est rigoureusement assujétie à celle du trou des vertébres qui l'environnent. Ainsi, le trou vertébral est arrondi chez tous les ophidiens, dont la moelle épinière est un cylindre assez régulier : il est arrondi dars toutes les vertèbres dorsales ct caudales des mammifères, dans les vertèbres dorsales et cervicales des oiscaux, parties chez lesquelles la moelle épinière se rapprochè du cylindre régulier des ophidiens. Le trou vertébral est, au contraire, triangulaire dans toule l'étendue de la colonne vertébrale des batraciens, dans les vertèbres cervicales et lombaires des mammifères, dans les vertèbres 
qui encaissent le renflement inférieur des oiseaux, des chéloniens, des lézards, des crocodiles, toutes parties sur lesquelles la moelle.épinière s'aplatit d'avant en arrière.

De là dérivent plusieurs des rapports importans de la moelle épinière : plus l'anneau que forme la vertèbre est grand, plus le segment de la moelle épinière qui lui correspond est volumineux, plus les vertèbres dorsales sont développées, plus les côtes qui viennent se placer sur leurs côtés sont prononcées, plus les muscles, plus les nerfs intercostaux, plus les arlères intercostales, sont volumineux. On voit donc que chaque segment de la moelle épinière est développé en raison directe du volume des vertèbres qui l'environnent; celles-ci en raison directe des côtes qu'elles supportent; les côtes en raison directe des muscles qui s'y insèrent; les muscles en raison directe des artères et des nerfs. Tous ces rapports se suivent, se lient, s'enchaînent; ils dérivent tous les uns des autres. Ce qui est vrai pour un segment de la moelle épinière, pour la vertèbre qui la revêt et ses dépendances, est également exact pour tous les segmens, pour toutes les vertèbres, pour toutes les parties qui les environnent.

De ces rapports partiels résulte donc le rapport général suivant: le volume de la moelle épinière est rigoureuscment proportionné au volume des différentes régions du corps de l'animal. Ainsi, plus le tronc d'un animal est volumineux, plus 
la région dorsale de la moelle épinière est volumineuse; plus la queue est forte, plus la région caudale est développée; plus le col est développé, plus la région cervicale de la moelle épinière est développée aussi.

Les anguilles (1), les lamproies (2), chez lcs poissons; le protée, l'orvet (3), les vipères (4), les couleuvres (5), chez les reptiles, ont le pl:1s petit corps, les plus petits nerfs, et la moelle épi-: nière la plus grêle.

Chez les autres poissons osseux et cartilagineux, l'augmentation de volume de la moclle épinière suit la progression de la masse du corps et des nerfs, ainsi qu'on le remarque chez le rouget (6), le gronau ( 7 ), la morue ( 8 ), le brochet (9), la tanche (10), le merlan (11), le turbot (12), etc., parmi les osseux; et chez la raie ronce (13), la raie

(1) Pl. VII, fig. 190, $\mathrm{n}^{\circ} 1$.

(2) Pl. II, fig. $226, \mathrm{n}^{\circ} 1$ et 2.

(3) Pl. V, fig. $109, \mathrm{n}^{\circ} 1$.

(4) Pl. V, fig. $126, \mathrm{n}^{\circ}$ a et $\mathbf{3}$.

(5) Pl. V, fig. $100, n^{\circ} 5$ et 6.

(6) Pl. VII, fig. $158, \mathrm{n}^{\circ} 6$.

(7) PI. VII, Gig. 155, n० 1 et 2.

(8) Pl. VII, fig. $168, \mathrm{n}^{\circ} 4$.

(9) Pl. VII, fig. $169, \mathrm{n}^{\circ}$.

(10) Pl. VII, fig. $187, n^{\circ}$.

(i1) Pl. VII, fig. $195, \mathrm{n}^{\circ} \mathrm{s}$

(12) Pl. VII, fig. 191, n०14.

(13) Pl. VI, fig. $138, n^{\circ} 1$. 
bouclée (1), l'ange (2) et le requin (3), parmi les cartilagineux. Mais parmi ceux-ci, l'esturgeon (4) fait à ce principe une singulière exception : il est remarquable par le volume de son corps et l'exiguité de sa moelle épinière.

La moelle épinière et le tronc suivent le même rapport chez les reptiles, comme chez le caméléon (5), le tupinambis (6), le crocodile vitlgaire (7), le crocodile à deux arêtes (8), la tortue grecque (9) et la tortue franche (10).

On trouve le même résultat chez les oiseaux, en suivant la progression de la moelle épinière dư roitelet (11) à l'autruche (12) et au casoar (13). On la voit augmenter progressivement chez l'hirondelle. (14), le verdier, les moineaux, les pigeons, les perdrix, la poule (15), les faisans, le

(1) Pl. VI, fig. $162, n^{\circ} 1$.

(2) Pl. VI, fig. $149, n^{\circ} 1$.

(3) Pl. VI, fig. $142, n^{\circ} 1$.

(4) PI. XII, 6g. 235, A

(5) Pl. V, fig. $111, \mathrm{n}^{\bullet} 1$ et 2.

(6) $\mathrm{Pl} . \mathrm{V}$, fig. $114, \mathrm{n}^{\circ} 5$.

(2) Pl. V, flg. $115, \mathrm{n}^{\circ} 1$.

(8) Pl. V, fig. $116, \mathrm{n}^{\bullet}$ 1.

(9) PI. V, fig. $125, \mathrm{n}^{\circ} 5$.

(10) Pl. V, fig. $119, \mathrm{n}^{*} 1$ et 2.

(11) $\mathrm{PI} . \mathrm{IV}$, fig. $94, \mathrm{n}^{\circ} 1$.

(12) Pl. IV, fig. $9^{8}, n^{\circ} 1$.

(13) Pl. III, fig. 83, $\mathrm{n}^{\circ}$ i.

(14) Pl. IV , fig. $9^{2}, \mathrm{n}^{\circ} 1$.

(15) $\mathrm{Pl}$. III, fig. $87, \mathrm{n}^{\circ}$. 
perroquet (1), la bondrée (2), la cicogne (5), et l'aigle (4).

Chez les mammifères, le volume du tronc et des nerfs correspond rigoureusement à celui de la moelle épinière. Les ruminans et les pachydermes, qui ont le corps le plus gros, ont la moelle épinière la plus forte, ainsi qu'on le voit chez le chameau (5), le cheval (6), l'âne, le boeuf, le mouton, le lama (7), le pécari (8), le bouc de la Haute-Égypte (9). Les carnassiers, dont le corps est plus svelte, ont aussi la moelle épinière moins forte, ainsi qu'on peut le remarquer chezl'ours (10), le raton (11), le lion (12), le coati (13). Chez les rongeurs, en général, la moelle épinière prédomine sur le volume du corps, si on les compare aux animaux précédens, ainsi qu'on le voit chez

(1) PI. III, fig. 8z, $n^{n}$.

(2) Pl. IV, fig. 91, $\mathrm{n}^{\circ} 1$.

(3) Pl. IV, fig. 99, $\mathrm{n}^{\circ} \mathrm{1}$.

(4) Pl. IV, fig. $101, \mathrm{n}^{\circ} 1$ et 2.

(5) PI. XIII, fig. 248, B.

(6) Pl. XV, fig. $274, \mathrm{~A}$.

(7) Pl. XVI, fig. $295, \mathrm{n}^{\circ} \mathrm{i}$.

(8) PI. XVI, fig. $300, \mathbf{n}^{\circ} 11$.

(9) Pl. XIV, fig. $261, n^{*} 1$.

(10) PI. XI, fig. $230, A$.

(1i) PI. VIII, fig. 202, A.

(12) PI. XIV, fig. 264, no 1 .

(13) Pl. XII, fig. 240, A.

II. 
le castor (1), le porc-épic (2) et l'agouti (3). Les phoques (4) et les cétacées (5) ont la moelle épinière, surtout dans la région cervicale, plus développée que ne le comporterait la masse du corps; mais cette prédominance tient peut-être à l'absence ou à la faiblesse des renflemens épiniens.

Enfin, chez les quadrumanes (6) et l'homme, la moelle épinière, les nerfs du tronc et le volume du corps sont dans le même rapport que chicz les autres mammifères.

Remarquons, à ce sujet, un contraste bien frappant entre les régions cervicale et caudale des mammifères et des oiseaux. Chez les oiseaux; la longrieur de la région caudale est fixe; chez tous, elle est composée du même nombre de vertèbres, et la moelle épinière se termine d'une manière presque uniforme; chez les mammifères, au contraire, rien de plus variable que la queue : elle diffère d'étendue, de volume, de forme; la moelle épinière partage toutes ces modifications. Le nombre de ses vertèbres est illimité, comme l'étendue des cordons nerveux qui les protègent : l'inverse se remarque dans la région cervicale.

(1) Pl. XIV, fig. $265, \mathrm{n}^{\circ}$.

(2) Pl. XIII, fig. $252, \mathrm{~A}$.

(3) Pl. IX, fig. 213, A.

(4) Pl. X, fig. $216, \mathrm{n}^{\circ} 1$; pl. IX, fig. $209, \mathrm{~A}$.

(5) Pl. XI, fig. 225, A.

(6) Pl. VIII, fig. $19 \vec{b}, \mathrm{~A}$; fig. $199, \mathrm{~A}$. 
Tous les mammiferes, à une ou deux exceptions près, sont fixes, quant au nombre des vertébres de cette partie et à la longueur de la moelle épinière qui lui correspond; les oiseaux, "au contraire, ont leur région cervicale aussi variable que la région caudale des mammifères : la longucur du col des oiseaux est aussi illimitée que la longueur de la queue des mammifères, te nombre des vertèbres cervicales est aussi variable chez les premiers que celui des caudales chez les seconds. La moelle épinière est effilée dans la région cervicale des oiseaux, comme elle s'effile au travers du canal caudal des mammifères : de là naît le contraste qui se rcmarque entre le volume de cette région dans les deux classes; très-forte et d'une étendue presque uniforme dans l'une, très-grêle et d'une étendue très-variable dans l'autre. Si la queue devient un puissant auxiliaire chez les mammifères, on n'a qu'à remarquer tous les mouvemens de la tête des oiseaux, les directions diverses que leur col lui imprime, pour apprécier chez eux tous ləs avantages de cette organisation. Et pour expliquer tous ces mouvemens, on n'a qu'à comparer la myologie cervicale des oiseaux à celle des mammiferes : on trouvera chez les premiers des muscles distincts, très-isolés, quant à leurs interstices, et l'on verra chez les mammifères les muscles confondus les uns avec les autres. La myologie cervicale des mammifères ne peut être expliquée que par celle des oiseaux : les digita- 
tions des inter-épineux cervicaux, des inter-transversaires, des complexus, des splénius, des scalènes, ne sont autres que les rudimens des muscles qui se rencontrent distincts chez les oiseaux, et qui permetteht à leur col cette diversité et cette précision de mouvemens qu'on admire chez eux.

Telles sont les métamorphoses de la moelle épinière, et les rapports qui en résultent. La plus singulière de toutes est peut-être celle de son ascensiou dans le canal vertébral, avec les effets qui en dérivent relativement au prolongement caudal des mammifères.

Chacun sait, et j'ai déjà dit, que l'embryon humain a un prolongement caudal qui persiste jusqu'au troisième mois de la formation; on sait aussi que jusquà cette époque la moelle épinière descend jusqu'au coccyx ou au sacrum. Du troisième au quatrième mois, la queue disparaît, et elle disparaît parce que la moelle épinière remonte dans son étui et s'arrête au niveau du corps de la deuxième ou troisième vertèbre lombaire; et si l'ascension de la moelle épinière n'a pas lieu, la queue persiste même après la naissance.

Il y a donc un rapport entre l'ascension de la moelle épinière et la persistance du prolongement caudal : plus la moelle épinière s'élève dans son étui chez les mammifères, plus la queue diminuc d'étendue, comme dans le cochon, le sanglier, lc lapin, le lièvre, et dans plusieurs espèces de singes; plus elle descend, au contraire, plus la 
queue se prolonge, comme dans le bœuf, l'écureuil.

L'embryon des chauve-souris sans queue ressemble, sous ce rapport, à celui de l'homme. Il a d'abord une queue, qu'il perd rapidement, parce que chez cet animal l'ascension de la moelle épinière est très-rapide.

C'est surtout chez le tétard des batraciens que ce double rapport est remarquable. Aussi longtemps que la moelle épinière se prolonge dans le canal coccygien, le tétard conserve sa queue : à l'époque où il se métamorphose, la moelle épinière remonte dans son canal, la queue disparaît, et les membres se prononcent de plus en plus. Si la moelle épinière s'arrête dans cette ascension, le batracien conserve sa queue, comme les chauvesouris, comme l'homme le font aussi dans le même cas. Le foetus humain et celui des chauve-souris se métamorphosent donc, sous ce rapport, comme le tétard des batraciens : ils perdent leur queue lorsque les membres acquièrent de la force.

En général, chez les reptiles et les poissons, plus les membres et les nageoires sont grèles, plus la queue est étendue, plus la moelle épinière est longue.

Le rapport de l'ascension de la moelle épinière avec le prolongement caudal offre plusieurs exceptions. Les hérissons, parmi les mammifères, ont une queue courte, quoique la moelle épinière se prolonge chez eux plus que ne le comporte cette brièveté du prolongement caudal; mais cette 
circonstance tient au développement de leur manteau. Parmi les poissons, la baudroie, la lamproic, l'esturgeon, le tétrodon-lune, ont la moelle épinière très-élevée dans leur canal épinien; quoique leur queue soit très-prolongée. Celte exception, non encore expliquée, tient peut-être à la disposition du système artériel (1).

Quoi qu'il en soit, cette ascension de la moelle épinière dans son étui rend parfaitement compte de la formation rle la queue de cheval : lorsque la moelle épinière s'étend jusqu'áu coccyx, chez l'homme, les batraciens et les chauve-souris, les nerfs lombaires, sacrés et coccygiens s'y implantent horizontalement, comme chez les animaux qui ont un prolongement caudal très-prononcé; mais à mesure que la moelle épinière remonte, l'insertion de ces nerfs devient de plus en plus oblique et s'éloigne de plus en plus. Enfin, quand la moelle épinière s'est arrêtée, les nerfs la remplacent dans la partie du canal vertébral qu'elle a laissée vide : ils forment, par leur adossement et leur direction, d'abord parallèle, puis divergente, un gros faisceau filamenteux, désigné sous le nom de queue de cheval. D'après cette explication, on voit que cette disposition nerveuse ne peut et ne doit exister que chez l'homme, les chauve-souris

(1) Je suis convaincu que l'étude des embryons de ces poissons et de leurs métamorphoses expliquera cette singulière exception. 
sans queue et les batraciens, parmi les mammifères; et chez l'esturgeon, la lamproic, la baudroie et le tétrodon-lune, chez les poissons. Elle est beaucoup plus prononcée chez ces derniers que chez les premiers, parce que chez les poissons la moelle épinière est beaucoup plus élevée que chez les mammiferes.

Cette explication, que n'ont pas admise tous les anatomistes, est rendue évidente par la terminaison de l'axe nerveux de certains animaux articulés. La queue de cheval existe chez quelques invertébrés, mais elle existe en sens inverse de celle des vertébrés. Chez ces derniers, elle n'existe pas chez les jeunes embryons, et elle se forme dans la série de leurs développemens; chez les articulés, au contraire, elle existe chez leurs embryons et disparaît dans le cours de leurs métamorphoses. Ramenons au même principe deux effets qui paraissent si opposés.

S'il est vrai que la queue de cheval soit l'effet de l'ascension de la moelle épinière, à cause de l'angle d'insertion ou de parallélisme des filets qui s'y reudent, il est évident que, si, après être remonté, cel axe nerveux descendait, la queue devrait se détruire. Elle se détruirait, parce qu'à mesure qu'elle descendrait, les filets obliques deviendraient transverses, les parallèles deviendraient obliques; et parvenus enfin au niveau de l'inscrtion de chaque filet, chacun d'eux prendrait une direction horizontale; il n'y aurait 
plus de queue de cheval. Nous aurions alors la preuve et la contre-preuve de ce singulier phénomène.

Or, il suffit de comparer les vertébrés aux invertébrés, pour voir cette formation et cette déformation complète de la queue de cheval. Nous venons de voir que, chez les vertébrés, elle se forme par l'ascension de l'axe nerveux; les invertébrés vont nous montrer sa déformation par la descente du même axe : il suffit de suivre pour cela la métamorphose dés larves de la mouche-asile et du scarabée monocéros. Chez les jeunes larves, l'axe nerveux est beaucoup plus court que la longueur totale du corps de l'animal; les filets qui viennent aboutir à sa terminaison dirergent dans tous les sens, pour s'y reñdre des parties moyennes et inférieures de la larve; ils forment, de cette manière, la plus belle queue de cheval qu'il soit possible de voir. Mais à mesure que la larve se développe, à l'époque où elle se change en nymphe, l'axe nerveux descend le long de la partie inférieure de l'animal : à mesure que cette descente a lieu, l'angle d'insertion des filets nerveux se redresse, les filets obliques deviennent horizontaux; les filets parallèles deviennent d'abord obliques, puis ils s'insèrent horizontalement comme les précédens, lorsque la terminaison de l'axe nerveux est descend uc jusqu'à leur niveau. Ce bel éventail que formait la divergence des filets, disparaît insensiblement, et à l'examen de la fin de 
l'axe nerveux des insectes parfaits, on ne se douterait jamais qu'il ait pu exister.

Ainsi, chez certains vertébrés, l'ascension de la moelle épinière donne naissance à la queue de cheval, et chez quelques invertébrés, la queue de cheval disparaît par la descente de l'axe nerveux. Les embryons des premiers correspondent à l'insecte parfait; les larves de ces derniers reproduisent l'état permanent de ces vertébrés arlultes. Si l'ascension de la moelle épinière s'arrête chez les vertébrés, il ne se forme pas de queue de chcval; si la descente de l'axe nerveux est suspendue chez les invertébrés, la queue dẹ cheval persistc. Des effets si opposés reconnaissent donc une cause bien simple, l'ascension ou la descente de l'axe nerveux du corps. Des phénomènes bien différens se trouvent donc rapprochés, ramenés les uns aux autres, et expliqués; et tel est, avons-nous dit, le but définitif de l'anatomie comparative.

Voila pour les rapports de la moellc épinière avec le tronc : ceux qu'elle présente avec les extrémités ou les membres ne sont ni moins importans, ni moins remarquables.

Les animaux, portant dans lcur intérieur le réservoir de lcur nutrition, ont été soustraits de cette manière à la dépcndance du sol sur lequel ils sc trouvent. En échappant à cette dépendance, ils sont tombés dans une autre; la nature ne leur 
offre pas, comme aux végétaux, une nourriture toute préparée et un magasin toujours pourvu, où ils puisent leur alimentation.

Détachés de la terre, ils la parcourent çà et là pour se procurer leur nourriture. Toutes les combinaisons possibles de formes et de moyens ont été données aux animaux pour faciliter leur locomotion, et assurer par là leur conservation et leur existence.

Parmi ces moyens, les pieds, les nageoires ou les aîles sont ceux qui frappent le plus les observateurs; ce sont les seuls qui doivent ici nous occuper, parce qu'ils cö̈ncident avec des modifications importantes de la moclle épinière chez les animaux vertébrés.

Pour saisir ces modifications, il était indispensable de suivre chez les embryons et les animaux parfaits les phases de la moelle épinière; il fallait voir si la moelle épinière conservait ses mêmes formes avant et après la formation des membres, ou si, avec la présence des membres, cctte partie éprouvait quelque transformation constante.

Dans son état le plus simple, la moelle épinière forme un cylindre d'uu calibre à-peu-près uniforme dans toute son étendue; c'est l'e cas des vipères, des couleuvres, de la lamproie, de l'esturgeon, des anguilles, de la syrène, du protée, animaux tous remarquables par l'absence des appendices latéraux qui constituent les membres ou 
Jeurs analogues (1). Les embryons des oiseaux, des reptiles, des mammifères et de l'homme, sont primitivement, comme les animaux précédens, dépourvus de membres; ils n'ont ni extrémités inférieures, ni stepérieures, leur moelle épinière est, comme celle de l'anguilie, du protée, de la vipère, sans aucune trace des renflemens qui plus tard doivent se développer : si on examine l'embryon du poulet des deuxième (2), troisième (3), quatrième (4) et cinquième jours, on ne remarque sur son trajet aucune trace des renflemens; le tétard des batraciens est dans le même cas les dixième, douzième (5) et quinzième jours (6) de sa formation. L'embryon du mouton au commencement. du deuxième mois ( 7$)$; celui des jeunes didelphes (didelphis virginiana) (8), du veau, à la cinquième semaine (9), de l'embryon humain au deuxième mois (10), du lapin au dixième ct au douzième jour,

(1) La syrène, le protée, l'esturgeon ont des appendices sur les côtés du trorc; mais ils sont si grêles, si rudimentaires, que l'on peut en faire abstraction dans les généralités.

(2) Pl. I, fig. 2.

(3) PI. I, fig. 3.

(4) Pl. I, fig. 4́.

(5) Pl. I, fig. $9, n^{\circ} 1,2$ et 3.

(6) Pl. I, fig. 10, $\mathrm{n}^{\circ} \mathrm{x}, 2 \mathrm{et} 3$.

(7) Pl. I, fig. 19, $n^{\circ} 1,2$ et 3 .

(8) PI. I, fig. $30, n^{\circ} x, 2$ et 3.

(9) PI. I, fig. $27, n^{n}$ i et 2.

(iv) Pl. I, fig. $24 \mathrm{et} 26, \mathrm{n}^{\circ} 1,2 \mathrm{et} 3$. 
du chièn au quatorzième, du chat au quinzième, ressemblent sous ce rapport à celui des reptiles ou des oiseaux ; point de membres sur les parties latérales du tronc, point de renflemens le long de la moelle épinière. Tous les animaux se ressemblent donc primitivement : dans leur état normal les ophidiens, les anguilles, le protée restent sans niembres et sans renflemens; les embryons des oiseaux, des reptiles et des mammifères en acquièrent par la série de leurs métamorphoses. Avec les membres apparaissent les renflemens qui leur correspondent; leur position et leur volume sont en raison directe de la position et du volume des extrémités. Chez l'embryon de l'oiseau, le membre inférieur se montre vers le sixième jour de l'incubation; le renflement inférieur paraît à celte époque (1); le membre supérieur ne devient visible que le huitième jour; ai huitième jour apparaît aussi le renflement supérieur (2). Les dixième (3), seizième (4), dix-huitième (5) et vingt-unième jours (6), les renflemens et les membres acquièrent une grosseur proportionnelle. Chez les batraciens, de même que chez les oiseaux,

(1) Pl. I, fig. $5, n^{\circ} 2$.

(2) PI. II, fig. 33, n० 4 .

(3) PI. II, fig. 34, n०2 el 4.

(4) Pl. II, fig. $38, n^{\circ} 4$ et 6 .

(5) PI. II, fig. 39, n० 4 et 6.

(6) PI. II, fig. $41, \mathrm{n}^{\circ} 5$ et 5 . 
le membre inférieur précède le supérieur; il en est de même des renflemens épiniens; l'inféricur se montre au dix-huitième jour de la formation du tétard de la grenouille et du crapaud (1); le supérieur ne se monire guère qu'un ou deux jours après. Suivez les métamorphoses des embryons des mammifères, vous voyez apparaître les membres et les renflemens sur la fin du deuxième mois, chez le mouton, le cochon, le chevreau; au commencement du troisième, chez le cheval, le veau et l'embryon humain; vous voyez ensuite le volume de chaque renflement suivre rigoureusement l'accroissement du membre qui lui correspond.

Quittons les embryons, et embrassons d'un coup d'œil l'ensemble des animaux vertébrés; mous allons voir se répéter chez eux les mèmes combinaisons, les mêmes formes et les mêmes rapports.

Parmi les reptiles et les poissons, nous trouvons d'abord les ophidiens, les syrènes, les protées et les anguilliformes constitués comme les trèsjeunes embryons par unc tête et une longue épine, et tous ces animaux nous présentent une moelle épinière effilée et d'un calibre régulier comme ces mêmes embryons.

Nous voyons ensuite des nageoires ajoutées chez les poissons comme des appendices pour aider

(1) Pl. I, fig. 15, n॰3. 
leur locomotion, et à chaque paire de ces appendices nous voyons correspondre un renflement sur la moelle épinière, proportionné par son volume au volume de la nageoire.

la position, le nombre et le volume des nageoires donnent la position, le nombre et le volume des renflemens de la moelle épinière des poissons.

Ainsi les poissons à nageoires abdominales ont la moelle épinière renflée dans la partie correspondante de l'abdomen.

Les poissons à nageoires pectorales ont leur renflement vers cette région; les poissons à nageoires cervicales les ont situés derrière la tête.

Les trigles, remarquables par les rayons détachés de leurs pectorales, le sont aussi par une série de renflemens (1) proportionnés pour le nombre et le volume, au nombre et au volume de ces mêmes rayons auxquels ils correspondent. J'ai compté chez le rouget (trigla cuculus) cinq rayons détachés; un nerf distinct pénétrait dans chaque rayon, un renflement (2) correspondait à l'insertion de chaque nerf. Chez le gronau ( $T$. lyra), j’ai vu six rayons, six nerfs et six renflemens (3), alignés symétriquement en arrière du cervelet (4). Les poissons électriques ont un

(1) Pl. VII, fig. $160, \mathrm{n}^{\circ} 1$.

(2) Pl. VII, fig. $159, \mathrm{n}^{\circ}$.

(3) Pl. VII, fig. $155, \mathrm{n}^{\circ} 3$.

(4) Pl. VII, fig. 158,159 et 160 
MOELLE ERINIERE.

renflement considérable, correspondant au nerf qui se distribue dans l'appareil électrique : j’ai observé ce nerf et ce renflement une fois chez la raie et le tétrodon électriques, et deux fois sur des silutes.

De plus, beaucoup de poissons ont un renflement terminal à l'extrémitć de la moelle épinière, parce que chez eux la queue est le moyen le plus puissant de la locomotion.

Les reptiles, chez lesquels la nature a le plus varié, après les poissons, les moyens de transport, nous offrent aussi après eux les variations les plus remarquables des renflemens de la moelle épinière.

Un grand nombre n'ont point de pieds, ni en avant, ni en arrière, et ils n'ont point la moelle épinière renflée.

D'autres ont des pieds de derrière et point de pieds de devant; ils n'ont alors de renflement à la moelle épinière que dans la partie postérieure.

D'autres, au contraire, n'ont que les pieds de devant et point de pieds de derrière; ils ont alors la moelle épinière renflée en avant.

Enfin, la nature accorde à un grand nombre ces deux combinaisons de pieds, et la moelle épinière présente en même temps les deux renflemens qui leur correspondent. Les reptiles quadrupèdes, depuis le lézard monodactyle et le tétradactyle, dont les pieds sont rudimentaires, jusqu'aux tortues et aux crocodiles, où ils sont si pro- 
noncés, ont les renflemens de leur moelle épinière proportionnés au volume de leurs pieds.

Ainsi, chez les reptiles, de même que chez les poissons, la position, le volume et le nombre des pieds, donnent rigoureusement le nombre, le volume et la position des renflemens de leur moelle épinière.

Chez les mammifères, la nature a beaucoup restreint ses moyens de locomotion. D'une part, nous ne connaissolis pas de mammifères sans pieds, comme sont les ophidiens et les anguilliformes; et de plus, en n'accordant que deux pieds à certains mammifères, la nature a été constante dans la position qu'elle leur a assignée. En effet, les cétacées n'ont que les deux pieds antérieurs, comme les reptiles bimanes; mais nous ne connaissons aucun mammifère qui n'ait que les pieds de derrière sans pieds de devant, ainsi que sont les bipèdes. Peut-être même cette combinaison estelle incompatible avec leur organisation (1).

Les modifications de la moelle épinière sont

(1) Le volume de la tête chez les mammifères, la brièveté du col et la concentration des poumons dans le thorax, par la présence du diaphragme, donnant un poids considérable à la partie antérieure du tronc, les pieds ont da être portés en avant pour la soutenir. S'ils étaient placés en arrière, la tête et la poitrine seraient nécessairement entraînées sur le sol, et ne pourraient être relevés et maintenus que par des puissances musculaires beaucoup plus fortes que celles que peut comporter l'organisation des mammiferes. 
aussi moins nombreuses chez les mammilères que chez les reptiles et los poissons; tous, à l'exception des cétacés', out les deux renflemens de la noellc épinière placés vis-à-vis des membres. Les cétacées, qui n'ont que ceux de devant ct point ceux de derrière, n'ont, comme les reptiles bimanes, que le renflement antérieur, et point le postérieur. A partir de la région pectorale, leur moelle épinière se comporte comme celle des poissons anguilliformes, et des ophidiens, parmi les reptiles. Les phoques, dont les membres empêtrés, selon l'expression des zoologistes, ne font guère que l'office des nageoires des poissons, sont remarquables par la faiblesse des renflemens épiniens qui leur correspondent. Du reste, dans cette classe comme dans les deux précédentes, les renflemens de la moelle épinière sont toujours proportionnés au volume des picds.

Enfin, les oiseaux sont, sous le rapport $d u$ nombre des renflemens, moins variables encore que les mammifères. Nous ne connaissons point d'oiseau privé de membres; nous n'en connaissons point qui n'aient ou que les pieds en arrière, ou que les ailes en avant : ces deux moyens de transport sont toujours réunis chez eux; toujours aussi leur moelle épinière est renflée en haut et en bas.

Mais a vec celte fixité des puissances Incomotrices, la nature a varié les movens de leui déplacement plus que dans toutes les autres classes réunies. II. 
Ainsi les uns ne peurent, comme les mammiferes, s'élever au-dessus du sol; les autres planent dans les airs, et y restent suspendus des journées entières; ceux-ci sont grimpeurs, ceux-là sont nageurs; un grand nombre se servent alternativement des ailes ou des pieds pour leurs moyens de transport.

Tous ont deux renflemens à la moelle épinière, ainsi que nous l'arons dit; mais le renflement inférieur prédomine sur le supérieur, ou le supérieur sur l'inférieur, ou bien les deux renflemens sont à-peu-près égaux, selon les habitudes locomotrices de l'oiseau.

Les oiseaux qui sont altachés au sol, comme les mammifères, ont des pieds très-développés et des ailes presque rudimentaires : tels sont l'autruche et lc casoar. Le renflement inférieur prédomine tellement sur le volume du supérieur, que ce sont, sous ce rapport, de véritables bipèdes.

L'aigle, la cigogne, le pigcon, l'hirondelle, s'élèvent, au contraire, dans les airs à une hauteur prodigieuse, et y nagent avec autant de facilité que les poissons dans leur ćlément. Chez cux, le volume du renflement supérieur l'emporte de beaucoup sur le volume de l'inférieur : celui-ci cst beaucoup moins développé. $\Lambda$ ussi ces oiseaux qui voguent avec tant de facilité dans les airs, nous paraissent-ils déplacés sur la terre.

Ceux qui grimpent habituellement le long des 
arbres, ou qui se meuvent dans les eaux au moyen de leurs pattes, ont. le renflement inféricur plus saillant que le supérieur.

Enfin, les oiseaux qui, comme la poule et l'oie, se trausportent d'un lieu à un autre, tantôt à l'aide de leurs pieds, tantôt à l'aide de leurs ailes, ou qui, comme le fou de Bassan, ont besoin d'une force égale dans les ailes et dans les pieds, sont remarquables par les dimensions à-pcu-près égales de leurs deux renflemens.

La mòlle épinière nous offre donc la répétition des différens moyens de transport que la nature a accordés aux animaux. La combinaison de ces moyens et les modifications de la mocl!s épinière qui leur correspondent, peuvent sc réduire à quatre :

Premièrement, les animaux sont privés de membres, et se meuvent par l'elfet des ondulations de leur longue épine; leur moelle épinière n'est renflée sur aucun point de son étendue.

Deuxièmement, la combinaison la plus générale est celle des deux pattes de derrière, et de deux pattes ou de deux ailes en avant: la moelle épinière est renfléc en haut et en bas.

Troisièmement, de dcux paltes en devant, saus pattes de desrière : la moelle épinière, renfléc en haut, ne l'est pointien bas.

Quatriemement, enfin, des deux paltes de derriłre, sans pattes de dlevant: la moelle épinière est renfléc en bas et ne l'est pas en haut. 
Telles sont les variétés de forme et d'étendue que présente la moelle épinière dans le grand embranchement des vertébrés. Ces variétés sont les mêmes chez les animaux parfaits et chez les embryons : chez les uns comme chez les autres, elles dérivent des mêmes causes et nous offrent les mêmes rapports. L'ophidien a sa moelle épinière répétée par celle des embryons des vertébrés les plus élevés, parce qu'à une époque de leur existence ces embryons sont, par leurs formes, de véritables ophidiens. Ce type simple et primitif de toute moelle épinière est modifié de différentes façons, parce que la série des métamorphoses développe sur ses flancs des appendices destinés à modifier, à accélérer, à varier le mode de locomotion des animaux; ces moyens nouveaux nécessitent pour leur action des modifications correspondantes de la moelle épinière. Ces modifications sont des renflemens qui rompent çà el là l'uniformité de son calibre : ces renflemens étant destinés à ces appendices, on voit donc que leur nombre, leur volume et leur position, doivent être nécessairement et irrévocablement fixés par la position, le volume et le nombre des nageoires, des ailes ou des pieds que la nature a accordés aux différens animaux pour se transporter d'un lieu à un autre, quelle que soit la différence des milieux où ils vivent, et quelles que soient les résislances qu'ils aient à vaincre pour le transport. Le spectacle de ces divers changemens et de leurs 
rapports est rendu plus incéressant ericore par les changemens et les rapports analogues qui se manifestent dans le système sanguin.

Chez les ophidiens, l'aorte ne fournit que des intercostales d'un calibre à-peu-près uniforme. Les embryons des oiseaux, le tétard des batraciens, l'embryon des mammifères et de l'homme, sont primitivement dans le inême cas que les ophidiens; mais à l'époque où les membres apparaissent, on voit apparaître aussi, en bas les artères fémorales, en haut les axillaires: Les cétacées n'ont que l'artère axillaire; les bimanes sont dans le même cas; les bipèdes, au contraire, n'ont que la fémorale, et point d'axillaire. Les embryons monstrueux n'ont que l'artère axillaire, ou la fémorale, selon que la paire de membres qui leur reste est située en haut ou en bas; le calibre des artères axillaires et fémorales est toujours rigoureusement proportionnel au volume des membres supérieurs et inférieurs, ou á la seule paire de membres chez les cétacées, les bipèdes, les bimanes, et chez les monstres qui nous offrent leurs conditions organiques.

Les oiseaux qui ont les ailes les plus fortes, ont. les artères axillaires les plus volumineuses; et aux plus faibles ailes, comme chez l'autruche et le casoar, correspondent les artères axillaires les plus grêles. Ces derniers oiseaux, au contraire, ont les fémorales et les jambes les plus fortes, tandis que nhez les premiers les fémorales ct les jambes s'atrom 
phient en raison directe du volume qu'acquièrent les aîles.

Cette coexistence de rapports entre le système sanguin et le système nerveux explique aussi les variétés du prolongement caudal des animaux. Tous lcs oiseaux ont la moelle épinière prolongée jusqu'à l'extrémité du coccyx; tous, à une ou à deux exceptions près, offrent le même nombre de vertèbres caudales; l'arlère sacrée moyenne partage cette fixité de rapports. Ricn de si variable, au contraire, que le prolongement caudal des mammifères; rien de si variable chez cux que le volume de l'artère sacrée moyenne et le prolongement de la moelle épinière dans le canal coccygien.

Jusqu'au troisieme mois de l'cmbryon humain, l'artère sacrée moyenne est véritablement la continuation de l'aorte; son calibre est plus grand relativement, qu'il ne le sera à aucune autre période de la vic. A partir de celte époque, l'artère sacrée va toujours en diminuant de volume, jusqu'aux huilième ou ncuvième mois, époque où elle a attcint le rapport qu'elle doit conserver. Il en est de même chez les embryons des chauve-souris sans queue: l'artère caudale diminue de calibre avec une rapidité qui est en rapport avec la prompte disparition de la queuc de l'embryon.

La métamorphose du tétard des batraciens a excité dans tous les temps la curiosité des zootomistes; un animal sans membres ct à longue 
queve perd tout à coup celle-ci, et des membres apparaissent. Ce spectacle si remarquable, et que nous avons tous les jours sous les yeux, nous frappe par la rapidité de ses variations et par les transformations complètes qu'éprouve l'animal qui en est le sujet; il est au fond le même que celui de l'embryon de l'homme et des autres mammifères sans queue, comme certaines chauvesouris, le magot, les orangs, etc.

La cause de ces étonnantes métamorphoses a son siẻge dans le système sanguin et le système nerveux. Ouvrez un tétard du douzième jour, vous lui trouvez une aorte d'un calibre presque uniforme de la. tête à la queue; il n'a ni artère axillaire ni fémorale, sa moelle épinière se prolonge jusqu'à l'extrémité du coccys, en conservant un calibre con. sidérable (1). Aux dix-huitième, dix-neuvième et vingtième jours, l'artère fémorale paraît, la queue s'atrophic, le membre postérieur se dessine sous les tégumens, l'artère sacrée moyennc diminue, la moelle épinière s'amincit à sa terminaison d'une manière remarquable (2). Observez le même animal au quarantième jour : à peine a-t-il une queue, à peine pouvez-vous distinguer l'artère sacréce, tant ellc est réduite à un pelit calibre; la moelle épinière (3), remontéc tout à coup dans

(1) PI. I, fig. $9, n^{\circ} 1$.

(2) Pl. I, fig. $15, n^{\circ} 1 \mathrm{et} \cdot 2$.

(5) Pl. II, fig. í, n 1 . 
le canal vertébral, n'est plus qu'un filet capillaire (1). Que sont devenus les muscles, les artères èt les vertèbres cartilagineuses qui formaient celte énorme queue? Ce riche appareil organique a disparu avec l'artère sacrée moyeune et la moelle épinière : en même temps les membres se sont développés, et leur accroissement s'est opéré aux dépens de la queue. J'ai suivi ce rapport inverse entre la queue et les membres dans les lois de l'ostéogénie; je me contente de le rappeler en ce moment.

La métamorphose de la queue du tétard et de celle des mammilères et de l'homme est donc sous l'influence de l'artère sacrée moyenne; plus l'artère sacréc moyenne est forte, comme chez le bouf, le cheval, le lion, le renard, l'écureuil, plus la queuc est volumineuse, plus son calibre se réduit, plus la queue s'atrophie, comme on l'observe chez le cochon, le lièvre et le lapin.

Le volume de la queue des animaux est donc rigoureusement proportionnel à celui de l'artère sacrée moyenne; l'absence des membres chez les ophidiens ct les juunes embryons coïncide donc avec le non-développement des arlères axillaires et fémorales, l'existence d'une scule paire de membres est donc en rapport avec le déreloppenent isolé, soit de l'artère axillaire, soit de la fémorale. Enfia l'antagonisme qui sc renouvelle

(1) PI. If, fig. $43, a^{\circ} 1$. 
surtout chez les oiseaux, entre la force des cxtrémités antéricures et postérieures, est donc aussi expliqué par l'antagonisme dés artères axillaires et fémorales.

Ces rapports, très-importans, sansdoute, puisqu'ils nous donnent l'explication du tronc des animaux vertébrés, le sont moins peut-être que ceux que la moelle épinière présente avec l'encéphale, ses nerfs et l'ensemble de la face où ccuxci prennent leur origine. Haller fut le premicr qui s'aperçut que l'encéphale ne suivait pas chez les mammifères la progression directe de la moelle épinière; Sœmmering et M. Cuvier exprimèrent ensuite plus nettemeni cette pensée, en disant que de l'homme aux mammifères inférieurs. on voyait accroître la moclle épinière et décroître Pencéphale. MM. Gall et Spurzheim tirèrent de ce rapport inverse une singulière déduction, comme nous le rapporterons plus bas. Carus dit que le cerveau seul y élait soumis, tandis que, selon cet anatomiste, le développement da cervelet suivait la progression croissante de la moclle épinière. Enfin liedemann fit une remarque bien ingénieuse, cn observan $\imath$ que ce rapport opposé avait principalement sa source dans le décroissement de l'encéphale.

En comparant a la moelle épinic̀re toute la masse encéphaliquc, on voit, en effet, cette deruierc décroître progressivement de l'homme aux poissons, tamilis gue l'axe nerveux du trone 
conserve ou accroîl même ses dimensious. On peut suivrc ce rapport opposé cn comparant le volume de ces deux parties chez l'homme, les singes, le drill (1), le mardrill (2), les cétacées (j), les phoques (4); chez les carnassiers, tels que lours (5), le raton (6), le lion ( 7$)$, la loutre (8), la marte (9); les pachydermes, comme le cheval (10), lc pécari (1 1); les ruminans, comme le lama (12), le chameau (13), le bouc (14); les rongeurs, comme lı porc-épic (15), lu castor (16) et le lapin (17). Ce même rapport se maintient d'une manière générale chez les oiseaux, les reptiles et les poissons, ainsi qu'on peut le voir chez le coq (18),

(1) Pl. VIII, fig. 199, A, B, C, D, G.

(2) Pl. VIII, fig. $196, \mathrm{n}^{\circ} 1, \mathrm{~B}, \mathrm{C}, \mathrm{G}, \mathrm{M}$.

(5) Pl. XI, fig. $225, \mathrm{~A}, \mathrm{~B}, \mathrm{C}, \mathrm{K}, \mathrm{N}$.

(4) Pl. IX, fig. $209, \mathrm{~A}, \mathrm{~B}, \mathrm{C}, \mathrm{G}, \mathrm{M}, \mathrm{N}$.

(5) Pl. XI, fig. $230, A, B, C, E, G$.

(6) Pl. VIII, fig. $202, \mathrm{~A}, \mathrm{~B}, \mathrm{C}, \mathrm{H}, \mathrm{K}, \mathrm{N}$.

(7) Pl. XIV, fig. 264, 1, 2, 5, 4, 6, 8 et 9 .

(8) Pl. X, fig. 223, A, D, H, F.

(9) Pi. XV, fig. $278, \Lambda, B, C, n^{\circ} 7,8,9$ et ro.

(10) Pl. XV, fig. 274, A, B, C, D, G, M, N.

(11) Pl. XVI; fig. $298, \mathbf{A}, \mathbf{D}, \mathbf{F}, \mathrm{G}, \mathbf{N}$.

(12) Pl. XVI, fig. 294, A, R, D, M, G, N.

(13) PI. XIII, fig. $248, A, \mathbf{B}, \mathbf{C}, \mathbf{E}, \mathbf{G}$.

(14) Pl. XIV, fig. $261, \mathrm{n}^{\circ} 1,2,5,4,7,9$ et 10.

(15) PI. XIII, fig. $252, \Lambda, B$, C, L, K:

(16) Pl. XIV, fig. $265, n^{\circ} 1,3,5,4,7$, s ct 9.

(17) Pl. II, fig. 57, $\mathrm{n}^{\circ}$ i , 2, 5, 5, 6 et 7 .

(18) Pl. II, fg. 41, n०5,6, 5 et 8 . 
la poule (1), le casoar (2), le perroquet (3), lat bondrée commune (4), l'autruche (5), la cigogne (6), l'aigle (7), l'hirondelle (8), le roitelet (9) ; l'orvet (10), le lézard vert (11), le camésléon (12), le tupinambis (15), le crocodile (14), la vipère hajé (15), la vipère à raies parallèles (16), la vipère de Fontainebleau (17), Ie caïman (18), la gronouille (19), la tortue grec que (20), la tortue franche (21), la perche (22),

(1) P1. III, fig. $85, n^{\circ} 1,5,5$ et 8 .

(2) Pl. III, fig. $77, \mathrm{n}^{\circ} 2,5,4$ et 7 .

(j) Pl. İl, fig. 84, $n^{\circ} 1,10,11$ et 12.

(4) Pl. IV, fig. ${ }^{\natural 1}, \mathrm{n}^{\circ} 1,2,7$ et 8.

(5) Pl. IV , fig. $9^{3}, n^{\circ} 1,5$, 4 et 6.

(6) Pl. IV , fig. 99, n० 1, 2, 4 et 5.

(z) PI. IV , fig. $101, n^{\circ} 1,2: 8,9$ et 10.

(8) Pl. IV, fig. 106, $\mathrm{n}^{\circ} \mathbf{1}, 5$, et 4 .

(9) Pl. IV, fig. $108, n^{\circ} 1,3$ et 4 .

(10) PI. V, fig. $\log , \mathrm{n}^{\circ} 1,2,3,4$ et 5 .

(11) Pl. V, fig. $110, \mathrm{n}^{\circ} .5, \mathrm{~b}, 9$ et io

(12) Pl. V, fig. 111, $\mathrm{n}^{\circ} 1,4,6$ et 7 .

(15) PI. V, fig. 114, n*5, 6, 8 et 9 .

(14) PI. V, fig, i i $5, n^{\circ} \cdot 1,3,5$ et 6 .

(15) Pl. V, fig. $126, n^{\circ} 2,5$ et 5 .

(16) Pl. V, fig. $133, n^{\circ} 2,5$ et $g$.

(17) Pl. V, fig. 152, $n^{\circ} 2$, 5 et 7 .

(18) Pl. V, fig. $135, n^{\circ} 2$ et 6.

(1,) Pl. I, fig. 16, $1^{\circ} 1,5$ et 5 .

(20) Pl. V; fg. 125, n $15,7 \mathrm{ct}$ ).

(21) P. V, fig. $11 !), 12^{\prime \prime}, 5.7,9+1 \mathrm{mo}$

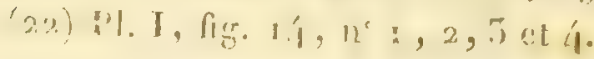


le brochet (1), le trigle (2), la morue (3), le turbot (4), l'égrefin (5), le barbeau (6), l'anguille $(7)$, la baudroie (8), la tanche (9), le merlaa (10), le congre (11), la lamproie (12), la raie 13$)$, le requin $(14)$, l'esturgeon (15), l'ange (16) ét l'aiguillat (17).

Ce rapport inverse se répète chez les embryons d'une manière admirable; plus ils sont jeunes, plus la moelle épinière est volumineuse, moins le cerveau est développé, plus ils se rapprochent conséquemment des classes inférieures. Ainsi les douzième (18), quinzième (19) et dix-huitième

(1) Pl. I, fig. i5, $\mathrm{n}^{\circ} \mathrm{x}, 2,3$ et 4 .

(3) Pl. VII , fig: $155, \mathrm{n}^{\circ} 2,4,5$ et 7 .

(3) PI. VII, fig. $163, n^{\circ} 4,7,8$ et 9 .

(4) Pl. VII, fig. $191, n^{\circ}$ i ; 5 et 4 .

(5) Pl. VII, fig. $177, \mathrm{n}^{\circ} 1,2$ et 3.

(6) PI. VII, fig. $183, \mathrm{BB}, \dot{\mathrm{n}}^{\circ} 1,2$ et 3.

(7) Pl. VII, fig: $190, n^{\circ} 1,2,3,4$ et 5 .

(8) Pl. VII, fig. $179, \mathrm{n}^{\circ} 1,2,3,4$ et 5.

(9) Pl. VII, fig. $185, \mathrm{n}^{\circ} 1,4,5$ et 6.

(10) Pl.VII, fig. 193, $\mathrm{n}^{\circ} 1,4$ et 6.

(1i) PI. VI, fig. 149, n 1, 5, 4 et 9 .

(12) Pl. XI, fig. $224, n^{\circ} 1,4,5,6$ et 7 .

(15) P1. VI, fig. 152, $\mathrm{n}^{\circ} 1,2,4,8$ et 14.

(14) Pl. VI, fig. 142, no $1, \mathrm{~B}, \mathrm{C}, 14$ et 15.

(15) Pl. XII, fig. 235, A, B, G, K.

(16) Pl. XII, fig. $237, A, B, D, E, F$.

(17) PI. XII, fig $236, \mathbf{A}, \mathbf{B}, \mathrm{G}, \mathrm{H}$.

(18) Pl. I, fig. 9, n* 5, 4, 5 et 7.

(19) Pl. I, fig. $10, \mathrm{n}^{*} 2,5,4$ et 5 . 
jours de sa formation (1), le tétard de la grenouille reproduit les proportions des poissons; aux quatrième (2) et sixième (5) jours de l'incubation, l'oiseau répète les poissons; au neuvième jour, les reptiles ( 4 ); aux quinziène (5) et seizième jours (6), il offre les rapports de sa classe. Ainsi le veau de la cinquième semane de formation est d'abord poisson (7), puis reptile (8), puis oiseau (9), puis rongeur (10). Ainsi, par le volume de sa moelle épinière et 'atrophie de l'encéphate, l'embryon humain de la cinquième semaine (11) se rapproche du pcisson; au milieu du deuxième mois, du reptie (i2); au commencement du troisième (13), de l'oiseau ; puis il devient rongeur (14), riminant (15), car-

(1) Pl. I, fig. 15, n० 3, 4, 5 et $?$.

(2) Pl. I, fig. $4, n^{\circ} 5$, 7 et 8.

(3). Pl. I, fig. 5, $\mathrm{n}^{\circ} 3,5,6$ et 8 .

(4) Pl. I, fig. $6, n^{\circ} 6,8$ et 10.

(5) Pl. II, fig. $38, \mathrm{n}^{\bullet} 6,8$ et 10 .

(6) Pl. II, fig. $39, n^{\circ} 7,8,9$ et 1 .

(7) PI. I, fig. 19, n० 3,4 et 5 .

(8) Pl. I, fig. 27, $\mathrm{n}^{\circ} 2,3$, 4 et 5.

(9) Pl. II, fig. $50, n^{\circ} 4,7,8$ et 9

(10) Pl. II, fig. 49, $n^{\circ} 4,5,7$ el.

(11) Pl. I, fig. 24, n:2, 3, 4 et i.

(12) Pl. I, fig. $25, \mathrm{n}^{\circ} 5,6,8$ et c.

(15) Pl. I, fig. $32, \mathrm{n}^{\circ} 4,5,6$, , t 8 .

(14) Pl. II, fig. 65, n०3 et 4 .

(15) Pl. II, fig. $70, n^{*} 1,2,3$, 5et 6. 
nassier (1), quadrumane (2), et enfin homme.

Mais ce rapport de la masse encéphalique avec le volume de la moelle épinière, chez les embryons et les animaux adultes, ne nous offre encore que les premiers pasde la science daus un de ses points les plus élevés tt les plus difficiles. Les rapports les plus féconds pour la physiologie et la pathologic sont ceux qui nous donneront la valeur positive des diveses parties de l'encéphale avec la moelle épinière là, nous verrons les tubercules quadrijumeaux développés constamment en raison dirccte de la mcelle épinière; nous trouverons, dans le corvelet, l'unc de ses parties suivre la progression croisan te de la moelle épinière, l'autre sa progression décroissante; nous trouverons, dans les hémisphères círćbraux, certaines parties développées en raison directe do l'axe nerveux du tronc, d'autres encaison inverse : nous puiserons, enfin, dans ce balincement continuel des élémens de l'encéphale, lixplication de cet organe dans les différeules clases, et dans les embryons qui reproduisent les ariations des classes.

Mais: pour suvre le développement du plan que nous nous simmes tracé dans cet ouvrage, nous ne pouvons, en ce moment, établix ce parallèle qu'entre lı volume de la moellc épinière et celui des nerf: encéphaliques. Il est connu,

(1) PI. II, fig. 72, $1^{\circ} 1,5,9,10$ et 11

(3) Pl. II, fig. $68,1^{\bullet}$ 1, 2, 3 et 4 . 
depuis les travaux de Sommering, de MM. Cuvier et Gall, que le volume des nerfs encéphaliques augmente à mesurc que le cerveau en masse décroît : de là ì la découverte du rapport direct du volume de la moelle épinière avec les principaux nerfs crâniens, il n'y avail qu'un pas; ce pas n'a pas été fait : il n'a pas été fait, parce que, d'une part, la véritable origine des nerfs était méconnue; de l'autre, parce qu'on n'avait pas distingué les rapports opposés des divers élémens de l'encéphale; et en troisième lieu, enfin, parce qu'on n'avait pas appliqué au système nerveux de la tête l'analogie aperçue entre les os qui composent cetto dernière partie et les vertèbres.

S'il est vrai, en effet, que la tête ne soit. qu'un assemblage de vertèbres, ainsi que l'ont établi MM. Duméril, Oken, Spix, Bojanus, Blainville, Genffroy-Saint-Hilaire, et moi-même (Lois de l'Ostéogénie); s'il est démontré, en sccond lieu, que le diamètre de la moelle épinière cst proportionnel à l'étendue de la vertèbre; s'il est prouvé, de plus, que les nerfs puisent leur origine dans l'étendue des organes, on voit que nécessaircment l'étendue des chambres des sens et. le volume de leurs nerfs doivent accroître dans la même proportion que le volume de la moelle épinière. Lc raisonnement conduit à ce rapport: l'observation, qui, dans les sciences exactes, n'est que le raisonnement décomposé, le justifie de point en point. Elle montre que, de 
même que les nerfs du tronc, les nerfs olfactif, optique et trijumeau, sont développés dans les quatre classes en raison directe de la moelle épinière, ainsi qu'on peut le voir en comparant ces nerfs à la moclle épinière, chez les quadrumanes (1), les cétacées (2), les phoques (3), les pachydermes (4), les ruminans (5), les carnassiers (6), les rongeurs (7), les oiseaux (8), les reptiles (9), les poissons cartilagineux (10), et les poissons osseux (11). Les nerfs acoustique et facial suivent, en général, le même rapport.

Si certains élémens de l'encéphale sont dans des rapports opposés avec la moelle épinière; si les uns sont développés en raison directe de cet axe nerveux, et les autres en raison inverse, on voit aussi que les nerfs crâniens devront partager le contraste de ces rapports; ils devront suivre le rapport direct des parties qui concordent avec la moelle épinière, et le rapport

(1) Pl. VIII, fig. 194 et 197.

(2) Pl, XII, fig. 234.

(3) PI. IX, fig. 208.

(4) Pl. XV, fig. 375

(5) Pl. XVI, fig. 295.

(6) PI. XIV, fig. 266.

(g) Pl. XIV , fig. 258.

(8) Pl. IV, fig. $88,96,97,98$ et 103.

(9) Pl. V, fig. 122 et 13 .

(30) Pl. VI, fig. 158, 142, 148 et 152 .

(11) Pl. VII, 6g. $162,164,163,178$ et 183. 
inverse de ceux qui sont en discordance de développement avec clle: c'est ce que prouve rigoureusement l'observation, ainsi que nous le montrerons en traitant des élémens particuliers de l'encéphale. La proposition qui établit que les nerfs des sens sont développés en raison inverse de l'encéphale, n'est̃ donc pas généralement exacte; elle ne l'est que pour certaines parties; et de même que, dans les rapports de la moelle épinière, il est de la plus grande importance, pour les progrès de la science, de préciser quelles sont ces parties, et de les distinguer soigneusement de celles qui présentent un rapport opposé, c'est pour arriver, autant que possible, à cette précision dans les rapports, que je me suis si particulièrement attaché à distinguer les élémens divers dont se compose l'encéphale dans les diverses classes.

Sont exceptés du rapport précédent les cétacées pour le nerf olfactif (1); la taupe (2), la musaraigne, le zemni (3), le rat-taupe du Cap, la chrysochlore, la cécilic et le protée, pour le nerfoptique.

Chez plusicurs animaux, tous les nerfs crâniens ne suivent pas un rapport direct entre eux : d'où naissent encore plusieurs exceptions. Ainsi, les oiseaux, qui ont le nerf optique si développé,

(1)Pl. XII, fig. 234, L.

(3) PI. XIV, fig. $260, \mathrm{n}^{\circ} 2$.

(3) Pl. XV,fig. $272, n^{n} .6$. 
ont la cinquième paire moins foric proportionnellement : au contraire, cirez le castor, la ciuquième paire est énorme et le nerf optique (1) trèsgrêle, quoique la moelle épinière soit très-développée. Il en est de même chez le raton (2). De plus, les nerfs de la troisième, de la quatrième et de la sixième paire, offrent des variations de volume qui ne sont point partagées par la moelle épinière, comme on le remarque chez le raton (3) et chez l'ours (4). Cette dernière observation est surtout a pplicable aux poissons, chez lesquels ces derniers nerfs, surtout la quatrième paire, sont très-faiblement développés, en comparaison du volume de la moellẹ épinière.

L'axe nerveux des invertébrés supérieurs est manifestement formé de deux cordons, tantôt réunis en un seul, tantôt séparés sur divers points de leur étendue, toujours disjoints pour livrer passage à l'oesophage : aussi les sentimens des auatomistes sont-ils unanimes sur la double origine de cet organe. Il n'en est pas de même de l'axe nerveux du tronc des animaux vertébrés : cette parlie est simple chez tous; elle forme l'un de ces organes auxquels les anatomistes ont donné le nom d'impairs. La moelle épinière n’est súparée

(1) Pl. XIV, fig. $258, \mathrm{n}^{\circ} 2$.

(2) Pl. VIII, fig. $200, n^{\circ} \cdot 2$.

(3) P.l. VIII, fig. $200, \mathrm{n}^{\circ} 3,4$ et 6.

(4) Pl. XI, fig. 231, n०5; 4 et 6. 
ni disjointe chez aucun d'eux; elle offre senlement un hiatus à la partie postéricure de la l'égion sacrée des oiseaux (1), hiatus formé par l'écartement en arrière des deux cordons primitifs qui la constituent.

Cette disposition permanente de la moclle épinière des vertébrés a été la source de plusicurs conjectures : les uns l'ont considérée comme un cordon unique; les autres, en beaucoup plus grand nombre, ont admis deux cordons pour sa composition, l'un droit, l'autre gauche. Quelques auteurs, enfin, comme Hygmore et Monro, ont avancé que chaque cordon se subdivisait en quatre cordons secondaires; ce qui porte à huit le nombre des faisceaux de la moelle épinière; nombre qui s'élèverait même à dix, si le faisceau admis par Charles Bell sur le flanc de chaque cordon s'y rencontrait réellement.

Dans l'examen de cetle question, dont il est inutile de faire sentir l'importance, à une époque où les travaux de tous les physiologistes marchent sur l'isolement des propriétés des diverses parties du système nerveux, il est nécessaire d'cnvisager cette pluralité des cordons sous deux points de vue: le premier, relatif à la formation de la moelle épinière; le second, à la structure qu'ellc présente chez les vertébrés parvenus au terme de leur développement.

(1) II. II, fig. $35, \mathrm{n}^{\circ} 2$. 
Quant à sa formation, l'étude de l'organisation pouvait seulc nous éclairer de ses lumières. Or, que pous enseigne cette étude? Elle nous apprend que, dans toutes les classes, la moelle épinière est primitivement formée de deux cordons (1), et uniquement de deux cordons; jamais il n'y en a plus, jamais il n'y en a moins. Il ne pouvait y en avoir moins, d'après la loi du double développement des organes, mais il pouvait y en avoir plus; et dès-lors il était important de s'en assurer par l'observation directe. Or, l'observation directe des jeunes embryons n'a montré que deux cordons primitifs, le premier (2), le second (3) et le troisième jour de l'incubation (4) des oiseaux; le douzième (5) et le quatorzième (6) de la formation du tétard des batraciens; le huitième du lapin ct du cochon-d'Inde; les quatrième et cinquième semaines du mouton ( 7$)$, du veau et de l'embryon humain (8). Il n'y a donc eu que deux cordons pour le développement de la moelle épinière.

Mais ces cordons ne restent pas dans l'état de

(1) Pl. I, fig. $1, n^{\circ} 5$ et 4 ; fig. $10, n^{\circ} 2,3$ et 4 ; fig. 25 , $\mathrm{n}^{\circ} 1,5 \mathrm{et} \cdot 5$.

(2) Pl. I, fig. 1, n ${ }^{\circ} 3,3$ et 4 .

(3) Pl. I, fig. 2, $n^{\circ} 2,3$ et 4 .

(4) Pl. I, fig. $3, n^{\circ} 4$.

(5) Pl. I, fig. 9, $\mathrm{n}^{\circ} 2,3 \mathrm{el} 4$.

(6) Pl. I, fig. $10, \mathrm{n}^{\circ} 1,2$ et 3.

(j) Pl. I, fig. $20, \mathrm{n}^{\circ} 1$ et 2 .

(8) Pl. I, fig. 23, $n^{\circ} 1$ et 3 . 
simplicité où on les observe à leur apparition; des faisceaux secondaires se développent dans leur épaisseur et compliquent la structure de la moelle épinière. Il arrive en partie à la moelle épinière ce qui survient dans la série des transformations du canal osseux qui l'enveloppe. Ce canal, membraneux d'abord, est formé de deux lames qui se réunissent en avant et en arrière; puis ces lames se transforment en cartilage, sur lequel nous ne remarquons aucune intersection dans le pourtour de l'anneau qui environne la moelle épinière; puis le rachis cartilagineux devient osseux, et nous voyons alors cet anneau être formé par la réunion de plusieurs pièces, toujours conformément à la loi primitive de conjugaison. Il y a une pièce distincte pour chaque masse transverse de la yertèbre, deux pièces pour former le corps, souvent deux pièces pour la composition des lames postérieures, et dans les vertèbres les plus étendues, deux noyaux osseux surmontant les apophyses épineuses; ce qui porte à huit le maximun des élémens osseux de chaque vertẻbre, qui, confondus par la séric des transformations, constituent un anneau unique, autour duquel on n'apcrçoit plus aucun indice de sa primitive composition,

Chaque segment de la moelle épinière répète t-il la composition de l'anneau osseux qui l'environne? Chaque noyau de la vertebre cst-il representé par un cordon distinct dans l'axc ncrveux 
du tronc? Chaque moitié de la moelle épinière se subdivise-t-elle en quatre faisceaux, comme chaque moitié de la vertèbre en quatre élémens osseux? L'analogie l'indiquait; c'était à l'obser-. vation à le prouver : or, l'observation le prouve.

Si vous considérez la moelle épinière des embryons des oiseaux, des reptiles et des mammifères, au dernier tiers de leur formation, vous. trouvez d'abord en avant un cordon central de chaque côté, marchant parallèlement à la suture antérieure des lames de la moelle épinière : ces deux cordons correspondent aux deux noyaux osseux du corps de chaque vertèbre. Vous distinguez ensuite deux cordons plus étendus sur chaque côté de ceux-ci; ce sont les représentans des masses transverses des vertèbres. Vous apercevez ensuite quatre semblables cordons en arrière, deux au centre et deux sur les côtés, qui correspondent aux lames vertébrales ct aux neyaux des apophyses épineuses. Il y a donc huit cordons a la moelle épinière, comme il y a huit noyaux osseux dans les vertèbres les plus composées.

Si des embryons vous passez aux animaux parfaits, vous trouvez chez les poissons osseux la répétition des huit cordous que vous ont offerts les premiers. Soit, par exemple, la morue : vous voyez en avant de la moelle épinière (1) quatre cordons très-distincts ct très-apparens, deux au

(1) II. VII. fig, $16 / 4, \lambda, 11$ ". 
centre (1) et deux sur les côtés (2); vous trouvez en arrière quatre faisceaux semblables, deux sur les côtés (3) et deux sur le centre (1). Pareillement chez le trigle, vous avez quatre cordons en avant (5) et quatre cordons en arrière (6) ; pareillement chez le turbot, vous rencontrez les quatre cordons antérieurs ( $(7)$ et les quatre postérieurs (8), formant un relief saillant sur la surface extérieure de la moelle épinière. De même chez la perche (9), de mème chez le congre (10), de même chez le merlan. Les poissons osseux reproduisent donc d'une manière permanente et très-distincte les huit cordons que l'on rencontre chez les embryons des autres classes.

Déjà chez les poissons cartilagineux les huit cordons sont moins distincts; il n'y a plus de visibles, sans préparation, que les quatre antérieurs (11), les postérieurs sont confondus en

(1) PI. VII, fig. 162, B.

(2) Pl. VII, fig. $162, A$.

(3) PI. VII, fig. $161, \mathrm{C}$.

(4) Pl. VII, fig: $161, \mathrm{~A}$.

(5) PI. VII, fig. $156, \mathrm{n}^{\circ} 9$ et ro.

(6) Pl. VII, fig. $157, \mathrm{n}^{\circ} 1$ et 2.

(7) Pl. VII, fig. $179, \mathrm{~A}, \mathrm{~B}$.

(8) PI. VII, fig. $174, n^{\circ} 2$ et 7.

(g) PI. VI, fig; 147 , et $136, \mathrm{n}^{\circ} 1 ; 4,7$ et 8.

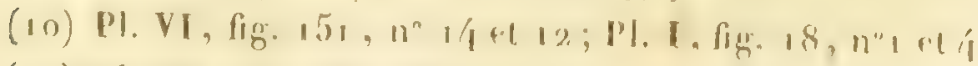

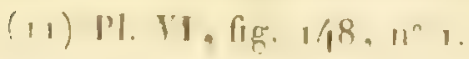


arrière (1). Remarquons, à ce sujet, que par la composition de la moelle épinière, les poissons cartilagineux occupent un rang plus élevé que les osseux, tandis qu'on les croirait plus descendus par la composition de leur rachis, celui-ci s'arrêtant à la transformation cartilagineuse. Quelle peut être la cause de cette opposition?

Chez les reptiles et les oiseaux adultes, les cordons ne font aucune saillie apparente sur la surface de la moelle épinière; mais en la déplissant avec soin, on y retrouve les quatre cordons en avant, et les quatre en arrière. Parmi les oiseaux, ceux chez lesquels leur manifestation m'a paru la plus apparente, sont l'aigle, lc milan, le vautour, la busc et le buzard; ils sont plus sensibles dans la partie dorsale et lombaire que dans la région cervicase.

L'inverse se remarque chez les mammifères parfaits; on n'aperçoit point les huit cordons à partir du tiers supérieur de la région dorsale; mais dans la région cervicale il est facile de les mettre en évidence, surtout chez le bouf, le cheval, le chameau, plus encore chez le marsouin, où ils sont moins confondus; chez l'agouti ils sont également faciles à distinguer. En général, plus on s'élève, dans cette classe, dans la région cervicale, plus les huit cordons deviennent distincts; plus on descend vers la région lombaire, plus ils sont

(1) Pl. VI, fig. 1 \&2, $\mathrm{rl}^{\prime \prime} 1$. 
confus : la moelle épinière ne paraît composée dans cette région que de quatre cordons, deux antérieurs et deux postérieurs.

De ces particularités diverses de l'organisation et du développement de la moelle épinière, dérivent les gouttières et les rainures qui en sillonnent la superficie.

Les cordons primitifs se réunissent d'abord en avant sur la ligne médiane par une suture trèsserrée; la moelle épinière offre un sillon à la partie antérieure chez tous les animaux, comme on le voit chez le trigle (1), la morue (2), le turbot (3), la tanche (4), l'anguille (5), la carpe (6), le brochet (7), la raie (8), le crocodile (9), la vipère hajé (10), la grenouille (11), la tortue franche (12), la poule (13), le casoar (14), le perro-

(1) Pl. VII, fig. $156, \mathrm{n}^{\circ} 9$ et 10.

(2) Pl. VII, fig. $162, A, B$.

(5) Pl. VII, fig. 176, B.

(4) PI. VII, fig. $186, \mathrm{n}^{\circ} 1$.

(5) Pl. VII, fig. $19^{2}, \mathrm{n}^{\circ} \mathrm{a}$

(6) Pl. VI, fig. $146, \mathrm{n}^{\circ} \mathrm{s}$

(7) Pl. X, fig. $217, \mathrm{~A}$.

(8) Pl. VI, fig. 148, A.

(9) Pl. V, fig. $118, \mathrm{n}^{\circ} 1$.

(10) Pl. V, fig. $127, \mathrm{n}^{\circ} \mathrm{x}$

(1i) Pl. V, fig. $131, n^{\circ} \%$

(12) Pl. V, fo. 122, $n^{\circ} 1$.

(13) Pl. III, fig. $85, n^{\circ}$.

(1i) Pl. III, fig. $79,1^{\circ} 2$. 
quel (1), la bondrée commune (2), l'autruche (3), la cigogne blanche (4), le drill (5), le mandrill (6), le raton (7), la marmotte (8), le phoque (9), l'agouti (10), la loutre (1 I), l'ours (12), le dauphin (13), le tatou (14), le chameau (15), le porc-épic (16), la taupe (17), le castor (18), le bouc de la Haute-Égypte (19), le lion $(20)$, le daman (21), le zemni (22), la

(1) Pl. III, fig. $82, n^{\circ}$.

(2) Pl. III, fig. $9^{1}, \mathrm{n}^{\circ} 1$ et 2.

(3) Pl. III, fig. $9^{8}, n^{\circ}$.

(4) PI. III, fig. $103, \mathrm{n}^{\circ} 2$.

(5) PI. VIII, fig. $194, \mathrm{~A}, \mathrm{~B}$.

(6) Pl. VIII, fig. $197, \mathrm{~B}$.

(7) Pl. VIII, fig: 200, $\mathbf{n}^{\circ}$.

(8) Pl. IX, fig. 205, $\mathrm{n}^{\circ} 9$.

(9) PI. IX, fig. 208, $\mathrm{n}^{0} 11$.

(10) Pl. IX, fig. 205, $\mathrm{n}^{\circ} 1$.

(ii) Pl. X, fig. $223, \mathrm{~A}$.

(12) Pl. XI, fig. 23I, A.

(13) Pl. XII, fig. $23 \dot{4}, \Lambda$ :

(14) Pl. XIII, fig. $246, n^{\circ} 1$.

(15) Pl. XIII, fig. 249, $n^{\circ} 10$

(16) PI. XIII, fig. 251, A.

(17) PI. XIV, fig. 260, A.

(18) Pl. XIV, fig. $258, \mathrm{~A}, \mathrm{~B}$.

(ig) PI. XIV fig. 262, A.

(20) PI. XIV fig. 266, A.

(2i) Pl. XV, fig. $275, A$.

(22) PI. XV , fig. $272, A$. 
loutre (1), le cheval (2), le lama (j), le kanguroo géant (4) et l'homme (5).

Après s'être réunis en avant, ces deux cordons se joignent en arrière et donnent naissance au sillon postéricur de la moelle épinière, sillon plus ou moins profond selon les animaux, et qui, comme l'antérieur, se remarque dans toutes les classes; chez les trigles (6), la morue (7), le congre (8), l'anguille (9), l'égrefin (10), la baudroie (11), le barbeau (12), le turbot (13), la tanche (14), la raie (15), l'esturgeon (16), l'orvet (17), le lézart vert (18), le tupinambis (19),

(1) Pl. XV, fig. $290, \mathrm{n}^{\circ}$.

(2) Pl. XV, fig. $275, \mathbf{A}$.

(3) PI. XVI, fig. $295, \mathrm{~A}$.

(4) Pl. X VI, fig. 300, A.

(5) PI. XIII, fig. 2/7, $n^{\circ} 1$.

(6) Pl. VII, fig. $155, n^{\circ}$, et $158, n^{\text {? }} 6$.

(2) Pl. VII, fig. 161, A.

(8) Pl. VII, fig. 168, A.

(9) Pl. VII , fig. $174, \mathrm{n}^{\circ} 2$.

(10) $\mathrm{Pl}$. VII, fig. $177, \mathrm{n}^{\circ} \mathrm{x}$.

(11) Pl. VII, fig. 179, $\mathrm{n}^{\circ} \mathrm{x}$.

(12) Pl. VII, fig. 185, B, B.

(13) PI. VII, fig. $181, \mathbf{n}^{\circ} 1$.

(14) Pl. VII, fig. $185, n^{\circ}$ 1.

(15) Pl. VI, fig. 158, n ${ }^{\circ}$.

(16) PI. XII, 6g. $223, n^{\circ}$ i et 2

(17) Pl. V, fig, $109, \mathrm{n}^{0} \mathrm{l}$

(18) Pl. V, fig. $110, n^{\circ} 5$.

(19) PI. V, fig. 114. $1^{\circ} 5$ 
la vipère de Fontainebleau ( 1 ), la vipère à raies parallèles (2), le caïman (3), la grenouille (4), la tortue franche (5), la poule (6), le casoar (7), le perroquet (8), la bondrée commune (9), l'aigle (10), le raton (11), la marmotte (12), le phoque (13), l'agouti (14), le mandrill (15), le coati (16), la taupe (17), le lion (18), le castor (19) et l'homme (20).

Il n'y a donc et il ne peut y avoir que deux sillons fondamentaux sur la surface de la moelle épinière; leur formation est aussi inévitable que

(1) Pl. V, fig. $132, n^{\circ} .1$

(2) Pl. V, fig. 133, $\mathrm{n}^{\circ} 1$ et 2.

(3) Pl. V, fig. 135, $\mathrm{n}^{\circ} \mathrm{i}$ et 2.

(4) Pl. I, fig. $16, \mathrm{n}^{\circ} \mathrm{i}$.

(5) Pl. V, fig. i19, $\mathrm{n}^{\circ} 1$ et 2.

(6) Pl. III, fig. $87, \mathrm{n}^{\circ} \mathrm{x}$ et $\mathrm{x}$.

(5) Pl. III, fig. 83, $\mathrm{n}^{\circ} 1$ et 2.

(8) P1. III, 6g. $84, n^{\circ}$.

(9) Pl. IV, fig. $9^{\circ}, n^{\circ} 1$.

(10) Pl. IV, fig. 101, $\mathrm{n}^{\circ} \mathrm{s}$

(11) PI. VIII, fig. 201, $n^{\circ} 1$ ot 2:

(12) Pl. IX, fig. 207 , A.

(13) Pl. IX, fig. 209 , A.

(14) Pl. IX, fig. $213, \mathrm{~A}$.

(15) Pl. XII, fig. 241, n* 1.

(16) Pl. XII, fig. $242, n^{\circ}$.

(17) Pl. XIV, fig. 256, A.

(18) Pl. XIV, fig. $264, n^{\circ} 1$.

(19) Pl. XIV , fig. $265, n^{\circ}$.

(20) PI. XIII, fig. $245, n^{\circ} 1$ et 2. 
celle des sutures qui unissent les os du crâne entre eux; mais, de même que celles-ci, ces sillons s'effacent plus ou moins chez les animaux adultes, et de là vient la dissidence des opinions émises à ce sujet. Il serait toutefois difficile de concevoir comment Vésale, Piccoluomini, Laurent, Bauhin et Spigel, ont nié leur existence, si l'on ne savait que ces anatomistes considéraient la moelle épinière comme un cordon unique.

Haller, Huber et Frotscher, n'ont admis que le sillon antérieur, parce qu'il est, en effet, plus apparent que le postérieur chez l'homme, les singes et quelques autres mammifères; mais sur beaucoup d'autres, tels que le cheval, le coati, le raton, le postérieur est aussi sensible que l'antérieur, et chez beaucoup de rats, chez la taupe, chez le lièvre et le lapin, le postérieur est plus prononcé que l'antérieur.

Chez les oiseaux, le sillon antérieur est constamment plus prononcé que le postérieur, qui même ne devient sensible que par l'effet de la macération, exceplé toutefois à la région sacrée, sur laquelle l'écartement des lames postérieures produit un hiatus (1), plus ou moins ouvert, selon la prédominance ou la faiblesse des extrémités postérieures, hiatus constant chez tous les oiseaux, et qui est le caractère classique de leur moellc épinière.

(1) PI. II, fig. $25, n^{\circ} 2$. 
Chez les reptiles, la profondeur des deux sillons est égale en avant et en arrière; les lames postérieures de la moelle épinière sont écartées en haut de la région cervicale (1), comme elles le sont en bas chez les oiscaux : le calamus scriptorius est alors beaucoup plus distendu chez les reptiles., à cause de cette circonstance, comme on l'observe chez la grenouille (2) et la tortue franche (3).

Chez les poissons cartilagineux, l'antérieur est plus prononcé que le postérieur; chez les osseux, au contraire, le postérieur est plus profond que l'antérieur. Ces derniers répètent, sous ce rapport, les embryons des mammifères jusqu'au commencement du dernier tiers de leur formation; souvent même les cordons postérieurs sont écartés sur divers points de leur élendue, de manière à former une ouverture qui pénètre en forme de scissure dans le canal épinien. On remarque particulièrement cet effet chez la morue, le congre. la tanche et la perche.

Chez tous les vertébrés, les deruiers ramuscules des artères spinales antérieures et postérieures pénètrent dans les interstices de ces sillons; cette disposition si manifeste chez les jeunes cmbryons, est d'autant plus sensible chez. les

(1) Pl. I, Gg. $16, \mathrm{n}^{\circ} 1$.

(2) Pl. I, fig. $12, \mathrm{n}^{\circ}$.

(3) Pl. V, fig. $119, n^{\circ} 2$. 
MOELIE ÉPINIERE.

animaux adultes, que les sillons sont cux-mônes plus apparens.

En outre de ces deux sillons fondamentaux, il existe d'autres rainures sur les côtés de chaque cordon, rainures dont l'existence est plus contestée encore que celle du sillon postérieur, parce qu'elles disparaissent en grande partie chez les animaux adultes. De ce nombre sont les rainures que l'on a nommées sillon latéral antérieur, sillon latéral postérieur. Pour les apercevoir, il faut se reporter, chez les embryons, à l'époque où les huit cordons sont apparens dans la moelle épinière, ou chez les poissons osseux, qui répètent ces embryons, comme déjà nous l'avons observé : on voit alors une rainure interposée entre le cordon antérieur (1) et l'antérieur latéral (2) de la moelle épinière, et une semblable dépression longitudinale entre le cordon postérieur médian (3) et le cordon latéral postérieur (4). On peut voir la terminaison de ces deux sillons chez la morue (5), les trigles (6), le turbot (7) et le congre (8). Chez les aiseaux et les reptiles on n'en aperçoit aucune

(1) Pl. VII, fig. 162, B.

(2). Pl. VII, fig. 162, A.

(3) Pl. VII, fig. $174, n^{n} 2$

(4) Pl. VII, fig. 174, n"7.

(5) Pl. VII, fig. 162 et 16 .

(6) Pl. VII, fig. 156 et $15 \%$.

(7) Pl. VII, fig. 174 et 176

(8) PI. I, fig. 18. 
I race, et chez les mammifères adultes on nc la met à découvert dans la région cervicale qu'après une macération plus ou moins longue dans un liquide préparé. Le postérieur se découvre plus facilement que l'antérieur, et l'un et l'autre, autant que je l'ai pu juger chez les embryons et les poissons osseux, correspondent à l'insertion des branches antérieures et postérieures des nerfs spinaux.

En général, chaque moitié de cordon de la moelle épinière est plus épaisse en avant (1) et en arrière (2) que sur sa partie moyenne (3). Il résulte de cette disposition une dépression sur le côté de chaque cordon, dépression que beaucoup d'anatomistes ont désignée sous le nom de sillon latéral de la moelle épinière. Celte différence d'épaisseur du cordon provient de ce que la matière blanche est plus épaisse en avant et en arriêre qu'au milieu; et elle n'est pas compensée totalement par la disposition de la matière grise, qui est, au contraire, en sens inverse de la blanche, plus épaisse au milieu, et plus mince en avant et en arrière.

Pour bien saisir ces faits, il faut, chez les mammifères, ouvrir la moelle épinière par le sillon postérieur, et renverser à droite et à gauche

(1) P]. IX, fig. $205, \mathrm{n}^{\circ} \mathrm{I}$.

(2) Pl. IX, fig. 205, $n^{\circ} 2$.

(3) PI. IX, fig. 205, n०3. 
lis deux lames. On voit, alors d'une part, la différence d'épaisseur que nous venons d'indiquer, et on remarque, de plus, quel'épaisseur s'étend plus loin sur la moitié antérieure que sur la postérieure; ce qui fait que la dépression latérale n'occupe pas le milieu du cordon, mais se déjette un peu plus en arrière qu'en avant.

On remarque toujours encorc, chez les mammifères, que cette différence d'épaisseur va en diminuant de la région cervicale à la région dorsale, au milieu de laquelle elle disparaît presque complètement; ce qui fait, d'une part, que la moelle épinière est plus aplatie à la partie supérieure qu'à l'inférieure, et de l'autre, que la dépression latérale n'est sensible que sur la région cervicale et sur une très-petite partie de la région pecrorale. On ne la voit plus dans les deux tiers de la région dorsale, ni dans la partie lombaire, ni dans la région caudale.

Dans les trois classes inférieures, la matière blanche de la moclle ćpinière allant foujours en croissant, la partie moyenne du cordon latéral devient plus épaisse, la dépression latérale disparaît; ce qui fait que cliez les oiscaux, les reptiles et les poissons, la moelle épinière est sans goultière latérale, commele sont les trois quarts inférieurs de cet organe chez les mammifères.

Laurent, Bauhiu, G. Bartholin, Blaes, Willis . Halpighi, Vicussens, s'expriment si claircment sur la nature fibrouse de la monlle épinière, et II. 
indiquent des procédés si simples pour mettreà découvert cette disposition, qu'on est véritablement surpris devoir cette vérité rejetée par Huber et Haller, et par plusieurs anatomistes modernes. Parmi ces derniers, Soemmering, M. Cuvier, Frotscher, M. Gall, Keuffel, Rachetti et Rolando, ont rendu évidente la texture lamineuse de cot axe nerveux des vertèbres. Les jeunes animaux, ou mème les foetus, au terme de leur naissance, sont les plus convenables à cette recherche. On développe encore les fibres en faisant macérer la moelle épinière. D'ailleurs, elles sont à nu dans les foetus hydromiellis, chez lesquels cette partie a éprouvé une macération naturelle; elles sont quelquefois évidentes sans aucune préparation au commencement des ramollissemens pathologiques qu'elle éprouve chez l'homme; enfin elles sont tout-à-fait à nu sur la surface extérieure de la moelle épinière de certains poissons osseux.

Quelle que soit la manière dont on les mette à découvert, on trouve la moelle épinière composée de trois ordres de fibres : les unes, parallèles', occupent principalement les côtés des sillons antérieurs et postéricurs; M. Gall les a indiquées : les autres, plus latérales et plus profondes, sont légèrement obliques; celles-ci se continuent en grande parlie avec les nerfs spinaux, et se joignent à leurs faisceaux antérieurs et postérieurs; elles sont plus nombreuses sur le cordon antérieur que sur le postérieur, et plus grosses sur le postérieur que 
sur l'antérieur; jamais elles ne se rendent jusqu'à la substance grise. Une variété de cet ordre, plus superficielle que les précédentes, forme des ares étenclus des faisceaux d'inscrtion d'un nerf aux faisceaux du nerf voisin: ces arcs se regardent par leur face convexe comme les deux parties de cette figure) (, et rendent continue la chaîne d'insertion des filets antéricurs et postérieurs. Le troisième ordre de fibres est transverse, il occupe spécialement le fond des sillons antérieurs et postérieurs, et réunit cn avant et en arrière les deux moitiés de la moelle épinière : on voit bien les fibres longitudinales sur le cheval, le bouf, le mouton, le chevreau, le chien, le renard, le cochon, le sanglier, le ralon, et les fibres transverses chez les reptiles, principalement chez les batraciens.

C'est ici le lieu de reproduire la question de la primogéniturc de l'encéphale ou de la moelle épinière. Quelques philosophes grecs, à la têle desquels se trouve Platon, imaginèrent que le cerveau provenait de la moelle épinière. En combattant cette opinion, Aristote tomba dans une ctrange erreur : il nia tout rapport physique entre ces deux parties. Celte question, délaisséc par les anatomistes i la renaissance des lellres, fut reprise par Bartholin et par Malpighi : par Bartholin, le premier des analomistes qui ait avancé que la moclle ćpinière élait la racinc de l'encéphale; et par Malpighi, qui en fournit les 
premières preuves en signalant l'apparition priz mitive de la moelle épinière dans la formation de l'axe cérébro-spinal du système nerveux. Néanmoins l'opinion contraire prévalut; on continua à dériver la moelle épinière du cerveau, et à la considérer comme la plus forte et la plus im. portante de ses productions.

L'anatomie avait placé la racine de l'axe nerveux des vertébrés dans la moellc épinière; la physiologie la transporta dans l'encéphale par les raisons qui suivent : toute altération du cerveau suspeud, modific ou anéantit l'action de la moelle épinière; les altérations de la moelle épinière n'influencent au contraire en aucune manière les fonctions spéciales du ccrveau; donc, physiologiquement, l'action nerveuse se propage du cerveau à la moelle épinière; celle-ci n'en paraît que le principal conducteur. La pathologie et l'anatomie pathologique vinrent confirmer les données de la physiologie: tout le monde crut, tout le monde adopta l'idée, que la moelle épinière dérivait anatomiquement du cerveau, comme elle semblait emprunter de lui son action.

Cette opinion était professée par toutes les écoles el par tous les anatomistes, lorsque MM. Gall et Spurzhcim reproduisirent celle de Platon, de bartholin et de Malpighi. Partant du fait que la moelle épinièrest dércloppéchez les animanx en raison inverse du rerveati, il parul ridiculr aux anatomisles allemands de dériver les 
Wus fortes muelles épinieres des plus petits cerveaux, et de faire produire aux plus grands cerveaux les plus petites moèlles épinières. Cette raison spécieuse ne souffre pas un examen approfondi : car qui ne voit qu'il est aussi difficile de faire produire les plus grands cerveaux par les plus petites moelles épinières, et les plus petits par les moelles épinières les plus développées? En raisonnant de cette manière, c'est renfermer le problème dans un cercle de propositions vicieuses; car depuis que j'ai démontré que certaines parties de l'encéphale sont rigoureusement développées en raison directe de la moelle épinière, on pourrait donc, et on devrait même, dans celle supposition, dériver l'axe nerveux du tronc, des parties qui lui correspondent dans l'encéphale pour le volume.

Je fus conduit par ces difficultés à ramener la question à son véritable point: cousidérée en cffet dans tous ses rapports, c'est un problème d'organogénie, commel'avait cntrevu notre illustre prédécesseur Malpighi. Il ne s'agit dès-lors que de déterminer par l'observation l'ordre de succession dans les développemens de l'ax c cérébro-spinal. Quelle est la parlic qui précèdle l'autre dans sa formation? est-cel'cncéphale, est-ce la moclle épinière?

()r, les faits fournis par l'embryogénic pronvent que dans sa formation la mocelle épinièce precide l'encéphate: sur les jouncs embryons 
des oiseaux (1), des reptiles (2), des mammifères (3) et de l'homme (4), les cordons qui circonscrivent la moclle épinière apparaissent avant ceux du cerveau. Celui-ci est encore à l'état de fluide, et vésiculcux, chez les oiseaux (5), les reples (6), les mammifères ( 7 ) et l'homme (8), lorsque les formes de la moelle épinière (9) sont déjà arrêtées (10); les pyramides (11), les olives (12), le corps restiforme ne deviennent distincts que lons-temps après la moelle épinière. Enfin, chez l'homme (13), les mammifères (14), les reptitiles ( 15 ) et les oiseaux (16), le cervelet n'apparaît que long-temps après que les évolutions de la moclle épinière sont terminées; ce qui établit, se-

(1) Pl. I, fig. 2, $n^{\circ} 3$ et 4 .

(2) Pl. I, fig. $9, n^{\circ} 5$ et 4 .

(3) PI. I, fig. $20, \mathrm{n}^{\circ} 1$ et 2.

(4) Pl. I, fig. $26, n^{\circ} 2,3$ et 4 .

(5) Pl. I, fig. $5, \mathrm{n}^{\circ} 6$ et 8 .

(6) Pl. I, fig. $15, \mathrm{n}^{\circ} 6$ et 7 .

(7) Pl. I, fig. 2x, $\mathrm{n}^{\circ} 6,7$ et 8.

(8) Pl. I, fig. 24, no 3,4 et 5 .

(9) Pl. I, fig. $5, \mathrm{n}^{\circ} 2$ et 3.

(10) Pl. I, fig. $15, n^{\circ} 2,3$ et 4 .

(ii) Pl. II, fig. $61, n^{\circ} 5$.

(12) Pl. II, fig. $67, \mathrm{n}^{\circ} 2$.

(15) Pl. I, fig. $25, n^{\circ} 6$.

(14) Pl. I, fig. $28, n^{\circ} 4$.

(15) Pl. I, fig. $12, \mathrm{n}^{\circ} 2$.

(16) Pl. I, fig. 5, no 5 . 
lon nows, que la moclle épinière précède l'encéphale dans sa formation.

Ce développement précoce est en harmonie avec toule l'organogénie; ainsi l'aorte précède lc cœur dans sa formation. La transformation cartilagineuse $\mathrm{du}$ rachis commence d'abord dans les vertèbres dorsales, puis dans les cervicales, et ne s'opère que long-temps après dans la région de la base du crâne. Il en est de même des points osseux primitifs; les vertèbres dorsales s'ossifient long-tempsavant les os du crâne. Tous ces effets ont leur cause dans le système sanguin : les artères intercostales centrales sont les premières formées; ce n'est que plusieurs jours après elles qu'on aperçoit les artères carotides, et plus tard encore les artères destinées au cervelet. L'ordre de succession des organes suit donc rigoureusement l'ordre de développement du système sanguin.

L'anatomie transcendante est une science toute nouvelle : l'angiogénie, qui en constitue la base, n'a pas encore été étudiée; la formation du coeur a seule jusqu'à ce jour fixé l'attention des zootomistes. On me permeltra donc de présenter un aperçu rapide de la formation des artères spinales, qui se licnt immédiatement au point que nous traitons en ce moment.

On donne, dans les livres d'anatomie, une idée si fixe sur l'origine des artères spinales, que pendant long-temps je ne songeai gucire a mettre en doute ce point do leur départ primilif; un fait 
cependant vint m'éclairer à ce sujet. J'avais injecté un foetus acéphale borné par la partie supérieure à la septième vertèbre cervicale; en examinant lá moelle épinière, j’aperçus l'artère spinale antérieure et la postérieure sur les régions dorsales el lombaires de cet organe qui étaient formées. D'où provenaient ces artères? Je ne pouvais ici les faire naître des vertébrales, puisque celles-ci n'existaient pas; ni des cérébelleuses, puisqu'il n'y avait ni vestige de la tête, ni aucune trace qui indiquât que le cervelet et ses artères eussent existé. Je fus donc réduit à faire former les artères spinales par les branches des intercostales qui pénetrent dans les trous de conjugaison, et avec lesquelles on dit ordinairement que les spinales communiquent.

Ce fut alors, et alors seulement, que se préscntèrent à mon esprit les objections qui se déduisent contre cette hypothèse de la formation même de l'axe cérébro-spinal du système nervcux. En effer, personne ne croit plus maintenant que la moelle épinière soit une production de l'encéphale, et cependant personne ne doute encore que les artères spinalcs ne proviennent des artères encéphaliques. On fait provenir l'encéphale de la mochle épinière, et les artères de lit moelle épinière de celles de l'encéphaic. Celte contradiction est choquante; elle est cependant Io resultat inciviable de l'étude isolée des diffívens systènes nrganiques tols que lesont presentés 
notre illustre Bichat, et scs successcurs, quil'ont si peu compris. Pour rétablir l'harmonie dans les diverses parties de la science, il est plus nécessaire que jamais de faire marcher de front l'étude des systèmes fondamentaux de l'organisation. C'est ce que je me suis efforcé de faire dans cet ouvrage.

Si la moelle épinière ne naît pas de l'encéphale, les artires spinales. ne proviennent donc pas res artères encéphaliques? D’où proviennent-elles? Des intercostales. Comment se forment-elles? C'est ce que nous allons dire.

Avant d'en développer le mćcanisme, je vais rappeler la formation de l'artère basilaire, dont la disposition est connue de tous les anatomistes. Celte artèrc occupe le sillon médian de la protubérance annulaire, comme la spinale antérieurc occupe le sillon antéricur de la moelle épinic̀re. Or la basilaire résulte de la jonction sur la ligne médiane des deux artères vertébrales; cette jonclion se fait tantôt plus haut, tantôt plus bas; quelquefois les deux artères, après s'être réunies, se séparent de nouveau, et se réunisscnt une seconde fois en avant du pont de varole : il existo alors un anncau artériel correspondant à la partic: moyenne de la protubérance. D'autres fois, enfiu, llles ne se réunissent pas du tout, comme je l'ai observé chcz des anencéphales.

Cola posi, passons di l'artere spiuale antéricurr.

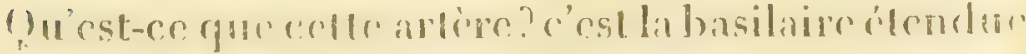

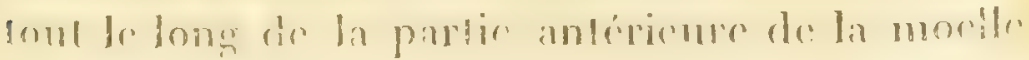


épinière; elle en diffère seulement par son long trajet : le mécanisme de sa formation est du reste le même que celui de l'artère centrale de la protubérance annulaire. Chaque artère spinale, après avoir fourni deux branches, dont l'une se distribue aux muscles, et l'autre à la vertèbre, en produit une troisième, qui est la continuation du tronc; celle-ci, après avoir traversé la dure-mère, se dirige obliquement en haut jusqu'au milieu de la moelle épinière; là, de même que l'artère vertébrale, elle rencontre la branche qui vient $\mathrm{du}$ côté opposé. Ces deux branches se confondent, et n'en forment plus qu'une, qui prend la direction verticale que l'on connaît à l'artère spinale antérieurc. La mème chose se passe à chaque vertèbre, à chaque artère spinale, d'où résulte la succession non interrompue de cette artère sur toute la parlie antérieure de la moelle épinière (1). Chaque artère inter-vertébrale se comporte donc comme les artères vertébralcs, ou plutôt ces dernières se comportent, sous ce rapport, comme les branches des intercostales, avec cette seule différence, qu'indépendamment de l'artère basi-

(1) Quelquelois l'artere calleuse est unique et centrale: alors le mécanisme de sa formation est analogue à celui de la basilaire et de l'artere spinale antérieure. Le plus ordinairement elle est double comme les spinales postéricures. lartere sacré moyeme résulte quelquefois aussi de lia remnion de deux bramehes. 
laire, elles produisent de plus les deux branches d'où l'on fait naître ordinairement la spipale antérieure. Le mécanisme de la formation de cette dernière artère est donc le même que celui de la basilaire. Comme celle-ci, la spinale antérieure est quelquefois double sur quelque partie de son trajet; il en résulte alors des anneaux artériels analogues à celni qui s'observe sur le centre de la protubérance.

Puisque les artères spinales ont une existence distincte indépendante de celle des artères encéphaliques, on conçoit donc maintenant comment la moelle épinière peut se former indépendamment du cerveau ; et pour expliquer les cas nombreux, que possède la science, de foetus anencéphales pourvus néanmoins de moelle épinière, il ne sera plus nécessaire de recourir à des suppositions de toute nature, à des maladies dont l'étiologie est plus obscure encore que celle du fait pour lequel on y a recours. Toute vertèbre renferme en ellemême les artèrcs qui produisent le segment nerveux qu'ellc doit revêtir. Que le foetus soit réduit à un tronçon de quelques vertèbres seulement, ce tronçon renfermera dans son centre la portion de moelle épinière qui lui correspond. Qu'il soit borné à la portion lombaire du rachis, comme jen ai vu un exemple, la moclle rachidienne scra néanmoins complètement lorméc. (üil n'ait que la portion thorachique, ainsi que j'on ai disséqui bux cxemples, dont l'un estau cabiuct d'anatomis 
du Iluséun d'histoire naturelle, ct toute la région lombaire et dorsale de la moelle épinière sera aussi bien constituée que si tout ce foetus s'étail régulièrement développé.

Je n'expose que des faits ou des corrélations d'existences organiques, et nous royons à chaque instant que ces faits expliquént le développement régulier et irrégulier des êtres. C'est là, si je ne m’abuse, le cachet des théories expérimentales. 
MOELLE EPINILIE.

TABLEAU COMPARATIH

Des Dimensions de la moelle épinière chez les Mammifères (I).

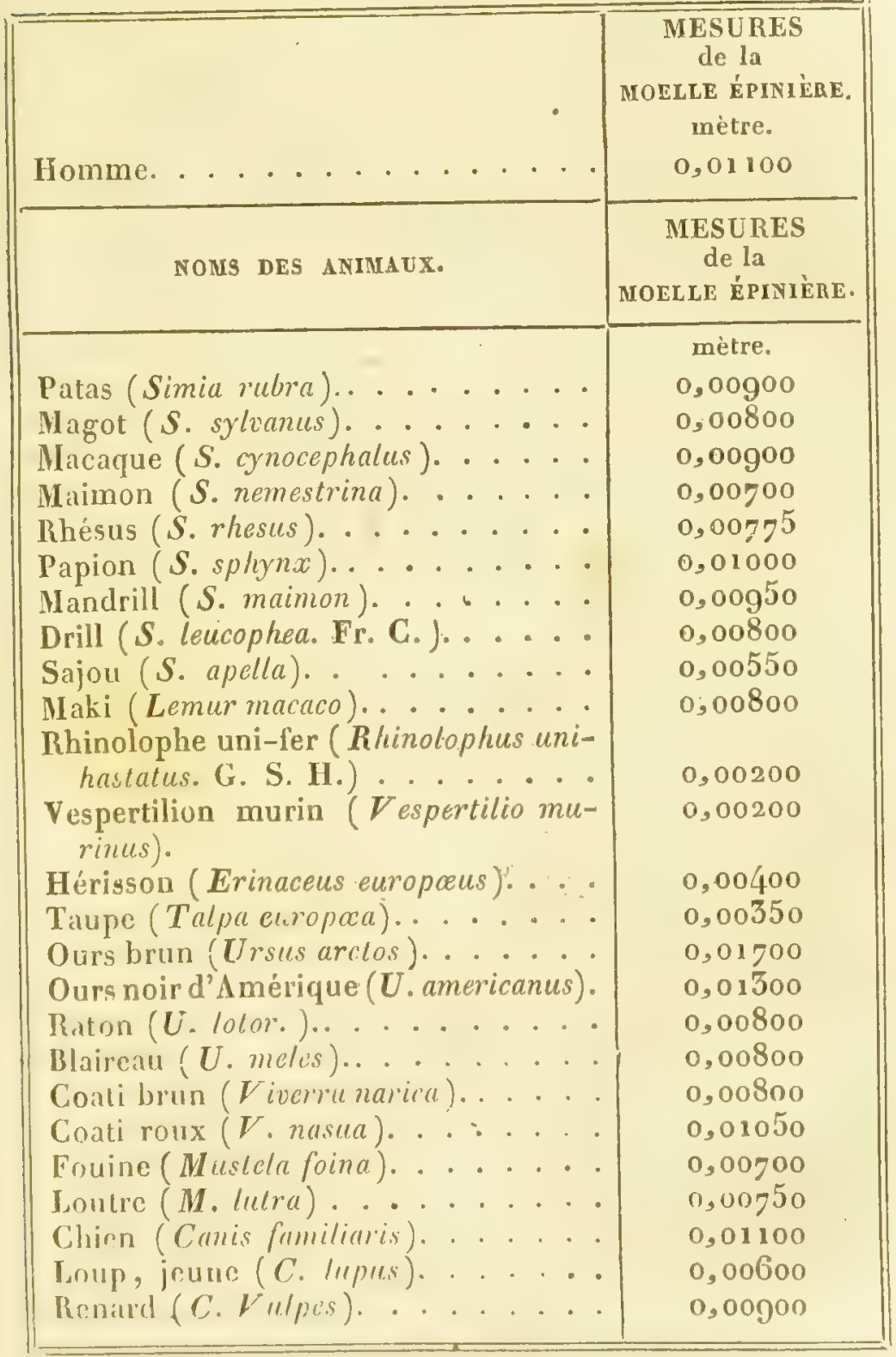

(1) Ia mesure a chi prise au niveau du segment correspondant, entre la premicre ct la deuxicme vertébre cervicale. 
Suite du Tableau comparatif des Dimensions de la moelle épinière chez les Mammifères.

\begin{tabular}{|c|c|}
\hline NOMS DES ANIMAXX. & $\begin{array}{c}\text { MESURES } \\
\text { de la } \\
\text { MOELLE ÉPIXIÈRE. }\end{array}$ \\
\hline 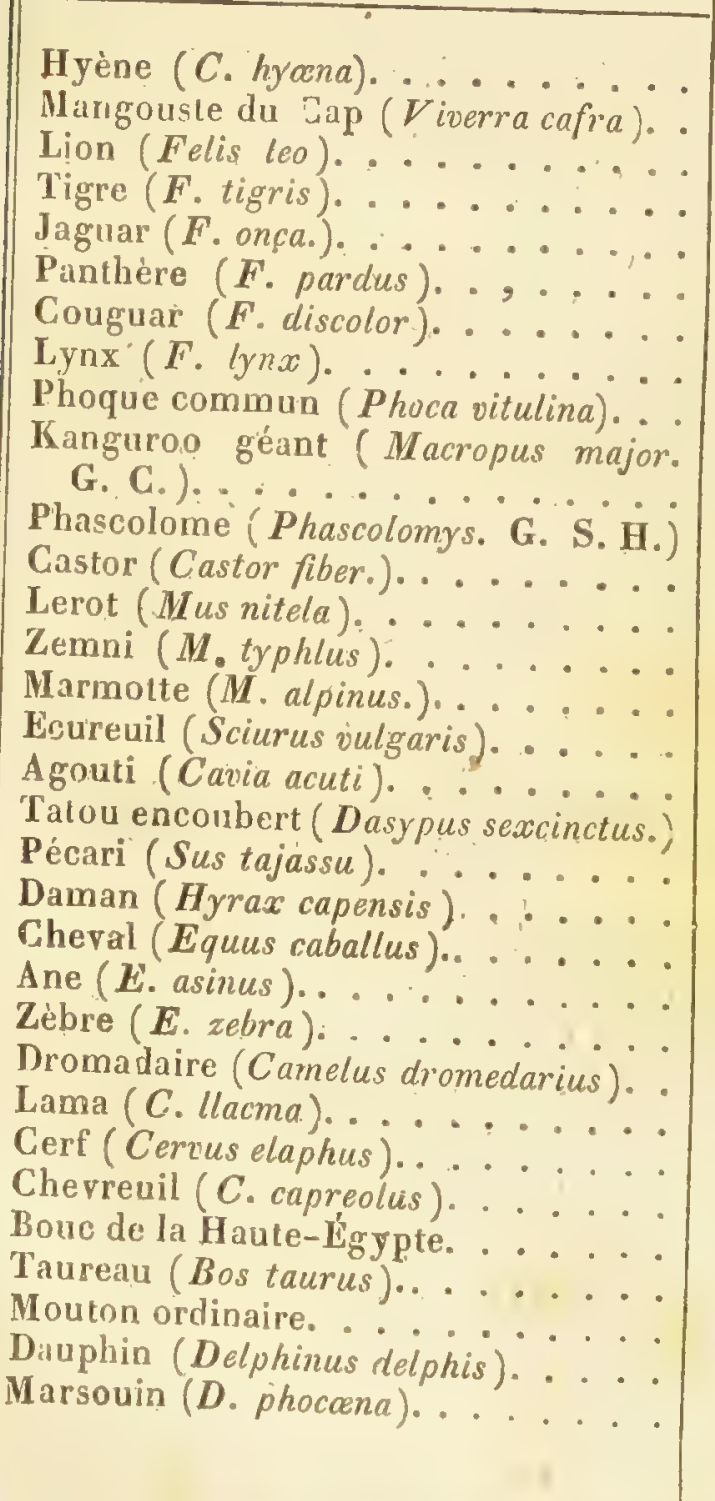 & $\begin{array}{l}0,01200 \\
0,00900 \\
0,00800 \\
0,00525 \\
0,00300 \\
0,00450 \\
0,00500 \\
0,00500 \\
0,00900 \\
0,01050 \\
0,00550 \\
0,02000 \\
0,01500 \\
0,01400 \\
0,01900 \\
0,01200 \\
0,00800 \\
0,01300 \\
0,01100 \\
0,01900 \\
0,00900 \\
0,01000 \\
0,00800\end{array}$ \\
\hline
\end{tabular}


Des Dimensions de la moelle épiniere chez les Oiseaux.

\begin{tabular}{|c|c|}
\hline NOMS DES ANIMAUX. & $\begin{array}{c}\text { MESURES } \\
\text { de la } \\
\text { MOELLE EPINIERE. }\end{array}$ \\
\hline 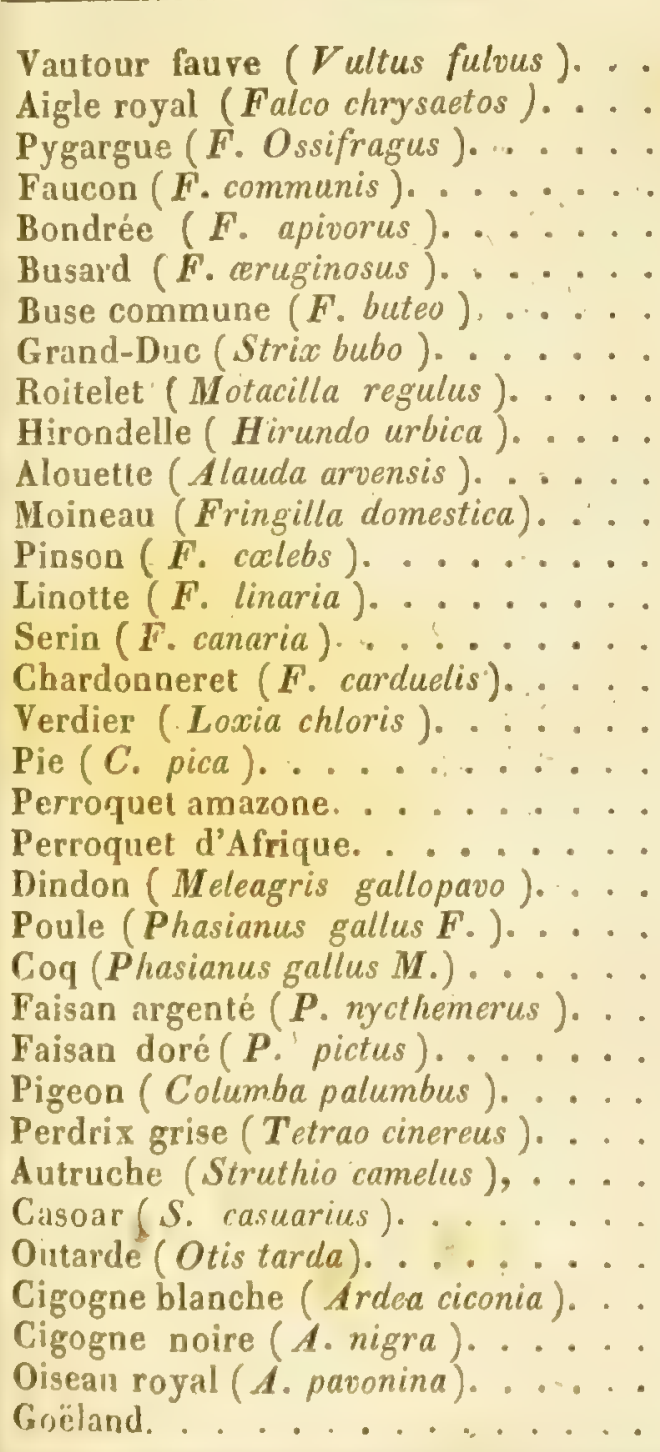 & $\begin{array}{c}\text { metre. } \\
\text { o, 00800 } \\
\text { o, } 00800 \\
0,00600 \\
0,00500 \\
0,00400 \\
0,00550 \\
0,00400 \\
0,00600 \\
0,00125 \\
0,00175 \\
0,00225 \\
0,00175 \\
0,00233 \\
0,00150 \\
0,00150 \\
0,00200 \\
0,00200 \\
0,00450 \\
0,00400 \\
0,00400 \\
0,00500 \\
0,00425 \\
0,00475 \\
0,00550 \\
0,00500 \\
0,00400 \\
0,00300 \\
0,00700 \\
0,00800 \\
0,00600 \\
0,00750 \\
0,00700 \\
0,00500 \\
0,00500\end{array}$ \\
\hline
\end{tabular}


176

MUELLE ÉPINILRL.

Suile du Tubleau comparalif des Dimensions de lu moelle épinière chez les Oiseaux.

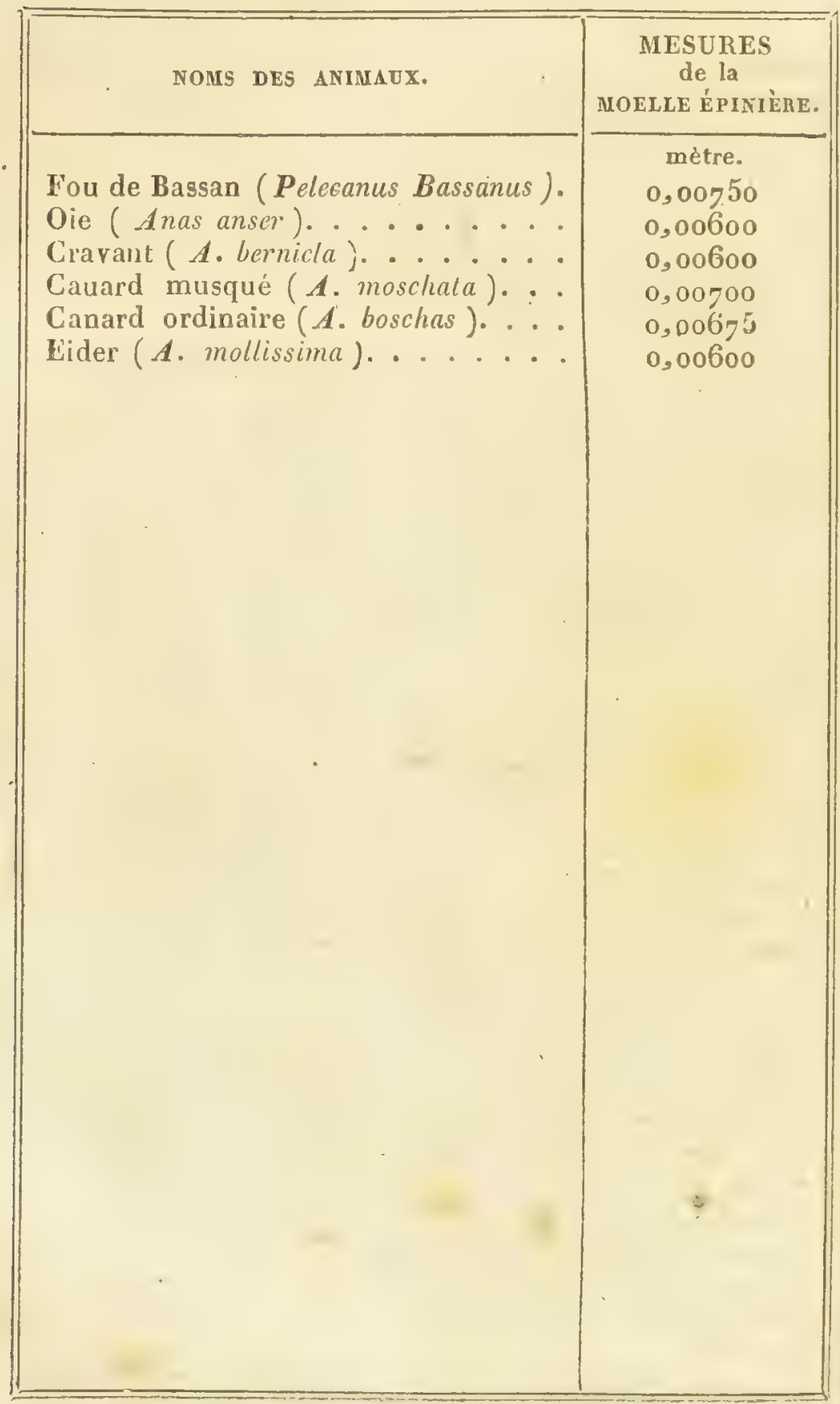


I A B I, EAL COMPARATIV

Des Dimensions de la moelle épiniere elerz les Reptiles.

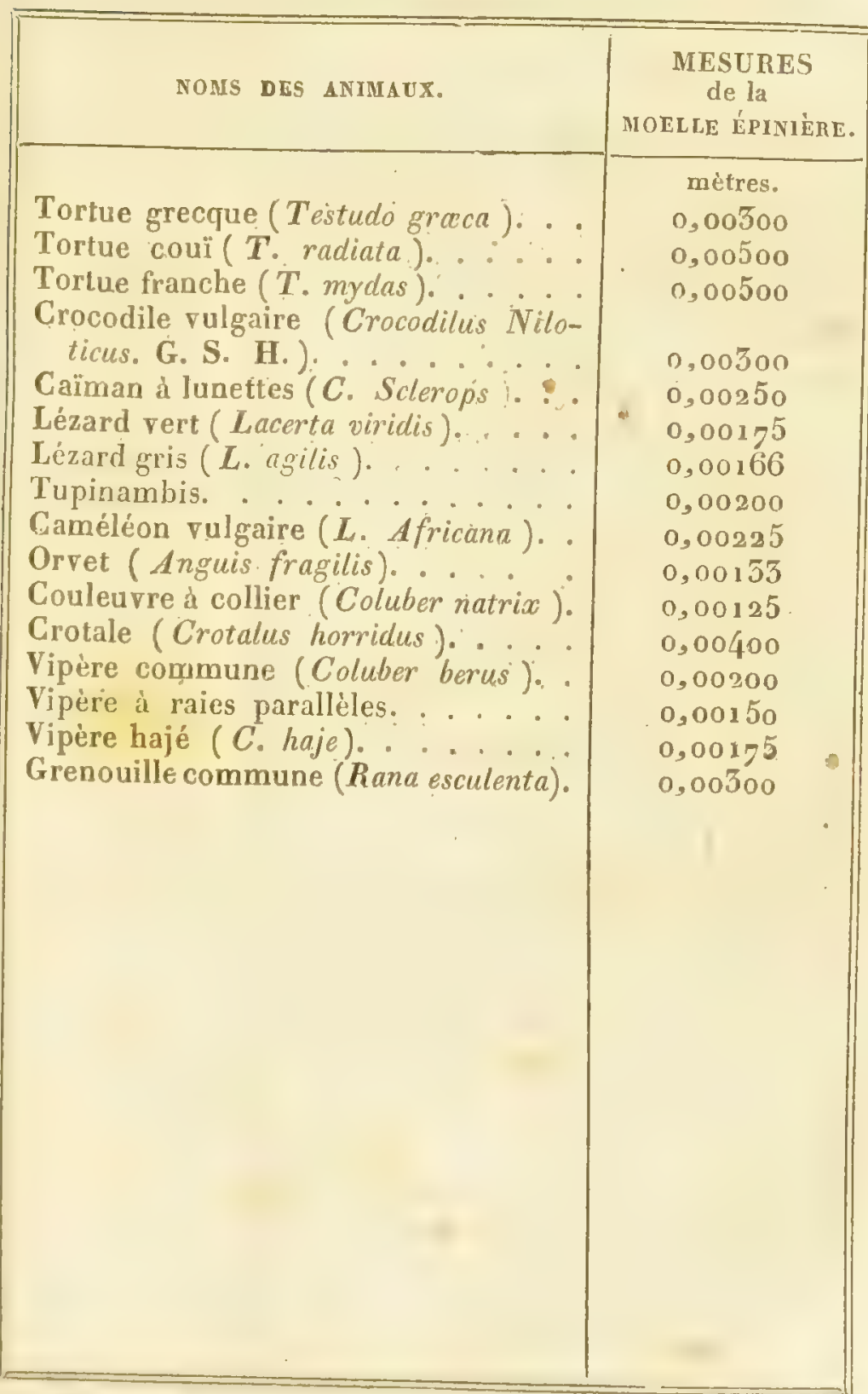


Des Dimensions de la moelle épinière chez les poissons.

\begin{tabular}{|c|c|}
\hline NOMS DES ANIMAUX. & $\begin{array}{c}\text { MESURES } \\
\text { de la } \\
\text { MOELLE EPINIÉRE. }\end{array}$ \\
\hline 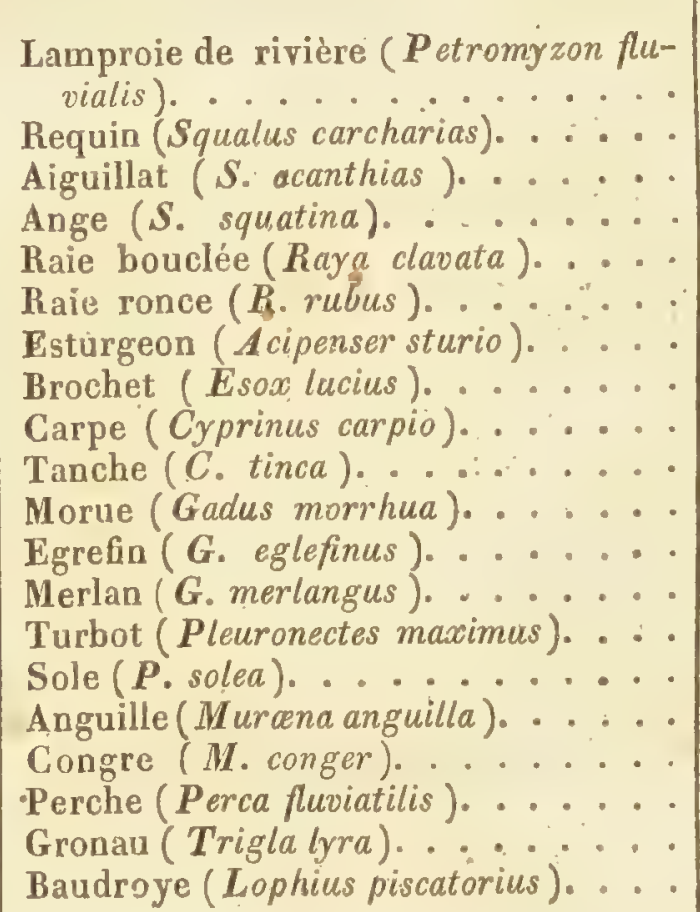 & $\begin{array}{l}\text { metres. } \\
0,00275 \\
0,00700 \\
0,00600 \\
0,00650 \\
0,00900 \\
0,00600 \\
0,00400 \\
0,00600 \\
0,00200 \\
0,00300 \\
0,00575 \\
0,00500 \\
0,00300 \\
0,00500 \\
0,00235 \\
0,00250 \\
0,00700 \\
0,00200 \\
0,00400 \\
0,00400\end{array}$ \\
\hline
\end{tabular}




\section{GHAPITRE IV.}

Anatomie comparative de la Moelle allongée dans les quatre classes d'animaux vertébrés.

La moelle allongée commence où finit la moelle épinière; mais quel eşt le point de son départ? quelles sont ses limites? L'anatomie n'a pu les assigner avec quelque certilude, parce qu'elle manque de lignes de démarcation distinctes : de là, l'arbitraire des auteurs dans les dimensions assignées à cette partie. Haller qui, le prenier, lui a consacré ce nom, la prolongeait sous le pont de varole, et fixait ses limites du trou occipital à la partie antérieure de la surface basilaire; il la faisait ainsi commencer à l'origine de la huitième paire, et finir un peu en arrière des tubcrcules quadrijumeaux et de l'insertion de la troisième paire crânienne. Après bien des variations à ce sujet, les anatomistes en sont revenus à l'opinion de Haller; mais ils y ont compris à tort la protubérance annulaire, qui, selon nous, ne peut être séparée du cervelet.

Quels que soient les noms divers par lesquels les auteurs ont désigné celte partie, tous se soňt accordés à la distinguer de la moelle épinière, à cause de la diversité de structure; tous l'ont re- 
garlée comme le point central de: l'encéphate. soit poury faire aboutir, soit pour en faire partir ses diverses radiations. Depuis Lorry, les physiologistes ont fait cause commune avec les anitomistes; ils ont reconnu que la meelle allongée étiit un des centres d'action les plus énergiques de l'encéphale. J'ai montré, il y a plusicurs années, que ses altérations étaicnt la source de beaucoup de maladies. L'anatomic, la physiologie ct la pathologie se réuniszent donc pour faire considérer la moclle allongre conme l'une des partics les plus importanics de l'axe cérébro-spinai dés animaux vertébrés.

La moelle allorf́gée fait donc suite ì la moelle epiniere. Primitivenent ces parties sont tout-àfait identiques chez les embryons de toutes les classes. La unoelle allongée, comme la moelle épinière, est forméc de deux lames isolées chez les oiseaux (1), les reptiles (2), les mammifères (5) et l'homme (4); leur réunion en avant continue le sillon antérieur de la moelle épinière; leur ćcartement en arricre produit l'excavation du quatrième ventricule et l'angle plus ou moins aigu qui le termine, et que l'on a désigné sous le nom de calamus scriptorius.

.(1) Pl. I, fig. 1, n $\mathrm{n}^{\circ} 5$ et 6 .

(2) Pl. I, fog. $9, n^{\circ} 5$.

(3) Pl. I, fig. $20, \mathrm{n}^{\circ} 2$.

(4) Pl. I, fig $26, n^{\circ} 5$ 
Lie Arenier est donc la fin de la jonction en arrière des lames de la moelle épinière; il termine le sillon postérieur, et il est situé plus ou moins haut, selon que les lames se réunissent plus tôt, comme chez les batraciens (1), ou plus tard, comme chez tous les mammilères (2) et tous les oiseaux (j). Le quatrième ventricule n'est, d'aprés cela, que la continuation du canal épinien, Irès-évasé en raison de la divergence des lames postérieures de la moelle allongée.

Comme sur la moelle épinière, on n’apercoit d'abord sur la moelle allongée que deux cordons étendus on membranes; cette parlie est lisse chez les jeunes embryons de toutes les classes; mais par la série des développemens, des faisceaux trèsdistincts se prononcent sur cette surface plane. et leur relinf les a fait soigneusement distinguer par le's ar!atomistes. Parmi ces faisceaux, il y en a quatrc principaux de chađjue côté, deux antérieurs (4) et deux postérieurs (5). Nous trourons donc réalisíe sur la moelle allongée l'une des stippositions qui ont été émises sur la structure de la moelle épinière; il n'est pas inulile de faire remarquer la liaison de ces idées. Les anatomistes

(1) Pl. I, fir. $16, \mathrm{H}^{\prime \prime} 1$.

(2) Pl. II, fig. $57, n^{\circ} 1$.

(3) Pl. III, fig. 83, $n^{\circ} 2$.

(4) Pl. XIII, fig. $249, \mathrm{~A}, \mathrm{O}$.

(5) PI. XIII, fig. $245, n^{\circ} 2$ et 5. 
ayant fait naître jusqu'à ces' derniers temps la moelle épinière de la moelle allongée, ont continué sur la première de ces parties les divisions tranchées qu'ils remarquaient sur la seconde : on a depuis bien exagéré cette analogie, en voyant dans la moelle épinière toutes les divisions et toutes les subdivisions du cervelet et des hémisphères cérébraux; mais toutes ces abstractions de l'esprit, nées de l'idée que le système nerveux est générateur de lui-même, s'évanouissent sous le scalpel.

La moelle allongée nous présente le premier degré de complication de l'axe cérébro-spinal du système nerveux; des huit faisceaux qui la composent, les antéricurs moyens ont reçu le nom de pyramides antérieures (1), les latéraux celui de cordons alivaires (2); les postérieurs moyens sont connus sous le nom de pyramides postérieures (3), et les latéraux désignés par la dénomination de cor dons restiformes (4). Ces faisceaux sont très-inégalement développés dans les diverses classes, et souvent dans les familles de la même classe : d ‘ l'inégalité de ce développement résultent nécessairement de grandes variations ảans son volume total et dans ses rapports. Pour arriver à quelques

(1) Pl. XI, fig. $251, n^{\circ} 1$; pl. XIV, fig. $289, n^{2} 4$.

(2) PI. XIV, fig. 258,0 ; fig. $289, n^{\circ} 5$ et 9 .

(5) PI. XIII, fig. $245, n^{\circ} 5$.

(i) Pl. XIII, fig. $245, n^{\circ} 2$. 
résultats certains, il est indispensable de considérer d'abord isolément chacun des quatre faisceaux qui constituent chaque moitié de la moelle allongée.

Des pyramides antérieures. Chez les poissons cartilagineux, les pyramides antérieures forment deux cordons rectilignes (1) qui, chez les raies, s'élargissent beaucoup en arrière des nerfs de la troisième paire (2) : chez la lamproie et l'esturgeon ils, sont si déliés, qu'on ne les distingue pas à l'extérieur (3). Chez les poissons osseux, leur apparence est en général plus. marquée que chez les chondroptérygiens. Chez la perche (4), le merlan (5), la carpe (6), le rouget (7), le gronau (8), les dẹux cordons, parallèles l'un à l'autre, sont visibles sur toute la base de l'encéphale; chez la morue (9) et le turbot (10), ils sont plus apparens encore, et sont légèrement renflés à leur partie la plus élevée.

Chez les reptiles, les pyramides sont si faibles

(1) Pl. VI, fig. 148, $\mathrm{n}^{\circ}$.

(2) Pl. VI, fig. $148, n^{\circ} 30$.

(3) Pl. XI, fig. $226, n^{\circ} 4$.

(4) Pl. VI, fig. $136, n^{\circ} 1$.

(5) Pl. VI, fig. $47, n^{\circ} \%$

(6) PI. VI, fig. $146^{\circ}, \mathrm{n}^{\circ} \mathrm{I}$

(7) PI. VII, fig. 156, n० 10.

(8) Pl. VII, fig. $157, n^{\circ} 2$

(9) Pl. VII, fig. $162, \mathrm{~B}$.

(10) PI. VII, fig. 176, B. 
qu'elles ne font aucunc saillie cxtérieure; la morlls. allongée reste toujours lisse, de même que che\% les jeunes embryons des mammifères; ainsi qu'on peut l'observer sur le camélćon ( $\mathrm{r}$ ), le crocodile (2), la vipère (3) et même la tortue franche (4). En ouvrant la moelle allongée, on les distingue cependant (5), de même que sur le fond du quatrième ventricule, tant sur la tortue (6) que sur la grenouille ( 7 ).

Haller a nié l'existence des pyramides antérieures chez les oiseaux, parce qu'en effet, chez le plus grand nombre, la moelle allongée est lisse dans toute son étendue, comme on le remarque chez le roitelet (8), l'hirondclle (9), la bondrée(10), la cigogne (11), l'autruche (12), le casaar (15); mais dans plusicurs espèces on découvre deux petites élévations longitudinales, parallèles soit entre

(1) Pl. V, fig. 112.

(a) Pl. V, fig. 117 et 118.

(5) Pl. V, fig. 127.

(4) Pl. V, fig $122, n^{\circ} 8$.

(5) Pl. XIV, fig. $288, n^{\circ} 4$.

(6) Pl. V, fig. $119, n^{\circ} 4$.

(7) Pl. V, fig. 134, a 1 .

(8) Pl. IV, fig. 94 .

(9) Pl. IV, frg. 92.

(10) Pl. IV, fig 91.

(11) Pl. IV, fig. 105.

(12) Pl. IV, fig: $9^{8 .}$

(13) Pl. III, fig. 79, n 2 et 3. 
MOELLE MLLONGIIE.

clles, soit avec le sillon antérieur, et qui corresponcient exactement à la position des pyramides antérieures des mammifères. Chez les aigles, le milan, les vautours, le dindou, les faisans, j'ai pu les suivre à l'oeil nu, après avoir légèrement râclé la pellicule blanchâtre qui les revêt, jusqu'au-delà de l'insertion des nerfs de la troisième paire. Chez les perroquets, après les avoir ainsi découvertes ( 1 ), on les voit d'abord se prolonger en bas, le long de la parlie antérieure de $1 a^{\circ}$ mocile épinière, en suivant une direction parallèle, puis s'élever en haut, en divergeant légèrement et en augmentant d'étendue (2), au niveau du pcint qui correspond au pont des manmifères (3). J eur divergence augmentc vis-à-vis l'insertion de la troisième paire, au moment où elles passent audessus de la base des lobes optiques (4) pour aller rejoindre la parlie postérieure des hémisphères cérébraux (5). Cette marche est la mème chez inus les oiseaux, et leur volume m'a paru suirre une progression croissante des passereaux aux gallinacés, aux aigles; aux buses, au milan, d' l'autruche et au casoar.

l.es pyramides antérieures sont ainsi, chez les

(1) Pl. III, fig. $82, n^{\circ}$ I.

(2) PI. III, fig. $82, \mathrm{n}^{0} 2$.

(3) Pl. III, fig. $82, n^{\circ} 9$

(4) PI. 11I, fig. $8 \mathrm{z}, \mathrm{n}^{\circ} 8$.

(5) PI. UII, fign $82, n^{n} /$. 
oiseaux, de même que chez les mammifères, İa continuation du cordon central antérieur de la moelle épinière.

Les embryons des jeunes mammifères et de l'homme sont, comme les reptiles, sans renflemens pyramidaux; ils ne deviennent apparens que vers le commencement du deuxième mois de la gestation, chez l'homme, le mouton, le veau et le cheval. Chez le chien, le chat, le renard, le cochon-d'Inde, le lapin, leur apparition est beaucoup plus précoce : ils sont d'abord aplatis et minces; ils augmentent ensuite par l'addition de nouvelles couches fibreuses, sans matière grise, les troisième, quatrième et cinquième mois de l'homme, du cheval, du veau et du cochon : leur saillie paraît alors plus forte chez les mammifères que chez l'homme, à cause de la formation du corps trapézoïde, qui les soulève chez les premiers.

Chez les animaux adultes, la proéminence des pyramides est beaucoup plus faible chez l'homme que chez les singes (1), les cétacées (2), les carnassiers (5), les ruminans (4) et les rongeurs (5). Cet affaissement est produit en partie, chez l'homıne, par la saillie latéralc des tubercules

(1) Pl. VIII, fig. 194, A; fig. 197. B.

(2) PI. XII, fig. 234, A.

(3) Pl. X, fig. $223, \mathrm{~A}$.

(4) PI. XIII, fig. $9 / 19, \Lambda$.

(5) PI. XIV, fig. 258, B. 
moyens de la moelle allongée ou des olives (1).

Dans l'hypothèse que les pyramides sont les racines des lobes cérébraux, leur développement devrait être en raison directe de ceux-ci ; or c'est ce qui n'est pas : les pyramides sont moins fortes chez l'homme que chezles singes, comme chezle drill (2) et le mandrill (3); elles sont plus prononcées aussi chez les cétacées (4), et à peine distinctes à l'extérieur chez le phoque (5). Cette prédominance des pyramides se continue chez les carnassiers, le lion (6), l'ours (7), le raton (8), la loutre (9); chezles pachydermes et les ruminans, le lama (10), le pécari (11), le cheval (12), le chameau (13), le bouc de la Haute-Egypte (14).

Les pyramides antérieures de la moelle allongée n'ont pas la même forme chez tous les mammifères : chez l'homme la ressemblance qu'on a cru

(1) PI. XIV, fig. $289, n^{\circ} 3$.

(2) Pl. VIII, fig. 194, A.

(3) Pl. VIII, fig. $197, \mathrm{~B}$.

(4) Pl. XII, fig, 234, A.

(5) Pl. IX, fig. 208.

(6) Pl. XIV, fig. $266, \Lambda$.

(z) Pl. XI, fjg. 231, A.

(8) PI. VIII, fig. $200, n^{\circ} \times$.

(9) Pl. X, fig. 223, $n^{\circ} \mathrm{A}$.

(10) Pl. XVI, fig. $295, \mathbf{A}$.

(ii) Pl. XVI, fig. $590, n^{\circ}$.

(12) PI. XV , fig. 275, A.

(13) Pl. XII, fig. $249, \mathrm{~A}$.

(14) I') XIV, fig. $263, B$. 
leur trouver avec une pyranide, leur a fail don. ner ce nom; mais déjà chr\% les singes (1) et les célacées (2), elles deviennent coniques; leur base arrondie este adossée contre le pont; celte forme se conserve chez certains carnassiers digitigrades, comme le lion (5), le tigre, la panthère. Chez les pachydermes ( 4 ), les ruminans (5) et les rongeurs (6), ils ont la forme d'un quadrilatère allongé, un peu plus large en haut qu'en bas. Cette forme est la même chez la marte $(\gamma)$, le daman (S), l'ours (9), le pécari (10) et le kanguroo (11).

Les pyramides ont une ascension directe chez les poissons(12) et les reptiles 13), elles marchent parallèlement l'une à l'autre chez tous les poissons, comme on le voit chez la raie bouclée (14),

(1) Pl. VIII, fig. 194, A.

(2) PI. XII, fig: $254, \mathrm{~A}$.

(3) PI. XIV, fig. 266, A.

(4) Pl. XIII, fig. 249, A.

(5) PI. XVI, fig. $295, n^{\circ} 1$.

(6) Pl. XIV, fig. $258, \mathrm{~A}, \mathrm{~B}$.

(7) PI. XV, fig. $290, \mathrm{n}^{\circ} \cdot 1$.

(8) $\mathrm{Pl}, \mathrm{XV}$, fig. $273, \mathrm{~A}$.

(9) PI. XI, fig. 251, A,

(10) PI. XVI, fig $300, \mathrm{n}^{\circ} 11$

(11) Pl. XVI, fig. 299, A.

(12) Pl. VI, fig. 14, no

(15) Pl. V, fig. 15. $111^{\prime \prime 2} \mathrm{ct}$.

(14) Pl. VI, lig. 148, n० 1 . 
MOEILE MITONGEI:.

chez la perche (1), chez le rouget (2), le gronau (3), la morue (4) et le turbot (5). Chez le merlan (6), on remarque de petits faisceaux transversaux qui les unissent en arrière de la sixième paire. Chez les reptiles ( $(7)$, leur marche est également directe.

Il n'en est pas de même chéz les oiseaux : la marche des pyramides dans cette classe m'a paru cntrecoupée à leur naissance par les faisceaux croisés situés vis-à-vis ou au-dessous de l'origine Ju nerf de la sixième paire, ainsi qu'on le voit chez le casoar (仓), l'autruche (9) et la cigogne blanche (10); de plus j'ai remarqué chez le casoar un enlacement des faisceaux vers l'insertion de la troisième paire (11). Cet enlacement est trèssurperficiel (12). Je ne l'ai jamais observé sur le trajet des pyramides découvertes profondément, comme chez le perroquet (13). Chez la poule,

(1) Pl. VI, fig. $156, \mathrm{n}^{\circ} \mathrm{l}$ et 2.

(2) Pl. VII, fig. $156, \mathrm{n}^{\circ} 10$.

(3) Pl. VII, fig. 156, $\mathbf{n}^{\circ}$ 2.

(4) PI. VII, fig. $162, \mathrm{~B}$; lig. $166, \mathrm{n}^{\circ} 1$.

(5) Pl. VII, fig. $176, \mathrm{~B}$.

(6) PI. VI, fig. $144, n^{\circ} 1$.

(7) Pl. V, fig. 124, $\mathrm{n}^{\circ} 1$ bis.

(8) PI. III, fig. $79, \mathbf{n}^{\circ} 2$.

(g) Pl. IV, fig. $9^{8}, \mathrm{n}^{\circ} 2$.

(10) Pl.IV, fig. 103, $n^{\circ} 2$

(1) Pl. III, fig. $80, \mathrm{n}^{\circ} 3$

(12) Pt. IV , fig. $9^{8}, \mathrm{n}^{\circ} 2$

(13) PI. III, fig. $82, n^{\circ}$ et 2. 
l'oie, le canard, le dindon, les faisans, je n'ai pas vu distinctement le passage des faisceaux superficiels d'une pyramide à l'autre.

Les pyramides, chez les embryons des jeunes mammiferes, sont, dans le premier temps de leur formation, comme chez les reptiles et les poissons; on peut les disjoindre sans apercevoir de croisernent; mais dès la sixième semaine du mouton, la septième du cochon, la huitième et la neuvième du cheval et du veau, on aperçoit des faisceaux croisés se porter de droite à gauche, ou de gauche à droite. Chez l'embryon humain j'ai aperç cet entrecroisement dès les septième et huitième semaines; le célèbre Tíedemann a fait la même observation. Il serait peut - être inutile de rappeler que cette disposition a été révoquée en doute par des anatomistes modernes très-célébres, si Rolando ne venait de la nier de nouveau, après les travaux de Sanctorini, de Mistichelli, de Duverney, de M. Gall, de M. Cuvier, de Tréviranus et de Tiedemann, sur ce sujet. Ce, doute, dans une question de. fait qui paraît si simple, provient de ce que les faisceaux, avant de se porter d'un côté de la moelle allongée à l'autre, s'enlacent les uns dans les autres : cet enlacement n'exisiant pas chez les embryons, on aperçoit sans interruption les faisceaux de droite (1) se porter à gauche, et vice

(1) PI. XIII, fig. $247, \mathrm{n}^{\circ} 8$. 
versi (1). Chez les embryons hydro-rachis, Ies faisceatxx étant tenus très-écartés, celte disposition est encore plus marquée, et ne saurait laisser le plus léger doute sur l'entrecroisement réciproque des pyramides.

L'homme seul a un entrecroisement très-distinct; chez les singes, le phoque, les cétacées, il est beaucoup moins apparent; il devient de moins en moins visible chez les carnassiers, les pachydermes (2) et les ruminans; chez les rongeurs (3) il est aussi peu sensible que chez les oiseaux; ce qui provient de l'épaisseur des pyramides à leur insertion avec la moelle épinière; il est, en général, beaucoup plus obscur chez les animaux oú les pyramides sont droites (4), presque parallèles (5) et quadrilatères (6), que chez ceux où elles se rapprochent de la forme conique ou pyramidale.

Les expériences physiologiques viennent confirmer ce point important de l'anatomie comparée. Chez les poissons et les reptiles, l'action du cerveau sur la moelle allongée et épinière est directe; chez les oiseaux et les mammifères elle

(1) Pl. XIII, fig. $247, n^{\circ} 9$

(2) PI. XIII, fig. $249, \mathrm{n}^{\circ} 10$.

(3) PI. XIII, fig. 251, A

(4) P1. XIII, fig. $290, n^{\circ} 1$.

(5) P1. XVI, fig. 299, A.

(6) Pl. XVI, fig. 295 , A. 
est croisée; c'esl-i-dire que si vous blessez le cerveau des reptiles et des poissous, vous affeclez le mouvement du même côté que la lésion : le contraire a lieu chez les oiseaux et les mammifères: le même résultal a licu pour le cervelct. J'ai démontré l'effet croisé de ce dernier organe dans les deux classes supérieures. Chez l'homme, les maladies organiques du cervelet et des lobes cérébraux prouvent irrévocablement ce croisement d'action.

Chez les poissons osseux (1) et cartilagineux (2), la marche des pyramides vers les lobes cérébraux est à découvert sur la base de l'encéphale; chez les oiseaux (3), elle est entrecoupée daus son tra-

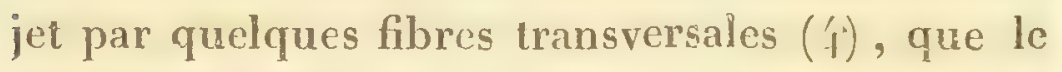
célèbre Haller a pris pour les analogues du pont; chez les jeunes embryons des mammifères, les pyramides sont égalemeut à nu jusqu'à la formation du corps trapézoïde et du pont, ce qui les rapproche des classes inférieures; chez les mammifères adultes, l'art seul peut mettre à découvert ce passage et suivre ces renflemens dans les lobes cérébraux.

Oria dit que les pyramides étaient développées en raisondirecte des lobescérébraux,qu'elles augmen-

(1) PI. VII, fig. 164, A.

(2) Pl. VI, fig. 148, $\mathrm{n}^{\circ}$.

(3) Pl. III, fig. $82, \mathrm{n}^{\circ} \mathrm{1}$.

(4) Pl. III, fig. 82, n². 
MOELLE ALLONGÉE.

taient des poissons aux oiseaux, et des mammilères inférieurs aux singes et à l'homme : ce rapport est inexact.

Les pyramides, très-faibles chez les reptiles, sont aussi développées chez les poissons osseux (1) et chez les cartilagineux (2) que chez les oiseaux (3) : quant aux mammifères, elles vont plutôt en croissant de l'bomme aux singes (4), aux phoques, aux cétacées (5), aux carnassiers (6), aux pachydermes $(j)$, aux ruminans $(8)$ et à certains rongeurs (9), cn raison inverse du cerveau, qu'elles ne suivent la progression directe de cet organe, comme l'avait fait supposer le système de Gall.

En traversant le pont, les pyramides antérieures divergent chez les mammiferes pour se rendre dans les pédoncules cérébraux. La divergence des pyramides suit en général une progression décrois sante de l'homme aux singes, aux cétacées, aux phoques, aux carmassiers, aux pachydermes, aux ruminans ct aux rongeurs; elles se rapprochent de plus en plus du parallélisme à mesure quon

(1) PI. VII, fig. 162, B.

(2) Pl. VI, fig: $148, n^{\circ} 1$.

(5) D. III, fig. $82, n^{\prime \prime} 1$.

(1) Pl. VIII, fig. $197, \mathrm{~B}$.

(5) Pl. XII, fig. $234, \Lambda$.

(i) P1. XI, fig. 231, A; pl. IX, fig. 225,1 .

(z) Pl. XVI, fig. $295,:^{\circ} 1$.

(8) PI. XIV, fig. 26 in, $\Lambda$.

(9) Pl. XIV, fig. $258, \mathrm{~B}$. 
descend des oiseaux aux reptiles et aux poissons.

La moelle allongée présente encore en avant les tubercules olivaires; ceux-ci sont situés sur la partie latérale et antérieure de la moelle allongée (1) ; leur forme chez l'homme les a fait comparer à une olive: de là leur nom, si peu convenable chez l'homme, si impropre dans les trois classes inférieures, dont les cordons olivaires ne sont point renflés. Une hypothèse avait fait supposer que les pyramides devaient être développées en raison directe du cerveau; on se hâta de le conclure : les faits prouvent le contraire, même chez les mammifères. Dans cette classe, les olives suivent au contraire ce rapport, et on l'a méconnu. Il est pénible, mais nécessaire, de faire sentir cette contradiction entre nos suppositious et les faits.

Les olives sont très-prononcées chezl'homme (2); elles forment encore chez les singes (3) un renflement saillant (4); leur proéminence diminue chez les cétacées (5) : chez le phoque (6) et l'ours (7) elles ne forment déjà plus une éminence distincte; on n'aperçoit chez eux qu'un faible renflement

(1) Pl. XIII, fig. 247, $\mathrm{n}^{\circ} 12$.

(2) Pl. XIII, fig. $247, \mathrm{n}^{\circ} 12$.

(3) P1. VIII, fig. $194,0$.

(4) Pl. VIII, fig. 197, 0.

(5) Pl. XII, fig. 234, 0.

(6) PI. IX, fig. $208,0$.

(7) Pl. XI, fig. 231, O. 
MOELLE ALLONGÉL.

sur les côtés des pyramides, renflement qui va en diminuant chez les carnassiers digitigrades, le lion (1), la loutre ( 2), la marte (3); chez les pachydermes, le pécari (4), le daman (5), le cheval (6); chez les ruminans, le chameau (7), le lama (8); chez les rongeurs et les insectivores, le castor (9), l'agouti (10), le porc-épic (11), la marmotte (12), la taupe (15), le zemni (14), le hérisson (15); et chez les chauve-souris (16). La décroissance des olives est donc en rais on directe de la décroissance des lobes cérébraux chez les mammifères.

Les faisceaux olivaires restent aplatis chez l'homme, les singes et les carnassiers, jusqu'aux

(i) PI. XIV, fig. 266,0 .

(2) PI. X , fig. 223, 0.

(3) $\mathrm{Pl} . \mathrm{XV}$, fig. $290, \mathrm{O}$.

(4) Pl. XVI, fig. 300, $\mathrm{O}$.

(5) Pl. XV, fig. 275,0

(6) Pl. XV, fig. $275,0$.

(7) PI. XIII, fig. 24! , O.

(8) Pl. XVI, fig. 295 , 0.

(9) Pl. XIV, fig. 258, 0.

(1.0) Pl. IX, fig. 21x, O,

(ii) PI. XIII, fig. 251,0 .

(i2) PI. IX, fig. 203, O.

(13) Pl. XIV, fig. 260.

(14) Pl. XV, fig. 272, 0.

(15) Pl. XVI, fig. 297.

(16) Pl. IX, 6g. 204, O. 
deux tiers environ de la gestation. Its som alors dans l'état où on les obscrve chez les nammiferes inférieurs. Aux deux ticrs d: la grestalion de's singes, de l'homme et des carnassicrs, il se forme au centre del'olive un amas de malièregrise font la disposition se rapproche beaucoup decolle du corps dentelé du cervelet. La saillie des lubercules est produite par la présence de celte matière grisc, dont la formation est de beaucoup postéricure, comme on le voit, à la matière blanche: sur la fin de la gesiation des singes, des carnassicrs et de l'homme, la matière grise sc dépose en abondance dans l'intérieur des tubercules olivaires; de là, le développement rapide de ces corps ì cette époque. Si la saillic des olives dépend de la matière grise, on voit de suite que celle-ci doit diminuer en raison directe de la décroissauce de ces tubcrcules. C'es?, en effet, ce qui existe: le corps dentelé gris del'olive, très-fort chezl'homme, l'est considérablement moins chez les singes, moins encore chez les cétacées, chez le phoque, l'ours, le lion, la loutre, la marte. Chez les pachydermes, les ruminans ct les rongeurs, la matière grise n'est plus festonnéc comme dans les précédens; on ne rencontre au centre de lolve qu'un pelit noyau de cette dernière substance.

Les olives ne sont point renfiées chez les oiseaux, ellcs sont par conséçacnt dépourvucs de matière grise; à la place oú devraient se trouver 
ces éminences, on rencontre dans cette classe deux cordons aplatis (1), longeant le côté cxterne des pyramides (2). Ces cordons vont en augmentant de la poule, de l'oie, des perruches, des perroquets, à la bondrée, aux oiseaux de nuit, à la cigogne, à l'aigle, à l'autruche et au casoar.

Chez les reptiles (3), les cordons olivaires sont très-petits; ils ront en augmentant de l'orvet au protée, aux ophidiens, aux vipères, aux sauriens et aux chéloniens; chez la tortue franche ils égalent presquue le volume qu'on leur remarque chez le pigeon, la perdrix et les faisans.

Chez les poissons, les cordons olivaires ont la même disposition que chez les reptiles et les oiseaux; ils sont en général moins gros chez les chondroptérygiens (4) que chez les poissons osseux (5). Chez les premiers ils augmentent de la lamproic à l'esturgeon, à l'ange, au requin et aux raies $(6)$ : chez les osseux, leg plus petits se rencoutrent chez les anguiliformes (7), et les plus volumineux chez les trigles (8), le tur-

(1) PI. III, fig. $82, \mathrm{n}^{\circ}$.

(3) Pl. III, fig. $82, \mathrm{n}^{\circ} 1$.

(5) PI. XIV , fig. $288, r^{\circ} 2 \approx 6$.

(4) P1. VI, fig. $148, n^{\circ} 50$

(5) PI. VII, fig. $162, A$.

(i) Pl. VI, fig. 14S, $\mathrm{n}^{\circ} 30$.

(7) Pl. VII, fig. 192, $\mathrm{n}^{\circ} 2$

(8) PI. VII, fig. $156^{\circ}, \mathrm{n}^{\prime \prime}$ ! 
bot (1), la morue (2); du reste, cette partie, comme loutes celles de l'encéphale, en gínéral, présinte dans cette classe de grandes variatiuns.

I.es olives ou les cordons qui leur correspondent, different donc beaucoup, dans les trois classes iuférieures, de ce qu'ils sont chez les mammiferes : chez ces dernicrs, leure faisceaux offrent des variétés essentielles à constiter; chez l'homme $(\bar{J})$ et les singes, ils embrassent le corps frangí grisâtre, en formant un arc autour de lui ; aux deux extrémités de ce corps, les fiisceaux des olives so croisent manifestement on haut et en bas chez l'homme, les singes, les célacécs et le phoque; chez les carnassiers, les ruminans ct les rongeurs, les faisccaux ne font que s'adosser en formant un entrelacement sans croisement distinct : celte disposition a élé indi quéc chez l'homme par Sancturini, Malicarne, Vicq-d'Azyr, Prochaska, Rolando. Les faisceaux des olives suivent dans le pont la marche indiquée par Reil, Gall et Tiedemann. Quelques fibres exicrnes se rendent vers les tubcrcules çuadrijumcaux (4); la plus grande parlie se porte de la base des tubercules vers le centre du lobe cérébral, pour

(1) Pl. VII, fig. $176, \mathrm{~B}$.

(2) Pl. VII, fig. $162, \mathrm{~A}$.

(3) Pl. XIII, fig. $247, \mathrm{n}^{\circ} 12$.

(4) Pl. XIV, fig. $289, n^{\circ} 3,13$ ct 14. 
aller se perdre dans la radiation de l'éminence mamillaire. Les fibres qui se dirigent vers les tubercules quadrijumeaux sont plus prononcées chez les rongeurs et les ruminans que chez les singes et l'homme; chez les cétacées, elles sont aussi prononcées que chez les.ruminans. Les faisceaux principaux des olives, qui se rendent dans la radiation mamillaire, vont en décroissant de l'homme aux singes, aux phoques, aux cétacées, aux carnassiers, aux ruminans et aux rongeurs.

Chez les oiseaux (1), deux ou trois petits faisceaux des cordons olivaires se portent, comme chez les mammiferes, dans les hémisphères cérébraux; mais la presque totalité des olives pénètre dans les lobes optiques (2), où elles s'épanouissent. Il en est de même chez les reptiles (3): à peine trouve-t-on un petit faisceau, chez les tortues, le crocodile et la grenouille, qui, des cordons olivaires, sé dirige vers les lobes cérébraux. Chez les poissons osseux et cartilagineux, tout rapport est rompu entre ces lobes et les cordons olivaires; ces derniers s'épuisent en entier dans les lobes optiques, de la manière que nous l'avons représenté chez le merlan (4), chez les

(1) Pl. III, fig. $82, \mathrm{n}^{\circ} 4$.

(2) PI. III, fig. $82, n^{\circ} 8$.

(3) P1. XIV, fig. $288, n^{\circ} 1$ et 5.

(4) Pl. VI, fig. $147, \mathrm{D}^{\circ} 2$ et $g$. 
trigles (1) et la morue (2). De cette diversité de structure dérivent la diversité de leurs rapports et la diversité des effets observés dans les expériences physiologiques.

Du cordon restiforme. Isa partie antérieure de la moelle allongée est donc formée par la pyramide antérieure et les faisceaux olivaires; la partie la. plus externe et la postérieure sont constituées par le cordon restiforme (3) et la pyramide postérieure (4); un tubercule formé par la matière grise (5) est interposé entre les cordons antérieurs et postérieurs de la moelle allongée. Le volume de ce tubercule, sa disposition et son étendue sont très-variables chez les mammifères et les oiseaux. En faisant partir le cordon restiforme du niveau de ce tubercule (6), on voit d'abord qu'il forme la plus grande partie du corlon postérieur de la moelle allongée; on remarque ensuite quen s'élevant il diverge au dehors, en formant un arc dont la convexité est en dehors (7), et la concavité en dedans (8); en dedans il correspond ì. la

(1) Pl. VII, fig. 156, $\mathrm{n}^{\circ} 2,3,5$ et 6.

(2) Pl. VII, fig. $-172, n^{\circ} \cdot 2$ et 4.

(3) PI. XIII, fig. $245, n^{\circ} 2$ et 3.

(4) Pl. XIII, fig. $245, n^{\circ} 1$ et 4.

(5) Pl. XIII, fig. $245, n^{\circ} 2$.

(6) PI. XIII, fig. $245, n^{\circ}$ vet. 5 .

(7) Pl. XIII, fig. $245, n^{\circ} .5$.

(8) Pl. XIII, fig, $245, n^{\circ} \mathrm{s}$ 
pyramide postérieure (1), en dehors il longe le faisceau olivaire (2), dont il est séparé, comme nous venons de le dire, par une traînée de matière grise. Par l'effet de la divergence que nous venons de décrire, le cordon restiforme se porte de la partic postéricure de la moelle allongée à sa partie externe (3), où on le voit faire saillie en dehors de l'olive (4). Le cordon restiforme ne suit donc ni le même développement ni les mêmes rapports que les pyramides antérieures et les faisceaux olivaires. Lorsque l'on a dęveloppé la moelle allongée, comme le représente la fig. 245 de la Pl. XIII, on voit que le corps restiforme augmente d'une manière progressive de l'homme aux singes, au phoque, aux pachydermes, aux ruminans, aux carnassiers et aux rongeurs. Il conserve chez les oiseaux une partie de cette prééminence, qu'il perd tout-à-coup chez les reptiles, pour la reprendre de nouveau chez les poissons.

Chez les poissons, !e cordon restiforme est en effet très-prononcé (5). Chez la morue (6), on voit que la partie postérieure de la moelle allongée représente exactement la disposition de celte

(1) Pl. XIII, fig. $245, n^{\circ} 4$.

(2) Pl. XIII, fig. 2 彳 3 , P.

(3) Pl. XIII, fig. $247, n^{\circ} 12$.

(4) PI. XIII, fig. 249, D.

(5) P1. VI, Gg. $147, n^{\circ} 8$.

(6) PI. VXI, fig. $161, n^{\circ} 1$. 
partie chez l'homme ( 1 ); on aperçoit la ligne de démarcation qui le distingue du cordon olivaire: en s'élevant il diverge comme chez l'homme, mais beaucoup moins, à cause de la moins grande capacilé, chez les poissons, du quatrième ventricule.

Chez les raies, on remarque sur la partie latérale un roflement elliplique considérable, sur lequel viennent prendre leur insertion les nerfs de la cinquième, de la septième et de la huitième paire. Les faisceaux olivaires marchent le long de ce renflement.

Pyramide postérieure. La partie interne et postérieure de la moelle allongée est complétée par un autre cordon très-distinct du précédent, et que je nomme, avec le professeur Rolando, $p y-$ rumide postérieure (2). Siluée au côté interne du cordon restiforme, il offre la même disposition que lui ; il diverge en s'élevant; il a sa face externe convexe, logée dans la concavité que présente en dedans le cordon restiforme; sa partie interne concave borde en dedans les lèvres du quatrième ventricule; sa partie supérieure est coupée à angle droit par le tania grisea (5) des frères Wenzel, couché en travers sur lui et le cordon restiforme : sa couleur est d'un blanc plus mat que
(1) Pl. XII, fig. 245.
(2) Pl. XIII, fig. $245, n^{\circ} 4$.
(3) Pl. XIII, fig. $245, n^{\circ} 6$. 
ce dernier. Le cordon restiforme et la pyramide postrieure reprísentent donc deux faisceaux enchâssés l'un dans l'autre; leur divergence réciproque (1) fait qu'en haut la pyramide postéricure forme seule le calamus scriplorius (2), qui, comme on le voit, n'est autre chose que l'angle résultant de cutte divergonce. Cet angle est rempli par un pelit ansas de matiere grise, qui est la terminaison de la lame de crute substance qui tapisse les cordons postéricurs de la moelle épinière.

La pyramide postérieure suit une progression croissante de l'homme aux singes, aux cétacées, aux phoques, aux ruminans (.j), aux carnassicrs (4) et aux rongeurs (5). Elle est beaucoup plus prononcće chezles oiseaux que chcz les mammifères, comme on le remarque chez la poule (6), le perroquet (7), le casoar (8) et l'aigle (9). Le calamus scriptorius est aussi beaucoup plus prononcé dans cette classe. Chez certains oiseaux,

(1) Pl. XIII, fig. $145, \mathrm{n}^{\circ} 1,3,4$ et 5 .

(2) Pl. XV: fig. $271, n^{\circ} 2$ :

(3) Pl. XV, fig. $2 z^{1}, \mathrm{n}^{\circ} 2$.

(4) Pl. XV, fig. $279, \mathbf{n}^{\circ} 2$.

(5) Pl. II, fig. $57, n^{\circ} 1$.

(6) Pl. III, fig. $87, \mathrm{n}^{\circ} 3$.

(7) PI. III, fig. 84, n' 15 .

(8) PI. III, fig. $83, \mathrm{n}^{\circ} 16$.

(9) Pl. IV, fig. 101, n० 15. 
comme le casoar (1), la pyramide postérieure forme un cordon festonné en dedans du quatrième ventricule.

Chez les reptiles, la pyramide postéricure est très-grêle (2); elle est très-faible chez les ophidiens (3) et les batraciens (4); elle acquiert un volume assez considérable chez la tortue franche (5), qui, sous ce rapport, se rapproche des oiseaux.

Chez les poissons, la pyramide postérieure devient aussi prononcée que chez les oiseaux. Chez les osseux (6), elles forment en arrière de la moelle allongéc un cordon aussi distinct que le sont en avant les pyramides antérieures chez les mammifères (7). Chez les osseux, elles sont beaucoup plus prononcées que chez les cartilagineux, comme on le voit chez la morue (8), l'anguille (9), le congre (10), comparés à la raie (11). Chez la

(1) Pl. III, fig. 83, no' 2 et 16.

(2) Pl. I, fig. 16, $\mathrm{n}^{0} \mathrm{1}$.

(3) Pl. V, fig. $110, n^{\circ} 6$.

(4) Pl II, fig. $48, n^{\circ} 3$.

(5) Pl. V, fig. $119, n^{\circ} 3$ et 4.

(6) Pl. VI, fig. $151, \mathrm{n}^{\circ} 12$.

(7) Pl. XVI, fig. $29^{5}, \mathrm{n}^{\circ}$.

(8) Pl. VII, fig. 16ı, A.

(9) PI. VII, fig. $174, \mathrm{n}^{\circ} 2$.

(10) Pl. VI, fig. 151, no 12.

(11) $\mathrm{Pl}$. VI, fig. $15_{2}, \mathrm{n}^{\circ}$. 
morue (1) et l'anguille (2), les pyramides postéricures ne divergent pas à lcur enitréo daus le quatrième ventricule; chez le congre, lcur divergence (3) suit celle du cordon restiforme; chez la tanche (4) et l'esturgeon (5), clles sont festonnées comme chez le casoar (6).

L'entrecroisement des pyramides antérieures est un fait constant chez les mammifères et les oiseaux; les pyramides postérieures sont toujours directes chez les mammifères (7), les oiseaux (8), les reptiles (9) et les poissons (10). Chez ces derniers, elỉes soni quelquefois réunies à leur entrée dans le quatrième ventricule par un faisceau blanchâtre transverse, qui d'une pyramidc se porte à l'autre, comme je l'ai fait représenter chez la morue (11) et chez le congre (12).

Le croisement des pyramides antérieures a servi à expliquer le croisement d'action des hémisphères

(1) Pl. VII, fig. $161, \mathrm{n}^{0} 1$.

(2) PI. VII, fig. $190, \mathrm{~B}$.

(3) Pl. VI, fig. $151,11^{\circ} 12$ et 15.

(4) Pl. VII, fig. 185, , "

(5) PI. XII, fig. $223, \mathrm{n}^{\circ} 2$.

(6) Pl. III, fige $83, n^{\circ} 16$.

(z) P1. XIII, firr, $245, n^{\circ}$ let 2.

(8) Pl. III, fig. $87, \mathrm{n}^{\circ} 3$.

(9) Pl. II , fig. 12, I" 1.

(10) Pl. VII, fig. $16 x, \Lambda$.

(11) Pl. VII, fig. 16i, n*

(12) Pl, VI, fig. $151, n^{\circ} 15$. 
cérébraux. Le non-croisement des postérieures a contribué à faire croire aux physiologistes que l'action du cervelet sur les mouvemens volontaires était directe : c'est une erreur. L'action du cervelet est croisée comme celle des hémisphères cérébraux. J'ai déjà fait connaître cette vérité importante, sur laquelle nous reviendrons ailleurs.

Telles sont les parties communes de la moelle allongće; elle offre en outre des parties propres, qui ne se remarquent que dans quelques classes en particulier. Ce sont le trapèze chcz les mammifères, et certains renflemens chez les poissons.

Le trapèze de la moelle allongée est un carré allongé, dont les bords internes et externes ne sont pas exactement parallèles. Il forme une espèce de ceinture à la moelle allongée, au-dessus des olives (1), et au-dessous ou en arrière de la protubérance annulaire (2). 11 part en dehors du tania grisea des frères Venzel, et se termine en dedans au sillon antérieur en se joignant ì celui du côté opposé. Le lieu de cette jonction offre une dépression qui correspond au sillon antérieur de la moelle allongée.

Le trapèze diffère de la protubérânce annulaire par son point de départ, par sa position et sa terminaison. Son point de départ correspond au

(1) Pl. XI, fig. $251,0$.

(2) PI. XI, fig. $231, \mathrm{P}$. 
tania grisea des frères Venzel. Sa position est plus profonde que celle du pont de varole; il se iermine en passant en arrière des pyramides antéricures (1), tandis que Ies fibres $d u$ pont passent constamment en avant et les recouvrent. Enfin, le trapèze et le pont sont développés en raison inverse l'un de l'autre.

Le trapèze est un ruban de matière blanche, formé par des fibres transversales et parallèles, non entrecoupées, comme dans le pont, par l'interposition de matière grise. Il est peu développé chezl'homme; il l'est beaucoup chez tous lessinges, comme on le remarque chez le mandrill (2) et le drill (3): il est plus faible chezle phoque (4), et disparaît tout-à-fait chezles cétacćes (5). Ala place quu'il devrait occuper, on trouve, chezces dernicrs animaux, une fossette assez profonde qui occupe toute l'étendue qu'il devrait avoir. Il augmente ensuite d'étendue des carnassiers (6) aux ruminans (7), et de ceux-ci aux rongeurs ( 8 ) et aux édentés; comme on leremarque chezles chauve-souris (9),

(1) Pl. XI, fig. $231, \mathrm{~T}$.

(2) Pl. VIII, fig. 194, T.

(3) Pl. VIII, fig. 197, T.

(4) PI. IX, fig. $208, \mathrm{~T}$.

(5) Pl. XII, fig. $234, \mathrm{~T}$.

(6) Pl. X, fig. $223, T$.

(7) Pl. XIV, fig. 26a, T.

(8) Pl. XIV, fig. $258, T$.

(9) Pl. IX, fig. 204, I. 
la taupe (1), l'ours (2), le raton (5), les coatis, la martc (4), le lion (5), la loutre (6), la mangouste du cap (7), le kanguroo géant (8), le daman (9), le cheval (10), le pécari (11), le chameau (12), le lama (13), le bouc (14), le mouton, le bouf, le castor (15), le zemni (16), les rats, le cabiai, le porc-épic (17), le tatou (18), la marmotte (19) et l'agouti (20).

En suivant ce développement progressif du trapèze de la moelle allongée, on voit qu'il a constamment lieu d'arrière en avant; il chasse devant

(1) Pl. XIV, fig. 260, C.

(2) Pl. XI, fig. $23 i, T$.

(3) Pl. VIII, fig. 200, T.

(4) Pl, XV, fig. $29^{\circ}, \mathrm{T}$.

(5) Pl. XIV, fig. 266 , 'T.

(6) Pl. X, fig. 223, T.

(7) PI. XIII, fig. $254, T$.

(8) Pl. XVI, fig. $290, \mathrm{~T}$.

(9) Pl. XV, fig. $27^{3}, \mathrm{~T}$.

(10) Pl. XV, fig. $275, \mathrm{~T}$ 。

(11) Pl. XVI, fig. 500, T.'

(12) PI. XIII, fig. $249, T$.

(13) Pl. XVI, fig. .295, T.

(14) Pl. XIV, fig. 262, T.

(15) Pl. XIV, fig. $258, T$.

(16) Pl. XV, fig. 272 , T.

(17) Pl. XIII, fig. 25 I, T.

(18) P. XIII, fig $24 \dot{u}^{\circ}, \mathrm{T}$.

(19) Pl. IX, fig. 205 , 'I'.

(20) Pl. IX, fig. $211, \mathrm{~T}$. 
lui de deux manières le pont de varule, comme on peut le remarquer sur tous les animaux précédens. Plusil s'élargit (1), plus la protubérance annulaire se rétrécit (2); l'un gague ce que l'autre perd.

Cet antagonisme répite chez les animaux adultes ceque nous offre la série des développemens chez les embryons; d'abord, chez le cheval, le veau, le cochon, le lapin, le trapèze est très-étendu en comparaison de la faiblesse du pont; puis le pont se développe, et il:dépasse de beaucoup par la suite l'étendue du trapèze. Ces mourcmens opposés indiquent donc l'indépendance et les différences de ces deux parties.

Quoique l'amas de matière grise que l'on a nommé ruban gris, ne se forme qu'après, et longtemps après le trapèze, il me paraît être la saillie de ce corps sur les côtés du quatrième ventricule. Cet'e matière grise est au trapize ce que la substance grise est à l'olive. De même que la matière grise de l'olive décroît dans les mêmes proportions quc celle - ci ; de mème, le ruban gris ou le tubercule qui le remplace chez les ruminans, croît dans les mêmes proportions que le trapèze. Le ruban gris est très-mince chez lc phoque, chez lequel le trapèze est pcu développé; il a disparu chez le dauphin de même que ce der-

(1) Pl. XIII, f g. 249, T.

(2) P1. XIII, fig. 249 , P.

II. 
nier. Le trapéze ne se remarque que sur la moelle allongéc des mammiféres; les mammifères seuls possèdent le ruban gris. Ces deux caractères sont propres à la moelle allongée de la classe supérieurc. Ceci continue a'expliquer ce que nous avons dit sur ce ruban grisâtre, à l'occasion des nerís de la septième paire, qui s’insèrent sur les côlés externes du trapèze.

Aiusi avec I'absence du trapéze de la moelle allongéc coincide, chez les reptiles (1) et les poissons (2), labscnce du rubar gris sur les côtés du quatriène ventricule, comme on peut le voir chez la grenouile (5), la tortue grecque (4), la torbue framehe (5), la poule (6), le perroquet $(7)$, le casoar (8) et l'aigle (9).

Le trapeze et le ruban gris manquent chez les poissong; mais dans celte classe la moclle allongée offre des particalariés qui ne se rencontrent pas ailieurs, et qui dérivent de leur organisation. Tout s'accumule vers la tête chez les poissons: les organes de la respiration; encaissés dans le

(1) Pl. I, fig. $12, \mathrm{n}^{\circ}$ :

(2) Pl. III, fig. $84, \mathrm{n}^{\circ} 15$

(3) Pl. V, fig. 134, n 5 .

(4) Pl. V, fig. $125, n^{\circ} 6$.

(5) Pl. V, fig. $120, \mathrm{n}^{\circ} 13$.

(6) Pl. III, fig. 8\%, $\mathrm{n}^{\circ} 2$ et 5.

(b) Pl. III, fig. $84, n^{\circ} 15$

(8) Pl. III, fig. 83, $\mathrm{n}^{\circ} 16$.

(9) Pl. IV, fig. $101, \mathrm{n}^{\circ} 15$ 
thorax chez les autres classes, viennent flanquer ici le derrière de la têle. Souvent les moyens de locomotion semblent avoir été entrainés par les poumons; les nageoires viennent se placer immédiatement derric̀re le crâne. Des organes spéciaux, tels que l'organe électrique ou les parties que, chez les squales, Jacobson et Treviranus considèrent comme des appareils nouveaux, tout en dernier lieu vient aboutir à ta moclle allongée. Ce mourement de concentration ver's la tête est ressenti par l'axe cérébro spinal du système nerveux, et par la moelle allongée plus spécialement. Des nerfs, qui dans les autres classes étaient tenus à disiance, sont ramenés chez les poissons au point de cortact, et sont confondus dans leur inserlion; lels sont les nerfs trijumeaux, l'auditif ct lc facial. Des renflemens, insolites dans les autres classes, viennent représenter ces organes sur les côtés de la moclle allongée.

De là les renflemeus qui, chez les trigles, sont situés cu arricre de cette partic (1), et qui, par leur volume (2) et leur nombre (j), répétent le volume et le nombre des rayons détachés de leurs nagcoires.

De lì le renflement qui se remarque chez les

(1) PI. VII, fig. 155, no 1, 3 et 4 .

(2) ग. VII, fig. $158, n^{\circ} 1$ et 2.

(3) PI. VII, fig. $159, n^{\circ} 1$ et 2. 
poissons électriques, à l'insertion sur la moelle allongée des nerfs qui se distribuent dans l'appareil électrique.

De là, chez beaucoup de poissons, le volume exagéré du nerf pnenmo-gastriqué (1) et le développenent latéral (2) da la partie de la moelle allongéc sur laquelle ce nerf s'insère.

De là, le renflement elliptique et très-étendu qui se romarque sur les flancs de cette partie chez beaucoup de poissons cartilagineux (3) et chez quelq̣ues osseux (4).

De là encore, la manifestation de tubercules particuliers (5) dans le quatrième ventricule des poissons (6), tubercules si développés chez la carpe (7), si développés chez la morue (8), qu'on les prendrait pour des lobules nouveaux du cervelet, et qui correspond ent a ruban gris des mammifères.

De là, enfin, les plis (9) et les replis (10) que

(1) Pl. XII, fig. $255, \mathrm{~A}$.

(2) Pl. XII, fig. 233, $\mathrm{n}^{\circ} 2,3$ et 4 .

(5) Pl. VI, fig. $148,13^{\circ} 16$.

(4) Pl. VII, fig. $164, n^{\circ} 17$

(5) Pl. VII , fig. $19^{3}, \mathrm{n}^{\circ} 2$.

(6) Pl. VII, fig. $174, n^{\circ} 5,6$ et 7

(7) Pl. VI, fig. $145,10^{\circ} 2$; fig。 $150, n^{\circ} 2$.

(8) Pl. VII, fig. $165, n^{\circ} 2,5$, 4 et 5.

(9) Pl. VI, fig. $138, \mathrm{n}^{\circ} 4$ bis et 9.

(10) Pl. VI, fig. 140, $n^{\circ} 5$. 
présentent les lames postérieures de la moelle allongée (1) chez les raies (2), chez l'aiguillat (3), l'ange (4) et l'esturgeon (5), plis et replis dont le but est évidemment de multiplier l'étendue des surfaces nerveuses pour les mettre en rapport avec le yolume des nerfs de la moelle allongće.

Avant de terminer la descriplion de la moelle allongée chez les animaux adultes, nous devons ajouter un mot sur son mode de continuité avec l'axe nerveux du tronc : cette continuité s'effectue de deux manières, en ligne directe et en ligne un peu courbe. La moelle allongée se continue en ligne directe chez tous les mammifères, comine on l'observe de l'homme (6) aux singes (7), aux carnassiers (8), aux ruminans (9) et aux rongeurs (10); chez tous les poissons osseux (11) et cartilagineux (12), et chez le plus grand nombre des reptiles, ainsi qu'on l'observe chez la gre-

(1) Pl. VI, fig. $152, n^{\circ} 1$ et 2.

(2) Pl. VI, fig. $152, \mathrm{n}^{\circ} 2,3,4,10$ et 11 .

(5) PI. XII, fig. $256, \mathrm{~B}, \mathrm{X}$.

(4) Pl. XII, ig. $257, \mathrm{C}$.

(5) PI. XII, fig. $233, n^{\circ} 2,3$, et 4 .

(6) Pl. XIII, fig. 247, n: 1.

(7) PI. VIII, fig. 194, B.

(8) Pl. VIII, fig. 200, $\mathrm{n}^{\circ}$.

(9) Pl. XIII, fig. $249, n^{\circ} 10$.

(10) Pl. XIV; fig. $258, A$.

(ii) Pl. VI, fig. 148, $n^{\circ} 1$.

(12) Pl. VI, fig. $146, n^{\circ} 1$. 
nouille (1), les vipères (2) et les tortues (3).

Chez les oiseaux, au contraire, il y a une courbure légère à l'endroit de la jonction (4); cette courbure existe chez tous les oiseaux à un degré à-peu-près semblable à celui que l'on voit chez l'autruche (5), le casoar (6), le perroquet ( $/$ ) et la cigogne (8) : elle est dans cette classe l'effet de la concentration générale de l'encéphale. On nc doit pas la confondre avec celle qui se remarque chez les embryons (9) des mammifères (10), à l'époque où la tête est profondément fléchie sur le thorax. Cette courbure existe aussi, à un degré plus faible, chez certains reptiles, notamment chez le camélćon (11) et le caiman (12).

Après avoir ainsi analysé toutes les parties constitutives de la moelle allongée dans les différentes classes, on explique, d'une part, les tentatives infructueuses qui ont été faites pour exprimer ses

(1) Pl. V, fig. $131, n^{0} \%$

(2) PI. V, fig. $127, \mathrm{n}^{\circ} \mathrm{l}$.

(3) Pl. V, fig. 122, $\mathrm{n}^{\circ} 1$.

(4) Pl. IV, fig. 104, $\mathrm{n}^{\circ} 1 \%$ :

(5) PI. IV, fig. 97, $\mathrm{n}^{\circ} \mathrm{l}$.

(6) Pl. III, fig. 78.

(7) Pl. IV, fig. 96, $n^{\circ} 1$ et 10 .

(8) Pl. IV, fig. 104, $\mathrm{n}^{\circ} 12$.

(9) PI. II, fig. 63, $n^{n} 2$ et 3.

(10) PI. 1I, fig. $64, n^{\circ} 2$.

(11) Pl. V, fig. 113, $n^{\circ} 1$.

(12) Pl. V, fig. $130, n^{\circ} 2$ 
rapports en masse; on juge, de l'aulre, combien ces rapports doivent être variés. On a dit qu'elle était développée en raison directe de la moclle épinière, ce qui est, en général, exact chez les poissons (1) et les reptiles, mais ne l'est plus chez les oiscaux, dont la moelle allongée suit d'autres rapports que la moelle épinière; ne l'est également plus chez les mammifères, puisqu'on a vu que l'olive, l'un de ses élémens principaux, suivait un rapport inverse.

Il en est de niême des hémisphères cérétraux et du cervelet : ces parties augmentent ou diminuent en masse, tandis que le plus souvent la moelle allongće reste invariable. L'inégalité de ces rapports résulte, comme nous l'avons dit, de l'inégalité de développement de ses diverses parlies, el souvent aussi du volume différent des nerís crâniens, qui font prédominer cerlaines partics et cn atrophient d'autres : c'est ce balancement alternatif qui rond les rapports en masse impossibles à déterminer.

Des risultats positils ne peuvent donc naître de la comparaison en masse de la moelle allongée; il fant les chercher daus les rapporis spéciaux de

(1) L'csturgonn, dont le volume prodigieux du nerf pneumo-gastrique fait prédominer braucoup la moelle allougé, et qui a une moclle épinière si grêle, fait une exception tranchée d ce rapport. 
ses élémens constitulifs. Je vais en présenter une esquisse.

$A$. Les pyramides antérieures sont, en général, dévcloppées en raison directe de la moelle épinière.

$B$. Chez les mammifères, les olives sont développées en raison inverse de l'axe nerveux du tronc.

C. Les pyramides pośtérieures et les cordons restiformes suivent, de même que les pyramides antérieures, la progression directe de la moelle épinière.

D. Le trapèze de la moelle allongée suit généralement les mêmes rapports que la moelle épinière.

$\boldsymbol{E}$. Il est constamment développé en raison directe de la pyramide antérieure, de la postćrieure et du corps restiforme.

${ }^{f}$. Le trapize est, au contraire, constamment dévcloppé chez les mammifères en raison inverse de l'olive. Sout exceptés de ce rapnort les cétacées et les phoques.

G. Les pyramides antéricures, les postérieures et le corps restiforme, sont développés en raison direcie du nerf olfactif, du nerf optique et des nerfs trijumeaux.

$H$. Les olives, chez les mammifères, sont développées en raison inverse de ces mêmes nerfs.

I. Le trapèze de la moclle allongèe suit la même progression que la cinquieme paire, que le nerf 
optique et que le nerf olfactif. Les phoques, et surtout les cétacées, font une étrange exception à ce rapport.

K. La taupe, les musaraignes, la chrysochlore du Cap, le rat-taupe, le zẹmni, sont nécessairement exceplés de tous les rapports précédemment énoncés entre le nerf optique et les diverses parties de la moelle allongée.

$L$. Chez ces mêmes animaux, le renflement spécial qui se trouve sur la moelle allongée, et qui se prolonge en bas jusques sur la partie antérieure de la moelle épinière, êst toujours développé en raison directe du volume de la cinquième paire.

$\boldsymbol{M}$. Le trapèze de la moelle allongée et le ruban gris sont dévcloppés en raison directe des nerfs acoustique et facial. Sont encore cxceptés de ce rapport les cétacées et les phoques.

$N$. En général, les nerl's acoustique el facial sont plus développés chez les mammifères quo: dans les trois autres classes. Serait-ce à cause de la présence du trapèze? ou le trapèze serait-il l'effet de ce surcroît de dévcl sppement des nerfs?

$O$. Les tubercules de l'intéricur du quatrième ventricule sont rigourcusement dévcloppés chez les poissons, en raison directe des nerfs de la cinquieme paire et du preumo-gastrique: de plus, on trouve, dans le quatrieme ventriculedes raies, des squales, de l'estirgeon, de la carpe, une sćrie de faisccaux médullaires Iransverses, qui du sillon 
médian se dirigent vers la cinquième ou la huitième paire.

$P$. Ces faisceaux et ces tubercules sont, à la cinquième et à la huitième paire des poissons, ce que sont le trapèze et le ruban gris pour le nerf facial et l'acoustique des mammifères. Les reliefs de ces nerf́s sont déplacés dans les deux classes : ils sont en arrière de la moelle allongée chez les poissons, et en avant chcz les mammifères.

Q. Du reste, le développement des tubercules du quatrième ventricule chez les poissons partage l'indépendance des rapports de la moelle allongée avec les différens élémens de l'encéphale. Ils augmentint, diminuent ou disparaisserst, sans que l'effet de ces variations soit spécialement ressenti par les parties constitutives de l'encéphale. 


\section{COROLLAIRES}

\section{PHYSIOLOGIQUES ET PATHOLOGIQUFS}

Sur la Moelle épinière et la Moelle allongée.

I. Une vérité bien ancienne dans la.science, et toujours nouvelle par lintérêt qu'elle présente, c'est que, si l'on comprime un nerf qui se rend à un muscle, le muscle perd aussitôt le mouvencnt et le sentiment : il en est de même si on le coupe. Ce qui prouve que c'est du nerf que le muscle recoit la faculté de sentir et celle de se mouvoir.

II. Mais le nerf reçoit-il ou possède-t-il cette double propriété? Il la reçoit et il la possède. Il la recoit, car un nerf étant coupé, nous venons de voir que la partic est comme séparée de l'animal; il la possède, car si on remplace ce stimulus rie l'animal par un stimulus artificiel, la parlic de ce muscle se contracte, s'ayile, entre cn convulsion. Ie nerf n'a donc ricn perdu par sa séparation; sa communicalion avec le tronc est seulement interrompuc : ce qu'il éprouve n'est plus perçu.

III. La moelle épinière comprinuéc, irritéc ou coupée, a produit des ellets analogues à l'action, ̀̀ la compression ou à l'irritation des nerfs en par- 
ticulier. Les parties sous-jacentes au point irıté ou détruit ont donné des convulsions, ou se sont paralysées; jamais les parties sus-jacentes n'ont été affectées.

IV. La sensibilité, ou la puissance qui transmet les sensations à l'âme, et l'irritabilité, ou la puissance de faire mouvoir, sont donc deux propriétés inhérentes au système nerveux. Quoique dépendanies l'une de l'autre, ces propriétés ne sont pas toujours confondues : on peut sentir vivement sans se mouvoir; on peut être agité convulsivemient, comme dans l'épilepsie, et ne rien sentir. Le sentiment peut être perdu dars une partie et les mouvemens conservés; et la paralysie so remarque très-fréquemment sans perte de la sensibilité. De cet isolement d'action quc la pathologie nous offre quelquefois, on a conclu à l'isolement du système nerveux; or: a pensé qu'il y avait des nerfs exclusivement sensibles, d'autres exclusivement irritables. Poursuivant ensuite cette distinction dans l'axe cérébro-spinal, on a divisé cet axe en parties principalement irritables et en parties principalement sensibles. Les faits n'ont pas encore entièrement cónfirmé cette division tranchće; mais, malgré les difficultés qui environuent les observaleura, la science a fait, dans ces dernicrs temps, des acquisitions importantes.

V. Ainsi, les branches postérieures des neris rachidiens ont paru plus sensibles qu'irritahles, el les antérieures plus irritables que sensibles. Les 
cordons de la moelle épinière ont paru aussi plus irritables ou plus sensibles, selon quils correspondent aux branches antéricures ou postérieures des nerfs rachidiens. C'est à M. Magendie que sont dues ces dernières idées; ses expériences sont connues de tous les physiologistes. Voici des faits pathologiques qui concordent avec elles.

VI. Une femme était périplégique depuis longtemps. La sensibililé était conservée dans les jambes et les cuisses : les cordons antéricurs étaicnt altérés, à partir du milieu de la région dorsale jusqu'a la terininaison du renflement inférieur; les postérieurs étaient intacts.

VII. Un homme avait perdu la sensibilité des jambes et des cuisses depuis onze mois; huit mois après il devint périplégique. Le renflement inférieur était désorganisé dans toute sa partie postérieure; antérieurement, une partie des cordons n'étaient pas altérés.

Voici un fait observé récemment dans ma division, qui leur est contraire :

VIII. Une femme était périplégique depuis environ deux mois : le sentiment était conservé aux jambes et aux cuisses; les cordons postérieurs étaient seuls altérés cn trois endroits différens, à partir du milieu de la région dorsale (1). Cette dern:ère observation confirmerait l'opinion de

(1) Je rapporte ces observations en abrégé; je les donnerai 
Rolando, qui pense que les cordons postérieurs sont les partics mobilcs de la moelle épiuière, et les antérieurs les parlies sensibles.

1X. Parmi les cas nombreux de maladies de la moelle épinicre que j’ai observés, je n'ai jamais vu se justifier les opinions de Ballingieri sur la moelle épinière. Je n'ai pas vu que les cordons antéricurs ou postérieurs fussent plus spécialemeut affectés aux moavemens d'extension ou de flexion.

$X$. Dans les cas où l'altération commence vers le centre de la moclic épinière, jai remarqué que lc mouvement n'était alYaibli que lorsqu'elle se rapprochait de la périphérie.

XI. Dans les cas opposés, c'est-à-dire lorsque c'est par la périphérie que conmence la maladie, toujours les mouvemens sont lésés.

XiI. Dans les expéricnces sur les animaux, plus on descend dans l'échelle, plus les différens effets de l'irritation ou de la section de la moelle épinière sont marqués.

XIII. Dans toutes les expériences et dans tous les cas pathologiques, l'action de la moelle épinière a été directe; c'est-à-dire quo les convulsions ou la paralysie du sentiment et du mouvement correspondaient toujours au côté de la moelle épinière blessé ou altéré.

dans leur plus grand détail dans un ouvrage qui suivra celuici, et qui sera destiné spécialement aux maladies du sy stème nerveux. 
XIV. L'action de la moelle épinière est intims-ment liée à l'intégrité dé a circulation. Si on lie l'aortc abdominale, on suspend le mouvement et le sentiment dans les extrémités inférieures. Il en est de même, si on intercepte la marche du sang par la ligature de la veine cave inférieurc.

XV. Après avoir suspendu la sensibilité et la contractilité dans les muscles inférieurs par la ligature, on rétablit l'une et l'autre de ces facultés, soit en injectant c'e l'eau tièc'e dans l'artère, soit par la transfusion du sang d'un autre animal. En comprimant simp!cment l'artère, on produit le mème effet que la digature; en lâchaut la compression, le mouvcment ct le sentiment se rétablissent, lentement si la compression est lâchée. graduelıment; si on lâche brusquement la compression et que lo sang entre ropidement dans l'artère, les mouvemens reviennent d'uue manière convulsive, comme tétanique; l'animal pousse des cris a:gus.

XV1. Haller n'admit aucune corrélation entre la sensibilité ct la contractilité : il fut inévitablement conduit, par ces idcécs, i nier toute influence de la moslle épinière cl du système nerveux sur l'action du coeur. Legallois rétablit cette influcnce: Wilson Philipp la combattit aussiôt et fit revivre l'opinion de Haller. M. Flourens a beaucoup éclairé cette question; et les contestations qu'clle a fait aaître, en montrant que plus on se rapproche de la naissance des animaux, moins la circulation 
est soumise à l'influence du système nerveux:

XV1I. Ce système lu i est tout-à-fait étranger avant la naissance. Ainsi, chez les foetus privés entièrement de moelle épinière, ou de moelle épinière et de l'encéphale, la circulation s'exécute jusqu'à la naissance et quelquefois au-delà..Ces fails, invoqués par Haller, et que Bidloo traitait de fables grossières, ne sont plus contestés de nos jours.

XVIII. Celte indépendance du cœur ne laisse aucun doute dans l'expérience suivante: Si on ouvre un ouf de poule, de canard ou de dinde, au troisième jour de l'incubation, on voit les mouvemens du coeur; si on détruit le cerveau et la moelle épinière, le cour continue à se mouvoir comme auparavant, l'incubation marche et le foetus continue ses développemens. Par celtẹ expérience on produit artificiellement ce que la nature nous offre dans les cas précédens, ainsi, peut-être . que chez certains poissons dans leur état normal.

XIX. La respiration est plus directement soumise à l'influence nerveuse que la circulation, ainsi que Haller, Legallois et M. Flourens l'ont établi, et que le prouvent les faits pathologiques.

XX. Chez l'homme, toute altération de la moelle épinière dans la région dorsale influe sur la respiration d'une manière d'autant plus prompte qu'elle est située plus haut, à partir du milieu de cette région.

XXI. Dans la région cervicale, toute affection de la moelle épinière est suivie d'une asphyxie d'autant plus prompte, qu'elle se rapproche da- 
vantage de l'insertion du nerf diaphragmatique. Au-dessus, l'asphyxic est instantanée si la maladie est brusque: au-dessous de cette insertion, elle survient promptement aussi, lors mème que l'altération ne s'est pas propagée jusqu'au niveau de l'insertion de ce nerf, surtout si elle affecte spécialement les cordons postérieurs.

XXII. La moelle épinière exerce-t-elle une action sur les intestins, la vessie et les organes génitaux? L'école de Haller a déjà résolu négativement cette question. Tous les physiologistes modernes ont sanctionné les assertions de Haller; il a fallu des faits bien positifs pour entrevoir l'opinion contraire.

XXIII. Ces faits m'ont été fournis par la pathologie : j'ai consulté ensuite la physiologic expérimentale. Voici les principales données que j'ai entrevues:

XXIY. Premièrement, il n'est pas exact que les animaux soient insensibles aux irritations des viscères : la vessie, les intestins, l'estomac, la vésicule du fiel, le foie même étant irrités avec le bistouri ou par les acides, les animaux ont manifesté par leurs cris et leur agitation des douleurs plus ou moins vives.

XXV. Secondement, dans les maladies de la moellc épinière, les intestins, la vessie, les ovaires, chez la femme, ont offert des effets provenant de la lésion de cet axe nerveux.

XXVI. Ainsi, dans les altérations du renfleII. 
ment inférieur, j’ai vu fréquemment des maladies consécutives de l'ovaire, une atrophie des testicules, la paralysie de la vessic.

XXVII. Si la désorganisation est bornée au renflement inférieur, les gros intestins sont spécialement atteints, paralysés, diminués dans leur calibre.

XXVIII. Au tiers inférieur de la région dorsale, la fin de l'intestin grêle ressent les effets de l'altération pathologique; au milieu, tout l'iléon est atteint; au haut, la moitié du jéjunum. Ces effets se manifestent par des rétrécissemens, des involvulus, et des inflammations consécutives, qui quelquefois sont portées au point de produire la perforation du canal.

XXIX. Si on ouvre le rachis d'un animal, et que l'on injecte un liquide irritant entre la duremère et la moelle épinière, on observe des mouvemens accélérés dans tout le canal intestinal, lorsque l'abdomen étant ou vert et le mésentère coupé, on a étendu les intestins sur un plan horizontal.

XXX. Si on imite la moelle épinière avec un acide, le renflement inférieur produit un mourvement sensible sur les gros intestins;

XXXI. La partie inférieure dela région dorsale, sur les gros intestins et la fin des intestins grêles;

XXXII. La région dorso-costale, sur tous les intestins grêles, et quelquefois sur l'estomac. Quelquefois aussi, l'irritation d'une partie de la moelle épinière produit des mouvemens divers et 
en sens opposé dans toutes les parties du canal intestinal.

XXXIII. La région cervicale agit sur l'estomac et les intestins grêles, et point sur les gros inteslins : mais l'estomac est surtout affecté lorsque l'on expérimente sur la moelle allongée et le cervelet.

XXXIV. Le canal intestinal est agité par deux mouvemens opposés, l'un dit péristaltique, l'aúu tre, antipéristaltique. Il devenait important de constater si ces mouvemens étaient différemment produits, selon que l'irritation était portée sur les cordons antérieurs ou postérieurs. J'ai fait des essais qui ne m'ont fourni jusqu'à ce jour aucun résultat şatisfaisant:

XXXV. Appliqués à la pathologie, ces résultats jettent une vive lumière sur les maladies aiguës et chroniques du canal intestinal. On n'a pas oublié l'effet que produisit, en 1813 , la publication de notre Ouvrage sur l'Entéro-mésentérite (1). Le domaine des fièvres était alors si paisible, on était si généralement convenu de les regarder toutes comme essentiolles; des hypothèses si ingénieuses avaient été imaginées pour en expliquer la théoric et la pratique; enfin, ce code d'erreurs était telJement cimenté, que l'on dut accueillir commc on le fit, un ouvrage rempli de faits qui sipaient dans ses fondemens un érlifice si colossal.

(1) Traite de la fieme cntero-mésenterique, in-8, arec planches coloriées, 1813 , par Mill. Petit el Serres. 
XXXVI. Cet ouvrage a produit ses effets. La doctrine des fièvres a changé de face, leur essentialité est maintenant rejetée universellement. Mais dans l'impossibilité de les localiser toutes dans l'état présent de la science, on invoque encore les sympathies; mot derrière lequel se retranche toujours notre ignorance médicale : on érige des systèmes sur lîrritation, les stimulus ; les contre-stimulus; on veut aller vîte, et le propre de l'observation est de marcher lentement ( 1 ):

XXXVII. Avant de passer outre, il n'est peutêtre pas inutile de remarquer que les fonctions de la moelle érinière se développent avant celles de l'encéphale. Ainsi, la digestion, la respiration, la circulation, s'exécutent librement et pleinement aussitôt après la naissance; tandis que, chez beaucoup d'animaux, et surtout chez l'homme, les fonctions du cerveau et celles du cervelet sont long-temps à se faire attendre.

XXXVIII. Nous ferons remarquer aussi que, quoique la moelle épinière et les tubercules quadrijumeaux soient parvenus au terme de leur développement ainsi que le cervelet, les mouvemens sont faibles, incohérens, chez beaucoup de mammifères et l'homme à l'époque de la naissance; ce

(1) Je devais ajouter ici une note qui m'avait été communiquée par M. A. Desportes; mais ce médecin distingué l'ayant déjà publiée, j'y renvoie le lecteur. (Revue médicale, annèe 1824.) 
qui prouve que le principe qui dirige les mouvemens volontaires ne réside exclusivement ni dans la moelle épinière, ni dans les tubercules quadrijumeaux, ni dans le cervelet. Plusieurs mammifères et beaucoup d'oiseaux, au contraire, marchent d'un pas assuré aussitôt après la naissance.

XXXIX. Plus nous nous élevons dans l'axe cérébro-spinal, plus ses fonctions deviennent compliquées et importantes. Il est bien difficile d'obtenir isolément; par l'expérience, sur les animaux, les effets des quatre parties principales qui la constituent : la mort survient si promptement lorsque l'on agit sur cette partie, qu'elle ne laisse ni le temps ni les moyens de bien analyser les effets qui se produisent.

$\mathrm{XL}$. Kau - Boerhaave était parvenu à déterminer expérimentalement que la moelle allongée est le siége de la sensibilité générale et des mouvemens.

XLI. Il avait remarqué, de plus, que l'animal étant mort depuis quelques minutes ct le cœur ayant cessé de battre, l'excitation de la moelle allongée faisait reparâtre ses mouvèmens : ces effets étaient surtout tranchés, lorsque préalablement il avait enlevé tout le cervelet.

XLII. Haller fut plus loin : il constata que la respiration et la circulation n'offraient aucun trouble chez les animaux dont il avait complètement enlevé le cerveau et le cervelet, la moelle allongéc restant intacte. Haller aurait pu conclure de ses expériences que la moelle allongée était le 
siége spécial dè ces deux fonctions; mais ses opinions le détournaient de cette conclusion.

XLIII. Elle fut déduite par Lorry, quant à ce qui concerne la respiration. Cet illustre physiologiste s'exprime ainsi : La division et lu compression de la moelle de l'épine, dans un endroit déterminé, produit la mort subite; inférieurement à cet endroit, cette moelle produit la paralysie; elle la produit de même supérieurement.

XLIV. Mais quel est cet endroit déterminé? Lorry ne le dit pas. M. Flourens, qui a suivi dans ses expériences une méthode plus sévère encore que Lorry, se borne à constater la paralysie de la respiration par l'ablation totale de la moelle allongée.

XLV. Le point indiqué par Lorry correspond aux éminences olivaires : la moelle allongéé, coupée transversalement à leur niveau, l'animal meurt aussitôt asphyxić. Au-dessus et au-dessious, la section de cette partie ne produit que la paralysie générale.

XLVI. La pathologie m'avait depuis longtemps indiqué ce fait. J'ai donné ailleurs (1) l'observation d'un homme qui mourut subitement à la suite d'un épanchement sanguin dans les éminences olivaires. Le cœur s'arrèta instantanément.

XI.VII. Depuis, d'autres faits mont fourni

(1) Annuaire medico-chirus gical des hopitaux, 18 in: p. 551 . 
quelques données sur les divers effets de la lésion de la moelle allongée.

XLIIII. La pyramide antérieure n'agit que sur les mouvemens volontaires.

XLIX. Les olives influent spécialement sur les mouvemens du cœur : j'ai vu les mouvemens de cet organe saccádés, irréguliers, dans les altérations isolées et chroniques de ces éminences. En répétant l'expérience de Kau-Boerhave, j'ai constaté que c'est en irritant les olives que l'on augmente ou que l'on ressuscite les mouvemens du cour.

L. Au contraire, les épanchemens brusques dans le corps restiforme paralysent le poumon; et c'est peut-être parce que souvent le corps reștiforme est lésé en même temps que le cervelet, que l'on a placé dans ce dernier organe le siége de la respiration. Dans ce cas, la mort arrive moins promptement que dans la destruction de l'olive.

LI. Dans les affections aiguës du bulbe rachidien, chez l'homme, les olives et le corps restiforme sont fréquemment atteints ; mais l'ołive résistant plus que le corps restiforme, les malades mểurent par la cessation de la respiration.

LII. Dans les affections chroniques de ces parties, les troubles de la circulation cö̈ncident avec les lésions des olives, et ceux de la respiration avec celles du corps restiforme. Le faisccau qui est intermédiaire a ces deux parties influe plus spécia- 
lement sur l'estomac. Les digestions sont troublées, la faim anéantię, l'estomac inerte, lorsque le siége de l'altération envahit les faisceaux qui séparent l'olive du corps restiforme.

LIII. Quand, après avoir enlevé le cervelet, on irrite la moelle allongée et que l'on parvient vers ce point, on détermine sur l'estonac des mouvemens très-remarquables : l'estomac se raccourcit, le pylore se rapproche de l'ouverture cardiaque, puis un instant après il reprend sa capacité primitive (1). Cet effet est produit aussi, mais plus faiblement, par l'irritation de l'olive et la partie interne du corps restiforme. Ces expériences et ces faits confirment l'opinion de M. le docteur Braschet, de Lyon, sur la cessation des mouvemens de l'estomac après la section des nerfs pneumo-gastriques.

LIV. Au-rlessous des olives, les affections chroniques de la moelle allongée produisent la paralysie générale, et de plus.l'immobilité de la langue et la perte de la parole. D'un seul côté la paralysie est directe, et la parole, cadencée, mal articulée, développe le bégaiement (2).

(1) C'est d'après cette expérience que j'avais cru pouvoir déduire que les cordons antérieurs de la moelle épinière sont je siége des mouremens antipéristaltiques.

(2) Jen'ai jamais observé le b’égaiement que chez l'homme. jamais chez la femme, dans les paralysies. Ce fait coüncide avec lo bégraicment naturel : ce défaut dans la liaison des 
LV. Le bégaiement est surtout produit quand la partie antérieure est affectée : je ne l'ai pas remarqué dans les cas de lésion à la partie postérieure; ee qui confirme l'une des vues de M. Magendie. Willis avait dit que l'olive était l'organe de la parole, partant du fait que cette éminence est plus développée chez l'homme que chez tous les autres animaux ( 1 ).

LVI. J'ai observé aussi le bégaiement dans les altérations supérieures des olives; mais comme ce point correspond à la protubérance annulaire, j'y reviendrai en présentant les corollaires physiologiques et pathologiques relatifs à cctte partie.

mots est particulier ả l'homme; dans les familles, il se trans- . met héréditairement de mâle en mâle; les femmes n'en sont point affectées. Je possède des notes sur près de cinq cents familles dont les hommes bégayent, et point les.femmes. Je n'ai encore rencontré qu'une seule femme qui bégayât naturellement : j'ai fait quelques recherches à ce sujet sur deux hommes de la même famille qui bégayaient; j'ai trouvé l'os hyoïde plus développé à droite qu'ì gauche, les muscles de la langue plus faibles à gauche qu'à droite; j'ai trouvé des noyaux osseux dans le ligament stylo-hyoïlien; le larynx était bien conformé, ce qui explique pourquoi les personnes qui bégayent en parlant n'uffrent pas le cadencement des mots quand elles chantent.

(1) Le plus grand nombre de ces corollaires sont extraits d'un mémoire déposé en octobre $182 \%$, à l'dcarlémie royale des Sciences. 
Histoire d'une maladie organique de la Moelle épinière.

Si ces corollaires sont exacts, il sera donc possible, pendant la vic, de déterminer par les symptômes le siége primitif d'une maladie de la moelle épinière et de la moelle allongée. Parmi les exemples que je pourrais présenter à l'appui de cette assertion, je choisis le suivant qui s'est présenté dernièrement à ma clinique, et dont plusieurs mé decins ont été les témoins.

Louis-Charles Gautier, âgé de vingt ans, tourneur, d'un tempérament lymphalique, d'une constitution grêle et délicate, et ne paraissant avoir que seize ou dix-sept ans, était adonné à la masturbation depuis son enfance. Il éprouva au commencement du mois de février 1815 une douleur légère à la région épigastrique, des digestions laborieuses et de la constipation; depuis quelque temps en outre, il avait de la gêne dans la respiration, déterminée par des palpitations que la marche et surtout la montée d'un escalier augmentait beaucoup. En état de repos, la respiration n'élait pas du tout troublée.

11 entra, le 28 avril, dans ma division, et nous offrit le lendemain à la visite les symptômes suivans:

F'ace légèrement animée, pommettes colorées. langue blanche, bouche pâteuse, soif modérée, 
point d'appétit, point de douleur à la région épigastrique, ni dans aucune autre partic de l'abdomen. Ventre souple, constipation : les urines étaient rares depuis quelques jours, mais rendues saus difficulté aussitôt que le besoin s'en faisait ressentir. La respiration était libre, égale; et conché, le malade n'éprouvait point d'oppression. Les pulsations du cour se font entendre dans toute la partie antérieure gauche de la poitrine, sur le côté et à la partie externe et postérieure de la même région; en outre, l'oreille appliquée sur la région du cœur est légèrement soulevée à chaque contraction. Le pouls est fréquent et assez fort, régulier et égal des deux côtés : il y a un peu de moiteur à la peau. La tête n'est pas douloureuse; le sommeil est bon et tranquille; les mouvemens des membres sont libres: toutefois la partie inférieure des jambes et les pieds sont légèrement infilirés.

D'après cet état, nous n'hésitâmes pas à portẹ le diagnostic d'une dilatation avec hypertrophie des cavités gauches du cour, et nous prescrivîmes en conséquence les boissons diurétiques nitrées, une potion antispasmodique avec quelques gouttes de teinture éthérée de digitale pourprée. Par ces moyens, et par le repos que nous avions expressément recommandé et auquel le malade s'assujélit, il y cut une rémission asscz mrompte; car, des le 5 ou 6 mai suivant, l'infiliration des jambes et des pieds avait disparu, les 
battemens du eceur n'étaient plus aussi forts et ne se faisaient pas ressentir dans une aussi grande étendue; l'appétit était revenu, la constipation avait cessé. Cette amélioration dans son état dura jusqu'au 12 mai. Ce jour-là le malade mangea beaucoup; il eut une indigestion. Alors des douleurs aiguës se manifestèrent dans l'abdomen : le ventre était balloné, tendu et un peu renittent; la constipation reparut. Le pouls était fréquent et concentré comme dans les affections abdominales. Je prescrivis les boissons mucilagineuses, les lavemens émolliens, et les fomentations de même nature sur l'abdomen.

Le 13 , le ventre était plus dur et plus tendu encore que la veille; le météorisme s'était développé : il n'y avait point eu de selles, même à la suite du lavement. La figure était un peu altérée. Le 14, les évacuations alvines se manifestèrent, la douleur de l'abdomen diminúa; le pouls ne fut plus aussi concentré ni aussi fréquent; la face restait dans le même état. Le 15 , le météorisme se dissipa et fut remplacé par un empâtement de l'abdomen, qui persista les 16,17 et 18 .

Depuis le jour de son indigestion, Gautier ne s'était levé que pour satisfaire à ses besoins. En descendant et.en remoutant à son lit, il avait remarqué qu'il se soutenait difficilement sur les jambes, effet quc l'on attribua d'abord à la phlegmasie abdominale; mais dès le 18 mai, la faiblesse des membres inféricurś étant très-augmen- 
téc, ses jambes ne purent plus le supporter; on garnit son lit, et. e malade ne se leva plus : du reste, les fonctions digestives et respiratoires étaient en bon état; le pouls s'était relevé et était moirs fréquent. Le 19 , nous portâmes notre attention sur les membres inférieurs : leurs muscles étaient flasques; il les remuait à peine, ne pouvait les porter au-dehors; mais quand on les avait déplacés, il les ramenait en.dedans avec beaucoup de difficulté. La sensibilité était párfaitement conservée; la plus légère irritation était ressentie. Nous examinâmes avec soin la colonne vertébrale, et nous n'aperçûmes ni déviation, ni saillie des apophyses épineuses.

Le 20 , la paralysie des extrémités inférieures était complète, et pour la première fois le malade nous dit éprouver une vive douleur dans les lombes, et de la difficulté à évacuer les urines; il n'y avait pas de constipation. Ce jour et les suivans, nous fîmes faire des frictions le long de la colonne vertébrale arec la teinture de noix vomique.

Du 21 au 25 cet état persista; la sensibilité des membres abdominaux était si vive, que ce jeune homme poussait des gémissemens et des cris pour peu qu'on les déplaçât. Les articulations étaient surtout le siége de vives douleurs; la vessie était romplètement paralysée : on fut obligé dès ce moment de sonder le malade.

Pendant tout le mois de juin et la moitié de 
juillet, les accideus persistèrent et furent même en s'aggravant insensiblement; les membres inférieurs devinrent complètement immobiles et douloureux; les pied's, les jambes, puis les cuisses, s'infiltrèrent: la vessie était complètement paralysée; on fut obligé de placer une sonde à demeure. Les fonctions digestives étaient assez bien conservées; il n'y avait ni constipation, ni devoiement, quoique cependant l'empâtement de l'abdomen persistât toujours. La face était pâle, un peu terreuse et tachetée; la peau était généralement flasque et un peu jaunâtre; la respiration était libre, les mouvemens du cour naturelș ; lc pouls était petit et un peu accéléré, les fonctions intellectuelles étaient dans la plus parfaite intégrité. La tête n'était pas douloureuse, quoiqu'elle tût fixe, déjetéc à gauche, et qu'elle restât toujours dans la même position.

Le malade restant constamment couché sur le dos, la peau du sacrum s'enflamma : il s'établit sur cette partie une ulcération qui fit des progrès très-rapides, ef dont aucun moyen ne put arrêter ni suspendre la marche; la partie inférieure des muscles sacro-lombaires et longs dorsaux fut mise à nu, l'os lai-même était dénudé sur le centre.

Le 22. nous nous aperçumes que la sensibilité des jambes et des cuisses était émoussée ; on pinçait fortement la peau sans que le malade en eût la sensation : la douleur ne se faisait ressentir que 
lorsque l'on cherchait à fléchir les articulations pour le changer de situation. Les déjections étaient difficiles depuis quelques jours, même à la suite des lavemens; la vessie était douloureuse, et plusieurs fois en le sondant précédemment, l'interne de la division, M. Ménétrier, s'était aperçu que la verge était en érection.

Du 22 au 26, les accidens inflammatoires de la vessie étant augmentés par la présence de la sonde, on la retira; le malade ne rendit plus les urines que par regorgement. Au milieu de ce déplorable état, les facultés intellectuelles étaient libres; ce jeune homme voyait arriver la mort avec un calme et une résignation que la religion seule peut donner, et que scule elle peut entretenir. On le plaçait sur un fauteuil : il y passait une partie de la journée, et paraissait un peu distrail de ses souffrances. Mais bientôt cette dernière consolation lui fut enlevée; les jambes et les cuisses s'infiltrèrent outre mesure par cette position; des douleurs lancinantes se manifestèrent dans l'ulcère qui recouvrait le sacrum, et on ne pouvait les diminuer, comme on le faisait sur son lit, en le changeant de situation.

Le 7 août, le malade pousse de temps en temps des cris lamentables; on l'interroge sur la cause et le siége de ses douleurs, il ne peut plus répondre. En palpant l'abdomen, la vessic nous parut très-distendue : on le sonda, ce qui n'apporta aucun amendement à ses souffrances. Le 8 , 
il est dans un état désespéré : le pouls est imperceptible, les extrémités supérieures et la tête sont froides; il est couvert d'une sueur visqueuse; le râle trachéal est très-prononcé; il nẹ voit ni n'entend. Le 9 , le malade sort tout-à-coup de cet état; il semble s'éveiller d'un profond sommeil; il n'a aucune connaissance de la position où il était la veille; il est étranger à tout ce qui se fait autour de lui; il voit, entend et parle avec assez de facilité. Le 10, il est dans la même position. Le 11, il retombe dans l'état oú il était le 8 , et meurt à trois heures de l'après-midi.

En résumant les symptômes que le jeune Gautier nous avait présentés dans le cours de sa maladie, on voit d'abord une maladie du cœur trèsprononcée, des palpitations, des battemens qui se font ressentir dans la plus grande étendue du côté gauche de la poitrine, accidens qui, joints à l'oppression et à l'oedème des pieds et des jambes, décèlent ordinairement une hypertrophie avec dilatation des cavités gauches de l'organe central de la circulation. Ces symptômes s'apaisent; et à la suite d'un léger excès dans le manger, il se déclare des accidens dénotant une péritonite et une entérite : ceux-ci diminuent à leur tour; mais au moment où l'on devait s'attendre à voir entrer le malade en convalescence, il déclare ne pouvoir se soutenir.sur ses jambes; et bientôt en effet il ne peut ni se lever, ni marcher, ni même soulever dans son lit les membres abdominaux. La péri- 
plégie est complète, les muscles des extrémités inférieures ont perdu leur action; la sensibilité persiste néanmoins dans ces membres paralysés, et quélque temps après y est exaltée au point de faire pousser des cris au malade par le plus léger attouchement. Tout dénote dès-lors une maladie organique de la moelle épinière, et la paralysie persistante de la vessie vient s'y joindre pour confirmer ce diagnostic.

Mais dans quelle partie de la moelle épinière et à quelle hauteur se trouvera la désorganisation, si clle existe? Telle est la question que l'on devait se faire, et que nous cherchâmes à résoudre avant de procéder à l'ouverture du cadavre. Deux groupes de phénomènes me portèrent à annoncer que l'altération organique devait être située très-haut dans la région dorso-costale de la moelle épinière. Premièrement, l'exaltation des mouvemens du cœur, par laquelle avait débuté la maladie ; secondement, la faiblesse et la lenteur des affections du canal intestinal.

Considérant ensuite que la perte du mouvement avait de beaucoup devancé la perte de la sensibilité, que celle-ci même avait été cxaltée pendant long-temps; considérant, de plus, que la respiration n'avait nullement été troublée pendant le cours de la maladic, j'ajoutai que, d'après les expériences de M. Magendie, les cordons antérieurs de la moelle épinière devaient être les plus affectés, à moins que nous n'eussions encore II. 
ici la répétition du cas de la femme $V$ iolet, dont nous avions ouvert le cadavre il y a quelque temps, et sur laquelle nous avions constaté la disposition inverse (1). Les bases du diagnostic ainsi établies, on procéda à l'ouverture du cadavre.

Après que le rachis fut ouvert dans toute son étendue, depuis la base de l'occipital jusqu'à l'extrémité du sacrum, on détacha de bas en haut la moelle épinière, en ayant soin d'enlever en même temps les ganglions intervertébraux. Parvenu à la hauteur de la troisième vertèbre dorsale, on remarqua d'abord que le corps de cette vertèbre était altéré légèrement; puis, portant son attention sur la portion antérieure de la moelle épinière qui lui correspondait, on aperçut une dégénérescence comme cancéreuse de toute la partie antérieure de la moelle épinière, ou plutôt de ses enveloppes, comme nous nous en assurâmes plus tard. Cette dégénérescence s'étendait depuis le corps de la troisième vertèbre cervicale jusqu'au niveau de la cinquième cervicale. Le corps de toutes les vertèbres, en rapport avec cette altération, était

(1) Ėtaient présens à l'autopsie cadavérique, MM. Fisher, Walter, Raleigh, Watson, membres du collège de Chirurgie de Londres; M. Ménétrier, interne de la division; MM. Martel, Crouzit, Soufflon, Martin (Joseph), élèves exlernes. M. le docteur Georget, que j'avais fait prévenir, n'arriva qu'après quel'altération organique fut découverte. MM. Fisher et Ménétrier écrivirent le diagnostic que j'avais établi avant qu'il fat procédé à l'autopsie cadavérique. 
Glanchâtre, un peu inégal, et ramolli à un faible degré : le ligament antérieur était détruit.

La moelle épinière, enlevée après avoir été coupée en arrière du trou occipital, l'enveloppe extérieure était fongueuse, dégénérée en une masse inégale d'un jaune verdâtre, ayant la consistance des tubercules pulmonaires non ramollis; elle était ainsi altérée dans l'étendue d'un décimètre cinq millimètres de long, sur quarante millimètres dans sa plus grande largeur : l'altération était bornée à la moitié antérieure de l'enveloppe fibreuse; la moitié postérieure était saine. Cette masse stéatomateuse incisée longitudinalement sur sa partie moyenne, on mit à découvert la partie de la moelle épinière contre laquelle elle était appliquée: on vit d'abord que l'arachnoïde, qui la tapissait intérieurement, était saine, ainsi que le ligament dentelé, dans toute la longueur correspondante à la désorganisation. Examinant ensuite la moelle épinière, nous reconnûmes au toucher qu'elle était ramollic, principalement dans la por tion située au niveau de la septième vertèbre cervicale et des trois premières dorsales; incisant ensuite le long du sillon antérieur, et divisant ainsi la moelle épinière en deux parties dans toute sa longueur, nous vimes que le ramollissement affectait principalement les cordons antérieurs, dont l'aspect était d'un blanc grisâtrı, qui devint rosacé après quelques lieures d'exposition à l'air; les cordons postérieurs étaient légìement ra- 
mollis au niveau seulement des trois premières vertèbres dorsales; ils avaient, du reste, leur couleur ordinaire, et ne devinrent pas rosacés par l'exposition à l'action de l'air. Après quatre jours de séjour dans l'alcohol, cette partie des cordons antérieurs est devenue grumeleuse; les postérieurs ont pris la disposition fibreuse.

Les branches antérieures et postéricures des nerfs rachidiens n'offrirent aucune altération sensible dans toute l'étenduc de l'altération organique; les ganglions intervertébraux de la septième cervicale et de la première dorsale, et les deux suivans inférieurement étaient confondus dans la masse désorganisée; mais en les disséquant avec soin, j’ai vu qu'ils n'avaient éprouvé aucune altération.

L'encéphale était sain : il y avait à la partie postérieure de l'hémisphère droit un tubercule jaunâtre, ramolli, de la grosseur d'un haricot; il paraissait d'abord siéger dans l'épaisseur de la substance corticale; mais en déplissant la circonvolution au fond de laquelle il était logé, on vit qu'il adhérait à la pie-mère. 11 y avait un peu de sérosité citrine dans les ventricules latéraux.

Les poumons étaient sains, crépitans; lè droit contenait supérieurement un petit tubercule non ramolli.

Le cœur était sain; son volume était normal les cavités gauches avaient leur ampleur et leur épaisseur ordinaires. 
Le péritoine offrait les traces d'une inflammation chronique très-intense; tous les viscères renfermés dans l'abdomen étaient adhérens entre eux, et réunis en masse par des pseudo-membranes; ceux renfermés dans le bassin étaient tellement agglomérés par ces productions organisées, qu'il devenait trèsdifficile de les distinguer. Il y avait un épanchement rougeâtre et purulent très-abondant dans la cavité péritonéale : intérieurement, l'estomac était un peu marbré de rouge dans le petit cul-de-sac. Le duodénum et le jéjunum étaient sains; l'iléon offrait vers son tiers inférieur un tubercule développé dans l'épaisseur de ses membranes; la portion de membrane muqueuse qui lui correspond, présentait une cicatrice, indice d'une ancieune ulcération. Du reste, l'intérieur de cet intestin offrait çà et là quelques plaques anciennes formées par les glandes de $\boldsymbol{P}$ eyer ; elles étaient très-nombreuses auprès de la valvule iléo-coecale. Les gros intestins étaient sains, le foie volumineux et un peu rámolli; la rate, le pancréas et les reins dans leur état normal; la vessie racornie, épaissie, d'un rouge noirâtre.

En outre de ces altérations, une glande bronchique, située à un pouce au-dessous de la bifurcation de la trachée-artère, s'était développée outre mesure et avait acquis lc volume d'un petil œuf; cette glande avait suppuré; le pus s'était logé en arrière de la plèvre, avait formé un pelit foyer s'n arrière du sommet du poumon droit: ce foyer 
avait fusé jusque dans la cavité du rachis, en pé-. nétrant par un des trous de conjugaison. Du reste, tous les autres organes étaient sains.

Un résultat nouveau ressort de cette observation : il est déduit de la coïncidence des mouvemens désordonnés du cour avec la lésion primitive des cordons antérieurs de la moelle épinière. Les expériences de Legallois ont établi que cet organe empruntait de tous les points de la moelle épinière une action qui favorisait ses contractions: mais d'après ce fait, et aussi d'après un autre assez analogue que j'ai observé, il paraîtrait que ce sont principalement les cordons antérieurs qui exercent cette influence sur le cour. Il paraîtrait aussi que les cordons postérieurs influencent plus spécialement les poumons, puisque, dans ce cas, la respiration n'a pas été sensiblement troublée, les. cordons postérieurs étant sains; dans d'autres cas, au contraire, les cordons postérieurs étant seuls. lésés, la respiration a été spécialement affectée, lorsque la désorganisation siégeait au-dessus ou. au-dessous de l'insertion des branches cervicales correspondantes au nerf diaphragmatique. 
TABLEA COMPARATIF

Des Dimensions du Bulbe rachidien chez les Mammifères (1),

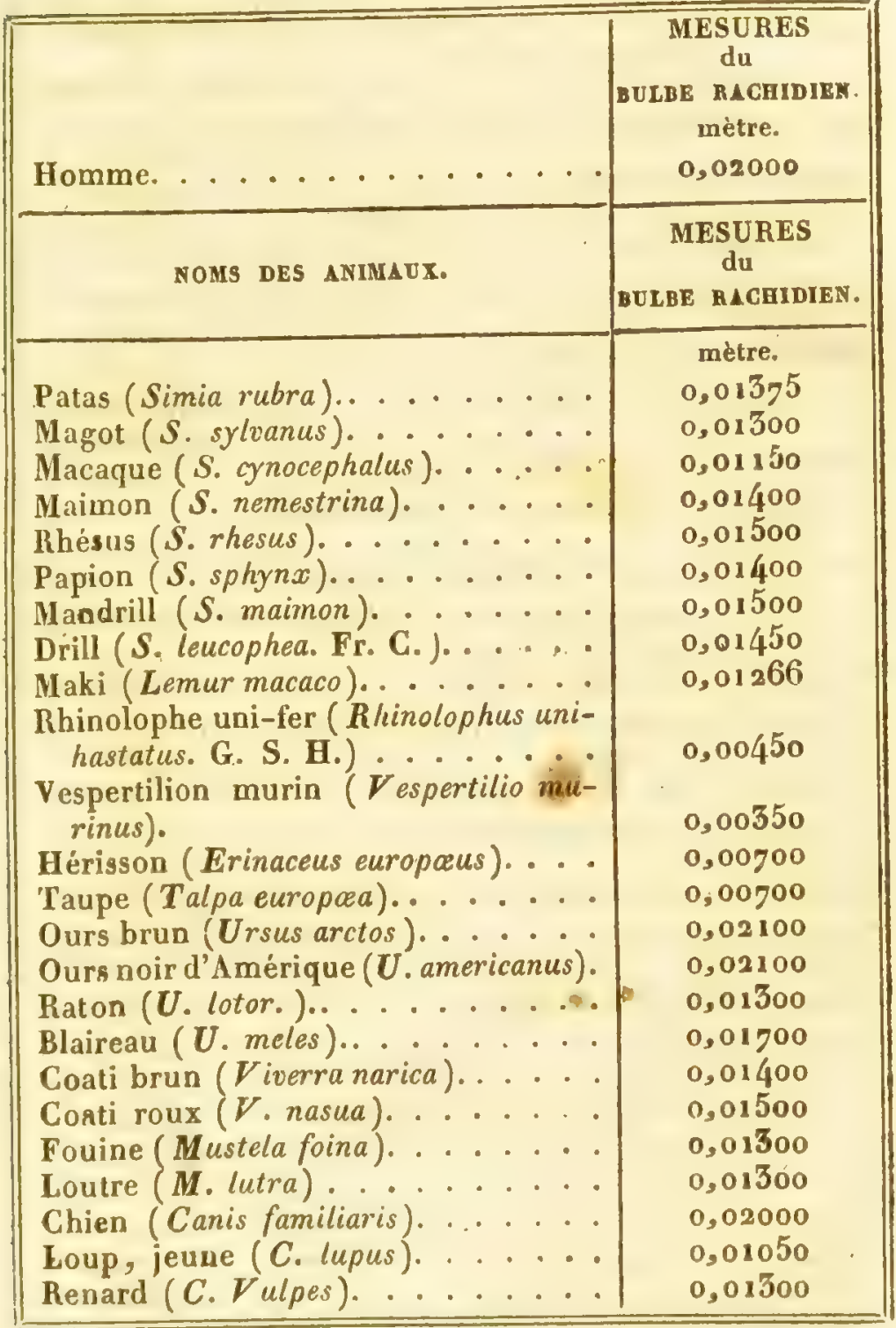

(1) J'ai séparé le bulbe rachidien du pont de Varole, parce que ces deux parties sont très-distinctes chez les mammifères. On verra plus bas les dimensions du pont et du trapéze, qui peuvent allssi êtro mis cn rapport avec la moclle allongee des autres classes. 
Suite du Tableau comparatif des Dimensions du Bulbe rachidien chez les Mammifères.

\begin{tabular}{|c|c|}
\hline TOMS DES AKIMAUX. & $\begin{array}{c}\text { MESURES } \\
\text { du } \\
\text { BULBE R LCHIDIEN. }\end{array}$ \\
\hline 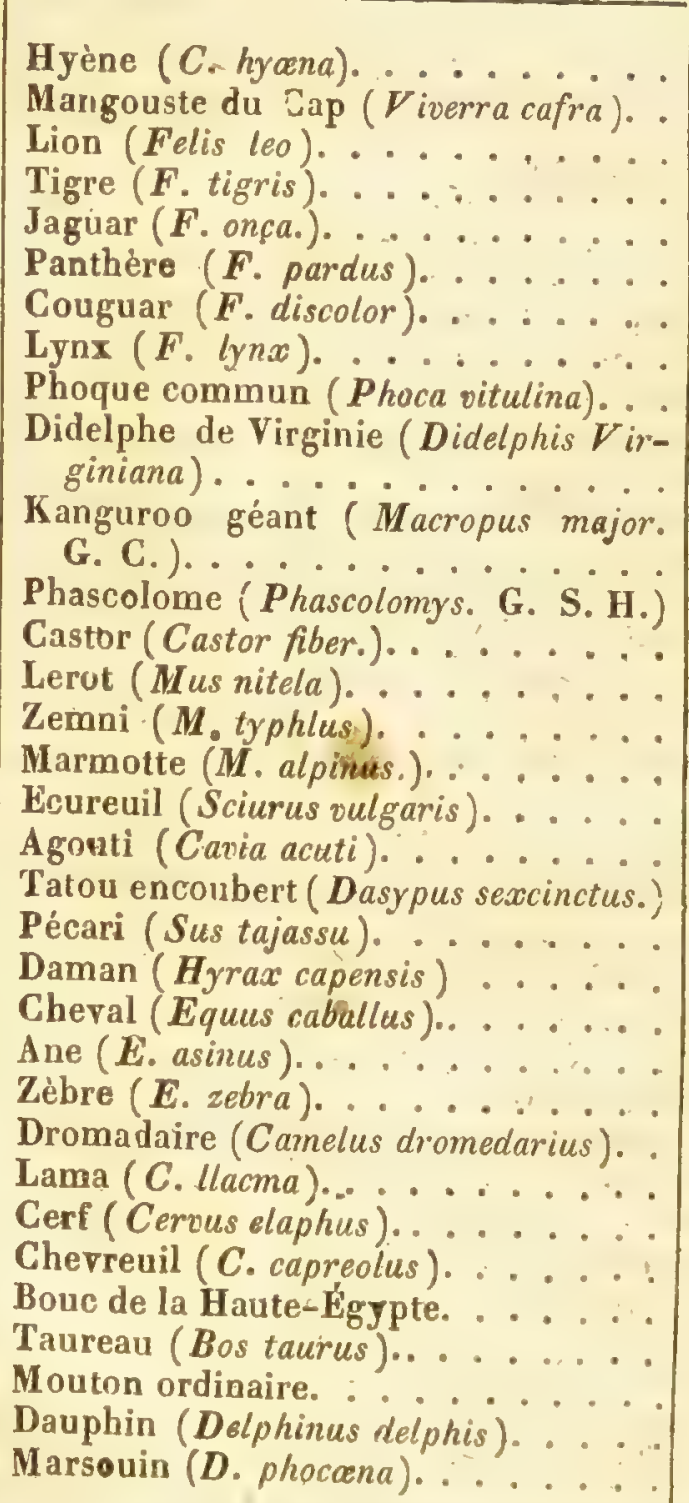 & $\begin{array}{l}0,02200 \\
0,01700 \\
0,01600 \\
0,00450 \\
0,00600 \\
0,01200 \\
0,00900 \\
0,01200 \\
0,01500 \\
0,02: 00 \\
0,00950 \\
0,03200 \\
0,02500 \\
0,02400 \\
0,03600 \\
0,02500 \\
0,01900 \\
0,02100 \\
0,01900 \\
0,03350 \\
0,01600 \\
0,01900 \\
0,01600\end{array}$ \\
\hline
\end{tabular}


NOELLE ALIONGEE.

TA BLEAU COMHA HA IY

Des Dimensions de la Moelle allongée chez les oiseaux.

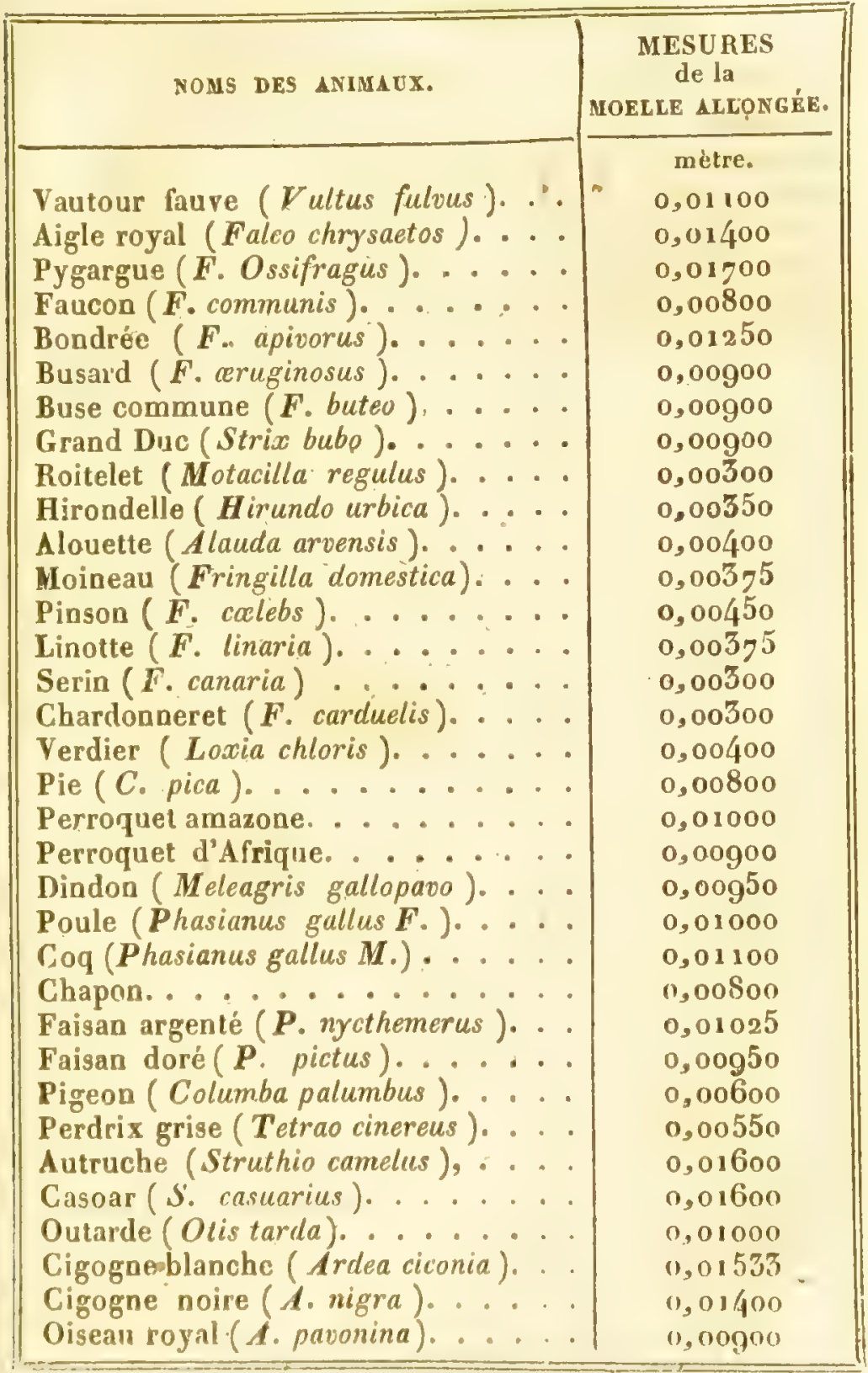


Suile du Tableau comparatif des Dimensions de la Moelle allongée chez les Oiseaux.

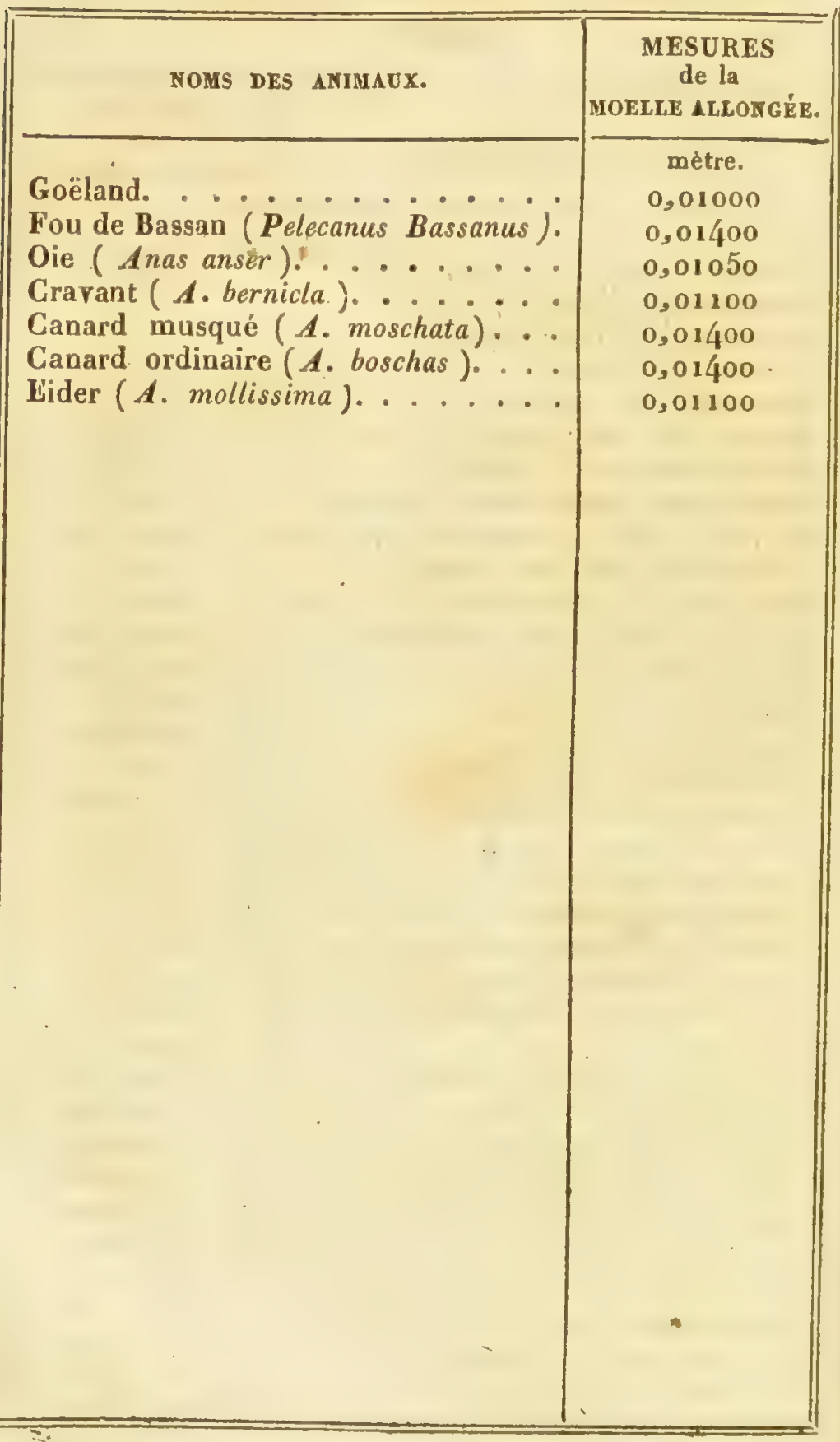


TABLEA U COMPARATIF

Des Dimensions de la Moelle allongée chez les Reptiles.

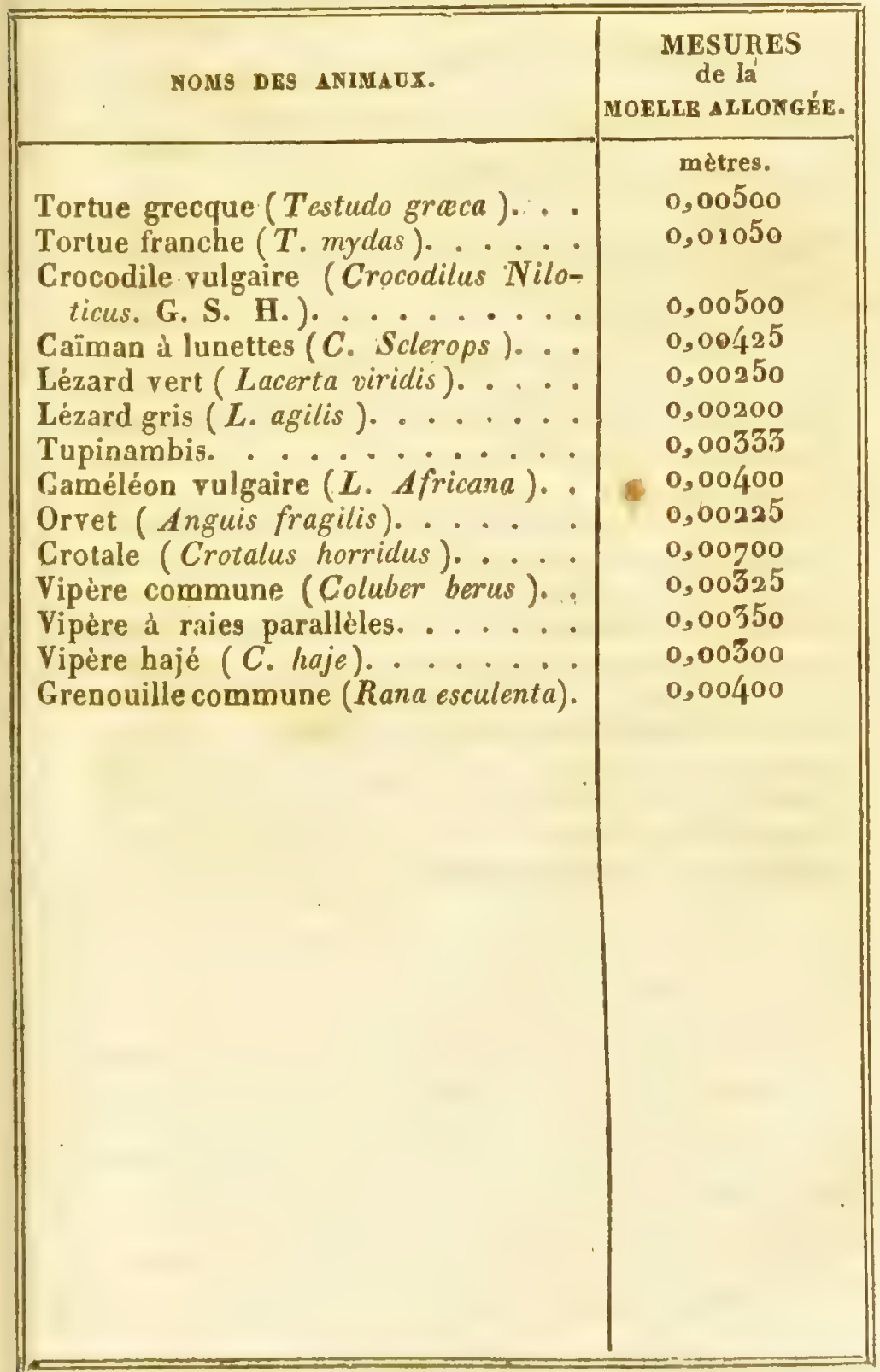


TABLEAL COMPARATIF

Des Dimensions de la Moelle allongée chez les Poissons.

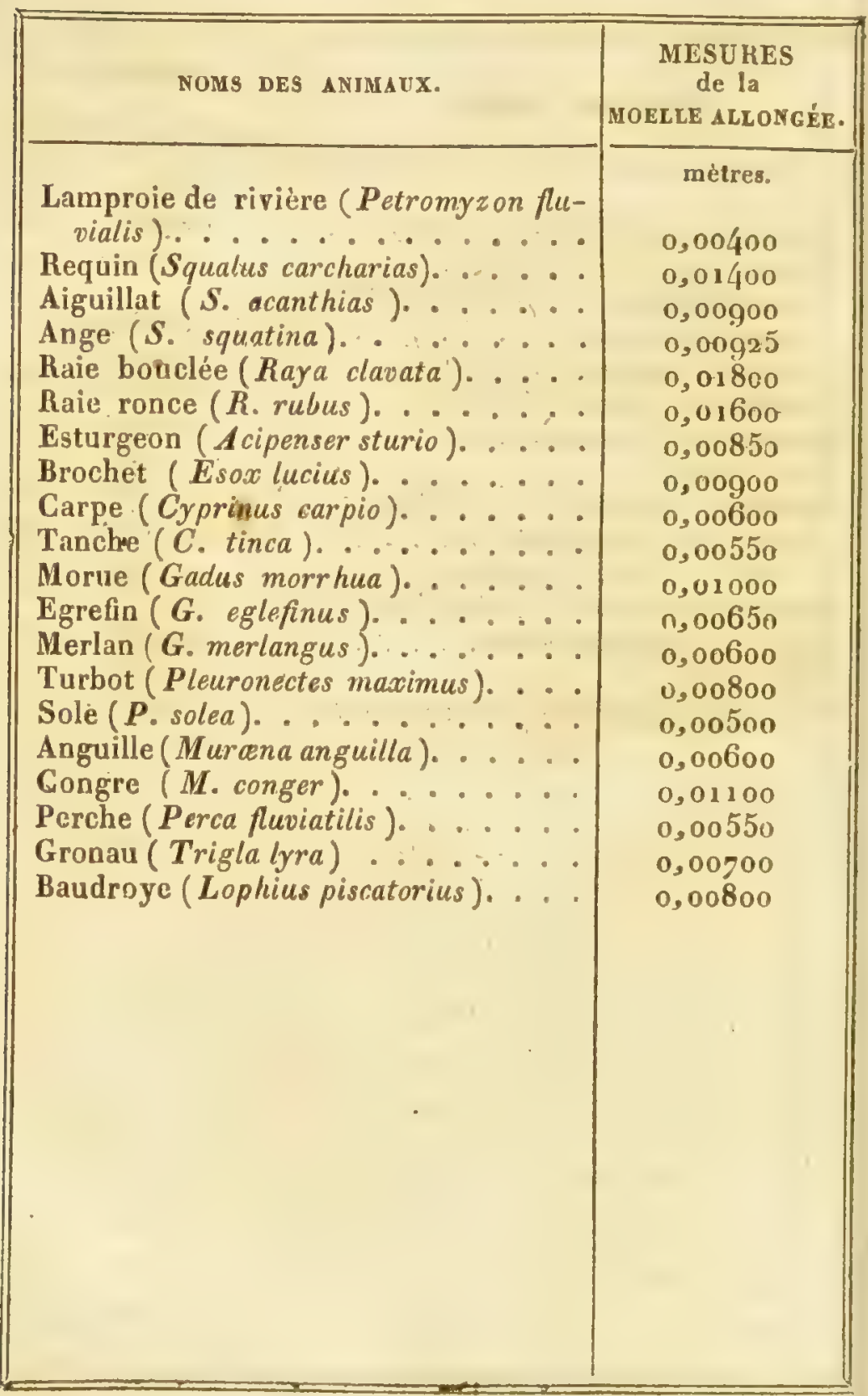




\section{CHAPITRE V.}

Des Tubercules quadrijumeaux, et de leurs analogues, les Lobes optiques, dans les quatre classes des vertébrés.

Jusqu'à ce jour les anatomistes avaient déduit leurs déterminations de la forme et de la position des parties, en choisissant l'homme pour point de départ, et les mammifères pour terme de comparaison des autres classes. La moelle épinière et la moelle allongée ayant une position constante dans toutes ces classes, et ne différant point ou presque point (le pont des mammifères excepté) quant à leur forme, on est généralement tombé d'accord sur l'analogie de ces parties chez tous les vertébrés. Les limites de la dernière ont seulement fait naître quelques controverses, mais elles n'ont eu aucune influence sur la science.

Il n'en est pas de même des tubercules quadrijumeaux, et des lobes qui leur correspondent dans les trois classes inférieures; lobes que j’ai nommés lobes optiques, le nerf de la vision n'étant en quelque sorte que leur continuation. Ici commencent la diversitéde forme, la diversité de position, pour les élémens de l'encéphale, et avec elles le doute, le vague et l'incertitude des déterminations. On conçoit en effet qu'en restant assujétis aux formes 
des parties, et en ne choisissant que les animaux adultes, on ne pouvait trouver similaires deux rangées de tubercules solides et une paire de lobes creux? Pareillement, en prenant pour règle la position, comment reconnaître à la base et sur les côtés de l'encéphale des oiseaux, les analogues des organes qu'on voyait constamment sur la face supérieure du même organe? On crut la chaîne des ressemblances rompues, on chercha à la renouer d'une autre manière : on aperçut des tubercules dans les lobes optiques des oiseaux; on n'hésita pas à les considérer comme les analogues des tubercules quadrijumeaux : on vit une voutte de couleur grise et blanche réunir les deux lobes optiques; on n'hésita guère moins à trouver en elle les caractères de la voûte à trois piliers des mammifères. On fut plus loin encore chez les poissons.

La richesse d'organisation de leurs lobes optiques, la prédominance qu'ils acquièrent sur les autres lobes qui composent l'encéphale dans cette classe, les fit considérer comme les analogues des hémisphères cérébraux des classes supérieures. Dès-lors on y trouva non-seulement, comme chez les oiseaux, les tubercules quadrijumeaux et la voûte, mais on y reconnut et on y plaça la couche optique, les corps striés, la voûte, et jusques au corps calleux. En arrivant à des rapports si peu vraisemblables, il semble qu'on eủt dû se meltre en garde contre les principes qui y condui- 
TUBERCULES QUADRLJUMEAUX.

saient : il n'en fut pas ainsi; plus ces rapprochemens parurent singuliers, plus ils piquèrent l'esprit des observateurs. Ce n'est pas, en effet, une chose facile que de trouver le cerveau de l'homme dans l'encéphale d'un poisson; mais pour arriver à ces conclusions, on choque toutes Ies vraisemblances, on délaisse toutes les connexions des parties, tous leurs rapports : on fait du cerveau dans cette classe une véritable monstruosité. Ce n'est donc pas une petite entreprise que de chercher à ramener par des voies simples et naturelles les lobes optiques des oiseaux, des reptiles et des poissons, à leurs correspondans chez les mammifères, les tubercules quadrijumeaux.

Certainement, si l'on bornait ces considérations aux animaux adultes, nul doute qu'on échouerait dans cette tentative. Considérez en effet à cet âge les animaux : les tubercules quadrijumeaux (1) des mammifères, et les lobes optiques des oiseaux (2), des reptiles (3) et des poissons (4), paraissent des organes tout-à-fait différens. Chez les premiers ce sont quatre tubercules (5) solides, ‘ symétriques, placés sur la face supérieure de l'en-

(1) Pl. XII, fig. $241, n^{\circ} 5$ et 6 ; fig. $242, n^{\circ} 6$ et 7 .

(2) Pl. III, fig. $78, n^{\circ} 7$; fig. $79, n^{\circ} 6$ et 7 .

(3) Pl. V, fig. 111, $n^{\circ} 6$; fig. $116, n^{n} 3$.

(4) PI. VI, fig. $139, n^{\circ} 5$; fig. $155, n^{\circ} 5$.

(5) PI. XI, fig. 225, E, G. 
céphale (1). Chez les oiseaux ce sont deux lobes creux (2), situés sur les côtés (3) et à la base du cerveau (4). Chez les reptiles (5) ces deux lobes restent dans la même position que chez les mammifères. Enfin chez les poissons osseux (6) et cartilagineux $(:)$, ils occupent la même place que. chez les reptiles; mais leur structure devient si compliquée, qu'on les a pris, comme nous venons de le dire, pour les hémisphères cérébraux. Leur organisation est en outre différente dans toutes les classes. Comment accorder toutes ces dissemblances? Comment ramener à l'unité de composition, et à la même signification, dans tous les vertébrés, des organes si opposés, par leur forme, leur position et leur structure.

L'anatomie comparative des embryons des quatre classes rapproche tous les animaux les uns des autres : il semble que ce ne soit qu'une grande famille, dont les genres éprouvent une série de métamorphoses plus ou moins nombreuses, plus ou moins variécs : les uns s'arrêtent plus tard dans leur évolution; les autres pius tôt,

(1) Pl. XI, fig. $232, n^{*}$.

(2) PI. III, fig. $83, n^{\bullet} 13$.

(3) Pl. III, fig. $78, n^{\circ} g$.

(4) PI. III, fg. $79, n^{\circ} \%$

(5) PI. V, fig. $119, n^{\circ} 7$.

(6) Pl. VII, fig. $163, n^{\circ} \%$

(7) Pl. VI, Gg. 138, $n^{*}$ \% 
d'autres enfin en parcourent toute l'échclle, en traversant tous les degrés intermédiaires des premiers. Je vais montrer une application de ce principe général des évolutions organiques, dans la formation des tubercules quadrijumeaux chez les vertébrés.

Et d'abord il pourra paraître singulier que leur étude précède celle du cervelet : ce dernier organe suivant immédiatement en arrière la moelle allongée, on croirait qu'il devrait être le premier formé après elle, et surtout long-temps avant les tubercules quadrijumeaux. L’inverse a constamment lieu : les tubercules et les lohes optiques se développent immédiatement après la moelle épinière, dont ils sont le bulbe de terminaison. Cetle succession dans la génération de ces parlies nesl pas seulement curieuse comme fait anatomique : clle devient en physiologie et en pathologie de la plus haute importance.

Comme la moelle épinière, les tubcrcules quadrijumeaux se montrent d'abord sous la forme de deux lames séparées sur la ligne médiane : ces lames sout distinctes chez les oiseaux, de la vingtcinquième à la trentième heure de l'incubation ( I) ; elles se réunissent en avant chez le mouton à la quatrieme semaine (2), chez le veau et le cheval ì la cinquicme, chez le lapin au dixicme jour,

(1) Pl. I, fig. 2, $n^{\circ}$.

(2) Pl. I, fig. $20, n^{\circ} 5$.

IT. 
chez l'umbryon humain vers la fin du premier mois (1), chez le tétard des batraciens du dixième au douzième jour de lcur formation (2). Dans cet état les lobes optiques et les tubercules quadrijumeaux forment dans les trois classes une large goutlière, dont les bords flottans sur le liquide ciủ la remplit se relèvent et marchent en se recourbant à la rencontre les uns des autres; lorsqu'ils sont parvonus au haut de l'encéphale, ils se recourbent verticalement, forment un sillon médian, qui est produit, clicz les mammifères et les oiseaux, par la présence de la faux de la duremire : cet effet esi très-apparent chez le poulet (5), loie, le canard, au commencement du cinquieme jour de l'incubation; chez le tétard des batraciens au quatorzième; chez le mouton de la cinquième semaine (4), chez le veau el le cheval de la sixième, etchez l'cmbryon humain de la même époque (5): les lames sont alors adossées, mais non réunies en arrièrc; cette réunion se fait plus tard par un mécanisme cntièrement scmblable à l'engronure postérieure de la moclle épinière. Cette réunion postérieure est effectuée chєz l'oiscau vers lc sixième jour de l'incubation (6); chez le tétard
( ) Pl. I, fig. $26, n^{\circ}$ ?.
(2) Pl. I, fig. $9, \mathrm{n}^{\circ} 5$ et 8 .
(5) Pl. I, fig. $4, n^{0}=$
(4) Pl. I, lig. $19, n^{\circ} 4$.
(5) Pl. I. fig. $2 i, n^{\circ} 4$.
(i) P1. I, fig. $5, n^{\circ} 6$. 
we s'opere du quinzième au dix-septieme (1); chez l'embryon du lapin, du onzième au douzième; chez le chat au vingtième, chez le unouton au commencement du deuxitme mois (2); chez le cheval, le veau et l'embryon humain, vers la septième semaine (5) : et si à cette ćpoque une maladie suspend le développement de l'encéphale, si le liquide qu'il renferme s'accumule en trop grande quantité, les lames nc sont pas jointes, elles restent écartées ou se déjettent sur les côtés, de la même manière que cela arrive pour la moelle épinière.

Arrêtons-nous un instant sur cet ćlat primilif des tubercules quadrijumcaux et des lobes optiques dans lẹs diverses classes. Nous voyons que chez l'homine (4), les mammiferes (5), les didelphes (6), les oiscaux (7) et les poissons (8) osscux et cartilagineux (9), ils ont rigoureusement la mème forme et rigoureusement aussi la même position, les mêmes connexions et les

(1) Pl. I, fig. $15, n^{\circ} 5$.

(2) Pl. I, fir. $28, \mathrm{u}^{\circ} 5$.

(3) $\mathrm{Pl} . \mathrm{I}, \mathrm{gg} .31, \mathrm{n}^{\circ} 4$.

(4) Pl. I, fig. 24, $n^{\mathrm{c}}$.

(5) Pl. I, fig. $21, n^{\circ} 8$.

(6) PI. I, fig. $30, n^{\circ} 4$.

(5) Pl. I, fig. $5, n^{\circ} 6$.

(8) Pl. I, fig. $15 \mathrm{et} 14,110, \mathrm{t}$

9) $\mathrm{Pl} . \mathrm{VI}, \mathrm{fig}, 1,7, \mathrm{n}^{\circ} \mathrm{3}$. 
mêmes rapports. Ce sout deux lobes symétriques séparés sur la ligne médiane par un silion plus ou moins profond, offraut une vaste carité dans leur intérieur, remplic chez les oiscaux, les reptiles, les mammifères et l'homme: par un liquide plıs ou moins épais; les tubercules quadrijumeaux de l'homme et des mammifères sont done primitivement une paire de lobes, comme chez les poissons, les reptiles et les oiseaux. C'est un organe identique en tout point dans toutes les classes; et s'il s'arrêtait au point où nous venons de l'observer, nul doute que celte identité ne fût reconnue par tous les anatomistes.

Mais il n'en est pas ainsi, les transformations que ces organes primitifs éprouvent, différant pour chaque classe, les formes quils revêtent, la position qu'ils affectent, la complication qu'ils acquiècut, doivent varier dans chacune d'elles. A la variation de ces métamorphoses correspondent les changemens que les tubercules quadrijumeaux et les lobes optiques subissent dans toutes les classes : pour les apprécier il est rigoureusement nécessaire de suivre avec précision ces métamorphoses, et les transformations qui en dépendent.

Les lobes optiques qui se métamorphosent le moins, sont ceux des reptiles. Suivez ces organcs chez les tétards des batraciens: jusqu'au quinzième jour, le lobe optique est unique de chaque côlé; 
du scizième au dix-huitième, la résicule qui le forme se subdivise par un petit sillon transversal(1), de telle sorte qu'on pourrait dire, à la rigucur, qu'il existe alors quatre tubercules quadrijumeaux; aux vingtième, vingt-unième et vingt-deuxième jours, ce sillon disparaît, et les lobes optiques deviennent jumeaux, comme ils l'étaient primitivement (2); leur forme est alors cellc d'un cône tronqué en devant (3) : ils restent quclques jours dans cet état, ils s'écartent ensuite en devant (4) du vingt-cinquième au trentième jour, dc manière à former entre eux un angle rentrant, dans lequel se loge la couche optique (5); cet angle s'agrandit dans le courant du deuxième mois, à mesure que les lobes optiques s'écartent (6) ct que la couche optique s'agrandit ( 7$)$. Ils restent dans cet état chez les batraciens adultes, un peu déjetés en devant sur les côtés (8) , et obliquement dirigés, par conséquent, de dehors en dedans. Chez le protéc, la cécilie, l'orvet (9), les deux lobes optiques semblent réunis en un seul, lo

(1) PI. I, $9 g .15, n^{\circ} 5$.

(2) Pl. I, Ag. 1 $1, n^{\circ} 5$.

(5) Pl. I, fg. $1, n^{\circ} 3$.

(4) PI. I, fig. $12, n^{\circ} 3$.

(5) PI. I, fig. $12, n^{\circ} 3 \mathrm{ct} 4$,

(6) Pl. I, fig. $36, \mathrm{n}^{\circ} 2$.

(z) PI. I, fig. $16, n^{\circ} 3$.

(8) P']. I, fig. $16, n^{\prime \prime} 2$.

(9) II. V, lier. $109, n^{\circ} 3$. 
sillon qui les divise supérieurement ne se voí qu'en arrière, où les lobes optiques sont trèspetits; ils sont presque parallèlcs, et à peine écartés en devant; chez la vipèrc hajé, la vipère de Fontainebleau (1), la vipère à raies parallèles (2), ils augmentent un peu de volume, et conservent en devant la même disposition à cause de la petitesse des renflemens optiques; chez le lézard gris, ils sont ovalaires (5); chez le lézard vert (4) ils se rapprochent beaucoup de la forme de ceux de la grenouille (5): chez le caméléon (6) ils sont trèsćcartés cn arrière et en devant, ce qui augmentc leur diamètre tranśversal; en devant ils s'écartent pour loger la couche optique $(7)$; leur ćcarlement postérieur sert à loger le cervelet (8). Chez lc crocodile à deux arêtes (9) ils s'écartent moins que chez le caméléon, et, comme chez ces derniers, leur volume est plus fort que chez l'orvet, les vipères ę les couleuvres; chez le crocodile vulgaire (10), l'ovale qu'ils représentent est légèrement déprimé
(1) Pl. V, fig. $126, \mathrm{n}^{\circ} 5$.
(2) Pl. V, fig. $155, n^{\circ} 5$.
(5) Pl: V, fig. 128, n०5.
(4) Pl. V, fig. 1.10, $\mathrm{n}^{\circ} 9$
(5) Pl. I, fig. $16,1 n^{\circ} 2$.
(6) Pl. Y, fig. $111, n^{\circ} 6$.
(z) P!. V, fig. $111,11 \times 8$
(8) Pl. V, fg. $111, n^{\circ} 4$.
(9) Pl. V, fig. $115, n^{0} 4$.
(10) P1, V, fig. $116,11^{\circ} \mathrm{J}$ 
sur les côles, et ils se louchent prescue on arriere el en avant. Chez le caiman (1) ils ont une forme splhérique; chez la tortue grecque (2) ils se rapprochent de la forme quadrilatire; ils offrent en arrière un sillon transversal (3) analoguc à celui du tétard du dix-huitième jour, de telle sorte qu'on croirait, chez ces animaux, à l'existence des tubercules quadrijumeaux des mammiferes. Chez la tortue franche (4), leur forme est très-diffé. rente de celle de la tortue grecque, ils représentent deux sphères arrondies, superposces sur la face supéricure de l'encéphale (5), séparées cn devant par la glande pinéale qui se place entre cux (6). Chez lo tupinambis (7) ils sont quadrilatères de même que chez la tortue grecque (8), et récouverts en arrière par une pelite languette du cervelet (9). Telles sont les diversités de forme que jai pu observer dans les diverses familles et les espèces de cette classe. Lcur cavité intérieure communique d'un lobe à l'autre: celte cavité, i peine distincte chez l'amphisbènc, est très-

(1) P1. V, 1ig $155,10^{\circ} 5$

(2) P1. V, lig. $12.5,11 " \mathrm{z}$

(3) PI. V, fig. 135, n" g et, - bis.

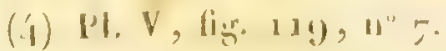

(5) Pl. V, fig. 120, $\mathrm{n}^{n} 5$.

(6) Pl. V, fig. $121, \mathrm{n}^{\circ} 10$ 。

(7) Pl. V, fig. 114, n" 8.

(8) Pl. V, fig. $125, \mathrm{n}^{\circ} \%$

(0) Pl, V, fig. 11 个, $n^{\circ} 1$. 
étroite chez l'orvet, le protée, les couleuvres, les vipères; elle augmente chez la grenouille (1), les crapauds, le camćléon, le tupinambis, les lézards, les crocodiles, le caiman, et surtout chez la tortue franche (2), où elle acquiert les plus grandes dimensions possibles dans cette classe. La lame qui les réunit l'un à l'autre est très-mince, son épaisseur suit la progression du volume des lobes et s'accroît insensiblement de l'orvet à la grenouille (3), au caméléon, au crocodile et à la tortue franche (4). Chez le protée, la syrène et la cécilie, le ventricule des lobes optiques paraît unique, la lame de jonction étant si étroite qu'on la distingue à peine en arric̀re. Sur le plancher de cette cavité qui est l'analogue de l'aqueduc de Sylvius des mammifères, on distinguc sur la ligne médiane le passage des pyramides, et l'on voit sur les côtés un petit renflement, imperceptible chez l'orvet, les couleuvres, les vipères, assez distinct chez la grenouille, le caméléon, le tupinambis, le crocodile, très-prononcé chez la tortue franche. Le volume de cerenflement est en raison directe du volume des nerfs optiques, et suit, comme ce nerf, une progression croissante de's ophidiens aux chéloniens. Quelques faisceaux très-déliés, de matière

(1) Pl. V, fig. 13, $\mathrm{B}^{\circ} 5$.

(2) Pl. V, fig. $121, \mathrm{n}^{\circ} \%$

(3) Pl. V, fig. 13 \%, $\mathrm{n}^{\circ} \mathrm{6}$.

(i) Pl. V, lig. $121,1^{\circ} 10$ 
blanche, en traversentla base chezla tortue franche.

La lame formant l'enveloppe de ces lobes est composée de deux couches, l'une interne, l'autre externe; l'interne, qui correspond au petit renflement, cst toujours grise; l'externe varie dans sa coloration : elle est presque grise chez le protée, l'orvet, les vipères et les couleuvres; d'un gris blanchâtre, chez les grenouilles et les crapauds; d'un blanc mat chezle crocodile, et d'un blanc assez pur chez la tortue franche. Cette couche formant l'extérieur du lobe, la couleur de celui-ci varie de la même manière qu'elle, chez les ophidiens, les batraciens, les sauriens et les chéloniens. L'épaisseur des parois des lobes augmente des ophidiens aux lacertiens, aux batraciens (1), aux sauriens et aux chéloniens (2).

Ainsi, chez les reptiles, les lobes optiques ( $t u-$ bercules quadrijumeaux ) restent ovalaires, creux; ils se dépriment en avant chez quelques-uns, pour loger la couche optique, mais ils ne se déplacent pas. On les voit chez tous sur la face supéricure de l'encéphale, jamais à leur base : ils conscrvent ainsi l'état embryonnaire permanent des mammifères et des oiscaux.

Dans cette deruière classc leurs évolutions sont beaucoup plus nombreuses; ils perdent quelquesuns des caractères embryonnaires, abandonnent

(1) Pl. V, Gg. $134, n^{\circ} 5$ et 6.

(2) Pl. $\mathrm{V}, \mathrm{fg} .121, \mathrm{n}^{\circ} 2$ et 10. 
la position constante quils ont dans loules les classes, passent de la lace supéricure i la partic latérale et à la base de l'organe; ce qui ne peut avoir lieu sans éprouver des changemens remarquables, qui non-seulement les ont fait méconnaître, mais les ont fait considérer comme les analogues de la couche optique en totalitê, ou de la moitié postérieure de cetle couche, ainsi que vicnt de le proposer le célèbre Treviranus. Enfiu, le volume qu'ils acquièrent dans celte classe leur a mérité d'être assimilés, par M. de Blainville, aux hémisphères cérébraux des mammifères. Ces opinions ayant entravé jusqu'à ce jour la marche de la science, il est indispensable d'en apprécier. la valeur pour justifier la détermination que j’ai donnée, et qui déjá est adoptéc par la plupart des analomistes: l'cxamen attentif des irausformations de ces organes chez l'embryon des niscaux, leurs connexions, leurs rapports chez les oiscaux adultes, ne laisseront, je l'espère, aucun doute sur la question fondamentale de l'anatomie comparative du cerveau.

La forme lobulaire quaflectent chez les viscaux les lobes optiques, se trouve déjì expliquéc pan l'état primitif de ces parhes chez les cunbryous d." l'homme et des manmiféres, par l'étal permanent de ces corps chez tous les reptiles. S'ils ne présentaicnt que celle dificulté pour éfre ramenés à leur analogic avec les tubercules quadrijuncaur. il nous sullirai do reprodure los rapports que 
nous avons exposés pour la classe des reptiles: mais leur changement de position cliez tous les oiseaux vicnt apporter une nouvelle inconnue dans le probleme; et comme rien d'analogue n'existe dans les autres classes, cette transposilion a été la source de toutes les erreurs dont l'encéphale de cette classc a été le sujct. Il est donc très-important, en suivant celte métamorphose, d'apprécier tous les changemens qui en sont l'effet.

Jusqu'aux cinquième ct sixième ( 1 ) jours de l'incubation, les lobes optiques sont doubles, symétriques, placés, comme dans les autres classes, sur la face supérieure de l'encéphale; aux seplième, huitième ou neuvième jours, selon qu'on observe la formation du poulet, des faisans, de l'oic, du canard ou du dindon, un sillon obliquc se place en arrière de chaque lobe (2), fl le divise en deux parlies inégales, l'une antéricure, plus grande (3), l'autre postérieure, plus pelite (4). Les lobes sont alors quadrijumeaux, de même que chez les tétards des grenouilles (5) et descropauds, du seizième au dix-seplième jour. Jusques-là ces lobes sont restés parallèles l'un à l'aulre, représen-

(1) PI. I, fig. $5, n^{\circ} 6$.

(2) PI. I, fig. $6, n^{\circ} 8$.

(3) Pl. I, fig. 6, $n^{\circ} 8$.

(4) Pl. I, fig. $6, n^{\circ} 7$

(i) PJ. I, fir. $1.5,0$. 
tant un ovoïde légèrement aplati sur les côtés (1), de la même manière que chez le tétard du vingtième jour (2). Les lobes optiques des oiseaux conservent leur forme aussi long-temps que la couche optique reste à l'état rudimentaire, ce qui s'observe jusqu'au huitième jour (3); mais aux neuvième et dixième jours, ce renflement augmente tout-à-coup de volume (4), de même que chez le tétard du vingtième au vingt-cinquième jour (5). L'effet produit par la présence de ce renflement est le même dans les deux clasśes; chez l'oiscau (6) comme chez la grenouille (7), les lobes optiques s'écartent en avant pour le loger: ils cessent d'être parallìles, ils deviennent obliques dans les deux classes (8), se bombent sur les côtés, au lieu de rester déprimés comme ils étaient auparavant. Si la métamorphose des lobes optiques s'arrêtait à ce degré chez les oiseaux, ils seraient entièrement analogues à ccux des reptiles, notamment de la grenouille adulte (9), du camé-

(3) Pl. I, fig. 5, n: 6 .

(2) Pl. I, fig. 11, n०3.

(3) Pl. I, fig. $6, n^{\circ} 9$.

(4) Pl. I, 0g. $7, n^{\circ} 8$.

(5) P1. I, 0n. $12, n^{\circ} 4$.

(6) Pl. I, fig. $7, \mathrm{n}^{\circ}$.

(7) PI. I, Ag. 12, $1^{\circ} 5$

(8) P1. I, fig. $7,1^{\circ} \mathrm{z}$

(9) Pl. I, fign, $16, \mathrm{H}^{n} 2$ 
TUBERGULES QUADRIJUMEAUX.

líon (1) et clu crocodile à deux arêtes (2). Chez certains oiseaux monstrueux ils conservent souvent cet état; je l'ai surtout observé sur des oufs de poule soumis par M. Geoffroy-Saint-Hilaire à des expériences dont le but était de produire des monstruosités artificielles. La ressemblance ne se borne pas aux lobes optiques, elle est encore partagée par!e cervelet, la couche optique et les lobes cérébraux : l'encéphale des oiseaux est l'analogue alors de celui des reptiles, et celui des reptiles est l'ćlat embryonnaire permanent des oiseaux. Ce parallèle est des plus curieux, el un des résultats les plus intéressans de l'anatomic comparalive des embryons:

Mais le cervcau des oiseaux ne s'arrête pas à cet ćtat, il continue ses évolutions dans l'élat normal; toutes ses parties se concentrent en même temps qu'elles se développent; la couche optique augmente en avant, le cervelet en arrière; les lobes cérćbraux se portent en arrière, le cervelet en avant; ils marchent ainsi à la rencontre l'un de l'autrc. Or, si on considère l'encéphale de l'oiseau aux sixième (5), huitième (4) et dixième (5) jours de l'incubation, on s'aperçoit de suite que cette

(1) Pl. V, fig. $111, n^{\circ} 6$.

(2) Pl. V, fig. $116, \mathrm{n}^{\circ} 5$.

(3) Pl. I, 'fig. $5, \mathrm{n}^{\circ} 5,6,7$ et 8 .

(4) P1. I, 4. 6, n० $7,8,9$ ct 10.

(i) Pl. I, fig. $7, \mathrm{u}^{n}(\mathrm{i}, 7,8 . \mathrm{ct} \mathrm{g}$. 
Iransformation ne saurait s'effectuer si les lubes opliques conservaicnt leur position et lcur saillic sur la face supéricure de l'organc; il faut nécessairemcnt qu'ils soiont déplacés. Voici le mécanisme de ce déplacement :

Du dixiène au douzième jour de l'incubation, les lobes optiques, écartés en avant pour la couche oplique, en arrière pour loger le cervelet, s'affaissent tout-à-coup dans leur partie moyenne et s'ćcartent l'un de l'autre; par cet écartement ils font une saillie très-prononcée sur les côlés, du treizième au quatorzième jour (1), et sur la base de l'encéphale, où ils deviennent visibles dès les dixième (2). et onzième jours. Les seizième (3) et dix-huilième (4) jours, le mouventent demi-circulaire des lobes continue, et ils s'arrêtent enfin vers le vingtième à la position quils conservent chez tous les oiseaux adultes (5); en même temps, la lame qui les réunit supérieurement s'amincit et s'allonge de toute la longueur dont les lobes s'écartent: lelle est la métamorphose qui donne aux lobes optiques des oiseaux leur caractère classiquc. Mais ce déplaccment a-t-il détruit leur analogic avec les lobes opriques des reptiles ot les tubercules qua-

(1) Pl. I, fig. $8, n^{\circ} \%$

(2) Pl. II, fig. 36, $\mathrm{n}^{\circ}$.

(5) Pl. II, fig. $58, \mathrm{n}^{\circ} 8$.

(4) PI. II, fig. $59, \mathrm{u}^{\circ} 8$.

(5) Pl. II, fig. $40, n^{\circ} 7$. 
Arijumcaux des mammifères à lcur ćpoque lobulaire? non sans doutc. Qui ne voit, en effet, que ce changement de position ninflue en rien sur leur signification? Qui ne voit que c'est le même organe, à la marche circulaire près, qui s'est effcctuće? Ces lobes sont donc un organe identique chez les reptiles, les oiseaux et les embryons des mammifères.

Chez tous les oiseaux adulies, la forme des lobes optiques est celle d'un sphéroïde saillant sur les côtés (1) et légèrement déprimé en dedans ct en haut (2), en dedans et en bas (5). Leur forme cst la mème chez la poule (4), les faisans, les perdrix, les pigeons, les canards, la bondrée (5), la cigogne (6), l'hirondelle (7), le roitelet (8), le verdier, le chardonneret, les moineaux, le serin, l'autruche (9), le perroquet (10) et le casoar (11). Leur volume va en augmentant graduellement du

(1) PI. III, fig. $84, n^{\circ} 5$.

(2) Pl. III, fig. $84, n^{\circ} 6$.

(3) PI. III, fig. $79, \mathrm{n}^{\circ} 6$.

(4) Pl. III, fig. $87, n^{\circ} 4$.

(5) Pl. IV, fig. 9o, $\mathrm{n}^{\circ} 4$.

(6) Pl. IV., fig. $105, n^{\circ} 6$.

(2) Pl. IV , fig. $9^{2}, \mathrm{n}^{\circ} 11$.

(8) PI. IV', fig. 94, $\mathrm{n}^{\mathrm{n}}$ 4.

(9) Pl. VI, fig. $9^{8}, n^{\circ} 5$.

(10) PI. III, fig. $84, n^{\circ} 6$.

(ii) Pl, III, fig. $79, n^{\circ} 7$. 
roitelet au casoar, c'est-à-dire des plus pelits aux plus grands oiseaux.

Leur couleur est d'un blanc mat analogue à celui de la moelle allongée et de la moelle épinière, couleur qui les distingue de celle du cervelet ct des hémisphères cérébraux.

De même que ceux des reptiles, les lobes optitiques des oiseaux sont creusés d'un ventricule. La capacité de ce ventricule, assez grande relativement à l'étendue des sacs membraneux dans lesquels il est renfermé, est toujours proporlionnée au volume de ces lobes, des nerfs optiques et de l'ocil ; très-petit chez le roitelet, l'hirondelle, le serin, le moineau, il devient très-spacieux chez la bondrée (1), l'aigle (2) et le casoar (3). Les deux ventricules s'abouchent dans la scissure de Sylvius par un petit canal court dirigé transversalement à la scissure, et que l'on distingue facilement sur la section longitudinale de l'encéphale, ainsi qu'on le voit chez le roitelet (4), chez l'hirondelle (5) et chez le perroquet (6).

L'ouverture de communication, très-étroite chez

(1) Pl. IV, fig. $88, \mathrm{n}^{\circ} 4$.

(2) PI. IV, fig $101, n^{\circ} 4$.

(3) Pl. III, fig. 83, $n^{\circ} 13$.

(4) Pl. IV, fig. $10 \%, n^{\circ} 5$

(5) Pl. IV, fig. $95, n^{\circ} 5$.

(6) PI. III, fig. $86, \mathrm{n}^{\circ} 3$. 
les petits oiseaux, s'agrandit comme les lobes : chez lc roitelet, le serin, le pinson, le chardonneret, elle reçoit à peine une soie de sanglier; chez l'oie, le canard, le coq-d'inde, le milan, les aigles, on peut y introduire la tête d'une épingle; chez le casoar et l'autruche elle a donné passage à un petit stylet. Si après avoir découvert le ventricule par sa partie supérieure, comme je l'ai fait chez le casoar (1), on insuffle l'ouverture mise à nu, alors on dilate la scissure de Sylvius, le ventricule du côté opposé, on soulève la lame transverse des lobes et mêne la valvule de Vieussers, ce qui prouve la libre communication de toutes ces parlies.

Le plancher des ventricules des lobes optiques est formé par une couche grise, cotonneuse, qui est évidemment la continuation de celle du quatrième ventricule. Le milien de ce plancher offre une rainure assez profonde, qui forme l'axe de la scissure de Sylvius. Celle-ci est très-longue cherz les oiseaux; elle s'étend du quatrième au troisième ventricule, et préscute, de chaque côté, à l'union du tiers postérieur avec les deux ticrs antérieurs, un renflement grỉsâtre, que Collins considérait comme le corps géniculé interne des mammiferes, et que depuis Haller et Malacarno on avait pris pour les tubercules quadrijumeaux

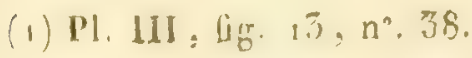

II. 
des oiseaux. Tous les oiseaux offrent en effet une saillie plus ou moins élevée aux pédoncules cérébraux en cet enciroit. Cettc saillie est un résultat physique de la métamorphose de l'encéphale pendant l'incubation. En effet elle n'existe pas à l'époque où les lobes optiques occupent la face supérieure de l'encéphale, parce que les pédoncules cérébraux sont droits jusqu'à cette époque. Mais au moment où l'encéphale se contracte, la ligne des pédoncules cérébraux est en quelque sorte brisée et ondulée. Il résulte de là, d'une part, l'excavation du quatrième ventricule, et la saillie si prononcée des pédoncules vers la moelle allongée; saillie que Haller comparait à la protubérance annulaire des mammifères. Après que les pédoncules ont formé cet arc, dont la convexitć est en bas et la concavité en haut, ils en forment un second opposé au premier. Celui-ci commence un peu avant linsertion de la troisième paire, et finit au niveau de l'infundibulum. La concavité est en bas, et correspond à celle du quatrième ventricule du premier contour; la convexité est en haut et fait une proéminence sur le plancher de la scissure de Sylvius (1). Cette proéminence est toujours proportionnelle au volume des pédoncules cérébraux. Elle est sitùée surchaque côté de la scissure de Sylvius; on voit donc que le 
même mécanisme qui creuse le quatrième ventricule, fait saillir considérablement les pédoncules cérébraux au niveau de la moelle allongée, et que la concavité qui se manifeste au niveau de la troisième paire' produit par la mème raison une proéminence des pédoncules cérébraux dans l'intérieur de la scissure de Sylvius. Ces deux proéminences ne sont pas plus les tubercules quadrijumeaux, que la saillie de la moelle allongée n'est la protubérance annulaire. Ces deux saillies et ces deux concavités sont un effet mécanique de la concentration de l'encéphale pendant l'incubation.

La lame de réunion des lobes optiques (i) sert de voûte à l'aqueduc de Sylvius, de même que chez l'homme et les mammifères; sa formation est la même? que celle des faisceaux transverses qui engrènent en arrière la moelle épinière. Da cinquième au huitième jour de l'incubation, on voit partir de la face interne du lobe optique, de petits faisceaux qui se dirigent vers ceux du côté opposé, de même que cela s'effectue chez les reptiles et chez les mammiferes: leur engrenure s'opère par l'enchâssement des faisceaux de droite avec ceux du côté gauche, et vice versâ. L'étendue transversale de cette voûte est proportionnée à l'écartement des deux lobes (2); son diamètre antéro-

(1) Pl. IV, fig. $90, n^{\circ} 3$.
(3) PI. III, fig. $84, n^{\circ} 6$. 
postérieur est un peu moins grand. (1); il est étendu depuis la valvule de Vieussens (2) jusqu'à la partie postérieure de la couche optique (3), qu'elle recouvre : sa grandeur absolue varie, du reste, comme celle des lobes optiques et des oiseaux; elle va en augmentant graduelleinent du roitelet (4), de l'hirondelle (5), aux pigeons, aux perdrix, aux faisans, au poulet, à la bondrée $(6)$, au perroquet $(7)$, à l'oie, aux canards, à l'aigle (8), à l'autruche et au casoar (9). Son épaisseur est moindre que celle des parois des lobes.

I.a couleur des parvis des lobes est grise chez l'embryon du poulet jusqu'au sixième jour; le feuillet est alors si mince, qu'on voit au travers le liquide dont la vésicule est remplie. Au dixième jour, ce même feuillet est blanc : comment s'est opérée cetle conversion du gris au blanc? le voici. Au septieme jour de l'incubation, on voit naître en dehors de chaque lobe des rayons de matière

(1) Pl. III, fig. $84, n^{\circ}$ \%

(2) PI. III, Gg. 84, $n^{\circ} 4$.

(3) PI. III, fig. 83, $\mathrm{n}^{\circ} 8 \mathrm{et} z$.

(4) Pl. IV, fig. $102, n^{\circ} 4$.

(5) Pl. IV, fig. 100, no"4.

(6) PI. IV, fig. $90, \mathrm{n}^{\circ} 3$.

(7) Pl. III, fig. 84, n'5.

(8) Pl. IV, fig. $101, n^{\circ} 5$.

(9) Pl. III, fig. $83, \mathrm{n}^{\circ} 6$. 
blanche, qui convergent de la circonférence au centre. On en compte trois ou quatre; au huiticme jour il y en a buit ou neuf (1). Ces stries blanches sont parallèles et concentriques entre elles : on distingue la lame qui est au-dessous et dont la couleur grise tranche avec celle des fibres médullaires. Au neuvième jour (2), ces fibres se rapprochent en s'étendant; au dixième, elles se touchent, et no forment plus qu'une lame blanche unique étendue sur toute la superficie du lobe (3). Cette couleur est la même chez tous les oiscaux adultes; mais chez ceux-ci, celte couche fibreuse blanche qui forme l'écorce des lobes optiques, se continue par sa base avec lo nerf optique; sa formation est de beaucoup postérieure à celle de ce nerf; ce qui prouve qu'elle ne lui sert pas d'origine.

Les parois des lobes opliques sont formées de quatre couches : l'une, interne, est grise (4); la soconde, blanche (5), envoic quclques faisccaux sur les pédoncules cérébraux (6); la troisième couche est grise, plus épaissc que la première (7); la qua-

(1) Pl. II, f.g. 33, n* 7.

(2) Pl. I, fig. $6, n^{\circ} 8$.

(3) Pl. I, fig. $5, n^{n} \%$

(4) PI. IV."fin. 101, n"

(5) Pl. IV, fig. $108 ; n^{n} 5$.

(6) PI. IV, fig. $91, n^{*}$ (1.

(c) Pl. IV, fig. $10 x, n^{0} 10$. 
trième, la plus extérieure, est blanche (1) : elle se prolonge en grande partie dans le nerf optique $(2)$; quelques-uns de ses faisceaux se jettent dans la partie postérieure des hémisphères cérébraux (3). On voit la disposition et les rapports de ces quatre couches dans les diverses coupes de ces lobes, chez l'hirondelle (4), le roitelet (5), le perroquet (6), la bondrée (7), l'aigle (8), et le casoar (9).

La lame rayonnée (10) qui forme la voûte de l'aqueduc de Sylvius, et qui sert de commissure aux deux lobes opiiques, est formée comme ceux-ci par la matière grise et blanche (11); son épaisseur étant moindre, on n'y trouve plus les quatre couches. Ces deux substances affectent la disposition striée (12), que l'on remarque sur les lobes du septième aux huitième (13) et neuvième jours (14)
(1) PI. IV, fig. 101, $\mathrm{n}^{\circ} 1 \mathrm{~F}$.
(2) Pl. IV, fig. 105, $\mathrm{n}^{\circ} 2$ et 5 .
(3) Pl. IV, fig. $9^{\mathrm{r}}, \mathrm{n}^{\circ} 4$ et 6.
(4) PI. IV, fig. $9^{3}, n^{\circ} 5$.
(5) Pl. IV, fig. $107, n^{\circ} 5$.
(6) Pl. III, fig. $82, \mathrm{n}^{\circ}$ i
() Pl. IV, fig. 91, n० 4,6 et 5 .
(8) Pl. IV, fig. $101, n^{\circ} 4,5,16$ et $1 \%$
(9) Pl. IV, fig. 105, $\mathrm{n}^{\circ} 2,8,9,1.0$ et' 5.
(10) Pl. IV, fig. $9^{\circ}, n^{\circ} 3$.
(ii) Pl. IV, fig. $100, \mathrm{n}^{\circ} 4$.
(12) Pl. IV, fig. 102, $n^{\circ}$ 亿.
(13) Pl. II, fig. 33, n ${ }^{\circ}$.
(4) PI. I, fig. $6, \mathrm{n}^{\circ} 8$. 
de l'incubation; elle est formée par des stries alternatives et parallèles de ces deux substances (1): les stries grises sont la continuation de la matière grise des lobes, les blanches font suite à leur matière fibreuse. La force et l'étendue de ces fibres sont proportionnelles à l'étendue de cette voûte et des lobes, ainsi qu'on peut le remarquer chez l'hirondelle (2), le roitelet (3), la bondrće (4), le perroquet (5) et le casoar (6). La formation des stries blanches n'a souvent lieu que quelque temps après la naissance.

Le nombre des stries blanchấtres varie donc chez les divers oiseaux : j'en ai compté quatre chez le roitelet, le rouge-gorge, le serin, le chardonnerct, le pinson; cinq chez la pie, chez le pigeon, les perdrix rouge et grise; six chez la poule, les faisans doré et argenté, l'orfraie, la bondrée, le milan, l'épervier: sept chez le canard ordinaire, le canard musqué, l'oie, le dindon, les cigognes blanche et noire, et huit chez le casoar et l'autruche. Ce nombre est du reste sujet à varier chez les individus de la même espèce. Ces fibres deviennent d'autant plus épaisses et plus solides

(1) PI. III, fig. 83, n" 6 .

(2) Pl. IV , fig. $100, n^{\circ} 4$.

(3) Pl. IV', fig. $102,1^{\circ} 4$.

(4) Pl. IV, fig. $90, n^{\circ} 5$.

(5) PI. III, fig. 8f, "1" (i).

(i) Pl. III, fig. 85, $\mathrm{H}^{\circ} \mathrm{6}$. 
qu'on les considère plus en arrière et plus en dehors vers les parois dés lobes.

Les tubercules quadrijumeaux des oiseaux recouvrent donc l'aqueduc de Sylvius, puis ils se portent sur les côtés des cuisses cérébrales (1), qu'ils embrassent, et avec lesquelles ils contractent en avant une adhérence qu'il faut détruire (2) pour découvrir les pyramides antéricures (3). Chez. certains oiseaux, ils se renflent de nouveau après avoir ainsi adhéré aux pédoncules (4); de iclle sorte que, considéré par sa base, l'encéphale de ces oiscaux, comme celui du casoar (5) et de l'autruche $(6)$, offre quatre lobules quadrijumeaux, deux antérieurs, sur les côtés de la jonction des nerfs opliques (7), et deux postérieurs, dont le milieu correspond au niveau de l'insertion de la troisième paire (8). Cés lobes sont séparés par une rainure, qui correspond au point où lcurs parois adhèrent aux pédoncules cérébraux; plus la paire antérieurc est léveloppéc, plus la rainure esı profonde: lorsque ces lobes s'affaisscnt la

(1) II. I1I, fig. 84, n"8.

(2) PI. III, fig. $82, n^{\circ} 5$.

(5) PI. III, fig. $\delta_{2}, n^{n ?}$

(4) PI. III, fig. $79, n^{\circ} 0$

(5) PI. III, fig. 79 .

(6) PI. IV, fig. $9^{8}$

(7) Pl. III, fi.

(8) Pl. III, fig. 79.11 is at 1 . 
rainure disparaît. On voit le commencement de cet effet sur la cigogne blanche (1).

'Tels sont les rapports naturels des lobes optiques des oiseaux avec ceux des reptiles et les tu bercules quadrijumcaux des mammifères: cependant, avant MM. Gall et Cuvier, tous les anatomistes avaient considéré ces lobes comme les analogues des couches optiques de l'homme et des mammifères. Les deux anatomistes que nous venons de citer fondèrent principalement leur détermination sur l'insertion des nerfs optiques, qui, chez les oiseaux, se continuent en totalité avec ces lobes, et en partie, chez les mammifères, avec les tubercules quadrijumeaux antérieurs : je crois l'avoir. démontré anatomiquement, par la comparaison de ces lobes chez les embryons des diverses classes, et par les métamorphoses qu'ils éprouvent pendant l'incubation, afin de répondre par des faits aux objections récentes du célèbre Treviranus.

Dans l'examen de cette détermination et do l'analogie primitive des lobes jumeaux des mammiferes et des lobes optiques des oiseaux, Treviranus a fait une omission importante : celle de comparer ces organes aux époques correspondantes le la formation des embryons des deux classes. Celte omission l'a conduit à signaler des différences qui ue sont que le produit des divers degrés de eléxelopuenent, et a domier des cxplica-

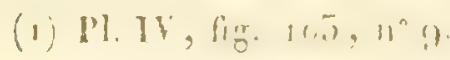


lions qui sont détruites par l'examen de la formation de l'encéphale pendant l'incubation.

Je ne m'arrêterai pas à la position différente des lobes optiques des oiseaux adultes; j’ai expliqué cette mutation, en montrant que primitivement ils occupent la même place que chez les reptiles, les poissons et les mammifères. Treviranus explique cette différence et l'affaissement des lobes optiques, par la faiblesse des cuisses cérébrales chez les oiseaux; mais cette explication est détruite par la considération de l'encéphale chez les embryons de cette classe; car jusqu'au douzième jour de l'incubation, les lobes optiques sont sur la face supérieure de l'encéphale, quoique les cuisses cérébrales soient plus faibles que chez les oiseaux adultes; bien plus, le déplacement des lobes optiques s'opère à l'époque même où les cuisses augmentent de volume.

Considérant néanmoins que l'enveloppe des lobes jumcaux de l'embryon des mammifères est formée par une lame mince, Treviranus ajoute que cette considération l'a conduit à rechercher les tubercules quadrijumeaux des oiseaux dans une lame semblable : cette analogie n'est pas heureuse, car Is degré d'épaisseur d'un organe ne peut jamais, ce me semble, servir de base de détermination. Quoi qu'il en soit, la bande transverse qui joint les deux lobes sur la partie moyenne (1) lui fournit

(i) Pl. IHI, fig. 85, $\mathrm{n}^{\circ} 6 ;$ fig. $84, \mathrm{~A}^{n}=$ 
une partie de ces tubercules (1). Une objection insurmontable s'offred'abord à cette première partic de la détermination de Treviranus. Nous avons vu, en effet, que les tubercules quadrijumeaux sont les premières parties qui se forment dans l'encéphale des embryons : or, celte lame transverse, de même que toules les commissures, ne se développe que très-tard; elle n'existe pas les troisième (2), quatrième (3), sixième (4) et huitième (5) jours de l'incubation (6), elle ne se forme que lorsque les lobes s'écartent, les dixiène (7), douzième, quatorzième (8) et dix-huitième

(1) Haller hésita beaucoup dans la détermination des tubercules quadrijumeaux des oiseaux. Nous avors déjà vu qu'il trouvait ces corps sur les côtés de la scissure de Sylvius, en remarquant toutefois que ces proéminences ne présentaient pas une élévation arrondie. En parlant du voile médullaire ou de la commissure des lobes optiques, il ajoute: Cette éminonce, qui néanmoins n'a pas une élévation do figure déterminée, est d'ailleurs analogue à l'eminence quadrijumelle de $W$ inslow. Ainsi voilà deux opinions très-distinctes émises par Hiller sur les tubercules quadrijumeaux des oiseaux. De ces deux opinions, Treviranus n'en a fait qu'unc, sans se rappeler peut-être les idées de Haller.

(2) Pl. I, fig. $5, n^{\circ} \mathrm{J}$

(3) PI. 1, fig. 4, $n^{\circ}$ \%

(4) PI. I, fig. .5, $n^{\circ} 6$.

(5) PI. II, fig. 55, n ${ }^{\circ}=$

(6) PI. I, fig. $6, n^{\circ} 8$.

(z) Pi. I, lig. $z+17^{\prime}=$.

-(8) P!. I, fig. $8, n^{n}:$. 
jours (1) : encore chez un très-grand nombre d'oiseaux, ct surtout chez la pic, le geai, la buse, les strics blanches ne paraissent que dans le premier mois de la naissance. Que sont jusquà cette époque les tubercules quadrijumeaux des oiseaux? Il remarque cnsuite que Haller et Malacarne ont observé au-dessous de celle bande, sur les côlés de la scissure de Sylvius (2), et en haut du quatrième ventricule (3), une paire de renflemens gris, qu'ils ont considérés i tort comme les tubercules quadrijumeaux en lotalité, tandis que, selor lui, ce ne serait quo les restes de la base de ces tubercules, dont il faut chercher la continuation dans la lame transverse. En lisant celte opinion, on est surpris qu'clle ait pu séduire un aussi bon observateur que Treviranus ; quil ait cherché $a$ former ur même organe de parties si dissimilaires, si élrangères les unes aux autres; qu'il n'ait pas vu enfiu que la lim: transverse n'est autre chose quo la commissure des lobes optiques, analoguc à la lame qui réunit les tubercules quacírijumeaux des mammifères.

Cela posé, l'auteur en vient à une nouvelle di:termination éces lobes opliques; il propose à leur égard une modification semblable a colle quil

(1) PI. II, 6.g. $79 \cdot n^{n} 8$.

(a) PI. III, fig. 8, $11^{\circ} 1 \%$

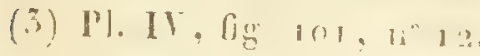


vient de faire à l'ancienne opinion sur les tubercules quadrijumeaux. Haller, Malacarne, avaient vu ces tubercules dans les renflemens grisâtres des côtés de la scissure de Sylvius; Treviranus ne leur accorde que la moilié de cetie destination : ces anatomistes avaient assimilé ces lobes à toule la couche optique; d'après le même système, l'analomiste allemand ne voit on eux que la moitié postérieure de cette couche des mammifères qui se serait affaissée chez les oiseaux. Il y a beaucoup de sagacité dans cette observation et dans ce rapprochement, mais il me paraît hors de toute vraisemblance : d'abord, ce morcellement des tubercules quadrijumeaux et de la couche oplique, cette séparation des parties qui les forment chez les mammifères, n'est qu'une supposition; aucun fait ne l'atteste, rien ne prouve même sa possibilité dans l'encéphale des embryons : en second licu, à aucune époque de la formation du cerveau, on ne trouve dans aucune classe que la partie postérieure de la couche optique des mammifères où les corps géniculés soient creux comme les lobes opliques. Comment se creuseraient-ils chez les oiseaux? autre proposition a démontrer avant d'admettre cette analogie. En iroisième licu (1), chez tous les vertébrés sans exception (2), la couche optique (3) ne se déve-
(1) Pl. I, fig. $5, n^{\circ} \%$
(2) Pl. I, fig. 15, $\mathrm{n}^{3} \mathrm{G}$.
(3) Pl. II, fig. 66, n"7. 
loppe que très-long-temps après les lobes optiques (1) ou les tubercules quadrijumeaux (2) : ce principe est constant chez les oiseaux (3), chez les reptiles (4), chezles mammifères (5) et l'honme(6). Comment accorderait-on ce fait général avec l'hypothèse qu'on propose? En quatrième lieu, les corps géniculés se montrent très-tard chez l'embryon humain; chez ceux du veau, du cheval, du mouton, du lapin, c'est l'une des dernières parties qui se développent. Les lobes optiques, au contraire, ou ce qu'on présume être chez les oiseaux les corps géniculés, sont les premières qui se forment après la moelle épinière. Comment accorderait-on cette opposition ou cette génération inverse chez les mammifères et les oiseaux? Enfin, si les lobes optiques des oiseaux étaient les corps géniculés des mammifères, leur commissure devrait être l'analogue de la commissure postérieure des couches optiques : mais cette dernière commissure existe chez tous les oiseaux au-dessous de la partie antérieure de la commissure des lobes optiques (7); elle n'a pas pu être méconnue, conséquemment elle n'a pas été déplacéc. Haller et Malacarne considéraient la commissure des

(1) Pl. I, fig. $5, n^{\circ} 7$.

(2) Pl. I, fig. $27, n^{\circ} 4$.

(3) Pl. I, fig. 5, $n^{\circ}$.

(4) Pl. I, fig. $9, n^{\circ} 5$.

(5) Pl: I, fig. $19, n^{\circ} 4$.

(6) Pl. : , fig. $24, n^{\circ} 4$.

(7) Pl. 1II, fig. $8 \%, n^{\circ} 5$. 
lobes optiques comme une partic spéciale des oiseaux, à laquelle ils donnaient le nom de voile médullaire, sans se demander comment la couche optique avait pu être transposée sans entraîner avec elle leurs commissures, et surtout la postérieure. La même objection est applicable à l'opinion de Treviranus. Comment la moitié postérieure de la couche optique se serait-elle portée en arrière en laissant en place la commissure postérieure? Tout est à prouver dans cette hypothèse : la forme, la position, le développement, la structure des lobes optiques, et même leurs rapports.

Remarquez, en effet, que si les lobes optiques étaient les corps géniculés des mammifères, la quatrième paire de nerfs naîtrait immédiatement derrière eux (1); le cervelet n'en serait séparé que par cette insertion (2); toute la partie moyenne de l'encéphale des oiseaux serait, pour ainsi dire, transposée. Observez aussi que les tubercules quadrijumeaux en totalité, d'après les anciens anatomistes, ou en partie, d'après 'Treviranus, seraient venus se loger dans l'intérieur des corps géniculés internes; de plus, la lame transverse des lobes optiques (5), interposée entre ces prétendus corps géniculés, devrait correspondre à la partie postérieure de la voûte à trois piliers, ses

(1) PI. III, fig. 84, nn 7 .

(2) PI. III, fig. $84, n^{n} 2$.

(5) Pl. III, fig. 83, in $^{\prime \prime}$. 
stries de matière blanche aux cordes de la lyre: ce sont des conséquences qu'il faudrait nécessairement accepter si l'on adoptait cette modification. Or, dans ce cas, les lobes optiques seraient plutòt l'analogue du lobe de l'hypocampe et de la corne d'Ammon, que des corps géniculés. Treviranus, pour éviter cette erreur, a morcelé lẹs tubercules quadrijumeaux : il sait, en effet, que l'un des caractères de ces corps est de former une espèce de voûte sur l'aqueduc de Sylvius : or, dans l'hypothèse des anciens anatomistes, leurs tubercules n'ofrraicnt pas ce caractère; telle me paraît être la raison pour laquelle cet anatomiste célèbre considère la lams transverse des lobes optiques comme une partie détachée des tubercules quadrijumeaux des oiseaux; teilles sont les objections insurmontables que présenterait cette détermination chez les oiseaux. En voici d'une autre force pour les reptiles. Dans cette classe, les lobes optiques conservent leur position sur la face supérieure de l'eiscéphale (1), de même que chez les oiseaux, jusqu'aux sixième (2), huitième (5) et neuvième jours de l'incubation (4). Les reptiles se distinguent donc des oiseaux par l'absence dc la lame transverse des lobes optiques (5); ils s'en

(1) Pl. V, fig. $119, n^{\circ} \%$

(2) Pl. I, fig. 5, $n^{\circ} 6$.

(3) PI. II, fig. 33, n० \%.

(4) Pl. I, fig. 6, $\mathrm{n}^{\circ} 8$.

(5) PI. HI, $14.84,1^{\circ} 6$. 
dislinguent aussi par l'absence des renflemens sur les còtés de la scissure de Sylvius (1). Il n'y a donc plus de tubcrculcs quadrijumeaux dans cetle classe. Treviranus ne s'explique pas à ce sujet, ce fait renversait son hypothèse; mais il considère les lobes optiques comme les corps géniculés. Cette détermination paraîtra moins vraisemblable encore que chez les oiseaux, si l'on fait attention ¿ la formation précoce de ces lobes chez le tétard (2), à l'apparition tardive de la couche optique $(\bar{\jmath})$, à son peu de dércloppement chez les ophidiens (4), les lacertiens (5), les sauriens (6), les cheloniens $(7)$, comparé au volume des lobes optiques de l'orvet ( 8 ), des vipères (9), de la grenouille (10), des crocodiles (11) et des tortues (12). Enfin, l'auteur abandonnant chez les poissons cette détermination, ne voit plus dans les lobes optiques de cotte classe la partie postérieure des couches

(1) Pl. V, fig $154, n^{\circ} 5$.

(i) Pl. I, fig. $9, \mathrm{n}^{\circ} 5$.

(3) Pl. I, fg. i5, $n^{\circ} 6$.

(4) Pi. V, fig. $126, n^{\circ} 6$.

(5) PI. V, fig. 100, $\mathrm{n}^{\circ} 10$

(6) Pl. V, fig. 111, $n^{\circ} 8$.

(5) PleV, fig. $121, n^{\circ} 5$.

(8) Pl. V, fig. $109^{\prime}, n^{n} 3$.

(9) Pl. V, fig. $126, n^{\circ} 5$.

(10) Pl. I, fig. $16, \mathrm{n}^{\circ} 3$

(11) Pl. V, fig. $115, n^{\circ} 4$.

(12) Pl. v, fig. 120, n० 5 .

IJ. 
optiques, mais bien les hrinisphires cérébraux des marnmifères et des oiseanx.

Si les. lobes optiques des oiseaux ne sont et ne peuvent être ni la couche optique des mammifères en totalité, comme le pense encore le professeur Rolando, ni la moitié de cette couche, comme le croit Trériranus, à plus forle raison ne pourrait-on les assimiler aux hémisphèrès cérébraux de la classe supéricure, comme l'a proposé M. de Blainville (1). Comment en effet les hémisphères cérébraux, qui sont toujours en avant des corps striés, de la couche oplique, des commissures antérieures el postérieures, seraient-ils passés chez les oiseaux, cn arrière de ces commissures, de la couche optique et du corps strié? Si le ventricule des lobes optiques était l'analogue des ventricules latéraux des mammifères, comment les tubercules quadrijumeaux seraient-ils venus s'y loger, tandis que toutes les parties qu'ils renferment ordinairement en auraient été exclues? Comment le cerrelet, qui, toujours chez les mammières supérieurs, est recouvert par les lobes cérébraux, serait-il au contraire, chez les oiseaux, superposé sur ces lobes et sur leur grande commissure? Rien dans l'encéphale mème des oiscaux ne pourrait jusificr un semblable déplacenent.

(1) Butletin des Scicnces, par la Société Philomalique, $n^{\circ}$. de mars 182.1 , pag. $5 z$. 
J'ai suffisamment établi, je pense, que les lobes optiques des oiseaux et des reptiles ne sont ni les corps géniculés des mammifères, ni leurs hémisphères cérébraux. J'ai aussi prouvé qu'on ne pouvait voir les analogues des tubercules quadrijumeaux dans les renflemens grisâtres des côtés de la scissure de Sylvius, et moins encore dans la commissure transverse des lobes optiques, puisque les renflemens de cette lame manquent chez les reptiles : j'ai démontré, d'un autre côté, que les lobes optiques sont les analogues des tubercules quadrijumeaux des mammifères et de l'homme. L'examen des tubercules chez ces derniers ne laissera, je l'espère, aucun doute dans l'esprit des anatomistes sur la valeur de cette détermination.

Accoutumés, comme nous le sommes, à voir dans les tubercules quadrijumeaux de l'homme et des mammifères adultes une masse renflée, solide, diviséc en quatre tubercules symétriques, l'esprit est peu disposé a leur trouver de l'analogie, soit avec les lobes optiques des oiscaux, soit avec ceux des reptiles. D'une part, il n'y a dans ces deux classes qu'un lobe de chaque côté, et tous les mammifieres ont quatre tubercules. De l'autre, les lobes sont creux, et les tubercules sont solides; enfin, chcz les oiscaux ils sont toutà-fait déplacés. Que de différence entre ces organcs! que de raisons pour écarter toute analogic entre cux! mais si, comuc nous l'avous prouvé 
dans les lois de l'organoginie, l'embryon des mammilères répète si fréquemment les formes organiques des classes inféricures, qui ne voit que nous devions cheriher chez lui los ressemblances qui s'elfacent chez los animaux adultes? Cette idée, vaguement conçne par plusicurs anatomistes, est devenue l'une des bases de la théorie des ana. - $\operatorname{logues}$ de M. le professeur Geoffroy-Saint-Hilaire. Les tubercules quadrijumeaux des mammifères vont nous cú présenter dans leurs transformations une belle application:

Wriberg, Haller, Blasius, Prochaska, Meckel, les frères Wenzel, Arsaki, avaicnt entreru la différence deces corps chez les embryons; Tiedemann et moi nous en avous suivi en même temps toutes les métamorphoses, Tiedemann, chez l'embryon humain seulement, et moi, sur les embryons de différentes familles; les lois de la zoogénie que je cherche à établir, m'cn imposaicnt l'obligation. De même que les mútamorphoses des lobes optiques des oiseaux nous les ontmontrés situés comme les tubercules quadrijumeaux des mammiferes, à une époque de leur développenent, de même chez les enbryons des mammiféres, nous allous trouver ces corps lobulaires et creux, ainsi que cela existe chez les oiseaux ct les reptiles. Ce double caractère des tubercules des mammiferes existe vers le quart de leur formalion, et se conserve plus ou moins long-temps, sclou la durec de la gestation. Cousidérez l'embryon du mouton de la fin de 
la quatrieme semaine (1), celui du veau de la cinquième (2), celui de l'homme du commencement de la sixième (5), celui du lapin du dixième ct douzième jour, celui du cochon-d'inde au quatorzième, celui du jeune embryon des didelphes (did. virg.) (4), vous verrez que leurs tubercules quadrijumeaux sont alors deux lobes jumeaux (5), situés sur la face supérieure de l'encéphale, de même que chez les embryons des oiseaux (6) et chez les reptiles à toutes les époques de leur vie (7). Ces lobes sont très-allongés chez le mouton (8), plus larges chez le veau (9), plus arrondis chez le didelphe (10), un peu déprimés chez l'embryon humain (11). Ce caractère lobulaire se conserve très-long-temps chez le mouton (12), le veau (15), le singe ouistiti (14). Chez
(1) Pl. I, fig. 19, $n^{\circ} 4$.
(2) Pl. I, fig. 2\%, $n^{\circ} 4$.
(3) Pl. I, fig. $24, n^{\circ} 4$.
(4) Pl. I, fig. $30, n^{\circ} 4$.
(5) Pl. I, fig. $21, \mathrm{n}^{\circ} 8$,
(6) Pl. I, fig. $5, \mathrm{n}^{\circ} 6$.
(7) Pl. I, fig. $16, \mathrm{n}^{n} 2$.
(8) Pl. I, fig. $19, n^{n} 4$.
(9) Pl. I, fig. $27, n^{\circ} 4$.
(10) Pl. I, fig. $30, \mathrm{n}^{\circ} 4$.
(11) Pl. I, fig. $24, n^{\circ} 4$.
(12) Pl. II, fig. 47, $n^{\circ} 7$.
(13) Pl. II, fig. 4! , n²
(14) PI, II, fig. fis, no 8 , 
l'embryon des didelphes ils deviennent pyriformes (1), puis arrondis (2); la forme lobulaire persiste, chez le lapin, jusqu'au vingtième jour (3); chez le chien, jusqu'au quarantième; chez le chat, jusqu'au trentième. Chez l'homme, les tubercules quadrijumcaux sont deux lobes optiques, les deuxième (4), troisième (5), quatrième (6) et cinquième mois de la vie utérine (7). Voilà donc les tubercules quadrijumeaux des mammifères ramenés à la forme lobulaire des oiseaux et des reptiles. Pendant le temps que les tubercules quadrijumeaux sont deux lobes jumeaux, ils offrent une cavité à leur intérieur, de même que chez les oiseaux, et un petit renflement grisâtre sur les côtés de la scissure de Sylvius. Cette cavité est très-vaste chez l'homme pendant le cours du troisième mois (8), chez les didelphes jusqu'aux trois quarts de l'incubation (9). Elle est d'autant plus grande qu'on l'ob-

(1) Pl. II, fig. $58, \mathrm{n}^{\circ}$ \%.

(2) Pl. II, fig. $49, \mathrm{n}^{\circ} 6$.

(3) Pl. II, fig. 55, $\mathrm{n}^{\bullet} 6$.

(4) PI. II, fig. 63, $\mathrm{n}^{\circ} 4$.

(5) Pl. II, fig. $65, \mathrm{n} \cdot 5$.

(6) Pl. II, fig. 58, $\mathrm{n}^{\circ} 3$.

(7) Pl. II, fig. $75, n^{\circ} 4$.

(8) P1. II, fig. $66, \mathrm{n}^{\circ} 5$.

(g) Pl. II , fg. 62 , nº 2. 
serve chez les embryons de l'homme, des singes, des pachydermes, des ruminans et des rongeurs. Chez les embryons des chauve-souris, la forme lobulaire des tubercules et des cavités persiste beaucoup plus long-temps que chez tous les autres manmiféres. Si les tubercules quadrijumcaux restaient dans cet ćlat, ils seraient donc scmblables aux lobes optiques des oiseaux et des reptiles. C'est le cas de certains embryons monstrueux, notamment des foctus monocles; leurs tubercules quadrijumeaux sont des lobes optiques jusqu'à la naissance, et au-delà, si l'animal offre les conditions organiques nécessaires à la vie extérieure. C'est aussi le cas des embryons monocéphales octopèdes; ils ont deux cervelets, et les tubercules quadrijumcaux sont deux lobes volumineux. J'ai vérifié ce fait chez l'homme, le veau, le lièrre et le mouton; M: le professeur GeoffroySaint-Hilaire l'a constalé sur plusieurs autres ( 1 ); ct il a parfaitement bien observé que dans ces cas les tubercules quadrijumeaux offrent l'état embryonnaire permanent des manmifères, et la forme permanente des classes inférieures.

Mais les lobes jumeaux des mammifères doivent devenir quadrijumeaux; de creux qu'ils étaient, ils doivent devenir solides; leur forme ct leur grandeur doivent varier selon les familles. Quelle est

(1) Voyez Philosephic analomique, tom. II. 
la cause de cette transformation? comment s'opère-t-elle? telle est la question que nous allons examiner maintenant.

On se rappelle que le canal épinien, si large dans les premières époques de l'embryon, se rétrécit, et enfin s'oblitère par la déposition intéricure de couches de matière grise; on se rappelle également que les tubercules quadrijumeaux ne sont que le bulbe de terminaison de la moelle épinière, et qu'ils ont le même mode de formation: nous compléterons cette analogie en faisant remarquer qu'ils se solidifient de la même manière. C'est, en effet, par la déposition de la matière grise dans les parois de la cavilé de ces tubercules, que cette cavité se rétrécit, puis s'oblitère. Cette oblitération a ordinairement lieu chez le mouton, - vers le quatrième mois; chezle lapin, du vingtième au vingt-cinquième jour; chez le chien, au quarantième; chez: le chat, au trentième ou trentecinquième; chez le veau et le cheval, elle ne s'effectue le plus souvent qu'à la naissance; chez l'embryon humain la cavité disparaît du quãtrième au cinquième, et au plus tard au sixième mois de l'cmbryon.

Voilà comment s'oblitère a cavité interue des tubercules quadrijumeaux des mammifères; voici comment ils se divisent :

Pendant tout le temps que les tubercules quadrijumeaux sont creux, leur surface extcrienre 
est lisse ; ¿l l'époque où ils se solidifient, un sillon transversal apparaît sur leur superficie : l'cffet de ce sillon est de diviser la masse tuberculairc en deux parties de chaque côté, l'une antérieure, l'autre postéricure; chaque tubercule devient double, de simple qu'il était précédemment : il y a alors quatre tubercules au lieu de deux. La présence et la permanence de ce sillon transversal sont donc le caractère classique des tubercules quadrijumeaux des mammifères.

Chez l'homme, il se dévelop pe vers la fin du cinquième mois ou au commencement du sixième(1); au septième il est toujours très-prononcé (2). Chez le chien, il est visible au trente-cinquième jour; chez le chat, au vingt-huitième on au vingt-neuvième; chez le lapin, il se montre du vingtième (5) au vingt-cinquième (4);-chez le veau et le cheval, au milieu du sixième mois; chez le mouton au quatrième.

De la position que ce sillon occupe en avant, au milieu et en arrière de la masse tuberculaire, dérivent le caractère parliculier de certaines familles de cette classe, et les variétés qui par-

(1) PI. IT, fig. 6g, $n^{\circ} \mathbf{3}$

(2) Pl. II, fig. $7 \mathbf{I}, 11^{\circ} 5$.

(3) Pl. II , fig. $55, n^{\circ} 6$.

(4) PI. II, fig. $56, n^{\circ} 5$. 
fois se manifestent dans les espèces d'unc même famille.

Chezl'homme il occupe ordinairement la partic moyenne (1); les tubercules antéricurs (2) sont égaux aux postérieurs (5). Chez les singes (4) il se porte un peu en arrière; les antéricurs (5) prédominent légèrement sur les postérieurs (6). Chez les cétacés il se replace sur la partie moyenne $(\tau)$, ce qui rétablit l'équilibre, comme chez l'homme, entre les tubercules de devant (8) et ceux de derrière (9). Chez les carnassiers, le sillon se porte un peu en avant; les lobes postérieurs sont plus grands que les antérieurs; chez le raton néanmoins il se reporte un peu en arrière (10), de même que chez les ours, ce qui fait prédominer de nouveau les tubercules antérieurs (11) et affaiblit les postírieurs (12). Il en est de même chez les coatis (15):

(1) Pl. II, fig. $71, n^{\circ} 5$.

(2) PI. II, fig. $71, n^{\circ} 6$.

(3) Pl. II, fig. $71, x^{\circ} \%$

(4) Pl. XII, fig. $241, n^{\circ} 6$ el 7 .

(5) Pl. XII, fig. $241, n^{\circ} \%$

(6) PI. XII, fig. $241, n^{\circ} 5$.

(z) Pl. XI, fig. 225.

(8) Pl. XI, fig. 225, F, G.

(9) Pl. XI, âg. 225, E.

(10) PI. VIII, fig. 201, n: 5 et 6 .

(11) Pl. VIII, fig. $201, \mathrm{n}^{\circ} 6$.

(12) Pl. VIII, fig. 201, $n^{\circ} 5$.

(13) Pl. XII, fig. $2 / 2, n^{\circ} 6$. 
chez les pachydermes et les ruminans, les antérieurs sont beaucoup plus forts que les postérieurs, comme on le voit chez la gazelle (1). Cette disposition est la même chez les rongeurs, ainsi qu'on l'observe sur l'agouti (2).

Remarquons toutefois que les rapports des tubercules quadrijumeaux entre eux sont trèsvariables, comme l'a déjà observé Treviranus : ainsi les antérieurs sont égaux aux postéricurs chez la mangouste (5) et chez la taupe (4); chez le zemni, les antérieurs prédominent sur les postérieurs (5); chez le renne, cette disposition est plus marquée que chez tous les ruminans; chez l'homme aussi, il arrive parfois que les postérieurs sont plus faibles que les antérieurs. Un effet asśez constant se remarque néanmoins dans toutes ces variations : c'est que presque toujours chez l'homme et les carnassiers, quand les tubercules perdent leur caractère de famille, la prédominance porte sur les tubercules antérieurs (6).

Le rapport des tubercules quadrijumeaux entre eux n'est donc pas soumis à une loi constante.

(1) Pl. XV, Gig. 271, n* 8 et 9 .

(2) Pl. IX, fig. $212, n^{\circ} 8$ et 9.

(3) Pl. XI, fig. $232, n^{\circ} 1$ et 2 .

(4) Pl. XV, fig. $291, n^{\circ} 1$ et 2.

(5) Pl. XV, fig. 292, $n^{\circ} 4$.

(6) PI. XIII, fig. 244, $\mathrm{n}^{\circ}$ / 5 ; pl. XII, fig. 243, n०6 et :; fig. $242, n^{\circ} 5,6$ et 5 . 
Leurs limites respectives ne pouvant toujours être précisćes avec rigueur, leurs connexions avec les partics environnantes variant quelquefois d'espèce à cspèce, il en résulte des modifications individuelles, qui rapprochent souvent des êtres situés aux deux cxtrémités de l'échelle des mammiferes. Ainsi, chez les rats, les souris, le hérisson, la masse des deux tubercules se rapproche beaucoup de la forme de celle de l'homme. Chez la taupe et les chauve-souris, l'étendue de leur diamètre transversal dépasse celui du diamètre longitudi nal, comme on le remarque chez le dauphin. Ces variations continuelles rendent raison du peu d'accord qu'il y a encore sur ce sujet entre MM. Ridley, Carus, Gall, Spurzheim, Cuvier, Treviranus.

En parlant des lobes optiques des poissons dans la Première Partie, j'ai montré ce qu'ils étáient; j'ai négligé toutes les suppositions que l'on a imaginées pour les assimiler aux hémisphères cérébraux : il nous reste maintenant à juger ces suppositions, et à faire connaître les principales variétés de forme et de structure que présentent ces corps chez les poissons osseux et cartilagineux.

Chez les oiseaux, les tubercules quadrijumeaux étaient pris pour la couche optique, parce queces hémisphères cérébraux sont méconnaissables dans cette classe; mais si ces hémisphères cusscnt étí atrophiés et déformés, comme chez les poissons; si dans leurs lobes optiques on rut rencontré des 
tubercules en forme de croissant, comme sont les corps striés chez les mammifères; si, de même: que chez ces derniers, ces tubercules avaient étć composés de matière grise et blanche; si les rayonnemens de la matière blanche avaient divergé du centre de ces tubercules à la périphéric des lobes, nul doute que l'on n'eût trouvé dans les tubercules quadrijumeaux des oiseaux les hémisphères cérébraux des nammifères.

Ce que l'ou n'avait pas fait jusqu'à ces derniers tempschez les oiseaux, a été tenté pour les poissons, chez lesquels les hémisphères cérébraux sont rudimentaires, et les lobes optiques portés à leur maximum de complication par une opposition trèssingulière. Ce sont donc ces complications qui doivent mainteuant fixer notre attention.

La forme générale des lobes optiques des poissons est celle d'un ovale, plus ou moins allongé chéz les cartilagineux (1), et plus ou moins sphérique chez les osseux (2); chez lous ils sont légèrement déprimés ca dedans à la partie par laquelle ils se correspondent. Chez la raie ronce (5) de même que chez le requin (4) el l'aiguillat (5), ils sont moins larges en arrìre qu'en avant, et un

(1) PI. VI, fig. $139, \mathrm{ni}^{\circ} 3$.

(2) Pl. VII, fig. $168, \mathrm{n}^{\circ} 4$.

(3) Pl. VI, fig. $139, n^{\circ} 3$.

(4) Pl. Vi, fig. 1/1, $n^{\circ} 6$.

(5) Pl. XII, fig. $236, \mathbf{E}$. 
peı aplatis à leur tiers postérieur, ce qui paraît dépendre, comme chez le tupinambis, de la juxtà-position d'une partie du cervelet. Cette dépression et cettc diminution des lobes en arrière sont surtout remarquables chez le squale glauque, dont le cervelet est en grande partic remonté sur ces corps. Chez l'esturgeon ( $\mathrm{r}$ ) ils sont au contraire beaucoup plus larges en arrière qu'en avant. Chez l'ange (2) ils sont sphériques, bombés, surtout i leur partie moyenne, et un peu déprimés aussi cn arrière par le cervelet; chez la torpille et la larnproie (5) ils sont arrondis et déprimés en dedans.

Chez les poissons osseux, ils se rapprochent beaucoup plus de la forme sphérique, comme on le voit chez le gronau (4), la morue (5), le congre (6), le brochet (7), l'égrefin (8), la baudroie (.9), la tanche (10), le merlan, l'espadon. Chez la carpe ils sont sphériques (11), offrent au milieu une dépression, en arrière deux

(1) Pl. XII, fig. 255.

(2) Pl. XII, fig. $257, E$.

(3) PI. Xr, fig. $228, n^{\circ} 3$

(4) Pl. VII, fig. $155, n^{\circ} 6$ ct 9 .

(5) Pl. VII, fig. $163, n^{\circ} z$.

(6) Pl. V.II, fig. $168, n^{\circ} 4$.

(a) Pl. VII, fig. $1\left(39, n^{\circ} 5\right.$.

(8) Pl. VII, fig. $177, n^{\circ} 3$.

(9) Pl. VII, fir $159, n^{\circ} 4$.

(10) Pl. VII, fig. $19^{1}, n^{0} 5$.

(11) Pl. VI, fig. $145, n^{\circ} 5$. 
petits tubercules, et en avant deux bandelettes médullaires qui s'élèvent de la dépression centrale.

Leur partie postérieure offre, chez certains osseux, des modifications toujours produites par le cervelet: en effet, lorsque chez les cartilagineux unc partie du cerveles surmonte les lobes optiques, ils sont amincis et déprimés en arrière. Chez aucun poisson osseux, le cervelet ne surgit au-dessus des lobes optiques; mais comme ses pédoncules supérieurs sortent de l'intérieur de ces lobes, ceux-ci sont ćcartés l'un de l'autre en arrière, d'une étendue proporlionnelle au volume de ces pédoncules. Cet écartement, déjà sensible chez le turbot (1); la mustelle commune, l'espadon, le sparus raii, beaucoup plus prononcé chez l'égrefin (2), les mulles (M. surmulettuis), le bogue commun, est porté à son plus haut degré çhez le sargue et le Caranx trachurus, chez lequel le cervelet se loge dans l'écartement de toute la longueur des lobes optiques.

On voit donc pourquoi les lobes opliques des poissons cárilagineux sont déprimés par le cervelet en arricere, et jamais ćcartés l'un de l'autre danscescns; pourquai au contraire, chez les osseux, ils sont souvent ćcartés postérieurement et jomais déprimés sur lcur face supérieurc. Cos elfels difierens dans ces deux grandes familles liennent

(1) Pl. VII, fig. 101.

(2) Pl. VII, lig. 1:- 
invariablement aux dispositions différentes que présente le cervelet.

Les lobes optiques, très-peu développés chez la lamproie, l'esturgeon, les anguilles, les silures, la torpille, vont en augmentant des raies aux squales et aux poissons osseux ( I ). Cet accroissement devient, surtout sensible, quand on compare la masse de ces corps aux autres parties de l'encéphale. Ainsi, coustamment chez les cartilagineux, le volume des lobes optiques est dépassé par celui des hémisphères cérébraux et du cervelet; au contraire, chez les osseux, ce sont les lobes optiques qui dominent le cervclet ct les hémisphères cérébraux.

La membrane nerveuse qui forne l'enveloppe des lobes optiques est formée, chez les poissons, de trois côuches : deux blanches, une en dehors (2), lautre en dedans (5); et une couche grise, trèsmince, interposée entre elles (4). La couche blanche extérieure est la plus épaisse, elle est lisse et ne se dédouble pas; la couche blanche interne est striée (5); les stries qui la composent ne sont souvent qu'adossécs les unes aux autres, de telle sorte qu'elles peuvent être isolées tout au pourtour du tubercule auquel clles adhe-

(1) Pour le volume absolu, voyez le Tableau.

(2) Pl. VII, fig. $165, n^{\circ} \%$

(3) Pl. VII, fig. $165, n^{\circ} 8$.

(4) Pl. VII, fig. $165,1^{\circ}$ 10.

(5) Pl. VII, fig. $170, \mathrm{n}^{\circ} 3$. 
rent, et présenter ainsi des espéces de feuillets. Cette disposition, que Malpighi comparait à des tuyaux d'orgue, à cause des cannelures qui les séparent quand on les déplisse, est surtout visible chez les poissons osseux : elle est à peine distincte chez les cartilagineux, à cause de l'épaisseur moins grande des faisceaux striés. La couche grise est apparente entre l'espace qui sépare les faisceaux striés, son épaisseur est à celle de la blanche che" les cartilagineux : : $1: 4$, et chezles osseux $:: 1: 5$. Il existc en outre quelquefois une seconde couche grise placée intéricurement.

Cette enveloppe circonscrit une cavité lont la capacité est généralement proportionnelle au volume des lobes optiques, et qui, chez les jeunes poissons, se continue avec le canal du nerf optique, de même que chez les jeunes embryons des mammifères le canal du pédicule olfactif communique avec les ventricules latéraux.

C'est cette cavité que les anatomistes out considérée tantôt comme les grands ventricules des hémisphères cérébraux, tantôt comme des cavités creusées dans la couche optique, d'autres fois enfin, mais plus rarement, comme l'analogue de la cavité qui se rencontre dans le lobule olfactif de certains rongeurs. Je l'ai nommée ventricule des lobes optiques. Ce ventricule est unique chez tous les poissons cartilagineux; chez le silure (1) $\mathrm{ct}$

(1) Les deux silures électriques que j'il exanines avaient II. 
la torpille électriques, il est obliteré, de même que chez l'amphisbène. Les lobes optiques sont alors solides ainsi que les tubercules quadri-jumeaux des mammifères; sur le plus grand nombre des poissons osseux le ventricule est unique aussi; chez quelques-uns il est double. Il y a alors deux cavités cnchâssées l'une dans l'autre. Nous expliquerons bientôt la formation de ce second ventricule, dont nous retrouverons d'autres exemples dans le quatrième ventricule des poissons.

Le plancher du ventricule optique est formé par la scissure de Sylvius, divisé sur la ligne médiane par unc rainure (1), en deux parties symétriques; les bords de la scissure sont formés de chaque côté par les pédoncules cérébraux, sur le milieu ct dans la profondeur desquels on voit le passage des cordons antérieurs moyens de la moelle allongée (2); au-dessus, et un peu au côté externe, se trouvent les faisceaux olivaires (3), qu'il est souvent difficile d'isoler. C'est sur cette partie des pédoncules cérébraux que se développent, chez les poissons, des tubercules particuliers, dont on ne trouve que de faibles vestiges chez les reptiles

été rapportés d'Ëgypte par M. le professeur Geoffroy-SaintHilaire, et conservés longtemps dins l'alcool. Cette circonstance a peut-être influé sur l'oblitération du ventricule.

(1) Pl. VI, fig. 151.

(2) Pl. VII, fig. $166, n^{\circ} 2$; fig. $189, n^{\circ}$ x et 6 ; pl. VI, fig.' 136 .

(5) Pl. VI, fig. 14 $7, n^{\circ} 2$. 
et les oiseaux, et qui varient de tant de manières le plancher du ventricule optique des poissons.

Ces tubercules, que Haller nommait Tori, les comparant sans doute au relief du soubassement des colonnes, n'existent pas chez la lamproie, le silure électrique, ni la torpille : ils sont peu développés chez les rayes et les squales, comparés à ceux des poissons osseux; le peu de relief qu'ils font, les a fait méconnaître chez les premiers de ces poissons. On les voit néanmoins chez l'ange ct l'aiguillat : chez certaines rayes (1), ils forment un croissant dont la concavité regarde la scissurc de Sylvius, et la convexité la périphérie des lobes : de cette dernière partent des stries médullaires minces.

Les tori sont beaucoup plus évidens et beaucoup plus développés chez les osseux; ils sont doubles chez la plupart, et leur position permet de les distinguer en postérieurs (2) et antérieurs (3). Les postérieurs, peu développés chez les rayes (4), les pleuronectes (5), le congre (6), la caranx, la tanche(7) labaudroie (8), les anguilles,

(1) Pl. VI, fig. $152, n^{\circ} 6$.

(2) Pl. VII, fig. $181, n^{\circ} 3$.

(3) Pl. VII, fig. 181, . ${ }^{\circ} 4$.

(4) Pl. VI, fig. $152, n^{\circ} 5$.

(5) Pl. VII, fig. $175, \mathrm{n}^{\circ} 5$ bis.

(6) Pł. VII, fig. $167, \mathrm{n}^{\circ}$ \%。

(7) PI. VII, fig. $187, n^{\circ} 4$.

(8) Pl. VII, fig. $180, n^{\circ} 4$. 
les harengis, devicnnent très-saillans chez les truites, les saumons, la perche (1), le brochet (2), l'igrefin (3), la morue (4), le gronau (5), la carpe (6), les spares et le ruban.

Leur forme varie singulièrement chez ces divers poissons : chez la perche, le congre, la morue, l'égrefin, ce sont deux bulbes allougés servant en quelque sorte de pédoncule au cervelet, dont ils cmbrassent la partic supérieure chez le lophius piscatorius (7); chez le copola tonia, c'est un tubercule échancré en arrière: chè la tanche, le tubercule est pisiforme et sans échancrure (8); chez le merlan ils ont la forme d'un rein, dont la partie concave regarde la scissure de Sylvius (9); Cietle formest plus prononcée encore chez le sparus raii. Chez le brochet (10), chaque tubercule est divisć par un sillon dans sa partie moyenne; il y a alors quatre tubercules distincts en arriere du ventricule du lobe oplique: chez le gronau, les tori postérieurs sont pisióormes, leur grosse extrémité

(1) Pl. VI, fig. $145, \mathrm{n}^{\circ} 5$.

(2) PI. VII, lig. $1=0, n^{\circ} 4$.

(5) Pl. VII, fir. $181, n^{\circ} 5$.

(4) Pl. VII, fig. $165, \mathrm{n}^{\circ} 9$.

(j) Pl. VII, firo. $159, n^{\circ} 4$.

(6) Pl. VI, fig. 153.

(7) Pl. VII, fig. $181, n^{*} 3$.

(8) Pl. VII, fig. $18-$.

(9) Pl. VII, ก… I0J.

(io) Pl. VII, fig. 1:0, 1." 
est tournéc en avant, leur pointe cu arriere: (1); le cetle derniere se létache de chaque côtó un faisceau, qui, d'abord réfléchi d'arrièc cn avaut, combrasse le sommet des tubercules, puis se refléchit de nouveau, et se porte d'avanten arrière, se dirige vers la partic supérieure du cervelet, où il se plonge (2). Ce faisceau blanchâlre est aiısj roulé deux fois.sur lui-même avant de se porter dans le cervelet.

Chez la carpe les tori postérieurs offent l'une des plus singulières complicationg de- l'encéphaic des poissons osseux. D'abord aumiliou on trouve un tubercule arrondi (5), qui sert cn quelpue sorle de chapiteau à la moclle médullairequi lewr serta base; ce tubercule, arrondi cn arrière, cst échancré en devant, où se trouve unc rainure assez profonde. Des côlés de chaque échancrure part une lame épaisse, arrondie, d'un blanc grisâtre; celle lame, rouléc, se dirige en devant, puis sitôtaprès se contourned'avant en arricre, ct forme un croissant autour du tubereule central, qu'elle cmbrasso des dewx côtés (4). Après avoir débordé ce tubercuke en arricre, cette lane se rélléchit de nouveau de dedans en dehors; elle se dirige ensuite d'arriore en avat, en formant un second arc concentrique au premier, qui, de cedc maniche, est embrassi

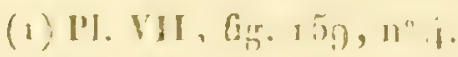

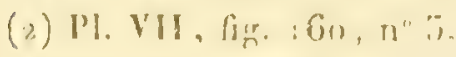

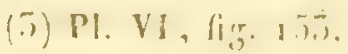

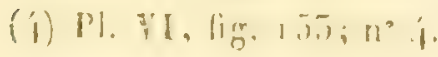


par ce sccond contour. La partic antérieure de La seconde lame de réllexion, arrivée en avant du point de départ de la première, s'enfonce audessous d'elle, en allant gagner la base de la masse médullaire d'où proviennent les tori postérieurs. En outre, au point de la seconde réflexion, il se détache une partie de la membrane nerveuse, qui vient se joindre à l'enveloppe externe des lobes optiques. Les tori postérieurs forment aussi chez la carpe deux circonvolutions libres et flottantes dans la cavité du ventricule optique, adhérentes par leur base à la masse médullaire, dont elles paraissent une expansion. Ces deux circonvolutions sont séparées par deux rainures, ou mieux encore par deux anfractuositćs, dont l'une est interne êt l'autre externc. Si on place l'encéphale dans l'cau, on voit flotter cetle membrane roulée, que rien n'assujétit dans ses trois quarts antérieurs à l'enveloppe externe des lobes. Ces circonvolutions remplissent non-seulement le ventricule optique, mais leur relief est tel dans cette cavité, qu'clles écartent dans leur partie moyenne le plancher des lobes optiques, et donnent à ces lobes la forme particulière qui les distingue de tous les autres poissons osseux.

Les tabercules postéricurs sont principalement en rapport avec le cervelet, comme on l'aperçoit chez la carpe (1), la perche (2). la tan-

(1) Pl. VI, fig. 15\%, 117.

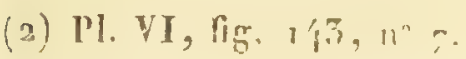


che (1), le congre (2); le merlan (3), la morue (4), le brochet (5), l'égrefin (6) et le gronau ( ᄀ). Leur superficic est souven: recouverte d'une petite pellicule grisâtre.

Les tori antérieurs (8), plus étendus souvent que les postérieurs, ont constamment la forme d'un croissant, embrassant par leur concavité les postérieurs, excepté chez la carpe : très-affaissés chez les cartilagineux (9), leur saillie est à peinc sensible sur le plancher du ventricule : beaucoup plus développés chez la perche (10), le merlan (11), le brochet (12), la sole (15), le gronau (1 亻), ils le sont plus encore chez la morue ( 15 ), où ils forment deux cylindres sur le côté externe des tubercules postérieurs, et chez l'égrefin, oùils ont la forme

(1) Pl. VII, fig. 18 \%

(2) Pl. VII, fig, $16 \overline{7}$.

(3) PI. VII, fig. 195.

(4) Pl. VII, fig. 165, $\mathrm{n}^{\circ} 9$.

(5) PI. VII, fig. 1 go, $\mathrm{n}^{\circ} 4$.

(6i) Pl. VII, fig. $181, n^{\circ} 5$.

(7) Pl. VII, fig. $160, n^{\circ} 3$ et 4 .

(8) Pl. VII, fig. $165, \mathrm{n}^{\circ} 10$

(9) PI. VI, fig. $152, n^{\circ} 6$.

(во) PI. VI, fig. $143, n^{\circ} 4$.

(1) Pl. VII, fig. $19^{3}, n^{\circ} 5$.

(12) Pl. VII , fig. $150, n^{\circ} 3$.

(13) PI. VII, fig. $175, n^{\circ} 5$.

(84) Pl. VII, fig. $159, n^{\circ} 5$.

(15) Pl. VII, fig. $165, \mathrm{n}^{\circ} 8$. 
d'un rein (1). Autant les tori postéricurs sont développés chez la carpe, autant sont simples et affaissés les antérieurs (2). Pour les apercevoir il est nécessaire d'enlever la double circonvolution que forment les premiers : on distingue alors sur la base de l'excavation du ventricule optique un tubercule blanchâtre, en forme de croissant, dont la convexité est en dehors, et la concavité en dedans; de la convexilé partent les faisceaux radiés, qui, par leur épanouissement, forment l'enveloppe du lobe, qui est très-mince chez la carpe, circonstance de structure qui prouve que les tori postérieurs ne concourcnt point à sa formation.

Les ,lubercules antérieurs, principalement formés par la malière médullaire, donnent naissance, par leur expansion, à la membranc interne des lobes optiques; le point de départ de cette lame étant pris au devant du tubercule (5), on la roit contourner d'abord 'les tubercules postérieurs, puisse réflćchir d'arricre en avant tapisser toute la partic interne du ventricule en formant des stries plus ou moins saillantes, comme il a déja été dit. Celte lame interne peut facilement êlre séparée de l'externe, à laquelle clle ne paraît adhérer dans la parlic moycnne que par une

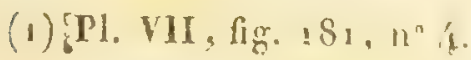

(2) Pl. VI, Gg. $155, \mathrm{n}^{\circ} 5$.

(5) Pl. VIJ . fig. 16.5. $n^{\circ} 10$ 
couche mince de substance grise: parvenue par cette réflexion vers le point de son départ, elle se réunit à sa congénère en formant sou vent une voute médullaire, ou une sorte de ruban blanchâtre en avant du lobe optique, comme on le remarque chcz la perche, le merlan, la tanche, la baudroie. le brochel et la solc. D'autres fois ce voilc médullaire ne se forme pas, et la membrane interne restestriéc dans tonte l'étendus du lobe optique; ce que l'on remarque chez l'égrefin : ou bien à sa place, il se forme un petit amas de matièrc blanche, séparée par une rainure sur la ligne médiane, comme on l'observe sur le gronau. Il est à remarquer que chez plusieurs poissons les plicatures de cette lame interne se prolongent jusqr:e dans le nerf optique de la manière que je l'ai indiqué chez la morue (1). Asscz souvent le voile médullaire, où se termine antérieurement la mcmbrane, est le point où s'arrête la plicature; de trlle sorte que l'on ne peut cn suivre la continuation dans le nerf de l'oeil. On voit spécialement cet effet chez le rascasse.

Chezd'autres poissons osseux, cette lame interne, tout-i-fait libre de l'externe, se plisse ou sc roule sur elle-même de manière à produire, sur le fond du ventricule oplique, des reliefs qui donnent à cet organe une nouvelle apparence. licst le: cas du congre, de lir duréc, des spares ret du ca-

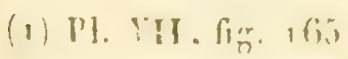


ranx. Comme c'est le plissement le plus simple de la membrane nerveuse des vertébrés, nous allons en suirre le mécanisme chez ces poissons.

Chez le congre (1), la membranc médullaire, parlie de son petit pédicule situé au-devant des tori postérieurs, embrasse d'abord ccux-ci par sa concavité ; puis se réfléchissant d'arrière en avant, elle suit la marche inverse de la premièrc en la côtoyant, et va se fixer au-devant du ventricule optique. La double circonvolution qu'elle forme est séparée par une gouttière fermée à l'endroit où la membrane se réfléchit. Ces deux circonvolutions sont libres.

Chez la dorée, la membrane n'est pas roulée cn circonvolution, ainsi que chez le congre et la carpe : partant du même point et allant aboutir au même endroit, elle forme une espèce de coquille froncéc dans sa parlic moyenne, où existe une cavité spéciale qu'elle cirçonscrit; dans l'eau cette membrane se relève, et lorsque les parois en sont rapprochées, c'est un second ventricule. dansle premier. La même disposition existe chez. le sparus raii et le bogue ordinaire, dont la caviti est plus prononcée que chez la dorée. Chez tous ces poissons le froncement de la membrane interne s'est opéré lo long de l'axe du veutricule optique; le second ventricule a la mème dircclion que le premier. Mais chez le sarge commun

(1) Pl. VII, Cig. 16 - 
(sparus sargus), le cervelet ayant écarté, comme nous l'avons dit, les lobes optiques en arrière, la membrane interne se fronce transversalement; le double ct le triple plissement qu'elle présente cn arrière s'opère horizontalement; la cavité qu'elle circonscrit a son grand diamètre transversal. Chez l'cspadon, ce plissement tient le milieu pour sa direction entre celui du sargue et de la carpe. Chez le caranx et le thon commun, la membrane interne est plissée dans toute sa périphérie; il y a six plissemens chez le caranx, et neuf chezle thon. Chaque pli a son anfractuosité intérieure, sc rendant dans l'anfractuosité centrale, qui, sclon Arsaki (1), est convertie en ventricule, chez ces derniers poissons, par un voile médullaire qui réunit les lames plissées en manière de pont. La plicature de la membrane interne des lobes optiques ressemble, dans ces derniers cas, aux plicatures dı nerf optique de l'espadon, du pagre (sargus argenteus) et du thon (scomber thynnus).

(1) Arsaki, auquel je rends un hommage tardif, parce que je n'ai eu sa dissertation que depuis peu (4 septembr: 1825 ), a représenté ce plissement chez le zcus faber, le sparu.s sargres, Io sparus raii, le caranx trachurus et le xiphias gladius. On l'a accusé comme moi (et, soit dit en passant, avere antant de raison) d'avoir trop grénéralisé ses idées, ou, en H'autres termes, d'avoir dit ce qu'il a vu. Cela doit être, et cera, toutes ies fois que les fitits observés ont le inalheur de ne pas se trouver on hamonic aree les hypotheses; car il fant que les hypotheses aycut aussi leur ristence! 
La formation de ce sccond ventricule dans to centre du premicr se forme par la conjugaison des deux lames des tori antéricurs, comme le ventricule de la moelle épinière, celui du cervelet, le troisième ventricule et les ventricules latéraux des mammifères. C'est une nouvelle cavité de conjugaison. Un fait que nous devons nous borner à bien constater pour le moment, c'est que la lame interne du ventricule optique des poissons osseux est distincte de la lame externe; que lorsqu'elle est appliquée contrc cette dernière, elle est simplement striée, et que, devenue libre, elle se roule en forme de circonvolution, ou se plisse en développant des anfractuosités plus ou moins profondes.

Ces tubercules, sur-ajoutés aux pédoncules cérébraux, forment le centre de la radiation médullaire la plus importante et la plus compliquéc chez les poissons osseux. Les faisccaux postéricurs de cette radialion se rendent dans le cervelet, les antérieurs dans le nerf optique, et les moyens radient vers la périphérie des lobes, ou bien forment une partie distincte ct isolée dans l'intérieur du ventricule optique.

Au centre de la deuxieme cavité, ou à la joncfion des tori en avaut, lorsque cettc cavité n'est pas formée, se trouve une petite dépression qui communique sourent dans le canal de la tige pituitaire, el quelquefois dans la cavitic res lobules ou lubercules optigurs. Chez beaseonp de pois- 
sons la cavité de la lige piluilaire ne communique pas avec celle du ventricule optique. Chez d'aurres, comme chez l'égrefin et la morue, elle s'ouvre dans la scissure de Sylvius, ct s'abouche immédiatenent dans le quatrieme ventricule prolongé le long de cette scissure. Chez le gronau les lames de la tige pituitaires"insèrent en avant des lobes optiques, et communiquent avec les hémisphères cérébraux. Quelquefois, en avant de l'excavation où s'ouvre la tige pituitaire, on rencontre une petite commissure (1) qu'Arsaki nomme. encore commissure antérieure. Quelquefois, comme chez la morue, il y en a deux : l'une superficielle, servant de réunion à la tête des tori antérieurs (2); l'autre profonde, réunissant les deux pédoncules cérébraux avant leur sortie de la base des lobes optiques (3). Je n'ai jamais aperçu de commissure en arrićre du point de jonction du canal pituitaire avec le ventricule optique. Il ne faut pas confondre ces commissures, comme l'ont fait quelques anatomistes, avec les lames de jonc tion de la lame interne, lames qui quelquefois sont situées en arant, comme chez la morue, ou en arriére des tori, comme chez l'espadon.

Les lobes optiques dominent toutes les autres partics de l'encéphale, chez les poissons osseux,

(1) Pl. VI, fig. $15 i, n^{\circ} 2$.

(2) I'l. VII, fig. 165, $1^{\prime \prime} 10$.

(i) VI. VII, fg. $166 \%, 11$ ' 
de même que le font les hémisphères cérćbraux chez les mammifères; leur intérieur offrant des doubles tubercules, qui rappellent les corps striés et la couche optique, leur cavité communiquant souvent par une espèce d'entonnoir avec l'intérieur de la tige pituitaire, comme cela existe encore chez les mammifères; on voit donc comment, en ne prenant que les formes pour guide, cette richesse d'organisation se prêtait à la comparaison de ces lobes avec les hémisphères cérébraux.

Tantôt, comme chez les pleuronectes, lecycloptère lump, le saumon, et surtout le brochet, les tori postérieurs étant doubles ou quadruples, rappelleut jusqu'à un certain point les tubercules quadrijumeaux. D'autres fois, comme chez le merlan et certains spares, leur disposition en forme de reins les rapproche de la couche optique; quelquefois enfin la double circonvolution qu'ils présentent chez le gronau et la carpe, prète à quelque rapprochement avec la corne d'Ammon. Aussi les a-t-on comparés à la corne d'Ammon, à la couche optique ou aux corps striés, selon les espéces qui servaient de terme de rapport. M. Cuvier est le premier qui par une observation ingénieuse ait délogé les tubercules optiques de l'intérieur du ventricule de ces lobes chez tous le poissons; c'est le promier pas vers une meilleure détermination.

Les tori antérieurs fayorisaicnt encore cette il- 
lusion. Le croissant qu'ils forment autour des postérieurs sur le plus grand nombre des poissons, et la disposition radiée de leur convexité, simulent jusqu'à un certain point les cannclures des corps strićs. Les prolongemens de leur membrane, lcur plissement, et leur conjugaison sur la ligne médiane, reproduisent encore les cornes d'Ammon, divisent le ventricule commun cn deux chambres, une supérieure, qui représenterait la partie supérieure des ventricules latéraux, et une inférieure, qui rappelleraitle prolongement dc ces ventricules vers les cornes d'Ammon, chez les mammifères. Quelquefois même, comme chez le thon, le sargue, le sparus raii, le caranx et l'espadon, on aurait pu trouver dans le second ventricule l'analogue du troisième des mammifères, cnchâssé dans le grand ventricule commun : dans la lame médullaire, qui quclquefois se ferme supérieurcment, on aurait pu voir la voute; et dans les faisceaux de conjugaison, soit de la lame plissée, soit des tori, soit des pédoncules, on aurait pu trouver les représentans des commissures antéricures et postérieures. En prenant ainsi tous les poissons osseux, en les considérant d'une manière abstraite, empruntant à l'un ce qui manque à l'autre, on serait parvenu à simuler les hémisphères cérébraux de la classe supérieure, dont les carlilagineux eussent été privés: car la simplicité rle structure de leurs lobes optiques no se prêtc nullement à tous ces rapprochemens. 
La structure de la lame qui forme la coquil!e des lobes optiques des poissons, les rapproche d'une part des tubercules quadrijumeaux des autres classes, et repousse de l'autre leur assimilation aux hémisphères cérébraux.

Dans les trois classes supérieures, et chez les chondroptérygiens, où les hémis phères cérébraux ne peuvent être méconnus, la matic̀re grise forne toujours l'enveloppe extéricure de ces hémisphères : non-seulement il n'existe dans ce rapport de position aucune exception dans les trois classes supérieures; mais, ce qui est plus remarquable, la couche grise extérieure augmente d'épaisseur à mesure qu'on descend vers les mammifères inférieurs; elle forme même, chez les oiseaus et les reptiles, la presque totalité de ces organes; et chez les chondroptérygiens, le requin excepté, à peine distingue-t-on uné traînée de faisceaux médullaires dans leur intérieur. La matière blanche qui forme l'enveloppe extérieure des lobes optiques des poissons, exclut donc l'analogic qu'on a cherché à leur trouver avec les hémisphères cérébraux.

Au contraire, dans les lobes optiques ( tubercules quadrijumeaux y des oiseaux et des reptiles, la matière grise et blanche affecte un rapport inverse : la maticre blanche en forme l'enveloppe extéricure, comme dans la moelle épinière, dont ces lobes sont la terminaison. Les tubercules quadrijumeaux ( lobes optiques) des poissons ren- 
zrent donc dans la structure générale que présentent ces parties dans les classes supérieures.

Ainsi, d'une part, ces parties ont une structure inverse de celle des hémisphères cérébraux dans toutes les classes; de l'autre, leur struclure est identique avec celle des tubercules quadrijumeaux (lobes optiques) des reptiles et des oiscaux. Tout confirme donc leur analogie avec ces dernières parties, en même temps qu'elle exclut tout rapport avec les hémisphères cérébraux.

L'explication de l'encéphale des poissons est toute entière dans la détermination de ces organes, qui en forment la partie principale : une fois méconnus, il était impossible de ramener leur encéphale à celui des autres classes; on tombait inévitablement dans un labyrinthe d'incertitudes dont on n'a jamais pu sortir, quelques efforts qu'on ait faits, quelques suppositions qu'on se soit permises; car le nerf optique, qui naît de la partie antéricure de ces prétendus hémisphères, l'epitclium du cervelet, qui sort de ce qu'on regardait comme les analogues des grands hémisphères, présentaient seuls dies ciifficultés insurmontables, et qu'on n'a jarnais essayé de surmonter. Ajoutons cncore que, dans celte supposition, l'encéphale des chondroptérygiens présentait une exception si extraordinaire, que seule clle suffisait pour la renverser; car dans ces poissons, où les hémisphères céréhraux ne penyent être míconnus, on

II. 
était conduit à admettre quatre hémisphères cérébraux, dont la paire antérieure, entièrement distincle de la postérieure, en différait sous tous les rapports. La dernière classe des vertébrés eût éte celle dont ces hémisphères eussent été les plus richement développés; ce qui est contredit par loute leur organisation.

On n'était guère plus heureux chez les poissons osseux; car comme on trouvait tout l'encéphale des mammifères dans ces prétendus hémisphères, il ne restait plus rien pour les lobes quiles suivent: ces lobes, tantôt au nombre de deux, tantôt au nombre de quatre, forment le tiers ou la moitié le l'organe. D'où il résultait que chez les poissons même qui avaient servi de type de comparaison, la plus grande partie de l'encéphale était inexpliquée et restait inexplicable.

Telles sont les variations des tubercules quadrijumeaux dans les quatre classes des vertébrés.

Ces variations influcnt d'une manière trèsmarquéc sur la disposilion générale de l'encéphale. Chez l'homme, les quadrumanes, les cétacées, les ruminans ct les carnassiers, ils sont tout-à-fait couverts, ot par le cervelet el par les hémisphères cérébraux. Chez les mammifères inféricurs, Jeur saillie semble avoir arrêté le développement de ces deux organes : ils paraissent à nu sur la face supérieure de l'encéphale; ils restent ainsi interposés cutre le cervelet ct les hémisphères cérébraux, quils maintiennent à distance. Il cn 
serait de même sans doute chez les oiseaux, sans lc déplacement quils éprouvent; mais en se plaçant sur les côtés et la base de l'encéphale, ils laissent libre l'espace qu'ils occupaient jusqu'aux deux tiers de l'incubation; ce qui donne au cervelet et aux hémisphères du cerveau la faculté de se rejoindre et de s'appliquer étroitement l'un contre l'autre.

Chez lus reptiles, leur déplacement n'ayant point lieu, ils offrent une paire de lobes visibles chez tous sur la face supérieure de l'organe (1), et constituant déjà une de ses parties fondamentales; enfin, chez les poissons, notamment chez les poissons osseux, ils forment véritablement la partie principale de l'encéphale.

Ces divers états nous reproduisent donc exactement et d'une manière permanente les formes fugitives et transitoires de l'encéphale des cmbryons : c'est, en grande partie, par les transformations des tubercules quadrijumeaux, que l'encéphale d'un embryon d'oiseau (2) répète l'encéphale du reptile; le reptile (3), celui du poisson: c'est pour la même cause, et par les rapports analogues qui en dérivent, que l'embryon d'un maunmifère supérieur(/) reproduit successivement

(1) Pl. V.

(2) Pl. VI et VII.

(3) Pl. I, fig. 5, 6 ct 7 .

(4) PI. I, fig. 12 et 14. 
les formes encéphaliques du poisson, puis du replile, puis des mammifères inférieurs (1), avant d'arrêter définitivement celles qui doivent le caractériser. Les cubryons donnent donc l'explication des formes permanentes de l'encéphale des qualre classes, puisqu'elles sont la répétition les unes des-autres.

Ccla posé, considérons les rapports des tubercules quadrijumeaux avec les parties que nous avons déjà examinées. Remarquons auparavant que jamais on n'a essayé de le faire, par là raison qu'ils étaient méconnus daus les trais classes inférieures, ct quil est impossible de les assigner en adoptant l'une ou l'autre des suppositions que lon a émises à leur sujet.

Ouvrons ces rapports par un résultat général qui nous soit déjà connu. Nous avons vu que plus les embryons sont jeunes, plus la moelle épinière, plus les tubercules quadrijumeaux sont développés : ce fait prouve donc que les tubercules quadrijumeaux et la moelle épinière sont dans les mêmes conditions d'existence. Or, si les animaux permanens répètent les cubryous, ce rapport doit done être chez les premiers comme chez les derniers; le raisonnement l'indique, et cet te fois encore l'ubscrvation directe confirme le raisonnement.

Dans toutes les classes, en effet, le volume des abercules quadrijumcaux suit celui de la moelle

(1) Pl. I ct II. 
épinière : on voit ce rapport chez les poissons osseux (1) et cartilagineux (2), chez les reptiles (3), les oiseaux (4) et les mammifères (5).

L'embryon humain du deuxième, du troisième et du quatrième mois, ressemble aux familles inférieures des mammifères; il a une moelle épinière très-forte, des tubercules quadrijumeaux énormes, un cervelet et des hémisphères cérébraux très-grêlés. L'embryon du lapin du douzième au quinzième jour est surtout remarquable sous ce double rapport (6).

Les microcéphales sont dans le mẻme cas; ils ont une moelle épinière et destubercules quadrijumeaux d'autant plus développés, que le cervelet et les hémisphères cérébraux le sont moins. Tous les idiots sont remarquables par le volume de leur moclle épinière et de leurs tubercules quadrijumeaux.

Les animaux qui ont la moelle épinière la plas grểe, comme le protée, la sirène, la lampraie, ont les tubercules quadrijumeanx les plus déliés; aprés cux viennent les anguilliformes, chez les

(1) PI. VII, fig. 163 ; fig. $169,176,188$ ct s go.

(2) Pl. VI, fig. 158, 1.42, $148 \mathrm{ct} 151$.

(5) Pl. V, fig. 104, $110,111,114,119,126,128,152$, 133 et 155 .

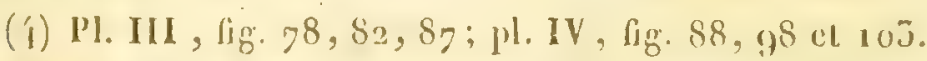

(j) P1. IX, fig. $205 \mathrm{el} 213$; pl. Xl, fig. 232 ; PI. XII

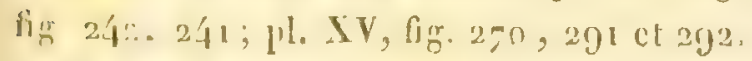

(i) $\mathrm{II}, 11$. 
poissons, ct les ophidiens parmi les reptiles. Chez les sauriens et les batraciens, le volume de la moelle épinière prend de l'accroissement, et on en observe un semblable dans le volume de leurs tubercules quadrijumeaux. Les poissons osseux sont daus le même cas. 11 y a peut-être, sous ce rapport, une légère exception pour les poissons cartilagincux ; car, quoiqu'ils aient la moelle épinière plus développée que les poissons osseux, leurs tubercules quadrijumeaux n'ont pas grossi dans la même proportion.

Enfin, chez les mammifères, cette proportion est exactement la même; la moelle épinière et les tubercules quadrijumeaux vont en augmentant graduelicment de l'homme aux siuges, aux carnassiers, aux ruminans et aux rongeurs.

Ce rapport constaté nous en indique d'autres: car si, d'une part, la moelle épinière est développée en raison du volume des nerfs aiéraux du trouc, en raison directe des nerfs de l'olfaction, de la cinquic̀me paire, et du trapèze de la moelle allongée; et si, de l'autre, les tubercules quadrijumeaux répètent les rapporis de la moelle épinic̀re, il suit nécessairement que ces tubercules, les nerfs latéraux du tronc, les nerf's olfactifs, les trijumeaux et le trapèze, doivent ôtre et sont réellement développés en raison directe les wins des autres (1).

(1) Voge: les plunches. Jy reuroic sourent Ir loctent 
Les tubercules quadrijumeaux ou les lobes optiques sont le renflement de terminaison de la moelle épinière dans le crâne: or, les renflemens supérieurs et inférieurs de la moelle épinière sont rigoureusement proportionnés aux membres auxquels ils correspondent. Pareillement, chez les poissons, les renflemens qui apparaissent en arriere de la moelle allongée suivent le développement des nerfs qui s'y insèrent. En sera-t-il de même du renflement de terminaison de l'axe nerveux du tronc ou des tubercules quadrijumeaux? Le nerf qui s'y insère, l'organe auquel il est principalement destiné, suivront-ils les variations diverses du volume de ces tubercules et des lobcs optiques dans les trois classes inférieures? $\mathrm{I}_{A}$ 'oil, le nerf optique et les tubercules quadrijumeaux, seront-ils enfin développés en raison directe les uns des autres? C'était à l'observation à répondre : or l'observation a montré un rápport constant entre les dimensions des yeux, des nerfs optiques et des tubercules quadrijumeaux, dans les quatre classes. A de petits yeux correspondent des nerfis optiques grêles et des tubercules quadrijumeaux peu prononcés; des yeux volumineux, au con traire, coïncident avec des nerfs opliques trèsforts et des tubercules quadrijumcaux énormes.

L'anguis fragilis est celui de tous les reptiles qui

afin d'eiviter d'interrompre par des chilfies l'expose des idées générales. 
a les plus petits yeux, les plus petits nerfs optiques et les plus petits tubercules quadrijumeaux. Les vipères et les couleuvres ont déjà les yeux, leurs nerfs et leurs tuberculcs plus développés. Le crocodile, le tupinambis, le caméléon et les tortues de mor, sont les reptiles dont les yeux sont les plus forts; ils ont aussi les nerfs optiques et les tubercules quadrijumeaux les plus volumineux (1).

Les anguilliformes, chez les poissons, sont comme les ophidiens chez les reptiles : ils ont les plus petits yeux, les plus petits lobes et les plus petits nerfs optiques. Viennent ensuite les pleuronectes, puis les poissons cartilagineux (2); puis, parmi les osseux, les gades et les trigles (3), chez lesquels ces trois parties augmentent dans un rapport proportionnel.

On sait que les oiscaux ont des yeux si développés, et certains d'entre eux une vue si perçante, qu'un anatomiste célébre ( Oken ) a proposé dernièrement de les désigner par le nom d'ocutiers. Or, toute cette classe est remarquable par le volume de ses nerfs optiques et de scs tubercules quadrijumeaux (4); elle l'est aussi par le plissement de la rétine, qui en augmente la surface, selon l'ingénieuse remarque de M. le docteur Desmoulins.

(1) Pl. V, fig. 112, 113,122, 120, 129 et 155.

(2) Pl. VI.

(3) PI. VII.

(4) Pl. III et PI. IV. 
Enfin, chez les mammifères, l'oil augmente de volume, de même que le nerf optique et leș tubercules quadrijumeaux, des quadrumanes aux carnassiers, aux pachydermes, aux ruminans et aux rongeurs: les phoques et les cétacées sont surtout remarquables sous ce triple rapport (1).

D'après l'opinion de M. Gall sur l'origine des nerfs optiques dans cette classe, il semble que ce rapport devrait coincider principalement avec les tubercules antérieurs. Cela cst d'une manière générale; ccpendant le blaireau, le renard, le putois, l'hyène, le raton (2), l'ornithorhynque, quelques rats et plusieurs rongèurs, ont la paire antéricure de ces tubercules (3) très-développée, quoique les nerfs optiques soient assez grêles en comparaison (4).

Sont exceptés de ces rapports les animaux privés de nerfs optiques, chez lesquels néanmoins les tubercules quadrijumeaux sont très-bien développés, tels que le protée et la cécilie chez les reptiles; la taupe (5), le zemni (6), le rat-taupe du Cap, les musaraignes et la chrysochlorc.

Remarquons à celte occasion unc discordance frappante entre les ronflemens de la moelle épi-

(1) Pl. XI, fig. 225, E, F, G ; P]. XII, fig. $234, \mathrm{~B}^{\circ} 2$.

(2) Pl. VIII, fig. $201, n^{\circ} 6$.

(3) Pl. YIII, fig. $201, n^{\circ} 5$ el 6.

(द) PI. VIII, fig. $200, n^{\circ} 2$

(5) Pl. XIV, fig. $260, \mathrm{n}^{\circ} 2$.

(6) Pl. XV, fi gro $2 z^{2}, \mathrm{n}^{n} 2$. 
nière ct son bulbe de terminaison. En effet, les renflemens supérieur et inférieur disparaissent avec les membres auxquels ils correspondent; il scmble, d'après cela, que les tubercules quadrijumeaux devraient cesser d'exister en même temps que le nerf optique disparaît. Mais il n'en est pas ainsi chez le protée; les lobes optiques n'ont rien perdu de cette absence des nerfs. Chez la taupe (1) et lc zemni (2), on peut voir également non-seulement que les tubercules quadrijumeaux ont survécu aux nerfs optiques (3), mais que leur dimension même n'en paraît pas diminuée. La mênı indépendance des tubercules quadrijumeaux, se reproduit sur quelques monstruosités animales; ainsi ces tubercules n'éprouvent aucun changement physique notable, soit que le nerf opliquene se forme pas, soit qu'après son développement un obstacle dépendant de la monstruosité l'empêche d'établir sa communication avec eux. Cette singulière exception est digne de fixer l'attention des anatomistes.

Signalons-en une autre non moins remarquable, quoique moins sensible au premicr aperçu. Nous avons vu que chez les reptiles et les poissons les cordons des olives s'épuisent en tolalité dans les lobes optiques; nous avons dit aussi qu'ils s'y

(1) Pl. XV, fig. $291, n^{\circ}$ ฯ.

(3) Pl. XV, fig. $29 ?, 11^{\circ} 4$.

(3) Pl, XIY, fig. $260, n^{\circ} 2 ; P l, X V$, fig, $2 \geq 2, n^{*} 2$. 
consument presque entièrement chez lcs oiseaux, tandis que chez les mammifères une grande partic de leurs faisceaux se portent dans les hémisphères. Ne semble-t-il pas, d'après cette disposition, que les éminences olivaires devraient s'accroître dans les mêmes proportions que les tubercules quadrijumeaux et les lobes optiques? Or, c'est ce qui n'est pas. Les éminences olivaires restent complètement affaissées chez les poissons, les reptiles et les oiseaux; elles sont affaissées aussi chez les mammifères inférieurs; elles ne deviennent saillantes que chez les supérieurs, et surtout chez les quadrumanes et l'homme. Cette discordance se reproduit aussi chez les embryons : ainsi, pendant la première moitié de la formation de l'embryon des quadrumanes et de l'homme, les tubercules quadrijumeaux étant très-développés, les olives. sont affaissées; pendant le dernier tiers, les olives deviennent de plus en plus saillantes à mesure que les tubercules s'affaissent et diminuent. Quelle est la cause de cet antagonisme? je l'ignore. Ce que nous avons dit du volume relatif de la moclle ipinière et des tubercules quadrijumeaux n'est donc point applicable aux éminences olivaires.

Du reste, les tubcrcules quadrijumeaux sont déveloprés chez les mammifères en raison direct: des pyramides antéricures, des postérieures êt du corps restiforme; ct che\% les oiseaux, dans un rapport propertionnd avec ces dexuices corps at los promides posterieures. Cher les poissons. 
toutes les parties de la moelle allongée semblent suivre assez exactement le dévcloppement des tubercules quadrijumeaux.

Les pyramides antérieures s'entre-croisent sur la moelle allongée : les olives ne s'entre-croiscnt point dans ce sens; leur croiscment se fait en avant par l'intermède des faisceaux du nerf optique. Sous ce rapport encore, les quatre classes présentent des différences très-marquées. Ainsi, plus l'entre-croisement des pyramides antérieures est distinct, comme chez l'homme et les quadrumanes, moins le croisement des faisceaux des olives est marqué à la jonction du nerf optique. Les mammiféres et les oiseaux sont les seules classes où le croisement des pyramides soit apparent : c'est aussi dans ces deux classes que l'on distingue si difficilement l'entre-croisement des nerfs optiques, qui n'a jamais lieu que pour une petite quantité de ses fibres.

Au contraire, chez les reptiles et les poissons, dont les pyramides anterieures sout directes, les nerfs optiques s'entre-croisent en masse; celui 1 i: droite passe à gauche, et celui de gauche à droite. sans confondre leurs fibres, dans un grand nombre de cas.

La liaison des deux systémes nerveux de droile et de gauche par leur cutre-croiscnent, a douc lieu à un mêne degré dans les quatre classes, quoique dans des points opposés. Lorsque l'entre-croisement est tres-prononcé cu arriere, il rest 
firible ch avant : il est très-manifeste en avant, quand il cesse d'exister en arrière.

Les tubercules quadrijumeaux ont donc avec les yeux les mêmes corrélations que les renflemens supérieurs et inférieurs de la moelle épinière avec les membres; ces corrélations du système nerveux central s'étendent beaucoup plus loin que les anatomistes ne l'ont pensé jusqu'à ce jour : nous allons en fournir une nouvelle preuve dans les rapports de l'os épactal de Fischer, ou des interpariétaux, avec les tubercules quadrijumeaux.

Ces os, que l'on pourrait aussi nommer pariétaux postérieurs, correspondent aux tubercules quadrijumeaux, comme les occipitaux au cervelet, les pariélaux et les coronaux aux hémisphères cérébraux : ils suivent, dans toutes les classes, les modifications de ces tubercules, grandissont et s'atrophient avec eux.

Ainsi, chez les poissons, nous trouvons les interparićtaux les plus grands, comme les tubercules quadrijumcaux les plus volumineux : après les poissons viennent les reptiles et les oiseaux, pour le volumie de ces os et de ces tubercules; puis, enfin, les mammiferes, chez lesquels l'os ćpactal est réduit dans ses dimensions, comme le sont les dimensions des tubercules quadrijumeaux. Cettc réduction est graduelle, comme la diminution successive des fubcrcules. L'os épactal est encore trè-prononcé chez les rongeurs; il l'est 
moins chez les ruminans et les pachydermes : il n'existe chez les carnassiers que dans le jeune âge. Enfin, l'homme occupant le bas de l'échelle des vertébrés par la faiblesse de ses tubercules quadrijumeaux, les interpariétaux n'apparaissent chez lui que comme des cas accidentels. Sur des sujets oú je les ai observés très-distincts et isolés des pariétaux et de l'occipital, j'ai remarqué, d'unc part, que les tubercules quadrijumeaux étaient irès-développés, et jai observé, de l'autre, que ce surcroît de volume leur était donné par la prédominance des tubercules antérieurs sur les postérieurs, double circonstance qui les rapprochait des rongeurs, des ruminans et des pachydermes (1).

En définitif donc, les lobes optiques des oiseaux, des reptiles et des poissons, sont les analogues des tubercules quadrijumeaux des mammifères.

Ils sont creux dans les trois classes inférieures, et solides chez les mammiferes.

Chez les embryons de ces derniers, ils sont creux comme dans les trois classes inférieures, et jumeaux comme les lobes optiques. Ies trois

(1) J'ai remarqué chez plusicurs idiots que les tubercules quadrijumeaux antéricurs sont plus développés que les posférieurs. J'ai encore vérifié ce fait ce matin (12 aont 1825) chez une fille de trentc-trois ans, idiote depuis son bas âge. J'ai constaté aussi une diminution sensible de la couche optique et des corps striés. Lc corvelet était très-petit, la moelle épiniere large, le trone énorme, la tête petite. 
classes inférieures sont donc, sous ce rapport, des embryons permanens de la supérieure, et ccux-ci reproduisent les formes permanentes des oiseaux, des reptiles et des poissons.

Les tubercules quadrijumeaux sont dominés chez les mammifères par les autres parties de l'encéphale. Chez les reptiles, ils dominent le cervelet; chez les oiseaux, ils forment une paire de lobes plus importans encore que chez les reptiles : enfin, chez les poissons, notamment les poissons osseux, ils forment la partie principale dc l'encéphale, et dominent toutes les autres.

De ces diverses conditions d'existence des tubercules quadrijumeaux dans les quatre classes dérivent donc leur forme, leur position, la structure ct les rapporis qui viennent de faire l'objet de ce Chapitre. 


\section{TABLIAU GOMPARATIF}

Des Dimensions des Tubercules quadrijumeaux chez les Mammifères.

\begin{tabular}{|c|c|c|}
\hline \multirow[b]{2}{*}{ Homme. } & \multicolumn{2}{|c|}{$\begin{array}{c}\text { MESURES } \\
\text { DO TUBBRC. QUADR. }\end{array}$} \\
\hline & $\begin{array}{c}\text { mùtre. } \\
0,01000\end{array}$ & $\begin{array}{c}\text { mètre. } \\
0,01100\end{array}$ \\
\hline NOMS DES ANIMAUX. & & \\
\hline Patas (Simia rubra)... . & 0,00625 & 0,00900 \\
\hline 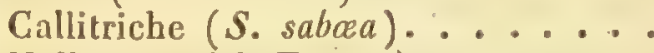 & 0,00600 & 0,00750 \\
\hline Malbrouck (S. Faunus)......... & 0,00700 & 0,00835 \\
\hline 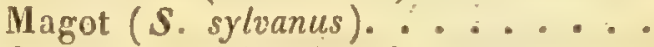 & 0,00650 & 0,00900 \\
\hline Ouanderou ( $\boldsymbol{S}$. silenus)...... & $0,005,5$ & 0,00800 \\
\hline Macaque (S. cynoeephalus). . : . & 0,00600 & 0,00733 \\
\hline Papion (S. sphynx) . . . . . & 0,00625 & 0,01000 \\
\hline Mandrill (S. maimon). . . . . & 0,00650 & 0,01000 \\
\hline Saï $(S$. capucina $) \ldots \ldots$ & 0,00450 & 0,00850 \\
\hline Maki (Lemur macaco)...... : & 0,00550 & $0,009_{2} 5$ \\
\hline 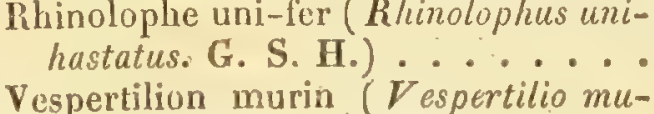 & 0,00300 & 0,00300 \\
\hline rinus). . .......... & 0,00250 & 0,00250 \\
\hline Taupe (Talpa europoa)........ & 0,00335 & 0,00500 \\
\hline Ours brun (Ursus arctos)....... & 0,00850 & 0,01200 \\
\hline Ours noir d'A mérique.(U. americanus). & 0,00875 & 0,01200 \\
\hline Raton (U. lotor). . . . . . & 0,00625 & 0,00900 \\
\hline Blaireau (U. meles)....... & 0,00600 & 0,01000 \\
\hline Fouine (Mustela foina). . . & 0,00566 & 0,00800 \\
\hline Marte ( $M$. martes ) . . . & 0,00550 & 0,00725 \\
\hline Loutre $(\boldsymbol{M}$. lutra) . . . & 0,00500 & $0,00 \$ 00$ \\
\hline Chien (Canis familiaris). & 0,00700 & $0,0097^{5}$ \\
\hline Loup, jeune (C. lupus).. & 0,00700 & 0,00950 \\
\hline Ilyine $(C$. hyona)....... & 0,01000 & $0,0127^{5}$ \\
\hline Civette ( $V$ iverra civelta ). . . & $0,0077^{5}$ & $0,00,50$ \\
\hline Genelte $(V \cdot$ senclta $) \ldots$ & $0,0,0650$ & $0,008-5$ \\
\hline Lion (Felis leo ) , . . . . . & $0,01200)$ & 0,01700 \\
\hline
\end{tabular}


Suile du Tableau comparalif des Dimensions des Tubercules quadrijumeaux chez les Mammifëros.

\begin{tabular}{|c|c|c|}
\hline \multirow{2}{*}{ noms deg A NIMATX. } & \multicolumn{2}{|c|}{$\begin{array}{l}\text { MESURES } \\
\text { DU TOBERC. QOADR. }\end{array}$} \\
\hline & $\begin{array}{l}\text { dianeitre } \\
\text { taansererse. }\end{array}$ & $\begin{array}{l}\text { dianeitre } \\
\text { anteropost. }\end{array}$ \\
\hline 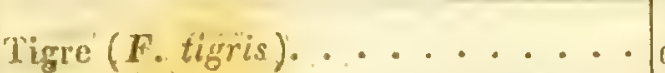 & $\left|\begin{array}{c}\text { mèlre. } \\
0,01000\end{array}\right|$ & $\mid \begin{array}{l}\text { mitre. } \\
0,01500\end{array}$ \\
\hline Jaguar $\left(F_{\text {. onga. }}\right) \ldots \ldots \ldots$ & 0,00950 & 0,01200 \\
\hline Panthere (F. pardus).......... & 0,00950 & 0,01200 \\
\hline Ocelot $(\boldsymbol{F}$ paradalis $) \ldots \ldots$ & $0,00775 \mid$ & $0,01 \% 50$ \\
\hline Couguar (F. discolor) $\cdots \cdots \cdots$ & $0,01000 \mid$ & 0,01200 \\
\hline Guépard (F. jubata) $\ldots \ldots$ & 0,00850 & $0,0: 150$ \\
\hline $\operatorname{Lyn} x(\boldsymbol{F} \cdot \operatorname{lyn} x) \ldots \ldots$ & 0,00900 & 0,01200 \\
\hline Chat $(\boldsymbol{F}$. catus $) ..$ & 0,00700 & 0,01000 \\
\hline $\begin{array}{l}\text { Phoque commun (Phoca vitulina)... } \\
\text { Didelphe de Virginie (Didelphis Vir- }\end{array}$ & 0,00950 & 0,01500 \\
\hline $\begin{array}{l}\text { Didelphe de virginie (Didelphis } \\
\text { giniana) } \ldots \ldots\end{array}$ & 0.00600 & $0,00,50$ \\
\hline Kanguroo géant (Macropus major. & & \\
\hline SH., G. C. ) . . & 0,00800 & 0,01400 \\
\hline Phascolome (Phascolomys. G. S. H.) & $|0,00700|$ & | " \\
\hline Castor (Castor fiber.). : . . . & $0,00700 \mid$ & 0,01000 \\
\hline Utia (Capromys furnieri. Desm.) . : & $|0,00450|$ & 0,00700 \\
\hline Zemni (Mus typhlus). . ..... & 0,00400 & 0,00600 \\
\hline Marmotte $(M$ : alpinus.) $\ldots \ldots$ & $0,00550 \mid$ & 0,00900 \\
\hline Porc-épic (Hystrix cristata).... & 0.00600 & $0,008,5$ \\
\hline Lapin (Leniss cuniculus)...... & $|0,00425|$ & 0,00700 \\
\hline Agouti (Cavia acuti)... & 0,00750 & 0,00700 \\
\hline Paca (C. poca.).... : - & $0,00775 \mid$ & . \\
\hline Cochon d'inde ( $C$. Cobaya $) \ldots$ & $|0,00400|$ & 0,00750 \\
\hline Tatou encoubert (Dasypus sexcinctus.) & $|0,00550|$ & 0,00650 \\
\hline Pécari (Sus tajassu). . . . . & $0,00850 \mid$ & 0,01500 \\
\hline Sanglier (s. scropha)......... & 0,01000 & 0,01500 \\
\hline Daman (Hyrax capensis) ..... & 0,00450 & 0,00700 \\
\hline Cheval (Equus caballus).. ..... & 0,01960 & 0,02275 \\
\hline Dromadaire (Camelus dromedarius). & $|0,01250|$ & 0,02125 \\
\hline Lama (C. llacma) . . . . . . & 0,01125 & 50,01800 \\
\hline Kevel (Antilope kevella).. & 0,00950 & 0,01700 \\
\hline $\begin{array}{l}\text { Gazelle (A. Gazella)...... } \\
\text { Chamois (A. rupioapra) }\end{array}$ & $\mid \begin{array}{l}0,00933 \\
0,00925\end{array}$ & $5 \mid \begin{array}{l}0,01475 \\
0,00750\end{array}$ \\
\hline Biche (Cervus claphus)....... & 0,01400 & \\
\hline $\operatorname{Dam}(C$. dama $) \ldots \ldots$ & 10,01300 & \\
\hline
\end{tabular}


Suite du Tableau comparatif des Dimensions des Tubercules quadrijumeaux chez les Mammiferes.

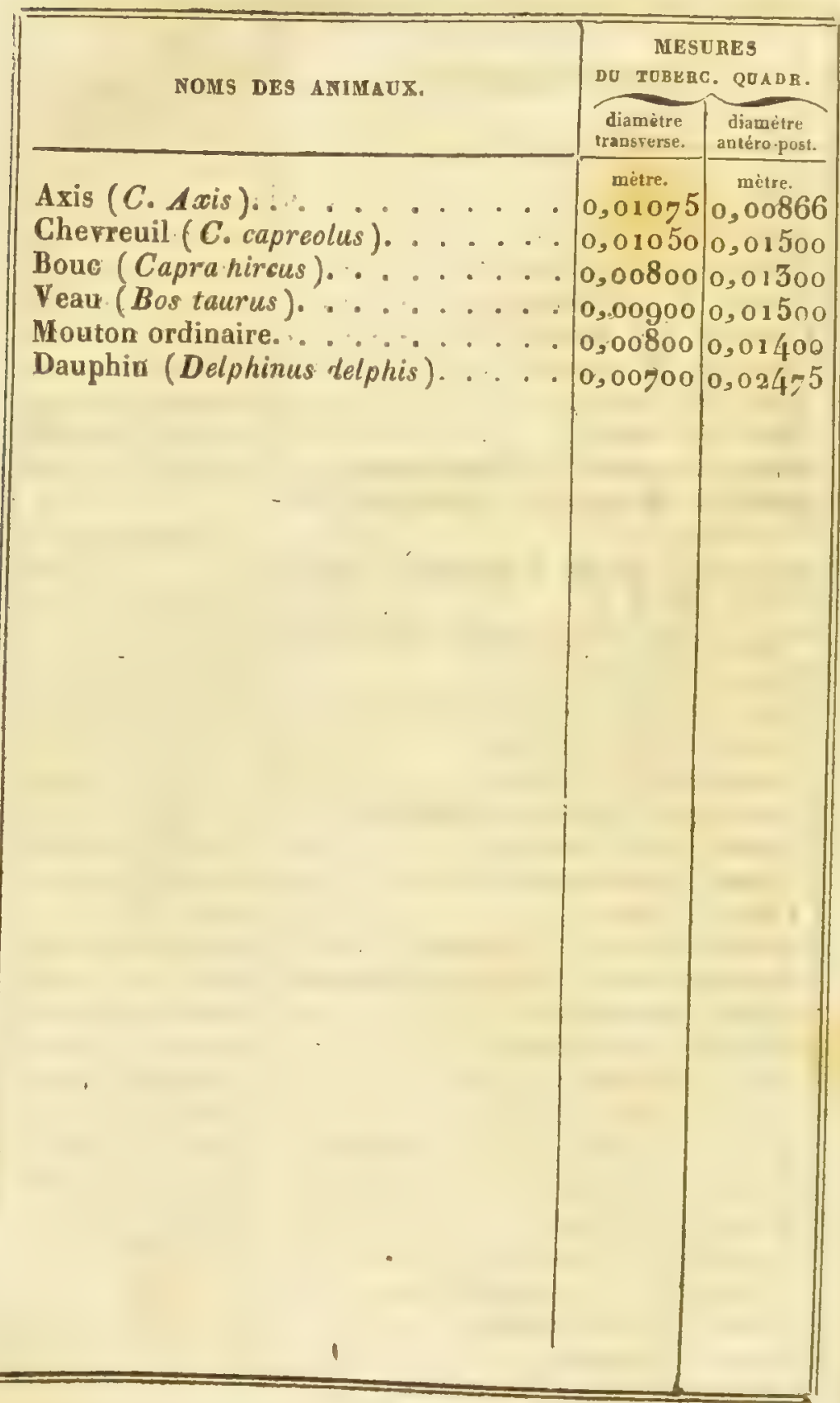


T A B I. E A L C O M P R ATIE

1) es Dimensions des I'ubercules quadrijumcaux chez les Oiseanx.

\begin{tabular}{|c|c|c|}
\hline NONS DES ANIMACX. & $\mid \begin{array}{c}\text { MENU } \\
\text { DK TUBERC } \\
\begin{array}{c}\text { dimmitte } \\
\text { transirbe. }\end{array}\end{array}$ & $\begin{array}{l}\text { URES } \\
\text { c. QUADR. } \\
\text { diametre } \\
\text { anteropost. }\end{array}$ \\
\hline Vautour fauve (Vultus fulvus). & 0,00800 & $\begin{array}{l}\text { mètre. } \\
0,01200\end{array}$ \\
\hline Aigle royal (Falco chrysaetos). . . & 0,00800 & 0,01200 \\
\hline Pygargue (F. Ossifragus ). : ‘ & 0,01100 & 0,01100 \\
\hline Faucon ( $\boldsymbol{F}$. communis) . . . . & 0,00725 & $0,007-5$ \\
\hline Bondrée ( $\boldsymbol{F}$. apivorus ). $\therefore$ & 0,00800 & 0,01000 \\
\hline Busard ( $\boldsymbol{F}$. ceruginosus). . .... & 0,00550 & 0,00900 \\
\hline Buse commune ( $\boldsymbol{F}$. buteo ) $\therefore$ & 0,00600 & 0,00900 \\
\hline Roitelet (Motacilla regulus). . . & 0,00500 & 0,00250 \\
\hline Hirondelle (Hirundo urbica). .... & 0,00475 & 0,00450 \\
\hline Alovetle (Alauda arvensis) . . . & 0,00425 & 0,00400 \\
\hline Moineau (Fringilla domestica) $\therefore$ & 0,00400 & 0,00350 \\
\hline Pinson ( $F$ calebs). . . . . & 0,00400 & 0,00400 \\
\hline Linotte $(\boldsymbol{F}$. linaria $) \cdot \ldots$ & 0,00300 & 0,00300 \\
\hline $\operatorname{Serin}(\bar{r}$. canaria) $\cdots \cdots$ & 0,00325 & 0,00300 \\
\hline Chardonneret ( $\boldsymbol{F}$. carduelis). : & 0,00325 & 0,00300 \\
\hline Verdier (Loxia chloris)....... & 0,00400 & 0,00350 \\
\hline $\operatorname{Pie}($ C. pica). . . . . . & 0,00600 & 0,00600 \\
\hline Perroquel amazone ..... : . & 0,00625 & 0,01000 \\
\hline Perroquet d'Afrique. ....... & 0,00600 & 0,00700 \\
\hline Dindon (Meleagris gallopavo)... & 0,00725 & $0,008,5$ \\
\hline Poule (Phasianus gallus)...... & . 0,00800 & 0,00900 \\
\hline Faisan argonté ( $P$. nycthemerus $)$. . & . $|0,00850|$ & 0,00900 \\
\hline Faisan doré ( $\boldsymbol{P}$ pictus $) \ldots$ & 0,00700 & 0,00800 \\
\hline Autruche (Struthio camelus), ... & $(1,01125$ & 0,01100 \\
\hline Caspar (S. casuarius). . . . & 0,01000 & 0,01050 \\
\hline Ontarde (Olis tarda). . . . . & 0,00 Soo & 0,00775 \\
\hline Cigogne blanche. (Ardea ciconin). . & 0,00800 & 0,01200 \\
\hline Oisealı royal (A. pavonina)..... & 0,00900 & $0,00,000$ \\
\hline 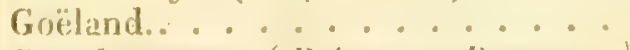 & 0,00800 & 0,00800 \\
\hline loun de bassan (Pelecemus Passmmus) & 0,00900 & 0,01100 \\
\hline Oie (Anas anser) . . . . . . . . & 0,00700 & 0,00850 \\
\hline Cravant (A. bernicla)...... & 0,00650 & 0,00900 \\
\hline Canard musqué (A. moschata). & 0,00800 & 0,00975 \\
\hline Lider (A. mollissima) ...... & . 0,00600 & 0,00625 \\
\hline
\end{tabular}




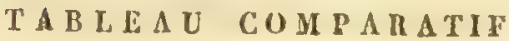

des Dimensions des Tubercules quadrijumeaux chez les Reptiles.

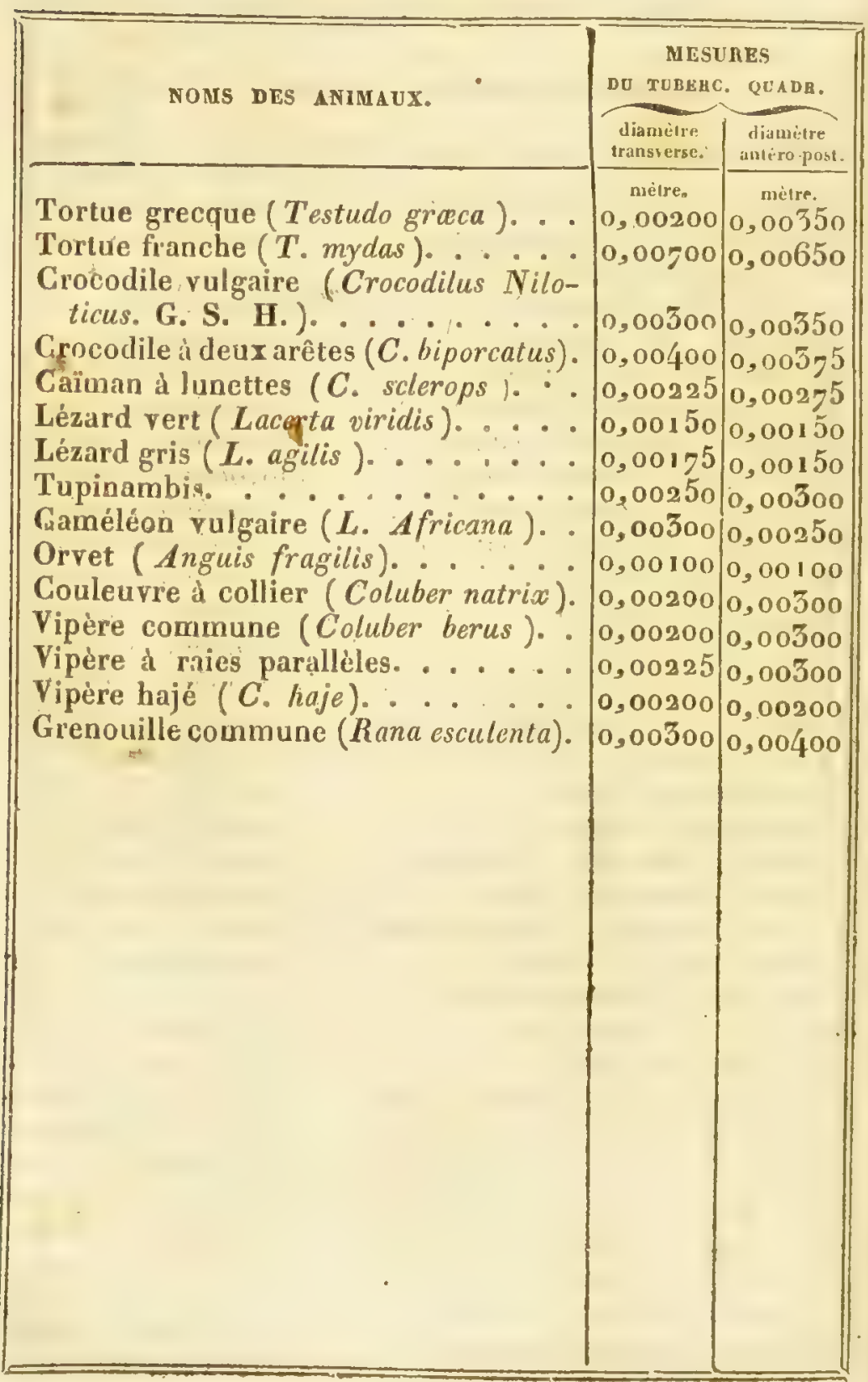


TUBERCLLS QUADRIJUMEAUX.

TA BLEAU COMPA IA A TIF

Des Dimensions des T'ubercules quadrijumcaux chez les Poissons.

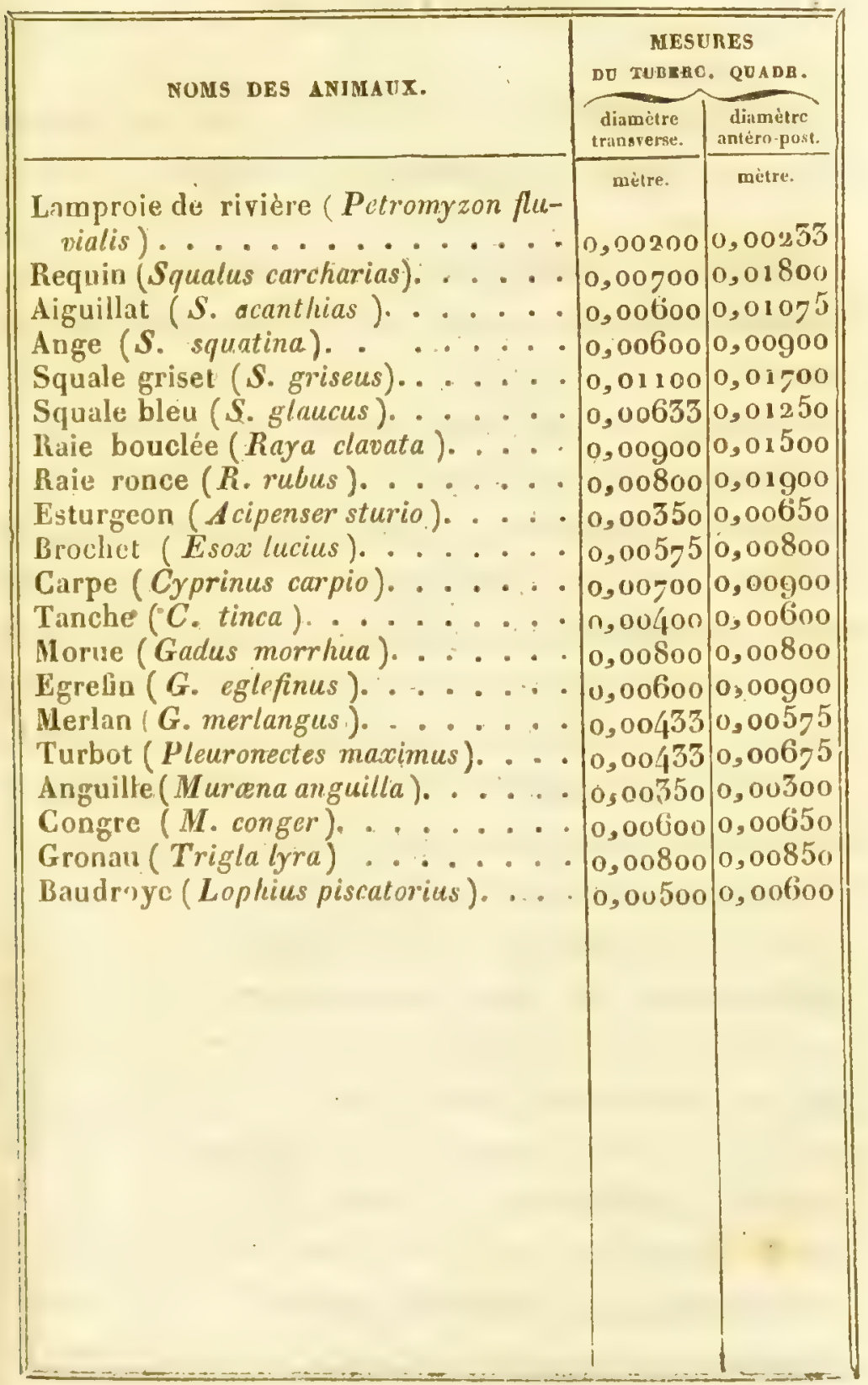




\section{CHAPITRE VI.}

Analomie comparée du Cervelet, dans les qualve classes des animaux vertébrés.

Parmi les résultats inattendus fournis par l'encéphalogénic, il n'en est pas de plus remarquable que celui de l'apparition tạrdive du cervelet clans les embryons des quatre classes des vertébrés.

Chez les oiscaux on ne distingue le cervelet qu'à la fur du cinquième et au commencement du sixieme jour de l'incubation; il est formé par deux feuillets isoles, l'un droit, lautre gauche; du huitième aux neuvième et dixième jours, ces fouillets se réunissent sur la ligne médiane; cette réunion s'opère par une espèce de suture analogue à la suture postérieure de la moelle épinière. Lc cervelet cst alors un organe impair.

Chez les reptiles son développement est plus tardif encore que chez les oiseaux; je l'ai cherché en vain sur les tétards des grenouilles et des crapauds avant le vingt-cinquième jour de leur formalion. Il est composé à cette époque par deux feuillels pelliculcux, partant des bords de la moelle allongée; ces feuil!ets sont isolés, et flottans sur les côtés du quatrième ventricule. Du irentecinquième au quaranticme jour, la rémnion s'opere. 
on arrière, comme chez les oiseaux au neuvième jour de l'inc ubation.

Le type de formation est le mème chez les nammifères et l'homme; sur l'embryon du cheval et du veau on n'aperçoit pas le cervelet avant la fin du deuxième mois; à cette époque, et au commencement du troisième, on trouve à sa place deux lames détachées, non réunies sur la ligne médiane. L'embryon du lapin du quatorzième jour est dans le même état, ainsi que plusieurs embryons de didelphes.

Chez l'embryon humain ce n'est qu'au commencement du troisième mois qu'on distingue les deux lames cérébelleuses; ces lames se dirigent l'une vers l'autre sur le plancher du quatrième ventricule; il existe alors deux cervelets, l'un droit, l'autre gauche. A la fin du troisième ou au commencement du quatrième, les lames se rapprochent sur la ligne médiane; leur jouction s'opère par une espèce d'engrenure qui resle apparente ¡usqu'à la fin du quatricme mois : le cervelet est alors un organe impair. Cette engrenure des deux parties du cervelet reste apparente chez. l'embryon du veau jusqu'au commencement du cincuième mois; cher le chat et le chicn jusqu'à Id fin du premier mois; rt sur l'embryon du lapin, jusqu'aux scizieme et dix-septicone jours de sil formalion.

Aussitòt que le cervelet est développé chez les 1'mbryons de lil classe supéricure, ou cel organe 
est le plus compliqué, on voit se manifester d'abord le lobe médian ou le processus vermiculaire supérieur. La saillie du lobe médian coincide alors avec la saillie des tubercules quadrijumeaux el l'atrophie des hémisphères du cervclet; puis les tubercules quadrijumeaux et lo lobe médian s'affaissent, el les hémisphères latéraux se développent: enfin, plus ces dernicrs se développent . plus le premier s'atrophie.

A l'ćjoque où le cervelet est devenu un organe impair chez les mammifères, il n'établit d'abord aucune communication avec la moelle allungée; lorsque le lobe médian est très-saillant, le trapèze commence à embrasser cette moelle. Cela a licu virs le tiers de la gestation. Vers la moilié de la formation dez cmbryons, on voit apparaître les faisccaux du pont, qui viennent former une cspéce d'arc très-saillant sur la partie antérieure de la moclle allongée. Le développement du trapèze coincide donc avec celui du lobe médian du cervelet, et le développement du pont de varole avec les hémispheres du même organe. Plus ceuxci accroissent, plus, arons-nous dit, le lobe médian s'affaisse; d'où il résulte que plus le pont de varole angmente, plus le trapize diminue.

Daus la premispe apparition du ceryclet; la marche est arrêtéc par le volume que présentent alors les tubercules quadrijumeaux; il semble mêne, chrz les embryons de toutesles classes, se digager do dessous ces tubercules, quilin domt- 
nent dans tous les sens. Tn parcourant la séric de ses déreloppemens, le lobe médian arrive en premier lieu au niveau des tubercules quadrijumeaux; en second lieu, il les déborde et leur devient supérieur; en troisième lieu, enfin, il les domine, et ces tubercules s'enfoncent au-dessous du cervelet, comme le cervelet paraissait d'abord cnfoncé sous les tubercules quadrijumeaux : d'où il résulte que ces deux organes se dominent tourà-tour.

En considérant chez les embryons l'ensemble des développemens du cervelet, nous voyons donc $\imath^{\circ}$. que d'abord cet organe est double ou pair; $2^{\circ}$. que lursqu'il est arrivé à former un organe impair, il est constitué par une lame mince faisant suite áur tubercules quadrijnmeaux; $3^{\circ}$. qu'ensuite le lobe médian surgit sur la face supćlicure de l'organe, dominant ainsi les lobes latinux, et qu'alors apparaît le trapèze de la morlle alongée; $4^{\circ}$. enfin, que les hémisphères latéraux deviennent dominateurs à leur tour, ainsi que les prolorgemens qu'ils envoient autour de la moelle allongée.

Considírant maintenant lis formes permanents du cervelet dans les quatre classes des vertébra, leur trouverons-nous quclque analogie avec es formes primitives? La sćrie de ses complicatins coüncidera-t-clle avec la séric de ses devclopemens? linfin, les animanx adultes nous repeteroteils le cervelet des embryons? The est lo 
point de vue d'après lequel nous allons examiner le cervelet des vertébrés, en commençant par les reptiles.

Dans cette classe, le cervelet descend a ux plus petites dimensions possibles; il s'arrête à la seconde série de ses développemens : il ne forme, sur la plupart des reptiles, qu'une petite lame triangulaire placée en travers sur le haut du quatrième ventricule.

Chez l'orvet cette lame est si petite et si mince $(1 j$, qu'à peinc la distingue-t-on en arrière des tubercules quadrijumeaux, auxquels elle adhère (2). Sur le lézard vert (3), elle est comme chez l'orvet : sa forme est celle d'un triangle dont la base est adossée aux lobes optiques (4), et dout la pointe est flottante au haul du quatrième ventricule. Chè la vipère de Fontainebleau (5), la vipère hajé (6), la vipère à raies parallèles $(7)$ et les couleuvres, la feuile triangulaire qui constitue le cervelet est un peu plus étendue. Chez les crapauds et les genouilles (8), la pointe du triangle est moinsal-

(i) Pl. V, fig. $109, \mathrm{n}^{\circ} 2$.

(2) PI. V, fig. $109, \mathrm{n}^{\circ} 5$.

(3) PI. V, fig. $110, \mathrm{n}^{\circ} 1 \mathrm{et}$.

(4) Pl. V, fig. $110,11^{\circ} 9$.

(5) PI. V, fig. $132, n^{\circ} 3$.

(6) Pl. V, fig. 126, n" 5

(z) Pl. V, fig. 133, n०3.

(8) Pl. I, fig. 16, $\mathrm{u}^{\circ} \mathrm{2}$ 
tongere que chez les vipères. I, cervelet n'est, chez les batraciens, qu'unc pelite commissure transverse placée en forme de poutie au-dessus du quatrième ventricule ( 1 ) ; disposition très-apparente, quand on a ouvert les lobes opliques et dejeté sur les côtés les feuillets qui les forment (2) et qui recouvrent en partie le cervelet.

Chez le lézard vert (3) et lc caïman (4) (5), lc

(1) Pl. V, fig. $134, n^{\prime \prime} 5$

(2) Pl. V, fig. 154, $n^{\circ} 5$ et 6 .

(5) Pl. V, fig. 128, $\mathbf{n}^{\circ} 2$.

(4) Pl. V, fig. $155, n^{\circ} 5$.

(5) Jusqu'à ce jour je n'avais eu à ma disposition yue de: trés-jeunes crocodiles et un jeine caïman à lunettes; j u'avais pu donner avec précision l'itendue du cervelet chez. ees animaux parvenus au terme de leur développement. J" me hâle de le faire, ayant maintenant sous les yeux l'cncéphale d'un raiman i museau de brochet, ardulte, de six piecis de long, et mort il y a quelques jours it Paris.

Chez co ciäman, le cervelet s'élive au-dessus des lobe. optiques; il a la forme d'un bonnet renver'sé sur le quatricuse ventricule, d'où il résulte que sa cavité est indépendante de celle du quatrieme ventricule. Il se termine en pointe, ei ne recouvre pas en tolalité ce dernier ventricule. Les raiaures sont lues-superficielles sur sa face externe; mais en dessons il y en a deux, plus profondes, qui viennent brider en arrićv: la cavilá cérébelleuse. La base du cervelet repose sur ses pédoncules, réunis en forme de commissuce transverse iumédiatement en arrière de l'inserlion de la quatrieme paire d. nerf. C'est atu-dessous de celte commissure tres-épaisse que so trouve l'onverture de l'aqueduc de Sylvius, nu que s'établit la communicatinu ayec le quatrieme, ventricule. Cielle 
ccrvelet, très-petit encore, se bombc légèrement à sa partie moyenne, ce qui lui donne une forme globuleuse lorsque l'on insuffle le quatrième ventricule; à la loupe, on distingue sur la superficis de l'organe une ou deux petites rainures trissuperficielles. Ces rainures sont beaucoup plus distinctes chez le caméléon (1), le crocodile vulgaire (2), et le crocodile à deux arêtes (3), reptiles chez lesquels le cervelet a pris beaucoup plus d'étendue que chez les précédens: chez les cro-

forme conique et creuse du cervelet apparlient a la seconde formation de cet organe chez les vertébrés supérieurs, disposition su' laquelie le professeur holando vient de donner des aperçns très-intéressans.

Quand on a soulevé le cervelet d'arrière en arant, on observe sur les côtés du quatrième ventricule deux tuberculcs volumineux, coinme on en remarque si fréquemment chez les poissons. Ces tubercules correspondent a l'insertion des nerfs acoustique et facial; ils sont, je pense, les analogues du tonia grisea des frères Wenzel. L'ouverture du quatrième ventricule se prolonge en arrière du cervelet; le rapprochement des deux tubercules précédens sur la ligne médiane le divise en deux parlies: une antérieure, plus large; une postéricure, plus étroite. La cinquième paire est énorme; le volume de chacun de ses ganglions dépasse de beanconp celui de chargure lobe optipue; te volume de ce nerl' coüncide avec l'étenâne considérable de ha face chéz ces animaux.
(1) Pl. V, fig. $111, n^{\circ} 4$.
(2) Pl, V, fig. 115, n'5 bis.
(5) Pl. V, figio $118, \mathrm{n}^{\circ} 2$ 
codiles sur-tout: le quatrième ventricule en est recouvert en totalité. Les petites rainures sont plus visibles quand on considère l'encéphale par sa partie latérale (1), que lorsqu'on l'examine supérieurement. Chez les ophidiens, les lacertiens ct les batraciens, le cervelet ne s'élève jamais au niveau des lobes optiques; ceux-ci le.dominent dans toute son étendue (2). Chez le caméléon et les crocudiles, le cervelet se rapproche plus que chez les reptiles précédens du niveau des tubercules quadrijumeaux (3).

Telle est la forme générale du cervelet chez les reptiles : cet organe affecte chez le tupinambis et les tortues une disposition qui s'en écarte et qui se rapproche de celle que nous rencontrons chez quelques poissons cartilagineux.

Chezle tupinambis, le cervelet a la forme de deux triangles (4) adossés au niveau de la partie postérieure des lobes optiques. Le triangle postéricur se place au-dessus du quatrième ventricule (5), l'antérieur recouvre la partie postérieure des lobes optiques (6), sa pointe se prolonge jusqu'en avant de ces lobes ( 7 ). Cet effet est

(1) Pl. V, fig. i15, $\mathrm{B}^{0}$.

(2) Pl. V, fig. $109,100,136,132$ et 133.

(3) PI. V, fig. 115 et 116.

(4) Pl, V, fig. $114, n^{\circ} 6$ et 1.

(5) Pl. V, fig. $114, n^{\circ} 6$.

(6) Pl. V, fig. 114, $\mathrm{n}^{\circ} 1$.

(g) Pl. V, fig. 114, $n^{\circ} 8$. 
produit par l'affaissement de ces derniers en arrière. Chez la tortue grecque, le cervelet forme d'abord deux feuillets festonnés sur le côté du quatrième ventricule (1), comme cela existe chez quelques poissons; ces feuillets se réunissent ensuite en arrière des lobes optiques et y produisent une lame elliptique (2) un peu bombée. Chez la tortue franche ce dernier effet est porté à son plus haut degré; ce qui donne au cervelet une configuration particulière. En effet, chez la tortue franche le cervelet a une forme globuleuse (3); sa surface est d'un tiers plus étendue que celle des lobes optiques, dont il dépasse un peu le niveau vers sa partie moycnne. A l'œeil nu cette surface paraît lisse; mais à la loupe on y distingue quelques rainures concentriquies, dans la dépression desquelles sont logés ces petits vaisseaux. Si on insuffle le quatrième ventricule, le globe du cervelet s'élère beaucoup au-dessus des lobes optiques. Du reste, on n'y distingue ni traces du lobe médian, ni aucun vestige des lobes latéraux. Quand on incise sur la ligne médiane le feuillet qui forme le cervelet globuleux de cette tortue, on voit que ses parties latérales sont festonnées (4), et que sir

(1) Pl. V, fig. 125, $\mathrm{n}^{\circ} 14$.

(2) Pl. $V$, fig. $125, n^{\circ} 6$ bis.

(3) Pl. V, fig. $119, \mathrm{n}^{\circ} 5$.

(4) Pl. V, fig. $120, \mathrm{n}^{\circ} 13$ et 3 . 
partie la plus élevéc est sous les lobes optiques (1). Ce feuillet cérébelleux est mince et ne se dédouble pas.

La couleur du cervelet est d'un gris cendré chez les ophidiens, les sauriens èt les batraciens. Chez les tortues de terre il est d'un gris un peu rosacé; mais chez la tortue franche il est d'un rouge assez foncé, comme chez les oiseaux, les poissons cartilagineux et les mammifères. Par sa couleur, sa forme et son étendue, le cervelet de la tortue franche s'élève donc beaucoup au-dessus des autres reptiles.

Après les reptiles, les poissons osseux sont, de tous les vertébrés, ceux dont le cervelet est le moins développé : il consiste toujours en une lame triangulaire superposée sur le quatrième ventricule et plus ou moins étendue selon les familles. Chez la plupart, cette lame s'enfonce dans l'intérieur des lobes optiques (2), sa partie moyenne paraît se détacher des tubercules postérieurs de leur ventricule (3). Ses parties latérales sont formées par les côtés de la moelle allongée (4); ces deux parties sont toujours réunies de manière à former chez tous un organe unique et impair, comme chez les reptiles.

(1) Pl. V, fig. $121, \mathrm{n}^{\circ} \mathrm{x}$.

(2) Pl. VII, fig. 165, $\mathrm{n}^{\circ} 9$.

(3) Pl. VII, fig. $19^{3}, \mathrm{n}^{\circ} 3$ et 4 .

(4) P. VI, fig. $150, n^{\circ} 2$ 
Cet organe est très-peiit chez la baudroie (1), le barbeau (2), l'anguille (3), le gronau (4), la sole (5) et le brochet (6). Chez ce dernier il se détache des quatre tubercules postérieurs du ventricule des lobes optiques. Sa forme est celle d'un triangle dont le sommet libre est très-obtus.

Chez le turbot il est plus allongé ( $)$ ) : il présente sur les côtés deux sillons, au côté externc desquels se voyent les feuillets de la moelle allongée. Cette disposition est la même chez le congre (8) : la partie médiane (9) est ainsi distincte des parties latérales (10). Chez la morue, les parties latérales sont un peu plus saillantes (11), sa pointe se prolonge au-delà du quatrième ventricule (12); la lame qui le forme adhère peu aux feuillets latéraux de la moelle allongée, elle semble provenir en très-grande partie de l'intérieur des lobes optiques (13). Chez le merlan le cervelet offre

(1) Pl. VII, fig. 179, n०2 et 3.

(2) Pl. VII, fig. $183, \mathrm{n}^{\circ} 1$.

(3) Pl. VII; fig. $190, \mathbf{n}^{\circ} \mathrm{z}$.

(4) Pl. VII, fig. $159, \mathrm{n}^{\bullet} \mathrm{s}$

(5) Pl. VII, fig. $173, \mathrm{n}^{\circ} 3$.

(6) $\mathrm{Pl}$ : Vu, fig. $173, \mathrm{n}^{\circ}, 2$.

(2) Pl. VII, fig. 19!, $\mathrm{n}^{\circ} 2$.

(8) Pl. VII, fig. $168, n^{\circ} 3$ et 2.

(9) PI. VII , fig. 168, n².

(10) Pl. VII, fig. $168, n^{\circ} 3$.

(11) PI. VII, fig. 163, $n^{\circ} 14$.

(12) Pl. VII, fig. 163, $n^{\circ} 4$.

(13) Pl. VII, fig. $165, \mathrm{n}^{\circ} 9$. 
unc disposition semblable (1). Chez l'égrefin (2), la lame qui se détache des tubercules postérieurs des lobes optiques se bifurque, en sortant de leur ventriculc (5), elle embrasse dans son écarieınent la partic moyenne du cervelet (4). Chice la tanche (5), le cervelet est globuleux, comme chez la tortue franche; il provicnt aussi en grande partie des tubercules postérieurs des lobes optiques (6), et il est embrassé à sa terminaison par les deux cordons roulés de la moelle allongée (7). Chez la carpe le cervelet est plus compliqué que sur les autres poissons osseux. Supérieurement il présente d'abord la lame triangulaire (8), formant une espéce de couverclesur ce quatrième ventricule; puis sur les côtés une lame frangée (9), se joignant en haut à la lame précédente. En troisième licu il y a en arricre une paire de lobes de cliaque côté (10), qui simulent jusquàà un certain point les hémisphères latéraux du même organe chez les mammiferes : ces lobes sont les tubercules du quatrième

(1) Pl. VII, fig. $19^{3}, 10^{\circ} 5$.

(2) Pl. VII, fig. $184, n^{\circ}$.

(3) PI. VII, fig. $181, n^{*} 3$.

(4) PI. VII, fig. $181, \mathrm{n}^{*} \mathrm{z}$.

(5) PI. VII, fig. $187, n^{\circ} 5$.

(6) Pl. VII, fig. $18 \eta, n^{\circ} 4$.

(7) PI. VII, fig. $185, n^{\circ} 5$.

(8) PI. VI, fig. $145, n^{\circ} 3$

(g) Pl. VI, fig. $150, n^{\circ} 2$.

(10) P1. VI, fig. 1/5, 11" 3

11. 
ventricule dont nous avons déjá parlé. La forme du cervelet varie donc chez les poissons osseux de famille à lamille, d'une espèce à l'autre, et quelquefois même elle n'est pas identique sur tous les individus d'une même espèce; ce que j'ai surtout vérifié chez la caịpe. Jamais chezles poissons osseux, de même que sur la plupart des reptiles, le cervelet ne surgit au-dessus des lubercules quadrijumeaux.

La couleur du cervelei est d'un rouge incarnat sur tous les poissons osseux: crlle couleur tranche sur la plupart, à côté du blanc mat des lobes optiques. Chez le merlan, la lame du cervelet est violacée à sa surface cxterne. La lame extérieure est formée par une couche mince de substance corlicale; quand ọn l'enlève avec précaution on distingue au-dessous des stries blanches (1) formant des arcs dont la convexité regarde les lobes optiques. Ces stries blanches se réunissent sur la ligne médiane, tantôt immédiatement, tantôt en chevauchant les uns sur les autres (2); le nombre de ces strics varie selon l'étendue du cervelet. Lorsque, comme chezla carpe, le cordon restiforuse est festonné, on distingue au dedans un semblable feston dentelé formé par la matière médullaire (5).

Le cervelet des poissons cartilagineux appartient à un degré plus élevé dans l'échelle animale que

(1) PI. VII, fig. $167, n^{\circ} 9$

(2) Pl. VII , fig. $174, n^{\circ} 4,5$ et 6.

(3) Pl. VI, fig. $150, \mathrm{n}^{\circ} \mathrm{2}$. 
celui des reptiles et des poissons osseux, quoique, sous quelques rapports et dans plusieurs espèces, une partie reproduise l'état primitif et disjoint de cet organe. Loin de présenter les variations continuelles que l'on remarque chez les osseux, il semble se compliquer graduellement, et s'élève, de la simplicité qu'il présente chez les reptiles, à la complication qu'il atteint chez les oiseaux. La comparaison de cet organe, de la lamproie au requin, va nous montrer ces divers degrés de déveInppement.

Chez la lamproie, le cervelet consiste en une pctite bande transverse (1), réunissant en haut les lames de la moelle allongée, qui sur les côtés du quatrième ventricule sont disjointes (2) et festonnées à-peu-près de la même manière que nous l'avons observé sur la tortue grecque. Il résulte de là que ce ventricule est tout-à-fait à découvert sur la face supérieure de l'encéphale. La lame transverse est si étroite et si mincc, que Tréviranus lui refuse le caructère et le nom de cervelet, et la con. sidère comme la commissure transverse du quatrième ventricule. C'est sans doute par extension de l'opinion de Tréviranus que d'autres anatomistes ont privé du même organe les ophidiens et les batraciens, comme si le plus ou moins de volume d'une partic devait entrer pour quelque chose dans sa détermination.

(1) PI. XI, fig. 224, n०9.

(2) Pl. XI, fig. $224,11^{\circ} 2$. 
Chez l'esturgeon, le cervelet offre d'abord deux lames roulées en forme de corde (1) sur les côtés du quatrième ventricule, non réunies sur la ligne médiane, et dont l'épaisseur coïncide avec le volume énorme des nerfs pneumo-gastriques. Il y a ensuite cn haut du quatrième ventricule une lame triangulaire très-épaisse (2) qui s'abaisse comme l'épiglotte sur la glotte, et qui recouvre la moitié de cettc cavité (3). Ce feuillet céré̉elleux est divisé en trois parties ou en trois lames secondaires, de telle sorte que la plus élevée(4) recouvrela moitié de la moyenne (5), et celle-ci la moitié de l'inférieure (6). Cette partie du cervelet est' ainsi d'autant plus épaisse qu'on l'observe plus près de son adossement aux lobes optiques, qu'elle déborde légèrement. Cette prédominance du cervelet de l'esturgeon sur celui de la lamproie est d'autant plus remarquable, que ces deux poissons se ressemblent par l'exiguité de leur moelle épinière.

Chez la raie ronce le cervelet est plus développé encore que chez l'esturgeon, quoiqu'il soit divisé en deux parlies, l'une droite, l'autre gauche. En effet, non-seulement chez ce poisson (7), les lames

1) PI. XII, fig. $233, n^{n} 2,3$ et 4 .

(2) Pl. XII, fig. $235, n^{\circ} 5$.

(3) Pl. XII, fig. $235, \mathrm{E}, \mathrm{D}, \mathrm{C}$.

(4) Pl. XII, fig. 235, E.

(5) Pl. XII, fig. $235, \mathrm{D}$.

(6) PI. XII, fig. 235, C.

(z) Pl. VI, fig. 152, no2. 
de la moelle allongée sont plissées, et dcux fois roulées sur elles-mêmes (1) dans l'intéricur du quatrième ventricule qu'elles remplissent; inais encore le lobe médian (2), qui vient cn couvrir la partie moyenne, est divisé en deux parties symétriques par une ligne moyenne (3), qui règne lans toute son étendue. Celte partie médiane, ainsi que les feuillets les plus extérieurs de la moelle allongée (4), viennent puiser leurs doubles racines sur les tubercules de l'intérieur des lobes opliques, comme cela a lieu chéz les poissons osseux (5).

Sur la raie bouclée les feuillets cérébelleux de la moelle allongée sont échancrés en dehors (6), mais ils ne sont ni roulés comme chez la raie ronce, ni aussi nombreux, ni aussi épais. D'un autre côté, le lobe médian (7) a, ché la raic bou-

(1) Pl. VI, fig̣. $152, n^{\circ} 3$

(2) Pl: VI, fig. $152, n^{\circ} 5$.

(3) Pl. VI, fig. $152, n^{\circ} 4$

(4) PI. VI, fig. $152, a^{\circ} 3$ et 5 .

(5) Ce rapport des tori postérieurs avec le cervelet n'est qu'une corrélation organique. Le volume des Lubercules, loin d'être en rapport avec celui du cervelet, paraît au contraice. cn sens inverse chez les poissons osseux et cartilagineux. Chez les osseux, le lobe inćdian, entrant daus la cavité des tubercules quadrijuncaux, ecarte leur partie postéricure; ce qui, chez beaucoup d'entre cux, déplace l'inserlion de la

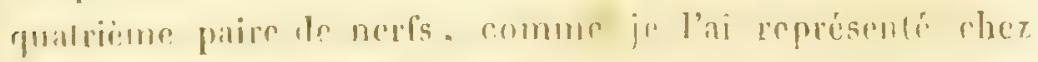

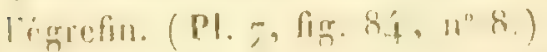

(6) PI. VI, fig. $140,1^{n} 5$.

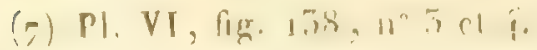


clée", deux fois plus de volume que celui de la raie ronce; de telle sorte que la masse totale du cervelet dans ces deux espèces est balancée par la différence d'étendue des feuillets cérébelleux et du lobe médian. En outre, ce lobe offre une disposition remarquable: Chez les poissons osseux le lobe médian est toujours un organe impair: il offre aussi ce caractère chez l'ange, dont nous parlerons plus bas. Mais chez toutes les raies que j'ai examinées, cet organe est pair. Chez la raie ronce il est divisé en deux parties, l'une droite, l'autre gauche. Chez la raie bouclée il y a quatre parties distinctes (1), deux d'un cốé (2), deux de l'autre (3); profondément divisées par un sillon médian (4), ces parties sont de plus séparées par un sillon transverse : le sillon médian est aussi distinct en dessous (5) qu'en dessus. De ces quatre lobules qui composent le lobe médian de cette raie, les postérieurs (6) recouvrent le quatrième ventricule; les antérieurs (7) se prolongent au-dessus des lobes optiques, qu'ils recouvrent en partie (8). Le cervelet de l'aiguillat

(1) Pl. VI, fig. 138, $n^{\circ} 3$ et 4 .

(2) Pl. VI, fig. $138, n^{\circ} 3$ et 3.

(3) Pl. VI, fig. 138, n० 4 et 4 .

(4) Pl. VI, fig. 138, $n^{\circ} 13$ :

(5) PI. VI, fig. $140, n^{\circ} 7 \mathrm{ct} 8$.

(6) Pl. VI, fig. 138, n० 4 ct 4 .

(7) P!. VI, fig. 158, n" 33.

(8) Pl. VI, fig. 158, $n^{n}$ ?. 
est la répétition exacte de celui de la raic boucléc, les feuillets de la moelle allongée (1) sont roulés sur les côtés du quatrième ventricule; le lobe médian est aussi quadri-lobé (2); il y a sur sa face extérieure un sillon vertical et un sillon transverse. Des quatre lobules, les deux postérieurs (3) se prolongent sur le quatrième ventricule, les deux antérieurs (4) recourrent la moitié des lobes optiques. Cet effet des lobules antérieurs chez ces deux poissons cartilagineux rappelle celui que nous avons observé sur le tupinambis, et coincide, comme chez ce reptile, avec une atrophie de la partic postérieure des lobes optiques.

Si le double développement du cervelet, ou la loi de symétrie appliquée à cet organe, avait besoin encore d'être appuyé sur d'autres faits, la raie ronce nous montrerait en permanence le mécanisme de la formation de cet organe chez tous les embryons. Chez cette raie, en effet, le cervelet bifide est réuni sur la 'igne médiane par un réseau très-délié de la pie-mère, qui se détache de cette inembrane au moment oú elle se réfléchit de dehors en dedans (5).

(1) PI. XII, fig. $236, X$.

(2) Pl. XII, fig. 236, B, D.

(3) PI. XII, fig. 236, B.

(4) Pl. XII, fig. $236, \mathrm{D}$.

(5) Le cervelet de la raic ronce est pour la double division 
Le cervelet de l'ange se rapproche plus de celui de l'esturgeon que de celui des raies. II a uné disposition globuleuse (1); son lobe médian présente un petit lobule qui lui semble sur-ajouté $(2)$; sa pointe se prolonge jusqu'au bas du quatrième ventricule, et se loge dans une gouttière formée par les deux cordons cérébcllcux (3) de la moelle allongée, qui sont trèsépais, mais non tordus conıme une corde; à leur réunion en has il présente encore un autre petit lobule vésiculeux (4), que je n'ai observé que ches ce poisson et le requin.

Cette disposition globuleuse du cervelet, imparfaite chez l'ange, est parfaitement développéechez le griset $(s q$. vacca) : le cervelet de ce squale rappelle on tout point celui de la tortue franche, nonseulement par sa forme, mais par son étendue, par ses rapports avec les lobes optiques, et le quatrième vent ricule, qui, chezce poisson de même que chez la tortue, se prolonge bien au-delà du cervelet. De plus, comme chez la tortue, la surface exterue du cervelet est sillonnée par de pctites rainures concentriques qui se rejoignent à peine sur

de cet organe, ce que l'hiatus du renflement inférieur des oiseaux est pour la moelle épinière.

(1) PI. XII, fig. 257, D.

(2) Pl. XII, fig. $23 z$, D.

(3) Pl. XII, fig. $237, \mathrm{C}$.

(4) Pl. XII, fig. $25 \overline{7}, \mathrm{~B}$ 
la ligne méliane. Il y a un icl rapport entre les lobes uptiques et le cervelct du griset et le cervelet et les lobes optiques de la tortue franche, que, sans les hémisphères cérćbraux dont les différences sont considérables, on confondrait facilement l'encéphale de l'un avec l'encéphale de l'autre.

Jusquici les reptiles et les poissons ne nous ont offert que des formes primitives du cervelet encore très-éloignées de la forme de cet organe chez les oiscaux; le squale bleu ( $s q$. glaucus) et le requin vont nous donner ce passage intermédiaire des classes inférieures aux supéricures.

En effet le cervelet du squale bleu et du requin (1) est un organe impair, sa forme est cylindroïde comme chez tous les oiseaux : sa superficic est sillonnée par des rainures concentriques (2) superficielles chez le bleu, beaucoup plus profondes chez le requin. Chez ce dernier, ces sillons sont au nombre de neuf; il y en a quatorze chez le bleu. En exarninant cet organe par sa partie latérale, il présente, chez ces. poissons, de même que chez les oiseaux, la disposition de eertaines coquilles, c'està-dire que toutes les rainures du cervelet convergent en différens sen's vers le pédoncule de cet organe. Chez le bleu, de même que chcz le requin (3), le cervelet s'élève sur les tubcrcules
(1) PI. VI, fig. $142, \mathrm{~B}$.
(2) PI. VI, fig. 1.1\%, 13, C,
(5) Pl, VI, fig. 1/12, B. 
quadrijumeaux et en recouvre la moitié posiérieure, comme cela aurait lieu chez les oiseaux, si ces tubercules restaient constamment sur la face supérieure du cerveau. Chez les poissons, de même que chcz les oiseaux et les mammifères, le développement du cervelet s'opère donc d'arrière en avant, dans le sens de la marche de ses artères, ce qui explique pourquoi, chez la raie bouclée, l'ange, l'aiguillat, le bleu et le requin, cet organe se dirige en avant au lieu de se prolonger en arrière. Il recouvre en partie les lobes optiques chez les poissons, et, comme chez les oiseaux, il viendrait rejoindre la partie postérieure des hémisphères cérébraux, si les tubercules quadrijumeaux s'atrophiaient au même degré que ceux des mammifères, et s'ils éprouvaient le même déplacement que cher les oiseaux. Quoique ces effets n'aient point lieu, on peut remarquer néanmoins que chez ces poissons, et surtout chez ces deux derniers squales, le cerveau se dirige déjà d'avant en arrière pour se rapprocher du cervelet, comme cela a lieu chez les oiseaux pendant le dernier tiers de l'incubation, et chez les embryons des mammifères, à partir de la moitié de leur gestation (1). La cause de ce changement est donc la même chez les poissons que chez les cmbryons des deux classes supérieures : en se développant, l'encéphale se concentre; l'effut de cette concentration

(1) PI!. I et II. 
est de rapprocher ses divers élémens, de mênu: que cela a lieu pour les ganglions céphaliques des insectes dans le cours de leurs métamorphoses.

La couleur extérieure du cervelet des poissons cartilagineux est d'un rouge léger, comme celui des poissons osseux; la substance corticale qui en forme la couche extérieure, est beaucoup plus épaisse que chez les reptiles et les poissons osseux, et dans une proportion très-supérieure à la substance médullaire : celle-ci offre une disposition sur laquelle il est essentiel de nous arrêter.

Chez la lamproie, il n'y a qu'une bande transverse de substance médullaire aussi petite que chez les batraciens; chez l'esturgeon, les raies, l'aiguillat, la matière blanche forme sur les côtés du quatrième ventricule un cordon plissé en divers sens : il est à remarquer que ce plissement est indépendant de tout moyen mécanique; il n'y a aucun faisceau transverse qui puisse contribuer à la formation de ces contours qui sont si nombreux sur les raies. Chez la raie ronce et l'aiguillat, les quatre lobules du lobe médian sont formés dans leur centre par une lame de matière blanche, qui, partant de la circonférence, se replie sur elle-même. Il résulte de ld un canal inégal dans la partie moyenne du lobe médian, canal dont l'ouverture s'aperçoit en soulevant le lobe médian (1). Chez les squales bleu et griset, les con-

(1) Pl. V1, fig. $140,11^{n} 6$. 
tours de la substance médullaire sont beaucoup plus profonds que chez les raies. Ie plissement de la lane médullaire du cervelet ressemble au plisscment de la lame médullaire du nerf optique du pagre, du thon et de l'espadon. Il a pour effet immédiat de multiplier l'étendue de la surface nerveuse. En incisant le cervelet du bleu, on forme une vaste vésicule par le déplissement de ce cordon, de même que chez les poissons précédens on donne au nerf optique la forme d'un intestin par le même procédé. Chez le requin, la lame médullaire est plus épaisse que chez le bleu; elle forme, comme chez ce dernier, une multitude de contours, de plis et de replis, qui forment des saillies et des enfoncemens sur les parois latérales du quatrieme ventricule. Ces plis et ces replis existent indépendamment des faisceaux transverses. On observe en outre à la face supérieure du cervelet un cône mince de matière médullaire, auquel viennent aboutir les segmens qui correspondent aux rainures de cet organe, comme nous verrons que cela a lieu pour les oiseaux.

Par sa forme, sa disposilion extéricure et sa structure, le cervelet du requin sert donc d'intermédiaire cntre le cervelet des poissons cartilagineux et celui des oiseaux. Dans cette dernière. classe cet organe continue son développement; il ne présente plus les variations que nous avons remarquées chez les reptiles, les poissons osseux et cartilagincux; ses formes sont arreters 
ct fixes, il ne differe que par plus ou moins d'étendue selon les familles.

Le cervelet des oiseaux se rapproche beaucoup de la formed'un cylindre (1). Il est logé en avant dans une courbure semi-lunaire forméc par la partie postérieure des lobes optiques et leur commissure (2); sa face supérieure (3) s'élève à la hautcur et quelquefois dépasse le niveau de la face supérieure des hémisphères cérébraux (4); il repose en quelque sorte sur l'intervalle des lobes optiques, áu-dessus desquels il fait toujours une saillie très-marquée (5). Ce vapport du cervelet avec ces lobes offre, chez les oiseaux, des variations qui modifient l'aspect extérieur de l'encéphale dans cette classe. Cette modification est produite par la saillie plus ou moins grande que font les lobes optiques en arrière et sur les côtés des hémisphères cérébraux. Chez l'autruche (6), les lobes optiques se portant très en arrière, semblent appartenir extérieurement au cervelet et en former les lobes latéraux ( 7$)$. Chez la cigogne blanche (\$), au contraire, ils sont enfoncés au-

(1) Pl. IV, fig. $9^{5}, \mathrm{n}^{\circ} 3,4$ et 5 .

(2) PI. IV, fig. $90, \mathbf{n}^{n} 2$.

(3) Pl. IV, fo. $104, n^{\circ} 4$.

(4) Pl. IV, fig. $97, \mathrm{n}^{\circ} 4$ et 12.

(5) Pl. IV, fig. $9^{5}, \mathrm{n}^{\circ} 4,3$ et 5.

(6) Pl. IV , fig. $95, \mathrm{n}^{\circ} 5$.

(z) Pl. IV, fig. y $y^{5}, n^{\circ} 4$ ct 5 .

(8) Pl. IV, fig. $103, \mathrm{n}^{\circ} 6$. 
dessous des hémisphères cérébraux (1) qui les débordent légèrement; il résulte de là que les lobes ne sont pas visibles extérieurement, l'encéphale ètant considéré par sa face supérieure (2): on n'y distingue que le cervelet (3) et les hémisphères du cerveau (4). La bondrée commune (5) et le casoar (6) tiennent le milieu entre ces deux extrêmes de la position des lobes optiques. Aussi ne voit-on qu'une partie du segment postérieur de ces lobes entre le cervelet et le cerveau. Toutes les variations possibles de la forme extćrieure de l'encéphale des oiseaux sont renfermées dans ces trois modifications principales.

Le cervelet, chez tous les oiseaux, est adossé contre la partie postérieure du cerveau; j’ai déjà expliqué cet effet par le déplacement des tubercules quadrijumeaux; et ce déplacement influe encore sur la hauteur à laquelle s'élève le cervelet.

En général, plus les lobes optiques.se portent en arrière, plus le cervelet est soulevés supérieurement, comme on le remarque chez l'autruche (7); plus ils s'enfoncent sous les hémisphères céré-

(1) Pl. IV, fig. 103, $n^{\circ} 5$.

(2) Pl. IV, fig. 99.

(3) Pl. IV, fig. 99, $n^{\circ} 2$.

(4) Pl. IV, fig. $99, n^{\circ} 3$ et 4 .

(5) Pl. IV, fig. $89, n^{\circ} 8$.

(6) Pl. III, fig. $77, \mathrm{n}^{\circ} 4$ et 3 .

(z) Pl. IV, fig. $9^{5}, n^{\circ} 3$ et 4 . 
braux, moins la saillie du cervelet est prononcée, comme on le voit chez la bondrée commune (1). Le cervelet s'élève sensiblement au-dessus des hémisphères cérébraux chez le plus grand nombre des oiseaux de proie, chez le milan, l'orfraie, la bondrée, la buse, l'aigle (2). Chez le coq, la poule, le pigeon, les perdrix, le faisan doré et argenté, l'hirondelle (3), le cervelet dépasse ausși le niveau du cerveau. Chez l'oie, le canard ordinaire, le canard musqué, le fou de bassan, le cervelet est déjà plus affaissé; chez les pies, les bécasses, les étourneaux, les merles, les perroquets, l'alouette, le roitelet (4), il reste au dessous du niveau des hémisphères cérébraux.

Le cervelet de tous les oiseaux, sans exception, est sillonné par des rainures plus ou moins profondes, étendues en forme d'arc sur sa face supérieure : ces sillons divisent le cervelet en segmens parallèles les uns aux autres (5): les segmens les plus larges sont au milieu (6); les plus étroits sont à la partic postérieure (7), et à la pointe qui s'enfonce en arrière de la commissure des lobes

(1) Pl. IV , fig. $89, \mathrm{n}^{\circ} 6$.

(2) PI. XI, fig. 101, $n^{\circ} 4$.

(3) Pl. IV, fig. 106, $n^{\circ} 6$.

(4) Pl. IV, fig. 108, $n^{\circ} \%$

(5) Pl. IV, fig. $99, \mathrm{n}^{\circ} 2$.

(6) PI. IY, fig. $9^{5}, \mathrm{n}^{\circ} 3$ et 4 .

(2) Pl. IV, hig. 99. 
optiques. Les segmens sont en général proportionnés au volume du cervelet. Leur nombre est variable comme celui des sillons.

Tous ces sillons convergent les uns vers les autres en se détournant sur la facc latérale du cervelet (1); ce qui donne à cet organe, placé sur le côté, l'aspect des cannelures de certaines coquilles bivalves (2).

En général, plus les oiseaux sont grands, plus les sillons sont nombreux. Cette règle éprouve néanmoins beaucoup de variations à cause de la différence de largeur des segmens, et du rapprochenient plus ou moins grand des sillons entre eux. J'ai compté huit sillons chez le pinson, le moineau, le verdier, le chardonneret, le rougegorge, l'hirondelle (3); neuf chez le roitelet (4), dix chez la fauvette, le rossignol, le canard musqué; douze chez le canard ordinaire, la bernache, la bondrée commune (5), le pigeon, les perdrix; quatorze chez le coq, la poule, lc faisan doré et argenté, le grand milan, l'épervier, le perroquet d'Afrique (6), l'aigle royal (7), l'au-

(1) Pl. IV, fig. 97, $\mathrm{n}^{\circ} 4$.

(2) Pl. IV , Bg. 104, $n^{\circ} 4$.

(3) Pl. IV, fig. 106, n ${ }^{\circ} 8$.

(4) Pl. IV, fig. $108, n^{\circ}$ \%

(5) Pl. IV , fig. $89, n^{\circ} 6$.

(6) PI. III, 6g. $84, n^{\circ} 2$.

(5) PI IV, fig. $101 ; \mathrm{n}^{\circ} 14$. 
truche de l'ancien continent (1), les cigognes blanche (2) et noire, le pygargue, la buse commune; seize chez le casoar (3), le fou de bassan, et dix-huil chez le perroquet amazone. On' voit, d'après ce tableau, que le nombre des sillons du cervelet donnerait un rapport très-imparfait du volume de cet organe chez les oiseaux.

Mais ce nombre, qui, du reste, est sujet à beaucoup de variations chez les individus de même espèce, est toutefois important à constater, à cause de ses rapports avec les radiations médullaires de cet organe. Le nombre de ces radiations est toujours dans un rapport rigoureusement proportionnel à celui des sillons. Plus les sillons sont prononcés, plus les segmens qu'ils circonscrivent sont larges, plus sont fortes et étendues les radiations médullaires du cervelet, ainsi qu'on le remarque chez le casoar (4), le perroquet (5), la bondrée (6) et l'aigle royal ( 7$)$, après avoir incisé verticalement le cervelet d'avant en arrière, et dans toute son épaisseur.

La couche extérieure du cervelet des oiseaux

(1) PI. IV , fig. $96, n^{\circ} 4$.

(2) PI. IV , fig. $104, \mathrm{n}^{\circ} 4$.

(5) Pl. III, fig. $77, \mathrm{n}^{\circ} 3$.

(4) Pl. III, fig. $83, n^{\circ} 3$ et 4 .

(5) $\mathrm{Pl}$. III, fig. $84, \mathrm{n}^{\circ} 2$.

(6) Pl. LV, fig. 9o, $\mathrm{n}^{\circ} \mathrm{a}$

(7) Pl. IV , fg. 101, $\mathrm{n}^{\circ 3}$

II. 
est'formée par la matière grise, sa couleur est celle d'un rouge incarnat, ce qui, comme chez certains reptiles et les poissous, distingue cet organe des tubercules quadri-jumeaux. Cette matière grise est superposée sur la blànche : de même que dans les deux classes précédentes, celte dernière offre, chez les oiscaux, une disposition remarquable.

L.es pédoncules inféricurs du cervelet (1), dont le volume est toujours très-considérable (2), réunis aux supírieurs ( 3 ), qui, de même que chez les poissons, descendent de la partic internodes lobes optịucs, forment un cône médullaire (4) occupant le centre de toute la hauteur du cervelet des oiseaux. De ce cône, partent en avant, en arrière, sur les côtés et en haut; des radiations médullaires aplaties, minces, disposées horizontalement (5), de manière que de leur bord libre elles regardent la périphérie du cervelet, et que de l'autre elles adhèrent aux parois du cône qu'elles constituent par leur réunion. D’après cette disposition, si l'on fait au cervelet des oiseaux une coupe horizontalc au niveau de son tiers supérieur, on développe une espèce d'arbre de vie, comme le représente la

(1) Pl. III, fig. 85, $\mathrm{n}^{\circ} 16$.

(2) Pl. IV , fig. $101, \mathrm{n}^{\circ} 15$.

(3) PI. III, fig. $83, \mathrm{n}^{\circ} 14$; fig. $84, \mathrm{n}^{\circ} 8$.

(4) Pl. IV, fig. $90, \mathrm{n}^{\circ} 2$.

(5) PI. IV, fig. $90, \mathrm{n}^{\circ} 2$. 
préparation; si on incise le cervelet sul sa partie médiane (2), on divise cet arbre en deux (2); si, au contraire, on coupe le cervelet de manière que la section passe dans le milieu des faces latérales, on n'aperçuit ni arbre ni rameaux : on n'en distingue pas également ì la base du cône.

Ce cône, de substance médullaire, est creux (3); cette cavité est aussi conique : sa base plonge dans le quatrième ventricule, son sommet correspond au haut du centre du cervelet.

C'est un nouveau ventricule, particulier aux oiseaux, que Malacarne a le premier fail cornaître, et dont nous exposerons bientôt le mode de formation. Nous devons remarquer pour le moment que c'est précisément dans le bas de ce ventricule que se développe le corps ciliaire du cervelet, qui conséquemment n'existe pas chez les oiseaux. A la place que doit occuper le corps ciliaire, il y a un vuide, comme on peut le voir chezle roitelet (4), l'hirondelle (5), l'aigle royal (6), le perroquet (7) et le casoar. (8).

Le cervelet des oiseaux cst formé presqu'en en-

(1) PI. III, fig. $86, \mathrm{n}^{\circ} 2$.

(2) Pl. IV, fig. 101, no 3 , 4 et 14 .

(3) Voyez son ouverture, Pl. IV, fig. 90, $\mathrm{n}^{\bullet} 2$.

(4) Pl. IV , fig. $10 z, n^{\circ} \cdot 2$.

(5) Pl. IV, fig. $9^{3}, \mathrm{n}^{\circ} 2$.

(6) Pl. IV, fig. 10i, $n^{\circ} 3$.

(7) Pl. III, fig. 86, n० 3 .

(8) Pl. III, fig. $83, \mathrm{n}^{*} 15$ 
lier par le lobe médian qui correspond an res supérieur des mammifèrcs. Ce rapport, qui avait ćté entrevu par Halitr, fut rejeté par Malacarue, et négligé par les anatomistes qui le suivirent. De là vient quie dans celte classe cet organo s'élève souvent, comme nous l'avons vu, au-dessus des hémisphères cérébraux, et il les dépasse d'autant plus que le lobe médian se porte plus en haut. Cependant le lobe médian ne forme pas à lui seul le cervelet des oiseaux, on entrevoit déjà dans cetteclasseles hémisphères laléraux de cet organe, qui cn rendent l'organisation si compliquée chez les nammilères supérieurs.

Lies petits hémisphères se remarquent sur le flanc du demi-cône que présente la face externe du lobe médian, ils sont à peine visibles chez la poule, les canards, les oies, les beruaches, lecoq, le roitclet, le serin, le moineau; mais ils sont trèsprononcés chez les perdrix, les pigeons, l'hirondelle (1), lesoiseaux de proie, la bondrée (2), l'autruche (3), le casoar (4) et les cigogncs (5). En général, les oiseaux qui s'élèvent et se souliennent long-temps dans l'air, comme les cigognes, ceux dont les ailes ou les pieds ont une force prodigieuse,

(1) Pl. IV, fig. $106, \mathrm{n}^{\circ} 8$.

(2) Pl. IV , fig. $89, n^{\circ} 6$.

(3) Pl. IV, fig. $9^{5}, n^{\circ} 3$.

(4) Pl. III, fig. $77, n^{\circ} 3$.

(5) Pt. IV, fig. 99, n². 
comme le fou de bassan et les perroquels, sont ceux chez lesquels les hémisphères mont paru les plus développés.

Leur position offre des variations soumises aux variations des lobes optiques. Lorsque les lobes optiques sont portés très en arrière, les petits hémisphères sont rejetés en bas du cervelet, comme on le remarque chez les pordrix, les pigcons, les faisans, la buse, la pie, les aigles, la bondréc (z) et l'autruche (2). Mais à mesure que les lobes optiques se retirent et se portent au-dessous des lobes céróbraux, les hémisphères du cervelet s'élèvent, comme on le remarque déjà chez le casoar (3). Enfin chez le fou de bassan, le perroquet d'Afrique et les cigognes (4), les tubercules quadri-jumeaux s'étant tout-à-fait enforicés sous le cerveau, les hémisphères du cervelet sont veI: us rejoindre la partie postéricure des hémisphères cérébraux. I.e perroquet amazone est de tous les oiseaux que j'ai examinés, celui sur lequel cet effet est le plus marqué. Il est à remarquer que chez tous les oiseaux, sans exceplion, les silions des hémisphèes se continucnt immudiaicment avec ceax du lobe médian. On voil bonc quor les principales variations des hémisphères du

(1) Pl. IV, fig. 8 n.

(2) Pl. IV, lig. !)

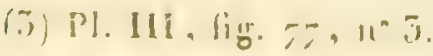

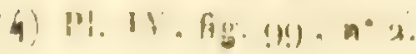


cervelet les oiseaux, de mênc que celles de leur lobe médian, sont rigourcusemeut soumises aux modifications que les lobes optiques éprouvent dans cette classe: on voit de plus qu'elles sont la répétition de celles que nous avons signalées dans le cours de l'incubation.

En outre, du lobe médian et de ces petits hémisphères latéraux, le cervelet des oiseaux offre sur la base de sa partie latérale deux peitis appendices tuberculeux (1), un de chaque côté. Ce pet it tubercule, quel'on trouve chez tous les oiseaux, est développé en raison directe de la masse du cervelet; il est superposé sur la facc externe des pédoncules de cet organe, immédiatement au-dessous des lobes optiques, et correspond aux sinus latéra ux du ven tricule du lobe médian du cervelet. Il est, à l'extérieur, d'un gris moins rosacé que le reste de l'organe: ses pelits sillons ne sont pas toujours concentriques à ceux du cervelet, ils ont souvent une direction opposée, comme on le voit chez la cigogne blanche (2), et comme souvent aussi nous le remarquerons chez les mammifères. Son centre est formé par un petit noyau de matièrc médullaire, d'où parlent de pclites radiations qui suivent la marche et la direction de ses sillons.

Puisque les tubercules lacéraux du cerrelet des oiseaux naissent des pédoncules du cervelet im-

(1) Pl. IV, fig. $104, n^{\circ} 15$.

(2) Pl. IV, fig. $104, n^{\circ} 15$ 
médiatement au-dessous des lobes optiques, on voit que, comme les hémisphères dont ils semblent être la racin'e, ils doivent s'élever ou s'abaisser, selon que ces lobes se portent en avant ou en arrière. C'est ce qui doit être, d'après la connexion des partics; c'est ce qui est, d'après l'observation directe. Chez les poules, les canards, les oies, les perdrix, les pigeons, les faisans, la pie, les aigles, la bondrée, oiseaux chez lesquels les lobes optiques se prolongent en arrière, de même que chez le casoar, les tuberculés latéraux sont, ainsi que chez ce dernier, descendus très-bas (1). Au contraire, ces tubercules viennent s'arlosser contre la base des hémisphères cérébraux, chez les perroquets (2), le fou de bassan, les cigognes (5), el en général chéz tous les oíseaux dont les lobes optiques, portés en avant, viennent se loger sous les hémisphères cérébraux. On voit donc que ces tubercules et les hémisphères suivent les mouvemens des lobes optiques; ces derniers semblent entraîner les premiers dans leurs divers mouvemens. Ce mouvement du cervelet est déterminé peut-être par la liaison des lohes optiques avec les pédoncules supérieurs de cetorgane : en effet, chez tous les oiseaux ces pédoncules se continuent avec les parois des lobes optiques (4), en passant au-

(1) PI. III, fig. $77, \mathrm{n}^{\circ} 5$.

(2) HI. IV , fig. $96, n^{\circ} 12$.

(3) PI. IV , fig. 104, $n^{\circ} 15$.

(i) Pl. III , fig. 8í, $n^{\circ} 5$. 
dessous de la partie postérieure de leur commis. sure. De la partie interne de chaque pédoncule se détache la valvule de Vieussens, véritable commissure de ces gros faisceaux (1). A cause de la position de ceux-ci, on voit que cette dernière commissure est sur un plan inférieur à celui de la commissure des lobes optiques. Sa couleur est d'un gris qui ressemble à la lame cornée des mammifères. Elle présente chez beaucoup d'oiseaux plusieurs segmens de matière grise, qui s'enfoncent dans le haut du quatrième ventricule, et y forment quelquefois un petit mamelon situé audessous de la partie antéricure du cervelet.

Le cervelet est, de toutes les parties de l'encéphale, celle qui a le plus occúpé les anatomistes. Depuis le médecin Pourfour-Petit, qui a eu la patience de compter les feuillets qui le composent, et qui le premier a beaucoup, accru le nombre des lobes que les anatonistes y observaient avant lıi ; depuis Malacarne, qui, avec plus de patience que Pourfour-Petit, a multiplié plus cricore et le nombre des lobes et le nombre des feuillets de cet organe, les anatomistes ont porté unc attention-sérieuse à son organisation compliquée. M.r. Chaussier, les frères Wenzel, Meckel. Reil, Carus, Gall, ont présenté à ce sujet des aperçrs ingenieux et piquans, qui n'ont pas donné la solution de la question, parce que lion bornait

(1) Pl. III, fig. 85, ri" lí. 
trop la question au cervelet de l'homme, et presque loujours au cervelet de l'homme adulte. Un hasard heureux pouvait seul y conduire dans celte direction; et l'on sait que ces résultats heureux sont rares dans les sciences, quand une bonne méthode d'observation ne dirige pas l'esprit. En effet, on a cherché à expliquer le cervelet le plus compliqué de tous, celui de l'homme, avant de s'être rendu compte de celui des mammifères qui Iui est inféricur; de celui des oiseaux, qui est moins compliqué que celui des deruiers mammifères; avant même d'avoir trouvé l'explication du cervelet des poissons et des reptiles, qui est plus simple encore que celui des oiseaux. Il n'est donc pas étonnant que l'on ait échoué dans cette tentalive; il l'eût été davantage que l'on y eût réussi.

La mélhode inverse, joiute à l'élude approfon die du cervelet dans les diverses périodes de la formation des embryons, nous place sur la seule et véritable route qui puisse nous conduire à l'cxplicalion difficile du cervclet des mammifères, et qui puisse nous y conduire par l'observation, dégagée de toute hypothèse. Jusqu'à présent l'état primilif du cervelet des embryons nous a fait concroir celui des reptiles, des poissons osseux et cartilagineux; lo cervelet de la tortue franche nous a donné la clef de celui des squales, tels quo les grisuls ct les milanilres; ceux-ci nous ont conduit an wrun, qui nous a offert un verilable cervelet d'ni5r.u. X.1s sommes arrivé, conduii pour ainsi 
dire par la nature, au cervelet déjà compliqué de la seconde classe des vertébrés, et nous avons trouvé dans son organisation même la source et la cause des variations qu'il nous a présentées dans les diverses familles de cette classe. Nos explications ne sont donc que des comparaisons : nous appliquons ì une classe les faits et les rapports que nous avons aperçus dans celle qui la précède; nous marchons ainsi avec des faits et des rapports connus, sur des rapports et des faits qui nous restent à connaître. C'est ainsi que nous allons procéder à l'explication du cervelet des mammifères, en commençant par les inférieurs, et remontant vers les supérieurs.

Le cervelet des mammifères inférieurs, tels que les rats, les chauve-souris (1), les didelphes, etc. (2), est remarquable par l'écartement qui existe entre cet organe et les hémisphères cérébraux (3). On croirait, au premier examen, que leur encéphale appartient à un développement inférieur à celui des oiseaux, ce qui n'est pas. A quoi tiennent douc ces apparences? Quelle est la cause de cet écartement? C'est ce que nous allons chercher à apprécier pour le cervelet.

Nous avons fait remarquer chez les oiseaux que

(1) Pl. IX, fig. $215, \mathrm{~B}$.

(2) Pl. II , fig. 55, ri 4; fig. 54, n` 6 .

(3) PI. IX, fig. 206, D, F. 
Iorsque les lobes optiques dépasseut les lobes cérébraux, les hémisphères du cervelei sont rejelés en arrière (Qu'arriverait-il dans ce cas, si les tubercules quadrijumeaux s'étendaient sur la ligne médians; si, au licu de la dépression formée par leur commissure, il y avait une proémincnce? Il est évident que chez les oiseaux, de mêtne que cela a licu chez le requin, tout le cerrelet serait repoussé en arrière et maintenu à unc distance plus ou moins grande du cerveau. Cet effet serait produit pour le lobe médian, comme il a lieu pour les hémisphères et les tubercules latéraux du cervelet; on verrait alors les lobes optiques des oiseaux interposés transversalement entre ce dernicr organe et le cerveau. $\mathrm{Or}$, ce qui ne se remarque pas chez les oiseaux, à cause de la position latérale des lobes optiques et de la dépression centrale de leur commissure, produitc par leur demi-rotation, existe chez les mammifères inférieurs, où ce déplacement n'ayant point lieu, les tubercules quadrijumeaux encore très-développés restent à leur place, et servent cu quelque sorte de barrière à la progression du cervclet en avant, comme ils arrêtent la marche res lobes du cerveau en arrière. Que doit-il résulter de là? Il doit en résuiler nécessairement que les iubercules quadrijumcaux restent en grande partie à découvert sur la face supérieure de l'encéphale, comme nous l'avons déjà dit, et 
comme on le voit chez le rhinolophe unifer (1), chez le vespertilion murin (2) et chez toutes les chauve-souris, chez le zemni. (3), la marmotte (4) et la plupart des rats, chez le didel phe de Virginie (5), la marmose et les dasyures, le tatou à six bandes, le fourmilier à deux doigts, etc. Ce caractère spécifique de l'encéphale de ces animaux n'est lui-même que la reproduction d'un état embryonnaire du lapin (6), du veau (7) et de l'homme (8), à l'époque où les tubercules quadrijumeanx sont très-saillans. La même cause produit les mèmes effets chez les animaux adultes et les embryons.

De plus, en ce qui concerne le cervelet, ces manmiferes se rapprochent des oiseaux, chez les. quels les lobés optiques débordent le cerveau et arrêtent le cervelet; mais ils en diffèrent par des particularités très-remarquables, dépendantes de la différence des tubcrcules quadrijumeaux dans les deux classes. Chez les vistaux l'affaissement de leur partie moyenue permet au lobe médian du

(1) PI. IX, fig. $206, \mathrm{~K}$.

(2) PI. IX, fig. $215, \mathrm{~B}$

(3) Pl. XV, fig. $270, n^{\circ} 2$.

(4) PI. IX, fig. $207, \mathrm{~T}$.

(5) PI. II, fig. 53, $1^{\circ} 4$.

(6) Pl. II, fig. $56, \mathrm{n}^{\circ} 5$.

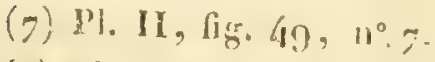

(8) IP. II, lig. 68, 11". 
cervelet de se porler en avant, les hémisphères seuls sont arrêtés et tenus à distance du cervertu, ì cause de la saillie des lobes optiques sur les cồ'és. Chez ces mammifères, au cøntraire, les tubercules quadrijumeaux sont saillans sur le milieu et affaissés sur les côtés. On voit déjà ce qui va résulter de cette disposition inverse. On voit que chez les mammilères ce sera le lobe médian qui sera rejeté en arrière, et les hémisphères qui se porteront en avant. Nulle part cette disposition curieuse du cervelet des mammifères inférieurs n'est plus marquée que chez les chauve-souris (1). Chez le rhisolophe unifer et le vespertilion murin le lobe médian du cervelet (2) est rejeté très en arrière, et les hémisphères, portés en avant (3), rejoignent la partie postérieure du cerveau. C'est l'inverse des oiseaux. Il résulte de là que le bord antéricur du cervelet forme une courbure elliptique dans la concavité de laquelle sont logés les tubercules quadrijumeaux; cette courbure, trèsmarquée chez les chauve-souris, est très-prononcée aussi chez le didelphe, la marmose et le tatou à six bandes, dont le lobe médian est considérablement rejeté en arrière, quoique les hémisphères, très-développés, soient déjả recouverts par les lobes cérébraux. Remarquons un autre contraste bie $\mathbf{n}$

(1) PI. IX, fig. 206 ett 215.

(2) PI. IX, fig. 206, C ; fig. 215, C.

(3) Pl. IX, fig. 206, D; fig. $215, \mathrm{~B}, \mathrm{~A}, \mathrm{C}$. 
singulicr dans la disposition de ces organes chez les oiseaux et che\% crs mammifères. Chez les oiseaux, les tubercules quadrijum eaux forment une courbure clliptique, concave en arrière, et dans laquelle vient se loger le lobe médian du cervelet : chez les mammifères, au contraire, c'est le cervelet qui forme celte courbure concave en avant, et dans laquelle viennent se placer à leur tour les tubercules quadrijumeaux. Dans le premier cas, le lobc médian du cervelet est inévitablement porté en avant, comme il cst inévitablement aussi porté en arrière dans le second. Les différences du cervelet qui en résultent dans les deux classes sont trèssaillantes, quoique la cause qui les produit soit d'une simplicité véritablement admirable.

Mais à mésure que les tubercules quadrijumeaux diminuent de volume, la courbure elliptique du bord antérieur du cervelet se redresse, et le lobe médian de cet organe se porte en avant à la rencontre des hémisphères cérébraux : on suit cette diminution des tubercules, et cette marche progressive du cervelet, chez la marmose, le fourmilier à deux doigts, le zemni (1) et la marmotte (2). Par ce moyen le lobe médian (3) prend donc peu-à-peu la position qu'il occupe chez tous les oiseaux; plus il s'avance vers le cer-

(1) Pl. XV, fig. $250, \mathrm{n}^{\circ} 2$ et 4.

(2) Pl. IX, fig. $207, \mathrm{~F}, \mathrm{~A}, \mathrm{C}$.

(3) Pl. IX, fig. 20g, C. 
veau, moins il se prolonge en arriere, comme on peut le voir en comparant celte partie du cervelet, chez les chauve-souris, le tator, la marmose, le zemni et la marmotte.

C'est par suite de ce mécanisme que le lobe médian du cerrelet parvient à sa place; mais il n'y parvieut et ne peut y parvenir qu'en chassant devant Jui les tubercules quadrijumcaux. L'effet de cette progression est donc de faire disnaraître ces tubercules de la face supéricure de l'encéphale en les enfonçant sous les hémisphères cérébraux, de même que nous l'avons obscrvé chez les oiseaux, toujours avec celte différence, que ce changement est produit par les hémisphères du cervelct, dans cette dernière classe, et par le lobe médian chez les mammifères. On peut remarquer en effet que les tubercules quadrijumeaux deviennent de moins à moins visibles, à partir des chauve-souris (1), des rats, des didelphes, du tatou, au fourmilier, au zemni (2), à la marmotte (3), et enfin au castor (4), mammifére chez lequel ce mouvement s'arrête, et où pour la dernière fois, dans cette classe, on distingue encore entre le cervelet et les hémisphères cérébraux une petite partie des tubercules quadrijumeaux.

Si comme déjà nous l'avons remarqué, la nudité

(1) Pl. IX, fig. 206 et 215.

(2) Pl. XV , fig. 270.

(3) PI. IX, fig. 207.

(4) PI. XIV, fig. $265, \mathrm{C}$. 
des tubercules quadrijumeaux, chez ces mammifères, répète un état embryonnaire, nous devons faire observer maintenant que leur disparition graduelle de la facesupérieu re de l'encéphale reproduit le même phénomène que nous avons suivi dans les diverses périodes de la formation des embryons, et le reproduit par les mêmes causes. Ainsi, sous ce rapport, les chauve-souris correspondent à l'embryon du lapin du quinzième jour, et à l'embryon humain du quatrième mois; les rats, le zemni, les didelphes et le tatou, conservent d'une manière permanente la disposition transitoire de l'embryon de l'homme du cinquième mois, de celui du lapin du vingtième jour. Enfin, après le vingt-cinquième jour de la formation du lapin, et a la fiu du sixième mois de l'homme, le cervelet et le cerveau cachent presqu'en totalité les tubercules quadrijumeaux, comme on le voit chez la marmotte et le castor. Causes et effets, tout se ressemble, tout se répète chez les embryons et les animaux adultes.

Lorsque chez les oiseaux les tubercules quadrijumeaux ont tout-à-fait disparu de la face supérieure de l'encéphale, les hémisphères du cervelet s'avancent à la place qu'ils occupaient, se rapprochent, comme chez les cigognes (1) et le fou de bassan, du niveau de la partie antérieure du lobe médian, et quclquefois se placent sur la même

(1) PI. IV , fig. 99. 
ligne que lui, comme on l'observe chez le perroquet amazone. Il est à remarquer que pendant que ce mouvement s'opère chez les oiseaux, les hémisphères acquièrent de l'accroissement, et le lobe médian diminue au contraire de volume. C'est ce double mouvement, si simple chez les oiseaux à cause de la faiblesse des hémisphères du cervelet, qui, se reproduisant d'une manière plus compliquée chez les mammifères, diversifie de tant de manières la surface externe du cervelet des rongeurs, des carnivores, des pachydermes et des ruminans.

Aussitôt, en effet, que les tubercules quadrijumeaux ont cessé d'être visibles extérieurementchez les mammifères, on voit le lobe mérlian se porter en avant, se loger, comme chez les oiseaux, dans une échancrure formée par l'écartement des hémisphères cérébraux, laissant ainsi en arrière de lui les hémisphères du cervelet. Cette tendance du lobe médian du cervelet à se porter en avant, déjà manifeste chez la marmotte (1), plus encore chez le castor (2), est si prononcée chez le kanguroo géant (3) et l'agouti (4), que, comme chez les cigognes, les hémisphères du cervelet restent en arrière de lui. Puis chez le lièvre, le lapin (5), le

(1) P1. IX, fig. 20 \%.

(2) Pl. XIV, fig. 265.

(3) Pl. XIII, fig. 250.

(4) P1. IX, fig. 213.

(5) Pl. II, fig. 5 y.

II. 
daman (1), le porc-épic (2), le lérot (3), les hémisphères du cervelet acquérant de l'accroissement, leurs rapports antérieurs avec le lobe médian deviennent semblables à ceux du cervelet $d u$ fou de bassan et du perroquet amazone.

Cics rapports entre le cervelet des oiseaux et celui de ces mammifères tiennent à une cause première, identique dans les deux classes. Qu'est-ce qui détermine la forme générale du cervelet des oiseaux? C'est évidemment la prédominance du lobe médian sur les hémisphères du cervelet. $\mathrm{Or}$, c'est la prédominance du lobe médian qui se continue chez ces mammifères, et qui donne à leur cervelet une physionomie qui le rapprochẹ de celui des oiseaux. Remarquez, en effet, que chez les chauve-souris, les rats, le fourmilier à deux doigts, la marmotte (4), le daman, le kanguroo géant, le porc-épic, et surtout l'agouti (5), la forme du lobe médian du cervelet est celle d'un demi. cône ainsi que chez les oiseaux; ses rainures se continuent, de mème que dans cette dernière classe, avec ceux des hémisphères du cervelet; enfin le ventricule du lobe médian, caractère classique des oiseaux, se retrouve en petit dans le lobe médian des mammifères inférieurs.

(1) Pl. XV , fig. 269.

(2) Pl. XIII, fig. 252.

(3) Pl. XV, fig. $2 ; 6$.

(4) Pl. IX, fig. 207, Co

(5) Pl. 1X, fig: $210, \mathrm{C}$ 
Après avoir déterminé ces analogies et montré la transition du cervelet des oiseaux à celui des mammifères, comme nous avions indiqué celle des reptiles aux poissons, et le passage des poissons cartilagineux aux oiseaux, indiquons d'une manière générale les modifications que cet organe éprouve dans la classe supérieure des vertébrés, et cherchons, s’il est possible, à en assigner les causes. II est presque inutile de dire que ce sont les mêmes que celles que nous avons suivies chezles embryons, et qui diversifient aussi de tant de manières le cervelet des différentes époques de la gestation des mammifères supérieurs. A quoi tiennent toutes ces variations embryonnaires? Elles sont évidemment le résultat de l'antagonisme qui s'établit entre le lobe médian du cervelet et les hémisphères du même organe. D'abord le lobe médian domine Tes hémisphères, puis les hémisphères égalent le lobe médian, puis le lobe médian est dominé par les hémisphères. Parcourez mainteuant l'ensemble des formes permanentes du cervelet chez les mammifères, et vous verrez cet antagonisme se reproduire des chauve-souris et des rongeurs, aux pachydermes, aux ruminans, aux carnivores, aux quadrumanes, aux phoques, aux cétacées et à l'homme.

En effet, chez les chauve-souris le lobe médian du cervelet ( 1 ) est très-développé en comparai-

(1) Pl. IX, fig. 206, C, D. 
son des hémisphères (1); chez la taupe (2), le lapin (3), la marmotte (4), le kanguroo-géant (5), le porc-épic (6), la mangouste du Cap (7), l'unau (8), le lérot (9), l'agouti (10), c'est toujours le lobe médian qui forme la partie principale de la face externe du cervelet; chez le daman ( 11 ), le coati roux (12), la marte (13), le lobe médian est égal aux hémisphères; chez le castor (14), les hémisphères dépassent déjà le lobe médian; chez le bouc de la haute Égypte (15), le chien, le chat, le lion (16), la loutre (17), l'ours (18), le chameau à deux bosses (19), le cheval (20), le pé-

(1) Pl. IX, fig. $215, \mathrm{C}, \mathrm{B}$.

(2) Pl. XIV, fig: 256, C.

(3) Pl. II, fig. $57, \mathrm{n}^{\circ} 2$.

(4) Pl. IX, fig. $207, \mathrm{C}$,

(5) P1. XIII, fig. 250, C.

(6) PI. XIII, fig. $252, \mathrm{C}, \mathrm{B}$.

(7) PI. XI, fig. $232, \mathrm{C}$.

(8) PI. XI, fig. 229, B.

(9) Pl. XV, fig. $269, n^{\circ} 2$.

(10) Pl.'IX, fig. 213 , B.

(11) Pl. XV, fig. $269, n * 2$.

(12) Pl. XII, fig. $240, n^{\circ} 5$.

(13) PI. XV, fig. $278, \mathrm{~B}$.

(14) Pl. XIV, fig. 265 , B. D.

(15) Pl. XIV, fig. $261 ; \mathrm{B}, \mathrm{n}^{\circ} 3$ et 4 .

(16) Pl. XIV, fig. 264, $n^{\circ} 2$ et 3.

(17) Pl. X, fig. $220, \mathrm{n}^{\circ} 1$ et 3 .

(18) Pl. XI, fig. 230 , B. C. D.

(19) PI. XIII, fig. 248, B.

(20) Pl. XV, fig. 274, E, C, K. 
cari (1), le lama (2), le mouton (3), la prédominance des hémisphèrés sur le lobe médian devient de plus en plus marquée. Chez le phoque (4), le dauphin (5) et les singes (6), les hémisphères forment la partic principale du cervelet; le lobe médian, réduit à peu de volume, ne constitue plus qu'une partie accessoire de l'organe. Enfin, chez l'homme, les hémisphères sont si développés, et le lobe médian est tellement réduit dans ses dimensions, qu'à peine a-t-il été considéré comme un des élémens du cervelet. Cette force du lobe médian coïncide avec le volume du pédoncule supérieur du cervelet : plus le lobe médian est développé, plus ce pédoncule augmente; d'où il suit que son accroissement est donné par les dimensions du lobe médian de l'homme aux rongeurs. Les faisceaux composant ce pédoncule se continuent supérieurement avec les tubercules quadrijumeaux, ainsi que je l'ai représenté chez l'agouti (7); d'où il suit encore que les conditions d'existence du lobe médian, de son principal pédoncule et des tubercules quadrijumeaux, doivent
(1) Pl. XVI, fig. 290, C. D.
(2) PI. XVI, fig. 294, R. A. D.
(3) Pl. X, fig: $221, \mathrm{D}, \mathrm{E}, \mathbf{n}^{\circ} 3$ et 4 .
(4) Pl. IX, fig. 209, C, B, C.
(5) Pl. XI, fig. 235, B, C.
(6) Pl. VIII, fig. 195, B, C, D; Fig. $19^{8}, \mathbf{B}, \mathrm{E}, \mathbf{P}$.
(7) Pl. IX, fig. $205, \mathrm{n}^{\circ} 5$. 
suivre et suivent en effet le même rapport de l'homme aux singes, aux carnassiers, aux ruminans et aux rongeurs.

Si cela est, on voit donc la raison pour laquelle les tubercules quadrijumeaux n'ont pas disparu chez la taupe, le zemni, la musaraigne, le rattaupe du Cap, quoiqu'ils n'ayent plis de nerf optique; puisque le lobe médian et le pédoncule supérieur du cervelet ont conservé leurs dimensions, les tubercules quadrijumeaux ne pouvaient cesser d'exister chez ces animaux.

De la face interne des pédoncules supérieurs se détache la lame médullaire nacrée, désignée sous le nom de valvule de Vieussens. Cette lame suit et doit suivre le rapport du lobe médian, des pédoncules supérieurs et des tubercules quadrijumeaux: ce qui est en effet, comme on l'observe de l'homme aux rongeurs et aux insectivores.

On conçoit que ce balancement alternatif dans les dimensions du lobe médian et des hémisphères du cervelet ne peut s'établir sans que leur forme extérieure soit modifiée; et il est inutile de remarquer que ces variations doivent nécessairement en produire qui leur correspondent dans la configuration générale du cervelet. Pour apprécier les diversités de cet organe chez les mammifères, il faut donc les considérer dans les parties qui les constituent.

Nous avons vu que chez les oiseaux le lobe médian n'étant nullement gêné dans son déve- 
loppement à cause de la faihlesse des hémisphères, sa forme est celle d'un demi-cône bombé à sa partie moyenne et plus effilé à ses deux extrémités; nous arons remarqué aussi qu'il conserve en partie cette même disposition chez les mammifères inférieurs, et qu'il la conserve par les mêmes raisons. Mais à mesure que les hémisphères se développent, la forme du lobe médian change. Le premier changement qui se manifeste, est celui de l'aplatissement de sa partie moyenne: cette partie, qui 's'élevait au-dessus deșémisphères chez la taupe (1), la marmotte (2) et l'agouti (3), s'affaisse, comme on le remarque chez le lièvre, le lapin, le daman (4), l'unau (5) et la mangouste du Cap (6); le lobe médian est alors au même niveau que les hémisphères. Malgré cet affaissement il perd peu de sa prédominance, par la raison qu'il s'élargit et qu'il gagne latéralement ce qu'il perd en hauteur; disposition que l'on remarque surtout chez la mangouste, le porc-épic ( 7 ), l'unau, le coati roux (8), et jusque chez la marte $(9)$.

(1) P1. XV, fig. $256, \mathrm{C}$.

(2) Pl IX, fig. 207, C.

(3) Pl. IX, fig. 213, C.

(4) Pl. XV, fig. $269, n^{\circ} 2$.

(5) Pl. XI, fig. $229, \mathrm{~B}$.

(6) Pl. XI, frg. $232, \mathrm{C}$.

(7) Pl. XIII, fig. 252, C.

(8) PI. XII, fig. $240, n^{\circ} 4$.

(9) Pl. XV, fig. $278, \mathrm{~B}$. 
Mais cet effet n'a lieu que tout autant que les hémisphères du cervelet ne prennent pas un grand accroissement. Aussitôt que par cet accroissement, les hémisphères dépriment le lobe médian, celui-ci est comprimé : placé entre les deux hémisphères qui le pressent latéralement, et ne pouvant plus remonter, il présente d'abord des dépressions sur ses côtés, dépressions dans lesquelles s'enfoncent les hémisphères; puis les hémisphères sc développent inégalement, l'un prenant plus d'accroissement que l'autre; le lobe médian est détourné et déjeté sur les côtés, de droite ou de gauche, selon l'hémisphère ou la partie de l'hémisphère qui se développe le plus. Enfin complètement dominé chez le phoque, les cétacées, le singe et l'homme, il est réduit à ne former qu'un appendice sur la face supérieure de l'organe, appendice que l'on a nommé vermiculaire, à cause des sillons transverses qui le divisent.

Les dépressions latérales du lobe médian sont en arrière chez la mangouste du Cap, parce que c'est à l'union de son tiers postéricur avec les deux tiers antérieurs que les hémisphères, se développent le plus (1). Le lobe médian, large en avant et rétréci à son tiers postérieur, est plus mince en arrière. Chez l'ours, au contraire, l'étranglement est en avant (2); le lobe médian,
(1) Pl. XI, fig. 232, B, D.
(2) PI. XI, fig. 25o, C. 
rétréci en cet endroit, est dilaté en arrière. Chez le castor, l'étranglement a lieu au milieu (1); le lobe médian, dilaté en arrière et en avant, présente un rétrécissement à sa partie moyenne. Chez le mouton, la chèvre, le bouc de la HauteÉgypte (2), le lobe médian est ondulé, il se déjette alternativement à droite et à gauche; chez le pécari (3), le lama (4), le chameau à deux bosses (5), il forme une courbure très-marquée. Cette courbure est aussi très-sensible chez les carnassiers, le chat, le chien, le renard, le loup, la marte (6), le raton (7), le lion (8). Dans cette famille celte courbure du lobe médian offre de grandes variations, selon l'âge des animaux. Sur plusieurs chats et plusieurs chiens aucun ne l'avait semblable : sur quatre cerveaux de lion que j'ai examinés, et dont trois sont encore au cabinet du Jardin du Roi, elle différait sur deux : un n'en avait pas, c'était le plus jeune. Chez le cheval, la courbure du lobe médian est double : ce qui le rapproche de la forme d'une S (9). Enfin,

(1) PI. XIV, fig. 265, B, ñ 2 .

(2) Pl. XV , fig. $261, B$.

(3) Pl. XVI, fig. $29^{8}, \mathrm{C}$.

(4) Pl. XVI, fig. 29' , R.

(5) P]. XIII, fig. $248, \mathrm{~B}$.

(6) Pl. X V , fig. $378, \mathrm{~B}$.

(7) Pl. VIII, fig. 202, C.

(8) PI. XIV, fig. $264, n^{\circ} 2$.

(9) Pl. XV, fig. 274, E, C. 
quoique très-réduit chez le phoque, le dauphin (1), le mandrill (2) et le drill (3), il est néanmoins encore légèrement ondulé.

Ces variations du lobe médian sont la cause de la non-symétrie de la face supérieure du cervelet de certains mammifères. Cet organe est symétrique chez tous les poissons, chez tous les reptiles et chez tous les oiseaux, c'est-à-dire que l'un de ses côtés est la répétition exacte de l'autre. Cette symétrie-se conserve chez les insectivores et les rongeurs, et chez certains carnassiers, comme chez les ours. Elle disparaît dans le genre félis, chez la plupart des ruminans, quelques singes, le phoque, les cétacées et l'homme.

Ainsi, plus on s'élève des mammifères inférieurs aux supérieurs, plus le lobe médian s'atrophie, plus les hémisphères du cervelet se developpent et se compliquent.

Chez le didelphe (4), les rats, les chauvesouris (5), l'unau (6), la mangouste du Cap (7), la taupe (8), un seul lobe forme la face supérieure des hémisphères du cervelet. Chez

(1) Pl. XI, fig. $225, \mathrm{~B}$.

(2) Pl. VIII, fig. 195, C.

(3) PI. VIII, fig. $198, \mathrm{C}$.

(4) PI. II, fig. $53, \mathrm{n}^{\circ} 3$.

(5) PI. IX, fig. $215, \mathrm{~B}$.

(6) Pl. XI, fig. 229, B, C.

(7) Pl. IX, fig. 232, D, C, D。

(8) Pl. XIV, fig. $256, \mathrm{D}$. 
l'agouti (1), le lièvre, le lapin (2), le porcépic (3), la marmotte (4), le zemni (5), le coatiroux (6), la marte $(7)$, le daman (8), le raton (9), le tatou (10), le hérisson (11), on en remarque deux de chaque côté du lobe médian. Chez le mouton (12), la chèvre, le bouc de la Haute-Égypte (13), le pécari (14), le cheval (15), le chat, le chien, le loup, le renard, l'ours (16). le tigre, le lion (17), il y en a trois. Chez le castor (18), le kanguroo géant (19), le lama (20), on en distingue quatre. Il y en a cinq sur chaque

(1) Pl. IX, fig. 213, C, D.

(2) Pl. XI, fig. $57, \mathrm{n}^{\circ} 2$ et 3 .

(3) Pl. XIII, fig. $252, \mathrm{~B}, \mathrm{C}$.

(4) Pl. IX, fig. $207, \mathrm{D}, \mathrm{F}$.

(5) PI. XV, fig. 2 go, D, C.

(6) Pl. XII, fig $240, n^{\circ} 3$ et 4 .

(7) Pl. XV , fig. $278, \mathrm{~B}, \mathrm{C}$.

(8) Pl. XV, fig. $269, n^{\circ} 3$ et 4 .

(9) Pl. VIII , fig. 202, B , C.

(10) P1. XIII, fig. $244, n^{\circ} 2$ et 3.

(i1) Pl. XVI, fig. $296, B, D$.

(12) Pl. X, fig. 22r , E, n 3 et 4 .

(13) Pl. XIY, fig. 261, $\mathrm{n}^{\circ} 2,3$ et 4 .

(14) Pl. XVI, fig. $29^{8}, \mathrm{D}, \mathrm{C}$.

(15) Pl. XV, fig. 254, B, K, D, C.

(16) Pl. XI, fig. $230, \mathrm{~B}, \mathrm{C}, \mathrm{A}, \mathrm{C}$.

(17) PI. XIV, fig. 264, $\mathrm{n}^{\circ} 2,3$ et 4 .

(18) Pl. XIV, fig. $265, \mathrm{D}, \mathrm{n}^{\circ} \mathrm{5}$ et 4 .

(19) Pl. XIII, fig. $250, B, C, A, C$.

(20) Pl. XVI, fig. 294, D, C, A, D. 
hémisphère chez le phoque (1), le dauphin (2), le chameau à deux bosses (3), les singes (4) et l'homme.

Ainsi, en y comprenant le lobe médian, la face externe et supérieure du cervelet est formée d'abord de trois lobes, puis de cinq, puis de sept, puis de neuf, et enfin de onze lobes. Les lobes composant les hémisphères du cervelet sont très-inégaux entre eux, chácun d'eux prédomine tour-à-tour sur son voisin; et de là résulte encore une nouvelle source de variétés extérieures pour cet organe; variétés produites par la position des rainures plus ou moins profondes qui séparent ces lobes, et par la direction différente des sillons qui parcourent leur superficie.

Le nombre des lobes composant la face supérieure des hémisphères du cervelet se reproduit exactement sur sa face inférieure, de telle sorte que l'une dés faces est la répétition de l'autre. Il y a cependant cette différence, que sur la face inférieure tous les lobes sont rangés concentriquement les uns aux autres et d'une manière assez régulière. Chaque lobe est séparé de son voisin par un sillon en forme d'arc, dont la concavité regarde le bulbe rachidien ou le pont de

(1) Pl. X, fig. $216, n^{\circ} 3$.

(2) Pl. XI, fig. 225, C, A, D.

(3) Pl. XIII, fig. $248, \mathrm{~B}, \mathrm{C}, \mathrm{I}$.

(4) Pl. VIII, fig. $19^{5}, \mathrm{~B}, \mathrm{C}, \mathrm{P}$. 
Varole. Pour connaitre la complication des hémisphères du cervelet, c'est donc cette face qu'il importe surtout de considérer, et non la partie supérieure, comme on l'a fait jusqu'à ce jour.

Ainsi, "d'après ce principe, on compte cinq lobes sur la face intérieure de chaque hémisphère dans ce cervelet de l'homme, des sínges, du chameau à deux bosses (1), du dauphin (2) et du phoque (3) ; quatre chez le kanguroo (4), le castor (5) et le lama (6); trois chez le lion (7), l'ours (8), le cheval (9), le pécari (10), le mouton, le bouc de la Haute-Égypte (11); deux chez le daman (12), le coati roux, la marte (13), le raton (14), le zemni (15), la marmotte (16), l'agou-

(1) Pl. XIII, fig. 24, 8, B, C, I.

(2) PI. XII , fig. $234, \mathrm{~A}, \mathrm{~B}, \mathrm{C}$; D, $\mathrm{O}$.

(3) Pl. IX, fig. $208, \mathrm{n}^{\circ} 8,9,12$ et 14.

(4) Pl. XVI, fig. $299, n^{\circ} 8$.

(5) PI. XIV, fig. $258, n^{\circ}$.

(6) Pl. XVI, fig. $295, \mathrm{~N}$.

(7) Pl. XIV, fig. $266, \mathrm{~A}$.

(8) Pl. XI, fig. $23 i, n^{\circ} 8$.

(9) Pl. XV, fig. 274, $\mathrm{n}^{\circ} 8$.

(10) Pl. XVI, fig. $300, \mathrm{n}^{\circ} \mathbf{1} 3$ et 8.

(11) Pl: XIV, fig. $262, n^{\circ} 8$ bis.

(12) Pl. XV, fig. $273, \mathrm{~B}$.

(15) Pl. XV, fig. $290, \mathrm{n}^{\circ} 8$.

(14) Pl. VIII, fig. 200, N, A, C.

(15) Pl. XV, fig. $272, n^{*} 8$.

(16) Pl. IX, fig. 203, $0, \mathrm{n}^{\circ} 8$ bi6 
ti (1) ; et un seul chez les chauve-souris (2), les rats, la taupe (3). Quelquefois, cependant, chez ces mammifères inférieurs, on aperçoit à la face inférieure deux lobes, quand il n'y en a cncore qu'un de visible sur la face supérieure; mais alors le second est rudimentaire, et si faible, que son relief n'a pu être marqué sur cette dernière face.

Cette complication régulière de la base du cervelet pourrait servir de base à la division des familles de cette classe, d'une manière plus positive que celle de la face supérieure, qui varie de genre à genre, souvent d'espèce à espèce, quelquefois même, comme chez les félis, d'individu à individu. Tréviranus a néanmoins cru que les mammifères pourraient être classés d'après les variations de la face supérieure du cervelet, ce qui me paraît difficile, d'après les observations que j'ai faites.

Une particularité que nous ne devons pas omettre, quoique j'en aie en vain cherché la cause, c'est que le cervelet de l'homme présente seul le petit lobule que Malacarne a désigné sous le nom de tonsillaire. Je ne l'ai aperçu ni chez les singes, ni chez les phoques, ni même chez les cétacées, dont le cervelet est si développé (4). La présence

(1) Pl. IX, fig. 211, $n^{\circ} 7,9$ et 8.

(2) PI. IX, fig. $214, n^{\circ} \%$.

(3) Pl. XIV, fig. 260 , C.

(4) $\mathbf{Y}$ aurait-il quelque rapport entre le développement considérable du cervclét chez le phoque et la faiblesse de ges 
de ce lobule coïncide chez l'homme avec les stries blanchâtres du quatrième ventricule, quoiqu'il n'y ait aucun rapport immédiat entre ces deux parties.

On se tromperait beaucoup cependant, si l'on croyait que la masse des hémisphères du cervelet s'accroît chez les mammifères en raison du nombre des lobes qui entrent dans leur composition. Cela serait, si, à mesure qu'un nouveau lobe paraît, ceux qui l'ont précédé conservaient le même volume. Mais en général les lobes semblent se développer aux dépens les uns des autres: si l'un prend un grand accroissement, celui qui l'avoisine s'affaiblit. Cette diminution n'est pas toujours proportionnelle; et de là dérivent les inégalités dans la masse des hémisphères. Considérée en général, cette masse va en augmentant des didelphes, des insectivores aux rongeurs, aux pachydermes, aux ruminans, aux carnassiers, aux quadrumanes, aux phoques, aux cétacées et à l'hoinme.

C'est surtout en considérant l'encéphale par la base, que l'on juge facilement de cet accroissement, parce que la moelle allongée qui en recouvre le centre sert de point de comparaison; chez les chauve-souris, les rats, le didelphe, la moclle allongéc est débordée à pcine latéralement par les hémisphères; chez les rongeurs, le lièvre,

renflemens épiniens? entre l'absence du renflement inféxicur les cétacée et le prodigneux accroissement du cervelet? 
le lapin, les insectivores, la taupe, le zemni, on voit les hémisphères s'étendre à droite et à gauche de la moelle allongée; on les voit déborder de plus en plus dans ce sens, chez le mouton, le cheval, la chèvre, le bouc, le lion, la marte, l'ours, le pécari, le chameau, le lama, les singes, le phoque, le dauphin et l'homme. Il est à remarquer que lés mammifères sont les seuls vertébrés chez lesquels la moelle allongée soit ainsi débordée sur les côtés par le cervelet; chez les reptiles, les poissons osseux et les cartilagineux, et même chez les oiseaux, on n'en voit aucun vestige, l'encéphale étant placé sur sabase ( 1 ). On conçoit que cela doit être ainsi, puisque la partie visible dans ce sens correspond aux hémisphères qui manquent chez les poissons et les reptiles, et qui sont représentés chez les oiseaux par le petit tubercule situé sur la partie latérale du cervelet, lequel est en quelque sorte étouffé par la moelle allongée.

Ce que nous venons de dire.sur l'antagonisme du lobe médian du cervelet et de ses hémisphères, explique les variations de ses bords antérieurs et postérieurs chez les mammifères. Ceux chez lesquels le lobe médian est très-développé, les extrémités de ce lobe font en arrière et en avant une saillie qui déborde les bémisphères dans ces

(1) Sont exceptés de cette disposition le squalus griscus, les cigognes et les perroquets. 
beux sens, commie on le remarque surtout chez les rongeurs et les insectivores, comme chez le castor ct ln taupe. Lorsqu'au contraire les hémisphères ont pris un grand accroissement, comme chezles ours, les singes, les cétacées et l'homme, les cxtrémités du lobe médiau sont rentrées en dedans, elles sont logées dans un angle rentrant sur les deux bords du cervelet, au lieu d'y faire une saillie.plus ou moins avancée. Chez les ruminans et la plupart des carnassiers, Ic lobe médian et les hémisphères étant médiocrement développés, lcurs extrémités antérieures et postérieures se trouvent àpeu-près sur la même ligne. Sont exceptés de cette disposition les mammiferes (tels que les chauresouris) dont la partie antćrieure du lobe médian est rejetéc en arrière par la saillie des tubercules quadrijumaux. Ce qui prouve que ces variations sout bien dépendantes de cet antagonisme, c’est qu'elles se présentent chez les embryons aux époques qui correspondent à la préciominance du lobe médian ou des hémisphères du cervelet, comme nous l'avons déjá vu dans la première partie.

Telles sont les causes qui, chez les mammiferes, font varier la surface extricure du cervelet. Mais pour cet organe, comme pour les autres, les différences sont i l'cxtéricur, ct les ressemblances à lintéricur. C'est, cn cffet, une chose assez rentarquable que la disposition presque uniforme: du noyau medullaire des himisplicars de cet organe, 
$\therefore$ côté des dissemblances do Leur superficie?

Une partie nouvelle et spéciale à la classe des mammifères se manifeste à la partic interne dece noyau médullaire, c'est le corps rhomboïdal, le corps ciliaire, le corps dentelé ou le ganglion du cervelet, dont nous allons étudier la formation.

On a déjà vu que dans toutes les classes la matière grise forme une enveloppe i la matière blanche; la première est toujours beaucoup plus énaisse que la seconde. Chez les reptiles la matière frrise est à la blanche :: $5: 1$, chez les poissons $:: 4: 1$, chcz les oiseaux :: 3:1, et chez les mammileres : : $2: 1$ ou 1 1/2:1. Ces rapporls sont inverses de ceux que nous avons observés à la moelle épinière, puisque, dans cette dernière partie la matière blanche est d'autant plus épaisse, et la matière grise d'autant plus rare, que l'on descend davantage dans l'échclle des vertébrés. Or si, comme nous l'avons dit, celte pelite couche de matière grise chez les vcrtébrés inférieurs ne peut être supposéc l'origine, la matrice ou l'organe de nutrition de la blanche dans la moelle épinière, comment, dans le cervelet, une si grande quaniité de matièregrise produira-t-cllc une si faible couche blanche? Comment, à mesure que celle-ci se dévcloppe, la matière grise diminue-t-clle d'épaisseur? Quoi qu'il en soit, cette disposition inverse des masses de ces deux snbstances est d'autant plus curieuse, que leur position respeclive est égan lement opposée dans les deux organes. Ainsi la 
matière blanche est toujours extéricure sur la moelle épinière, et la grise forme les couches intérieures; au contraire, dans le cervelet, la grise est en dehors et la blanche en dedans.

Pour suivre la disposition, la forme et l'accroissement de cette dernière, on est donc obligé d'ouvrir le ccrvelet en pratiquant une section longitudinale, qui chez les mammifères diviscrait le lobe médian dans sa partie moyenne. On suit ainsi son accroissement des poissons aux mammifères supérieurs, en considérant chaque moitié du cervelet par sa partie interne.

Chez les poissons cartilagineux à cervelet impair, comme le squalus griseus, le squalus glaucus et le requin, on voit les plis et replis de la lame médullaire formant des sinus creux que tapisse la pie mère, sinus qui tous viennent aboulir à une cavité commune et plus vaste, située vers le milieu et sur le flanc du quatrième ventricule. Chez les oiseaux ces sinus cérébelleux sont comblés par la déposition de couches blanches sécrétées ici comme dans la moelle épinière par le lacis vasculaire de la pie-mère; ces couches médullaires, réunies à celles du côté opposé, forment le cône médullaire du cervelet des oiscaux. Un seul sinus n'est pas comblé (1), c'est celui de la partic moyeuno. qui communique dans la cavité com-

(1) II. IV , fig. 88. 
mune, laquelle róste également vide (1). Cinqur moitié du cervelet des oiscanx offre ainsi, dans sa partic moyenne, un hiatus creusé dans le demirônc de substance médullaire; si on rapproche les deux paries du cervelet, les parois de cet hiatis s'uppliquantl'un contrel'autre, donnent naissance au ventricule parliculier des oiseaux, creusédans l'axe du lobe médian. Ge ventricule n'est donc, comme le quatrième lui-même, qu'un canal dr roujugaison. L'ouverture de ce ventricule dans l. srand ventricule du cervelet est souvent très-réirécie, comme ou le voit chez le casuar $(\%)$ et lo perroquod (3); d'autres fois plus large, comme choz l'ainle royal (4). Ciette ouverture est souvent ré. trécie par une petite valvule médullaire qui parl de ses bords, ou bien par un petit tubercule de malière grise, qui se trouve à son cntrée sur le plancher même du qualrième ventricule, ainsi quion le voit chez ce deruirr oiseau.

Chez les mammiferes le dernisr sinus qui reste vide chez les oiscaux est comblé par un scomblable. mécanisne; le ventricule du lobe médian disparaîl, sa base seule reste creuse chez quelquos inscetivores et quelques rongeurs. C'est au milicu de: cet hiatus cérbelleux des oiseaux ainsi combli

(1) Pl. IV, fig. 85, $\mathrm{n}^{\circ} 15$

(i) Pl. VI, fig. 83 .

(j) Pi. IV, fig. 88, " "2.

(4) P4. IV, fig. 101 . 
ehes les manmiferes, que se déreloppo le corps dentelé ou rhomboïdal, dont l'étendue s'accrốt conime les hémisphères de cet organe, des iuseclivores, des rongeurs, aux ruminans, aus carnassiers, aux singes, au phoque, aux célacées et a l'homme. A mesure que le corps dentelé se développe, unc lame jaunâtre, plissée ou denteléc, l'environue; cette lame n'existe ni chezles insectiwures, ni chez les rongeurs, ni chez les ruminans; elle commence chez les carnassiers, et devient cnsuite de plus en plus étendue jusqu'à l'homme. C'est encore la répétition de ce que nous présente. ce corps chez les embryons; vers le quatrième mois, époque de son apparition, il resscmble chez l'homme à celui des ruminans, puis à celui les carnassicrs, des singes, des phoques ou du dauphin. La masse uédullaire des noyaus du crrvelet resscmble successivement aussi à cette mene: partic. che les squales et les oiseaux, romme chez ces demiers le ventricule du lobe mirchan cxiste à unc corlatue époque, puis s'oblilive the la mene maniere que la cavile des lubervules quadrijumeaus.

On voit donc la raison pour laquelle les mammiferes sculs possédent le curps dentelé, puisque li place qu'il occupe est vide chez les poissons et les viscaux; on voil aussi que che\% les vertébxés adultes, de mêne que chez le cmbryons, l'appasition de la matione grisatre ou jumâtre qui en forme la frange eatcrue, est de beaucoup posté- 
rieure à la masse médullaire qui l'environne et Ic circonscrit de toute part (1).

Les siuus cérébelleux des poissons cartilagineux, le ventricule médian des oiseaux sont donc comblés par la déposition de couches de matière blanche, de même que le canal épinien est rétréci et presque oblitéré par la déposition de couches appartenant à la matière grise. Cet effet est, dans tous ces cas, produit par le systeme sanguin, quoique dans le cervelet, ce soit par l'intermède de la

(1) Ce mécanisme du développement du cervelet des animaux adultes n'avait point été aperçn, et ne pourait l'être aussi long-tempg. que l'on conservait l'hypothèse de la formation centrale des organes. Si vous admettez, en effet, que le cerveletse développe du centre à la circonférence, le corps ciliaire derra se former le premler dans le noyan médullaire des hémisphères; ce quí est en opposition avec ce que nous offre l'embryogénie et la oomparaison du cervelet chez les mammifères, et dans les classes inférieures, qui répétent la formation embryonnaire des manmifères supérieurs. Vous ne pourrez conceroir ni le développement du ventricule particulier des oiseaux, ni les sinus des poissons, ni l'oblitération de ces sinus et de ce ventricule chez les mammifères. Vous trouverez toujours ces faits en opposition avec cette hypothèse. Sí, au contraire, vous appliquez au cervelet la loi générale du développement de la circonférence au centre, vous royez que nécessairement ce qui est doit être. Je ne saurais exprimer toutes les peines que je me suis données pour chercher à faire disparaitre cette contradiction des faits avec nos anciennes suppositions, avant de m'être aperçu que la nature avait été interprétée en sens inverse de sa manifestation. 
matière blanche, et dans la moclle épinière, par la matière grise. Pourquoi la pie-mère interne secrète-t-elle de la malière grise dans la moelle ćpinière, et de la matière blanche dans le cervelét? Je n'ai pu en découvrix la cause dans les nonbreuses recherches que j'ai faites sur le système sanguiu. En exposant le mécanisme de la for-mation du canal épinien, et du ventricule des lobes optiques, nous avons vu que ces cavités, d'abord trè-amples chez les jeunes embryons, s'oblitéraient d̀ mesure que leurs parois s'épaississaient de dehors en dedans. Il en est de même de la capacité du quatrième ventricule; il est d'autant plus vaste, considéré principalement par sa voûtc et ses parois latérales, que ses parois sont moins épaisses. Sous ce rapport, le quatrième ventricule des squalus glaucus, squalus carcharias, squalus griseus, reproduit celui des embryons des mannifères au premier tiers de leur gestation; puis celui des oiseaux représente le commencement du deuxième.

Le planclier du quatrième ventricule est tapissć, chez l'homme et les quadrumanes, par une petitc couche de matière grise: qui est la continuation de celle qui revêt les cordons antérieurs; au dessous de cette couche se trouve la saillic postéricure de ces cordons (1) : à nu'sure que l'on descend

(1) Pl, Xill, fig. $245, n^{n} 8$ 
drs quadrumaux aux carnassiers (1), aux ruminans (2) et aux rongeurs, la matière grise diminuant comme dans l'intérieur de la moello épinière, ces cordons deviennent visibles sans préparation. Ils sont plus visibles cncore chez les oiseaux (3) et les reptiles (4), que chez les rongeurs. Chez les oiseaux, ils traversent l'aqueduc de Sylvius et vont se rendre à la partie postérieure des couches optiques, en passant audessous de la commissure postérieure. Chez la cortue franche (5), le plancher du quatrième ventricule est cannelé par la saillie die quatre cordons blancs de chaque côté (6), dont le relief n'est point apparent dans l'épaisseur de la muelle alongée. La couche de matière srise qui revêt les cordons postérieurs, se ternine brusquement à l'entrée du quatrième ventricule, où elle forme un petit tubercule à la partie interne du calamus scriptorius. Le volume de ce tubercule est proportionné à l'épaisseur de la couche grise de la moelle épinière : très-développé chez l'homme ( 7$)$, il est beaucoup plus faible chez les qua-

(1) Pl XV, fig. $259, \mathbf{n}^{\circ} 5$

(2) Pl. XV, fig. 271 .

(5) PI. III, fig. 83 et 8 ; ; Pl. IV, fig. a. a.

(4) PI. V, fig. 154.

(5) Pl. V, fig. $129, n^{\circ} 5$.

(6) Pl. V, fig. $121, n^{\circ} 1$.

(7) PI. XIII, fig. $245, n^{n} 5$. 


\section{CERV ILIST.}

frumanes, ef ne se trouve déji plus chez lo phoque (1), ni chez les carnassiers (2) et les ruminans (3). Enfin, chez les oiseaux ct les reptiles, animaux ohez lesquels, comme nous l'avons dit, la couche grise de la moelle épinière devient de plus en plus mince, il n'existe pas de vestige de ce tubercule grisâtre, comme on lc voit chez le casoar (4), le perroquet (5), la poule (6), l'aigle royal (7), l'orvet (8), le lézard vort (9), les vipères (10), la grenouille (i1), le caïman (12), la tortue grecque $(13)$ et la tortue franche (14).

I.e quatrième ventricule de l'homme offre des stries blanchâtres, dout rous avous déjà parlé; ces stries ne se rencoutrent sur aucun mammifère, sur aucun oiseau, ni sur aucun reptile; la tortue frauche seulc présente quatre stries blau-

(1) Pl. X, fig. $218, \mathrm{n}^{\circ}$ z et 5

(2) Pl. XV, fig. $279,11^{\circ}$.

(5) Pl. XV, fig. 2, 1, n 2 .

(4) Pl. III, fig. $83, n^{\circ} 16$.

(5) Pl. III, fig. $84, n^{\circ} 15$.

(6) Pl. III, fig. $87, n^{\circ}$.

(7) Pl. IV, fig. $10 x, n^{\circ} 15$.

(8) Pl. V, fig. $109, n^{\circ} 1$.

(9) Pl. V, fig. $110, n^{n} 6$ et 7.

(10) Pl. V, fg. 155, n.2.

(11) P1. V, fig. 134, $n^{\circ} 2$

(12) Pl. V, fig. 135, $n^{n} 2$

(15) P. V. fig. 125, n" 14

(14) Pl, V, fig, $119, \mathrm{n}^{\circ} 5$ 
châtres de chaque côté (1), dont la direction est oblique, et situées très-près. les unes des autres, ce qui les distingue de celle de l'homme. Le quatrième ventricule de l'embryon humain est non strié jusqu'à la naissance, ainsi que celui des autres vertébrés. Je ne les ai aperçues que du huitième au dixième mois après la naissance; elles se développent de dehors en dedans ou de la circonférence au centre. Leur nombre et leur direction sont très-variables. Le plus grand nombre que j'ai rencontré est de dix, cinq de chaque. côté, ou six d'un côté et quatre de l'autre. Le plus petit chez l'adult: est de deux, le nombre moyen est de six. Sur trois nègres je n'en ai trouvé que trois; sur deux négresses, deux seulement, un de chaque côté. I es stries supéricures sont obliques, les inférieures horizontalcs, quelquefois elles sont légèrement arquées. Souvent celles de droite correspondent à celles de gauche sur la ligne médiane, d'autres fois elles ne se correspondent point. Une ou deux fois j'en ai vu quelques-unes qui s'entrccroisaicnt.

L.e quatrième ventricule est recouvert cn entice par le cervelet chez les manmifères', les oiseaux et la plupart des poissons; parmi les cartilagincux, la lamproie (2), l'esturgeon (5), le squale bleu

(1) II. V, lig. 121, n" I bis,

(3) II. XI, fir. $228, \mathrm{n}^{\prime \prime} 1$.

(3) PI. XII, lig. $235 . B$. 
et le squale griset ont la partie inférieure à nu; les individus de ces deux dernières espèces sont remarquables, parce quils se rapprochent beaucoup des reptiles sous ce rapport, et surtout de la tortue franche (1). Tous les reptiles ont leur ventricule découvert inféricurement, ce qui dépend, chez eux, du peu d'étendue de leur cervelet. Les crocodiles (2) et le caméléon (3) sont les seuls animaux de cette classe chez lesquels j'aie trouvé le quatrième ventricule entièrement recouvert par le cervelet.

Pour saisir maintenant la raison des rapports des divers élémens du cervelet avec les autresparties de l'encéphale, il est nécessaire de se rappeler que des trois pédoncules qu'on y remarque de chaque côté, le supérieur est en rapport avec les tubercules quadrijumeaux postérieurs, l'inférieur avec le cordon restiforme, et le moyen avec le corps ciliaire des hémisphères. Ces trois pédoncules se réunissent séparément, deux en avant en contournaut la moelle alongée, et un en arrière, en suivant la marche ordinaire des commissures.

La commissure du pédoncule inférieur est formée par le corps trapézoïde; celle du pédoncule supérieur, par la valvule de Vieus-
(1) Pl. V, fig. $119, \mathrm{n}^{\circ} 4$
(2) PI. V, fig. $115 \mathrm{el} 116, n^{0} 2$
(5) Pl. V, fig. $111, n^{\circ} 4$. 
sens, et les fibres de la protubérance annulaibr sont évidemment la continuation: du pédoncul. moyen.

Cela explique, d'urie part, les rapports du corps trapézoïde, que nous avons dejjà cxposés; cela montrc, de l'autre, quela valvule de Vieussens devra. suivre les mêmes rapports; or, comme les pédoncules supérieur et inférieur suivent la mêm: progression que le lobe mćdian du cervelet, on voit donc que toutes ces parties devront être. soumises aux mêmes rapports.

Ainsi, la valvule de Vicussens est développéc: en räison directe de la moelle épinière, des pyramides antérieures et postéricures, du zurps resliforme, des pédoncules supérieurs du cervelé el des tubercules quadrijumcaux. Elle va donc 'n diminuant de volume, des quadrumanes aux carnassicrs, aux ruminans ct aux rongeurs.

Cetle commissure, cst, au contraire, dévelopgice? ('n raison inverse des corp's olivaires.

Le lobe médian du cervelet, en nous renfemant: plus spícialement dans la classe des manmifires. scra donc dévcloppé aussi en raison directe de lis monlle épiniere, des pédoncules supérieurs et iufé ricurs du corvelel, de leurs commissures, du trijize, de la valvule de Vieussens, ct des tribcreale's quadrijumeaux.

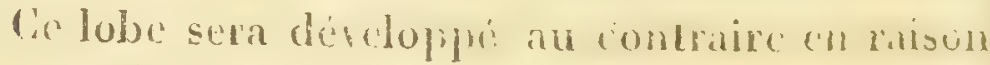
inverse de lolire; lous ces rapports sout mon- 
wes-rigoureusement exacts par la comparaison k: ces diverses parties de l'homme aux insectifores et aux rongeurs.

Les hémisphères du cervelet étant en rappor: arec le pédoncule moyen, comme le lobe médiau est en rapport avec les pédoncules supérieurs el inléricurs, il en résulte que le pédoncule moyen. sál commissure et la protubérance annulaire deront suivre la progression de ces mêmes hémis= phères.

Or, les himisphères du cervelet sont dévelopfís cn raison inverse de son lobe médian; il suit donc quic los dépendances de ces hémisphères devront être dans un antagonisme de développenient avec le lobe médian et ses dépendances: c'est en effet ce qui est.

Les hémisphères du cervelet sont développés cn raison inverse de la moelle épinière, des tubercules quadrijuneaux, des pédoncules supericurs et inléricurs, de la valvule de Vicussens, du corps trapézoïde, des nerfs de la cinquième paire, du nerf optique et du nerf olfactif.

Par la même raison, les parties qui sont développés en raison inverse du lobe médian, devraient l'être en raison directr des hćmisphères; c'est ce gui est. Pour le monent, nous ne pouvons que signalex le rapport direct de développenent qui existe entre le curps olivaire ct ces hémisphères; rapport que nous avons déjà suivi chez les emhrous, et qui se reproduit d'une maniere admi- 
rable chez les animaux adultes, de l'homme aux quadrumanes, aux cétacées, aux phoques, aux carnassicrs, aux ruminaus, jusqu'aux chauvesouris et aux rongeurs.

Nous avons déjà vu que certaines parties de la moelle alongéc produisent un relief plus ou moins grand dans le quatrième ventricule: ainsi les nerfs de la cinquième paire et le nerf de la huitiéme sont représentés chez les poissons par des tubercules particuliers; ainsi le tania correspond au corps trapézoïde. Sous ce rapport, le corps ciliaire du cervclet peut être considéré comme le tubercule du pédoncule moyen et de la protubórence annulaire.

Si cela est, le corps ciliaire devra suivre la même progression que les hémisphères du cervelet et la protubérance annulaire : ce que l'on remarque, en effet, de l'homme aux mammifères inférieurs, selon les observations faites par M. Gall, chez les animaux adultes; par Tiedmann, et par moi, chez l'embryon de l'homme; et par moi, chez les embryons des mammifères supéricurs.

Les hémisphères du cervelet et la protubérance annulaire étant développés en raison inverse $\mathrm{du}$ corps trapézoïde, il suit donc aussi que le corps ciliaire et le tania grisea du quatrième ventricule devront suivre une progression inverse. Comparez en effet ces deux parties de l'homme aux ruminans, ct vous verrez le tania décroîtrc 
¿ mesure que le corps ciliaire se développe. l.es cétacées sont exceptés de ce rapport.

Par la même raison, le corps ciliaire doit êtro, développé en raison directe du corps olivaire. Suivez ces deux corps chez les embryons des mammifères supérieurs et les animaux adultes, vous les verrez toujours se suivre progressivement. dans leur développement. Vous verrez aussi que l'olive et le tania du quatrième ventricule sont. dans une discordance qui répète celle du corps ciliaire et des hémisphères du cervelet.

Quclles que soient néanmoins les limites de ces variations, le pont est toujours projeté en avant, et il est projeté cn avant par la même raison que le cervelet lui-même; comme ce dernier il se développe sous l'influence de l'artère basilaire et des artères cérébelleuses, dont la direction marche du trou occipital aux apophyses clinölles postérieures. Nous verrons le corps calleux qui lui correspond, suive une marche inverse, parce que la marche de ses artèrcs est opposée à celles du pont. Les fibres de la protubérance annulaire ne sont donc pas directes comme celles du trapèze, les arcs qu'clles forment autour de la moelle alongéc sont obliques et concentriques d'arrièrs en avant.

Nous avons réjà dit que le cervelet el le cerveau so forment en sens inverse. L'un marche d'arriere en avant, l'autre d'avant en arricre. Lo, développement des hémisphères cévébraux se 
mesure par le prolongement do leur partic postéricure sur le cervelet, consćquemment par lit considération de la face supérieurc de l'encéphale. Celui du cervelet est surtout sensible à la base. D'après cette marche opposée, ces deux parties semblent rentrer l'une dans l'autre. Cette idéc générale peut seule donner la clef des variations multipliées que présente la base de l'encéphale rles mammifères. Ainsi, plus l'encéphale sera développé, plus la protubérance sera enfoncée sous les hémisphères cérébraux, comme on l'observe de l'homme, des dauphins, des phoques, des singes, des carnassiers, des ruminans, jusques aux chauve-souris et aux rongeurs. Plus cette progression antérieure du pont sera marquée, moins sera étendu l'espace qui sépare la protubérance annulaire de la jonction des nerfs optiques. C'est cette surface, bornée sur les côtés par le lobe de l'hippocampe, que j'ai nommée champ mamillaire.

Ces rapports nous fournissent les bases de ceux du pont de Varole, sur lequel nous devons nous arrêter un instant. Premièrement son ćlendue suil l'accroissement des hémisphèrés du cervelet, dont il forme la grande commissure. C'est chez l'homme qu'il est porté au maximum de sa grandeur (1); puis viennent les cétacées (2). le phoque (j),

(1) Pl. XIII, fig. $247, n^{\circ} 5$ ct 6 .

(2) P!. XII, îg. 25 , P.

(5) P'. IX, lig. 208. I? 
ct en troisième lieu les singes, le drill (1), la mandrill (2); les carnassiers, le lion (5), la

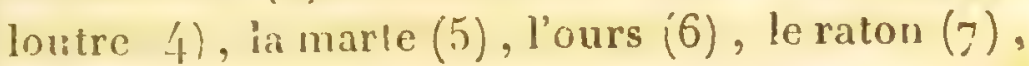
le kanguroo (8); les ruminans, le chameau (9), le cheval (10), le lama (11), le daman (12), le pécari (15), le porc-épic (14), le castor (15), la marmotte (16), l'agouti (17), les rats et les chauve-souris (18).

Les fibres composant le pont de Varole sont sur un plan plus anterieur que celles du trapèze, ce qui explique pourquoi ce dernicr passe toujours derrière les pyramides antérieures '19),

(1) Pl. VIII, fig. 197, P

(2) Pl. ViII, fig. 194, P.

(3) Pl. XIV, fig. $266, \mathrm{P}$.

(4) Pl. X, fig. 223, P.

(5) PI. XV, fig. 29o, P.

(6) Pl. XI, fig. $231, \mathrm{P}$.

(7) Pl. VIII, fig. 200, P.

(8) Pl. XVI, fig. 299, P.

(9) Pl. XIIl, fig. 249, P. (10) Pl. XV, fig. 275, P. (11) Pl. XVI, fig. 295, P. (12) Pl. XYI, fig. $273, P$. (13) Pl. XVI, fig. 300, P. (14) Pl. XIII, fig. $251, \mathrm{P}$. (15) Pl. XIV, fig. $258, \mathrm{P}$. (16) Pl. IX, fig. 203, P。 (17) Pl. IX, fig. 211, P. (18) Pl. IX, fig. 204 et 214, P. (19) PI XVI, fig. 299, T, 14. 
tandis que la protubérance annulaire recouvre la gerbe de leur épanouissement. Celte disposilion en explique une autre. A mesure que le pont se développe, il couvre'aussi le trapèze; ce que l'on voit surtout chez les phoques (1) et chez l'homme, sur lequel le trapèze n'est plus à nu sur la face externe de la moelle alongéc.

On a dit que le pont s'étendait en avant jusqu'à l'insertion de la troisième paire. Rien de moins généralcment vrai que cette mesure. La troisième paire est très-rapprochée du bord antérieur du pont chez l'homme, les singes (2) et les cétacées (3); elle le touche chez les ploques (4), seuls mammifères chcz lesquels cettc assertion soit exacte. Elle s'en écarte beaucoup chez les carnassiers digitigrades, le lion (5), la marte (6), la loutre (7), la mangouste (8); chez les plantigrades, le raton (9) et l'ours (10); chez les pachydermes, les ruminans (11) et les rongeurs (12):

(1) Pl. IX, fig. 208, P, T.

(2) PI. VIII, fig. 194 et 197, P, 3.

(3) Pl. XII, fig. $234, \mathrm{P}, 3$.

(4) PI. IX, fig. 208, P, 3.

(5) PI. XIV, fig. 366, P, 3.

(6) Pl. XV , fig. 2yo, P, 5.

(7) Pl. X, fig. 223, P, 3 .

(8) Pl. XIII, fig. 254, P, 3.

(9) Pl. VIII, fig. $200, \mathrm{P}, 3$.

(10) Pl. XI, fig. 23r, P, 3.

(11) Pl. XV, fig. $290, \mathrm{P}, 3$.

(12) Pl. XIII, fig. 251, r, 3. 
ces trois derniers ordres sont ceux où cet écartement est le plus prononcé, ainsi qu'on l'observe chez le cheval (1), le chameau (2), le lama (3), le bouc de la haute Égypte (4), le porc-épic (5) et le castor (6). Le rapprochement s'effectue de nouveau chez le tatou (7), la marmolte (8) et l'agouti (9).

En général, cet effet est produit par l'allongement ou la concentration des hémisphères du cerveau et de ses pédoncules, comme on peut le remarquer chez l'ours (10), le daman (11), le lama (12), le pécari (15), le bouc (14), comparés au pheque (15) et à l'homme.

Le pont de Varolen'a pas la même forme chez tous lcs mammifères. Tantôt il est quadrilatère; tantôt il se rapproche d'un cône tronqué à son sommet.

(1) PI. XV, fig. $27^{5}, \mathrm{P}, 3$.

(2) Pl. XIII, fig. 249, P, 3.

(3) Pl. XVI, fig. $29^{5}, \mathrm{P}, 3$.

(4) PI. XIV, fig. $262, \mathrm{P}, 3$.

(5) Pl. XIII, fig. 251, P, 3.

(6) Pl. XIV, fig. 258, P, 3.

(2) Pl. XIII, fig. 246, P, 3.

(8) Pl. IX, fig. 203, P, 3.

(9) Pl. IX, fig. 211, P. 3.

(10) PI. XI, fig. 231.

(1i) Pl. XV, fig. 275 .

(18) Pl. XVI, fig. 295.

(13) Pl. XVI, fig. 300.

(14) Pl. XVI, fig. 262.

(15) Pl. IX, fig. 208. 
Il est quadrilatère chez l'homme (1), les quadrumanes (2), le phoque (3), les célacés (4), la loutre (5), le chat, le chien, le lion 6), le mouton, la chèvre, le bouc de la haute Égyple (7), le daman (8), le castor (9), l'agouti (10), la marmotte (11). Il est conique chez le raton (12), la marte (13), lc kanguroo géant (14), le pécari (15), le lama (16), le cheval (17) et le chameau à deux bosses (18).

Si, d'une part, le champ mamillaire se raccourcit à mesure que le pont se rapproche de la jonction des nerfs optiques; si, de l'aulre, en se dévelo ppant le cervelet se porte d'arrière en avant,

(1) Pl, XIII, fig. $24 \%$.

(2) PI. VIII, fig. 194 et 197.

(3) Pl. IX, fig. 208.

(4) Pl. XII, fig. 234.

(5) PI. X, fig. 223.

(6) Pl. XIV, fig. 266.

(z) PI. XIV, fig. 262.

(8) Pl. XV, fig. 273.

(9) Pl. XIV, fg. 258.

(io) Pl. IX, fig. 211.

(11) Pl. IX, Gig. 203.

(12) P!. VIII, fig. 200.

(i3) Pl. XV, Gg. 290.

(14) Pl. XVI, fig. 229.

(15) PI. XVI, fig. 300.

(16) Pl. XVI, fig. 295.

(17) PI. XV, fig. 275.

(18) $\mathrm{Pl} . \mathrm{XIII}$, fig. 249 . 
il en résulte que le pout sera d'autant plus isolé des hémisphères cérébelleux que l'on descendra plus bas vers les mammifères inféricurs. En suivant ce rapport, on trouve que chez le porcépic (1), le chameau (2), le bouc de la haute Egypte (3), les hémisphères du cervelet s'élèvent à peine au niveau de l'insertion de la septième paire, puis au niveau de la cinquième chez les carnassiers plantigrades et digitigrades (4); puis enfiu ils rejoignent le bord autérieur de la protubérance annulaire chez le dauphin (5), où ce mouvement cst le plas prononcé. hez le plus grand nombre des mammilères supérieurs, le cervelet s'arrête au nivcau de la partie antérieure de la cinquième paire, comme on le remarque chez l'homme, le phoque (6) et les quadrumanes (7). C'est lc moyen terme de co, mouvement entre le dauphin et les rongeurs. Les hémisphères du cervelet sont étrangers à cette variation de leur commissure. La forme conique du pont de Varole a sa cause dians une modification de la moelle alongée. En effet, chez tous les mammicères où
(1) Pl. XIII, fig. 251.
(2) Pl. XIII, fig. 24.9.
(3) Pl. XIV, fig. 262.
(4) PI. XIV', fig. 266.
(5) Pl. XII, fig. 234.
(6) Pl. IX, fig. 208.
(c) Pl. VIII, lig. 194. 
il offre cette disposition, la moelle alongée est bombée au niveau de l'extrémité externe du trapèze. Or, l'effet de la saillie vers cet endroit est d'élargir la base de la protubérance annulaire , et d'effiler son sommet. Plus la moelle alongée est saillante, plus est prononcée la forme conique du pont, comme on peut le remarquer chez le cheval (1), le porc-épic (2), le raton (3) et la marte (4). On voit par ce nourcl exemple avec quelle facilité la corrélation des parties de l'encéphale explique leurs diverses modifications, non-seulement selon les classes, mais dans les familles, les genres ou les espèces de la même classe. ("'est là, je le répète encore, le véritable but de l'anatomie comparative.

De tout ce qui précède dérivent les rapports du pont de Varole chez les mammifères. Ce pont est développé en raison directe des hémisphères du cervelet, en raison directe du corps ciliaire, en raison directe des olives. Il est développé, au contraire, en raison inverse de la moclle épinière, des pédoncules inférieur et supérieur du cervclet, du irapèze, de la valvule de Vieussens, des tubercules quadrijumeaux et du lobe médian du ccrvelet.
(1) PI. XIII, fig. 275 .
(2) Pl. XIII, fig. 251.
(3) Pl. VIlI, fig. 200.
(4) Pl. XV, fig. 200. 
Des Dimensions du Cervelet chez les Mammifères.

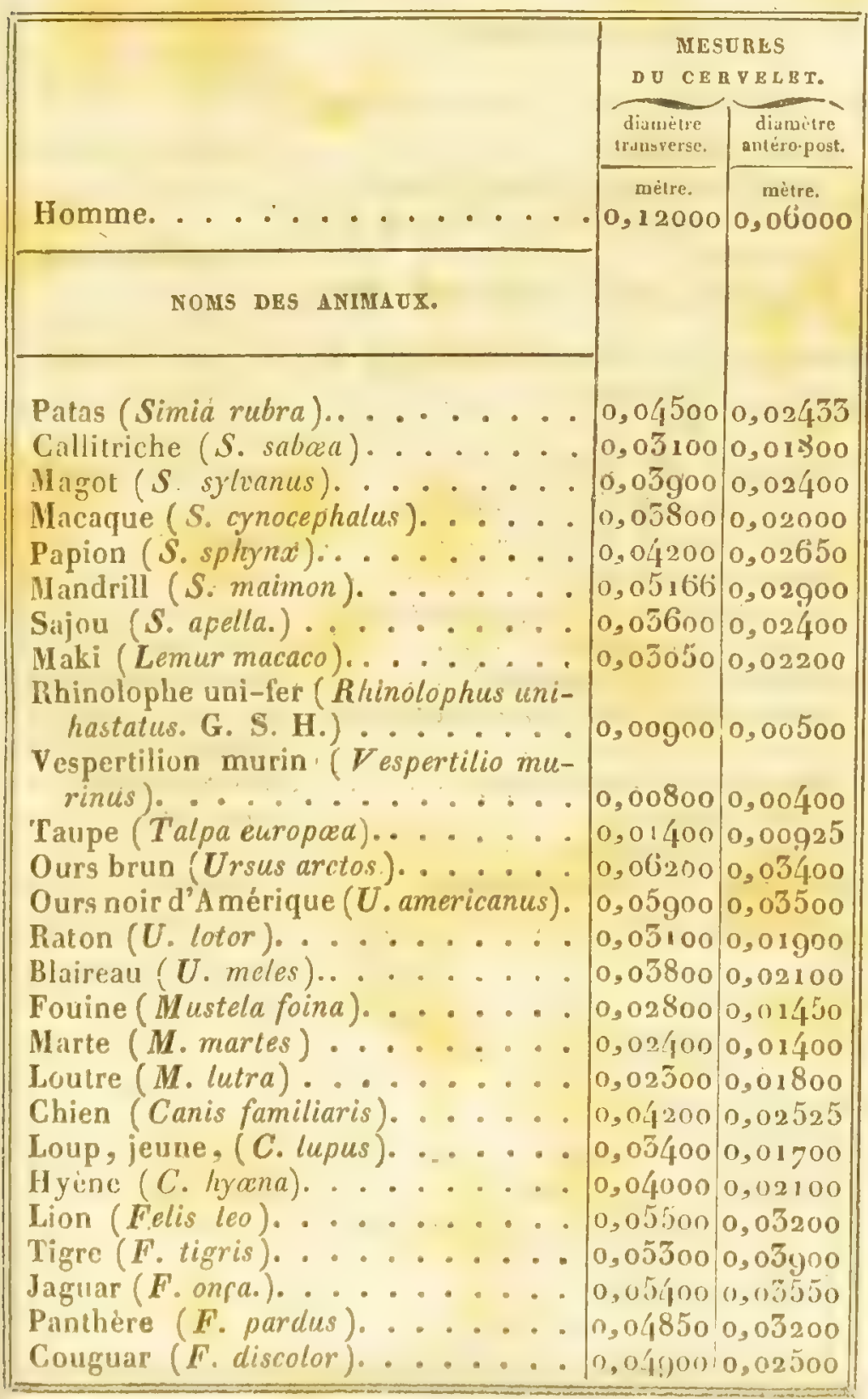


Suite du Tableau comparatif des Dimensions du Cervelet chez les Mammifëres.

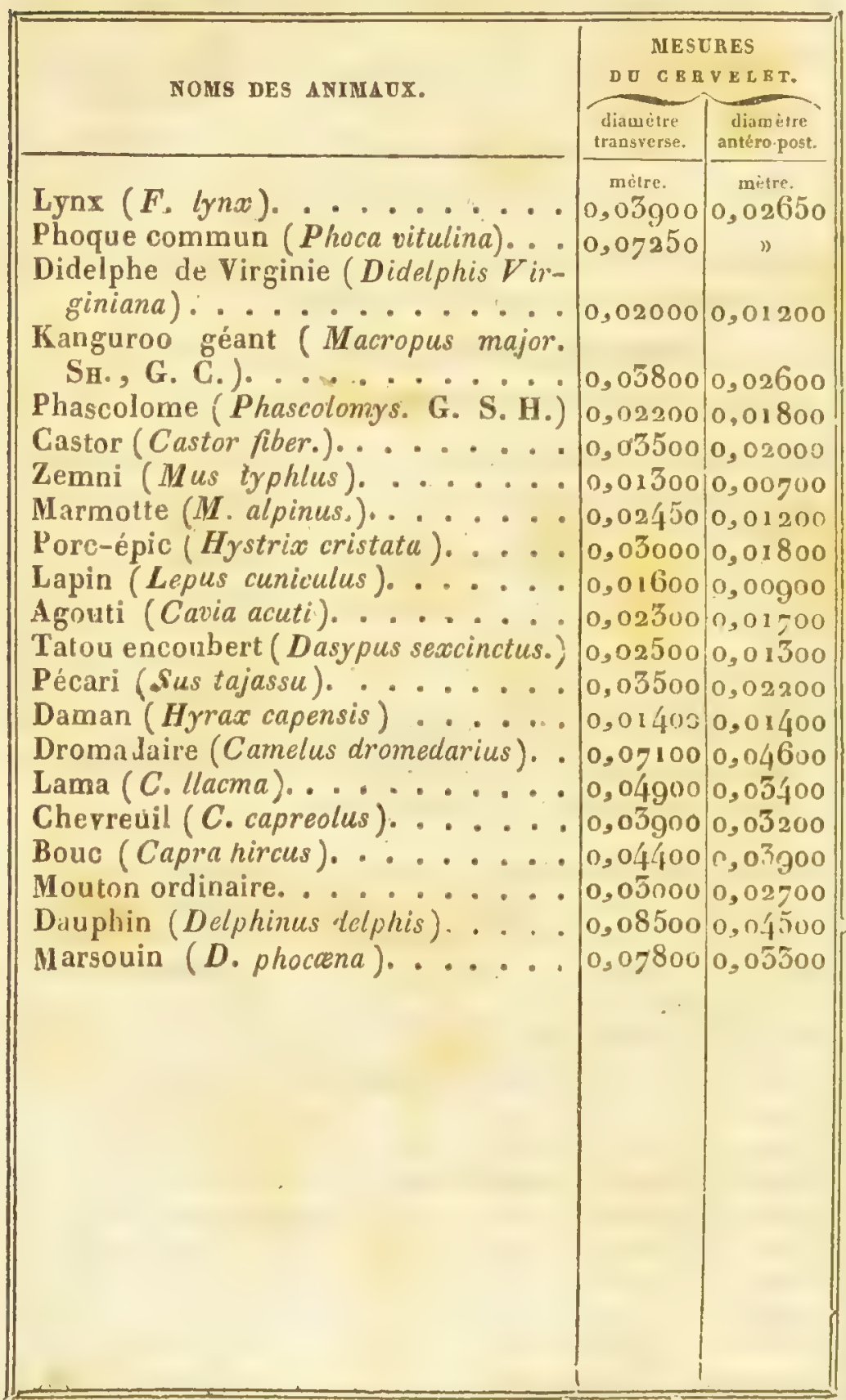


TABLEAU COMPARATIF

Des Dimensions du Cervelet chez les Oiseaux.

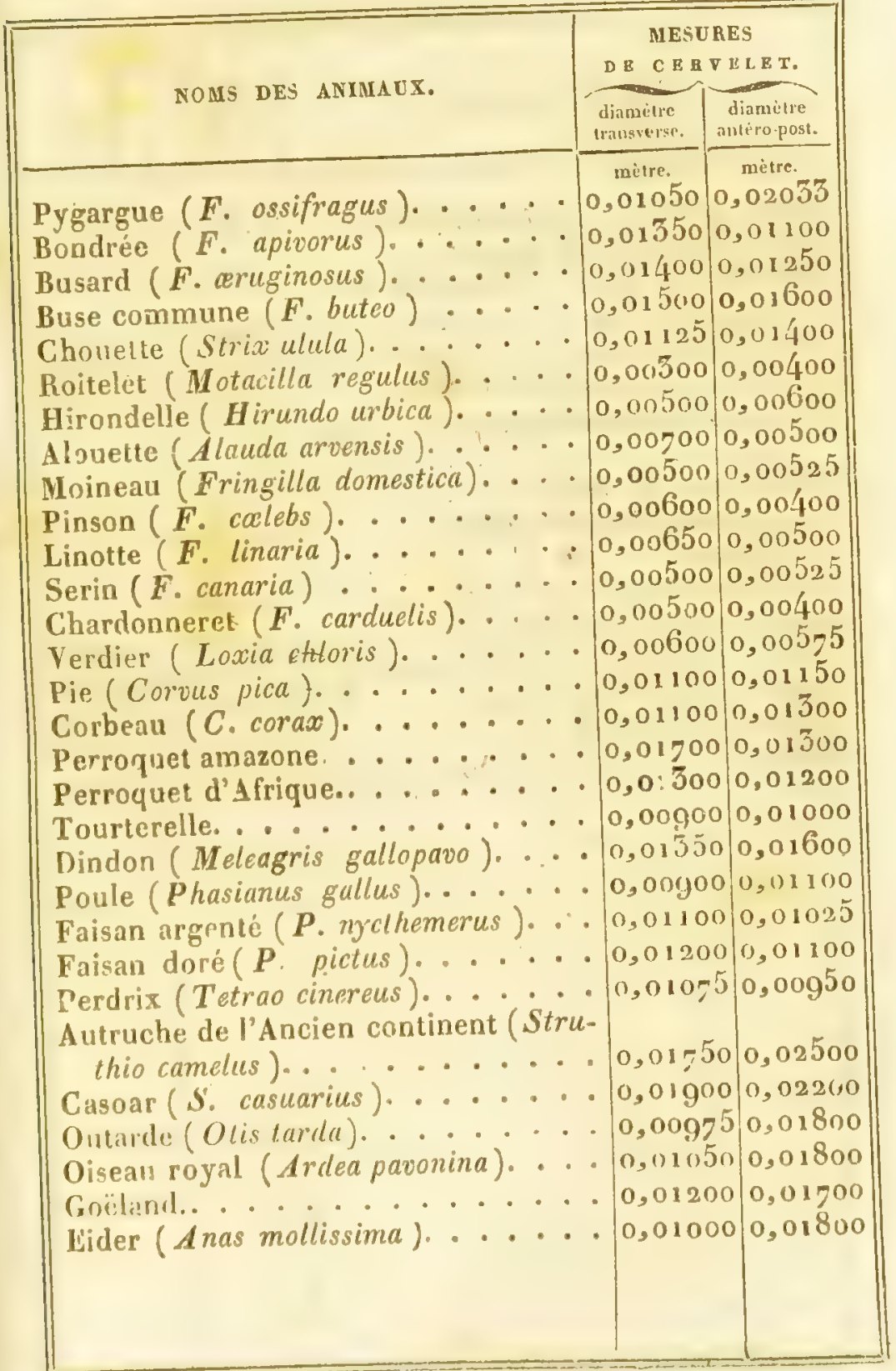




\section{TABLEAU COMPARATIE}

\section{Des Dimensions du Ciervelet chez les Reptiles.}

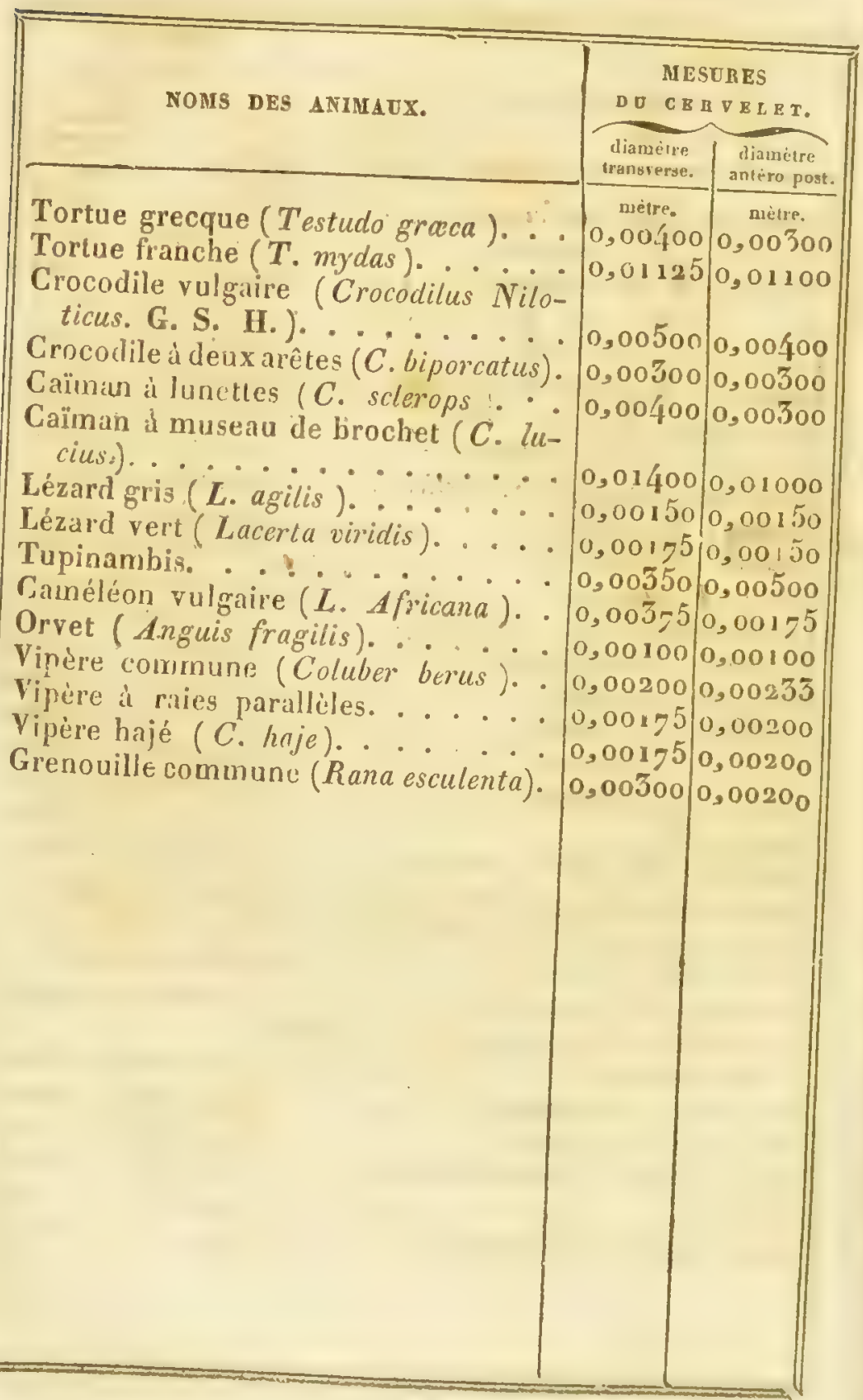


TABLEA COMPA A TIF

Des Dimensions du Cervelet chez les Poissons.

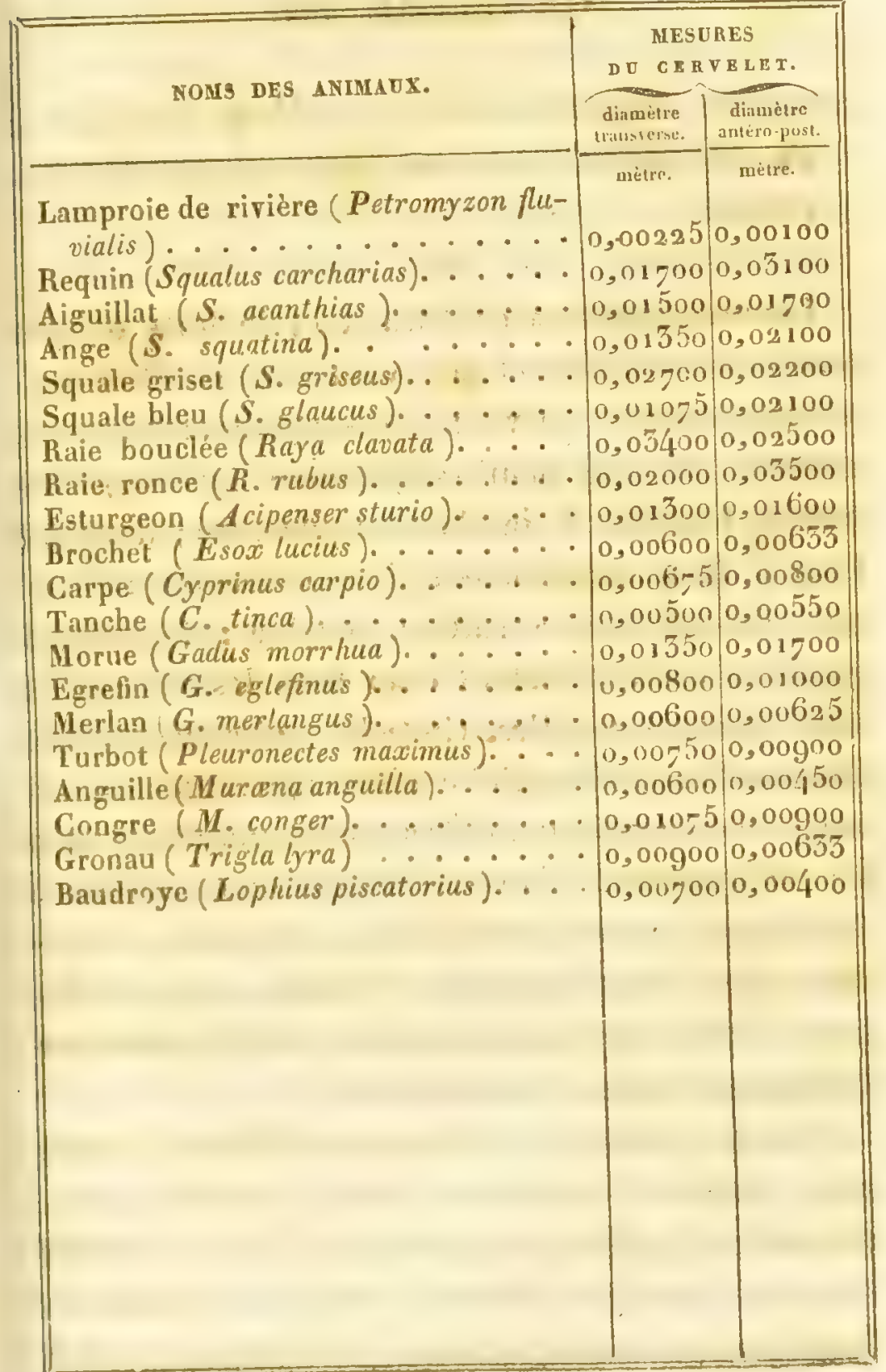




\section{CHAPITRE VII.}

Anatomie comparative des hémisphères cérébraux et du lobe olfactif dans les quatre classes des vertébrés.

Les hémisphères cérébraux et le lobe olfactif terminent en haut et en avant l'axe cérébro-spinal du système nerveux des vertébrés : les hímisphères ne sont semi-sphériques que dans les hautes familles de la classe supérieurc; dans les basses, ils se rapprochent de la forme d'un triangle, dont la base serait en arrière : disposition gévérale qu'ils affectent chez tous les niseaux et chez certains reptiles. Chez les poissons osseux, chacun d'eux esí unglobe solide, légèrement aplati sur la face par laquelle ils se correspondent; chez les cartilagineux, ils sont généralement aplatis et confondus en une seule masse compacte : considérés aux deux bouts de l'échelle, leurs différences extérieures sont extrêmes, et leur structure n'est guère moins dissemblable.

Chez les poissons, leur masse solide est homogène : chacun d'eux cst un bloc de matière grise. sillonné en arrière par quelques stries de matière. blanche; tout est confondu dans leur épaisseur. Dans les trois aulres classes, les hémisphères se 
creusent, l'écartement de leurs lames permet à des tubercules intérieurs de se développer et de s'accroître: ces tubercules, d'abord simples, sc compliquent par la manifestation de faisceaux médullaires, dont les uns les réunissent sous le nom de commissures, et dontles autres, partis du pourtour de leur surface externe, se dirigent en rayonnant vers la périphérie dess lobes. Celte membrane interne, simplement épanouie dans leur épaisseur chez le: oiseaux, se roule daus la classe supérieure, se replie sur elle-même, et se joint à celle du côté opposé en formant les plus grandes comme les dernières commissures de l'encéphale. Par cet accroissement, ses plicatures multiplient sa surface, et donuent naissance aux circonvolutions qui sillonnent la face extérieure des hémispheres cérébraux. En se réunissant à celle du côté opposé, elle forme un double plafond sur les tubercules des pédoncules cérébraux, et donne ainsi naissance aux doubles ventricules des mammifères, dont le supérieur, cloisonné dans sa partie moyenne, est séparé de cetle manière en ventricule droit et ventricule gauche.

Sur les tuberculcs inférieurs, désignés sous le nom de couche optique, vient s'implanter la glande pinéale, dont la constance a lieu de surprendre, quand on coinsidère sa complète inutilité, suivant l'état présent de la science : leur base s'implante, à son tour, sur la glande pituitaire, non moins constante et non moins mystérieuse 
que la glande pinéale; enfin la base des hémisphères présente, en outre, un ou deux tubercules en rapport avec le kiasma des nerfs optiques, que l'on a confondus avec les éminences mamillaires de l'homme.

Tout-à-fait en avant, les hémisphères cérébraux se terminent par un lobe particulier, inaperçu, pour ainsi dire, jusqu'à ce jour : c'est le lobe olfactif. Ce lobe est si petit chez l'homme, qu'il forme en quelque sorte un hors-d'oeurre de son encéphale : or, comme on cherchait toujours l'homme chez les animaux, cet organe se trouva en dehors de la science, et devint par cela même une source d'incertitude, à l'égard des classes oú son accroissement le plaça presque sur la même ligne que les hémisphères eux-mêmes. Un examen spécial et approfondi était donc nécessaire pour reconnaître au milieu de leur atrophie ou de leur hypertrophie, des parties si variées, si variables et toutefois si importantes, et pour apprécier leur influence sur les variations des hémisphères qui en sont la conséquence.

En effet, toutes ces variations se répètent et se reproduisent dans la série successive des formes embryonnaires. Les lobes cérébraux des poissons sont d'abord creux comme ceux dés autres classes; ceux de li classe supérieure sont d'abord pareillement sans éminences intérieures. Ces éminences sont dépourvues de commissures; il n'y a nivoûte, ni corps calleux, ni circonvolutions visibles à l'ex- 
térieur : tout se rapproche, se confond et s'unit dans le cours de celte création, miniature peutêtre de la grande création primitive.

Ce rapprochement inattendu des formes embryonnaires avec les formes permanentes de l'axe cérébro-spinal, qui a frappé tous les esprits, eût peut-être paru plus singulier que vrai, si nous en eussions commencé l'exposilion par les hémisphères cérébraux. Qui eût voulu croire que des lobes cérébraux des poissons dérivent les lobes si compliqués des nammifères supérieurs? Mais accoutumé, comme nous le sommes, par ce qui précéde, à celle transformation, on n'y verra qu'une application de plus des principes généraux de cet ouvrage: application que nous verrons se reproduire dans les couches optiques, les corps strićs et les autres parlies que nous allons examiner,

\section{De la Couche optique et du Corps strié.}

Dégagés de la moelle allongée, ou de l'épaisseur du pont, chez les mammifères, les cordons primitifs de l'encéphale prennent le nom de pédoncules cérébraux. D'abord désunis, ils se réunissent en avant comme les cordons antéricurs de la moclle épiniere, et un sillon profond analogue du sillon antérieur de cette moclle, atteste dans l'encéphale des adultes le mécanisme de celle formation dans toutes les classes : dans le premier jet de l'encé- 
phale des mammifères supíricurs, ces pédoncules sont aplatis en haut, et tout-ì-fait à nu sur la face supérieure de l'népéphale, en avant des lobes optiques qui les dominant; les hémisphères ne les recouvrent pas encore: plus tird ces pédoncu. les se gouflent, et il se forme sur leur plateau deux épiphyses solides, qui sont la couche opüique et le corps strié. La couche optique et le corps strié sont donc aux pédoncules cérébraux ce que les renflemens sont à la noelle épinière, l'olive et les tubercules du quatrième veniricule à la moelle allongée. Aussi long-temps que les tuberculessont aplatis, une simple rainure les sépare; mais en se bombant ils s'écartent, et de cet écartement naît linfundibulum du troisième ventricule. Ce sont ces divers états embryonnaires qui se reproduisent chez certains animaux adultes.

Ainsi, chez certains poissons cartilagineux, les couches optiques sont aplaties comme dans la première phase de l'embryon, et visibles à nu sur la face supérieure du cerveau entre les lohes optiques et les hémisphères cérébraux. C'est le cas du squalus griscus, du squalus glaucus, de l'aiguillat (1), de l'ange (2); chez la lamproie (3), l'esturgeon (4), elles sont plus saillantes; et chez la raie

(1) PI. XII, fig. 236

(2) Pl. XII, fig. $25 \%$.

(3) Pl. XI, fig. 224 et $228, n^{\circ} 4$.

(4) Pl. XII, G. 
bouclée (1), on remarque déja un rurliment du iroisième ventricule. Chez les poissons osseux, les pédoncules cérébraux sont aplatis (2), et sans aucune trace d'épiphyse (3). Les lobes cérébraux sont immédiatement adossés aux lobes optiques. Chez les vipères et les couleurres, les couches optiques sont développées au même degreé que chez les poissons cartilagineux; chez les lézards elles sont un peu plus déyeloppées; cheł les batraciens elles sont déjà très-saillantes, quoique non cachées sous les hémisphères (4); chez le caméléon, les crocodiles, les caïmans, la couche optique devient de plus en plus saillante. Cihez le caiman à museaude brochet, quoiqu'entourées par la saillie des lobes optiques, elles forment deux tubercules solides très-prononcés; chez la tortue de terre elles sont pyriformes et yisibles en dehors comme chez la grenouille, à cause de l'écartement des hémisphères en arrière (5). Chez la tortue de mer, elles sont plus arrondies et beaucoup plus volumineuses (6). Imperceptibles chez les poissons osseux, les couches optiques se sont donc peu-à-peu montrées des poissons cartilagineux, aux ophidiens,

(1) Pl. VI, fig. $138, n^{\circ} 9$.

(2) Pl. VII, fig. $166, n^{\circ} \%$

(3) Pl. VII, fig. $165, n^{\circ} 11$; fig. $180,11^{\circ} 8$; fig. 187 ; fig. $189, n^{\circ} 6$.

(4) Pl. I, fig. $16, n^{\circ} 3$.

(5) Pl. V, fig. $125, n^{\circ} 8$.

(6) Pl. V, fig. $121,10^{\circ} 5$.

II. 
aux lacentiens, aux batraciens, aux sauriens et aux chéloniens; elles deviennent plus saillantes encoro chez les oiseaux.

Dans toute cette classe, ces deux épiphyses son deux tubercules solides, arrondis, recourerts dans leurs quatro cinquièmes antérieurs par les hémisphères cérébraux, et dans leur cinquième postérieur, par la commissure des lobes optiques (1). Leur écariement spr la ligne médiane est plus grand que chez lés reptiles ( 2 ), ce qui augmente l'excavaliou du truisic̀me ventricule (5). Indépendamment de lour volume, les couches optiques des oiseaux se dislinguent de celles des reptiles par deux cordons blanchâtres (4), situés à leur partie interne, et qui sont la continuation des pédicules de la glaude pinéale, comme on le voit chez le casoar (5), le perroquet (6), la bondrée commune (7), et l'autruche (8). Ies oiscaux nous conduisent ainsi aux conches optiques des mammifères.

La couche optique restée dans les trois classes précédentes daus un état inférieur de développe-

(1) PI. III, fig. $85, n^{\circ} \mathrm{F}$.

(2) PI. III, fig. $8 y, n^{\circ} 6$.

(3) Pl. III, fig. $34,1^{\circ} 8:$ : $4 \mathrm{~g} .3,-, 11$ (

(4) Pl. 1II, fig. 85, $11^{\circ}=$

(5) PI. 1II, lig. $83,11^{\circ} \%$

(6) Pl. IIl, fig. $84, n^{\prime \prime} 8$.

(z) Pl. IV, fig. gu, $\mathrm{n}^{n}$ i.

(8) Pl. 1Y, lig. 101, 10 O. 
unent, s'eleve tout-a-coup chezles mamunicres (I) a son maximum de volume (2). Cul accroissement a une cause non aperçue jusqu'a ce jour, c'est celle de l'iuserlion de la plus grande partic des nerís optiques. Los tubercules que l'on a nommés genoux interne et externe sont évidemment nux nerfs optiques ce que les tubercules du quatrième ventricufe des poissons sont aux nerfs qui vicnnent y aboutir. Ces tubercules occupent la partie postérienre de bi coucíc optique; at comme le nerf optique va en augmentant de volume de l'homme aux quadrumanes, aux ruminans rt aus ronguars, de même la partie postéricure de la conche optirue suit dans son développement la même progression (3). Une pelice bandelelts d'un blanc: mat sépare la couche optaruen deux panties, l'une postórieure, c'est celle don i nous venons de parler ( 4 ), ei l'autreantcyeure, qui paraît tout-à-fait étraugere au nerfie la vision (5); la moitié posiérieure est recouverte d'une frellicule blanchâtre, qui se joint en arrière. aux faiscoaux qui, dés tubercules quadrijumeaux anlérieurs, se portent vers le nerfoplique. I a moilié antéricure est plas grise et moins blanche; de:

(1) P. IX, fig. $218,1^{\circ}$, ..

3) P. XII, fin. 24, $110=28$.

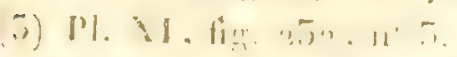

(4) Pl. IX, fig. $205, n^{\circ} 10$

(5) Pl. IX, lig. 295, $11^{\circ} 11$. 
mème que la postérieure suit le rapport du vo. lume du nerf de la vision, de même l'antérieure m'a paru suivre le rapport du demi-centre ovale des hémisphères.

Cette démarcation superficielle de la couche optique chez les manımifères, a servi de point de départ à Tréviranus pour sa délermination des lobes optiques des oiseaux. Selon cet anatomiste, ce serait, comme nous l'avons vu, la partic postérieure qui aurait servi à la composition de ces lobes. Aux raisons que nous avons rapportées, et qui nous paraissent devoir faire rejeter cette opinion, nous en joindrons une dernière : c'est que chez les grands oiseaux, la couche optique est diviséc. de la nême manière que chez les mammifères, en deux parties, l'une antérieure (I), l'autre postérieure (2). Cette disposition, distinctesurtout chez l'autruche et le casoar, est le passage intermédiaire de celte classe à celle des mammifères, et une preuve sans réplique du non-morcellement de la couche optique des oiscaux.

Chez les mammilères, dè même que chez les oiseaux, deux rubans blanchâtres en ceignent le bord interne (3), et se réunissent en arrièrc à la glande pinéale, comme on le voit chez la man-

(1) PI. III, fig. $85, n^{\circ} 9$

(3) Pl. IX, fig. 85, $n^{\circ} \%$.

(5) Pl. III, fig. $205, \mathrm{n}^{\circ} 12$. 
gouste (1), le mandrill (2), le coati roux (3), la gazelle (4) et l'agouti (5). Ces cordons sont en général plus larges et plus ípais chez les nammiferres que chez l'homıne; chez les rongeurs ils s'épanouissent sur le plateau supérieur de la couche optique, ct forment uue lame blanchâtre qui en couvre la plus grande parlic. C'est au dessous de cette lame que se remarquent les faisceaux blanchâtres, qui, du bord antérieur des tubercules quadrijumeaux, se dirigent vers les nerfs optiques.

La gerbe des faisceaux médullaires qui divergent de la couche optique, diminuc d'épaisseur des chauve-souris, des rongeurs, des ruminans, à l'homme : la diminution a lieu d'arrière en avant; c'est-à-dire que les faisceaux postéricurs disparaissent successivement. Celte disparition fait que la couche optique a d'autant moins de connexion avec les circonvolutions postérieures, que l'on s'écarte davantage des quadrumanes, pour se rapprocher des rongeurs. Enfin chez les oiscaux et les reptiles supéricurs, tels que les crocodiles, les caïmans, les tortues, la gerbe de ces

(1) Pl. XI, fig. $2.52,11^{\circ} 5$.

(3) PI. XI, fig. 241, no 8.

(3) Pl. XI, fig. $242, n^{\circ} 10$

(i) PI. XV, fig. $278, n^{n} 11$

(5) PI. IX . fig $20,5, n^{2} 12$. 
faiscenux nalus ancun rafport aree la coquille extérieure des hémisphères.

Cette gerbc des rayons médullaires émanés de la couche optique forme une mombrane rayonnante qui s'étale dans les hémisphères; placez sur celle membrane un amas de matière grise, et vous aurez le corps strié, situé conséquemment dans tout le pourtour et au côté externe de la couche optique. La grosse extrémité est en avant, et la pointe se termine en mourant en arrière, et après avoir contourné toute la couche optique, dans l'espèce de cul-de-sac de l'étui de la corne d'Ammon. De plus, en avant, la grosse extrémité du corps strié s'enfonce dans l'écartement antérieur des deux lames de la membrane rayonnante. Plus les faisceaux médullaires qui forment la membrane sont épais et nombreux, plus la matière grise qui les revêt est abondante; d'où il suit que la diminution du corps strié devra suivre cclle de ces mêmes faisceaux : donc le corps strié devra aller en diminuant d'épaisseur et de volume de l'homme, des quadrumanes et des carnassiers, aux ruminans, aux rongeurs, aux insectivores et aux chauvesouris, ce qui, chez les mammiferes, ne soufre pas d'exception, comme l'ont reconnu MII. Cinvier, Gall, Tréviranus, Tiedemann et Rolando.

Il y a donc une opposition chez les mammiferes entre le dévcloppement général du corps stric at celui de la couche optique : phas la partice pastiyieure de la couche oplique acquicrt de: volume. 
cumme chez les ruminans, phus la patic antérieure ¿lu corps strié diminuc. Que doit-il résulter de ce double effet? Il doit en résulter nécessairement que, plus on descend dans l'échelle des mammifères, plus le plateau résultant de la réunion de la la couche optique et du corps strié doit grossir en arrière ct diminuer en avant, plus conséquemment il doit se rapprocher de la forme triangulaire. Au contraire, plus on s'élève vers les quadrumanes et l'homme, plus la partie postérieure diminue, plus la partie antérieure augmente, plus ce plateau se rapproche de la forme sphérique. Or la lorme générale des hémisphères se moule su: celle de ce plateau; donc plus on descend vers les rongeurs et les chauve - souris, plus les hémisphères doivent tendre vers la forme triangulaire; plus on s'en éloigne au contraire, plus ils doivent deveuir sphériques. C'est ce qui est; et d'apprès la seule considération de ce plateau, nous pouvons ajouter, c'est ce qui doit être. Chez le phoque, lr: marsouin et le dauphin, ce plateau devient presque sphririque à cause de la direction plus transversale de la couche optique et du corps strié; de lá, la forme plus glolsuleuse de leurs hémisphères cérébraux.

De cette.disposition du corps striéché les man milieres, du rapport constant qui se remarqur antre le volume des faisceaux of lo volume di: lit maliore grise qui les couvrs, on a conclu que lis matire blanche derive de la grise, on lui en-. 
prunte les fonctions dont on l'a douée : cette opí nion ex primée différemment aux diverses époques de la science, par Willis, Vieussens et M. Gall, suppose ce qui était à prouver, savoir, que la matière blanche n'existait qu'à cause ou par l'inter mède de la matière grise. Nous avons vu dans l'encéphalogénie, qu'il en était différemment: nous allons montrer que cette opinion n'est pas plus vraisemblable dans l'encéphale des oiseaux adultes; leur corps strié va nous en fournir la preuve.

Si le décroissement progressif du corps strié et des radiations qu'il recouvre, continuait chez les oiseaux, nous devrions rencontrer une gerbe médullaire très-faible, et un corps strié au-dessous du volume où il s'observe chez les insectivores et les chauve-souris. Nous pourrions supposer alors, comme on l'a fait chez les mammifères, que la matière grise est la matrice de la matière blanche : mais si nous trouvons la matière blanche entièrement réduite dans ses radiations, et si nous voyons la matière grise plutôt accrue que diminuée dans sa masse, que devient notre supposition? Quel rapport y aura-t-il entre l'organe nutritif et l'organe nourri? Entre la masse de renforcement et la faiblesse de la masse renforcée? Ne serons-nous pas forcés de convenir, de mème que tous l'avons fait dans la moclle épinière, que cette hypothèse n'est point l'expression des faits? Mais que doit-on considérer comme le corps strié chez les oiseaux? 


\section{E'T LOBH OLFAC'TIF.}

Les tubercules quadrijumeaux des oiseaux ayant sté pris pour les couches optiques, cette crreur devait conduire d'une part à méconnaître ces couches, comme on l'a fait, et de l'autre, à les considérer comme les analogues du corps strié. C'est cn effet aux conches optiques des oiseaux que Collins, Haller et Malacarne ont donné le nom de corps strié: l'idée que toute la partie moyenne de l'encéphale de cette classe avait été déplacée, les maintenait dans cette croyance, et leur faisait négliger les connexions qui auraient pu les éclairer. Willis hésita sur la détermination du corps strié chez les oiseaux; il penchait à les trouver dans la inembrane rayounante dæs hémisphères; opinion qu'eût partagée Malacarne, s'il ne lui eût paru démontré que ce que nous nommons les couches optiques, était les véritables corps striés des oiseaux. Haller avait signalé dans les hémisphères un énorme tubercule, qu'il appela innominé, et auquel Malacarne donna le nom de tubercule de IIaller. C'est ce mème tubercule que Vicq-d'Azyr, MM. Cuvier, Blainville et Tielemann ont considéré avcc raison comme le corps strié de l'encéphale des oiseaux. Tréviranus n'adopte pas en entier cette détermination; il pense que l'on doit seulement considérer comme le corps strié, la partie de ce tubercule située en arricre de la commissure antéricurc. Dans celle supposition Io corps stric serait singulièrenent réduit dans 
ses dimeusions; la partic antéricure du grand tuberculc hémisphérique serail, d'après l'analomiste que nous venons de citer, la partie postérieure du bulbe olfactif, ou le commencement de ce que i'ai nommé lobe de l'hippocampe chez les mammifères; tet la partie postéricure bosselée chez les grands oiseaux, représenterait la corne d'Ammon : ce qui resterait entre ces deux parties, pour le corps strié, se trouverait réduit à presque rien. Le tubercule hémisphérique des oiseaux renfermerait donc, en abrégé, tout ce que l'on trouve dans les hémisphères de la classe supérieure, la couche optique exceptée. Mais si l'on considère l'uniformité intérieure de ce tubercule, son défaut de connexion avec le vérilable lobe olfactif, dont on retrouve le pédicule sur la base de l'encéphale, comme chez les mammifères, on ne trouvera pas, je pense, que ces déterminations soient justifiées.

Araut Tréviranus, M. de Blainville avail soupçonné que l'hémisphère entier des oiseanx pourrait bien être le lobe olfactif des mammiferes; ce célèbre zootomiste se fonde sur le volume énorme du corps strić. Mais comment le corps strié se serait-il logé dans le lobe olfactif? Chez les mammifères, plus le lobe olfactif augmente. plus le corps stric diminue; il faudrait donc sup. poser que chez les oiseaux l'inverse a lieu, el fur le corps strić el le lobe olfactif so développont 
simulanément el dans un rapport direet? De plus, eomment le lobe olfactif serait-il double de chaque côté chez les oiseaux?

J'ai moi-même long-temps hésité sur la détermination du corps strié des oiseaux : en effet, avant que mes recherches sur les cmbryons m'eussent dévoilé l'indépepdance réciproque des deux substances qui composent l'encéphale, je ne pouvais comprendre un corps strié si volumineux chrez les oiseaux, à côté de si faibles radiations médullaires; il me semblait que le volume des radiations devait nécessaircment être proportionué à celui de la matière grise. Je ne considérais en conséquence comme le corps strié, que la pointe du tubercule hémisphérique qui se trouve chez les grands oiseaux séparée de son corps par une petite dépression; mais celte sćparation n'existant que sur un petit nombre d'oiseaux, et se trouvant en-dehors de l'are des radiations médullaires sur lesquelles est toujours placé le corps strié, j'en revins au tubercule hémisphérique, qui les enveloppe de la même manière que chez les mammileres, ainsi que nous allons le dire.

En effel, détachons par la pensée le corps strié de la couche oplique des mammilères, nous verrons gu'il forme autour des radiations un are en fer à cheval, dans la concavité duquel pénèire la substance hanche. Getedisposition est celle quil wifecte chez les rongeurs, l's irsectivores al leg rhauve-sonris. Considerons maintenaut le tuber- 
cule hémisphérique des oiseaux : nous verront qu'il forme un arc semblable, dont la concavité, tournée en-dedans, reçoit les radiations émanées de la partie antérieure de la couche optique; mais ces radiations étant plus faibles encore que chez les derniers mammifères, et la matière grise ćtaut hors de toute proportion, cet arc sera une masse solide, ayant la forme d'un rein, et occupant les dcux tiers de la capacité du ventricule de l'hémisphèrc. 'Toutes les radiations postérieures de la couche optique ayant disparu chez les oiscaux . non-seulement elles ont perdu leur connexion avec la coquille des hémisphères, comme chez les insectivores et les chauve-souris; mais le corps strie lui-même a perdu dans cette classe ses comnexions arec la couche optique qui est restée en arrière, le corps strié étant projeté en avant sur le trajet des radiations. Pour rendre au corps strié des oiseaux les relations qu'il a perdues avec la conche. optique, il suffit de rapprocher ces deux parties l'une de l'autre, et de les ramener au point de contact; on voit alors le corps strié formant un arc, et cnveloppant la couche optique dans sa concavité, comme chez les mammifères. A la vérité. le corps strié paraît toul-à-fait déplacé chez les oiseaux; il semble qu'en venant se loger dans le ventricule hémisphérique, il a changé ses rapports habituels; mais ce changement qu'on a opposé à sa déterminatiou, n'est qu'apparent, l'uisque. comme je rais ir dire. le feniltet hémisphériquet 
qui sunterpose entre la couche optique et lui, me paraît être l'analogue de la lame cornée qu'on sait ètre interposée, chez tous les mammifères, entre ces deux parties.

Entrés par la partic concave du corps strié, les rayons médullaires divergent en tous sens dans l'épaisseur de la matière grise qui le constitue; parvenus dans sa partie moyenne, ils forment un arc médullaire, dans l'épaisseur duquel on observe plusicurs rayons qui le traversent directement (1); le plus grand nombre s'arrête à la concavité de l'arc; puis de la convexité partent de nouveaux faisceaux beaucoup plus dèliés, et qui se terminent insensiblement à la périphéric da tubercule, comme je l'ai représenté chez le casoar (2). L'extérieur du corps strié est concave en dedans, convexe en dehors; en arrière il offre une, deux ou trois bosselures, selon les espèces que l'on examine: dans sa partie postérieure il est libre; dans sa convexité externe il est adhérent avec la coquille de l'hémisphère; celte adhérence est une véritable continuité de substance chez les oiseaux adultes; antéricurcment sa pointe se recourbe en bas. En dcdans le tubercule est libre dans toute son étendue. Il résulte donc de cette disposition. que le corps strie des oiseaux forme un tubercule considćrable, libre en dedans, et dans sa moitić

(1) PI. IV, fig. $105, \mathrm{n}^{\circ} 6$.

2) PI. IV , fig. 105, us 5, 6 e 7. 
postérieure, continu, au contraire, par une portion de sa convexilé supérieure, avce la lame externe de. l'hémisphère, et strié dans toute sa profondew par le rayonnement de la matière médullairc.

Ainsi le tubercule hémisphérique des oiseaux renferme le corps strié dans son épaisseur; sa limite me paraît correspondre à l'are médullair. qui traverse sa partic moyenne (1) : sa partie supérieure serait la masse correspondante à ce que l'on nomme denu-centre ovale chez les mammilères. Cettc délimination est beaucoup plus sensible chez les reptiles supérieurs que chez les oiscanx. Si on ouvre, en cffet, chez le crocodile, le caiman a muscaú de brochet re les tortues, le ventricule hémisphérique, on le trouve presque combli, commc chezles oiseaux, par un tubercule considérable, solide, d'un gris plus blanc que la substance corticalc des mammifères. Vu par la partic interne, où il est entièrement libre, $c$ tubercule est divisé par une rainure, qui lo sépare en deux parties, l'une inférieure, l'autre. supérieure : l'inféricure forme un croissant, dont l'extrémité postérieure part de la partie antérieure de la conche oplique, dont clle est séparée par le fenillet de l'hémisphère qui représente Ia lame cornée; dans sa concavilé il cmbrasse les rayons médullaires émanés de la couchc optique;

1) P. 11. fig. 105, n"1;. 
il s'arrète en devant à trois ligues cuviron de la terminaison de l'hémisphère. Au-dessus de ce tubercule se trouve un second tubercule plus considérable, plus étendu en haut, cn avant et en arrière que le précédent, et recouvrant en quelque sorte ce dernier. Cette disposition est surtout distincte chez les crocodiles et les caïmans : le tubercule hémisphérique s'arrête antérieurement une ou deux lignes avant la terminaison du ventricule hémisphériquequi, en cet endroit, se prolonge dans le ventricule du lobule optique, si prononcé chez le caïman à museau de brochet et la tortue franche. Le tubercule hémisphérique de ces reptiles est tout-à-fait libre en dedans, tout-à-fait libre supérieurement; il n'adhère que par sa base et sa face externe, au feuillet externe de l'hémisphère.

Le tubercule hémisphérique de ces reptiles est donc composé du corps strié et de la masse grise et striée qui cnveloppe ce corps, et qui correspond au demi-centre ovale des mammifères. C'est donc et par le demi-centre et par le corps strié que le ventricule hémisphérique est rempli. Déjà chez la tortue grecque, el chez le tupinambis et le caméléon, le tubercule du demi-centre ovale s'atrophie, et le corps strié, réduit lui-même dans son volume, se dessine plus neltement que chez les crocodiles ét les caïmóns; le ventricule devient de plus en plus libre: chey les lízards, on ne trouve plus que le corps strié formant un petit croissant autour des radiatious de la couche optique, croissant: qui rappelle celui que forment. 
les tori dans le ventricule optique des poissons. Enfin, chez les ophidiens et les batraciens, le corps strié devenu rudimentaire, forme à peine une saillie scnsible sur le fond du ventricule hémisphérique. Il résulte de là qu'ì mesure que le tubercule du demi-centre ovale diminue, la cavité du ventricule hémisphérique est dégagée: lorsqu'il a disparu chez les lézards, le ventricule est toutà-fait libre; chez les ophidiens et les batraciens, il n'y a plus que l'épaisseur du feuillet externe qui rétrécisse cetle cavité.

Tel nous a paru le corps strié llans les trois classes supérieures; il n'existe pas chez les poissons : la masse solide qui forme leurs hémisphères cérébrạux paraît entièrement due au feuillet hémisphérique externe.

Il est à remarquer que chez les oiseaux et les reptiles supérieurs, le volume du corps strié ne suit pas celui des pédoncules cérébraux; ce qui d'ailleurs se remarque déjà chez les mammifères: car l'agouti, le blaireau, le bouc de la haute Egypte, ont les pédoncules cérébraux beaucoup plus développés que les ours, quoique ces derniers aient un corps strié beaucoup plus considérable que les mammifères précédens.

Système des commissures des hémisphères cérébraux.

Les commissures sont l'intermède à l'aide duquel les diverses parties des hémisphères communiquent les unes avec les autres. Sous ce rapport 
viles sont de deux sortes. Les unes mettent en rapport l'hémisphère d'un côté avec l'hémisphère du côté opposé. Je les nomme commissures similaires, parce qu'elles conjuguent toujours les parties homologues des lobes cérébraux. Les autres lient les parties dissemblables d'un même bémisphère : ce sont les commissures dissimilaires. Parmi les premières, on distingue les commissures postérieures et antérieures, le corps calleux, et les commissures transrerses de la voûte ou les cordes de la lyre. Dans la seconde classe, on peut ranger la voûte, la bandelette demi-circulaire, les pédicules de la glnnde pinéale et leз nombreux faisceaux des plexus raycnnans, qui forment un tout des centres médullaires divers dont chaque hémisphère se compose.

Sous le rapport de la substance qui les forme, les commissures diffèrent également entre elles; le plus généralement, ces organes sont composés par de la matière blanche dont la disposition est le plus souvent fibreuse. Plus rarement c'est la matière grise qui établit le moyen d'union, comme dans la commissure molle des couches optiques. Une seule tient le milieu entre le gris et le blanc et offre un ligré de consistance plus ferme que 1. reste do l'encéphale : c'est la lame cornée et le septum lucidum.

Primitivement toutes les parties sont disjointes dans l'eucéphale des jeunes embryons; les commissures, quelles qu'elles soient, sont toutes des II. 
organes de seconde formation : cette proposition est rigoureusement exacte pour toutes celles des hémisphères cćrébraux, pour celles du cervelet, pour celles de la moelle épinière et des lobes opiiques. C'est le terme le plus élevé du développement de chaque partie.

Si , comme nous ne cessons de l'établir dans cet ouvrage, les classes inférieures répètent les phases divers que parcourent les embryons des inummifères supérieurs, nous derrons donc voir les commissures diminuer ou disparaître, à mesure que nous descendrons l'échulle des vertébrés, et que nous nous rapprocherons davantage des formes cmbryonnaires. Nous en avons déjà acquis la preuve dans les commissures du cervelet; nous avons vu le pont et le trapèze disparaître complètement chez les viseaux, et ne plus se reproduire dans les autres classes. Les oiseaux conservent encore la commissure des pédoncules supérieurs du cervelet, ou la valvule de Vieussens; mais on ne la trouve plus ni chez les reptiles, ni chez les poissons. C'est la répétition du même décroissement de l'encéphale, que nous allons retrouver dans les commissures des hémisphères cérébraux.

\section{Commissure antéricure el posterieure.}

Ces deux faisceaux médullaires conjuguent, en arrière et cn avant, les pédoncules cérébraux, lours renfemens et leurs radiations. Cie sont deux 
commissures similaires, quoique l'antérieure offre de grandes variations dans les différentes classes. La postérieure est le moyen d'union de la couche optique droite avec la couche optique gauche; elle manque chez les poissons, et son absence est la couséquence du défaut de développeneut de la couche optique. Elle manque également chez la plupart des reptiles, comme chez les ophidiens, les lacertiens et les batraciens, chez lesquels la couche optique est arrêtée lans son premier état embryonnaire. Les premiers rudimens de celle commissure ne se montrent que chez les sauricns et les chéloniens. Sa situation est plus profonde que dans les autres classes. Elle est très-épaisse, assez large chez les crocodiles, le caïman à museau de brochet et la tortue franche; sa partie externe se continue avec l'enveloppe des lobes optiques, dont ello est aussi le lieu de conjugaison. Chez les chélonieus, elle est un peu plus superficielle ct plus intimement liée à la couche optique, ce qui dẻjà se rapproche des oiseaux. Dans cette dernière classe, la commissure postérieure devient plus superficielle; elle abandonne ses rapports avec les lobes optiques et devient exclusivement propre à la couche optique. Elle cst située au-devant et un peu au-dessous de la commissure transverse des lobes optiques (1), bornant en arrière le troisième ventri-

1) PI. IV , lig. $989,11^{\circ} 8$. 
cule, et en avant la scissure de Sylvius (1); directe au milieu, elle s'incline un peu en avant, en s'enfonçant dans la masse grise de la couche optique (2). Chez la plupart des oiseaux, elle est formée de deux plans de fibres; un, superficiel, qui sc confond avec le bord antérieur de la commissure des lobes optiques; et un, profond, qui est toujours le plus considérable; quelques fibres de ce dernier pénètrent dans les pédoncules cérébraus. Haller méconnut catte commissure, Malacarne la décrivit, tout en prenant pour le corps strié des oiseaux leur véritable couche optique.

Tous les mammifères, sans exception, possèdent la commissure postéricure; chez eux comme chez les oiseaux, elle réunit la partie postérieure de la couche optique, ot borne en arrière le troisième ventricule. Ce rapport constant dans ces deux classes établit donc que chez les oiscaux la partie postérieure de la couche optique n'a point été déplacée, comme le croit Tréviranus. Ln général, la commissure postérieure est plus forte chezles mammifères (et surtout chez les ruminans) que chez l'homme. Cette augmentation cn rolume de cette commissure tient évidemment à l'accroissement de la partie postérieure de la couche optique. Chez les carnassiers, on peut y distinguer

(1) Pl. III, fig. $87, \mathrm{n}^{\circ} 5$.

(2) Pl. IV, fig. $101, n^{\circ} 1 \%$ 
irois lames appliquées l'une sur l'auire; chez les ruminans, il y en a quatre distinctes, comme je l'ai vu principalement chez le mouton, le chevreau et le bouc de la Haute-Égypte : leur division n'est sensible qu'à la partic postérieure de la commissure ; en avant, elles sont étroitement collfondues. Chez quelques ruminans, cependant, la bande poslérieure est séparée des autres; ce qui ferait croire à l'existence d'une double commissure postérieure.

Les lames superficielles sont celles qui s'étendent le plus profondément dans l'épaisseur de la couche optique; les plus profondes sont toujours plus courtes et en même temps plus blanches que les autres. Dans l'épaisseur de la commissure postérieure, on ne trouve de matière grise ni chez los mammifères, ni chez les oiscaux. A la différence de volume près, différence qui même n'est piss très-saillante, la commissure postérieure a la mêrne disposition chez tous les mammiferes; il n'en est pas de même le l'antéricure : sa disposition offe des dissemblances assez remarquables.

Tous les poissons et la plupart des reptiles sont donc privés de la commissure postéricure; l’antricure se retrouve dans tontes les classes, mai: avec des différences remarquables qui cö̈ncideni avec le développement divers des hemisphères cérébranx. Je n’ai tronyr la commissure anićximure chez aucun poisson carlilagincux; ce qui provient sans doute do la fusion des deux couches 
optiques en un plateau conique (1) : elle n'existe que chez quelques osseux, où, comme chcz le congre (2), elle forme une bande transverse réunissant les deux pédoncules cérébraux à leur sortie des lobes optiques. Celle commissure se rencontre chez presque tous les reptiles : elle devient sensible chez les batraciens; chez les lézards elle est plus prononcée encore; chez les crocodiles et les caimans, elle est située en avant de la couche optique ct dans le sillon qui la sépare du corps strié : à droite et à gauche, elle pénètre dans le tubercule hémisphérique, dans lequel elle se perd presque aussitôt son entrée; sa partie postérieure correspond à l'ouverture du ventricule hémisphérique. Elle est le point de réunion de la lame externe des hémisphères. Chez les tortues, mais surtout chez la tortue franche (3), elle se porte plus en arrière, comme chez les oiseaux, ct pénètre plus profondément que chez les sauriens, dans le tubercule hémisphérique, où elle se joint à quelques faisceaux des pyramides; les tortues ressemblent, sous ce rapport, aux oiseaux. Dans cette dernière classe, la commissure antérieure est située immédiatement en avant de la couche optique (4): sa position est plus pro-

(1) PI. VI, fig. 158, $n^{\circ}$ !)

(2) PI. VII, fig. 16;

(3) Fl. V, fig.:20, $\mathrm{n}^{\circ} \mathrm{z}$ bis.

(i) P. 111 , fig. $8=, n^{\circ} 8$. 
fonde que chez les reptiles; ce qui fait qu'elle devient visible à la base de l'encéphale (1), quand on a enlevé le kiasma des nerfs optiques, ct écartéles pédoncules. Sa position en arrière des piliers de la lame rayounante est constante chez tous les oiseaux. En pénétrant dans le tubercule hémisphérique, elle concourt à la formation de l'ouverture postérieure de son ventricule; arrivée dans la masse du tubercule, elle s'unit aux faisceaux des pyramides, et concourt à la formation de leur plexus médullaire; on suit aisément son trajet dans l'épaisseur du tuberculc, à cause du blanc mat de son tissu, qui tranche sur le fond grisâtre de ce dernier.

Chez les mammifères, la commissure antéricure paraît destinée à réunir les radiations du lobe olfactif et du corps strié; droite chez les oiseaux, où ce corps et ce lobe sont atrophiés, elle s'incline, chez les mammifères, en avant ou cn arrière, selon que les lobes olfactifs ou les corps striés sont prédominans dans l'encéphale où on la considère. Celte double conjugaison qu'clle est destinée à former, fait que dans cette classe le volume de la commissure antéricure reste à-pcuprès le même au milieu des variations nombrcuses des deux parties, dont elle est le moyen d'union: lorsqu'en effet le corps strié diminue, le lobe olfactif augmente; leur commissure doit done rester et reste en effet slationnaire. 
De ce donble rapport de la commissure antérieure dérivent aussi los variations qu'elle présente dans cette classe. Lorsque les lobes olfactifs sont portés au maximum de leur grandeur, et les corps striés réduits dans leur volume, la commissure antérieure est entraînće vers les lobes, et alors elle représcnte un arc dont la concavité est cn avant et la convexité en arrière : c'est le cas des ruminans, des rongeurs, des insectivores, des didelphes. Dans l'état moyen de développement du corps strié et du lobe olfactif, la commissure devient presque droite; c'est le cas des carnassiers plantigrades et digitigrades. Au contraire, chez l'homme, les singes, le phoque, et surtout les cétacés, chez lesquels le corps strié est parvenu au summum de son développement, et le lobe olfactif à son minimum, la commissure antérieure s'incline en arrière, et forme ainsi un croissant inverse de celui des premiers mammifères. Telles sont les dispositions générales de la commissure dans cette classe, et, selon nous, telles soni aussi les causes qui les déterminent.

la commissure antérieure recoit donc les radiations du lobe olfactif et de son pédicule; chez les mammifères, tels que l'homme, les singes et le phoque, chez lesquels les racines des nerfs sont filiformes, elle plonge principalement dans les circonvolutions postérieures du lobe antérieur, et dans les lames plissées de la partic antéricurc du lobe sphénoïdal. En traversant la frofondeur du вoyau mérlullaire de la scissure do 
Sylvius, elle reçoit les faisceaux du pédicule olfac. tif, principalement chez les carnassiers et les ruminans. Chez les mammifères à lobe olfactif très-prononcé, sa communication avec ses radiations présente des différences importantes. Tantôt, comme chez le mouton, le chevreau et le lapin, un des faisceaux principaux de la commissure longe la racine interne du pédicule olfactif, et s'étale dans la substance blanche de son lobule: tantôt, avant de former cet épanouissement, cetle branche, la plus forte de la commissure, parcourt le centre du pédicule olfactif, ce qui a lien surtout chez le cochon d'Inde : tantôt enfin, comme chez l'ornithorhynque, remarquable par le volume démesuré de cette commissure, la branche interne située au milieu du pédicule olfactif, est la plus faible, ci la branche externe, beaucoup plus considérable, envoie ses nombreuses radiations dans toute la circonférence du lobe de l'hippocampe. Les deux tiers des radiatious de chaque hémisphère convergent vers le faisceau de la commissure antérieure. Il est à remarquer que chez beaucoup de mammifères le trone de la commissure antérieure est logé dans une espéece de gaine? forméc par la pic-mère, qui semble l'isoler ainsi de la substance propre des hémisphères; il est i remarquer aussi que chez lous les embryons la commissure est parfaitement formée à l'ápoque ou le curps strić est remangubble par l'absence de strics méclullaires qui lui ont mérité son nom.

Fon se rendani d'un corpestuir a l'anter, la com- 
missure antérieure passe derrière les piliers antérieurs de la voûte, et concourt à la formation dè l'ouverture comparée, on ne sait pourquoi, à la vulve. Les oiseaux n'ont pas cette ouverture, ce qui tient à la position inverse des piliers de la lame rayonnante. En effet, des différences très-remarquables résultent de cette variation de la position de la commissure antérieure dans les deux classes. Chez les mammifères, les pédoncules antẻrieurs de la glande pinéale, après avoir débordé la couche optique, viennent s'adosser aux piliers de la voûte; chez les oiseaux, au contraire, les piliers de la lame rayonnante étant postérieurs à la commissure, c'est vers cette dernière que se portent les pédoncules de l'épiphyse cérébrale. Antérieurement le troisième ventricule est borné chez les mammifères par les piliers antérieurs d'abord, puis par la commissure au milieu; chezles oiseaux, c'est la commissure, puis les piliers qui limitent cette cavité. Ce reculement de la commissure des oiseaux fait aussi que dans toute la classe l'entonnoir est situé immédiatement au-dessous d'elle, tandis qu'il en est éloigné chez les mammifères.

\section{De la commissure molle.}

()n a nomme commissure molle, ou mieux commissure grise des cosiches optiques, un petil amas de matière grise formant une espèce de diaphragme Iorizontal dans le troisième ventriculc : elle part du miliou de la profondeur de la couche optipur. 
et ne s'étend qu'à sa surface interne. Elle manque quelquefois chez l'homme. Chez les mammifères, clle va en croissant des quadrumanes aux rongeurs; chez ces derniers, la moitié du troisième ventricule est comblée par sa présence. Elle offre néanmoins beaucoup de variétés dans son volume chez tous les mammiferes.

La commissure grise est plus faible chez les oiscaux que chez les mammifères; elie est aussi situéc plus bas que dans cette dernière classe. Chez les tortues, les crocodiles, les caïmans, elle est plus forte que chez les oiseaux, et située aussi plus bas que chez les mammifères; chez les batraciens et les ophidiens, elle est plus épaisse que chez les reptiles précédens. Chez les poissons cartilagincux, elle paraît avoir comblé en eutier le troisième ventricule, d'où résulte lc non-isolement des̉ couches opliques; d'où résulte aussi le reculement de l'entonnoir et son embouchure dans la partie antérieure du ventricule optique.

De la bandelette demi-circulaire.

Un sillon assez profond en avant, plus superficiel en arrière, sépare la couche optípue du corps strié chez les mammifères. Un rubar médullaire etroit, mince, d'une couleur plus ou moins grise chez lhomme ot les singes, les camassiers et les ruminans, blanc chez les rongenas, vient oceuper

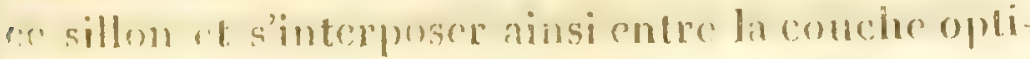


que, qu'il laisse en dedans, et le corps strié, quiesk situé en dehors. Ce ruban est ce que l'on a nommé bandelette demi-circulaire, lame cornée, centre demicirculaire. Sa consistance est plus résistante que la matière blanche; et, détachće de l'encéphale, ellea une espèce de trausparence nacrée : ses limites, son étendue, son but et son analogue chez les autres animaux u'ont été ni indiqués, ni entrevus. Je vais essayer de suppléer à cet oubli, en indiquant d'abord la partie qui me parait lui correspondre chez,les reptiles et les oiseaux.

Les hémisphères cérébraux des reptiles et des oiseaux, tout-à-fait isolés l'un de l'autre par leur partic supérieure à cause de l'absence du corps calleux et de la voûte, viennent se fixer ou s'assujétir par leur base aux pédoncules cérébraux; la lame externe, qui seule les constitue chezles ophidiens, après s'être réfléchic en arrière sur ellemême, se lirigye en dedans el s'ỉnsère sur les pédoncules cérébraux, immédiatement en arant de la couche optique. Chez les crocodiles, les tortues, 't chez le caïman à museau de brochet, cclte même lame, après avoir recouvert el enveloppéle unbercule hémisphérique. se réfléchit an-dcssous re liai, et, se dirigeant en dedins, vient fixer Théuispuiere entre fa couche oplique ot le corps strié. La conche oplique se trouve aunsi calehors de la cavité hémisphérique, ses radiations passant au-dessous de celte partie de la laue externe. Considérćc dans le but qu'clle parât rm- 
plir, cette lame peut être nommée ligament postéricur des hémisphères cérébraux. Chez tous les oiseaux, mais surtout chez cenx d'un grand volume, chez les aigles, les vautours, le casoar et l'autruche, la lame externe de l'hémisphère, après arvir contournéle tubercule quicomble leur cavité, se réflechit en bas, se porte aussi en dedans, et, comme chez les reptiles, vient s'insérer dans le sillon assez marqué qui sépare la couche optiqué du corps strić. Son usage paraît être, dans cette classe, le même que chrz les reptiles, quoique déjà la lame rayonnante vienne placer d'une manière plus étroite les hémisphères sous la dépendance des. pédoncules cérébraux.

Pour concevoir maintenant comment ce ligament, dont la couleur est d'un blanc mat chez les reptiles et les oiseaux, peut devenir la bandelette demi-circulaire des hémisphères de la classe supérieure, il faut se représenter la marche du développement progressif du corps strié, et les changemens qui surviennent dans ses rapports avec la couche oplique. Chez les reptiles et les oiseaux, le corps strié est situé en avant de la couche op-. tique, et sans conrexion a vec ellc; ce ligament est. interposé comme une cloison cutre eux. Dans.le: premier tiers de la formation des cmbryons des mammifères, la partie antérieure du corps strié existe seule; un sillon la sépare de la conche op-tique : une petite lame mince réflćchie do la co quillo intéricure de l'hémisplsère vient s'insérer 
dans ce sillon; cette lame est floltante et libre à cettc époque de la formation des embryons, ellé n'a ni la forme ni les rapports généraux de la bandelette demi-circulaire des mammifères adultes, et voilà pourquoi elle a été méconnue par les frères Wenzel et par Tiedemann. Mais à mesure que le corps strié se dévcloppe d'avant en arrière, il chasse devant lui le petit ligament; lixé en avant dans le sillon de séparation de la couche optique et du corps strié, libre en arrière, où il suit la marche progressive de l'hémisphère, l'effet du dévelappement du corps strié est'donc de l'appliquer entre lui et la couche optique; position danslaquelle il est d'ailleurs maintenu par ses adhérences avec les pelits vaisseaux qui passent au-dessous de lui comme sous un pont. D'après ce mode de développement on voit que la bandelette demi-circulaire n'est et ne peut être à sa place que lorsque le corps strié a parcouru ses principales évolutions, ce qui n'a lieu que vers le commencement du dernier tiers de la formation des embryons. Avant cette époque je la cherchais en vain, comme les autres anatomistes, et je ne l'ai reconnue que lorsque, pour répondre à l'appel de l'Académie des sciences, j'ai cherché à déterminer, comme je viens de le faire, à quelle partie elle correspondait dans l'encéphale des autres classes.

En avant, la bandelette demi-circulaire se joint à la parlie exturne du pilier antéricur de la roûte; puis sa lame épanouie tapisse la paroi externe du septum ou de la cloison transparente. 11 est à re- 
marquer que la cloison, chez les mammifères, n'à ni la couleur de la voûte, ni celle de ses piliers, ni celle du corps calleux, parties avec lesquelles elle est cn rapport; tandis que chez tous les mammifères elle offre la même nuance de coloration que la bandelette demi-circulaire. Dans les altérations pathologiqucs de ces partićs de l'encéphale, la cloison reste étrangère aux changemens que peurent éprouver la voûte ou le corps calleux, tandis qu'elle partage toujours ceux de la lame cornée. La bandelette demi-circulaire se termine donc en avant, en s'épanouissant sur la surface externe de la cloison, par l'intermède de laquelle elle se joint au corps calleux; en arrière, elle présente un épanouissement analogue, et offre un rapport important qui n'a pas encore été aperçu.

On a dit que la bandelette demi-circulaire s'arrêtait en arrière au niveau de la partie postérieure de la couche optique. Tarin la perdit de vue en cet endroit, et Lancisi ayant fait du corps calleux le sensorium commune, la considéra comme une continuation de ce corps qui venait se joindre par son intermède aux pédoncules cérébraux. Vicqd'Azir et les frères Wenzel la conduisirent plus loin, jusqu'à l'entrée du plafond de la corne descenlante du grand ventricule. Elle s'étend bien auIelá cricore; c'est même au point où l'ont conduite ces dernicrs anatomistes, et Meckel, qu'elle augmente de volume, et que commence le rapport important que nous allons faire connaitre. 
En effet; chez l'homme; les singes, les carnassiers, les ruminans et les rongeurs, lorsque la bandelette demi-circulaire est arriyée sur le plafond de la réflcxion de la corne descendante, elle grossit beaucoup, et forme un arc dont la convexité est en dehors et la concavité en dẹdans: elle change ainsi de direction, et se rapproche de la convexité des nerfs optiques, un peu avaut leur entrée, dans le corps genouillé inférieur. Un peu plus avant encore, la bandclette se joint intimement au nerf optique, aqquel elle adhère trèsfortement, ainsi qu'aux pédoncules cérébraux. Chez l'homme et chez quelques ruminans, j'ai vu un filament très-prononcé se détacher en cet endroit de la lame cornée, et se joindre aux faisceaux qui composent le nerf optique. Après avoir contracté cette union avec le nerf đe la rision, union qui simule cette figure $\mathrm{x}$, la bandelette se rend dans le fond de la corne descendante; là elle s'épanouit sur un tubercule volumineux de matière grise, qui occupe la terminaison du ventricule, et correspond en dehors à la partie postérieure et externe de la scissure de Sylvius. Cet amas de matière grise, que je nomme tubcrcule de la commissure demi-circulaire ou de la lame cornée, est circonscrit par une coquille mince, de matière médullaire : la commissure antérieure vient le pénétrer en dehors chez l'homme et quelques singes. Chez l'homme, ce fubercule paraît ĉtre le point de départ de la commissưre antérieure et de la 
commissure demi-circulaire. On voit, d'apris la marche de celte dernière, qu’elle est desiince à meltre en rapport les parlies posléricures des loćmisphères aves les piliers antéricurs de la voùte et le corps calleux; ce qui me paraît justifier le nouveau nom que je lui ai donné.

Avant de s'épanouir dans ce tubercule, la commissure demi-circulaire, cn abandonnant le ucrf oplique, laisse libre un pelit espace dont le plancher est formé prar les pédoncules cérébraux, le côré interue par le uerfoptique, le côlé externe par la bandelctle, et le plifond par la terminaison du corps frangé ou d!u pilicr postéricur de la voûte. Celte ourcrture, que je nomme fissure ammonique, est irès étroite chez l'homme, les singes, les phoqueset les cétacés; clle est occupée par des vaisseaux qui pénètrent par cet onároit dans le ventricule. Chez les carnassiers ct les rumiuans, clle est plus larse; enfin, chezles rongeurs ct les chauve-souris, la fissure ammonique est une fento assez large. Quelqu'élroile que soit colte fissure chez l'homme, les singes et les carmassicrs, on comçoil que la scirosilé qui s'y trouve a l'état de vapeur, pourrait s'en celapper; une petite valvule met obstacle a celle sorlic, ou à colle de lout aulre liquide accidentellement accumulé daus le veraricule. Cotlevalvule est formée par wne polibe lume grisatre et tros-résistante, qui de ha commissure demi-circulaire se portesur la erminaison de la hame frangéce ou du pilice postéricur de la voúle. Le bord bibre ast en II. 
dedans, le bord adhérent est uni à la terminaison de la corne descendante du ventricule latéral. L'existence de cette valvule est constante, quoique l'usage que nous lui attribuons ne soit qu'une conjecture.

Le corps frangé considéré dans l'ensemble des mammifères, fait partie du pilier postérieur de la voûte. Longeant le côté concave de la corne d'Ammon, il pénètre jusqu'au fond de la corne descendante, où il se réunit, par l'intermède de la valvule, avec la commissure demi-circulaire : ce mode de terminaison, peu prononcé chcz l'homme et les singes, est beaucoup plus apparent chez les carnassiers (surtout chez les digitigrades), chez les pachydermes et chez les ruminans. Chez les rongeurs, son bord tranchant est à nu, hors du ventricule, au niveau de l'adhérence de la commissure demi-circulaire, ce qui élargit la fissure ammonique; on peut mème chez ces derniers animaux le suivre au-delà du ventricule jusqu'à la partic postéricure du champ olfactif, où il communique avec un ou deux faisceaux de la commissure antéricurc. Il est à remarquer que depuis les carnassiers jusqu'aux rongeurs ct aux chaure-souris, les dentelures du corps frangé disparaissent successivement; ct c'est peut-être à cause de leur déplissement chez ces lerniers animaux, queson bord tranchant débovie l'intericul de la corne descendante. Vers te milien de la hauteur de la corne descendante du ventriculc latéral, 
le pilicr postérieur se confond arec le cylindre de la corne d'Ammon, dont le plus grand nombre des faisccaux se joint à lui : ainsi renforcé, le pilicr se place au-dessus de la couche oplique, communique arec son congénère par des fibres transverses, et ne forme plus qu'un organe unique, étendu en forme de plafond sur l'ouverture du troisième ventricule et le plateau des couches optiques; la voùte se recourbe légèrement en bas, au-devant des couches opliques, et sedivisc de noureau en deux cordons fibreux, qui sont les piliers antérieurs (1), situćs en arrière de la commissure anlérieure (2), que l'on aperçoil sur la lignne médiane dans l'intervalle qui les sépare. De même que les piliers pustirieurs s'enfoncent dans la corne descendante dugrand ventricule, jusqu'au niveau de la surface exierne de l'encéphale; de mêmn les piliers antéricurs descendent dans la profondeur de la couche oplique, jusque daus le noyau gris des éminences mamilhires, chez l'homme, et chez les carnassicrs jusque sur le pelit amas médullaire qui, l'après Vicq-d'Azyr, Soemmering cl M. ('uvier, constitue le rudiment de ces émirences. et que l'on rencontre aussi au-dessous du tubercule optique des ruminans (5). La voûte, en outre, se joint en arricre au corps calleux, au point de con-

(1) PI. IX, firg. 205, n०14.

(a) P. IX, ng. $205, n^{\circ} 15$ \& t 1 -

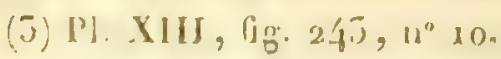


rergence des denx cylindres de la corne d'Ammon. Eu avant des pilicrs antéricurs, on roit unc exp usion membraucuse qui monte vers le corps calleux, cxpansion qui, lapisséc en dehors parl'épanouisscment de la commissure demu-circulaire, forme le srptum lucidum. La voûte réunit ainsi les principales parties de la rigion inférieure de chaque hémispi:ère, et plus spécialement celle que nous arous nomméc lobe de l'hippocampe.

On a dit que la roûte suivait chez les mammiferes les memes rapports que la poutre; proposilion qui, vaic en apparence, me paraît dé. mentic par l'examen de cette partic, suivi des quadrumanes aux rongeurs. Elle cst vraic, si on n'a égarl qu'a la longucur : on observe ca clict, sur une scclion verticale de l'encéphale (1), que l'étendue longitudinale de la voúte décroît successivement, el proporlionnellement au décroissement du corps calleux, de l'homme aux qualrumancs, aux phoques, aux célacés, aux carnẹssiers, anx pachydermes, aux ruminans, et cufin aux rongeurs et aux chanve-souris; décroisscment qui n'est que relalif à la diminution du prolongement des lićmispheres cérébraux "cn arriere. Mais l'ilunduc de ha voulo: doit être mesurice gur lia lirgeur de son platcau; ct, considérie dans ac sens, on la voil varier dos rongeurs à l'homme de la manicre suirante. Chez

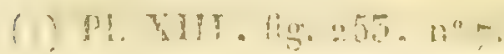


l'homme et les singes, elle n'occupe qu'un espace éroit, en arricre des couches optiques; dejisechez le phoque on la roit s'élargir beaucoup en cet endroit (1), plus cucore chez les carnassicrs (2); cnfin chez les ruminans, les rongeurs et les chanre-souris, clle ne couvre pas seulement les couches opliques, mais mente la commissure deni-circulaire et l'extrimité postéricure du corps strić. On doit remarquer i ce sujet que l'évasenent du troisième ventricule a uquel elle sert en quelque sorte de plisfond, loin de s'agrandir dans la même proportion, diminue au contraire en sens inverse de son élargissument. A cet élargissement correspond une disposition importante, a laquelle n'ont point fait allention les analomistes; c'est que la membrane interne qui la constitue, se plisse daus le point qui correspond à son plus grand diamétré, et furme deux circonvolutions qui muliiplicnt beaucoup sa surface. Ces circonvolutions de hroute, deja Iris-distincies chez le phoque (J). plus prononcées chezles auires carnussiers (4), vontenangmentant chez les pachyderine's, les ruminans of l:'s rongeurs. Eilles sont toujours proportionuclles an
(1) Pl. X, fig. $2.18, n^{\circ} 10$.
(2) Pl, XII, fig. $2.12, n^{\circ} 8$.
(ii) Pl. X, lig. $218, n^{\circ}$ so et 11 .
(4) Pl. XII, fig. $24^{2}, n^{\circ} 8 \mathrm{ct} 9$. 
développement du lobe de l'hippocampe et de la corne d'Ammon.

La corne d'Ammon suit les mêmes rapports que la voûte; clle augmente des quadrumanes aux carnassiers, aux ruminans ct aux rongeurs. Le petit pied d'hippocampe, ou le relief de l'anse d'une anfractuosilé dans la corne postérieure du grand veniricule, n'a encore ćlé aperçu que dans l'homme; on l'efface en le dépliseant par le procédé que l'on met cn usage pour développer le grand rentricule latéral; je l'avais moimême mécoanu en procédant à sa recherche de cette manière. Je l'ai découvert au contraire chez les singes et les phoques, en pratiquant sur le lobe postéricur une section rerticale au niveau du genou postéricur du corps calleux : on enlére de colte manière toute la cavité ancéroïde, ct en l'entrourrant, en comprimant légèrement le dobe postéricur, on roit le pelit picd d'hippocampe. Dans les cerveaux qui ont élé durcis par l'alcuhol, on le met à decourert par crtte sestion et par le procédé ordinairc. Je l'ai maintenant suus les yeux, chez le papion (simia sphynx), chez le rhésus, chez une autre cspice de macaque, et chez le mandril! (s. maimon). Chez le phoque, il est plus court que chez les singes, el ne se prolonge que dans une partie de l'étui de la corne postéricure. Chez le mersonin, le clauphin et les carnossiers, il n'en existe plus aucune trace. 
Plus on s'éloigne de l'homme, et plus on s'avance vers les rongeurs, plus la forme de la voûte se rapproche de celle d'un triangle, dont la base serait en arrière. Cette disposition tient, d'une part, à l'accroissement successif de la partie postérieure, et, del'autre, au développenent de la partie postéricure de la couche optique, dont nous avons déjà parlé. Ce qui confirme ces données sur le rapport progressif de l'étendue de la voûte, c'est que ses piliers antérieur et postérieur vont en augmentant de volume, de l'homme aux singes, aux phoques, aux carnassicrs, aux ruminans et aux rongeurs.

La commissure demi-circulaire est donc une dépendance de la voûte; et celle-ci, conjuguant les parties d'un même hémisphère, est donc aussi une commissure dissimilaire. C'est surtout chez les oiseaux que la voûte présente ce caractère.

La membrane rayonnante (1) qui la constitue, a été prise par Willis pour le corps strié des mammilères; Haller la compara à la voûte, fondant principalement sa détermination sur ses piliers antéricurs; Malacarne rejeta celte comparaison et l'assimila au corps calleux; d'autres anatomistes, depuis Haller, ont cru qu'ellc correspondait à la cloison trausparcnte; enfin, on a rejeté tous ces rapports, et on n'admet chez les oiscaux ni cloison transparente, ni voutte, ni corps callcux. En réfutant l'opinion de Haller, et en établissant

(1) PI. III, fig. 85, $n^{n} 11$ et 12. 
ses nourelles vues, Malacarne ne s'arrêta que sur ses conuexions avec la partic interne dres hémisphières; il nígligea et sa partic postérieure el ses piliers : c'est lii sans doute ce qui dernicrement a porlé Trériranus à voir tout-ì-la-fois dans la lame rayonnaute et le corps calleux, par sa partie supéricure, e: la voûte, par sa base. En repoussant la comparaison que l'on avait établic arce la cloison transparcnte, co célèbre anatomiste fait usage d'un argument qui, appliqué au corps calleux, me parât également détruirc l'idée qu'il propose. Après avoir fait observer arec raison, que fil cloison transparente diminue chez les rongeurs, les inscctirores, ef devicut toul-à-fait rudimentaire chez les chauve-souris: il n'est donc pas probable, ajoute-t-il, qu'une parlic qui avait presque disparu chez les dernicrs mammiferes, reparaisse tout à-coup dans le cerveau des oiscaux avec plus de force el d'étendue que chez les animaux de la classe supéricure. Mais le corps calleux n'est-il pas chez les derniers mammifères dans les mêmes conditions que la cloison? N'est-il pas réduit à une lame mince ct courte, dont la force et l'élendue ne peuveut être comparéc's à celles de la lame rayonnante des oiscaux? La direction transversale du corps calleux, le caractere qu'il présenie, de conjuguer les partics similaires des deux hémisphères, ne sont-ils pas opposés à la direction verlicale de la lame rayonmante, et surtout à son but, analoguc à celui de la voûte, 
el qui est de rémnir les parties dissimilaires d'un même hémisplière? D'après ces considérations, ct surtout d'après la disposition qu'elle affectedans toute la classe, la lame rayonnante des oiseaux me paraît, comme je l'ai déjà dit, correspondre à la voute des mammifères. Je vais justifier ma détermination par la description de celte partic importante de l'encéphale des oiseaux.

Si vous écartez supérieurcment les hémisphères cérébraux des oiscaux, vous apercevez sur leur partic interne une lame argenline ct rayonnée (1) d'un blanc très-pur, ayant la forme d'unc faux, pour me servir d'une comparaisou usitée en anatomic. Colte lame s'étend de la partic antéricure (2) à la partic postéricure (5) de la face interue de l'hémisphère. Tous ses rayons convergent, d'arrière en avant pour les postérieurs (4), et d'avant en arrièce pour les antérieurs (h), et vicnnent se réunir cu un grand laisccau arrondi (j) qui s'enfonce dans la profondeur de l'hémisphère au-dessus de l'entrecroisement des nerfs optiques, cn passant au-devant de la commissure anté. ricure (7). Chez grand nombre d'oiscaus, chez
(1) Pl. 1II, fig. 83, $\mathrm{n}^{\circ} 12$.
(2) Pl. 111, fig. 83, $n^{\circ} 11$.
(3) Pl. III, fig, 85, $n^{\circ} 9$.
(4) Pl. III, Gin. $86, n^{\circ} 10$
(5) Pl. III, fig. $86, n^{\circ} 9$.
(6) Pl. III, fig. 86, n० 8 .
(7) Pl. 111, fig. $86,11^{\circ} 4$. 
le coq, le faisan doré, le faisan argenté, les canards, et surtout le canard musqué, la bondrée commune (1), le perroquet (2) et le casoar (3), ce pilier de la lame rayonnante devient visible sur la base de l'encéphale et au-devant des nerfs optiques (4), de la profondeur desquels il semble se détacher. Chez le plus grand nombre des espèces, comme chez les oiseaux de proie, les perdrix, les pigcons, les oics, les cigognes (5) et l'autruche (6), le nerf optique déjeté eu avant, cache le pilier que l'on découvre en renversant le nerf en arrièrc. Ce pilier s'enfonçe en s'amincissant audessous de l'entrecroisement des nerfs de la vision. Son insertion à la partie moyenne de la lame rayonnante la divise en deux partics que l'on peut nommer ses ailes, l'une antérieure el l'autre postérieure.

L'aile postérieure, étendue du pilier à la parlie postéricure de l'hémisphìre, s'élève au-dessus de la commissure antéricure ( 7 ), se dirige ensuite d'avant en arrière, et se place au-dessus de l'excavation du troisième ventricule, auquel il sert d'epithelium ou de couvcrcle. Sa structure est moins

(1) Pl. IV , f.g. $88, \mathrm{n}^{\circ} 6$.

(2) P1. IV , fig. $g^{6}, n^{\circ} 7$.

(i) Pl. III, fir. $79,11^{\circ} 10$; fig. $78, \mathrm{n}^{\circ} 31$.

(4) Pl. IV, fig. 103.

(5). Pl. IV , figr. 98.

(6) PI. MII, fir. $85, n^{\circ} 10$.

(g) Pl. III, Gig. $85, \mathrm{n}^{\circ}$ : 0 . 
argentine que celle de l'aile antérieure; dans l'interralle de ses rayons il existe une certaine quanlité de matière grise. Son bord inférieur, quand les hémisphères sont écartés, ou externe, quand ils sont rejoints, est formé par unc petite bande d'un blane mat (1), qui bride les rayons, et que j'ai comparée à la lame cornćc des mammifères. Les rayons qui forment cette aile sont froncés chez beaucoup d'oiscaux, spécialement chez les aigles, l'autruche, les cigognes et le casoar (2), et simulcnt, jusqu'à un certain roint, les dentelures de la lame cornće en arrière, ou même, comme chez le casoar, les circonrolutions de la parlie postéricure de la vonte des mammifèrs. Telle est l'apparence de cetleaile de la lame raynnante, quand les hèmisphères sont écartós l'un de l'autre; mais quand on les laisse en pusition, et qu'on examine celte partic d'arriere en avant après avoir renversé le cerrelet, on observe alor's que ses rapports arec li voutc sont beaucoup plus évidens que daus la préparation précédente. Si en effet on soulère alors la parlic postérieure dos hémisphìres, on aperçoil gue les deux ailes pnstérieures, adossécs sur la ligne médiane, sont unics loujours par de pelits laisccaux de la pie-mère, ou, plis rarement, comme chez les perroquets, les cigngnes, l'autruche et le casoar, par un ou deux

(1) P1. I11, fig. $85, n^{\circ} 9$

(2) Pl. III, fir. $85,11^{\circ}$ !) 
filcts extrêmement miuces de substance mé.lullaire. Ainsi réunies, les deux ailes simulent assez bien la tente du cerrelet; elles forment un véritable plafond, étendu jusqu'au tiers antérieur de la commissure des lobes opliques (1), et couvrant toute la surfice supéricure de la conche optique. Le froncement des rayons postéricurs est rejeté en dehors et un pcu au-dessous de l'hémisphère, comme le sunt chez les mammifères iuféricurs, la lame cornée c.t la partic la plus interne de la corne d'Ammon.

Immédiatement au-dessous de cette tentc, ou de cellevoûte (carc'est vicritablement une voûte), on trouve, conme chez les mammilères, un lacis vasculaire des plus richement organisćs : c'est le tronc des plexus chorö̈dicus, qui de là pénètrent dans le troisième ventricule ef le veutricule hémisphérique, de même que chez les mammifères. Ce lacis vaseulaire forme une membrane sur laquelle repose cette partic de la roûte, toujours a ussi comme la voûte de la classe su! éricure. Au milicu de ce lacis se trourent aussi la glande pinéale et ses pédoncules que la tente rayonnée recourre, ainsi que le troisieme ventricule. L'ailc postéricure de la lame rayounante des oiseaux représente donc la partie postéricure de la voûte des mammiferes, à l'époque embryonnaire, où

(i) Pl. III, fig. $85, n^{\circ} 5$ et 6. 
el!c n'est pas réunic sur la ligne médiane. Lcs fibres transverses que l'on trouve quelquefois en arrière sont évidemment les premiers rudimens des filisceaux de la lyre.

L'aile antíricure de la jame rayonnante se dirige en avant, et occupe les deux tiers de la fice interue de l'hénsisphère (1). Ses fibres sont d'un blanc plus brillant que les postíricures, et la matière grise intercalće est moins abondante. Partant de la partic antericure du pilič, ses faisceaux divergent en manière de flammes (2); les plus antéricurs s'étendent jusqu'a la base du petit lobe olfaclif (3) et vers sa parlic inférieurc. Ces fibres représentent la cloison trausparente dont cll's ont tout-à-fait la dircclion. Quand les deux hímisphères sont appliqués l'un contre l'autre, c'est un double diaphragme qui isole les deux rentricules la!ćraux ou hémisphériques. En dehors, comme chez les mammilères, se trouve lut corps st icdes oiseaux; enfin, les piliers de la lame rayonnante ressemblent tellement aux piliers antérieurs de la voute, chez l'autruche, le perroquet, les cigogues, le casoar, et le plus grand nombre des oiscaux, qu'on cst surpris que celle analogie n’ait pas encore été signaléc. La seule différence qu'ils offrent (différence à laquelle per-
(i) Pl. III, fig. $85,11^{\circ}$ I\%
(a) H. II, fig. 8. , n० 11 .
(5) PI. III, fir. 9 i, 11019. 
sonne, que je sache, $\mathbf{n}$ a fait attention), c'est qu'ils s'insèrcnt en avant de la commissure antérieure chez les oiscaux, et en arric̀re chez les mammifẻres. Cette différence tiendrait-elle à la concentration de la couche optique ct du corps strié chez les mammiferes? Pour saisir ces rapports avec la voûte, il faut élablir la comparaison chez les mammifèreśs inférieurs. surtout chez les chauresouris; et, pour en embrasser l'ensembledans une seule préparation, on doit détacher la lame rayonnante dans toute sa circonférence, de masière à découvrir le ventricule hénisphérique des deux côtés, puis la soulever par la partie supérieure, comme on a coutume d'agir pour examiner daus l'anatomie de l'homme la cloison transparcute: on voil alors se manifester tout ce que uous venons d'exposer. L'amincissement de la lame interne de l'hémisphère forme, dans toute l'élendue de la lame rayonnaute, une dépression qui en dessine les contours (1): colle dépression correspond à la partie interne du ventricule lateral, dont la surface externe est comblćc par le tubercule hémisphérique.

Les reptiles ont, eomme les oiseaux, celte parlie de la lame interne qui, dans celte classe, forme la lame rayonnante: conme shez les oiseaux, clle sert do cloison interue au veutricule latéral, ct forme une dépression sensible a la partic

(1) Pl. III, fig. $83, \mathrm{n}^{0} 11$ ct 12. 
interne de l'hémisphère (1). Chez le crocodile, le caiman à museau de brochet, et surtout chez les tortues (2), on y distingue quelques stries médullaires; mais ces stries n'ont ricn de constant dans leur disposition, et ne rappellent en aucune manière l'organisation radiée de la membrane rayonnante des oiseaux. En arricre, le bord interne du feuillet hémisphćrique sc rapproche de la parlie que nous avons comparée à la lame corrée. Du reste il n'y a a cun vestige des piliers. Ites parties de la lame hémisphérique qui correspondent à la lame rayonnante, viennent aboutir, en avant et en arrière, à la commissure antćrieure, commissure qui, sur un grand nombre d'entre eux, est remplacée par un plateau de matière grise, qui est aux hémisphères ce que la commissure molle est aux couches opliques.

Tout annonce donc que la voûte a disparu chez les reptiles; et ce serait en vain que nous en cliercherions les moindres vestiges dans les hémisphères des poissons. Dans les lobes opliques mème, où plusieurs anatomistes l'ont placée, on ne trouve rien qui en rappelle la disposition dans les deux classes supericures; il me scmble même que Tréviranus l'a plutôt soupçonnéc que rue, quand il dit que de si fortes éminences mamillaires (parties qui, sclon nous, ne sont point crs émincnces) chez les poissous, peu-

(1) Pl. V, fir. $120, n^{\circ} 9 \mathrm{rt} 10$.

(8) Pl. V, lig. $120,00^{\circ} 106$ 
vent faire croire à l'cxistence d'unc voûte. Si d'aillcurs la commissurc postéricure a disparu ch'z les poissons, comment lit voûte, qui apparticnt à un degré plus élevédel'organisation, s'y trourcrait-clle?

\section{Du Corps Calleux.}

A mesure que nous nous élevons dans le sy'stème des commissures, nous voyons cerlaines d'entre elles disparaître complètement dans des classes entières : la commissure postéricure monque chez les poissons el la plupart des repliles; la roûte ne se trouve ni chez les reptiles ni chez l's poissons, les oiscaux la possèdent encoic avec les différences que nous avons exposées; cufin nous arrivons à la dernière et à la plus importante des commissures des hímisphères, le corps callcux, et nous la royons mauquer dans les trois classes inférieures. Le corps calleux forme le caractère classique des lobes cérćbraux des mamnifères, comme la prolubérance annulaire furme celui de leur cervelet. De même que la commissure du cervelet est déreloppće en raison direcle du volume de ses hémisphères, de même le corps callcux suit progressivement le rapport des lobes latéraux et postéricurs. Ce n'est done pas toute la masse des lobes cérébraux qui suit l'accroissenent du corps calleux, comme ce n'est pas toute la masse du cervelet qui suit le développement de la protubérance. Nous avons remarqué en effet que le lobe médian du cervelet suivait une marche inverse de la protubiranco; or, il y a un 
lobe cérébral qui répète dans les hémisphères cérébraux le lobe médian du cervelet, et qui, comme ce dernier aussi, est développé en raison inverse du corps calleux. C'est le lobe de l'hippocampe.

Le corps calleux diminue progressivement de l'homme aux singes (1), aux phoques.(2), aux cétacés, aux carvassiers (5), aux ruminans, aux rongeurs, aux insectivores et aux chaure-souris. Chez l'homme, les singes et les phoques, il forme un carré allongé, à-peu-près de la même largeur dans toute son étendue; chez les singes, il devient déjà plus ét roit en devant, comme on le voit chez le drill (4); et il l'est plus encore chez les carnassiers, ainsi qu'on l'observe chez le coati roux (5). En général, en descendant des quadrumanes aux rongeurs, plus les hémisphères cérébraux se rétrécissent en avant, plus la partie antérieure du corps calleux diminue d'étendue. Maiș sa largeur présente encore des différences plus tranchées que sa longueur. C'est surtout par la comparaison de cette partie chez les quadrumanes, les rongeurs et les insectivores, que l'on peut apprécier les pertes que fait le corps calleux dans cette dimen-

(1) Pl, VIII, fig. $19^{5}, \mathrm{H}, \mathrm{K}$.

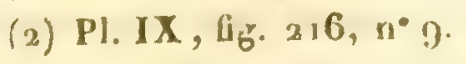

(3) Pl. XII, fig. $240, n^{*} 10$.

(4) PI. VIII, fig. $19^{8}, \mathrm{~K}$.

(5) Pl. XII, Ag. $340, n^{\circ} 10$ et 11.

II. 
sion. Le septum lucidum suit rigoureusement celte réduction progressive. En même temps que cotte commissure se rétrécit, en même temps qu'elle se raccourcit, son épaisseur et le nombre des plans fibreux qui ln constituent, diminuent tellement que, chez les rongeurs, le hérisson, la laupe et les chauve-souris, ce n'est plus qu'un pelit plateau très-mince, étendu de la partie interne d'un hémisphère à l'autre. Le corps calleux est une commissure similaire, ce qui le distingue de: la voùte: d'après sa disposition, il représente une seconde voûte superposée sur la voûte inférieure; le septum est interposé entre elles comme une sorte. de ligament.

\section{De l'Épiphyse et de l'Hypophysc cérébrales. (Glande piuéale et Glande pituitaire.)}

Je réunis l'examea de ces deux corps placés en quelque sorte hors de l'ensemble de l'encéphalt' des vertébrés, auquel ils ne sont réunis que d'une. manière médiate. Pendant le règne de l'hypothèse. des esprits animaux, on leur altribua plusieur. usages; mais le temps ế les progrès de la physio. logie ne nous ont donué que des doutes à leur égard : ils sont au systèmc cérébro-spinal ce que les capsules surrénales sont aux organes génitourinaires, la rate au sysleme digestif, le corps thyroide et le thymus aux organes respiratoires : le but de tous ces organes est encore un mystère. 
Au milieu de cette profonde incertitude, la constance de l'épiphyse et de l'hypophyse cérébrales chez les vertébrés, est un fait bien digne d'attention : elle semble nous indiquer que leurs usages sont d'une nature moins élevée qu'on ne l'a présumé dans tous les temps; et c'est dans cette vue qu'il est surtout important de constater leur existence dans toutes les classes de vertébrés.

Malgré les assertions contraires de Sténon et de Haller, la glande pinéale (épiphysecérébrale) existe dans toutes les classes de vertébrés. Chez les poissons osseux, elle cst le plus souvent si petite et si profondément cachée entre les hémisphères et les tubercules quadrijumeaux, qu'il est nécessaire, pour la distinguer, d'employer une loupe et d'éclairer d'une vive lumière le lieu qu'elle occupe : par ce procédé on parvient à la voir chez la plupart des poissons; je l'ai dernièrement montrée sur la lotte, le rouget, le hareng et le maquereau commun. Chez les cyprins, elle est beaucoup plus distincte; et il est étonnant que Haller, après l'avoir signalée dans cette famille, l'ait entièrement méconnue chez les oiseaux. Chez la morue et le congre, elle est distincte sur la face supérieure de l'encéphale (1), et plus volumineuse que chez beaucoup de reptiles. Je ne l'ai pas aperçue sur les raies que j'ai examinées; chez l'esturgeon, elle

(1) Pl. VII, Gg. $168,1105$. 
ressemble à un grain de millet implanté sur les pédoncules cérébraux (1); chez l'ange, elle est d'un volume qui dépasse toutes les proportions que nous lui connaissons dans les autres classes (2). Enfin, chez la lamproic de rivière (3), elle est immédiatement adossée à la partie postérieure des hémisjphères cérébraux, au-dessous desquels on distingue ses pédoncules ( 4 ), et leurimplantation sur la couche oplique (5). Chez tous les poissons chez lesquels la glande pinéale devient superficielle, l'ange exceptée, elle est tellement enchâsséc dans le lacis vasculaire qui l'cnvironne, et dans la matière gélalineuse qui recouvre la face supérieure de l'encéphale, qu'il esı absolument nécessaire de faire les recherches dans l'eau pour pouvoir la distinguer. 'l'elle est sans doute la cause qui l'a dérobée aux recherches de Camper, de Vicqd'Azyr, d'Arsaky, et, dans ces derniers temps, à celles de Tiedemann.

L'épihyse cérébrale est bien plus apparente chez les reptiles que chez les poissons : je l'ai rencontrée chez tous ceux dont j'ai disséqué l'encéphale. Elle est située en arrière des hémisphères cérébraux, et sur le renflement des couches optiques, dont

(1) Pl. XII, fig. 235, G.

(2) Pl. XII, fig. $237, \mathrm{~F}$.

(3) Pl. XI, fig. $224, n^{\circ} 3$.

(4) Pl. XI, fig. $228, n^{*} 5$.

(5) PI.XI, fig. 228, n०4. 
ses pédoncules dépassent quelquefois les limites. Chez l'orvet (1) elle cst d'unc ténuité extrême; chez les couleuvres, les vipères et les lézards, elle est un peu plus volumineuse, quoique toujours réduite à de très-petites dimensions. Chez le caméléon elle est plus prononcée (2), ainsi que chez le tupinambis (3); chez les crocodiles (́) elle est d'un volume considérable (5), comparativement à la masse totale de l'encéphale; elle est de forme allongée, et un peu bifurquée en devant. Chez le caïman à museau de brochet elle est plus forte encore, et divisée jusqu'à son sommet. Chez la tortue grecque elle est bifide (6) dans toute son étendue. Chez la tortue franche elle est ovalaire ( 7$)$, interposée entre les tubercules quadrijumeaux et les hémisphères; ses pédoncules, plus tranchés et plus courts (8) que chez les crocodiles et le caïman à museau de brochet, se prolongent jusqu'à l'entrée des pédoncules cérébraux dans les hémisphères, sur le plateau de matière grise qui joint ces derniers en cet endroit. Chez les reptiles, où il existe un rudiment de la commissure postérieure, la

(1) Pl. V, fig. $109, \mathrm{n}^{\bullet} 3$.

(2) Pl. V, fig. $111, n^{\circ} 8$.

(3) Pl. V, fig. $114, n^{\circ} 8$

(4) PI. V, fig. $115, n^{*} 5$.

(5) PI. V, fig. $116, n^{\circ} 6$.

(6) PI. V, fig. $125, \mathrm{n}^{\circ} 8$.

(7) Pl. V, fig. $119, n^{\circ} 9$

(8) Pl. V, fig. 12I, $\mathrm{n}^{\circ} 5$. 
glande pinéale n'a avec elle aucun rapport; disposition que j'ai surtout vérifiée sur les crocodiles et sur le caìman à museau de brochet.

Quand un homme dont le nom fait autorité dans la science, a émis une opinion erronée, il est rare qu'elle ne soit pas reproduite, après même que l'opinioncontraire aété prouvée jusqu'à la démonstration; c'est pour quelques auteurs une manière de faire croire à leur indépendance dans les sciences. Haller avait dit que les oiseaux manquent de glande pinéale; Vicq-d'Azyr, Malacarne, M.Cuvier ont redressé Haller à ce sujet; Tiedemann, Tréviranus, et tous les anatomistes qui ont disséqué avac soin le cerveau des oiseaux, ont confirmé la justesse des observations de M. Cuvier, de Malacarne et de Vicq-d'Azyr. Il était donc piquant de se mettre de nouveau en upposition avec ces hommes célèbres et avec la vérité; c'est ce qu'on a fait : néanmoins l'épiphyse cérébrale existe bien chez les oiseaux, et elle se trouve chez tous, en arrière de l'extrémité postérieure du sillon qui divise les hémisphères cérébraux, et en avant $\mathrm{du}$ cervelet, dans le licu où se rencontre le vaisseau analogue à la grande veine de Galien des mammifères. Je l'ai rencontréc chez le roitelel, lc chardonneret, le serin, le pinson, l'aloucte, le verdier, la linotte, le moineau, les mésanges, les perdrix, le pigeon, le merle, la poule, les faisans, les oies, les canards, le dindon, la pic, le geai, le hibou, le nilan, l'épervier. les 
aigles, le fou de Bassan, l'autruche et le casoar.

Le volume de la glande pinéale est en général proportionnel à celui de l'encéphale et de l'oiseau. Chez le coq d'Inde cette partie égale presque le cœur de la grenouille, dont elle se rapproche par la forme, ainsi que l'a remarqué Malacarne. Chez l'épervier elle est très-large et un peu aplatie d'avant en arrière. Chez les perroquets elle est arrondie (1), ainsi que chez les aigles et l'autruche de l'ancien continent. Chez le casoar (2) elle est aussi arrondie, très-volumineuse; un petit disque arrondi proémine sur sa face supérieure. Chez le plus grand nombre des oiseaux elle est d'un rouge vineux, de même que chez la plupart des reptiles, excepté à sa base, où se remarque un très-petit noyau de matière blanche. Chez d'autres, comme chez la pie, elle est d'un gris transparent.

L'épiphyse cérébrale des poissons et des reptiles n'est assujétie faiblement à l'eucéphale que par deux filamens déliés, dont l'insertion est trèsvariable. Chez les oiseaux, ses connexions sont plus fixes el plus multipliées; elles ont lieu, de même que chez les mammifères, par deux ordres de pédoncules, les uns postérieurs, les autres antéricurs. Les premiers partent du noyau blanchâtre situé à sa base; ils sont très-rléliés, el souvent plus

(1) PI. III, fig. 84, n० 7 .

(2) PI. III, fig. $83, n^{\circ} 8$. 
longs que leurs analogues chez les mammifères : chez certains oiseaux, et, par exemple, chez l'émerillon, ils jouissent d'une véritable élasticité. Leur implantation a lieu sur la commissure postérieure; ct cettc insertion est un guide assuré pour distinguer cette commissure de la bande antérieure de la commissure des lobes optiques, avec laquelle la glande pinéale n'est jamais en rapport.

Les pédoncules antérieurs de l'épiphyse cérébrale des oiseaux sont plus marqués que les postérieurs: ils se dirigent en avant, se placent, comme chez les mammifères, sur la face internedes couches optiques qu'ils circonscrivent (1), et se prolongent tantôt jusqu'en arrière de la commissure antérieure, comme on le voit chez lc casoar (2), tantôt jusqu'à cette nême commissure, ainsi que je l'ai fait représenter chez le perroquet (3); ils s'avancent quelquefois aussi jusqu'à l'entrée des pédoncules cérébraux dans les hémisphères, comme le montre l'isolement de ces pédoncules chez la poule (4). Si l'insertion des pédoncules postérieurs est un moyen de reconnaître dans cette classe la commissure postérieure, la position constante qu'affectent les pédoncules antérieurs est un caractère plus

(1) PI. III, fig. $84, n^{\prime} 8$.

(2) Pl. III, fig. $83, n^{\circ} 7$.

(3) PI. III, fig. $84, \mathrm{n}^{\circ} 8$.

(4) PI. III, fig. $87, n^{\circ} 7$ 


\section{E'T IOBE OLFAC'IT.}

certain encore pour préciser l'aualogic de leurs couches optiques avec ces mêmes parties chez les mammifères.

Chacun sait quel usage Descartes attribua à lit glande pinéale: le temps a fait justice de son hypothèse, mais la science a conservé et mis à profit les nombreuses recherches que l'on fit pour la justifier. De cette époque seulement datent les idées précises que l'on a eues sur ses connexions chez les mammifères. Comme chez les oiseaux, la glande pinéale a une double connexion arec l'cncéphale, en arrière par l'intermède des pédoncules postérieurs, en avant par celle des pédoncules antérieurs. Son volume dans cette classe n'oftre pas de très-grandes variations; cependanl chez les carnassiers elle est en général plus petit: que chez l'homme; chez le phoque elle est beaucoup plus étendue; chez les ruminans et les rongeurs, quoique moins grande que chez le phoque, elle dépasse le volume de celle des quadrumanes et de l'homme. On peut voir l'ensemble de ce rapport chez le phoque (1), le raton(2), la mangouste (3) et la gazelle (4). Chez beaucoup do mammifères, la glande pinéale est allongée, de même que chez les reptiles; c'est particulièrement

(1) Pl. X, fig. 218, n $^{\circ} 6$

(2) Pl. VIII, fig. 201, D.

(3) Pl. XI, fig. $2.32,11^{\circ} 5$.

(4) PI. XV, fig. $271, n^{\circ} 10$ 
ce que l'on remarque chez le phoque (1), le cas. tor (2) et le bouf; chez le mouton, le bouc ct le chevreau, elle se rapproche de la forme d'un triangle; chez les carnassiers elle est arrondie; elle est un peu conique chez certains singes (3). Enfin chez l'agouti, le fourmilier à deux doigts, la marmotte et le tatou, elle représente assez exactement une poire ayant sa grosse extrémité en arrière. Les graviers, que l'on rencontre dans son épaisseur chez l'homme et la plupart des singes, seraient un caractère distinctif de ce corps dans cette classe, si on les rencontrait chez tous; mais je ne les ai trouvés ni chez les rongeurs, ni chez le cerf et le mouton; le lieu qu'ils devraient occuper chez ces derniers animaux est remplacé par une très-petite cavité; Wenzel, Soemmering et Ticdemann ne les ont trouvés chez aucun mammifère; Malacarne, au contraire, a trouvé chez. le chevreau de petits cubes de matière calcaire, très-durs et à angles coupés : j’ai également rencontré ces cubes sur un chevreau, mais chez un autre ils n'existaient pas. Chez les oiseaux, les reptiles et: les poissons, on ne trouve aucuue trace de ces indurations; ces êtres sont, sous ce rapport, dans lo même cas que les foetus de tous les mammifères.

Trćviranus croit avoir remarqué que les animaux aquatiques ont la glande pinéale plus développér

(1) PI. XIV, fig. 265, (;

(a) Pl. XII, fig. 2/3. 
que ceux qui ne fréquentent pas habituellement les eaux. Je n'ai rien trouvé qui confirmât cette observation, exceptéchez les phoques. Les diverses espèces de canards n'ont pas ce corps plus étendu que les oiseaux qui ne recherchent jamais l'eau. Iues tortues m'ont paru même dans une disposition inverse; car les espèces terrestres ont cette épiphyse plus développée que les aquatiques. Enfin les poissons, qui sont les animaux açuatiques par cxcellence, ont la glande pinéale dans un lel état d'exiguité, qu'il 'st ordinairement très-difficile de la trouver chez ceux qui en sont pourvus, et que. malgré les recherches les plus minutieuses, je ne suis pasencore parvenu à l'a percevoir chez les raies.

Les pédoncules antérieurs de la glande pinéale viennent de la couche optique, comme déjà nous l'avons exposé. Ses pédoncules postérieurs s'implantent au rebord transversal qui limite en arrière le troisième ventricule, au-dessus de la commissure postérieurc. Chez beaucoup de mammifères, ils s'insèrent sur la couche optique même; je ne les ai jamais vus se porter jusque sur les tubercules quadrijumeaux antérieurs, comme quelques anatomistes disent les aroir aperçus.

Nous devons signaler une différence très-remarquable cntre les oiseaux et les mammiferes, dans l'insertion des pédnncules antérieurs de l'épiphyse rérbrale. Che\% les mammiferes, ces peidoncules s'adossent aux piliers antérieurs de la voùte; che\% les niscaux, col adossemonl a lieu sur la commis- 
sure antérieure. Cette différence tient, d'une part, au reculement de la commissure antéricure ché. les oiseaux; elle provient de l'autre, de ce que, par ce reculement, les piliers de leur lame rayonnante, au lieu de passer en arrière de cette commissure, comme chez les mammifères, se placent au contraire en avant. Ce déplacement de la commissure et des piliers est la différenct la plus notable du troisième ventricule dans ces deux classes.

L'hypophyse cérébrale (glande pituitaire) est encore plus isolće de l'encéphale que la glancie pinéale. Logée chez les mammifères et les oiseaux dans une fossette particulière, protégée par une lame de la dure-mère, qui lui forme une espèce de tente, elle communique avec la base du cerveau par un cordon creux que l’on a nommé sá tige. Organe impair chez les mammifères, les oiseaux et beaucoup de reptiles, ellc est double chez les jeunes embryons et quelques poissons.

L'hypophyse est ronde chez l'homme, les singes, les phoques, la plupart des carnassiers et des ruminans. Chez les carnassiers inférieurs, comme la fouine et le putois, elle prend la forme d'un champignon, sa queue étant formée par la tige. Chez les rongeurs, les chauve-souris et le hérisson, le disque de son plateau est plus aplati que chez les avinaux précédens. Quant à sa structure, clle est formée, chez les mammifères, d'une couche de matière griso . et d'un petit amas 
de matiere blanche, qui occupe ordinaircment le milieu.

Chez beaucoup de carnassicrs, comme chez l'ours (1), le noyau de l'hypophyse (2) est environné par deux lames qui l'enveloppent comme la coquille d'une amende (3): la tige marche entre ces deux lames, et va s'implanter à sa partie supérieure. Chez le castor, la forme de l'hypophyse est celle d'une bouteille, le col étant re-

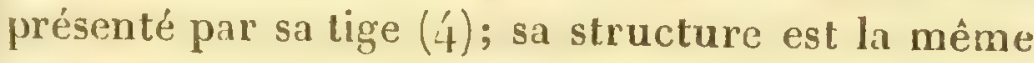
que chez l'ours (5) : le noyau est libre el comme enchâssé (6) dans les lames; le pédicule forme une espèce de gaine qui reçoit l'infundibulum ( $(7)$ : les lames ne descendent pas jusqu'à la partie inférieure du noyau (8), dont une partie est à nu, comme on le voit, l'hypophyse étant à sa position (9). Chez certains ruminans, comme chez le lama, la disposition est à-peu-près semblable : le noyau, à-peu-près pyriforme (10), est situéau milieu d'une cavité formée par les deux lames qui le circonscrivent (11) : l'infundibulum paraît

(1) Pl. XII, fig. $239, n^{\circ} 1,2$ et 3.

(2) Pl. XII , fig. $238, n^{\circ} 3$.

(3) PI. XII, fig. 238, $\mathrm{n}^{\circ} 1$ et 2.

(4) Pl. XIV, fig. $258, n^{\circ} 3$.

(5) PI. XIV, fig. $263, n^{\circ} 1,2$ et 3.

(6) Pl. XIV, fig. $263, n^{\circ} 2$.

(2) Pl. XIV, fig, $263, \mathrm{n}^{\circ}$.

(8) Pl. XIV, fig. $263, n^{*}$.

(9) Pl. XIV, fig. $258, n^{*} 3$.

(10) Pl. XVI, Aig. $293, n^{\circ} 4$.

(11) Pl. XVI, 6g. $1 g^{3}, n^{\bullet} 3$ et 3 . 
comme euchatonné à sa partie supérieure (1). Ia tige creuse de l'hypophyse s'implante le plus généralement dans cette classe sur la pariie moyenne du tubercule optique, comme on le remarque chez le lion (2) ef le castor (3): quand cette insertion se déplace, elle tend constamment à se rapprocher du kiasma des nerfs optiques, ainsi qu'on le voit chez le chamcau (4), le cheval (5) et le lama (6); enfin chez le hérisson (7) et le pécari (8), l'hypophyse vient adhérer à la partie postérieure du kiasma. Il est à remarquer que plus l'hypophyse se rapproche du nerf optique. plus sa tige se raccourcit.

La glande pituitaire exisle chez tous les oiseaux. Chez les gallinacés elle est ronde : chez quelquesuns, comme chez la poule, elle paraît composée de deux ou trois petits tubercules étroitement unis entre eux. Chez les cigognes elle est presque lenticulaire. Sa couleur est d'un gris analogue à celui des ganglions intervertébraux. La tige est trèscourte, unique etcreuse, comme chez les mammifères; clle s'implante, comme chez ces derniers,

(1) PI. XVI, fig. $2 g^{3}, \mathrm{n}^{\circ} 5$.

(2) Pl. XIV, fig. $266, n^{\circ}$.

(3) Pl. XIV, fig. $158, n^{\bullet} 3$.

(4) Pl. XIII, fig. $249, \mathbf{R}$.

(5) Pl. X V, fig. 275 , K.

(6) Pl. XVI, fig. $29^{5}, \mathrm{n}^{\circ} 18$.

(7) Pl. XVI, fig. $297, n^{\circ} 4$.

(8) Pl. XVI, fig, $300, n^{\circ} 16$. 
sur le milicu du lobule optique, mais un peu plus près de la jonction des nerfs visuels. Tréviranus a vu des filamens médullaires unir la tige au kiasma; je les ai cherchés en vain chez la poule, les oies, le dindon, le canard, les perdrix ct le pigeon. Le troisième ventricule se prolonge dans la tige, de même que chez les mammifères.

L'hypophyse est en général très-petite chez les reptiles : chez les grenouilles, elle est arrondie, trés-petite, d'un rouge jaunâtre; chez les ophi-diens et les lézards, clle est grise; son volume est plus considérable chez le caméléon, les crocodiles et le caïman à museau de brochet; chez ce dernier, olle est d'un gris rosacé ; sa tige est courte, mais très-distincte; elle s'implante à la partie moyenne du lobulc optique ; sa cavité communique dans celle de ce lobule, et par son intermèdc dans le troisième ventricule. Chez les grenouilles, les serpens et les ripères, je n'ai vu à la place de la tige qu'un filament très-mince; c'est sur les côlés de ce filament, et en arrière du lobule optique, que se trouvent les deux bandes médullaires que 'Tréviranus a décriles chez la grenouille, et les deux petits tubercules blancs que Malacarne a rencontrés chez les lézards et quolques vipères; res deux petits tubercules, ainsi que ces filamens, sont flottans sur la base de l'encéphale; ils n'ont. avec cedornieraucune communication immédiate. Ces deux petits tubercules seraient-ils des appendices de l'hypophyse? Seraient-ce les deux lames 
roulées qui forment l'enveloppe de son noyau cher les mammiferes? L'hypophyse m'a paru dépourvue, chez les reptiles, de la matière médullaire qu'elle présente chez les mammifères.

La disposition des lobules optiques dont nous allons bientôt parler, a fait naître diverses conjectures sur les corps particuliers que l'on rencontre chez les poissons, en arrière de l'entrecroisement des nerfs optiques. L'hypophyse a ainsi été sou vent méconnue; pour la distinguer, on doit d'abord la considérer chez les poissons, où ses rapports avec le tubercule optique sont les mêmes que chez les mammifères : tel est le griset (squalus griseus.) La forme de cette partie est oblongue, et sa tige très-épaisse s'implante sur le tiers antérieur du tubercule optique. Chez le squale bleu, elle est plus arrondie; sa tige, plus longue et toujours très-forte, s'adosse immédiatement au kiasma. Chez le brochet, elle simplante sur le tiers antérieur du lobule optique; elle est creuse et communique dans la carité de ce lobule. Chez le turbot, l'hypophyse a la forme d'un champignon (1); la tige très-courte s'unit à la partie moyenne du tubercule optique (2). Chez la baudroie, l'hypophyse, oblongue (3), rejetée en avant et très-éloignée de l'encéphale, communique avec

(1) Pl. VII, fig. $175, \mathrm{n}^{\circ} 5$.

(2) Pl. VII, fig. $17^{5}, \mathrm{n}^{\bullet} 4$ et 5 .

(3) PI. VII, fig. $179, \mathrm{n}^{\circ} 6$. 
te dernier par un rédicule grêle et trés-long (i): A l'endroit wà clle s'impiante sur le lobule optique; il semble se bifurquer et recevoir le pédicule d'un autre tubercule pyriforme (2), qui me parait êtrè une seconde hypophyse restée à sa place accoutumée. C'est poul-être la niême disposition, au pédicule près, que l'on remarque chez la morue, sur laquelle on trouve deux hypophyses distinctes, l'une postérieure, oblongue, et plus volumineuse (3), l'autrc anlérieure, plus arrondie et plus petite ( $\left(\begin{array}{l}\prime \\ )\end{array}\right)$. Chacune d'elles communique immédiatement avec une rainure particulière qui se voit au côté interne du tuberculc optique (5). Chez. la plupart des raies, l'hypophyse rejetée en arrière (6), est très-écartée díu nerf optique. Chez l’aiguiliat, elle est arrondie (7), ct son pédicule, très-grêle, s'implante dans la rainure des tubercules optiques. Chez le gronau, la glande pituitaire cst arrondie et appliquée immédiat’ment à la parlie postéricure du kiasma; son pédicule se pro. longe au-dessus du nerf optique jusqu'au-rlevant de la cavité des tubercules quadrijumeaux, et dans l'espace qui sépare cc lobe des hémisphères céré-
(1) Pl. VII, fig. $180, n^{\bullet} 10$
(2) Pl. VII, fig. $182, a^{\circ} 4$.
(3) Pl. VII, fig. $162, n^{\circ} 5$.
(4) Pl. YII, fig. $162, n^{*} 6$.
(5) Pl. VII, fig. 164, n* get 10.
(6) Pl. VI, fig. 148.
(2) Pl. X, Gg. 222, n*5. 11. 
braux; cette disposition est à-peu-près la meme chez la plupart des poissons osseux.

Les frères Wenzel ont fait la remarque, qu'il existe une corrélation très-ćlroite cutre la maripre d'être de la glande finćale et colle de la glante pilutaire. Conte observation, grécratement raic chezles mammiferes el clacz la phopert des replikes, troure quedinues execplions chez les oiscanx ct parmi les poissons: les rayes forment une exception remarifuble; car, qucicue chez :outes celles que jai cxaminces, lhypophyse soit trèsprononcée, je n'ai jamas aperçu la glande pinćale, quclque soin que j’aic apporté is cclic rccherche:

\section{Du Lobule oplique.}

Le lobule oplique, élément particulier de l'cncéphaic, est silué cn arrière du fiasma dos nerts opliques, auquel il est intlmement uni. Il a unc forme aplatie et triangulaire chez l'homme; sa base est adossćc aux cmincuces mamillaires; son sommet est enchissé derriere les nerf́s opliques : la cauche qui le forme est peu épaisic. Chez les singes, il est beaucoup plus dérloppé que che z l'homme (1); sa forme csi arrondic chez le mandrill (2), un pea ovaliare chez le drill (j),

(1) H. VIII, fir. 19. , $11^{\circ} 10$.

(2) PI. VIII, fig. 19, $n^{\circ} 10$.

(5) Fl. VIII, fig. 19z, u० 10. 
où il cst un peu moins développé : chez tous les quadrumanes, il ofreconstammenl'une oul'autre de ces aleux disposilions, et la saillic qu'il fait sur la basc de lencephate est loaucoup plus prononcée que chez lhomme. Chez le phoque (1), it présente le double en volume de celui du mandrill, quoique sa forme soil arrondie comme che\% lui. Chez les carnassicrs, tantôt il conscrve ccte méme forme, comme cher le lion (2) et la lon-

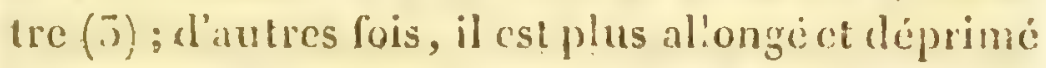
surses côtís, comme chezl'ours (4) el le raton (5). Chezle cheval (6), le chamean a deux bosses (7), le bocuf, l'ine, le mouton, il affecte la forme d'un cône tronqué, dont le sommet arrondi est tomrné vers les netfi de la trodsième pairc. Chez le kanguroo géant (\$), lc lama (9) et le pécari (10), la dépression latírale que nous avors remarquéc chez cortains carnassicrs, se reproduit, et son yolume continue toujonrs de s'uccroitre. Chacz de

(1) 陪, $1 \mathrm{X}$, fig. $208, \mathrm{~K}$.

(2) Pl. IIV, fig. $266, \mathrm{n}^{\circ}$.

(3) Pl. X, fir. $22 \overline{3}, \mathrm{~V}$.

(i) Pl. XI, Gg. $251, \mathrm{~V}$.

(5) Pl. VIII, fig. $200, \mathrm{n}^{*}, 0$.

(6) PI. XV; fig. 2 z5, n 2 .

(2) Pl. XII, lig. $249, \mathbf{R}$.

(8) PI. XYI, fig. $299, n^{\circ} 4$.

(9) Pl. XVI. fin, $3 y^{5}, n^{\circ} x$.

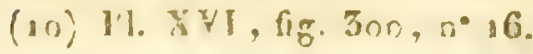


porćépic (1), le castor (2), et la plupart des rongeurs, il augmente de plus en plus, et s'arrondit de noureau, comme chez les singes. Chez certains carnassiers, comme chez le raton (3) ct l'ours (4), son extrémité postérieure offre sur la ligne médiane une échancrure superficielle. Chez le bouc de la haute Égypte (5), il est bifide antérieurement, et tout-à-fait arrondi en arrièrc. Clicz la taupe (6), le zemni (7), la musaraigne musette, la chrysochlore du Cap et le rat-taupe, le lobule oplique (qui ne mériterait plus ce nom chez ces animaux privés des norfs de la vision) est formé par deux tubercules arrondis, situés immédiatement en avant du pont, sur le point que devrait occuper le nerf moteur commun des yeux. Enfin je dois faire remarquer, comme une particularité digne d'attention, que je n'ai point aperçı ce lobule chez le dauphin (8) ct le marsouin, quoique les nerfs optiques soient d'un volume considérable. Si cette dernière observation se confirme, comment le lobule optique

(1) Pl. XIII, fig. $251, n^{\circ} 3$.

(2) Pl. XIV, fig. $258, n^{\circ} 3$.

(3) Pl. VIII, fig. 200.

(4) Pl. XI, fig. $25 \mathrm{I}$.

(5) PI. XIV, fig. $262, \mathrm{R}$.

(6) Pl. XIV, fig. $260, \mathrm{n}^{\circ} \mathrm{2}$ :

(c) Pl. XV, fig. $272, \mathrm{a}^{\circ} \%$

(8) PI. XII, fig. $254, n^{\circ}$. 
a-L-il abandonné les nerfs de la visiou chez les cé tacés, taudis qu'il a persisté chez les animaux précédens après la perle des nerfs optiques? Serait-ce à cause de ses rapports avec le lobe olfuctif? Serait-il anéanti, chez les cétacés, avec ce dernier lobe? et son volume, chezles mammifères privés des nerfs de la vision, serait-il cause de la persistance du lobule optique? Appuyons dès à présent cette conjecture, en faisant remarquer que, chez les mammifères, le lobe olfactif, le champ olfactif et le lobule optique sui. vent un rapport progressif de développenent de lhomme ct des quadrumancs aux rongeurs, comme on le voit chez le drill (1), le mandrill (2), le phoque (3), l'ours (4), le lion (5), la loutre (6), la marte (7), le raton (8), la mangouste (9), le cheval (10), le chameau à deux bosses (11), le daman (12), le bouc de la haute Égypte (13), le

(1) PI. VIII, fig. 197

(2) Pl. VIII, Ug. 194.

(3) PI. IX, fig. 208.

(4) Pl. XI, fig. 231.

(5) PI. XIV, fig. 266.

(6) PI. X, fig. 223.

(z) Pl. XV, fig. 290.

(8) PI. VIII, fig. 200.

(9) PI. XIII, fig. 254.

(10) Pl. XV, fig. 275.

(12) Pl. XIII, fig. 249.

(12) Pl. XV, fig. 273.

(15) Pl. XIY, ig. $26 \%$ 
lama (1), le pécari (2), les didelphes, le kanguroo géant (3), le tatou (4), le porc-épic (5), le castor (6), le lapin (7), la taupe (8), le zcmni (9) etl’agouti (10). Répétons cucore que ce même rapport se reproduit dans les phases divẹrses que parcourt l'embryon des mammiféres supérieurs; phases pendant lesquelles le lobule optique s'atroplaic dans le méme rapport que le lube olfaclif; enfin nous devons observer que chez licancoup de mammiferes inféricurs, chez lesquels le champ oifictif s'ifiece, le lobule optique cesse de s'accroitre, commc on le remargue chez le herisson ( 1 i), le vespertition marin (12), le rhinulophe unifer (15) ct les atatres chaure-souris.

La couleur du lobule oplique cọ d'un gris cendré chez l'homme, d'un gris rosace chez le drill, le mandrill, le papion, le rhésus, lia plupart des autres singes, el chez le phoque. Clixz

(1) Pl. XVI, fig. $29 \%$

(2) Pl. X'I, fig. 3oo.

(i) Pl. XVI, fig. 299

(i) Pl. XIII, lig. 2fí

(5) PI. XIII, fig. 255.

(6) Pl. XIV, fig. 258

(i) Pl II, figs. 52 .

(8) PI. XIV, fig. 260 .

(9) P1. XV , fig. $2 \% 2$.

(10) Pl. IX, fig. 211.

(ii) Pl. XVI, fig. $2 y^{-}$.

(i2) Pl. IX, tig. 214.

(15) PI. JX, fig. 204. 
les carnassiers, sa teinte se rapproclie de celle de la substance corlicale, ainsi que chez les ruminans ct les rongeurs. Chez le cheval, elle cst violacese, ct d'un blanc sale à sa terminaison posiéricure; cotic même couleur blanche se remarque aussi chezle raton, et se trouve chez d'autres mammiferes, comme chez le bouc de la haute Eigyple, rapprochéc de la jonclion du lobule optique sтес le kiasma.

Le nerfoptique est dans un rapport trìs-ćtroit arec le lobule optique. Chez l'homme, on rencontre plasieurs filamens médullaires qui, de son épaisscur, se portent dans le kiasma des nerfs de la vision. Cilucz les singes et te phocque, ces faisccaux sont plus prononcés que chez l'homme : chez beancoup de carnassicrs ils sont trì-forts, commc chez le lion, le raton; ils sont plus fuibles chez le renard, le loup et lies ours : chez les rumiuans, ils sont it-peu-pres comme chez les carnassicrs; mais chez les rongeurs, les insectirores et les chaure-sonris, lo lobule oplique est si choitcment uni arec le kiasma, qu'il scmble confordu avec lui.

En metlant i découvert ces faisceaus, on net ì $n$ la scissure médiane qui divise la profonderer du lobule oplique, el qui paraît ctre la suito du troisieme ventricule; scissure dont on roit quelquefois les traces à l'exléricur, tantôt au milicu, comme chez le cheval, tantôt on avant, comme ehez le bouc de la haute Egypte; tantôt, et c'est 
le cas le plus fréquent, en arrière, conmr chex quelques carnassiers et chez cortains ruminans. Comme cette petile rainure postérieure est le caractère d'après lequal on a assimilé ce lobule aux éminences mamillaires, il est essentiel de faire remarquer les variations qu'clle présente.

En outre, en arrière de la profondeur du lobule oplique, se trouve un petit amas de matière blanche, assez apparent chez lcs singes, Ics carnassiers ct les ruminans, qui me parât être le rudiment des émincnoes blanches, dont la saillie a été couverte par le dóvcloppement du lobule optique. Cet effacement dos émincnces mamillaires cst le résultat du balancement alternatif des élémens de l'encéphale, dont nous avons déjà rencontré un si grand nombre d'exemples. L'accroissement du lobule oplique atrophie l'éminence mamillaire; de même que dass l'embryogénie de l'homme, ces éuninences ne se montrent que lorsque le lobule optique est réduit daus ses dimensions. Rappelons, en effet, que ce n'est que dans le cours du septième mois de la vie utérine que ces émincnces deviennent distinctes.

Arant de passer à l'examen du lobule ou tubercule optique chez les oiseaux, nous devons dire que, chez les mammiferes, son volume suit celui du nerf optique de l'homme aux rongeurs. Ce rapport général éprouve cependant de singulières exceptions : indépendamment de celles des cétacés dont nous avons parlé, de celles des mam- 
mifères privés des nerfs de la vision, et qui montrent l'inverse des cétacés, on trouve chez le raton (1), le castor (2) et le porc-épic (3), des nerfs optiques très-gréles à côté des lobules optiques les plus forts qui existent dans cette classe; ces exceptions nous préparent à l'explication de ce tubercule chez les oiseaux.

Chez tous les oiseaux le lobule optique existe, et il existe à la même place que chez les mammifères, en arrière de l'entrecroisement des nerfs optiques; mais, loin d'avoir suivi le développement du nerf optique si prépondérant dans cette classe, il s'est, au contraire, réduit dans ses dimensions, comme on peut le remarquer chez la roitclet (4), l'hirondclle (5), la boudréc commine (6), le perroquet ( 7 ), les cigognes ( 8 ), l'autruche (9) et le casoar (10). Si dans cette classe, comme on le voit manifestement, le lobule optique a perdn son rapport de développement arce le nerf de la vision, quel est l'élément
(1) Pl. VIII, fig. 200.
(2) Pl. XIV , fig. 258.
(3) Pl. XIII, fig. $25 x$.
(4) Pl. IV , fig. 9.1.
(5) Pl. IV , fig. $9^{2}$.
(6) Pl. IV, fig. 88.
(g) Pl. IV , fig. $9^{6 .}$
(8) Pl. IV , fig: 103.
(9) Pl. IV , fig. $9^{8 .}$
(in) M. 111, fig. $=8$ et 79 . 
de l'encéphale dont il aura, pour ainsi dire, partagé la sort? C'est évidemment le lobe olfaclif, qui est si réduit dans cclte classe, que beaucoup d'umatomistes l'ont méconnu ct le mécomnaissent cncore. Obserrons, à l'appui de celle asscrtion, que les oiseaux chez lesquels le lobe olfactif derient le pliss saillant sont précisément cenx chez lesquels le lobule oplique oflie le plus de ro?ume, ainsi qu'on peut s'en convaincre prar bexamen de la base de l'encephale du perroquet (1), de l'autruche (2), et sur-lout du casone (j). Il cst it remarquer que: Huller avait pris les lubules op̣tiques, dans celle classe, pour les éminenes mamillaires, ct que Nalacane lenr avill donne le nom d'apophyses michlullaires des nerps epliques.

La forme du lobule optique des oiscaux n'ofre pas lis mêmes varhllions qua nous arons remarqućes chez lis mammiferes; il est, cn génciral, areondi, quelquefois ovalaire, comme chez lautruche, et le plus someut dirisé en deux par un sillon sur la ligne méliane, comme on lobserve sur-tout chez le casoar. Sun aspect est rosace chez tous lesoiscaux; je ne lui ai ru wue couleur grisâlre que chez le perroquet. Ce lobule est soliele, blanchâtre intérietrement, et sa substauec se continue immédialement arec l'culiecroiscnent des

(1) Pl. IV , fig. $9^{6}, n^{\circ} 6$ et 15.

(2) Pl. IV , fig. $9^{8, n^{\circ}} \mathrm{z}$ ct $n$.

(5) Pl. Int, fig. $=8, n^{\circ} y$ ct $x 2$. 
nerfs opliçues; contimulé qui semble contredire ce que neus arons remarque sur ses rapports arec les neres de la vision.

Chez les reptiles; le tubcreule optique cst, cn géncial, plus restreint que chez lis cricaux; chez l.'s ecrpens, les conleures, les halracicus el Ies lizards, il est ires pelil, cl orcupe l'angle rutiont formé par l'entrecroisement des nerfs opliques. Chrz le comolion (1), il est plus volumincux ct plus arrondi; chez les crocorlites ctdes caïmans, il forme un globe saillant en arriere des nerfs opliques et en arant de la moclic allongée. Chez lit tortuc franche, son rulume st considérable; sa forme est celle d'un oxale allonge. Clace les crocodiles (2), les caïmins el les tortues, son volume est proporliomué a colinides lobes ulliclifs. Chez les tormes, sa masse csi presque solide, excepté an centre, de même que cincz les oiscaux. Chez les crocediles, et sur-tout le coiman i muscau de brochet, c'est unc ré biule membraneuse crense, qui communique, cu hut dans le troisieme ventricule, on bas dans lo ligge piluitaire ou de l'hypophyse; la meablane nerreuse qui la forme, ma paru composée de deux conches blanclies, dans liuberralle desquelles est une petite quantité de matiere grise.

En outre, jai trouvé chéz quelques lézards et

(1) P1. V, fig. $11 \%$

(2) Pl. V , fir. 117 et $118, \pi^{n}$ i. 
sur-tout chez la grenouille, deux petites vésicules. blanchâtres, situées en arrière du tubercule optique. Malacarne a pris ces vésicules pour les éminences mamillaires : Tréviranus fait observer qu'elles ne sont que contiguës à l'cncéphale, à la. base duquel elles sont appliquées par un repli de la pie-mère; elles m'ont paru unies, au contraire, avec la partie postérieure du tubercule optique.

Suivons attentivement le lobule optique chez les poissons, afin de ne pas le méconnaître dans. les diverses métamorphoses qu'il subit dans cette classe. Tous les anatomistes, jusqu'à ce jour, ont pris ce lobule pour les éminences mamillaires: les uns, à cause de sa forme, quoiqu'clle diffère essentiellement de celle des éminences mamillaireß de l'homme; les autres, à cause de sa position; et ces derniers raisonnent dans l'hypothèse que les lobes optiques sont les hémisphères cérébraux : tous sont entraînés dans celte détermination, pour avoir méconnu le lobule optique et ses étroiles. connexions avec le nerf de la vision. D'abord, chez l'anguille (1) et la lamproie (2), it se présente sous ld forme simple qu'il offre chez les reptiles ct les oiseaux; chez le griset (squalus griseus), i) est quadrilatère, à angles arrondis, et déprimé an milicu, comme nous l'ont offert plusicurs nammifères; du tiers antérieur s'élève la tige pi--

(1) PI. VII , fig. 193.

(3) PI. XI. fig. 2 ait. 
initaire; en avaut, il est étroitement lić à l'entreicroisement des nerfs optiques. Chezla tanche (1), il est simple aussi, et sa couleur rougeâtre le lait distinguer des parties environnantes. Chez lc turbot (2), il est très-volumineux, simple et ovalaire; comme chez les crocodiles et les tortues; du milieu s'élève le pćdicule qui l'unit à l'hypophyse cérébrale (3). Comme chezles reptiles, il est embrassé parl'écartement postérieur des nerfs optiques après leur entrecroisement. Chez le brochet; sa forme globulcuse le rapproche de celui du caïman, et sa cavité se prolonge, comme chez ce dernier reptile, dans le tuyau de la tige pituitaire (亿). Chez le squale rochier, il y a deux lobules optiques, un de chaque côté; et, comme ils sont presque sphériques, ils ne se touchent que par un point sur la ligne médiane. Ils sont ovalaires, doubles ausși chez l'ange (5), mais contigus en dedans sur une plus grande surface que chez le rochier : la tige part du point de leur jonction en arric̀re (6); leur forme rappelle celle des lobes optiques de certains reptiles et d'un grand nombre de poissons. Chez les raies (7), il y a deux lobules optiques, de
(I) Pl. VII, fig. $x 86$.
(2) PI. VII, fir. $176, n^{\circ}$.
(3) Pl. VII, fig. $175, n^{\circ} 4$.
(4) PI. X, fig. $217, \mathrm{~B}$.
(5) Pl. X, fig. 222.
(6) Pl. X, fig. $222, n^{\circ} 5$.
(7) $\mathrm{PI}$, VI, fig. $348, \mathrm{n}^{*} 8$. 
forme ovalaire, comme chez l'ange : ils sont sćparćs l'un de l'autre à cause de l'hypoplyyse qui vient en arriere so placer ontre eux. Chezle squale blew, lo lobule uphique est quadrilobé, cfet qiai est proluit, de même que rans les tubercu'es quadrijumcan des manmóeres, par un sillon lateral qui ne pendere pas dans toute la profondeur du lobule. Cus lobules sont situés à la suite l's uus des itutres; les atiticurs sont plus grands que les postéricurs : la lige réunie an nerf oplique communique avec l'mbérieur. Chez le gronau (1) c'est linrresc: les lobules postéricurs ont teilcmont prédomine sur l's antírieurs, que ceux-ci sont pefoulés dans l'angle rentrant des verfs cpliques. La meme chose a licu chez le merlan (2), la carpe (j), la morue (i), chla baudroic, chez laqunlle fos iobules sunl néimmoins très âlfaissés (5). On voit donc comment, chezles poissons, le lobule optique s'eliere graduclioment de l'étal de simplicité qu'il préseute dans quelques-uns d'entre cux, au plas haul degré de complication quill atdeigne clez les animax verlćbrés. On roit aussi que c'est loujours lomème organc, quelque simple qu'il soit, quelque compliqué qu'il nous paraissc.

Les lohules opliques sont tantôt presque so-

(1) Pl. VII, fig. 15 .

(2) PI. VI, fig. 你, $\mathrm{n}^{\circ} 4$.

(3) Pl. VI, fig. $146, n \cdot 5$.

(4) Pl. VII, fig. 164, n* 9 .

(5) PI. VII, fig. I $\delta_{2}$. 
lides, comme chez les oiscaux, tautôt crensés d'une cavilé, quelquefois mique, comme chez certains repliles, ct d'autres fois double, ce qui a licu quand les lobules sont séparés: dans les lubules guadrilobés il u'y a qu'une cavilé pour chaque lobulc. Chez les puissons a lobules solides, la masse

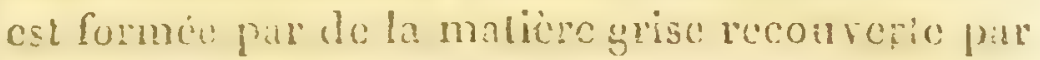
ruc couch : blandur; chez ceux a lobale creur, i la coucie blanche externe sen cst joint: une interee, comme on lo roit che\% les cyprins (1); confre les deux conclics est une lame de matic̀n grise plus ou moins épaisse sclon les espècos.

D'apres la posilion de cr lobulc, sa base alhere atu point d'insertion du nerfoplipue el da nerf olfaclif chcz bcaucoup de poiszons (2); c'ost sans doute d'apress s:e ripport, qui, ì ce qu’il paraît, cst cucorc plus prononé chez li: cyeloplerus lume pus, lecycloplerus glulinosus, le salmo salur, les plinronectes, eteontainstrighs, que Pallaş cl'Prórirmus ont ile conduils a penser que ehez les poissons ce lobule est l'originc des nerfs oplique ct olfichif $(\bar{n})$.

La cavite du lobule oplique communique dans celle classe arec la cavili des lubercules qualrijumcaux cn arant, el au-dessus de loutrecruise-

(1) P!. VI, fig. 150, $\mathrm{n}^{\mathrm{n}} 4$ his.

(2) PI. VI, fir. 136 et 145 .

(3) Cette opinion, chez le célébre Tréviranus, me parait tre la conséquence de l'idéc dans liquelle il est, que lez labes opliques sont les hémisphères civébraus des poissons. 
ment des nerfs optiques, avec le petit espace interposé entre ces tubercules et les hémisphères cérébraux, et qui correspond au troisième ventricule des mammifères ( 1 ), des oiseaux et des reptiles.

Si les rapports généraux précédemment énon. cés entre le lobe olfactif, les nerfs de la vision et le lobule oplique sont exacts, on voit donc que ce lobule doit être porté à son maximum de grandeur chez les animaux où le nerf de la vision et le lobe olfactif sont eux-mêmes parvenus au summum de leur accroissement. C'est ce qui a liea chez les poissons. De lá le volume du lobule optique, de là son indépendance dans cette classe.

\section{De l'éminence mamillaire.}

Nous sommes ainsi conduits par la méthode d'abstraction à la connaissance exacte de l'ćminence mamillaire : nous savons que ce qu'on a pris pour elle chez les mammifères, est le tubercule optique. En suivant les métamorphoses de ce tubercule dans les diverses classes, nous le voyous se convertir chez certains poissons en deux lobules qui sont aux lobes optiques ce que ces éminences sont aux hémisphères cérébraux. Ces lobulcs sont creux, les éminences blanchâtres sont solides: ces lobules sont étroitement unis et souvent con-

(1) P!. 11I, fig $16 i-, n^{\circ} 4$. 
fondus dans toutes les classes avec le kiasma des neris optiques; les éminences n'ont avec lui aucuu rapport : la cavité du lobulc optique se prolouge le long de la tige jusque dans l'épaisscur de l'hypophyse cérébrale; les éminences n'ont aucun rapport, aucune connexion soit arec la tige, soit avec la glande pituitaire. Eufin les émiucnces mamillaires ne se rencontrent saillantes et visibles à l'extérieur que dans l'encéphale le plus descendu pour le volume du nerf de la vision et le lobe olfactif, tandis au contraire que ce que l'on prend pour elles chez les mammifères, les oiscaux, les reptiles et les poissons, est développé en raison directe du volume, et du lobe olfaclif, et du nerfoptique. Tant de différences saus a ucune analogie ne permettent douc pas de méconnaître que les éminences blanchâtres sont un des caraclères spécifiques de l'espèce humaine, et qu'clles dis. tinguent éminemment l'homme de tous les animaux. Sous ce rapport, elles sout à la base de l'encéphale, ce que le lobule consillaire est au cervelet, ce que les cordons médullaires sont au quatrième ventricule,

Cette spécificité d'existence débarrasse la science de beaucoup de conjectures. En les royant disparaître clıez les singes, ce serait en wain que nous chercherions un foudement à l'iclée si positivonicnt énise par Haller et Malacarne, sur l'indépendance de ces éminences chez los oiseaux; quel- 
que confuses que soient les notions qu'ils nous ont laissées à ce sujet, il me paraît évident qu'elles se rapportent au petit lobule optique. Si ces éminences ont disparu de la surface externe de l'encéphale chez les mammifères, les oiseaux et les reptiles, comment se reproduiraient-elles chez les poissons? Cette reproduction eût été si singulière dans cette classe, que cette singularité me parut à priori un motif pour rejeter cette analogie, en admettant même la supposition que ce que nous regardons comme les lobes optiques des poissorıs, fût leurs hémisphères cérébraux. Par ce doute, je fus conduit à la détermination de la partie que l'on prenait dans toutes les classes pour les éminences blanchâtres, et le résultat de mes recherches a été d'étabiir, comme élément particulier de l'encéphale, le tubercule ou lobule optique qui avait été méconnu jusqu'à ce jour. C'est aux anatomistes à juger les raisons et les faits qui m’ont dirigé dans ce point difficile de l'encéphalotomie.

Quoi qu'il en soit, l'èminence blanchâtre dans l'espèce hunaine, est le relier de l'une des radiations médullaires les plus impartantes de l'encéphale, portée chez l'homme au maximum de son développement, et beaucoup plus imparfaite chez les autres mammifères. Les épanchemens de cette radiation que Reil a désignés sous le nom de substance médullaire innominée, sont totalement 
méconnus áaus la méthode de dissection de Varole et de Gall; il faut nécessairement recourir à celle de Vicq-d'Azyr, si amèrement censurée par ce dernier anatomiste. On suit alors les trois principaux faisceaux mis à découvert dans les planchę de notre illustre anatomiste, auquel Tréviranus rend un hommage qu'on se plaît à voir sortir de la bouche d'un homme de ce haut mérite. De ces trois faisceaux, l'antérieur passe sous le kiasma des nerfs optiques et se perd dans la partie antérieure du corps calleux; le postérieur se répand dans la profondeur des pédoncules cérébraux; le latéral se dirige vers le genou interne, et s'unit is une parlie cies fibres qui, de la couche optique, se rendent dans le nerf de la vision. Tréviranus croit, en outre, que chez les mammifères, un des faisceaux de la promière radiation va se rendre a la strie médullaire qui divise la superficie de la. couche optique en deux parties. Sanclorini a nommé ces éminences blanchâtres tubercule des piliers antérieurs de la voûte, parce que c'est en effet chez l'homme un de ses plus importans rapports: mais si l'on considère que ces piliers sont beaucoup plus forts chez les mammiferes que chez l'homms, on concevra que leur saillic est indépendante de cette partie du trigone céréhral. Je fais cette remarque parce que Tréviranus com clut de la prétendue existence des éminences mamillaires des poissons, à l'existence de la voûte dans les lobes optiques, ou, selon lui, daus les hs s-.. 
misphères cérébraux de cette classe. Si ccla élail! de quelle large voûte les poissons auraient dû être pourvus? Et dans cette hypothése, comment les repliles en eussent-ils été privés?

\section{Des Lobes cérébraux.}

A quoi se réduisent donc les lobes cérébraux des poissons, puisqu'ils ne renferment ni la couche optique, ni le corps strié, ni les commissures, ni la voûte, ni le corps calleux? Organes tout-à-fait rudimentaires dans cette classe, ils constituent une espèce de tubercule solide, formé dans sa presque totalité par la matière grise; à pcine remarque-t-on chez certains d'entre eux quelques stries blanchâtres, formées par la terminaison des pyramides : leur structure contraste avec l'organisation riche et variée que nous avons trouvée dans les lobes optiques. A une époque où la science n'avait point encore arrêté les bases de ses déterminalions, on ne pouvait donc reconnaître les hémisphères dans des organes si atrophićs, comme on ne pourait voir dans les lobes opliques, si compliqués, lcs analogues des tubercules quadrijumeaux, si simples chez les mammifères. Complication, simplicité des organes, tout devenait sujet de confusion dans cette absence d'un bon sysic̀ne de détermination, et lout le deviendrait encore, si nous abandonnions fes règles qui nous ont dirigés jusqu'a présent.

Lorsqu'on prenait les lobes optiques des pois- 
sans pour les lobes cérébraux, les véritables hémisphères étaicnt regardés comme les nocuds o!factifs; alors on comptait une ou deux paires de ces nouds, sclon que l'on trouvait deux ou quatre. lobes on avant des lobes optiques. Il n'y avait guère que les raies et quelques squales dont les lobes, réunis cn une seule masse, fussent assimilés ou aux hémisphères on à leurs lobes antérieurs; et, chose remarquable, c'est maintenant les seuls hómisphères de ces squales que l'on confond avec le lobe olfactif, tandis que ccux des poissons osscux con̉servent leur véritable détermination. Tréyiranus étant revenu à l'opinion qui fait des lobes optiques les hémisphèrẹs, se trouve embarfassé pour déterminer la paire qui les précède, où les lobes cérćbraux; il se borne à faire remarquer que ce sont deux masses solides, appendices latéraux des cuisses cérébralos, sans rechercher à quelle partie de l'encéphale des autres classes ces appendices correspondent. Quant à la paire qui les suit en avant, il la reconnait pour les lobes olfactifs. D'après cet anatomiste, il y aurait donc entre les hémisphères cérébraux et les lobes olfactifs une paire de lobes indéterminés. Ces lobes indéterminés chez les poissons osseux deviendraient, chez les cartilagineux, les lobes olfactifs, et alors l'encéphale de cette grande famille serait composée, en ouire du cervelet, des hémisphères cérćbraux et du tobe olfactif, ou du lobe olfactif et des lobes optiques, pour les ana- 
tomistes qui ont adopté nos déterminations. Ces deux hypothèses me, paraissent inadmissibles.

Et d'abord sur quoi se fonde-t-on pour assi. miler les hémisphères cérébraux des squales au lobe olfaclif? Sur ce que le pédicule olfactif s'insère immédiatenent sur la partie antérieure de ces hémisphères, et se continue avec eux; mais ce rapport, que j'ai déjà signalé chez les raies, le requin, le griset, la lamproie, l'esturgeon, l'ange et l'aiguillat, cesse d'exister chez le squale bleu, sur lequel le pódicule olfactif longe la partic latérale des hémisphères, et se prolonge directement sur les pédoncules cérébraux. Le squale bleu aurait dónc des lobes cérébraux, et l'aiguillat, l'ange et le requin en seraicnt dépourvus? La lamproie et l'esturgeon n'auraient aussi que des lobes olfactifs à la place de leurs hérrisphères', et comme, chez ces poissons, de mème que chéz l'ange, la glande pinéale est très-distiricte, il faudrait admettre que cette épiphyse a survécu à l'anéantissement des lobes cérébraux, et s'est fixée en arrière du lobe olfactif. Celte assertion me paraîtrait difficile à établir. - De plus, parmi les poissons osseux que jai disséqués, l'égrefin a son pédicule olfactif (1) en continuation immédiate arec la base des lobes optiques (2). D'après cette hypothèse, ce poisson

(1) Pl. VII, fig. 184, n*b.

(2) Pl. VII, fig. $184, n^{\circ} 3$. 
perdrait dunces deruiers lobes, comme les squales leurs hémisphères cérćbraux?

'Parmi les reptiles, les uns ont le lobe olfactit pédiculé; tels sont les crocodiles, la plupart des lézards et des vipères : ce pédicule se continue immédiatement dans les hémisphères cérébraux, comme chez les raies et les autres squales : ces reptiles seraient donc aussi dépourvus de lobes cérébraux. Nous nommerions lobe olfactif chlez ces reptiles, ce qui serait les hémisphères cérébraux du caïman à museau de brochet et de la tortue franche, parce que chez ces deux derniers le lobe olfactif se serait rapproché et confondu avec les lobes cérébraux. Mais ce rapprochement et cet éloignement du lobe olfactif ne change rien aux connexions du nerf de l'olfaction. Ce nerf se contiuue immédiatement dans les hémisphères (1) chez la tortue franche et le caïman à museau de brochet, comme il le fait chez les lézards, les vipères et les crocodiles; comme il le fait chez les oiseaux; comme il le fait dans les mammifères inférieurs, chez lesquels le pédiculc olfactif, son lobe et le champ olfactif, sont confondus en une seule masse. En admettant cette hypothèse, nous verrions donc la plupart des vertébrés perdre leurs lobes cérébraux, à moins que par une seconde hypothèse, nous ne rapportions au lobe olfactif ce que nous convien-

(1) Pl. V, fig. $121, n^{\circ} 12$. 
drions d'enlever aux hémisphères : car ce lobc olfactif restc toujours en avant des lobes cérébraux dans toutes les classes; les squales l'ont plus développé que pas un des animaux composant les Irois classes supéricures; et, quoiqu'il le soit un peu moins que chez quelques osseux, son yolume dépasse souvent, comme chez le requin, la masse des lobes optiques.

Cettc opinion, qui prive lcz squales de lcurs hémisphères cérébraux, cost eqnprunléc ì $M$. de Blainville; mais ce célébre anatomiste conserve l'unité de détermimation dans loules les classes, tandis que la confusion est inévitable dans le syslème de détcrminalion que paraît adopter Tréviranus. Quoi qu'il en soit, nos détcrminations restent telles que nous les avons élablies; les poissons cartilagincux, de même que lcs osseux, conscrvent et leurs hémisphères cérébraux et leurs lobes olfaclifs.

Les plus petils lobes cérébraux que je connaisse, sont ceux de l'égrefin (1). Ce sont deux petits tubercules arrondis, placés en avant des lobes optiques, et égalant le volume d'un gros grain de millet (2); ils sont séparés l'un de l'autre par les deux uerfs olfactifs, qui paraissent sorlir de la base des tubcrcules quadrijumeaux. Viennent ensuite Its anguilliformes, chez lesquels toutefois les lobes

(1) PI. VII, fig. $157, \mathrm{n}^{\circ} \mathrm{F}$

(a) PI. VII, fig. 28, , no 8 . 


\section{F.T LOBE OLFACTIF.}

cérébraux se mettent en proportion avec les aures lobes cncéphaliques, comme on le remarque ch:z l'anguille (1); chez le congre, ils sont globuleux (2), aplatis par leur face interne (3), et formés par une masse grise, dans laquelle on suit la terminaison des pyramides (4). Chez les trigles, leur globe est légèrement déprimé en dehors (5). Chez tous ces poissons, les lobes cérébraux sont immédiatement précédés par les lobes olfactifs; chcz lous, ils ont une forme globuleuse asscz parfaite, caraclire qui s'efface plus ou moins, lorsque, comme chez les gades, les tétrodons ei les silures, le lobe olfactif, projeté en avant, ne communique avec l'encéphale que par un pédoncule dont la longeur est proportionnée à son écartement. On voit commencer cette déformation chez le merlan (6), la tanche (7), le barbcau ( 8 ), la carpe (9), le brochet ( 10 ), et la norue (11); chez quelques-uns de ces poissons,

(1) Pl. VII, $\mathrm{f}_{\mathrm{j}^{\circ}} \mathrm{rg0}, \mathrm{n}^{\circ} 4$.

(2) PI. VII, fig. $16 \mathrm{~s}, \mathrm{n}^{\circ} 6$.

(5) PI. VII, fig. $167, n^{\circ} 6$.

(4) PI. VII, fig. $16 ;, n^{\circ} 5$.

(5) PI. VII, fig. $155, \mathrm{n}^{\circ} 7$.

(6) PI. VII , fig. $195, n^{*} 6$

(7) PI. VII, fig. $185, n^{\circ} 6$.

(8) M. Y11, lim. 185, 110

9) PI. VI, fig. $x 45,1^{\circ}(i$.

(in) H'l. VHI, fig $169, n^{\circ} 4$.

(1) Pl. VII, fig. $163, n^{n} \mathrm{~g}$ 
comne chez la carpe el la morue, la surface externe et supéricure des lobes cérébraux est bosselée, et présente des inégalités (1) que Vicqd'Azyr a assez improprement comparées aux circonvolutions cerébrales des mammifères supérieurs.

Saisissons ce caractere chez les poissons ossenx, pour l'appliquer aux cartilagineux, et nous verrons aussi chez ces derniers, les lobes cérébraux se rapprocher d'autant pius de la forme sphérique, que le lobe olfactif sera plus rapproché des lobes córebraux, ainsi qu'on l'observe chez la lamproie (2), dont le lobe cérébral est pyriforme (3); chez l'esturgeon (4), où il devient plus globuleux, quoique ce ne soit que le lobe cérébral de la lamproie, beaucoup plus volumineux; chez le squale griset et le requin (5), où le lobe cérébral est demi-sphérique, et le lobe olfactif à peine écarté de sa face antérieure. Plus, au contraire, ce lobe s'écarte, plus son pédicule d'union s'allonge, plus nous royons le lobe cérébral s'aplatir et s'écarter de la forme sphérique ou globuleuse. Cet effet, qui est déjà très-sensible chez le squale bleu, le devient sur-tout chez

(1) PI. VII, fig. $163, \mathrm{n}^{\circ} 8$.

(2) PI. XI, fig. $226, n^{\circ} 7$.

(5) PI. XI, fig. $224, n^{*} 8$.

(4) PI. XII, 6g. $235, \mathrm{~K}$. 
l'ange (1), l'aiguillat (2), et toutes les raies (3). Chez ces derniers poissons, le lobe cérébral n'a ni la forme globuleuse des poissons osseux, ni la forme demi-sphérique des autres cartilagineux; c'est un tubercule aplati, en quelque sorte quadrilatère, à angles arrondis. Remarquons, toutefois, que sa surface externe se bosselle, ou devient irrégulière, comme cela a lieu chez certains osscux à lobe olfactif écarté. Ces bosselures ou ces irrégularités, légères chez l'aiguillat (4), plus prononcées chez le squale bleu, forment de véritables scissures chez le rochier.

Le lobe cérébral, solide chez tous les osseux, presque solide aussi chezla lamproie et l'esturgeon, se creuse d'une petite cavité chez l'ange, l'aiguillat, le squale bleu, le griset, le requin et les raies; le plus souvent même cette cavité est ouverte en dehors, en arrière du lobe cérébral (5), comme on le remarque sur-tout chez la raie bouclée (6). En insufflant par cette ouverture, on dilate ce petit ventricule, et on renfle le pédicule du lobe olfaclif; ce qui prouve la libre communication de l'une à l'autre: en prolongeant beaucoup l'in-

(1) Pl. XII, fig. $257, n^{\circ} 6$.

(2) Pl. XII, fig. 236, G, H.

(3) Pl. VI, fig. $152, \mathrm{~B}$; fig. 148, D; fig. $138, \mathrm{n}^{\bullet} 14$.

(4) Pl. XII, fig. 236, G, H.

(5) Pl. XII, fig. 236, F.

(6) Pl. VI, fig. 138, n` 9 . 
sufflation, on finit par disjoindre l'adhérence interne de chaque feuillet hémisphérique; et l'on voit alors que chaque lobe cérébral est formé par le recourbement de dehors en dedans, de son feuillet, ainsi que nous l'arons observé chez les embryons de toutes les classes. Ainsi ces poissons cartilagincux conservent les traces de la formation primitive des lobes cérébraux, comme ils nous ont montré d'une manière permanente Ia disjonction première des lames du cervelet.

Puisqu'il y a deux lames cérébrales distinctes, jointes sur la ligne médiane, le lobe cérébral des poissons carlilagineux est donc double commc celui dles osseux. Ce caractère est plus ou moins prononcé selon les espèces, et les lobes cérébraux sont plus ou moins séparés et distincls. Ainsi, chez la lamproic (1) et l'esturgeon (2), les lobés sont aussi distincts que chez les poissons osseux; ils ne se confondent que par un plateru de matière grise, situé à leur base: Chez l'arguillat et lis raies, la scissure médiane qui les sépare se prolonge dans toute l'ćpaisseur dos lobes; on ha trouve sur la face inférieure (5) de méme que sur la supéricure (4): quoique plus serréc chez le griset, le squale bleu et le requin sur lequel elle

(1) PI. XI, fig: $224, \eta^{\circ} 6$ et 7 .

(s) Pl. XII, Gs. $255 . \mathrm{H}$.

(j) Pl. V1, firg. $148, \mathrm{D}$.

(1) Pl. VI, fig. $152, \mathrm{~B}$. 
trace si peu (1), elle reste néanmoins visible sur les deux faces, de même que chez les poissons précédens.

Quel que soit le point où l'on fasse insérer les nerfs olfactifs des poissons, toujours est - il que chez les osseux, ils se glissent en dedans des lobes cérébraux, et côtoient leur face interne. Chez les cartilagineux, au contraire, l'insertion du pédicule olfactif a lieu le plus souvent sur l'angle antérieur et externe du lobe cérébral (2), ou même tout-à-fait en dehors, comme chez le bleu. La lamproie et l'esturgeon tiennent le milieu entre ces extrêmes du déplacement de cette insertion: chez ces poissons, le pédicule olfactif est la continuation immédiate de la pointe du lobe cérébral, comme cela existe chez la plupart des reptiles. Sous ce rapport, la lamproie et l'esturgeon sont aux poissons cartilagineux ce que le congre est aux poissons osseux. Or, chcz les osscux, cette position du nerf de l'olfaction tieut les lobes écartćs, ct s'oppose à leur jonction inmédiate; de là, leur séparation complète. Chez la lamproie et l'esturgeon, il abandonne cette position, et se porte en avant du lobe : rien ne séparant plus les lobes sır la ligne médiane, leur réunion commence à s'effectuer : clle devient plus complete encore lorsque l'iusertion a passé de la partie interne à l'ex-

(1) PI. VI, fig. $142,14^{\circ} 17$.

(2) PI. Vi, Gg. $148, \mathrm{C}$. 
terne. On voit donc de cette manière, comment s'opère, chez les poissons, la fusion des deux Jobes cérébraux.

Comment les lobes cérébraux, si simples cher les poissons, deviennent-ils si compliqués chez les mammifères? C'est par le même mécanisme que les lobes optiques si compliqués chez les poissons deviennent des organes si simples dans la classe supérieure; seulement l'ordre des décroissances et des développemens est inverse. Suivons ce double rapport. Nous trouvons d'abord que, dans leur état le plus descendu, chez les poissons osseux, les lobes cérébraux sont solides comme les tubercules quadrijumeaux chez les mammifères supérieurs, où ils sont réduits à la condition la plus minime de leur existence. En second lieu, les lobes cérébraux des cartilagineux, réunis par leur base, se creusent d'un petit ventricule, comme déjà dans les tubercules quadrijumeaux des rongeurs, on aperçoit le rudiment du ventricule optique des classes inférieures. Considérés aux deux extrémités de l'échelle des vertébrés, ces deux ordres d'organes sont donc solides. A quoi tient cette solidité? Éridemment, à l'épaisseur de la coquille externe qui les forme primitivement. Quelle est maintenant la cause de la cavité qui s'y manifeste? C'est évidemment aussi la diminution d'épaisseur de cette. lame externe, qui, en se dilatant, forme un vide vers son centre. Ce mécanisme est évident, pour 
les lobes cérébraux, chez les poissons cartilagineux; pour les tubercules quadrijumeaux, chez les rongeurs, les insectivores et les chauve-souris, parmi les mammifères, l'amphisbène et les ophidiens, parmi les reptiles.

Chez les reptiles, les lobes cérébraux et les lobes optiques sont creux; mais les deux ventricules sont d'abord très-étroits chez les ophidiens et les batraciens, dont les enveloppes externes restent toujours très-épaisses. Ils se dilatent ensuite beaucoup chez les sauriens ct les chélonicns; mais à peine sont-ils dilatés, que nous voyons apparaître sur leur plancher des tubercules qui, en élevant leur fond, diminuent leur capacité. Dans l'ordre des développemens, ces tubercules exercent la même influence et sur les lobes optiques et sur les hémisphères cérébraux. Continuons maintenant d'examiner les conditions diverses d'existence de ces deux ordres de tubercules.

Ln premier lieu, dans les lobes optiques, leur masse compacte donne naissance à des stries médullaires qui, chez les sauriens, les chéloniens et les oiseaux, viennent se joindre à la lame blanche intérienre des tubercules quadrijumeaux; puis, chez les poissons cartilagineux, nous. en voyons partir une lame radiée qui tapisse et renforce l'intérieur de la lame externe. Enfin, chez certains poissons osseux, ces tubercules, portés au maximum de leur grandeur, deviennent le point de départ d'une lame interne plissée, 
indépendante de la coquille du bobe optique: cette lame interne forme un contour et des circuils qui produiraicnt sur la coquille cxterne de véritables circonvolutions, si elles étaient appliquées l'une contre l'autre.

Suivez maintenant les tubercules de l'intćricur des lobes cérébraux : très-faibles d'abord chez les ophidicns et les batraciens, vous les voyez augmenter chez les lézards, et rester, dans ces diverses familles, dans une indépendance complète de la coquille extérieure des lobes; puis tout-àcoup, chez les sauriens, les chéloniens et les oiseaux, le tubercule antérieur remplit le ventricule hémisphérique, en comble presque la cavilé et se confond avec une partic de l'enveloppe du lobe. Enfin, chez les mammiferes, les radiations qui se rendent à ces tubercules forment, dans les familles inférieures, une membrane rayonnante, qui, tapissant l'intérieur de la coquille, ne produit point de relief sur sa surface, à cause, d’une part, de sa faiblesse, ct, de l'autre, de l'épaisseur de la coquille elle-même; enfin, chez les mammifères supérieurs, cette membrane rayonnante forme des feuillets plissés dont les circuits et les contours donnent naissance aux circonvolutions qui distinguent les hémisphères cérébraux de ces mammifères.

Que sont ces lames plissées des mammifères? Les lames circonvolvulées des tori des poissons osseux. Pourquoi, dans un cas, les circonvolutions 
se manifestent-elles sur la coquille du lobe, et pourquoi, dans l'autre, ne se manifestent-elles pas? Elles ne se manifestent pás sur la surface du lobe des poissons, parce que la coquille est séparće des feuillets roulés, et qu'un espace vide ou rempli par un liquide se trouve placé entre elles. Appliquez artificiellement cette coquille sur ces lames, et vous la voyez répéter les contours de la lame plissée, ou, en d'autres termes, vous voyez paraître les circonvolutions sur le lobe optique des poissons. Vous avez imité par cctte expérience ce qui se passe chez les mammifères supérieurs, lors de la manifestation des circonvolutions. Faites maintenant l'expérience inverse, c'est-à-dire mettez les lames plissées des mammifères dans les conditions où se trouvent celles des poissons, et vous verrez les circonvolutions disparaître. Cette expérience est toute faite chez les jeunes embryons dans le premier tiers de leur formation; la coquille extérieure des lobes cérébraux cst lisse comme colle des autres classes ou des mammiféres inférieurs. Ouvrez ces lobes, vous voyez dans leur intérieur des circonvolutions nombreuses, des lames plissées et ondulées, comme le seront plus tard les circonvolutions de la surface externe de la coquille. Pourquoi ces plis et ces contours ne sont-ils pas dessinés sur la coquille? Par la même raison que chez les poissons; parce qu'à celte époque l'enveloppe extérieure est séparée des lames plissées par un intervalle plus II. 
ou moins grand selon les espèces, intervalle occupé par un liquide : à cette époque donc, le lobe cérébral des mammifères répète la disposition du lobe optique de certains poissons osseux; tous les deux sont privés de circonvolutions extérieurès, parce que, dans les deux cas, la coquille du lobe est séparée des lames ondulées. La même cause produit les mêmes effets dans les deux classes extrêmes. Si vous pouviez renfler les circonvolutions des tori des poissons, vous verriez leurs lobes optiques vous offrir les mêmes ondulations que les lobes cérébraux des mammifères; de même que si vous pouviez suspendre le développement des lames plissées des mammifères, vous verriez leurs lobes cérébraux présenter chez tous la surface lisse et polie des lobes optiques des poissons.

Un mécanisme si simple a été méconnu même de Tiedemann, qui cependant a si bien anatomisél'encéphale des embryons où il est le plus manifeste: à sa place on a mis des suppositions. Willis avait admis dans l'encéphale des fibres ascendantes, descendantes et transverses : ces dernières formaient spécialement le corps calleux; elles prenaient leur origine dans la matière grise des circonvolutions : chaque circonvolution envoyait son trousseau de fibres. Reil admit en partie ces idées, dont MM. Gall et Spurzheim ont formé leur système des fibres rentrantes. Willis faisait former le corps calleux par les circonvolutions; M. Gall fait former les circonvolutions par le corps cal- 
leux. Cette idée est ingénieuse, mais elle n'est pas exacte; et, pour montrer son peu de fondement, il suffit d'examiner l'embryon d'un quadrumane au tiers de son développement, et celui de l'homme aux troisième et quatrième mois de la conception. Les circonvolutions manquent, et le corps calleux existe: donc le corps calleux ne produit point les circonvolutions. Celte observation que je trouve dans Tiedemann, peut donner lieu à quelques contestations; mais, pour trouver une objection sans réplique, il faut s'élever un peu plus haut dans la vie utérine, et observer l'encéphale immédiatement avant l'apparition du corps calleux. Les circonvolutions sont toutes formées dans l'intérieur des hémisphères; elles sont libres et flottantes dans sa cavité : il n'y a encore ni fibres transverses, ni fibres rentrantes, et conséquemment ni corps calleux : le plissement des lames hémisphériques est donc étranger à cet ordre de fibres, quelque nom qu'on leur donne. Cen'est, en effet, que quelque temps après que les lames plissées sont formées, que l'on voit celles de gauche s'incliner vers celles de l'hémisphère droit, et que l'on remarque les rudimens des faisceaux transverses, d'où doit provenir le corps calleux. A l'époque où celui-ci commence à se former, on suit immédiatement les faisceaux dans le contour supérieur des lames plissées; faisceaux qui, à cette époque, sont sans communication directe arec lá coquille extérieure du lobe. On 
voit donc que le plissement des lames cérébrales est un effet primitif et indépendant de tout moyen mécanique, de même que le plissement des feuillets cérébelleux; de même que celui des lames internes du nerf optique de certains poissons; de même que le plissement de la rétinè; de mème, enfin, que les ondulations de la lame intérieure des lobes optiques de quelques poissons osseux. Dans tous ces cas, le but évident de la nature est de multiplier l'étendue des surfaces nerveuses.

Les circonvolutions sont donc le relief extérieur des lames plissées de l'intérieur des hémisphères, ou de la terminaison des pédoncules cérébraux. Si ces lames sont simplement rayonnées, ou si un intérvalle se trouve entre elles et la périphérie de la mombrane externe, comme cela se voit chez divers mammifères inférieurs, ce relief manque, le lobe cérébral est lisse.

Appliquez maintearaint ces idées au lobe cérébral des diverses classes, et vous voyez que chez les ophidiens, les batraciens et les lacertiens, ce lobe est presque en entier formé par la coquille externe; à peine distinguez-vous sur son plancher le croissant du corps strié et les faibles radiations ani s'y readent. 11 n'y a donc pas et il ne saurait y avoir de circonvolutions. Chez les reptiles supërieurs, tels que les crocodiles, les caïmans et les tortues, à la place des lames ondulées des mammifères, vous trouvez un tubercule brut, sillonné par des stries médullaires, et continu 
dans une partie de son contour avec la coquille externe du lobe. ll n'y a donc là ni matière à produire les ondulatious, ni matière à produire le relief des circonvolutions; l'coveloppe externe de l'hémisphère s'applique sur cc tubercule et en dessine les contours dans les parties où il lui adhère. Tous les oiseaux répètent l'organisation intérieure de ces derniers reptiles: chez tous, le tubercule hémisphérique remplit la presque totalité de son ventricule; chez tous aussi les radiations médullaires s'arrêtent à la périphérie du tubercule. Cette classe est donc et doit être sans circonvolutions; il y a seulument chez quelques espèces des dépressions sur la face supérieure des lobes , dépressions qui répètent le point de jonction de la coquille avec le tubercule. Celui-ci est bosselé en arrière sur un grand nombre d'oiseaux, et nul doutc que ces bosselures ne se répétassent sur la coquille, si celle-ci n'en était séparée par un intervalle, et par les divisions artérielles de la branche externe du plexus choroïde.

Si j’ai bien exprimé ce que j’ai vu, on conçoit donc pourquoi les mammiféres supéricurs ontleurs lobes cérébraux circonvolutionnés, el pourquäi les circonvolutions manquent dans les trois classes inféricures; si jai également bien exprimé ce que m'ont offert les embryons des mammiferes inférieurs, on voit aussi que l'absence du plissement de leurs feuillets hémisphériques est cause de l'absence des circonvolutions; onfin on presscnt 
la conclusion qui en dérive, c'est que plus les plicatures des lames hémisphériques sont nombreuses et profondes, plus sont profondes et nombreuses les circonvolutions dans les diverses familles supérieures des mammifères. Si ces idées sont l'expression de ce qui est, supposez que nous enlevions toute la masse médullaire du demi-centre ovale : la partic interne de la coquille du lobe devra alors répéter et reproduire, mais en sens inverse, les circonvolutions, c'est-à-dire qu'elle sera bosselée aux points répondant aux excavations des plicatures, et enfoncée et déprimée aux endroits placés vis-à-vis de leurs saillies. C'est ce qui arrive dans les apoplexies que l'on nommait sanguines, et que j'ai appelées hémato-encéphalies ; toute la matière médullaire est quelquefois enlevée sur un lobe; il ne reste plus que la matière grise ou la coquille circonvolutionnée, comme nous venons de le dire. Le même effêt sc remarque chez les embryons hématocéphales, quand l'hématocéphalie est survenue postérieurement à l'adhérence des feuilles plissées et de la coquille, ainsi que je l'ai dernièrement observé sur un foetus de cheval. Supposez au contraire que rous insufliez la cavité de l'hémisphère à l'époque du plissement isolé des lames, vous voyez aussitôt les lames s'étendre, les plis se déformer, et une vaste vessie se déployer; à la place de l'air, supposez de la sérosité ou de l'cau, comme nos pères qualifiaient la sérosité, et vous verrez les 
circonvolutions disparaître plus ou moins, selon la quantité de liquide épanché. Tel est l'effét et le résultat de la maladie connue sous le nom d'hydro-encéphalie; sur le cervelet ou dans l'hydrocérébellie, la distension graduclle par le liquide produit également l'effacement de ses scissures et de ses sillons. Ainsi la formation, la déformation, l'absence et la présence des circonvolutions sur les lobes cérébraux sont le résultat du plissement ou du non-plissement des feuillets internes des hémisphères. Ces données étaient nécessaires pour concevoir ce qui nous reste à dire sur les lobes cérébraux des reptiles, des oiseaux et des mammifères.

Les lobes cérébraux des reptiles, plus parfaits que ceux des poissons osseux et cartilagineux, sont moins variables dans leur forme que dans cette dernière classe. Chez les ophidiens et les lacertiens, chacun d'eux est pyriforme, ayant la grosse extremité arrondie en arrière, et la petite se prolongeant insensiblement dans le pédicule olfactif, comme on le remarque chez l'orvet (1), la vipère commune (2), la vipère hajé (3), la vipère à raies parallèles (4), le lézard vert (5) et

(1) Pl. V, fig. $109, \mathrm{n}^{\circ} 5$.

(2) P]. V, fig. : $26, n^{\circ} 6$.

(5) Pl. V, fig. 127, n०4 et 5 .

(4) Pl. V, fig. $155, n^{n} 5$.

(5) Pl. V, fig. $100, \mathrm{n}^{\circ} 10$. 
le lézard gris (1). Chez les batraciens (2), ils sont plus globuleux et moins allongés que chez les reptiles précédens; chez le tupinambis (3) ils ou: la même disposition que chez la grenouille; enfin, chez les sauriens, les crocodiles (4), le caïman à museau de brochet, le caméléon (5) et la tortue franche (6), on les voit se rapprocher de plus en plus de la forme qu'ils affectent chez tous les oiseaux. .

Chez les reptiles, de même que chez les poissons, tantôt le lobe olfactif est ramené au point de contact, de même que chez les poissons osseux, comme on le voit chez la grenouille (7), le caïman à musear de brochet et la tortue franche (8); tantôt il est tenu à distance ainsi que chez les cartilagincux: le plus grand nombre des reptiles est dans ce dernier cas, comme on l'observe 6 hez tous les ophidiens ( 9 ) et les lacertiens; mais le résultat relativement à l'accroissement des lobes cérébraux, n'en est pas le même que. chez les poissons. Ainsi, dans cette dernière classe, c'est
(1) Pl, V , fig. $128, n^{\circ} 4$.
(2) P. I, Gg. $16, n^{\circ} 4$.
(3) PI. V, fig. 114, $n^{\circ} 9$.
(4) PI. V, fig. $116, n^{\circ} 5$.
(5) Pl. V, fig. $111, n^{\circ} \%$
(6) Pl. V, fig. $119, n^{\circ} 11$.
(द) Pl. I, fig. $26, n^{\circ} 5$.
(8) Pl. V, fig. $122,12^{\circ} 15$ et 16.
(9) Pl. V, fig. $109,110,826,127,138 \mathrm{et} 135$. 
lorsque le lobe olfactif est adossé aux lobes cérébraux, que ces derniers sont les plus descendus, tandis que chez les cartilagineux leur accroissement commence avec son éloignement; 'chez les reptiles c'est presque l'inverse. Ainsi les plus petits lobes cérébraux dans cctte classe sont chez les ophidiens; les plus développés sont chez le caïman à museau de brochet et la tortue franche, reptiles cher lesquels le lobe olfactif est, pour ainsi dire, confondu avec le lobe cérébral. Les crocodiles, le caméléon, le caïman à lunettes (1), la tortue grecque (2), font néanmoins exception à ce principe.

La masse du lobe cérébral diffère beaucoup chez les diverses familles de reptiles, et cette différence tient, comme nous l'avons vu, aux conditions diverses de la coquille du lobe et de son noyau intérieur. Constamment, l'enveloppe extérieure se détache du pédoncule cérébral, immédiatement en avant de la couche optique; elle se recourbe ensuite de dehors en dedans, et enferme la masse du corps strié. D'où il suit que jamais, dans cette classe ni dans les suivantes, le corps strié ne se trouve hors de son enceinte, comme l'ont dit quelques anatomistes. La couche optique peut ne pas être recourerto dans son enticr par cette enveloppe, comme on
(1) Pl. V, fig. 135, $n^{\circ} 9$.
(2) Pl. V, fig. 125, $n^{n} 11$ el 12. 
l'observe chez certains batraciens; mais d'après l'ordre constant des développemens, il est impossible que le corps strié ne le soit pas. C'est la partie postérieure de cette lame qui, dans cette classe, de même que chez les oiseaux, correspond à la lame cornée des mammifères.

En se roulant de cette manière, la coquille $d u$ lobe forme une cavité, ou une coiffe autour du. corps strié et du plexus choroïde : on conçoit, d'après ce que nous avons dit, que la capacité de cette cavité doit être proportionnée, d'une part à la moindre épaisseur de la coquille, de l'autre au volume moindre du noyau central. Aussi chex les ophidiens, les lacertiens et les batraciens, cette cavité ou ce ventricule hémisphérique est trèsspacieux, à cause de la faiblesse du noyau central; au contraire, chez les sauriens et les chéloniens, le ventricule est presque comblé par l'accroissement énorme du tubercule hémisphérique. Dans aucun cas ce ventricule ne saurait être assimilé au ventricule latéral des mammifères : pour peu que l'on réfléchisse à la formation de l'un et de l'autre dans les deux classes, on ne saurait confondre deux espaces si différens. C'est cependant de cette fausse analogie que l'on s'est servi pour attaquer un de mes principes.

Le soupirail de ce ventricule se voit sur la face interne du lobe el en arrière; il est situé immédiatement en avant el un peu au côté externe de la couche optique, en arrière de la commissure an- 
térieure, chez tous les sauriens et les chéloniens; c'est chez ces reptiles une ouverture très-étroite, et bouchée dans l'état normal par un riche plexus choroïde qui s'insinue dans le ventricule. Chez les batraciens et les ophidiens, l'ouverture est plus large, le plexus choroïde beaucoup plus mince. Cette différence du soupirail tient à la différence de volume du noyau central. En avant, ce ventricule se continue dans la cavité du pédicule olfactif, chez les reptiles où ce lobe est pédiculé, ou immédiatement dans la cavité du lobe, comme chez le caïman à museau de brochet et la tortue franche, chez lesquels le lobe olfactif est joint au lobe cérébral. Chez les sauriens et les chéloniens, le ventricule se réfléchit en arrière au pourtour de la partie du tubercule hémisphérique, qui n'a point adhéré à la coquille. Chez tous les reptiles sans exception, la face externe du lobe cérébral est lisse; chez tous aussi, il est adossé en arrière à la face antérieure du lobe optique, dont la saillie semble arrêter son développement. Tel est le lobe cérébral dans cette classe.

De la considéralion de cet organe chez les sauriens et les chéloniens, mais principalement chez le caïman à museau de brochet, les crocodiles et les tortues, nous sommes conduits d'une manière presque insensible aux lobes cérébraux des oiseaux. C'est la même forme renfléc. Au premier aperçn, cette ressemblance chliape par des canses étrangères au lobe cé- 
rébral; ainsi l'affaissement subit de la partie moyenne des lobes optiques le reléve, ou plutôt paraît le relever en arrière beaucoup plus que cela n'a réellement lieu; ainsi l'atrophie du lobe olfactif, sa position constante au-dessous de sa pointe, lui font perdre définitivement le caractère qu'il avait chez tous les reptiles.

Le lobe cérébral des oișeaux, mousse, large et bombé en arrière, affaissé légèrement en avant, ot se terminant en pointe obtuse, représentc assez bien la figure des gousses de certaines plantes. Cette forme est identique dans toute cette classe., dont Í tixité organique paraît être, comme nous l'avons si souvent remarqué, le caractère distinctif. Supérieurement il ne présente point de traces de circonvolutions; seulement, chez beaucoup d'oiseaux, mais parliculièrement chez les cygnes (i) et les oies, on y distingue deux sillons longitudinaux correspondant au point où la lame externe s'applique sur le tubercule hémisphérique : sa base nous offre les rayons médullaires du nerf olfactif, et une scissure (2) où s'arrêtent ces rayons, et qui est bien évidemment l'analogue de la scissure de Sylvius. Pour la première fois nous voyons la base du lobe cérébral divisée en deux lobes distincts: le lobe antérieur, placé au

(1) PI. IV, fig. y9, n"4.

(a) Pl. IV , fig. 103, $\mathrm{n}^{\circ} 10$. 
devant de cette scissure (1); et le moyen, placé en arrière (2). Ce dernier est-il l'analogue du lobe moyen des mammilères, ou la première apparition du lobe de l'hippocampe? je penche pour cette deruière asscrtion. C'est surtout en considérant la face latérale, que l'on distingue bien les lignes de démarcation de ces deux lobes, comme on peut le voir chez le casoar (3), le perroquet (4), la cigogne (5) et l'autruche (6). Par le côté interne les deux lobes se touchent; un sillon trèsmarqué sur la face supérieure indique leur adossement, comme on le voit chez le casoar (7), la bondrée commune (8), l'autruche (9) et la cigogne (10). Ce sillon s'étend de haut en bas dans le sens de l'épaisseur; on le trouve en écartant les lobes interposés entre les deux lames rayonnantes, ainsi qu'on l'observe chez le casoar (11), le perroquet (12), la poule (13): en disjoignant.les

(1) Pl. IV , fig. $103, n^{\circ} 17$.

(2) Pl. IV, fi ․․ $9^{8}, n^{0} 12$.

(3) Pl. III, fig. $78, \mathrm{n}^{\circ} 13$.

(4) Pl. IV, fig. 96, $\mathrm{n}: 4$ et 8.

(5) Pl. IV, fig. 104, $\mathrm{n}^{\circ} 9$ et 10.

(6) Pl. IV, fig. 97, $n^{\circ} 9$ et 12.

(7) Pl. III, fig. 77.

(8) Pl. IV, fig. 80.

(9) Pl. IV , fig. $9^{5}$.

(10) Pl. IV, fig. 99.

(11) Pl. III, fig. 83, $n^{\circ} 11$.

(12) Pl. III, fig. 84, $n^{\circ} 14$.

(13) Pl. III, fig. $87, n^{\circ} 11$. 
lobes et les écartant l'un de l'autre d'avant on arrière, comme je l'ai représenté chez la poule (1), on observe que cc sillon est limité en avant de la commissure antérieure par un petit plateau de matière grise qui joint les lobes en cet endroit, comme cela a lieu chez les reptiles. Le sillon se continue ainsi jusqu'à la base des lobes, comme on le remarque chez le casoar (2), le perroquet (3), la bondrée (4), l'autruche (5), la cigogne (6), le roitelet (7) et l'hirondelle (8).

C'est sur la face par laquelle les hémisphères se touchent, que se trouve la lame rayonnante dont nous avons déjà donné la description : cette face est lisse et plane de haut en bas; quaud on écarte les hémisphères elle se bombe, mais cet effet est dù à la mollesse de son tissu. Ces deux faces sont en haut accolées l'une à l'autre par l'intermède de l'arachnoïde; dans le reste du sillon vertical, leur union a lieu par un tissu cellulaire et vasculaire assez résistant.

Le lobe cérébral est plus élevé en arrière qu'en

(1) Pl. III, fig. $85, n^{\bullet} 5$.

(2) Pl. III, fig. 79.

(3) Pl. III, fig. 8x.

(4) Pl. IV , fig. 88.

(5) Pl. IV , fig. $9^{8}$.

(6) Pl. IV , fig. 103.

() Pl. IV, fig. 94.

(8) Pl. IV, fig. g2. 
avant; les oiseaux chez lesquels il m'a paru le plus bombé en ce sens, sont les perroquets, les merles, les étourneaux, les bécasses, les pies; puis viennent le fou de Bassan, les canards, les oies, les faisans, les perdrix, les pigeons, la poule et les oiseaux de proic. La masse du lobe est presque formée dans son entier par de la substance grise, plus blanche dans la coquille, et plus rouge dans le tubercule hémisphérique. En coupant le lobe horizontalement par sa partie moyenne, on ne développe point de demi-centre ovale, comme chez les mammifères; on met à découvert lc centre du tubercule hémisphérique, qui est d'un rouge grisâtré, pointillé de blanc; les points blancs sont évidemment les rayons médullaires du tubercule coupé en travers (2). Ainsi, la partie correspon. dante au demi-centre ovale de la classe supérieure est presque entièrement formée par la substance grise. On ne trouve un petit noyau médullaire que lorsqu'on est arrivé dans la profondeur du tubercule au niveau du point de départ de la commissure antérieure.

Le ventricule hémisphérique des reptiles supérieurs, tels que le caiman à museau de brochet et la tortue franche, se compose, comme nous l'avons dit, d'une partie interne et antérieure, qui se prolonge dans le lobe olfactif, et d'une partie postérieure, qui se réfléchit en arrière du tuber-

(1) Pl. IV , fig. 105, $n^{n} 7$. 
cule hémisphérique. Fermez la partie antérieure de cette cavité; comblez par de la matière grise sa communication avec le lobe olfactif, et vous aurez le ventricule hémisphérique des oiscaux. La partie antérieure de ce ventricule forme une espèce de croissant (1), dont la concavité est en dehors, la convexité en dedans; en arrière il se réfléchit au pourtour du tubercule hémisphérique, et c'est en bas de ce tubercule que sa capacité est la plus grande : en haut il est plus limité que celui des reptiles, parce que sa continuité avoc la coquille a lieu chez les oiseaux par une surface plus étendue que chez les reptiles supérieurs. La partie antérieure de ce ventricule est la seule qui, chez les oiseaux et les reptiles, corresponde au ventricule latéral des mammifères. Le soupirail de ce ventricule est situé au devant de la couche optique; il est plus profondément caché que chez les reptiles, et donne entrée, comme chez eux, au plexus choroïde; plexus qui, dès son entrée dans le rentricule, se divise en trois branches, l'une interne, d'autre postéricure, et la troisième inférieure. Ce plexus choroïde, qu'on ne peưt méconnaître pour l'analogue de celui des mammifères, me paraît prouver l'analogie réclle des lobes cérébraux des oiseaux avec ceux de la classe supérieure.

Les lobes cérébraux des oiseaux ressemblent

(1) Pl. III, fig. $81, n^{\circ} 5$. 
tellement à ceux de certains mammifères inférieurs, spécialement à ceux des chauve-souris, de la taupe, du zemni, des didelphes et des lièvres, que si nous pouvions développer le lobe olfactir, lui faire quitter la base de la pointe du lobe sous laquelle il est caché, et le faire saillir en avant du lobe cérébral lui-même, nous a urions une ressemblance extérieure parfaite. Ce que nous voudrions opérer chez tous les oiseaux, la nature l'a exécuté chez le casoar (1), le canard musqué et la bernache. Ice lobe olfactif, projeté chez ces oiseaux en avant du lobe cirébral (2), reproduil ce que nous offrent tous les mammifères inférieurs. C'est le passage intermédiaire d'une classe à l'autre, en ce qui concerne la configuration extérieure de ce lobe.

Chezles oiseaux, le lobe olfactif n'a plus aucune influence sur les lobes cérébraux; il a perdu celle qu'il avait exercée chez les poissons et les reptiles: il la reprend à un degré plus marqué encore, et sur-tout d'une manière plus constante chez les mamnifères. Ainsi les mammifères peuvent être livisés en trois seclions, d'après le nombre de lobes que l'on aperçoit sur la face supérieure de leurs hémisphères cérébraux. La première, qui est trèspeu nombreuse, a trois lobes, un anlérieur (3).

(1) PI. III, fig. $78,11^{\circ} 1 \%$

(2) Pl. III, 6g. 75, $n^{\circ} 6$.

(3) PI. V II, fig. 196, G, F. II. 
un moyen (1) et un postérieur (2). Elle comprend l'homme, les singes ct les phoques; et, comme nous l'avous déjì vu, la disposition qui la caractérise, coïncide avec l'absence du pédoncule olfactif, et l'insertion filiforme du nerf de l'olíaction. Ja seconde, qui a deux lobes, beaucoup plus nombrcuse que la précédente, a le pédicule olfactif très-prononcé el ses racines distinctes; ce sont les manmifères à champ olfactif, tels que les carnas:iers plantigrades et digyitigrades, la plupart des pachydermes et des ruminans. La troisieme section, trùs-nombreuse aussi, n'a qu'un lobedistinct sur la face supérieure de l'hémisphère; le lobc offactif confondu avec son pédicule, se continue immédiatement avec le lobe de l'hippocampe: tels sont les rats, les chauve-souris, les didelphes, la taupe, le zemni, la marmolte, l'agouli, etc.

Les célacés folmont unc singulière exception à ces données; car chez eux il n'y a que deux lobes sur chaque hémisphère, quoique le norf et le lobe olfactifs aient disparu.

On vemarque ces dispositions différentes chez le drill (5), le mandrill (4), le phoque (5), le

(i) Pl. VIII, fig. 196 , H. M.

(2) Pl. VIII, fig. 196, B. C.

(3) Pl. VIII, fig. 199 .

(4) Pl. VIII, fig. ${ }_{196}^{6}$

(5) Pl. IX, fig. 209 . 


\section{ET LOBF OLPICTIP.}

lion (1), la loutre (2), le raton (3), la marte (4), l'ours (5), le chameau (6), le cheval (7), le lama (8), le pécari (9), le mouton (10), le bouc (11), le castor (12), le porc-épic (13), l'agouti (14), la marmotte (15), le vespertilion murin (16), le rhinolophe uni-fer (17) et le dauphin (18.)

C'est sur-tout sur la base des hémisphères cérébraux que cette influence est marquée; outre le lobe antérieur, que tous les anatomistes y ont reconnu, il en est deux qui ont peu fixé leur attention, parce qu'ils ont été méconnus, c'est le lobe sphénoïdal et le lobe de l'hippocampe. J'ai nommé

(1) Pl. XIV, fig. 264.

(2) PI. X, fig. 220.

(3) Pl, VIII, fig. 202.

(4) Pl. XV, fig. 278.

(5) Pl. XI, fig. 230 .

(6) Pl. XIII, fig. 248.

(7) Pl. XV, fig. 274.

(8) Pl. XVI, fig. 294.

(9) Pl. XVI, fig. 298.

(10) Pl. X, fig. 221.

(ii) Pl. XIV, fig. 26 .

(12) Pl. XIV , fig. 265.

(13) Pl. XIII, fig. 252.

(14) Pl. IX, fig. 213.

(15) Pl. IX, fig. $20 \%$

(16) Pl. IX, fig. 215.

(1 7$)$ Pl. IX, fig. 206.

(18) PI. XI, Gir. 225. 
lobule sphénoïdal (1) la saillie inférieure du lobs. moyen dans la fosse sphénoïdale de la base du crâne, et lobule de l'hippocampe (2), le lobe que circonscrit en dehors la racine externe du pédicule olfactif. Ces lobes sont dans un antagonisme de développement dont la considération seule peut expliquer les transformations considérables qu'éprouve celte partie des hémisphères cérébraux, des rongeurs aux quadrumanes et à l'homme.

Ainsi, chez l'homme et les singes, le développement prodigieux du lobule sphénoïdal étouffe et fait en quelque sorte disparaître de l'extérieur de la base des hémisphères le lobule de l'hippocampe, comme on le remarque chez le mandrill (3) et le drill (4). Chez le phoque, l'affaissement subit du lobule sphénoïdal (5) fait déjà saillir considérablement le lobule de l'hippocampe (6), dont la forme est presque quadrilatère; chez les carnassiers le lobe sphénoïdal réduit encore dans ses dimensions, comme on l'observe chez le lion (7),

(1) Pl. VIII, fig. 194, G.

(2) Pl. XIV, fig. 258, H.

(3) Pl. VIII, fig. 194, B, D, H.

(4) P1. VIII, fig. 197, G, D, H.

(5) Pl. IX, fig. 268, D.

(6) Pl. IX, fig. $208, \mathrm{H}$.

(7) P!. XIV, 6g. 266, K. 
Ia loutre (1), la marte (2) et le raton (3), dégage de plus en plus le lobe de l'hippocampe, dont la saillie plus prononcée lui donne la forme d'un demiovoïde, comme chez le lion (4), la loutre (5), la marte (6) et le raton ( 7$)$; ovoïde qui devient plus saillant encore chez le chameau (8), le cheval (9), le lama (10), le pécari (11) et le bouc de la haute Égypte (12), à mesure que le lobule sphénoïdal s'affaisse, comme on le remarque surtout chez ce dernier (13).

Ainsi, en descendant des quadrumanes aux ruminans, on voit que le lobule de l'hippocampe gagne tout ce que perd le lobule sphénoïdal. Ce dernier lobule inaperçu à l'ex térieur chez l'homme et les singes, se dégage d'abord chez les phoques, puis augmente graduellement chez les carnassiers et les ruminans. Jusque-là le lobule sphénoïdal

(1) Pl. X, 6g. 223, D.

(2) Pl. XV, fig. 290, D.

(3) Pl. VIII, fig. $200, a^{*} 5$.

(4) Pl. XIV, fig. 266, H.

(5) $\mathrm{Pl} . \mathrm{X}$, fig. $223, \mathrm{H}$.

(6) PI. XV, fig. $290, \mathrm{H}$.

(7) Pl. VIII, fig. 200, H.

(8) PI. XIII, \&g. $249, H$.

(9) Pl. XV , fig. $275, \mathrm{H}$.

(10) Pl. XVI, fig. $295, \mathrm{H}$.

(11) PI. XVI, fig. $300, n^{\circ} 1 \%$

(12) Pl. XIV, fig. 262, H.

(15) Pl. XIV, fig. 262, G. 
est encore assez apparent sur la base des hémisphères cérébraux; dont il va disparaître chez les mammifères inférieurs; cette retraite déjà visible chez le castor (!) et le porc-épic (2), est complètement effectuée chez l'agouti (3), la marmotte (4), les rats et les chauve-souris (5). En même temps aussi, la place qu'il occupait est envahie par le lobule de l'hippocampe, qui, d'accessoire qu'il était d'abord sur la base de l'hémisphère, devient lobe principal, puis en quelque sorte lobe unique de cette partie; il acquiert ce maximum de développement en se confondant arec le champolfactif, les pédicules et le lobe olfactif, comme on le voit chez le vespertilion murin (6), le rhinolophe uni.fer (7), l'agouti (8) et la marmotte (9). Ces derniers mammifères sont pour le lobe de l'hippocampe, ce que les quadrumanes et l'homme sont pour lelobesphénoïdal. Ce dernier a disparu chezles mammifères inférieurs, étouffé en quel que sorte par le lobe de l'hippocampe, comme le lobe de l'hippocampe disparait sous l'influence du développe-
(1) Pl. XIV, fig. $258, \mathrm{G}$.
(2) Pl. XIII, fig. $251, \mathrm{G}$.
(3) PI. XI, fig. $21 x, G$.
(4) PI. IX, fig. 203, G.
(5) Pl. IX, fig. 204 et 214.
(6) Pl. IX, fig. $214, \mathrm{~F}$.
(7) Pl. IX, fig. 204, no 5.
(8) PI. IX, fig. $211, \mathrm{H}$.
(9) PJ. IX, fig. $203, \mathrm{H}$. 
ment considérable du lobe sphénoïlal chez les mammifères supérieurs : chacun de ces lobes devient tour-à-tour dominateur et dominé, selon qu'on le considère aux deux extrêmes de la classe des mammifères. En même temps aussi, la scissure de Sylvius s'efface ou devient plus profonde; elle s'efface à mesure que le lobule de l'hippocampe s'accroît; clle se creuse à mesure que le lobule sphénoïdal se développe. C'est toujours la répétition de ce que nous ont présenté les métamorphoses des embryons.

Par la position du lobe sphénoïdal, par ses conncxions eu avant et en arrière, on voit qu'avec son atrophie doit coïncider, en premier lieu, la diminution de la hauteur de l'hémisphère cérébral; en second lieu, la diminution du lobe antérieur eu avant; et en troisième lieu, la diminution du lobe moyen en arrière.

On observe en effct que l'bémisphère diminue graduellenent en hauteur, à partir de l'homme, des quadrumanes et des phoques, aux carnassiers plantigrades et digitigrades, aux ruminans, aux rongeurs, aux insectivores et aux chauve-souris; c'est, en effet, lorsque par cette diminution de hauteur, les pédoncules olfactifs sont arrivés au niveau du lobe de l'hippocampe, qu'ils se réunissent et se confondent avec lui.

C'est aussi à cause de la diminution graduelle du lobe antérionr que l'on voit chez l'homme et 
les singes (comme thez le mandrill (i) ei k drill (2)) et chez les phoques (3), ce lobe recouvrir et cacher le lobe olfactif, puis le laisser un peu à découvert, conme chez les carnassicrs ef les ruminans, puis entin le mettre tout-à-fait à nu, comme chez le lapin (4), les didelphes (5), l'agouti (6), la marmolte (7), la taupe (8), le zemni (9), le lirot (10), le hérisson (11), le vespertilion murin (12) et le rhinolophe uni-fer (13).

L'effet de la diminution et de la retraite du lobe antérieur est done de décourrir le lobe olfactif, et de le dégager de dessous la partie antérieure de l'hémisphère où il est si profondément caché chez l'homme. L'effet de la diminution de l'hémisphère en arvière va être de dégager le cervelet, de le retirer de dessous le lobe postérieur, puis lorsqu'il sera tout-à-fait à nu, dc.

(1) Pl. VIII, fig. 19f, E.

(2) Pl. VIII, fig. 197, E.

(3) Pl. IX, fig. '208, E.

(4) Pl. II, fig. $57, n^{\circ} 5$.

(5) Pl. II, fig. 54, no 8.

(6) Pl. IX, fig. $211, \mathbf{E}$.

(z) Pl. IX, fig. 20\%, N, E.

(8) Pl. XIV, fig. $256, \mathbf{N}, \mathbf{E}$.

(9) Pl. XV , fig. 270, F, E.

(10) Pl. XV, fig. $276, B, E$.

(11) Pl. XVI, fig。 $29^{6}, \mathrm{~F}, \mathrm{E}$.

(12) Pl. IX, fig. 215, O, E.

(13) Pl. IX, fig. 206, N, E. 
dégrager encore les tubercules quadrijimanux.

Suivez en effet cette coüncidence de la diminution du lobe sphénoïdal avec l'hémisphère en arrière; vous verrez coincider avec son plus grand développemeni l'existence du lobe jostérieur de l'homme et des quadrumanes, et vous trouverez, comme chez le drill et le mandrill, le cervelet enlièrenent recouvert par lui. Puis chez le phoque, l'affaisscment subit du lobe sphénoïdal mettra à nu une partie du cervelet (1), par la retraite el la diminution du lobe postéricur de l'hémisphère: puis chez les carnassiers vous verrez le lobe postéricur disparaitre à mesure que le lobule sp̣hénö̈dal s'affaisse, comme chez la loutre (2), le lion (5), la marte (4), le raton (5), le coati (6) et l'ours (7); el ce raccourcissement de l'hémisphère découvrira la moitié et cnsuite les deux tiers du cervelet, puis le mettra entièrement à nu chez les ruminans: car le lobe sphénoïdal s'atrophiant de plus en plus, de plus en plus aussi les hémisphères se raccourcissent, et leur prolongement en arrière diminuc. C'est ce qu'on remarque chez le cheval ( 8 ), le

(1) P1. IX, fig. $209, \mathrm{~A}, \mathrm{C}$.

(2) Pl. XI, fig. $220, A, C, n^{\bullet} 3$.

(3) Pl. XIV, fig. $264, \mathrm{~A}, \mathrm{C}$.

(4) Pl. XV, fig. $278, A, C, C$

(5) Pl. VIII, fig. 202, C.

(6) PI. XII, fig. $240, n^{\circ} 3$.

(7) Pl. XI, fig. $2 \mathbf{3}_{0}, \mathbf{B}, \mathrm{H}$.

(8) Pl, XV, fig. 274, E, A, C. 
chameau (1), le lama (2), le pécari (3), le mouton, et sur-tout chez le bouc (4). Enfin ce mouvement rétrograde du lobe cérébral accompagnant toujours l'atrophie du lobule sphénoïdal, vous voyez découverts, non-seulement le cervelet, mais même les tubercules quadrijumeaux, comme chez les didelphes, les rats, le castor (5), l'agouti (6), la marmotte (7) et sur-tout les chauve-souris (8). Le cervelet et les tubercules quadrijumeaux sont donc mis à nu, en arrière des hémisphères, par le même mécanismc qui découvre en avant le lobe olfactif; et la cause de ces deux effets opposés en apparence, réside donc dans les divers degrés d'accroissement ou d'atrophie du lobule sphénoïdal.

Or le lobule sphénoïdal commande en quelque sorte le développement du corps calleux; d'où il suit que lc corps calleux devra suivre ses divers décroissemens; de là, la diminution progressive de celte grande commissure, de l'homme aux quadrumanes, aux carnassiers, aux ruminans, aux. rongeurs et aux chauve-souris.

(1) Pl. XIII, fig. $248, B, C$, D.

(2) Pl. XVI, fig. 294, A, C, R.

(3) PI. XVI, fig. $29^{8}, \mathrm{~A}, \mathrm{C}, \mathrm{D}$.

(4) PI. XIV, fig. $261, \mathrm{~B}, \mathrm{C}$.

(5) Pl. XIV, fig. 265, C.

(6) Pl. IX, 218, F.

(7) Pl. IX, fig. $207, \mathrm{~F}$.

(8) Pl. IX, fig. '206 et 215. 
Mais le lobule de l'hippocampe gagne tout ce que perd le lobule sphénoïdal; son accroissement est lié à celui du système de la voûte : aussi voyezvous, à mesure que ce lobule s'accroît, le corps frangé se déplisser et s'étendre, d'abord en partie chez les carnassiers et les ruminans, puis en totalité chez les rongeurs; de même, vous voyez aussi la voûte proprement dite recouvrir à peine la couche optique chez les quadrumanes, la cacher en totalité chez les carnassiers et les ruminans, et atteindre chez les rongeurs le corps strié, dont elle couvre de plus la partie postérieure. Ainsi toutes les parties se lient et s'enchaînent dans les lobes cérébraux des mammifères adultes; les grandes variations qu'ils nous présentent dans les diverses familles, et qui, au premier aperçu nous effraient par leur complication, se réduisent donc en dernière analyse à la discordance de développement, ou à l'antagonisme de deux de ses lobules, le lobule sphénoïdal et le lobule de l'hippocampe. Lit remarquez que la position de ces lobules est à la base de l'hémisphère, tandis que le lobule médian et les hémisphères du cervelet qui commandent ce dernier, sont sur la face supiricure; observez de plus que la grande commissure des hímisphères du cervelet occupe la hase do l'ucéphale, rt celle du lobule sphénoïdal lir fartic supérieure; yous concevrez ainsi la pensere que jai exprumée en disant que les parties

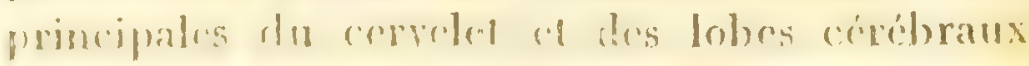


étaient renversées dans leur position, et que des données que nous fournissait la partie supérieure de l'un de ces organes, nous étaient fournies par la base de l'autre. C'est là ce qui est, et il faut bien le dire, que cela blesse ou ne blesse pas les idées préconçues de nos devanciers : car, en dernicr résultat, il ne reste dans les sciences qué ce qui se trouve réellement dans la nature.

Celte retraite antérieure et postéricure du lobo cérébral des mammifères influe d'une manière. différente sur son grand ventricule. Diminuez l'épaisseur des couches qui forment le lobule antérieur de l'homme, des quadrumanes et des carnassiers, en même temps qu'il se raccourcil, vous verrez sa base se rapprocher de plus en plus de l'anse antérieure du ventricule latéral; et comme le pédicule olfactif occupe cette base, vous le verrez marcher de plus en plus aussi vers la corne antérieure du même ventricule. Or, en sé développant, le pédicule olfactif se creuse d'un petit canal; il arrive donc un moment où ces deux cavités doivent s'aboucher, s'unir et se confondre: c'est ce qui doit être, d'après la disposition des parties, et c'est ce qui est; car déjà chez quelques carnassiers on distingue un petit hiatus en avant de l'anse antérieure du ventricule latéral, hiatus qui conduit dans le canal du pédicule. Ce hiatus s'ouve sie plus en plus chez les pachydermes ct les ruminans; et cnfin chez les rougeurs et les chaure-souris, c'est un canal continu 
qui, de la corne antérieure du ventricule, se prolonge dans le canal du pédicule jusqu'à la cavité du lobe olfactif. C'ęst la reproduction de ce que nous ont offert les embryons des mammifères supérieurs; chez ces derniers, cette communication s'effacé par un mécanisme inverse de celui qui la rélablit chez les mammifères inférieurs.

Maintenant, en arrière du lobe cérébral, avec la manifestation du lobule postérieur, vous verrcz apparaître chez les mammifères qui eu sont doués une troisième anse dans le ventricule latéral. Cette anse, ou la corne postérieure, sera donc soumise pour son existence et son étendue à l'existence et au volume du lobule postérieur. Aussi est-elle plus étcndue chez l'homme que chez les quadrumanes, et la voit - on diminuer rigoureusement chez ceux-ci comme le volume du lobule postérieur. Chez les phoques, l'anse postérieure est encore très-prononcée, ainsi que le lobule; chez le marsouin et le dauphin il n'y en a pas le moindre vestige, de même que chez les carnassiers et les ruminans. Chez les embryous des quadrumanes il n'y a également pas de traces de la corne postérieure avant l'ap parition du lobule postéricur. En suivant chez eux la marche de la corne antérieure et de la postérieure, on observe 'jue la première se rétrécit à mesure que la seconde se dilate; la capacité du ventricule gagne en arvière ce qu'elle semble perdre en a vant : enfin c'est torsque la corne postéricure a arrêté son dévelop- 
pement que l'antérieure perd définitivement sa communication avec le lobe olfactif. Observez maintenant que tous les mammifères chez lesquels cette communication existe sont privés de la corne postérieure.

De l'intérieur du lobe cérébral des mammifères, revenons à sa surface extérieure pour apprécier les variations opérées par la présence ou par l'absence des circonvolutions. Leur but évident est, comme nous l'avons déjà observé, de multiplier l'étendue de la surface du lobe; elles manquent chez les mammifères inférieurs, de mème que chez les poissons, les reptiles et les oiseaux; leur absence coïncide avec l'atrophie du lobule sphénoïdal et le grand développernent du lobule de l'hippocampe.

Sous ce rapport, les circonvolutions les plus étendues sont celles de l'homme; viennent ensuite celles des singes (1), des phoques (2) et des cétacés (3). Parmi les carnassiers elles vont en diminuant du lion (4) au loup, au renard, au chien . à la loutre (5), à la marte (6), à la fouine; elles décroissent chez les ruminans, du chameau (7), du

(1) Pl. Vill, fig. 194, 196, 197 et 199.

(2) Pl. IX, fig. 209.

(3) Pl. XI, tig. 225.

(4) PI. XV, fig. 264 et 266.

(5) Pl. X, fig. 220.

(6) Pl. XV, fig. 278.

(c) Pl. XIII, fig. 248 et 2.9 . 
bour, du lama (1) au cerf, au mouton (2), au bouc; enfin elles s'effacent d'une manière presque. insensible chez le kanguroo géant (3), le porcépic (4), les édentés (5), le castor (6), le lapin ( 7$)$, la taupe (8), l'agouti (9), la marmotte (10) et les chauve-souris (11). On roit d'après ce tableau que les circonvolutions sont développées en raison directe du lobule sphénoïdal, et inverse du lobule de l'hippocampe.

Les mammifères se distinguent entre eux par la symétrie ou la non-symétrie des circonvolutions. Les circonvolutions sont symétriques d'abord chez les mammifères où elles sont les plus faibles, comme on le voit chez l'agouti, la taupe, l'unau, le porc-épic, le castor, le kanguroo géant; clles sont au contraire tout-à-fait dissimilaires d'un lobe à l'autre chez l'homme, le phoque et les cétacés; et, chose remarquable, elles affectent

(1) Pl. XVI, fig. 994 et 295 .

(2) Pl. X, fig. 221.

(3) PI. XIII, fig. 250 ; pl. XVI, fig. 39 ?

(4) Pl. XIII, fig. 251 et $25 \%$.

(5) Pl. XI, fig. 229, $n^{\circ} 4,5,1$ et 2.

(6) Pl. XIV, fig. 258 et 265.

(7) Pl. II , G $5.57, n^{0} 6 \mathrm{el}$.

(8) PI. XIV, fig. $256, \mathbf{K}, \mathbf{N}$.

(9) Pl. IX, fig, $218, \mathrm{E}, \mathrm{N}$.

(10) Pl. IX, fig. $207, \mathrm{E}, \mathrm{N}$.

(11) PI. IX, fig. 204, 206, 214 et 215. 
une similitude presque parfaite chez les singes placés entre ces derniers mammifères.

Pour décider cette question à laquelle les îdées de Willis renouvelées de nos jours par M.M. Gall et Spurzheim donnent une certaine importance, il est nécessaire de considérer les circonvolutions sur la face supérieure du lobe, sur sa base et sur ses côtés; nous trouverons par cet examen que beaucoup de mammifères ont certaines circonvolutions similaires, et d'autres non-similaires. Remarquons en premier lieu que sur la base du lobe antérieur, la circonvolution qui loge dans sa goultière le pédicule olfactif, est similaire chez tous les mammiferes, des quadrumanes aux rongeurs; de plus observons qu'elle s'élargit en raison directe du développement de ce pédicule, des rongeurs aux quadrumanes, et qu'en s'élargissant, elle se déplisse, ce qui fait que son anfractuosité devient de moins en moins profonde. Suivons le mème rapport sur le lobule de l'hippocampe, et nous trouverons çue les circonvolutions s'effacent à mesure qu'il grandit : l'effet général du développement do celobule et du pédicule olfactif est donc de déplisser la base de l'encéphale, et de la rendre de plus en plus lisse et unie, comme on le voit des singes aux carnassiers, aux ruminans, et sur-tout, chez les rongeurs, du castor et du porc-épic à l'agouli et à la marmotte. Si ce que nous avons dit de l'antagonisme du lobule sphérioïdal et du lobule de l'hip- 
pucampe est exact, les circonvolutions de la base devront s'accroître cn raison directe du développement du premier de ces lobules. Remontez la séric des mammiferes yue nous venons de descendre, ct vous verrez se confirmer encore l'exactitude do cette proposition.

Chez l'homme, le phoque (1), les cétucés ( 2 ) ct le cheval (5), les circonvolutions sont dissimilaires sur toute la périphéric die lobe cérébral. Chez les singes, la similitude des circonvolutions n'est parfaite que sur les faces supérieurc et latérale; sur la base elles sont dissimilaires (4). Chez les carnassiers, les circonvolutions supérieures sont symeiriques et parallèles; chez le lion (5), le ligre, la panthère, le chat, le renard, le loup, la genette et les mangoustes, ces circonvolulions s'étendent sur toute la surface du lobe; chez la marte (6), la fouine, le coali et le blaireau, le parallélisme de ces circonvolutions est iuterrompu par une circonvolution transversalc, qui se place, lantôt en avant, comme cher la marte (j), tantô! au milicu, comme chez de rator (8), tantôt en

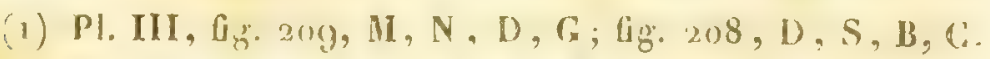

(2) PI. XI, fig. 225. I. K ; pl. XII, fig. 254. F, K, M

(3) Ii, XV. fig. $274, \mathrm{~b}, \mathrm{~F}, \mathrm{O}, \mathrm{f} ; \mathrm{fig}, 275, \mathrm{C}, \mathrm{G}$.

(4) 1'. VIII, fig. I!) 4, , , F: ig. 197, I, G, E.

(5) PI. XIV, fg. 264, $n^{\circ} 7$ et 8.

(6) Pl. XV, fig. $278, n^{\circ} 7 \mathrm{ct} g$.

(7) Pl. XV, fig. $278, n^{2} 10$.

(8) Pl. VIII, fig. ans, l; 
arrière, comme chez le blaireau. Chez d'autres carnassicrs, la symétrie et le parallélisme de ces circonvolutions s'effacent, comme on l'observe chez la loutre (1) et chez l'ours (2). Chez tous ces carnassiers, les circonvolutions de la base sont plus ou moins dissimilaires, comme on peut le voir chez le lion (3), l'ours (4), la loutre (5), le tigre, la panthère et la genette; chez le raton (6), au contraire, elles sont similaires, comme celle de la face supérieure et de la face latérale. Le kanguroo-géant (7) a également les circonvolutions symétriques dans tous les sens (8).

Parmi les ruminans et les pachydermes, la symétrie a lieu assez exactement, sur toutes les faces chez le mouton (9), sur les faces supérieure (10), latérale et inféricure (11) chez le chameau,

(1) Pl. X, fig. $220, n^{\circ} 9$ et 10.

(2) Pl. XI, fig. $220, E, \mathbf{F}$.

(3) Pl. XIV, fig. 266, K, G, S.

(4) PI. XI, fig. $251, H, L, N, S$.

(5) P1. XI, fig. 223, F, D.

(6) PI. VIII, fg. 200, G, F.

(5) PI. XIII, fig. $250, \mathrm{D}, \mathrm{K}$.

(8) Pl. XVI, fig. $299, \mathrm{n}^{\circ} 5$ et 18.

(9) Pl. X, fig. $221, \mathrm{n}^{6} 5,6,7$ et $g$ -

(10) PI. XIII, fig. $248, E, F, G$.

(11) PI. XIII, fig. 249, A. C, G. 
sur les mêmes faces chez le bouc de la haute Egypte (1), le daman (2), le lama (3) et le pécari (4). Enfin, chez les rongeurs, les circonvolutions sont parfaitement similaires dans tous les sens, comme on le voit chez le castor (5) ct le porc-épic (6). Renfermant tous ces faits dans une proposition générale, on peut donc dire que les circonvolutions sont d'autant plus symétriques, qu'elles sont moins nombreuses, et d'autant plus dissimilaires qu'elles sont plus étendues et plus multipliées; mais les singes font à ce principe une exception si remarquable, qu'il est important de la faire ressortir d'une manière spéciale

Willis avait dit vaguement que les circonvolutions se répètent dans les espèces d'une même famille; Soemmering et Monro avancèrent que la similitude était parfaite dans les familles et les es pèces; M. Gall le suppose sans l'affirmer aussi posivement, et Tréviranus adoptant l'opinion de: Soemmering et de Monro, croit que les circonvolutions pourraient fournir des caractères positifs pour la classification des mammiferes. Nous venons de voir combien celte proposition est loin

(1) PI. XIV, fig. $201, n^{\circ} 6,7,8,9$ et in; fig. $262, k$, (i. 11 .

(2). Pl. XV, fig. $269, n^{\bullet} 6,7$ et 8 .

(3) PI. X VI, fir. 294, D, E, F, G; fig. 295, li, G.

(4) Pl. XVI, fig. $29^{8}, \mathrm{D}, \mathrm{E}, \mathrm{F}, \mathrm{G}, \mathrm{fig} .300, \mathrm{M}, \mathrm{S}$.

(5) Pl. XIV, fig. $265, n^{n} 5$, 7 et 9 ; fig. 258, E, I. G, IK.

(6) Pl. XIII, fig. 252, K, I. ; fig. 251 , E, G. 
564

d'être exacte en ce qui concerne les familles; d'après nos observations sur trois phoques, quairc lions, six renards, deux loups, environ vingl chiens, dix à douze chats, cinq à six bœufs et moutons, nous pouvons assurer quelle ne l'est pas non plus en ce qui concerne ces espèces, si du moins les différences que nous avons remarquées, ne sont pas un effet de l'âge. Tous les anatomistes savent d'ailleurs que chez l'homme les dissemblances sont infinies, comme je l'ai constaté d'âge à âge, de sexe à sexe, de profession à profession, d'habitude à habitude, et aussi dans le même âge, le même sexe, la même profession et les mêmes habiludes. Rien n'est donc moins vraisemblale que cette pensée de Willis, développée par MM. Gall et Spurzheim, qui attribue au nombre et à la disposition des circonpolations une si puissante influmee sur la manifestation de l'intelligence, ou sur la ruse et les instincts des animaux. En laissant de côté l'homme, dont l'intelligence échappe à toutes les données de l'anatomie, on peut remarquer que le sanglier, le cochon, le mouton, le bouc, le bœuf ont des circonvolutions plus uombreuses et plus étendues que le lion, le tigre, le renard, le loup, le chcval, le chien, et sur-tout que le castor. Or, comparez Ics actes de ces animaux; quel est, du renard, dri chicn, ou du mouton, celui dont la rusc est la plus étenche? et quelle distance immense sépare les actes dn castor do ceux du bouc et du sanglier? 
II existe chez les mammiferes un rapport remarquable entre les circonvolutions des lobes cérébraux, el les divisions du cervelet; ainsi en embrassant ce rapport d'une manière générale, le cervelet est parfaitement symétrique chez les mammifères, dont les circonvolutions se répètent dans tous les sens d'un lobe à l'autre; le cervelet 'st au contraire non-symétrique chez les mammirères à circonvolutions tout-à-fait dissimilaires; la symétrie du cervelet s'efface chez les animaux lont certaines circonvolutions sont similaires et les autres dissimilaires. Remarqucz toujours que la non-similarité des circonvolutions commence par la base du lobe cérébral, et colle du cervelet, par sa face supérieure; l'inversion que nous avons observéc entre ces deux organes se reproduit ainsi jusque dans les moindres particularités de leur organisation.

\section{Du lobe olfuctif.}

J'ai déjà dit pourquoi et comment le lobe olfactif avait, pour ainsi dire, ité inapercu par les anatomistes; j'ai montre son importance et fait apprécier l'influence qu'il exerce sur les autres prarlies de l'encéphale, el plas spéchalement sur les lobes crébraux doni limiôt il s'écarte, comme chez cortains prissons an point do dépasser la rabite du crane, de dont it se rapproche elle-

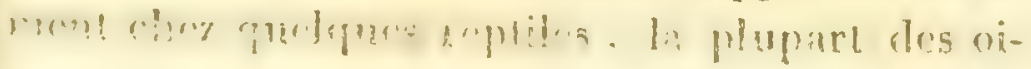


seaux et beaucoup de mammifères, qu'il semble en quelque sorte se confondre avec lui. La condition errante de ce lobe, comparée à la fixité des autres élémens de l'encéphale tient sans doute à sa position. Terminant en avant l'axe cérébrospinal, il peut se déplacer, et se déplace en effet sans troubler les connexions et les rapports des autres lobes de l'encéphale, ce que n'auraient pu faire ni le cervelet, ni les lobes optiques, ni les lobes cérébraux : sous ce rapport il se rapproche du point terminal de la moelle épinière, que nous avons trouvé errant dẹ la même manière à l'extrémité opposéc de cet axe; se prolongeant souvent et se fixant aux dernières vertèbres coccygiennes, flottant ailleurs dans le canal vertébral, et s'arrêtant plus ou moins haut ou plus ou moins bas, selon les classes, les familles et les espèces.

$\mathrm{Si}$, faisant abstraction des membres, nous bornons pour un moinent l'être animal au tronc et á la tête, nous trouvons qu'il se prolonge d'autant plus inférieurement que le point terminal de l'axe descend plus bas dans sou canal, et qu'il se raccourcit en proportion de son ascension dans le canal, ou de sa concentration. Un parcil rapport se répètera-t-il en avant du tronc? La face et le crâne éprouveront-ils du déplacement du lobe olfaclif une semblable modification? Le crâne s'affaissera-t-il, comme le bassin, à mesure que le lobe olfactif se projètera on avảnt? La face se prolongera-t-elle en avant, ou se concentrera-t-elle sous 
le crâue, sclon que le lobe olfactif sera eufoncć sous les lobes cérébraux, ou placé à distance de ces lobes? Pour répondre à cette question, dont le simple énoncé indique l'importancc, jetez un coup d'oeil sur l'inclinaison de l'angle facial des mammifères: d'après les belles recherches de Camper, de Blumenbach et de M. Cuvier, vous royez, en descendant des quadrumanes aux rongeurs, le crâne se déprimer et la face s'élendre, "n même temps que le lobe olfactif caché sous les lobes cérébraux s'en dégage de plus en plus, et vient saillir sur leur partie antérieure; l'affaissement du crâne coïncide avec le raccourcissement des lobes cérébraux, et la saillie du lobe olfactif avec la projection en avant de la face. Les cétacés font seuls une exception à cette règle.

Opposez à cette variation conlinuellc du crânc et de la face des mammifères la fixité des mòmes parties chez les oiseaux, et vous verrez l'immobilité de ces parties coïncider avec la position fixc rlans cette classc du lobe olfactif.

Yous trouvercz, chez les reptiles le crâne benucoup plus affaissé encore et la face plus relevéc ct plus étendue, selon que le lobe olfactif sera ráinevé au point de contact des lobes cérébrilux, comme chez les batraciens et les chélonicus, on rejeté à dislance, comme chez les ophidions, les lacerticns ct la plupart des saurions.

Enfin, vous remarquerez que cher leg poissons la face se place sur le mene nivean cuuc le crâne. 
parce que le lobe olfactif alteint presque les mêmes dimensions que les lobes cérébraux. 11 est vrai que chez les poissons osseux l'allongement de la face ne suivra pas exactement l'éloignement du lobe olfaclif des lobes cérébraux; que, par exemple, lorsque le lobe olfactif est projeté hors du crâne, comme chez les silures, les tétrodons, les gades, les cyprins, la face ne s'allonge pas dans la mème proportion; mais cette exception est la répétition de celle que nous a présentée le point terminal de la moelle épinière chez les lophius et les tétrodons. Vous trouverez une autre exception chez les poissons cartilagineux dont l'encéphale ne remplit point la capacité du crâne, et chez lesquels conséquemment le lobe olfactif peut s'éloigner ou se rapprocher des lobes cérébraux sans allonger ou affaisser la face. Ces exceptions, dont les conditions organiques nous échappent dans l'état actuel de l'anatomic comparative, finiront par rentrer dans les règles communes, lorsque l'étude des sens clans cette classe sera plus avancéc.

Quoi qu'il en soit de ces rapports sur lesquels nous avons cru devoir nous arrêter un instant, il nous reste à dire un mot du lobe olfactif considéré comme élémenl de l'encéphale dans les quatre classes des vertćbrés : nous avons déjà apprécić ses convexions, en parlant de son pédicule. it l'article du nerf de l'olfaction.

Ce lobrest phus ou moins volumincux chez lia

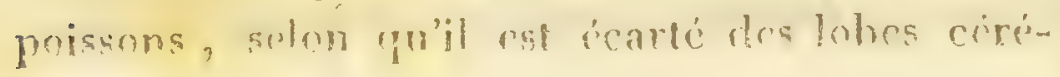


braux, ou qu'il léur est adossé. Ainsi, chezle congre (1) et l'anguille vulgaire (2), il égale presque en volume les lobes cérébraux, auxquels il est immédiatement adossé. Chez le gronau (3), il conserve également une grande dimension, qu'il perd chez le barbeau (4) et tous les poissons osseux chez lesquels il abandonne les lobes cérébraux. Sa structure, chez les premiers, diffère peu de celle du lobe cérébral; au contraire, en s'éloignant de lui, il se durcit, se déforme et se rapproche plus de la structure des sanglions intervertébraux des classes supérieures. Il revêt sur-tout ce caractère lorsque, sorti de la cavité du crâne, il se place dans les mèmes conditions queces ganglions eux-mêmes.

Chez les poissous cartilagineux, plus il s'éloigne aussi du lobe cérébral, plus il semble perdre sa forme arrondie, comme on le voit chez la lamproie (5), l'esturgeon (6) et le requin (7), comparćs aux raies (8), à l'ange et l'aiguillat, aux squalus glaucus, squalus galeus et squalıs griseus. En même temps qu'il se déforme chez ces derniers poissons, la cavité intéricure qu'il présente chez le requin,

(1) Pl. VII, fig. $168, n^{\circ} \%$

(2) Pl. viI , fig. $190, n^{\circ} 5$.

(3) Pl. VII, fig. $155, n^{\circ} 8$.

(4) PI. VII, fig. 183, I, I.

(5) Pl. XI, fig. $324,11^{n} 1$

(ii) PI. XII, fig , 235. 110

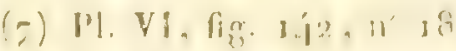

(8) 91 \% fin $158,11 \%$ 
se rétrécit et tend à s'oblitérer complètement. Cette variation de structure est beaucoup moins scnsible cependant que chez les poissons osseux.

Chez les reptiles il varie dans sa position; de même que chez les poissons, il s'éloigne et se rapproche alternativement du lobe cérébral. Quand il s'en éloigne, il communique toujours avec lui par un pédicule plus ou moins gros (1), de même que chez les poissons cartilagineux, et jamais par des rubans aplatis, comme cela a lieu chez ies osseux (2). Quand il s'en rapproche, cen'est plus une simple contiguité, comme chez les poissons osseux, mais une continuité immédiate, comme on le voit chez la tortue franche (3) et la grenouille (4): cctte continuité est moins complète toutefois chez les batraciens et les trigonocéphales, que chez cette tortue, et le caïman à museau de brochet. Le lobe olfactif, très-petit chez la tortue grecque (5), plus volumineux chez les vipères (6) et les lézards (7), reste allongé et en quelque sorte fusiforme, lorsqu'il est séparé du lobe cérébral; sa forme devient plus globuleuse lorsqu'il se rapproche de ce lobe, comme cela a lieu en partie

(1) Pl. V, fig. 135, n 9 .

(2) Pl. VI, fig. 149, $\mathrm{n}^{*} 1 \mathrm{I} \mathrm{ct}$ i3.

(5) Pl. V, fig. $12 n, n^{\circ} 14$.

(4) Pl. I, fig. $16,11^{\circ} 5$.

(5) Pl. V, fig. $125, \mathrm{n}^{\circ} 11$.

(6) Pl. V, fig. $126, n^{\circ} 9$.

(7) Pl. V, fig. $128, n^{\circ} 6$. 
chez le requin, la lamproie ct l'esturgeon, parmi les poissons cartilagincux. On voit sur-tout ce changement chez la tortue franche (1), la grenouille (2), les crapauds, l'amphisbene et certains trigonocéphales: il est particulièrenzent remarquable chez le caïman à museau de brochet comparé au crocodile vulgaire. De même que chez les poissons osseux, le lobe olfactif atteint ses plus grandes dimensions en venant s'adosser au lobc cérébral; sous ce rapport, la tortue franche, le caïman à inuseau de brochet et les batraciens, sont aux. reptiles ce que le congre, l'anguille et les trigles sont aux poissons osseux. Son rentricule s'abouche directement alors dans le ventricule hémisphérique des reptiles; quand il est éloigné du lobe cérébral il communique ałcc lui par la cavité du pédicule, connme cela a lieu chez les carílagineux, ct de même que chez ces poissons, son ventricule propre se rétrécit d'autant plus qu'il s'éloigne davantage. On peut sur-tout observer ces diverses modifications du lobe olfactif chez le caïman à muscau de brochet et chez le crocodile vulgaire. La différence de structure du lobe olfactif avec le lobe cérébral des poissons cartilagineux, ne peut donc scrvir à rejeter chez ces poissons la détermination th premicr de ces lobes, puisque nous trouvons retle mênue différence chcz les poissons osseux el

(1) PI. V. תin. 12\%, $11^{n} 16$

(?) Pl. I, fig. If, !" is 
chez les reptiles. Chez tous les reptiles où le lobe olfactif se continue avec le lobe cérébral, un ćtranglement semblable à celui de la tortuc franche (1) indique le point d'union des deux organes.

Il résulte de ce que nous venons de voir chez les poissons et les reptiles, que, plus le lobe olfactif se rapproche des lobes cérébraux, plus il augmente de volume. Si cette règle d'accroissement se continuait chez les oiseaux, et si, dans cette classe, le lobe olfactif se joignait constamment au devant de la base du lobe cérébral, son volume devrait donc s'accroître encore, et dépasser celui qu'il a chez la tortue franche et le caïman à museau de brochet. Or, cette position et la continuité du lobe olfactif avec le lobe cérébral sont constantes et presque invariables chez les oiseaux, et cependant les dimensions du lobe olfaclif dans cette classe sont si réduites qu'il est resté inaperçu par beaucoup d'anatomistes. On voit donc que les lois d'accroissement et de décroissement des élémeus del'encéphale varient quelquefois de classe à classe. Ce fait exceptionnel constitue lui-même une espèce de règle que ne doivent jamais perdre de vue ceux qui veulent traiter avec succes de l'anatomie comparative.

Le lobe olfaclil des oiseaux forme une espece de papille nerseuse arrondie, cl situéc au-devant ct à la base du lobe cérébral antéricur. comme

(1) Pl, Y, fig, 122, $1^{2} 15$. 
on le remarque chez la bondrée commune (1), l'hirondelle (2), le roitelet (3), la cigogne (4) et l'autruche (5). Continu chez tous les oiseaux avec la substance mème du lobe, il n'offre de rudiment de pédicule que chez le perroquet $(6)$, le canard musqué, la bernache et le casoar ( 7 ), chez lesquels seuls il constitue un lobe distinct du lobe cérébral. Alors il se place tout-à-fait en avant de la base de ce dernier lobe, et le déborde comme chez les rongeurs, ainsi qu'on le voit chez ce dernier oiscau (8). Chez les autres, tantôt il en occupe la pointe, comme chez la cigogne (9); tantôt comme chez l'autruche (10) et la bondrée (11), il s'enfonce plus ou moins sous sa base.

Son volume diffère peu chez les oiseaux, en comparaison des variations qu'il présente dans les autres classes; toutefois, il m'a paru plus développé chez l'oie, le canard, le dindon, le vautour, l'aigle, le hibou, l'épervier, le corbeau, la bé-

(1) Pl. IV, fig. $88, \mathrm{n}^{\circ} 9$.

(2) Pl. IV, fig. $9^{2}, n^{\circ} \%$

(3) Pl. IV, fig. $94, n^{\circ} \eta$

(4) Pl. IV, fig. $103, \mathrm{n}^{\circ} 12$.

(5) Pl. IV, fig. $9^{8}, \mathrm{n}^{\circ} 11$.

(6) Pl. IV, fig. 96, n' 15.

(z) Pl. III, fig. $78, \mathrm{n}^{\circ} 12$

(8) Pl. III, fig. $77, n^{\circ} 6$.

(9) Pl. IV, fg. $103, n^{\circ} \times 2$.

(10) Pl. IV, fig. $9^{8}, n^{0}$ u

(1) Pl. IV, fig. $88, n^{\circ} 3$. 
casse, que chez la poule, les perdrix, les merles, les étourneaux, le pigeon, la tourterelle, les pies et les pics. Il devient si petit chez le moineau, le rossignol, le pinson, le serin, le chardonneret, la fauvette, le roitelet et l'hirondelle, qu'on a beaucoup de peine à le distinguer du lobe cérébral. Les perroquets et le casoar sont les oiseaux chez lesquels je l'ai trouvé le plus volumimineux.

Quelque peu développé que soit le lobe olfactif chez les oiseaux, on trouve à son centre un petit vide, qui est l'analogue du ventricule qu'il présente chez les reptiles el les mammifères : cette cavité devient très-sensible chez les perroquets et le casoar. Chez ce dernier, j'ai cru la voir communiquer avec le ventricule hémisphérique (1).

(1) On aurait une fausse idée du sens de l'olfaction chez les oiseaux, si on mesurait son étendue d'après le volume dı lohe olfactif; en effet, d'une part, les ramifications des nerfs olfactifs sont beaucoup plus nombreuses que ne pourraient le faire supposer les dimensions de ce lobe; de l'autre, la branche supérieure de la cinquième paire fournit à ce sens une grosse branche, qui se distribue à l'orifice externe des narines, contourne l'os éthmoïde, s'enfonce dans ses cavités el se prolonge ensuite sous la voûte palatine jusqu'à l'extrémité du bec : c'est peut-être de cette disposition que naít l'affinité qu'on remarque chez les ciseaux, entre le sens du gont et celui de l'odoral. C'est du moins ce que peuvent faire présumer les nouvelles recherches sur la cinquième paire, et les expériences que je m'vccupe de faire sur la section de c'e 
Le lobe olfactif ne présente donc plus chez les oiseaux les mêmes variations que chez les poissons et les reptiles; mais nous les retrouvons en partie chez les mammifères, avec des différences qu'il est nécessaire de faire remarquer. D'abord, chez la plupart, il est pédiculé, comme chez certains reptiles et certains poissons; mais son pédicule devient moins apparent, à cause de l'étendue du lobe cérébral qui le recouvre presque complètement. Ensuite, lorsqu'il cesse d'être pédiculé, il se confond avec le lobe antérieur même, comme cela a lieu chez la tortue franche et le caïman à museau de brochet. Chez les reptiles et les oiseaux, il diminue de volume en s'éloignant đu lobe cérébral; chez les mammifères, il semble augmenter au coniraire. Plus il est enfoncé sous les hémisphères . comme cher les singes (1) et le phoque (2), plus

nerf dans cette classe; car dans aucune autre, la cinquième paire ne prédomine davantage dans le sens de l'olfaction : on en peut juger par le nombre des plis, des anfractuosités et des sinus ethmoïdaux, dans lesquels se ramifie le gros rameau de la cinquième paire; sinus, anfractuosités et plis qui diffèrent beaucoup seinn les familles. Ainsi ce plissement de la membrane olfactive, très-multiplié et très-étendu chez les aigles, les milans, les vautours, le corbeau, le hibou, les canards, l'est infiniment moins chez la poule, le perroquet, le pigeon, la perilrix, le moineau, l'alouetle, elc. Le nombre des plis de la membrane olfactive ine parait être à l'odorat ce que le pliszonent de: In rétine est an sens de la vue.
(1) P!. VIII, fig. $197,11^{\circ} 1$.
(2) PI. IX, lig. 208, E. 
il est réduit dans ses dimensions; plus il s'en écarte, comme chez le lapin (1), l'agouti (2), la marmolte (3) et les chauve-souris (4), plus it devient considérable. Néanmoins, chez les mammifères de même qque chez les reptiles, son plus grand volume existe chez ceux où il se continue immédiatement avec le lobe cérébral, comme on l'observe chez la taupe (5), le zemni (6), le lérot (7) et le hérisson (8). Entre ces deux extrêmes de grandeur, le lobe olfactif croît grad uellement des singes aux phoques, aux carnassiers digitigrades, aux plantigrades, aux ruminans, aux rongeurs, aux chauve-souris et aux insectivores. Son veutricule suit la mème progression, et il s'ouvre, comme nous l'avons dit, dans le grand ventricule latéral. Ses radiations se continuent, comme nous l'avons vu aussi, soit avecla commissure antérieure. soit avec l'hippocampe, mais jamais directement avec le corps calleux. Ce lobe manque complètement chez les cétacés.

S'il est vrai que l'anatomic comparative ait pour' but de déterminer la similitude des organes chez

(1) Pl. II, fig. $56, \mathrm{n}^{\circ} \mathrm{\eta}$

(2) PI.'IX, fig. 213 et $211, \mathrm{E}$.

(3) Pl. IX, fig. 203 et $20 \%, \mathrm{E}$.

(4) Pl. IX, fig 204 et 206 , E.

(5) Pl. XIV, fig. 256 et 260 , E.

(6) Pl. XV, fig. 2\%o et $272, \mathrm{E}$.

(z) Pl. XV, fig. $2,6, \mathrm{E}$.

(8) Pl. XVI, fig. 296 et 297, E. 
les animaux, et les rapports qu'ils ont dans les di. verses classes, on nous pardonnera les détails dans lesquels nous avons été obligé d'entrer pour déterminer avec précision les élémens du système ncrveux dans le règne animal, et établir les rapports des diverses parties de l'axe cérébro-spinal des vertébrés, objet spécial de ce concours. Tant que l'on a considéré l'encéphale en masse, on a erré dans ses rapports, ou l'on a désespéré de les décou. vrir. Cet organe étant le plus compliqué de toute l'organisation, la marche actuelle des sciences indiquait qu'il fallait d'abord le décomposer, et en comparer ensuite les diverses pièces une à une: c'est cctte marche analytique que je me suis efforcé de suivre; c'est elle qui m’a entraîné dans les recherches nombreuses et difficiles auxquelles j'ai dû me livrer. C'est aux anatomistes à juger mes efforts et les résultats comparatifs et généraux qui vont terminer ce chapitre.

1. Les lobes cérébraux chez les mammifères sont composés de plusieurs lobules ou lobes secondaires.

2. Les lobules les plus importans sont le lobule sphénoïdal et le lobule de l'hippocampe.

3. Ces lobules sont développés en sens inverse l'un de l'autre.

4. Plus le lobule sphénoïdal se développe, plus le lobe antérieur grandit dans tous les sens, plus le lobe postéricur devient manifeste. La plus grande. longueur dr l'hémispherecébébral, sa plus grande 
hauteur coincide done avec le plus grand développement du lobule sphénoïdal.

5. Au contraire, avec le développement du lobule de lhippocampe, coüncide la diminution dans tous les sens de l'hémisphère.

6 . Le lobe olfactif suit le rapport direct du lobule de l'hippocampe et le rapport inverse du lobule sphénuïdal.

7. Te lobule sphénoïdal est développé en raison dirccte du corps calleux, et inverse de la voûte et de ses dépendances.'

8. B'ou il suit que le lobule de l'hippocampe doit suivre la raison directe de croissance de la voûte, et l'inverse de celle du corps calleux.

9. Le lobule sphénoïdal est développé en raison directe des hémisplières du cervelet, en raison inverse de son lobule médian et des tubercules quadrijumeaux; d'où il snit qu'il est développé aussi en raison directe de la protubérance annulaire, en raison inverse du trapèze, de la moelle épinière, du nerf de la cinquième paire, du nerf olfactif et du nerf de la vision.

10. Le corps calleux, suivant le rapport du lobule sphénoïdal. est donc aussi développé en raison inverse des nerfs des sens, de la moelle épinière, des tubercules quadrijumeaux, du lobe. médian du cervelet, du trapize, cten raison directe. des hémisphères du cervelet et de la protubérance annulaire.

11. Le lobule de thippocampe élant l'antago- 
histe du lobul: sphéncidal est donc déreloppé en raison inverse dr la protubérance annulaire et des hémisphères du cervelet, et en raison directe des ierfs des sens, de la moelle épiniere, des tubercules quadrijumeaux, du lobe médian du cervelet, et du trapéze de la moelle allongée.

12. Le lobe olfactif répétant le lobe de l'hippocampe, on voit donc qu'ii doit être développé en raison inverse de la protubérance annulaire et des hémisphères du cervelet, en raison directe de son lobule médian, du trapèze, des tubercules quadrijumeaux, des nerfs des sens et de la moclle ćpiuiere. Tous ces rapports se suivent, se lient, s'euchaîuent dans ía classe la plus parfaite par son organisation; et cette liaison, ces enchaînemens constitueut la science.

\section{R LSUT:}

UES rARACTIRES PROPRES A L'ENCÉPHALE DES QOATRE CLASSLS D'ANIMAUX JERTÉBRÉS.

De l'examen que nous venons de terminer, il risulte donc :

$1^{\circ}$. Que l'onciphale des poissons est le plus simple de celui des vertebrés, quoique fomé dre ses quiatre démens fomlamentaux, le cervelet. leg 
lobes uptiques, les lobes cérébraux et les lobes olfactifs.

$2^{\circ}$. Que les lobes optiques dominent toutes les autres parties, et que dans leur intérieur on trouve des tubercules striés, des radiations et des feuillets qui ne se trouvent dans aucune autre classe.

3。. Que lc lobule optique est porté dans cette classe au maxinum de sa grandeur.

$4^{\circ}$. Que chez les poissons seuls on trouve des tubercules dans le quatrième ventricule, ou en arrière du cervelet.

$5^{\circ}$. Que les lobes cérébraux sont deux masses solides, arrondies et entièrement isolées chez les poissons osseux, mais réunies au contraire en un plateau unique chez les cartilagineux.

$6^{\circ}$. Que la lamproie et l'esturgeon forment l'intermédiaire de ces deux états opposés des lobes cérébraux dans cette classe.

$7^{\circ}$. Que le lobe olfaclif toujours très-développé dans cette classe, tantôt adossé aux lobes cérébraux chez les poissons osseux, et tantôt tenu à distance, est constamment pédiculé chez les cartilagineux.

8. Enfin, quoique l'on ait avancé le contraire, que les poissons sont. dépourvus de corps strié, de couche optique sćparée, de voûte, de corne d'Ammon, de corps calleux et d'éminences mamillaires.

$9^{\circ}$. L'atrophie des lobes cérébraux caractérise 
ainsi tous les poissons; l'atrophie du cervelet est le caractère de fous les reptiles.

$10^{\circ}$. Ce que les lobes cérébraux ont perdu chez les poissons semble acquis par les lobes optiques; les hémisphères cérébraux gagnent en développement chez les reptiles, tout ce que perd le cervelet.

$11^{\circ}$. Les lobes optiques ont perdu les tubercules internes qu'ils avaient chez les poissons : les lobes olfactifs diminuent de volume, sont à distance ou en contact avec les lobes cérébraux; dans tous les cas, leur cavité communique directement dans le ventricule hémisphérique.

$12^{\circ}$. La couche optique et le corps strié existent séparés; et on aperçoit déjà chez eux les premiers rudimens de la lame cornée.

$1^{\circ}$. Les oiseaux sont plus avancés encore que les reptiles.

$14^{\circ}$. Ils se distinguent d'abord par l'atrophic du lobe olfactif, comme les reptiles par l'atrophie du cervelet, et les poissons par l'atrophie des lobes cérébraux.

15\%. Les lobes optiques tout-à-fait affaissés sur la face supérieure, font saillie sur la fice latérale, en arrière des lobes cérébraux; ils sont réunis par une commissure large et striéc qui ne se remarque dans aucune autre classe.

${ }_{16}^{\circ}$. Leur couche oplique est plus développée, le troisième ventricule plus évasé, les pédicules antrieurs do la glande pinéale continus avec 
la couche optique, le corps strié beaucoup plus saillant, et les radiations qui le sillonnent, plus mullipliées que chez les reptiles.

$17^{\circ}$. Une lame rayonnante striée, comme la commissure de leurs lobes optiques, tapisse la face interne de leurs lobes cérébraux, et présente cn arrière des contours qui simulent ceux de la corne d'Ammon; au milieu les rayons se réunissent à deux pédicules qui sont les analogues des piliers de la voûte.

18. Ces piliers ont cela de remarquable, qu'ils passent au-devant de la commissure antérieure chez tous les oiseaux, au licu d'être situés en arrière d'clle, comme chez tous les mammifères.

$19^{\circ}$. Lc cervclet beaucou! plus dévaloppe que daus les deux classes précédenies, présente au milicu de son lobe médian un ventricule tont-àfait particnlicr.

$20^{\circ}$. Ce ventricule occupe la place où doit se développer le corps frangé des mammifères: les oiseaux sont donc nécessairement privés de ce. corps.

$21^{\circ}$. Le corps calleux, la protubérauce annulaire et le corps trapézoïd: manquent completement dans cette classe.

$22^{\circ}$. Le corps trapézoüde. la protubérance annulaire be corps calleux sont donc les caracteres classiques des manímifères.

$25^{\circ}$. Ies lobes optiques, que l'on nommo: chez cux mbercules quadrijumeaur, som atrophich 
et solides comme les lobes cérébraux des poissons

$2^{\prime}{ }^{\circ}$. Les hémisphères du cervelet et les lobes cérébraux sont portés à leur maximum de grandeur, ainsi que le corps strié et la couche optique:

$25^{\circ}$. Le corps frangé caractérise leur cervelet, comme le ventricule du lobe médian celui des oiseaux; ce corps et ce ventricule ne se trouvent que dans chacune de ces classes.

$26^{\circ}$. Le demi-centre ovale des lobes cérébraux n'existe que chez les mammifères; leur grand ventricule est divisé par une cloison qui sert en quelque sorte de ligament à la voûte et au corps calleux.

$27^{\circ}$. Tantôt ce ventricule se prolonge en avant jusque dans la cavité du lobe olfactif, comnie chez les reptiles, et alors il est moins étendu en arrière ; sa cornc postérieure manque.

$28^{\circ}$. Tantôt, au contraire, prolongé par cette corne: en arrière, il perd en avant la communication précédente.

$29^{\circ}$. Le pelit pied d'hippocampe qui se développe dans l'étui de cette corne n'est pas particulicr à l'hounme. comme nous l'avious tous cru juscru'a ce jour. 11 existe cher les singes ot be phocpue.

$30^{\circ}$. Enfin, l'homme seul possede k houlc tonsillaire du cervelet, les cordons medultaires th quatrieme ventricule, et des imineners m. 
millaires saillantes et distinctes sur la base de l'encéphale.

$31^{\circ}$. Ainsi chaque classe de rertébrés est caractérisée par l'atrophie de l'un des élémens fondamentaux de l'encéphale, les reptiles par l'atrophie du cervelet, les mammifères par celle des lobes optiques, les poissons par celle des lobes cérébraux et les oiseaux par celle des lobes olfactifs.

$32^{\circ}$. Chaque classe a un ou plusieurs de ces élémens, qui dominent les autres et semblent les affaisser par ce développement.

$33^{\circ}$. Ainsi les lobes optiques des poissons osseux surgissent sur la face supérieure de leur encéphale, arrêtent le cervelet en arrière et les lobes cérébraux en avant.

$34^{\circ}$. Chez les reptiles les lobes cérébraux dominent les autres lobes; les lobes optiques restés à leur place semblent anéantir le cervelet.

$35^{\circ}$. Chez les oiseaux, l'affaissement médian des lobes optiques fait surgir les lobes cérébraux ct le cervelet. Ces deux organes maintenus à distance l'un de l'autre chez les reptiles et les poissons, sont par ce seul effet ramenés au point de contact.

$36^{\circ}$. Ce rapprochement est facilití encore chez les mammifères par l'atrophie des lobes optiques. .$\overline{3} 7^{\circ}$. Le cervelet ne déborde jamais en avant les lobes optiques. il s'arrête à leur niveau. $58^{\circ}$. Il n'en est pas de mème des lobes cérébraux; 
en suivant leur marche d'avant en arrière, ils couvrent d'abord les lobes optiques, puis ils empiètent sur le cervelet, le cachent en partie, puis enfin dans sa totalité.

$59^{\circ}$. Il en esi du lobe olfactif en avant, comme du cervelet en arrière; il s'enfonce et se cache sous le lobe antérieur, comme le cervelet sous le lobe postéricur.

$40^{\circ}$. Or on conçoit que cet effet ne peut avoir lieu sans que les lobes cérébraux augmentent de hautcur en même temps qu'ils se prolongent en arrière et en avant.

$41^{\circ}$. On voit ainsi comment et pourquoi tous les lobes de l'encéplale sont à au sur sa face supérieure chez les poissons ct les reptiles, pourquoi jamais ils ne se recouvrent inutuellement;

$42^{\circ}$. Pourquoi au contraire la partie moyenne des lobes optiques et le lobe olfactif sont cachés chez les oiseaux;

$43^{\circ}$. Comment et pourquoi enfin chez les mammifères supérieurs, lo lobe cérébral est le seul que l'on distingue au haut de l'encéphale.

1 f $^{\circ}$. Tels sont les caracteres de cet organe dans les quatre classes d'animaux vertébrés, et ces caraclieres sout arrêtés par chacune d'clles.

$45^{\circ}$. Ja nature rétrograde dans ses développemens; elle n'avance jamais.

$46^{\circ}$. De lá l'espéce de barrière posée sur les liunites de charque classe, barriere que la nature ue depasso jamais ni dars l'élat normal, ni dans ses 
586 HWMISPHLRES CEREBRAX

aberrations, quelque étonnante fécondité que souvent elle nous offre dans ces dernières produetions.

$47^{\circ}$. Si cette barrière, dont rien ue pent uous dévoiler la raison dans l'élat présent de l'organisation, n'existait pas, la nature aurait pu opérer la transilion d'une classe à l'autre; elle aurait pu, par de légères modifications, fairc de l'encéphale d'un poisson osseux celui d'un carlilagineux, de celui-ci un reptile, d'un reptile un oiseau, d'un oiseau un mammilère.

$48^{\circ}$. Ce que la nature n'a pas fait, ce qu'il semble même qu'elle ne pourrait faire sans s'écarter de la loi de subordination des caractères qu'clle a empreinte sur ses œuvres, l'art peut le tenter pour un ou plusieurs systèmes organiques.

$49^{\circ}$. C'est ce que j'avais fait dans mon grand ouvrage pour l'encéphale des vertébrés, dans la partie intitulée : Unité de composition abstractive de cet organe.

$50^{\circ}$. Par l'étude de l'embryogénie, j'avais ramené par l'analyse, l'encéphale de toutes les classes à un seul ct même type; par cetle espèce de synthèse artificielle je remontais l'śchclle que javais descendue : je ramenais l'encéphale d'un poisson à celui d'un mammiére, à l'aide des dessins que j'avais lait composer dans ce but (1);

(1) Je croyais pouvoir, daus cet ourrage, indifuer :"l unoins brievement celle parlie le non lrarail; jarais inseré 
je montrais à combien peu cle chose ticment les différences de famille à famille, d'ordre à ordre, de classe à classe, et je transformais un poisson en mammifère, sans irop choquer les rapports naturels existant dans chaque classe. Je donnais de cette manière la contre-épreuve de toutes les explications que j'ai déduites dans cet ouvrage de l'organisation normale de l'encéphale dans les quatre classes des vertébrés.

Ici se termine la tâche que je me suis imposée en répondant au concours ouvert par l'Académie royale des sciences: j'ai présenté des faits, j’ai montré leurs rapports, j'en ai déduit des conséquences; c'est à cette compagnie illustre à juger si les conséquences sont bien déduites, si les rapports sont bien exacts, si les faits sont bien observés.

dans l'atlas quelquez figures modifićes dins cet intention. $C_{0}$ : sunt les figures $18,66,85,121,149,1,8,252,243,24 \%$ 204 et 259 . 


\section{TABLUAU COMPARATIF}

Des Dimensions des Lobes cérébraux che: les Mammifères.

\begin{tabular}{|c|c|c|c|}
\hline \multirow[b]{3}{*}{ Homme. . . . . . . . } & DES LO & $\begin{array}{l}\text { MESURES } \\
\text { OBES CÉBB́BE }\end{array}$ & A Aux. \\
\hline & $\begin{array}{c}\text { diamitre } \\
\text { antera-post. }\end{array}$ & $\begin{array}{l}\text { dianuetre } \\
\text { transverse. }\end{array}$ & $\begin{array}{l}\text { diamètre } \\
\text { vertical. }\end{array}$ \\
\hline & $\begin{array}{c}\text { mètre. } \\
0,17000\end{array}$ & $\begin{array}{c}\text { mètre. } \\
0,07500\end{array}$ & $\begin{array}{c}\text { mitre. } \\
0,09000\end{array}$ \\
\hline NOMS DES ANIMAUX. & & & \\
\hline Patas (Simia rubra)...... & 0,07700 & 0,03300 & 0,05600 \\
\hline Magot (S. sylvanus)...... & 0,07200 & 0,02950 & 0,04300 \\
\hline Macaque (S. cynocephalus).. . & $0,0,000$ & 0,02825 & 0,04500 \\
\hline Papion (S. sphynx) . . . . & 0,08200 & 0,03400 & $0,05,00$ \\
\hline Mandrill (S. maimon)..... & 0,08100 & 0,05200 & 0,04900 \\
\hline 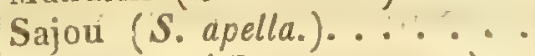 & 0,05900 & - & 0,04300 \\
\hline Maki Vari (Lemur macaco). . & 0,04500 & 0,02125 & 0,02900 \\
\hline Ours brun (Ursus arctos). . & 0,09500 & 0,04300 & 0,06100 \\
\hline 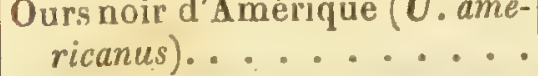 & 0,08300 & 0,03650 & 0,04800 \\
\hline 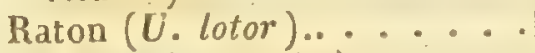 & 0,05000 & 0,02150 & 0,02900 \\
\hline Blaireau (U. meles)... .... & 0,05400 & 0,02400 & 0,03200 \\
\hline Coati brun (Viverra narica). . & 0,04900 & 0,01850 & 0,03150 \\
\hline Fouine (Mustela foina).... & 0,03900 & 0,01700 & 0,02400 \\
\hline Loutre (M. lutra) ..... & 0,05200 & 0,02400 & 0,03400 \\
\hline Chien (Canis familiaris). . & 0,06000 & 0,02950 & 0,04400 \\
\hline Loup, jeune (C. lupus).... & 0,05600 & 0,02550 & 0,03200 \\
\hline Renard (C. vulpes)..... & 0,05600 & 0,01750 & 0,02800 \\
\hline Hyène (C. hyana)..... & 0,06600 & $0,029^{50}$ & 0,04100 \\
\hline Lion (Felis leo)....... & 0,09100 & 0,04050 & 0,04800 \\
\hline Tigre (F.tigris)...... & 0,09400 & 0,04250 & 0,06400 \\
\hline Jaguar $(F$ onca. $) \ldots \ldots$ & 0,08100 & 0,03250 & 0,04800 \\
\hline Panthère ( $\boldsymbol{F}$. pardus)..... & 0,07800 & 0,05400 & 0,05000 \\
\hline Couguar ( $\boldsymbol{F}$. discolor)..... & 0,07100 & 0,02950 & 0,04200 \\
\hline $\operatorname{Lynx}(\boldsymbol{F} \cdot \operatorname{lyn} x) \cdots$ & 0,06100 & $0,02-50$ & 0,04200 \\
\hline 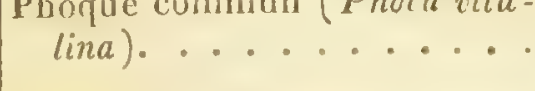 & $n, 10100$ & 0,04900 & 0,04400 \\
\hline
\end{tabular}


F.' LOBE OI.T.MTIF.

Suile du Tableau comparatif des Dimensions des Lobes cérébraux chez les Mammifères.

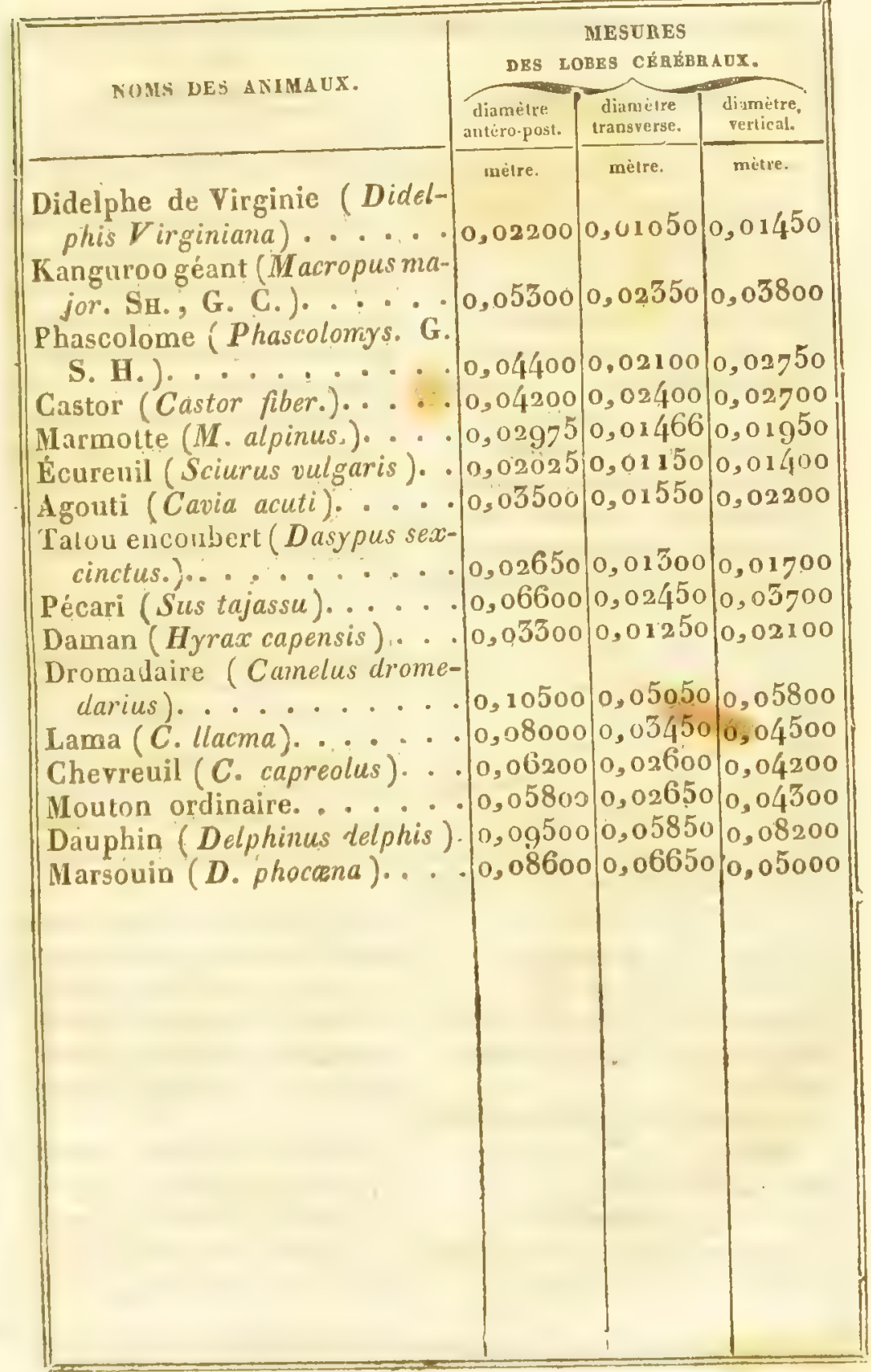


Des Dimensions des Lobes cérébraux chez les Oiseanx:

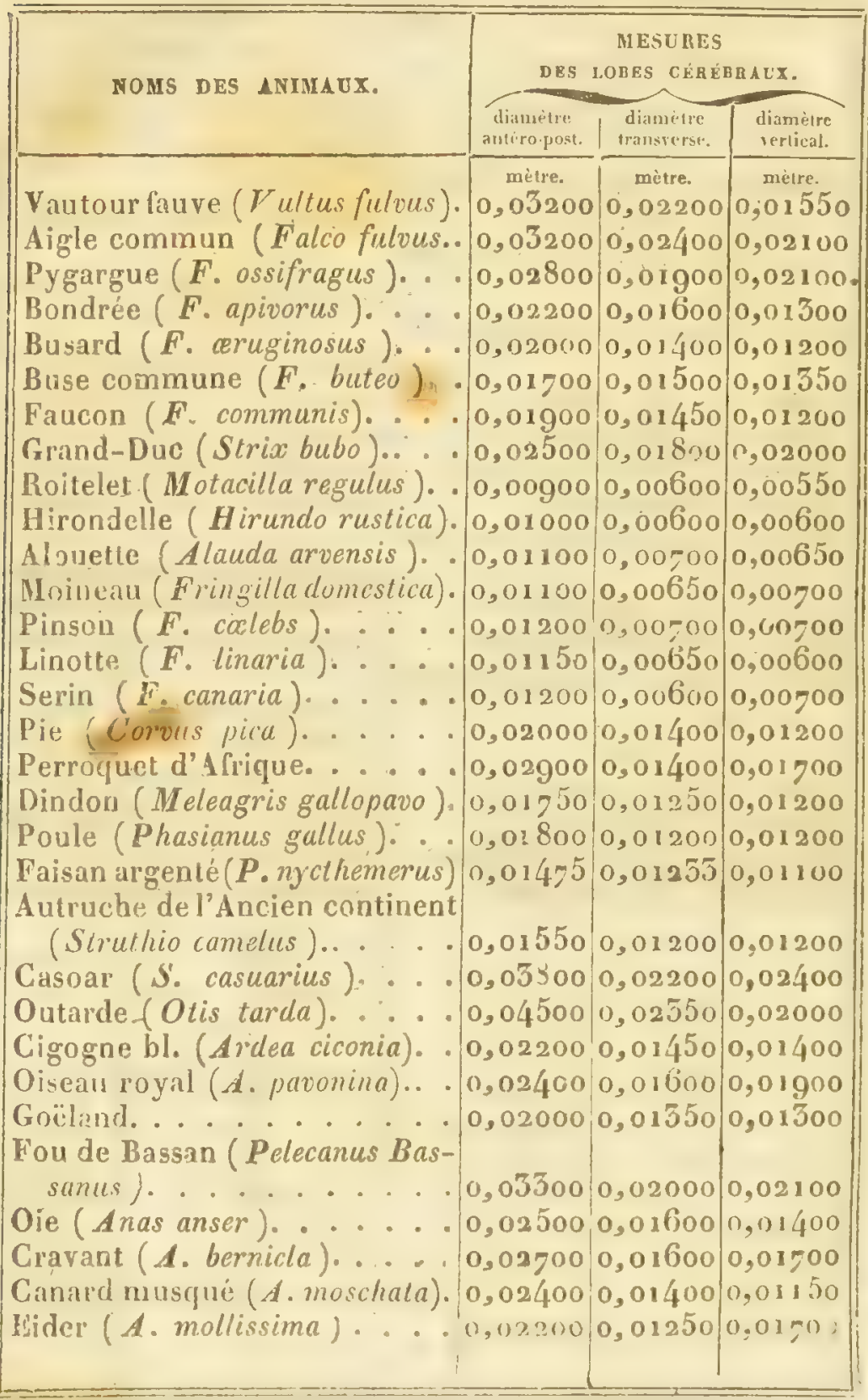


Des Dimensions des Lobes cénébraux chez les Ropliles.

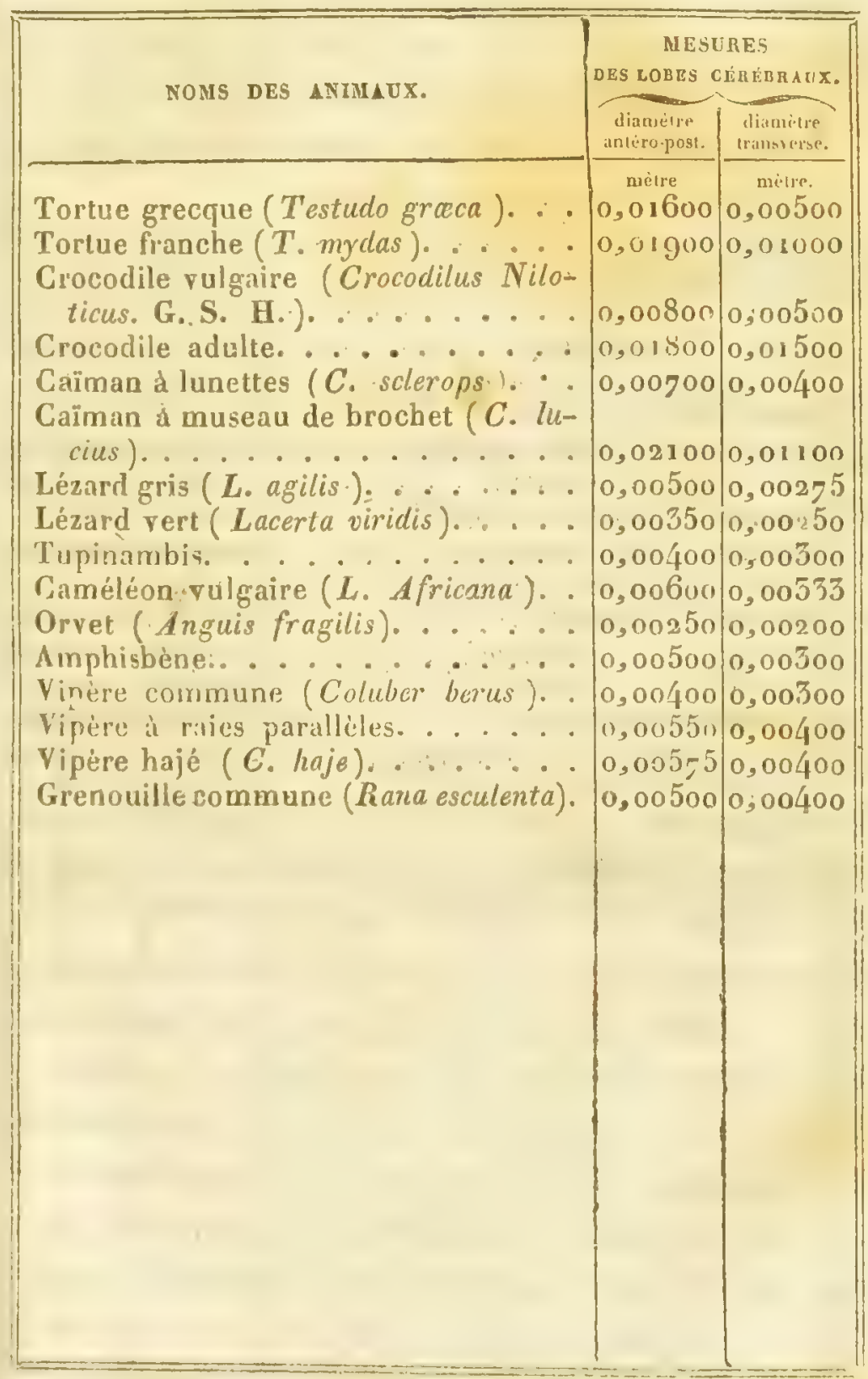


TABLEAU COMPA RATE

Des Dimensions des Lobes cérébraux chez les Poissons.

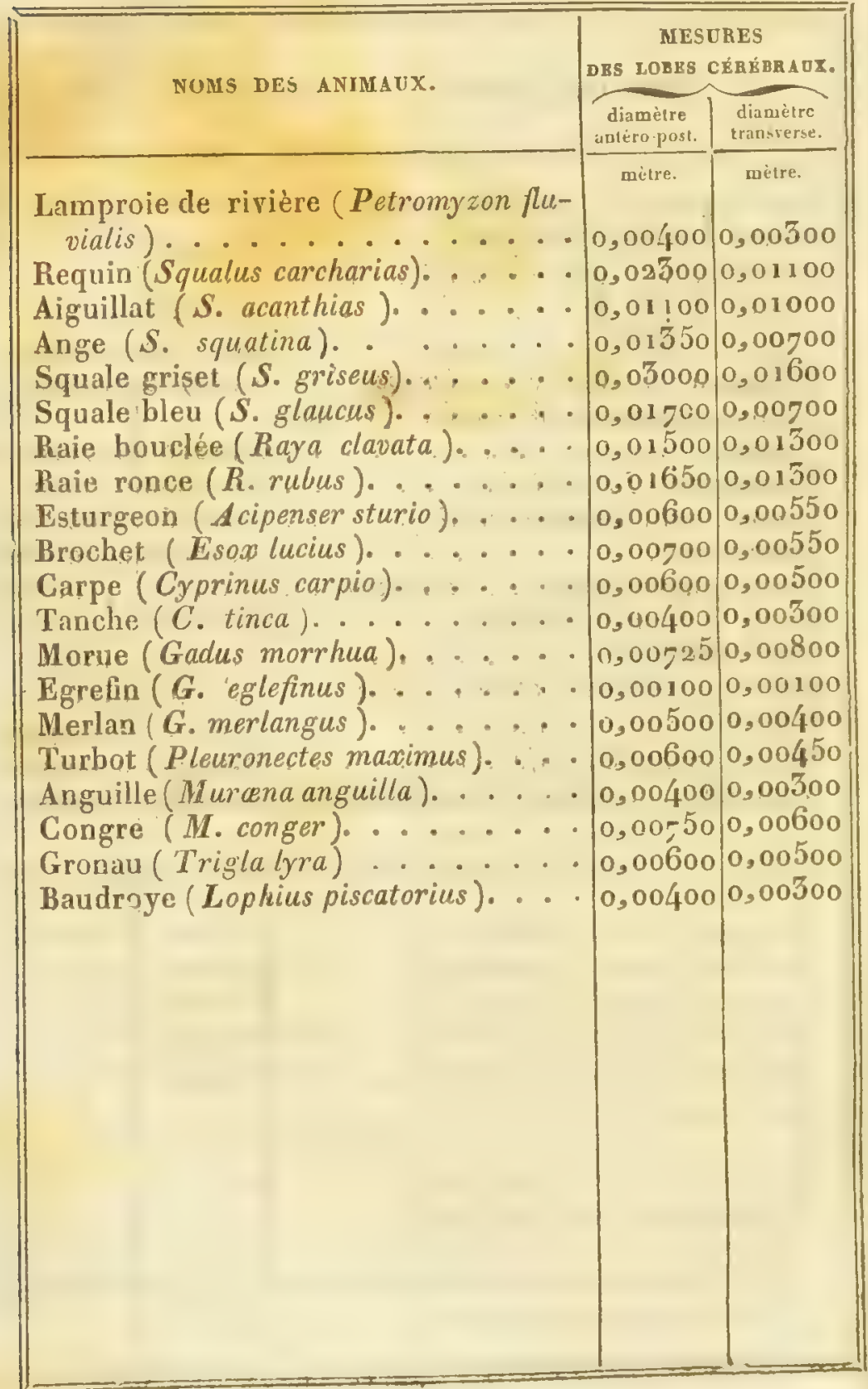




\section{CHAPITRE VIII.}

Corollaires physiologiques et pathologiques sur le cervelet, les tubercules quadrijumeaux et les he'misphères cérébraux.

Les fonctions et les maladies du système nerveux sont à la tête de la physiologie et de la pathologie. De là, trente siècles de recherches non interrompues pour en éclairer les routes difficiles; de là, des hypothèses sans nombre pour en découvrir l'essence. Parmi ces dernières, celle dont l'influence a été la plus pernicieuse, et dont nos explications et notre langage portent encore l'empreinte, est celle des esprits animaux. Imaginés d'abord pour aider à comprendre ce qui dépasse la portée de notre esprit, l'union de l'âme et du corps, on ne tarda pas à les admettre an nombre des principes constitutifs de l'organisation. Une fois admis, il fallut leur trouver une origine ou une source, pour reproduire l'expression dont on se servait au seizième siècle.

Galien les puisa dans l'air, et les fit arriver dans l'enećphale par l'ethmoïde et le poumon, pour les concentrer dans le quatrième ventricule. Riolan, surnommé l'intrépide, à cause de ses atlaques vigoureuses contre ceux qui n'adoptaicnt pas les vues de: Galien, leur assigna pour origine II. 
l'arachnoide; il les fit sécréter par cette membranc, et décrivil leur marche jusqu'au quatrième ventricule, avec la précision usitée en anatomie pour les organes les plus matériels. Wepfer, qui avait déjà réfuté l'opinion de Galien sur la prétendue noblesse du quatrième ventricule, combattit aussi l'opinion de Riolan, son dernier défenseur; et après avoir établi que l'arachnoïde ne peut ètre l'organe sćcréteur des esprits animaux, il doua de cette propriété la matière blanche du ccrveau, et plus spécialement le demi-centre ovale des hémisphères sur lesquels Vieussens établit presque toute sa doctrine physiologique du système nerveux.

Avant Vieussens, et à-peu-près à la même époque que Wepfer, Willis avait jeté les fondemens de cette belle doctrine de la spécialité d'actions des diverses parties de l'axe cẻrébro-spinal du système nerveux, dont le développement sera l'un des plus beaux titres de gloire de notre époque. Entraîné par l'exemple, il admit les esprits animaux, et leur donna pour organe la matière grise de l'encéphale, et plus spécialement la substance corticale; opinion que servit admirablement Malpighi en supposant cette substance un composć de glandes. L'hypothèse de Willis se trouvait par là mieux établie encore que celle de Riolan.

Mais par un cflort d'intelligence qui ne saurait trop être rcmarqué pour cette époque, toul en admethant lhomogenéité des csprits animaux, 
et de la matière du système nerveux, IVillis s'éleva à la pensée sublime de la diversité d'action de la moelle épinière, du cervelet et du cerveau; et aussitòt, par une extension prématuréc, il circonscrivit les diverses fonctions. Il créa un organe pour les mouvemens volontaires, et un autre pour ceux sur lesquels la volonté n'a point d'action. Une autre partie lui parut être l'organe de la conservation individuelle; celle-ci, l'organe de la propagation de l'espèce : la mémoire, l'imagination, la parole, furent aussi localisées. Enfin, l'organe de la musique couronna cet arbre des facultés instinctives et intellectuelles. Mais jc m'aperçois que je suis conduit à la doctrine psychologique de MM. Gall et Spurzheim, et l'espace mo manque pour en juger les bases, établir ses rapports et ses différences avec celle de Willis, et montrer par quel abus de l'analogie, de l'analyse et du raisonnement, ces hommes d'un rare mérite ont rendu invraisemblables les idées fondamentales de la spécialité d'actions du système nerveux (1).

Quoi qu'il en soit, avant d'entrer dans l'examen

(1) Quoique M. le docteur Juardan me paraisse opposé a celte spécialité d'actions, j'invite les hommes qui s'occupent de cette maticre à lire le Discours préliminaire que ce savant a placé en tête de sa traduction de l'ouvrage de Tiedemann. M. le docteur Jourdan a rendu et rend tous les jours a la science les plus grands services, en nous faisant connaître Ies dnetrines dea sivins étrangers; mais quand on a lu ce dis- 
de l'action du cervelet, des tubercules quadrijumeaux et des hémisphères cérébraux, je crois utile de présenter quelques considérations sur les rapports de développement de ces organes avec quelques autres parties. Nous avons déjà dit le rapport que présentaient, chez les mam mifères, le dévcloppement du cervelet et celui des membres supérieurs; nous avons vu que ces organes étaient de la seconde formation, parce que leurs artères apparaissaient en second licu dans l'embryogénie: il en est de même des membres inférieurs et des organes génitaux externes. L'artère hypogastrique et l'iliaque externe sont de seconde formation, comme l'axillaire et la vertébrale; la cuisse et les organes génitaux externes paraissent à la même époque que le cervelet et le membre antérieur : de même que le cervelet, les organes génito-urinaires sont rigoureusement soum is aux lois de formation que nous avons établies; ils se développent de la circonférence au centre; ils sont d'abord doubles; leurs canaux ne sont que des canaux de conjugaison. La matrice et la prostate sont primitivement des organes doubles, même chez l'espèce humaine : de la réunion des deux parties qui les constituent primitivement, résultent ces organes tels que nous les offre l'ana-

cours remarquable, on ne peut que regretter qu'un homme qui est si prodigue des idées des autres, sc montre si avare dans la publication des siennes propres. 
tomic normale, ot tels aussi que nous les présente quelquefois l'anatomic anormale (1).

L.e cervelet formé, nous avons vu avec quelle rapidité se développe le lobe médian; on voit aussi à la mêne époque les organes génitaux externes se dessiner avec la même promptitude. Les nucubres sont loin d'offrir la mềme rapidité dans leurs évolutions: ce n'est que lorque les hémisphères du cervalet deviennent le centre d'activité de cet organe, que les mombrēs prennent un grand accroissement. On serait tenté de conclure, d'aprés ces faits, qu'il y a un rapport physiologique constant cntre le lobe médian du cervelet et les organes génitaux, les membres supérieurs et inférieurs et les hémisphères des mêmes organes. Mais, d'une part, le cervelet termine ses développemens arant la naissance, et de l'autre, les organes génitaux sont très-tardifs à entrer en action; les membres cux-mêmes ne peuvent soutenir et porter l'animal qu'à une époque plus ou moins reculéc après la naissance. Enfin, on trouve dans les hémisphères cérébraux une autre source de rapports qui tendent à restrcindre ces corrélations anatomiques. De plus, chez les oiseaux, le lobe médian du cervelet le constitue presque en entier, et les organes génitaux ne font plus saillie à l'ex-

(1) Voyez l'application de ce point d'anatomie transcendante a la pathologie externe, dans l'ouvrage de notre célebre chirurgien M. Lisfranc. (Des Rútricissemens de l'urètre. Paris, 182年.) 
térieur; les hémisphères du cervelet sont trèsfaibles, quoique chez certaines espèces, les ailes et les pieds acquièrent une force prodigieuse: nous devons remarquer aussi que chez beaucoup de reptiles, l'exiguité du cervelet n'est plus en rapport avec le développement considérable des membres; tandis que chez les poissons, au contraire, l'exiguité des nageoires contraste avec le développement du cervelet. Comment a-t-on pu supposer, d'après ces variations continuelles, que la même partie du système nerveux conservait dans toutes les classes la même spécialité d'actions? Cela n'est pas, et l'anatomie seule prouve que cela ne pouvait être.

Indépendamment des rapports que les tubercules quadrijumeaux ont avec le volume du globe de l'oeil, il en est un autre, moins prononcé peutêtre, mais qui n'en mérite pas moins notre attention : c'est celui du volume de la queue.

Chez l'embryon humain, la queue persistc pendant tout le temps que les tubercules quadrijumeaux sont dominans dans l'encéphale; elle disparaît lorsque les tubercules s'affaissent. Chez les mammifères il existe un rapport général entre ces deux partics: les cétacées ont des tubercules quadrijumeaux enormes, et leur train postérieur n'est en quelque sorlc qu'une énorme queue. Chez les oisean, ce rapport ne se conserve pas; quant aux tétards des batracicns. Jeur lengue quene conaride ave le relume di 
Icurs lobes optiques, et il est à remarquer qu'à cette époque cet organe est le principal agent de leur locomotion; ils sont, sous ce rapport, comme les poissons. Or, dans cette classe, la queue est portée au maximum de son développement, et ce développement coincide avec la prédominance des. lobes optiques sur toutes les autres parties de l'encéphale (1) J'ai cru devoir indiquer ces données avant cae présenter les corollaires physiologiques et pathologiques qui concernent ces organes.

Les médecins grecs et arabes avaient placé le siége de la mémoire dans le cervelet: le nombre de feuillets dont il est composé leur semblait correspondre aux nombreux compartimens que paraît supposer l'exercice de cette faculté; plus tard, cette disposition l'a fait regarder par Malacarne, comme l'organe de l'intelligence; et de nos jours encore, le célèbre professeur Rolando a puisé dans cette disposition l'idée que le cervelet est une pile yoltaïque en action.

Hoffmann avait porté une attention sérieuse sur la dépendance et l'indépendance des mouvemens de la volonté; Willis assigna des siéges dif-

(1) Ce rapport est d'enscmble. On ne s'attend pas que j'énumere ici les nombreuses exceptions qu'il présente, exceptious auxquelles s'attachent toujours les hommes qui ont trop pen ril poun comprende les priacipes génciaux des sciences. 
férens à ces deux ordres de mouvement; il regarda les hémisphères cérébraux comme les organes $d u$ mouvement volontaire, et le cervelet comme celui des mouvemens involontaires. La circulation, la digestion, la respiration, se trouvaient dès-lors sous sa dépendance immédiate. Il est à remarquer que dans le cervelet, Willis comprenait la protubérance et la portion de la moelle allongée qu'elle embrasse ; circonstance à laquelle n'ont point èu égard Perrault, Vieussens, Riedley, Chirac, Senac, Kau-Boerrhave, Haller, Zimmermann, Zinn et Lorry, dans les expériences négatives qu'ils ont opposées à Willis.

Les anciens avaient divisé les nerfs en ceux du sentiment et ceux du mouvement; ils avaient placé l'origine des premiers dans les membranes du cerveaù, et celle des seconds dans cet organe même. Pourfour-Petit est le premier qui ait considéré le cervelet comme l'organe de la sensibilité. Saucerotte, Sabouraut et Choppart sanctionnèrent, dans un concours mémorable, l'assertion de Pourfour-Petit, qui fit croire, pendant qu'elle fut admise, à l'insensibilité complète des hémisphères cérébraux; enfin, de nos jours, le retour de cette opinion porte encore à dépouiller ces hémisphères de cette mystérieuse propriété.

En outre de ces facultés dont on a tour-à-tour dépouillé et gratifié le cervelet, Walstorf le considérait comme l'organe actif pendant le sommeil ; mais il est évident que dans ses expériences, il 
agissait médiatement sur la moelle allongée, puisque l'ablation complète du cervelet ne dévoloppait pas les mêmes effets que la compression.

Le cervelet a donc tour-à-tour été considéré comme l'organe de la mémoire et de l'intelligence, comme le siége et le régulateur des mouvemens de conservation; Willis en a fait l'organe de la musique; M. Gall, celui de la propagation; M. Rolando le considère comme générateur des mouvemens volontaires; M. Flourens, comme leur servant de balancier; enfin, Pourfour-Petit et quelques médecins placent dans le cervelet le siége de la sensibilité. Pour juger ce qui est, nous allons recourir, selon notre méthode, aux faits pathologiques et aux expériences sur les animaux.

De l'action du cervelet sur les organes génitaux.

I a nature a confié à chaque espèce le soin d'èterniser sa création. Après le désir de la conservation, il n'en est pas de plus impérieux que celui de la propagation. Willis est encore le premier qui ait cherché à localiser cette faculté : la moelle épinière est, selon lui, l'organe de la propagation. Tout le monde connaît à ce sujet les idées de M. Gall; mais elles avaient médiocrement frappé les physiologistes avant la publication des faits pathologiques que je vais reproduire en 
abrégé, et des expériences qui ont confirmé ceđ faits.

Un homme de trente deux ans fut frappé d'apoplexie dans l'acte du coït, et après avoir bu plus que de coutume. Aux symptômes ordinaires des apoplexies violentes, se joignait l'érection du pénis, qui persista jusqu'aux approches de la mort Le cerveau était sain; mais le lobe médian du cervelet était le siége d'une vive irritation; la substance cérébelleuse était brisée en plusieurs endroits; de petits foyers sanguins étaient creusés le long du processus vermiculaire supérieur.

Un journalier de cinquante-cinq ans, d'un tempérament sanguin, très-adonné aux plaisirs vénériens, après une journée passée au cabaret, fut pris, dans la nuit, d'une attaque d'apoplexie dont l'érection du pénis formait, comme daus l'observation précédente, le symptôme insolite. Il mourut deux jours après, ayant offert à chaque paroxysme la tension de la verge, et dans le dernier, une abondante éjaculation. Comme dans le cas précédent, irritation vive du lobe médian du cervelet avec érosion de sa substance. Foyer sanguin dans le centre de son hémisphère droit , qui s'ćtait fait jour dans le quatrième ventricule.

Un homme de quarante-six ans mourut à la suite d'une apoplexie violente, pendant laquelle le satyriasis et l'éjaculation sc manifestèrent, suivis d'un gonflement de toutes les partics ex- 
ternes de la génération. Le lobe médian du cervelet renfermait plusieurs petits foyers sanguins, l'irritation vive qui les environnait se propageait à droite et à gauche vers les hémisphères.

Un cadavre apporté de l'hospice de Bicêtre à l'amphithéâtre central des hôpitaux, présentait une tuméfaction considérable de la verge et des bourses; le cervelet était phlogosé dans toute son étendue.

Un écrivain public, de trente-deux ans, avait offert pendant le cours d'une apoplexie, la turgescence du pénis, et, dans certains momens, une érection complète. Dẹ petits foyers sanguins étaient situés le long du lobe médian du cervelet; un autre plus considérable occupait la partic postérieure de l'hémiphère droit.

L'observation suivante, recueillie à ma clinique, m'a été communiquée par M. le docteur Falret, connu dans la science par ses belles recherches sur le suicide et l'aliénation mentale.

- Je vicns de lire avec le plus grand intérêt votre promier mémoire sur les apoplexies céré- belleuses, inséré dans le journal de M. Magendic. - J'ai beaucoup regretté de ne pas y trouver l'ob- servation d'un apoplectique que j'ai eu occasion "d'observer dans votre division à l'hûpital dela Pilié.

"Lin 1820 , dans un arlicle sur l'Annuaire mé* dico-chirurgical des hôpitaux, qui, par des cir- constances indépendantes de ma volonté, n'a

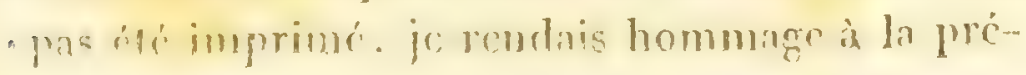


"cision de volre diagnostic dans les maladics or"ganiques de l'encépliale. Je rapportais à cett" * occasion l'entretien que nous eûmes dans une des - salles de votre division au sujet d'un apoplecti" que âgé de cinquante ans, entré de la veillè dans - l'hôpilal.

"Pourriez-vous déterminer, me dîtes-vous, le » siége positif que doit occuperl'épanchement chez - ce malade? Non, répondis-je; et il y aurait, ce - me semble, de la témérité à prononcer d'une » manière affirmative..Eh bien! reprîtes-vous, je "suis ce médecin téméraire. Je pense que l'épan" chement est dans le cervelet. J'en ai pour garant - l'érection du pénis, symptôme qui en effet avait \ beaucoup fixé votre attention et la mienne. Cette " preuve ne me parut pas d'abord sans réplique; - mais, comme vous annoncâtes en même temps "que le malade n'avait pas plus de trente-six heu* res à vivre malgré tous les secours de la méde* cine, j'attendais l'examen du cadavre avec im'patience, et, à mon grand étonnement, je vis * votre diagnostic parfaitement justifié.

- Je ne rapporterai pas ici tous les symptômes - apoplectiques que présentait ce malade; ils sétaient les mêmes que ceux des apoplexies céré- brales, mais de plus le pénis était gonflé, tendu, n rouge, en un mot dans l'état d'érection. Voici s en abrégé ce que nous trouvâmes à l'ouverturc - du cadavre.

"Développement considérable des parties géni- 
- tales; taches brunes sur le gland et sur les bour- ses; nembres rigides et dans l'extension. Les • tégumens du crâne n'offraient rien de remar- quable. Les sinus étaient gorgés de sang; trois - onces environ de ce liquide s'écoulèrent à * l'ouverture de la têle. Les vaisseaux de la pie^ mère étaient gonflés; la substance grise du cer- veau était rougeâtre, injectée; mais on ne - reconnut dans cet organe aucune trace d'épan* chement ancien ou récent. Il n'en fut pas de - même pour le cervelet; indépendamment de - l'injection de cet organe, nous remarquâmes - dans la partie centrale du processus vermiculaire - supérieur, un large foyer s'étendant à droite et à - gauche vers les hémisphères du cervelet; un - caillot sanguin le remplissait. Les processus cere- belli ad testes étaient très-rouges; le quatrième - ventricule contenait du sang. Les poumons * étaient gorgés de sang; les organes de l'abdomen -n'offraient rien de remarquable.

Une fille livrée de bonne heure aux plaisirs vénériens, sc prostitue pour assouvir ses désirs, et se livre à toutes les manœurres de la masturbation, pour suppléer à l'insuffisance des cohabitations journalières des hommes. Elle tombe dans la nym phomanie; honteuse elle-même de son état, elle supporte que l'on brûle le clitoris, ce qui n'a aucun résultat avantageux. Elle meurt, et on trouve une irritation chronique arce induration du lobe médian du cervelet : de petits foyers 
à bords calleux, comme le reste de l'induration, indiquent une phlegmasie ancienne de cette partie.

Un de nos élèves, M. le docteur Romet, observe à l'hôpital saint-Antoine une femme de soixantedix ans apoplectique; il remarque que l'écoulement des règles, qui avait cessé depuis longues années, se reproduit pendant le cours de la maladie; il soupçonne une apoplexie cérébelleuse: la femme meurt, et l'ouverture du cadavre offre d'une part un vaste foyer sanguin, ayant brisé tout le lobe médian du cervelet, et de l'autre la matrice remplie par un caillot sanguin; tout le tissu de cet organe était phlogosé, les ligamens larges, les trompes et les ovaircs étaient colorés comme si on les cût plongés dans un liquide rouge (Revue médicale, année 1824.$)$.

Elisabeth Blanchet, âgée de dix ans, d'un caractère sombre, adonnée à la masturbation, se plaignait depuis quatre mois de la tête; depuis trois semaines ces douleurs lui arrachaient des cris; alitée depuis douze jours quand on l'amena à l'hôpital des Enfans, elle avait eu des vomissemens bilieux suivis de somnolence; depuis trois jours elle cessait de parler, ou ne répondait que trìsdifficilement, en portant la main à sa tête qui était renversée en arrière; ses derniers jours furent marqués par un état comateux, un peu de strabisme convergent, la dilatation des pupilles. L'autopsie cadavérique offrit une inflammation avec infiltra- 
tion purulcnte de l'arachnoïdc à la parlic supérieure du cervelet, dans lequel existaient sept à huit tubercules autour desquels la substance de cet organe était ramollie et blanche; les parties moyennes étaient aussi ramollies; les ventricules contenaient trois onces de sérosité. Cette altération remarquable ne peut être considérée que comme une véritable inflammation de la substance cérébelleuse (Essais sur l'encéphalite, par M. le docteur Payen, pag. 25, 1826.)

Ces faits pathologiques offrent donc de commun l'excitation des organes génitaux, et une coïncidence remarquable de l'irritation du cervelet, et plus particulièrement du lobe médian de cet organe. Ce qui établit, selon nous, que l'aclion des organes génitaux est dans une corrélation très-ćtroite avec l'action du cervelet, ainsi que 1. Gall l'avait avancé le premier. Ces résultats constatés chez l'homme par la pathologie, il restait à voir si la physiologie expérimentale les confirmerait. (1)

(1) Depuis la publication de ces faits dans le journal de A. Nlagendie, j'ai eu occasion de voir deux apoplectiques, chez lesquels l'érection se manifestait pendant les paroxysmes. 'lous deux sont guéris à la suite de l'application de sangsues it la nuque, et de cataplasmes émolliens sur cette partie. M. le docteur Gensoult, chirurgien de l'Hôtel-Dicu de Lyon, a obtenu un résultat plus remarquable encore. Un homme d'environ trente ana arail trois ou quatre évacuations the sperme chaque nuil, evacuations pour lesquelles M. lo 
Sur les boufs abattus en portant des coups dis marteau sur la partie postérieure de l'occipital, j’ai rencontré le cervelet déchiré dans sa partie supérieure, chez ceux chezlesquels la verge avait offert pendant l'expérience un mouvement d'oscillation très-prononcé.

Sur un cheval entier dont la jambe avait été écrasée par une voiture, un couteau à amputation plongé sur le lobe médian du cervelet, d'avant en arrière, jusqu'au haut de la moclle épinière, détermina une érection très- prononcée.

Mais ce résultat a surtout été constaté depuis la publication de ces faits, par un de nos habiles physiologistes, M. le professeur Ségalàs.

Si sur un cochon-d'inde mâle dont on a mis le cerveau à nu, dit ce physiologiste, on plonge un stylet dans le cervelet, de manière à arriver à la partic supéricure de la moelle de l'épine, on produit lérection; si l'on pousse ensuite le stylet dans la colonne vertébrale jusques dans la région lombaire, l'éjaculation a lieu, tandis que la vessie, fût-elle pleine, n'en conserve pas moins son dépôt. Les mèmes phénomènes s'observent dans les cochons-d'inde décapités, quand on agit de même

professeur Lallemand de Montpellier avait tenté en vain la cautérisation des canaux éjaculateurs; M. Gensuult fit appliquer des sangsues et de la glace pilée a la nuque: les pollutions furent arrêtées comme par enchantement (Disscrtation inaugurale de $M$. le dorteur $V$ ial, pag. 24 et 25.) 
avec unstylet de haut en bas sur la moelle de l'épine. Gette dernière expérience, que j’ai répétée et dont chacun peut s'assurer par soi-même, prouve deux choses: la prenière, que l'irritation du cervelet détermine l'érection; la seconde, que la partie inférieure de la moelle épinière produit l'éjaculation, et agit plus spécialement sur les appareils sécréteurs et excréteurs du sperme.

Or nous avons déjà vu dans un des chapitres précédens que les maladies organiques de la partie inféricure de la moelle épinière déterminent des maladies de l'ovaire, suppriment les règles, ou, comme j'en ai maintenant un exemple sous les yeux, s'opposent chez les jeunes filles à leur manifestation. L'action des organes génitaux, chez les mammifères, a donc tout-à-la-fois son principe et dans le cervelet, comme nous le pensons avec M. Gall, et dans la moelle épinière, comme l'avait cru Willis, qui l'avait considérée comme l"organe de la propagation.

Il est vraisemblable que l'éjaculation est produite dans cette expérience, parce que l'irritation. de la moelle épinière développe la contractilité des vésicules séminales, soit que l'on admette ou que l'on n'admette pas leur structure musculeuse. La moelle épinière agit sur ces vésicules comme elle le fait sur la matrice. L'intégrité de la partio inférieure de la moelle épinières paraît nécessaire pour que la matrice se débarrasse du produit de la conception. Sur deux femmes parapléII. 
giques, arrivées au terme de l'accouchement, la matrice resta dans une telle inertie, que chez l'une, le col ne se dilata pas suffisamment pour pouvoir introduire le forceps ; chez l'autre, on fut obligé d'extraire l'enfant par l'opération césarienne. M. Brachet, de Lyon, cite le cas d'une dame devenue enceinte, quoique affectée de paraplégie. C'était une quatrième grossesse. Dans les trois premières, survenues avant sa maladie, l'accouchement avait été naturel : le forceps devint nécessaire dans celui-ci; il n'y avait point de douleurs expulsives; la matrice toujours assez ferne ne se contractait point ( 1 ).

Si sur des femelles pleines, de lapin, de cochond'inde ou de chien, etc., on fait la section de la moelle épinière à la région lombaire, quelque temps avant le terme de la parturition, celle-ci n'a point lieu. Ces animaux meurent sans mettre bas.

Si pendant le travail de la mise bas on fait la même section, les contractions s'arrêtent subitement, et la parturition ne s'effectue pas.

L'irritation de la moelle épinière dans cette même région produit, au contraire, l'avortement. Si après la décapitation des femelles pleines, on introduit un stylet dans la moelle épinière, et qu'on le dirige de haut en bas, arrivé à la région

(1) Voyez, sur ce fait et le suivant, la dissertation de M. le docteur Brachet, pag. 65. 
Jombaire, la contraction des cornes utérines se manifeste, et la parturition s'accomplit. Sur de.; femelles de lapin et de cochon-d'inde, l'injection de la teinture de noix vomique dans les veines crurales produit le même effet.

Des indications thérapeutiques importantes dérivent de ces expériences; dans les cas de gros-sesse avec paraplégie, on doit se garder de combattre celle-ci par la noix vomique, dans la craint: de provoquer l'avortement. Au contraire, le travail de l'accouchement étant commencé, son administration sera utile pour combattre l'inertic de la matrice. L'inertie de la matrice ne pourraitelle pas être combattue par ce moyen dans les cas si fréquens où on a recours au forceps (1)?

Si des mammifères nous passons aux trois autres classes, nous voyons que les variétés de volume du cervelet ne peuvent donner une raisun suffisante soit de la fécondité dont ils jouissent, soit de la volupté qu'ils paraissent goûter dans

(1) J'ai fait l'application de ce principe au traitement de la colique de plomb. Trois malades affectés de cette colique ont été guéris en quatre, cinq et six jour's de traitement. La teinture de noix romique a été employée en lavemens dins des potions, et en frictions sur la région lombaire et sur l'abdomen. Nul accident ne s'est manifesté, quoique le dernier se soit gorgé d'alimens le quatrième jour du traitement, et ait eu une indigestion, qui a manģué d'avoir les suites les plus graves. 
l'acte de la fécondation. A la vérité, la prédominance du lobe médian du cervelet des oiseaux rend comple jusqu'à un certain point de l'ardeur qu'ils présentent dans la génération; mais chez les poissons ossenx, et surtout chez certains reptiles, l'anatomie repousse cette corrélation jusqu'à ce que la physiologie l'ail établie.

Nous voyons, au contraire, la moelle épinière augmeuter de dimension à mesure que l'on s'éloigne des mammifères supéricurs, et que l'on se rapproche des poissons; en même-temps, plus la moelle épiniere augmente, plus nous voyons la fécondité s'accroitre, plus nous voyons la volupté lićc à cct acte diminuer (autant toutefois que nous pouvons juger des impressions des animaux). On peul donc présumer, et présumer seulement par l'aualogic de ce que nous montrent les mammifères que la moelle épinièresest le siége ou l'organe de la fécondité, et le ccrvelet, le siége de la volupté. Il est à remarquer sous ce rapport que la volupté de l'acte de la génération est essentiellement liée à la posilion quaffectent ses organes; or chrz les mammiferes seuls, les organes de la génération sont à l'extérieur. Leur situation plus ou moins intérieure, dans les autres classes, doit nécessairemcnt amortir l'impression que peuvent éprouver les animaux (1).

(1) Parmi les poissons, on sait que les raies et les squales 
Si, des animaux vertebrés, nous passons aux articulés et aux mollusques, nous trouverons que l'acte de la génération et la fécondation s'opèrent dans toutes ces classes hors de l'influence de la moelle épinière et du cervelet, puisque leur système nerveux ne présente ni cervelet ni moelle épinière.

Action du cervelet sur les mouvemens volontaires.

Depuis trois siècles le principe des mouvemens volontaires a beaucoup occupé les physiologistes et les pathologistes; après qu'on en eut placé le siége dans les membranes de l'encéphale, comme nous le dirons ailleurs, Willis, qui combattit cette opinion, en doua exclusivement les hémisphères cérébraux. Pourfour-Petit fit pour la sensibilité ce que Willis avait fait pour les mouvemens: rejetant l'idée que les nerfs sensibles puisaient leur origine dans les enveleppes du cerveau, il consacra le premier le cervelet à cette importante jropriété. I)ans les expériences qu'il tenta pour prouver son opinion, il ne tiut qu'un faible compte des effers qu'il produisil sur les mouvemens, regardant, comme Willis, les hémisphères cérébraux comme la source unique des mouvemens

s'accouplent, au contraire de ce gui a lieu dans presque Inute cetle classe. Or lés raics el les squales se rapproch ont déjả des oiseaux par Jeur ecrwelct. Leur aceouplement tiendrait-it at of developpement du eervelel: 
volontaires. Aussi, de son propre aveu, ces expériences ne lui laissèrent-elles que des doutes. Saucerotte, Sabouraut et Chopart, dans le concours dont nous avons déjà parlé, avaient pour but de constater les résultats du médecin de Namur. Le premier, seul, fit des expériences, et, tout en admettant que le cervelet était l'organe de la sensibilité, il porta une attention plus sérieuse sur l'effet que les lésions du cervelet déterminent sur les mouvemens; il détermina des convulsions géuérales, un opisthotonos assez constant sur les animaux dont le cervelet était profondément lésé, et une faiblesse plus grande du côté opposé où la lésion avait eu lieu. Malheureusement ces résultats furent gâtés par des explications, qui, les faisant méconnaître, les rendirent nuls pour la science. Sabouraut, plus diffus encore que Saucerotte, resta beaucoup au.dessous de lui. Enfin Chopart sanctionna l'ancienne erreur de Willis, que le cervelet n'exerçait aucune influence sur les mouvemens volontaires. Zinn, avant tous ces auteurs, avait produit aussi l'opisthotonos et la paralysie générale par les altérations plus ou moins profondes du cervelet; il avait même vu sur des pigeons que, malgré l'ablation complète du cerveau, le cervelet restant intact, l'animal continuait à marcher ; il mangeait aussi la nourriture qui lui était oflerte. Le résultat de ces expériences confirmait l'influence du cervelet sur les mouvemens; mais Zinu, l'un des plus zílés disciples de 
Haller, ne cherchait dans ces faits que des preuves à opposer à la doctrine de Willis concernant l'influcnce de cel organe sur les mouvemens involontaires. Au milicu de ce vague et de cette incertitude, le cervelet avait été reconnu comme une des sources du mouvement; mais son action avait été jugée directe, en sens inverse de celle des hémisphères cérébraux. Ce fut l'opinion de Morgagni qui cxerça sur les esprits celte fâcheuse influencc

Après avoir restitué au cervelet son influence sur les mouvemens volontaires, le point le plus important pour la paíhologie élait de déterminer si cette influence était directe ou croisée? Cette question a été résolue par les faits et les expériences que j’ai publiés, et dont voici l'exposé succinct.

Un marchand de fer, de soixante-un ans, affecté depuis long-temps d'une paralysie du côté gauche, paralysie dans laquelle le membre inférieur était plus immobile que le supérieur, succomba des suites d'une rétention d'urine. Les hémisphères cérébraux étaient intacts; la base de l'bémisphère droit $\mathrm{du}$ cervelet contenait un ancien foyer apoplectique dont les pourtours s'ćtaient ramollis, et le centre occupé par une certaine quantité de pus.

Sur un homme de soixante-huit ans, une hémiplégie du côlé gauche coïncidait avec un foyer sanguin creusé dans le noyau médullaire de l'hémisphère droit du cervelet.

Un journalier de suixante-six aus, hémiplégique du cóté dreit, uous offrit un ramollissement qui 
avait envahi et désorganisé dans presque toute son élendue l'hémisphère gauche du cervelet.

Dans tous ces cas et dans plusieurs autres que je néglige de rapporter, parce qu'ils sont la répétition de ceux-ci en ce qui concerne la perte du mouvement, on observe donc que l'altération de l'un des hémisphères du cervelet, produit l'abolition du mouvement dans les membres du côté opposé. Si l'altération organique est à gauche, la paralysie est à droile, ct vice versa. Parmi les faits analogues qui ont été observés depuis la publication de mes mémoires, j'extrais le suivant, rapporté dans une thèse soutenue dans le cours de l'année 1825 , à la Faculté de Médecine.

Un fabricant d'étoffes de soic, de Lyon, âgé de soixante-treize ans, entra à l'Hlôtel-Dieu de la même ville, le 18 octobre 1824 , pour une attaque d'hémiplégie qui affectait le côté gauche. I en avait déjà éprouvé deux semblables daus le courant de la même année. Il succomba après le troisieme jour de son entrée à l'hôpital. A l'ouverture du cadlavre on trouva les trois quarts du lobe droit du cervelet ramollis dans toute son épaisseur; le ramollissement s'étendait jusqu'à la protubérance annulaire. Altération de l'hémisphère droit, paralysie à gauche, résultat de l'action croisée du cervelet sur les mouvemens des membres. Telles sont les données die la pathologie, telles sont aussi celles de la physiologic expérimentale. 
Un chien de moyenne taille fut trépané à la partie postérieure du pariétal droit; le sang ayant été épongé, je plongeai un bistouri à lame très. mince dans l'ouverture; je le dirigeai de haut en bas et de gauche à droite, afin de faire la section de l'hémisphère du cerveau du même côté; cela fait, le chien fut dégagé de ses liens; tout le corps de l'animal était courbé du côté droit, la tête surtout était fortement penchée de ce côté. En cherchant à expliquer ce phénomène, nous aperçûmes qu'il était produit par la contraction des muscles de ce côté, ceux du côté gauche, qui sont leurs antagonistes, ayant éprouvé une diminution sensible dans leur action. Le chien restait d'abord immobile; excité par mes aides, il fléchit d'abord sur le côté gauche, tomba sur ce côté, et y resta environ trois minutes; il sereleva ensuite, marcha l'espace de vingt pieds, en décrivant un arc de gauche à droite, ce qui indiquait que les muscles du côté droit étaient beaucoup plus forts que ceux du côté gauche; il arriva alors contre un mur et appuya contre lui le côté gauche du corps. Lorsqu'on le retirait, et qu'on l'appryait du côté droit, il se retournait et appuyait le côté gauche; l'animal éprouvait le besoin d'être soutenu de ce côté. Il fit ce manége aussi souvent qu'on le retourna. Un peu plus tard on lc plaça au milieu de la cour, et on le contraignit de marcher : il se sombnait ì prine sur le côté gauche; les pattes anterieure et postririeure de creoté ćtaicnt 
légèrement fléchies; l'animal paraissait pouroir plus difficilement mouvoir les muscles extenseurs. Il resta jusqu'au soir dans cet état; le lendemain on ouvrit le crâne pour examiner sur quelle partie de l'encéphale avait porté le bistouri.

L'encéphale ayant été retiré du crâne avec beaucoup de soin, je trouvai que le bistouri avait divisé l'hémisphère droit du cervelet en intéressant légèrement le pédoncule du même côté, et passé à une ligne de la partie externe du corps ciliaire: je n'avais intéressé ni la partie postérieure de l'hémisphère droit du cerveau, ni les tubercules quadrijumcaux du même côté, accident qui m'élait fréquemment arrivé dans les autres expériences, et qui avait compliqué les effets que j'avais obtenus.

Un autre chien, trépané de la même manière, et aussi du côté droit, offrit les mêmes phénomènes, mais, de plus, il tournait constamment de gauche à droite; placé sur le dos, il roulait sur lui-mème, pivotant toujours sur le côté droit; il était plus faible que le précédent du côté gauche, se soutenait moins bien sur ce côté, et se couchait après quelques pas; laissé en repos, il présenta des mouvemens convulsifs des membres gauches, mouvemens plus for's dans la patte de derrière que dans celle de devant. Il mourut sept heures après l'opération. Sur celuici le cervelet avait été lésé plus profondément; l'hémisphèr" droit du cervelet avait élé incisé 
dans sa partie moyenne, el presque dans toute sa profondeur.

Un chien trépané rlu côté gauche, et opéré de la même inanière, nous offrit le même ordre de phénomènes, mais en sens inverse : le côté droit était celui qui fléchissait constamment; l'animal tombait sur ce côté quand il s'élançait pour marcher; il s'appuyait à droite et jamais à gauche; les membres du côté gauche étaient dans une demi-flexion, ce qui arrive fréquemment dans les hémiplégies, à cause de la prédominance des muscles fléchisseurs sur les exteuseurs; prédominance qui trouve sa cause dans le plus grand nombre de nerfs que reçoivent ces muscles, proportionnellement aux extenseurs. L'hémisphère gauche et le pédoncule du cervelet du même côté avaient été divisés par l'instrument tranchant; en arrière, l'hémisphère était coupé dans sa totalité.

A cette époque (1819), je me rendais fréqueminent il la grande voirie de Montfaucon, pour me procurer les embryons des animaux qu'on transporte cn cet endruit de Paris et de ses environs : je soumis à cette expérience cinq chevaux destinés à être abattus ; dans un seul je réussis à n'intéresser que l'hémisphère du cervelet.

Le cheval ctait de moyenne taille; j'enlevai avec deux couronnes de trépan la partic gauche de l'occipital; j’incisai la dure-mère et j'épongeai le sang résultant de la plaic: je portai ensuite le Listouri dans l'ouverture; je le portai dans une 
620 PHYSIOLOGIE ET PATHOLOGIE.

direction d'abord directe, puis un peu oblique de droite à gauche. On détacha l'animal; son corps se courbait en arc comme dans les expériences précédentes : la courbure avait lieu à gauche, par la contraction des muscles de ce côté, et à cause du relâchement de ceux du côté droit. L'animal était couché; on le releva avec beaucoup de peine; il fléchit aussitôt du côté droit et tomba sur ce côté. On le releva de nouveau, on le soutint du côté droit; il marcha, mais avec tant de difficulté des membres droits, que, sans plusieurs hommes qui le soutenaient, il serait tombé à chaque pas. On l'appuya à droite contre un mur ; il y resta plusieurs minutes dans un équilibre apparent : les muscles du côté droit fléchirent ensuite d'une manière graduelle, et il tomba à droite. Deux heures après cette première tentalive, je la fis réitérer; elle offrit les mêmes résultats. Mais, de plus, ayant voulu voir si l'animal se releverait seul, je l'excitai vivement : dans les efforts qu'il fit, il se roulait sur lui-même; la rotation avait lieu de droite à gauche, parce que les muscles du côté gauche étaient évidemment les plus forts; ceux du côté droit obéissaient à leur action. Il était sensible dans toutes les parlies du corps; la respiration ne paraissait pas gênée. Il aurait sans doute vécu quelques jours; mais il fallut tuer l'animal, les ouvricrs attendant après lui pour travailler.

J'enlevai la calotte du crâne avec une scie a 
amputation, et je détachai l'encéphale sans lui faire éprouver aucuue déchirure : les hémisphères cérébraux étaient intacts ; je n'avais pas même intéressé la partie postérieure de l'hémisphère gauche, qui, chez le cheval, déborde légèrement la partie supérieure du cervelet; l'instrument avait divisé l'hémisphère gauche de ce dernier en commençant en haut sur les feuillets transversaux du lobe médian antérieur; et intéressant dans la profondeur les faisceaux moyens du processus cerebelli ad testes : l'incision divisait ensuite un peu obliquement, de droite à gauche, toute l'étendue de l'hémisphère. Son lobule antévieur était coupé dans la partie moyenne jusqu'au tiers supérieur du pédoncule du cervelet : le lobule moyen était tranché ainsi profondément dans son tiers externe; enfin le petit lobule postérieur n'avait, été touché qu'à trois lignes en dehors. La moelle allongée et la moelle épinière n'avaient éprouvé aucune altération.

On voit donc que les résultats de ces expériences cöncident parfaitement avec ceux que m'avaient offerts les malades que j'avais observés. Si j’opérais sur l'hémisphère droit du cervelet, l'animal fléchissait du côté gauche; les jambes de ce côté supportaient difficilement le corps; en même temps l'animal se courbait en formant une espèce d'arc, dont la concavité correspondait à droite, et la convexité à gauche. Cet effet était surtout remarquable au cou. Fn cherchant à expliquer ce 
622 PHYSIOLOGIT ET PATIOLOGIS.

phénomène, on irouvera, je pense, que les muscles du côté gauche ayant perdu leur action en totalité ou en partie, les muscles antagonistes du côté droit conservant toute leur énergie, tout le corps se trouvait nécessairement entraîné de ce côté. L'inverse avait lieu sur les animaux dont l'hémisphère gauche du cervelet avait été lésé : la concavité de l'arc était à gauche, quand l'animal faisait des efforts pour se relever, et la convexité à droite : c'était du côté droit qu'il fléchissait, qu'il cherchait un appui. Plaçait-on l'animal sur le dos, et le forçait-on à se relever, dans les efforts qu'il faisait, les muscles opposés à la lésion du cervelet entraînaient ceux de l'autre côté; l'animal roulait sur lui-même: cette rotation avait lieu de droite à gauche, ou de gauche à droite, selon qu'on avait blessé l'un ou l'autre des hémisphères. Tout indiquait donc que le côté opposé à l'hémisphère lésé était celui qui était frappé de paralysie (1).

Cette rotation des animaux m'avait beaucoup frappé, parce que j’avais précédemment observé un malade qui, au lieu de voir tourner les objets comme cela arrive dans les prodromes de la plupart des apoplexies, tournait au contraire Juimême, et se roulait dans son lit pour peu qu'on

(1) I. Flourens ayant parlaitement établi le croisement d'action du cervelet chez les oiseaux, je m'abstiens de rapporter mes propres expériences. 
l'abandonnât. Ce fait étant encore unique dans la science, je vais le reproduire avec quelques détails.

Le nommé F. E. Duval, âgé de soixante-huit ans, cordonnier, d'une constitution faible, grand buveur, fut sujet dans sa jeunesse aux saignemens de nez, plus tard aux hémorrhoïdes, et toute sa vic à une faiblesse d'estomac qui rendait ses digestions pénibles : souvent il était pris de diarrhées, qui duraient huit ou dix jours et cessaient par lc régime. A l'âge de cinquante ans il eut dans l'articulation du genou gauche un gonflement que l'on combattit par les bains, et qui rendit les mouvemens difficiles pendant plusieurs mois; la jambe fut oedématiée; un ulcère situé à la malléole interne (suite d'un coup) fut très-long-temps à se cicatriser. Telles étaient les affections morbides dont le malade et ses amis avaient conservé le souvenir.

Le 5 janvier 1819 , le malade ayant dîné chez un de ses amis, et bu beaucoup, comme à son ordinaire, fut néanmoins plus étourdi par lc vin qu'il n'avait coutume de l'être. Une circonstance qui l'avait frappé dans cet état, c'est qu'il ne voyait pas tourner les objets comme cela arrive d'ordinaire, mais qu'il lui semblait au contraire qu'il tournait lui-même; ce qui porta ses amis à croire qu'il ćtait ivre, et le fit conduire chez lui. A peine y était-il arrivé, qu'il se mit à tourner réellement sur lui-même. Le tournoiement avait 
lieu de droite à gauche; on le coucha : peu de temps après il vomit quelques gorgées de vin. Dans la nuit il eut une attaque d'apoplexie avec hémiplégie du côté gauche; on la combattit par les saignées, les vomitifs et les purgatils; les symptômes apoplectiques se dissipèrent, mais il resta paralysé de la 'jambe gauche; le bras avait repris assez de force et assez d'agilité pour que le malade pût travailler sur son lit, ou dans un fauteuil où on le plaçait pendant le jour.

Néanmoins il s'affaiblissait de jour en jour: une diarrhée opiniâtre se manifesta; la maladie. s'augmentait encore par l'usage du vin et de l'eau-de-vie. Ce dévoiement l'obligea de garder le lit pendant tout le mois d'avril; les astringens les plus énergiques avaient été sans effet; le malade allait involontairement à la selle : ce fut cette dernière circonstance qui détermina ses parens à l'amener à l'hôpital de la Pitié, le 12 mai 1819.

Il était pâle, émacié, les muscles flasques, légèrement infiltrés, surtout ceux de la jambe gauche; la langue était rouge, racornie; l'abdomen douloureux principalement vers les fosses iliaques; le pouls |était petit, concentré et assez fréquent; la peau, aride, offrait une desquamation légère et générale; le dévoiement était continuel.

La jambe gauche était immobile: quelle que fût la volonté du malade, il ne pouvait lui faire exécuter le plus léger mouvement. Le sentiment était conservé; le bras gauche exécutait librement 


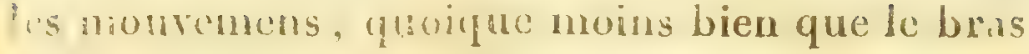
Iroit. Intorrow sur la cause de cette immobilité le la jambe, lo malade l'attribuait à la tumeur banche qu'il avait cue au genou gauche plusieurs anućes auparavant, et à l'habitude qu'il avait de frapper ce genou dans son travail.

On conçoit que dans cet état on ne put rien untreprendre contre cette hémi-paraplégie. Je portai toute mon attention sur la diarrhée chronique, que j'essayai de modérer, mais en vain, par l'usage de l'opium fractionné. Le malade succomba lc 2/ du mềme mois, après doùze jours de séjour dans ma division.

A l'ouveriure du cadavre, nous rencontrâmes des ulcérations chroniques dans tous les gros ıntestins, mais spécialement au coecum, à l'arc fransversal du colon et à l'S iliaque du même intestin.

Les hémisphères du cerveau étaient dans leur clat ordinaire; au cenire de l'entrée du pédoncule du cervelet dans l'hémisphère droit de cet srgane, il cxistait une excavation de neuf lignes le long, oblique de dehors en dedans, et large de cinq lignes dans son plus grand diamètre transversal. Au pourtour de ce foyer la substance blanche étail deveuue jaunâtre, plus consistante qure dans l'élat naturel. la foyer élait traversé par une bride jamnâlre, et divisé cn deux prites loges, une antérieure, l'autro postéricude; la lo postérieure, qui s'enfoncait le plus profondénioni 
dans l'hémisphère du cervelet, ét it remplie par une matière de la consistance de la bouillie, d'une couleur brune. La loge antérieure était remplie par une substance plus épaisse, d'un jaune brun, entièrement détachée des parois de la cavcrne; tout l'hémisphère droit du cervelet ćtait plus consistant que le gauche; les radiations de la substance blanche avaient une teinte jaunâtre qu'on ne remarquait pas dans les radiations du côté opposé. Le reste de la protubérance annulaire, la moel!e allongée et la moellc épinière, ne présentaient rien de particulier.

Dans les expériences sur les animaux, la rotation était l'effet de la section plus ou moins profonde des segmens qui recouvrent le quatrième ventricule; mais, dans ce cas, la tendance presque irrésistible qu'avait le malade à tourner, provenait évidemment de la lésion du pédoncule droit du cervelet. C'est ce qui, peu de temps après, a été mis hors de doute par les expériences de M. Magendie. Ainsi les animaux sur lesquels l'on coupe l'un des pédoncules du cervelet, se roulent sur leur axe et du même côté que la section, avec une vitesse qui va en diminuant à mesure que l'on s'éloigne du pédoncule. Ainsi, une fois encore, la physiologic expérimentale a confirmé une des données fournies par la pathologie.

Depuis douze ans j'ai varié de mille manières les expériences que j’ai tentées sur les animaux 
pour produire artificiellement les effets que me dévoilait l'observation des maladies organiques du système cérébro-spinal de l'homme. Un: fait que j'avais souvent remarqué dans les apoplexies cérébelleuses était le renversement et la chute des malades en arrière. Dans ces cas, le cervelet avait élé détruit dans la base des hémisphères et des deux côtés; quelquefois mème le quatrième ventricule avait été mis à découvert par l'étendue de la désorganisation.

Si sur un animal on blesse le cervelet horizonlalement et dans toute son étendue, la tête est renversée en arrière; si l'on se rapproche des pédoncules, il y a opisthotonos, le corps est courbé en un arc, dont la concavité est sur le dos et la convexité sur la face abdominale. Si dans cet état on le force à marcher, l'animal s'élance en avant; mais après quelques pas une force irrésistible lentraîne, et il recule. Cet effet a été produit avec diverses modifications, par Zinn, Saucerotte, MM. Magendie et Flourens.

La tendance au reculement est alors un cffet de la tendance invincible qui porte les animaux i) se maintenir en équilibre; ils cherchent cet équilibre en reculant: si dans cet élat, et lorsque la têle seule est renverséc, on coupe les muscles qui la portent en arrière, la tendance au reculement est arrêtéc, l'animal marche devant lui ayant sa tête entre les jambes de devant.

J'ai bien remarquí cet effet sur une jeune fille 
de douze ans, que MM. Magendie et Laurcnt placèrent dans ma division. Dans de courls accès d'épilepsie, qui se succédaient avec une grande rapidité, cet enfant était irrésistiblement entraîné à reculer. La tête était portée en arrière; le corps lui-même était légèrêึent incliné en ce sens. Si on s'opposait au renversement de la tête, le reculement s'arrêtait ; · lorsqu'on lui laissait parcourir ainsi un certain espace en la tenant par la main, elle décrivait une courbe, qui fut en augmentant à mesure que la malade approcha du terme de la guérison. Elle sortit deux mois après son entrée, très-bien rétablie, et depuis deux ans elle n’a éprouvé aucune rechute. Le renversement et la chute en arrière de certaines apoplexies cérébelleuses, et le reculement de cel enfant me paraissent donc expliqués par les expériences précédentes.

En outre, dans le cervelet réside réllement la force qui coordonne les mouvemens pour le saut. M. Flourens l'a dit; mais ses expériences ne me paraissent pas aussi décisives que les suivantes.

Si, un animal étant libre, on transperce le cervelet avec un poinçon très-acéré, au niveau de son tiers supérieur, l'animal fait un bond qui peut s'élever à plusieurs pieds de hauteur. Cet effet est si prompt, si instantané chez le lapin, que l'on croirait que le saut est produit par la détente d. un ressort. 
Cette propricté expligre ae que lon a cru remarquer de contradictoire dans la grenouille : la progression de cet animal est bien différente, selon qu'elle s'exécute dans l'eau ou sur le sol. Dans l'eau, elle nage; sur la terre, elle saute. Cette circonstance est donc bien propre à éclairer ce point de la physiologie du cervelet.

Si sur une grenouille on enlève cet organe, cc qui n'est pas très-difficile, et qu'on la plonge dans l'eau, elle nage comme avant cette ablation. Si on la met à terre, elle reste immobile : on a beau l'exciter, cllo remue les patles; mais elle ne marche point, parce qu'elle ne peut marcher sans sauter. En perdant son cervelet, elle a donc perdu cette faculté.

De ce que la grenouille ne peut plus sauter lorsqu'elle a perdu le cervelet, en conclure que la marche est abolie chez tous les reptiles qui ont subi cette mutilation; ce serait n'avoir pas saisi l'anomalie particulière de la marche de ce batracien. En effet, de même que la grenouille nage après cette ablation, de même les lézards et les couleuvres continuent de marcher, les oiseaux de voler, mais beaucoup plus faiblement, et sculement pendant quelque temps, après l'ablation $d u$ cervelet.

Le cervelet excree donc une influence très-puissantesur les mouvemens des membres; mais cette influence n'est pas la même sur les membres supiricurs nuanforicurs, en sur les inferiems ou posta 
ricurs. Les maladies et les mutilations artificielles de cet organe affectent plus les membres inférieurs que les supérieurs. C'est un résultat que j'ai constaté par la pathologie et la physiologie expérimentale, et dont l'application, comme je l'ai montré dans mes mémoires, est très-importante pour la pathologie (1).

Un homme était paralysé de la jambe gauche, le bras conservait quelques mouvemens, l'hémisphère droit du cervelet était détruit en grande partie.

Chez un maçon qui avait fait une chute sur la partie postérieure de la tête, le bras gauche exécutait quelques mouvemens., tandis que la jambe du mème côté était complètement immobile; l'hémisphère droit dù cervelet était aussi le siége de l'altération organique.

Sur deux femmes les résultats furent les mêmes, excepté que chez l'une l'hémi-paraplégie était à droite, et l'hemisphère gauche du cervelet avait seul été désorganisé. Chez une femme qui est en ce moment dans ma division depuis sept mois, la jambe et la cuisse gauches sont dans l'immobilité la plus complète; le bras gauche exécute tous ses mouvemens aussi bien que le droit, et avec la même force.

Chez les animaux que l'on expérimente, les

(1) Voyez trois Mémoires publiés en 1823 , dans le journat de M. Magendie. 
effets sont les mêmes, les membres postérieurs sont toujours plus affectés que les antérieurs. Nul n'est plus propre à cette expérimentation que le lapin : quand on lui enlève la partie moyenne du cervelet, le train de derrière est presqu'immobile, tandis que celui de devant est dans une agitation continuelle. Dans les efforts que fait l'animal, il se renverse souvent en arrière, en prenant son point d'appui sur le train postérieur resté fixe et immobile: d'autres fois l'animal pivote sur ses pattes postérieures, il décrit un cercle dont le centre correspond au train postérieur, qui ne se meut point.

Chez la grenouille, on remarque un effet à peu près semblable quand on la place sur le sol; souvent elle se renverse en arrière, et souvent aussi elle décrit un arc en s'appuyant sur les pattes de derrière.

Mais c'est surtout chez les oiseaux que cet effet est sensible. Après l'ablation du cervelet les paltes sont immobiles, tandis que les ailes se meuvent encore. Lancez l'oiseau en l'air, il vole; mais tombé à terre, il ne se relève plus, parce que les pattes étant paralysées, il ne peut plus prendre sur elles son point d'appui pour s'élancer de nou-veau : dans cet état, un oiseau est comme si on lui avait coupé les pattes; ct pour voir cette similitude dans ces effets, il est curieux de faire en même temps et comparalivement ces deux 
expériences (1). Le canard placé dans l'eau perd la faculté de nager, comme la grenouille placéc sur la terre perd la faculté de sauter; ces deux effets, en apparence si différens, proviennent donc de la même cause. Si on géuéralisait ces résultats, on pourrait donc dire que le cerveau est l'organe du vol, du saut et de la nage; mais ce qui est vrai pour la grenouille, le canard et le lapin, ne l'est plus pour d'autres animaux. C'est cette généralisation à laquelle nous sommes tous portés d'une manière presque involontaire, qui peut êtrc reprochée à MM. Rolando et Flourens, dont les ouvrages sont d'ailleurs si remarquables.

Action et Maladies de la protubérance annulaire.

Dans les apoplexies que j’ai désignées seus ic nom d'hémato-encéphalie, et de malaco-encéphalie: les symptômes les plus variés et les plus constans se manifestent, selon que la continuité des fibres de l'encéphale est interrompue sur tol ou tel point de sa surface. Avant la première pu-

(1) D'aprés cette influcnce du cervelet sur les membres postérieurs, les cétacées devraient avoir éprouvé une diminution notahle dans le volume de cet organe, ce qui nien pas. Bien plus, In cervelet a singulic rement accru de volume. Cette anomalis: me parait inexplicable dans liblat prosent d. la srienes: 
Wication de mes recherches, on négligeait conplètement leur étude; l'hypothèse de la compression de l'encéphale par les liquides en détournait les esprits. (1)

On sait aujourd'hui que les paralysies affectent lantôt la moitié du corps, tantôt une jambe ou un bras, ou qu'enfin tous les muscles subjaccus a la tête peuvent être paralysés instantanémem. Quelle est la partie du cerveau qui régit ainsi la presque totalité des mouvemens soumis à la volonté? L'anatomie aurait pu le dire; mais la patho-

(1) Culte hypothèse n'était que la conséquence rigoureusement déduite du système des esprits animaux. Ces esprits itaient si déliés, c'était une vapeur tellement subtile, que quelques gouttes de liquide suffisaient pour oblitérer les pores d'où ils transsudaient, ou interrompre la continuité des canaux dans lesquels s'opérait leur circulation; telle était la base sur laquelle était élayée la doctrine des maladies du système nerveux. Cette doctrine était admirablement liéc dans toutes ses parties; tout s'expliquait par elle, cumme on peut surtout en juger par l'ouvrage de Vieussens; mais on ne saurait trop le répéter, on n'avait qu'une doctrine de convention; tout le monde adencttait l'existence des esprits animaux, tout le monde devait nécessairement admettre les conséquences de ce système. Personne n'y croit plus de nos jours, et cepenilant la science a conservé et conserve encorc les explications qui sont nées de leur existence. Gette contradiction est choquante; n’a-t-on pas reproché it $\mathrm{Ml}$. Flourcns la mullite de la plupart de ses résultats, en se fondant sur ce fu'il me!tait ses animanx on anoplexie en laissant du santr

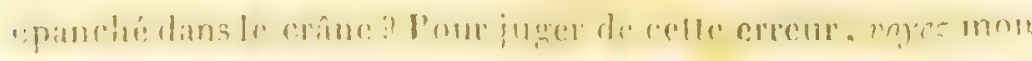

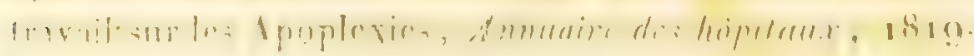


logie a encore ici devancé l'anatomie et la physiologie. Chez trois hommes dont j'ai rapporté l'histoire dans un autre ouvrage, l'immobilité générale avait été produite par la destruction presque complète de la protubérance annulaire ( $\mathrm{Hé}-$ mato-mésocéphalie).

Sur deuxautres, observés l'un en 1822 , l'autre en 1825 , un phénomène assez remarquable s'était manifesté. Au moment de l'attaque, ils ressentirent une douleur des plus vives, jetèrent des cris et coururent devant eux comme pour éviter un grand danger. Ils tombèrent après environ cent pas. Chez tous les deux, la tendance à se porter en avant avait été spontanée; la proıubérance annulaire avait été détruite dans toute sa profondeur, et la paralysie complète.

Chez une femme, dont la moitié postérieure du mésocéphale était squirrheuse, lardacée (scléroméso-céphalie), la marche avait d'abord été chancelante comme dans l'ivresse, puis la mobilité et la sensibilité furent entièrement perdues. On l'excitait, elle ne sentait point; on la mettait sur un côté, elle y restait jusqu'à ce qu'on la déplaçât de nouveau. Elle perdit la vue complètement sans opacité dans les cornées; elle perdit le goût, l'ouie et l'odorat; la vie de relation était tout-à-fait anéantie un mois avant sa mort. Ces phénomènes étaient évidemment le résultat de la désorganisation de la protubérance annulaire, et de l'insertion des nerfs trijumeaux. 


\section{PIXSLOLOGIE ET W'THOLOGIE.}

Un homme décédé le 20 novembre 1821 , dans les salles de M. le docteur Montaigu, à l'HôtelDieu, avait offert les mêmes phénomènes : insensibilité, immobilité absolues; perte du goût, perte de la vue, perte de l'ouie et de l'odorat. La partie inférieure de la protubérance annulaire était ramollie, réduite en bouillie (malaco-mésocephalie) le milieu était dur et lardacé (1).

Chez tous ces malades, des douleurs atroces avaient précédé la perte complète de la sensibilité. Cette propriété réside donc spécialement dans la protubérance annulaire et dans la partie de la moelle allongée qu'elle embrasse. Elle est donc aussi le centre du principe des mouvemens volontaires. Que conclure de cette coincidence de la perte de tous les sens, par la désorganisation de la protubérance annulaire? Ces faits n'expliquentils pas ceux que nous avons déjà cités à l'occasion de la section et de la désorganisation des nerfs trijumeaux?

Dans ces cas de ramollissement de la partie moyenne du mésocéphale, les mouvemens avaient d'abord présenté l'agitation de la danse de saintguy, puis l'incohérence de l'ivresse, puis la paralysie complèté.

Sur tous ces malades, les pupilles ont été contractées ou immobiles, selon que les muscles

(1) Cette observation a éte parfaitement rédigée par M. Ie. dncteur Pariset, interne de la division. 
étaient en agitation ou en repos. Dans ceux où la protubérance était plus affectée d'un côté que de l'autre, le nerf facial était paralysé du côté opposé, la commissure des lèvres déviée, la narine immobile; le bégaiement avait lieu.

Sur la plupart, tous les muscles inspirateurs étaient immobiles, et la respiration était interrompue par la cessation d'action des puissances inspiratrices, effet bien différent de la paralysie des poumons produite par l'altération du corps. restiforme ou de l'olive. J'ai vu souvent alors le rire sardonique coïncider avec l'agitation générale. Le rire sardonique était évidemment leffet de la convulsion du nerf facial.

Dans les encéphalites aiguës (fièvres ataxiques), I'inflammation de la protubérance annulaire produit la rigidité de tous les membres, la contracdion permanente des pupilles.

Si la protubérance est plus enflammée à droite qu'à gauche, il y a rigidité plus grande du côté gauche et constriction plus prononcée de l'iris à droite. Si l'inflammation est plus forte à gauche, les symptômes sont inverses; l'iris gauche est la plus resserrée, et les membres droits les plus contraclés. Souvent aussi il y a rigidité des mâchoires. Les phénomènes qui se développent du côté de l'iris sont expliqués par l'action de la cinquième paire, et ceux des mouvemens par cclle de la protubérance.

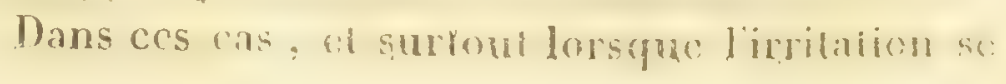




\section{PIYSIOLOGIT RT PSTHORGGT.}

(i),

jropage jusques sur le bulbe rachidien, on observe le vomissement, et les irrégularités de la respiration et de la circulation qui accompagnest si fréquemment les encéphalites.

Tels sont les phénomènes que développent les maladics organiques de la protubérance annulaire; la physiologie expérimentale les produit en grande partie sur les animaux. Lirritation artificielle produit la contraction des pupilles, l'agitation convulsive et générale de tous les muscles, leur paralysie complète, la paralysic des muscles inspirateurs, le vomissement, ot sa cessation par l'aplatissement du bulbe rachidien. L'irritation de la protubérance du côté droit délermine des conivulsions à gauche, et le resserrement de l'iris à droite; l'inverse a lieu quand on irrite le côté gauche.

Quand on a détruit complètement la protubérance et le bulbe rachidien, l'animal est complètement insensible; il est complètement immobile: on peut l'irriter de toutes les manières, il est insensible à tout; état qui reproduit si exactement ce que nous ont présenté nos malades. La respiration s'arrête, et elle s'arrête par la cessation seule des puissances inspiratrices, tant que les côtés de l'olive et la base du pont ne sont point intéressés dans la section. C'est d'après ces résultats que Iorry décirla que la moelle allongee était le seul organe actif du cerveau, et 
qu'elle était le principe et la source du mouvement et du sentiment.

Une de ses plus belles expériences, et que la plupart des physiologistes modernes ont répétée, esí celle qui consiste à enlever à un mammifère les lobes cérébraux, les tubercules quadrijumeaux, le cervelet, et de ne laisser de la protubérance que la partie qui correspond au-dessus de l'insertion de la cinquième paire. Dans cet état, l'animal, réduit au segment basilaire de l'encéphale, a une vie régulière et constante; la respiration et la circulation s'exécutent librement, quoiquavec un peu plus de précipitation que dans l'état naturel. La sensibilité est conservéc; l'animal sent quand on le pique; et il a la conscience de cette sensation, et la volonté de s'y soustraire, puisqu'il agite les paltes, le tronc et la queue avec régularité, sans néanmoins pouvoir marcher; il ouvre la gueule pour crier, sans produire de cris; il cntend, il sent, il paraît même goûter les substances que l'on place dans sa bouche. Mais la vue est perdue ou paraît perdue; sans cette dernière circonstance, cette expérience serait la contre-épreuve rigoureuse des effets obtenus par la section de la cinquième paire. C'est à l'intégrité de l'insertion de ce nerf qu'est due la persistance des sensations, car dans les maladies de la protubérance, tant que l'altération ne s'étend pas sur ces cordons d'inser- 
tion, les sens restent intègres; ils sont anéantis quand ils sont atteints. Cet effet est beaucoup plus prompt quand la maladie commence par les cordons supérieurs du quatrième ventricule, que lorsqu'elle débute par la partie antérieure. Aussi, chez les animaux, les faisceaux qui forment le plancher du quatrième ventricule étant enlevés jusqu'à la cinquième paire, on paralyse les sensations de la face, tandis que les faisccaux extérieurs intermédiaires aux deux nerfs laissent persister ces sensations (M. Desmoulins) (1).

Cette influence du segment basilaire de l'encéphale est plus prononcée chez les oiseaux que chez les mammifères; elle est plus prononcée chez les reptiles que chez les oiseaux, comme l'attestent les expériences de Redi, de Fontana et du professeur liolando. II paraît être le point de

(1) Je n'ai pas réussi à produire ces derniers effets dans mes expériences, quoique j'eusse préparé et fait représenter chez les animaux cette partie, pour montrer qu'elle est la répétition de celle de l'homme. En parcourant sur mes registres les expériences que j'ai tentées sur cette partie, j'en trouve une qui a beaucoup d'intérêt dans le moment présent: elle est de Cockburn. "Si j'cnfonce un stylet entre la première vertébre et l'occipital, je produis des mouvemens convulsifs généraux, et dans les yeux, au bout de trois jours la vue se trouble, parce qu'il survient des ulcères à la cornée, et que tout l'xil sc dítruit. D'où je conclus que les nerfs opliques ne finissent pas, ou ne commencent pas, comme on le dit, anx couches contiques. " 
Téunion des principales facultés des poissons. comme l'avaient soupçonné Collins et Manget, et comme peut le faire présumer l'anatomie de cefto partie, si richement organisée dans cetle classe; tandis que les hémisphères cérébraux sont si descendus dans leur organisation.

On a dit et on a cru, d'après Hippocrate, que la même lésion du cerveau pouvait produire la paralysie d'un côté, et les convulsions de l'autre. La paralysie était croisée et les convulsions étaient directes. Ces deux effets sont contradictoires et inconciliables, car ils se passent tous les deux dans les muscles; ils sont dépendans de la même propriété. Les convulsions sont l'irritabilité en aclion; et la paralysie, le repos ou l'anéantissement de l'irritabilité. Cela est si vrai, qu'en irritant le cerveau, par exemple, on détermine d'abord des coavulsions dans lè côté opposé; si l'irritation est long-temps continuée, et l'organe détruit, les divers muscles qui étaient en convulsion deviennent immobiles et paralytiques. Les convulsions et les paralysies sont deux états divers, ou deux modifications d'une même propriété. II est donc impossible que la même altération produise les convulsions d'un côté et la paralysie de l'autre.

Lorry s'aperçut de cette contradiction : en admettant les faits que l'on rapportait à son appui, il essaya d'en rendre raison en cherchant à produire arlificiellement cette allernative de convul- 
sions d'un côté et de paralysie de l'autre. Il crut que la moelle allongée les lui avait présentés, mais il hésita daus les conclusions. M. Flourens, dégageapt ces expériences de la complication qu'elles pouvaient offrir, a concla rigourcusement que la moelle allongée est directe dans son action, et il pense qu'Hippocrate a voulu parler des lésions complexes d'une partic croisée de l'encéphale avec unc lésion coïnciḍente de la moslle allongée. Mais les faits rapportés dans la science, par Baglivi, Bonnet, Morgagni et quelques modernes, sont tous des lésions simples, et des lésions qui intéressent uniquement les \émisphères cérébraux. En supposant donc que l'action de la moelle allongée soit directe, l'explication de notre habile physiologiste ne leur est point applicable.

Mais la moelle allongée a-t-elle une action directe? En bornant, comme je l'ai fail, et comuse l'a fait aussi M. Flourens, la moelle allongée ì la parie postérieure dés abercules quadrijumeaux, oџ voit que chez les mammiferes et l'homme les deux tiers de la prolubérance sont compris dans cette limite. Or, l'altíration de toute la prolubérance est croiséc; un foyer à gauche produit la paralysie à droite, et vice versui. Lit dans des cas où jai eu assez tôt les maladies sous les youx, jai vu des mouremens couvdosifs prócéder la perte croiséc du mouvement; jamais je n’aí remarqué de convulsion directe. Si on culeve ¿̀ un animal les hémisphères cérébraux, lesiuber- 
cules quadrijumeaux et le cervelet, de manière à conserver la protubérance, et que l'on irrite celleci, on détermine constamment les convulsions croisées, et aux convulsions succède la paralysie. L'effet n'est direcl qu'au delà de la protubérance, au point qui correspond au niveau des olives.

L'effet est un peu différent chez les oiseaux; l'action croisée de leur moelle allongée (la protubérance n'existant pas), ne se manifeste qu'au dessus de l'insertion de la cinquième paire. Audessous l'effet est direct; mais alors ce résultat s'étend et aux convulsions et aux paralysies. M. Flourens ayant expérimenté sur les oiseaux, a sans doutc été induit én erreur en voulant étendre aux mammifères ce que Jui avait offert cette classe. Car, comme je l'ai déjà expliqué, la moelle allongée et tout l'encéphale a une action directe chez les reptiles et les poissons.

Au reste, commençons par savoir s'il y a réellement dans une même affection de l'encéphale convulsion directe d'un côté, et paralysie croisée de l'autre. Il y a déjà plusieurs années que j’ai mis en doute ceux que l'on a rapportés; je ne sache pas que personne en ait vu depuis. Plus de deux mille paralytiques ont été soumis à mon examen; jamais je ne l'ai remarqué. Avant de vouloir expliquer le fait, il faut le constater.

Action et Maladies des tubercules quadrijumeaux.

La fréquence des maladies des organes est, dans 
l'ćconomie de l'homme, en raison directe de l'importance du rôle qu'ils jouent dans son organisation. Les tubercules quadrijumeaux justifient pleinement cette assertion dans l'encéphale; autant les maladies des lobes cérébraux sont communes, autant celles de ces tubercules sont rares. Les résultats de la physiologie et de la pathologie confirment, en ce point, les données de l'anatomie comparée, mais ne justifient pas sous d'autres toutes ses conséquences.

Ainsi, on aurait dù croire que la vision serait détruite par les altérations de ces tubercules; j'ai vu ces tubercules désorganisés, et je n'ai pas observé, dans ces cas, la perte de la vue.

A la suite de longs chagrins, un homme fut pris de la danse de St. Guy; cette affection revint d'abord à de très-longs intervalles; en $18_{11}$, elle devint continue; en 1812 , ne pouvant plus se livrer à ses occupations, il fut reçu à l'Hôpital de la Pitié. Les mouvemens étaient dans un désordre difficile à exprimer; la station était toujours agitée : si on forçait au repos ses nembres, la face était agitéc convulsivement, les yeux roulaient dans l'orbite avec une rapidité que l'observateur pouvait difficilement suivre. La marche était des plus bizarres : tantôt il levait les pieds plus qu'il n'était nécessaire, tantôt il les traînait ct chevaunchait par la rencontre de l'obstacle le plus líger. D'autres fois il levait trop une jambe, ot pas assez la jambe opposéc. Les nouvemens des bras étaient: 
aussi désordonnés que ceux des jambes. Voulait-il manger la soupe, il portait la cuillère au front, ou la renversait sur sa poitrine; ce n'était qu'après les efforts les plus pénibles qu'il parvenait à l'introduire dans la bouche. La langue ne partageait point cette irrégularité de mouvement.

Dans la colère, l'équilibre des mouvemens paraissait un instant rétabli; il marchait alors devant lui et ne s'arrètait que quand il rencontrait un arbre ou les murs de la cour; alors il se retournail tout d'une pièce et revenait sur ses pas : cet état était ordinairement suivi d'une attaque d'épilepsie, dont la durée moyenne était d'une demiheure ou de trois quarts d'heure. Nous trouvâmes, à l'ouverture du cadavre, un noyau dur et comme squirrheux dans la partie moyenne des tubercules quadrijumeaux; au pourtour et surtout en arrière, la substance cérébrale était ramollie et marbrée; le ramollissement s'étendait jusqu'à la partie interne des processus cercbelli ad testes.

Une femme hystérique et affectée d'un cancer de l'utérus, était vente dans rotre hôpital dans l'espérance d'entrer à l'hospice de la Salpétrière; mais dans une de leurs visites, les membres du bureau central la désignèrent pour être renvoyée: aussitôt elle devint taciturne, ne voulnt p̣lus.manger ni répondre aux questions qui lui étaient faites, ni même aux assurances qu'on lui donna, qu'elle scrait placée selon ses désirs. Elle restait immobile sur son lit; et quand ello se levait pour satisfnire 
à ses besoins, sa démarche était celle d'une personne irre; cinq jours après clle fut prise de mouvemens spasmodiques, sans attaque d'hystérie; ses jambes allaient involontairement dans son lit, ses bras étaient dans la même agitation. Ses yeux étaient tantôt fixes, tantôt dans la plus grande mobilité. Les pupilles étaient constamment contractées; le délire survint, et au délire succéda un état comateux et apoplectique, état comateux qui avait cela de particulier, que de loin en loin les jambes et les bras étaient pris de mouvemens spasmodiques et en apparence irréfléchis. A l'ouverture, nous trouvâmes un foyer sanguin situé au haut des cordons supérieurs du quatrième ventricule, au-dessous, par conséquent, des tubercules quadrijumeaux postérieurs.

Sur une autre femme de soixante-huit ans, qui nous avait offert, au plus haut degré, les mouvemens incohérens de la danse de St.-Guy, auxquels succéda une apoplexie foudroyante, toute la base des tubcrenles quadrijumeaux, principalement les tuborcules postérieurs, était le centre d'un foyer sanguin, qui, en avant, s'étendait jusqu'au dessous de la glande pinéale, et en arrière jusque dans l'épaisscur de la partic antéricure de la protubérance annulaire.

Une malade affectée de la danse de St.-Guy (1),

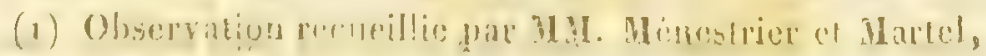
internen de ma divicinn. 
et décédée dans ma division en 1825 , nous a offert le premier degré de cette dernière altération. Les tubercules quadrijumeaux antérieurs et postérieurs étaient enflammés; les cordons internes du processus cerebelli ad testes l'étaient également et à un degré d'autant plus grand, qu'ils se rapprochaient davantage des tubercules quadrijumeaux (1).

Dans la danse de St.-Guy, les mouvemens sont involontaires, désoordonnés; ils pêchent par le défaut d'équilibre entre eux. Si, dans les cas que nous venons de rapporter, les symptômes de la danse de St.-Guy étaient le résultat de l'irritation des tubercules quadrijumeaux et de leurs dépendances, il était à présumer que l'expérience reproduirait ce qu'avait dévoilé l'observalion.

Il n'est pas de physiologiste qui, ayant expérimenté sur le cerveau, n'ait remarqué que la lésion la plus légère des tubercules quadrijumeaux est suivie de mouvemens convulsifs les plus énergiques et les plus désordonnés. En découvrant l'encéphale d'un chien et mettant à nu ces tubercules, on les produit et on les fait cesser à volonté. Mais ce n'était pas de cette manière que

(1) Je ne connais dans la science aucune observation de la danse de Saint-Guy, suivie de l'ouverture du cadavre; c'est pourquoi j'ai fait peindre avec le plus grand soin les altérations organiques rencontrées sur ces trois dernicrs malades. 
nous pouvions produire les symptômes que nous avions observés; il fallait que l'animal pût survivre un certain temps à l'expérience, résultat que l'on obtient par la ponction de l'encéphalc.

Sur un chevreau on fit traverser un bistouri très-effilé sur la base des tubercules quadrijumeaux : l'animal éprouva une douleur très-vive, suivie de mouvemens convulsifs dans les membres et dans les yeux, qui roulaient dans l'orbile. L'instrument fut laissé en place; l'animal s'apaisa au bout de deux heures; huit heures après l'opération on le fit lever. La démarche était on ne peut plus irrégulière, on eût dit qu'il ne savait plus marcher; souvent il remuait les pattes sans se mouvoir, d'autres fois il traînait celles de devant et levait outre mesure celles de derrière. On retira l'instrument, des mouvemens convulsifs se produisirent; les pupilles étaient contractées. Il resta daus cet état quatre jours, offrant, lorsqu'il était couché, des mouvemens spasmodiques dans les pattes, et marchant toujours avec la même irrégularité. Quand il voulait boire, il était longtemps avant de pouvoir mettre le museau dans le vase où était l'eau. Il avait également beaucoup de peine à saisir les herbes qu'il choisissait pour se nourrir.

L'instrument avait traversé la base des tubercules quadrijumeaux, et le gros faisceau sur lequel repose le processus cerebelli ad testes. Sur deux lapins ct un chien l'effet fut le même. Le profes- 
seur Rolando a obtenu aussi les mêmes résultats. J'ai observé, dit-il, sur quelques-uns des plus gros de ces animaux (cherreaux, agneaux, cochons, daims, chiens, chats), qu'après avoir déchiré tantôt les lubercules quadrijumeaux, tantôt une portion des couches optiques, il se manifestait des phéromènes qui démontraient que les muscles de l'animal ne se mouvaient pas dans un sens rigulier, mais avec une ugpèce d'irrégularité tout à fait semblable aux mouvemens d'un homme ivre. C'est pourquoi, quand ils marchaient, tantôt ils élevaient les pieds plus qu’il n'était nécessaire, tantòt ils marchaient en les traînant.

Si on traverse chez les oiseaux les deux lobes optiques par leur base, on produit les mêmes effets; mais comme alors on a compris dans la blessure les pédoncules cérébraux, on conçoit que le résultat est compliqué. Pour apprécier ce qui appartient aux lobes eux-mêmes, il faut les blesser isolémen et alternativement. La blessure du lobe droit fait tourner l'animal à gauche, en produisant de ce côté des mouvemens convulsifs; en blessant le gauche l'animal ne tourne plus, il danse sur ses pattes; jeté en l'air, son rol est très-irrégulier. ía nême agitation, lc mème décousu des mouvemens se remarquent chez les grenouilles. Il est à remarquer que chez les mammifères et les oiseaux la blessure ou l'ablation d'un seul tubereule fail comme lianimal sur le?

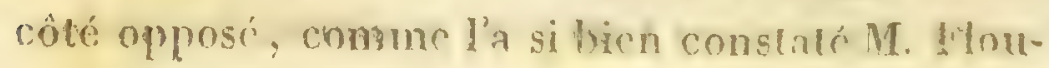


rens; tandis qu'il tourne sur le même cóté de la blessure chez les reptiles ( 1 ), toujours par la raison que j'ai donnée, du non-croisement des pyramides dans les deux classes inférieures.

Dans les altérations pathologiques des tubercules quadrijumeaux, je n'ai pas remarqué d'altération sensible de la vue; dans les expériences sur les mammiferes, elle m'a paru quelquefois troublée momentanément, mais à un degré si faible que l'on peut douter que ces corps exercent une action directe sur la vision. Il n'en est pas de même chez les oiseaux, les reptiles et les poissons : la vue est constamment affaiblie ou. perdue complètement, selon que les lobes optiques sont blessés, détruits ou enlevés en totalité ou en partie.

L'action de ces lobes est croisée, c'est-à-dire que constamment la blessure ou l'extirpation du lobe droit trouble ou anéantit la vision de l'oeil gauche, et celle du lobe gauche la vision de l'oeil droit. Dans ces opérations l'iris est sensiblement affectée; et ce qui paraîtra contradictoire à ce que déjà nous avons dit, liíis du côté droit est affectée par l'excitation ou l'ablation du lobe gauche, et celle du côté gauche par les semblables mutilations du lobe droit; mais dans ces

(1) Si los tuberculen quadrijumeans sont careilatemes de

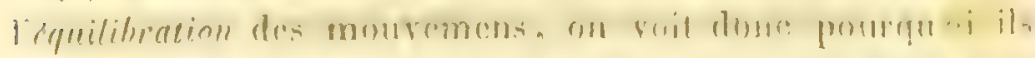

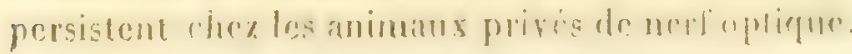


cas l'iris n'est pas affectée directement, clle no l'est que conséculivement au trouble porté dans l'action de la réline et du nerf optique, comme cela arrive tous les jours dans les paralysies de ce nerf (1). C'est donc à la sur-excitation ou au défaut d'excitation de la rétine que sont dus, dans ce cas, la constriction ou le relâchement de l'iris. Voici une expérience directe qui le prouve. Si dans la chambre obscure on fait tomber un pinceau de rayons lumineux sur la rétine, en le dirigeant par l'ouverture pupillaire, l'iris se contracte aussitôt; si on dirige le même pinceau sur l'iris, elle reste immobile; l'iris est donc insen- sible à l'action de la lumière : elle est insensible aussi aux irritations directes, comme je m'en suis plusieurs fois assuré chez l'homme. Cette propriété lui est commune avec larétine, ainsi que l'a constaté M. Magendie.

L'iris se meut chez tous les animaux vertébrés, excepté chez certains poissons et quelques reptiles, où elle est fixe. Comment se meut-elle? Cette question tant débattue pour et contre les opinions de Haller, est loin d'offrir encore une solution satisfaisante, Ce grand physiologiste ayant

(1) C'est là ce qui a fait dire à Haller que le nerf optique était le siége de la contraction de l'iris; en le plaçant, comme on l'a fait dernièrement, dans les tubercules quadrijumeaux, c'était donc reproduire sous d'autres termes l'opinion de $\mathbf{H}$ aller. 
attribué exclusivement l'irritabilité aux muscles, et tout mouvement devant être produit par eux, on lui opposa cette membrane, qui était, selon Whytt, mobile et sensible en même temps. Haller nia qu'elle fùt musculeuse. Il aurait pu conclure de ses travaux et surtout de ceux de Zinn, qu'elle était nerveuse; mais il se fût mis en contradiction avec lui-même; car une membrane nerveuse eût été sensible et irritable, ce qui répugnait autant à sa doctrine que d'admettre qu'une partie irritable était sensible. Les physiologistes modernes adoptant les vues de ce grand homme sur l'irritabilité, s'obstinèrent à y trouver des muscles, que cela s'accordâl ou non avec ses vues sur la sensibilité. Monro en trouva deux, l'un circulaire, l'autre à fibres longitudinales; M. Maunoir les retrouva; MM. Hoine et Bauer y ont ajouté le muscle circulaire de la membrane hyaloïde. Les fibres de l'iris existent circulaires et longitudinales, comme l'ont dit les deux premiers anatomistes, quoiqu'ils diffèrent sur la position des premières. Tous les anatomistes les ont vues ou peuvent les roir. Mais ces fibres sont-clles musculeuses? Leur continuité immédiate avec les nerfs ciliaires détruit cette supposition; la raison sur laquelle on se fonde, c'est qu'elles sont contractiles, ct il répugne à nos doctrincs actuclles sur le système nerveux d'admettre la contractilité au nombre des propriétés physiques des nerfs. Mais si cette propriété existe réellement dans les nerfs ciliaires, la question des 
mouvemens de l'iris me paraît rósolue, et résolue sans des suppositions quc repoussent son organisation et sa texture.

Ce phénomène de la contractilité des nerfs ciliaires est des plus intéressans. Si, après avoir ouvert l'oeil sur un animal, on enlève avec soin un nerf ciliaire, aussitôt qu'on le saisit avec des pinces par l'une de ses extrémités, l'autre s'en rapproche, en formant des spirales si rapprochées et si rapides, que dans moins de deux secondes le nerf est réduit au vingtième de sa longueur; il se roule sur lui-même, se pelotonne. Si dans cet état on le plonge dans l'eau, il reprend sa longueur primilive, et en le retirant il ze roule de nouvcau comme la première fois. On peut répéter cent fois ces expériences, cent fois le résultat sera le même. Sur l'homme elle réussit constamment aussi, et absolument de la mème manière, un, deux et trois jours après la mort. Chez les oiseaux, les reptiles et les poissons, la rétraction du nerf est aussi instantanée que chez les mammifères. Voilà sans doute un phénomèe inattendu dans la science; et si maintenant on se rappelle la disposition de ces nerfo dang l'iris, on verra qu'il n'est nullement nécessaire de l'intervention des muscles pour expliquer sa mobilité. (1)

Chez les animax à pupille fixe, il n'existe

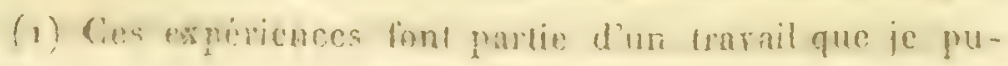


PIVSIOTOCIF HT MATIOLOGIE.

sans doute pas de nerf ciliaire; je l'ai constaté chez la grenouille; on sait que chez ce batracien la pupille n'éprouve aucune mobilité, et qu'une disposition particulière garantit la rétine de l'action trop vive de la lumière. Les recherches les plus soignées n'ont pu me faire découvrir les nerls ciliaires ni dans la texture de l'iris, ni hors du globe de l'øeil. En sera-t-il de même chez les poissons à pupille immobile? si cela est, la motilité ou l'immobilité de celte membrane serait due à la présence ou à l'absence des nerfs ciliaires.

Action et maladies des himisphires cirébraux.

En arrivant aux hémisphères córébraux, nous nous retrouvous en présence des grandes queslions dont se sont occupés les physiologistes relalivement au siége de la sensibilité et des mouvemens, des sensations et des facultés intellectuelles. Nous retombons, plus que dans toules les autres partics, dans le cercle des supposilions, iles hypothèses et des systèmes.

Duand les fluides jouissaient du privilege exclusif de iout produire daus le systeme merreux, ils étaicnt a-la-fois la causc essenticllo du senti-

Blierai insesaninent sur la contractilite du systeme norrens: of fles autres lisdus non muscthaires. 
ment et du mouvement; les nerfs $\mathrm{n}^{*} \mathrm{en}$ étaient que les dispensateurs. Lorsque les membranes du cerveau dépossédèrent les fluides, on transporta sur celles-ci tout ce que l'on avait attribué à ceuxlà. Ce transport ne se fit pas subitement; on supposa d'abord que les enveloppes étaient le siége de la sensibilité, que les nerfs sensibles y puisaient leurs racines, et que les nerfs moteurs tiraient les leurs de la masse cérébrale. Riolan spécifia cette idće en concentrant sur l'arachnoïde toutes les propriétés que l'on attribuait aux membranes en général et à la masse nerveuse; il assigna des facultés diverses à l'arachnoïde externe et à celle qui tapisse l'intérieur des ventricules. Baglivi, dont l'esprit ne pouvait s'accommoder de toutes les vues de Riolan, trouva plus facile de faire de la dure-mère l'organe unique du sentiment et du mouvement. Tout cela se passait à une époque où le fluide nerveux avait, par un changement de nom, remplacé les esprits animaux; on voulut le faire circuler comme le sang auquel on le comparait : il fallut dès-lors lui trouver un cœur; la dure-mère remplit cet office : cette membrane devint le principe de tous les mouvemens; elle agissait sur la substance corticale dont elle exprimait le fluide nerveux, qu'elle transportait ensuite avec la rapidité de la pensée jusqu'aux extrémités les plus éloignées. La sensibilité était étroitement liée à l'exercice des mouvemens : la dure-mère devint le foyer de cette propriété: 
PIYSIOLOGIE KT PATIOLOGIE.

comme elle était le principe de tous les mouvemens. On aperçoit dans cette hypothèse un mé. lange des idées de Riolan avec l'opinion de Malpighi, qui avait fait un organe sécréteur de la masse des lobes cérébraux, et qui, pour unc idée qui aujourd'hui nous paraît si peu raisonnable, avait fait rejeter les hautes pensées de Willis, son antagoniste. Ce fut contre ces idées que combattit avec tant de persévérance toute l'école de Haller.

Ainsi présentées dans toute leùr nudité, ces opinions répugnent présentement à notre esprit ; mais quand on lit avec attention ce que l'on a écrit depuis quelques années sur l'arachnitis, on trouve que nous avons peut-être dépassé Riolan dans les fonctions qu'il attribuait à l'arachnoïde. On ne voit pas en effet ce qu'il resterait à faire à l'encéphale, si l'arachnoïde exerçait l'action qu'on lui suppose. Pour rendre cet organe aussi nul qu'il l'était dans les vues de Baglivi et de Pacchioni, il ne nous reste qu'à généraliser quelques idées émises dans ces derniers temps à l'occasion d'un fait dans lequel l'intégrité des mouvemens paraît avoir survécu à la destruction re la partie matérielle de l'axe cérébro-spinal. Ainsi la science se replie sur ellemême de siècle en siècle, et les anciennes erreurs pèsent de lout leur poids sur notre esprit, à mesure que nous faisons un pas.

La pie-mère avait été nćgligée dins ces diverses hypothèses; mais elle eut son tour après les belles 
injections de Ruysch. Bocrhave, slipposant la matière médullaire de l'encéphale composée de vaisseaux, plaça toute l'action nervense au point de contact de ces vaisseaux avec les artères provenant de la pie-mère et de la substance corticale. Les autres membranes élaient étrangères à ces actions. Reil eût donné à cette iđée une grande vogue si ses opinions physiologiques eussent cu le succès de ses recherches anatomiques; il distingua dans le uerf l'enveloppe et la pulpe : la première était un étui formé par la pie-mère, la seconde élait le prolongement de la pulpe cérébrale; il doun l'enveloppe du privilége de prodnire les mouvemens, et fit de la pulpe l'organe de la sensibilité. Annemanu crut avoir parouvé cette assertion, en établissant qu'après la cicatrisation des nerfs, le mouvement seul se rétablissait, le sentiment étant pour toujours anéanti: a cotte supposition, Aruemann en ajouta une autre, en assurant que dans la reproduction des nerfs, l'enveloppe seulc se régónérait : il expliquait de cette maniere le retour du mouvement et lit perte de la sensibilité. Reil w'ayant fait sucune expériehce, Vicq-d'Azyr et Bichat supplérent à celłe onission : ils isolirent le prétcridu néritime de la puipe, l'irritèrent et ne prodiaisireat nien de ce que faisit espérer lilypolhèse de Reil. Ces expériences directes firent oublirr cette opinion déjà jugie, avant qu'alle parùt, par celles de Maller, de Castell da Walstonf ei sle Tuatui. 
Enfin, les idées de Willis, qui rétablissaient dans son empire l'axe cérébro-spinal, prévalurent; Boerhaave avait déjà préparé ce retour, dont la gloire restera à l'école de Haller. Un philosophe moderne a dit que l'homme était une intelligence servie par des organes : en traduisant cette haute pensée dans le langage anatomique, on peut ajouter que les instrumens de cette intelligence sont les diverses partics du système nerveux. Chercher ailleurs que dans ce système le siége du mouvement, du sentiment, des sensations et de l'intelligence, c'est rentrer dans les routes qui nous ont égarés jusqu'à ce jour.

Mais à peine eut-on entrevu cette grande vérité, qu'aussitôt on imagina de trouver dans l'encéphale un point qui régît toutes les autres parties. Cette prérogative fut accordée à la glande pinéale par Descartes, Muralt, Gaukes; au corps strié et à la couche optique par Willis, PourfourPetit, Saucerotte et Sabouraut; au septum lucidum par Digby, Kenalm, Duncan; au corps calleux par Bontekoë, Lancisi, Lapeyronie; à la moelle allongée par Hoffmann, Blancard, Schelhamer, Lorry : enfin de nos jours, Socmmering le père, interprétant une pensće de Boerhaave, a replacé dans les parois du quatrième ventricule le siége du sensorium commune; c'est revenir après de longs détours à l'idée favorite du médecin de Pergame. Une vérité fondamentale ressort cependant de ce déplacement allernatif du sensorium 
commune; c'est que les fonctions de l'encéphale sont réparties dans toute sa masse et qu'aucune partie ne les régit toutes : et une scconde vérité qu'on aurait pu déduire des recherches précédentes, c'est que chaque partie y concourt à sa façon. Or c'est précisément la part que chacune y prend, que nous cherchons présentement à déterminer.

Cette diversité d'action de l'encéphale avait hypothétiquement été entrevue. Boerhaave rejetant l'idée d'un sensorium commune, avança que chaque nerf devait avoir une portion de pulpe cérébrale qui lui fût propre; il regarda cette partie comme la limite fixe des impressions, des sensations et des actes que le nerf reçoit, conserve et exćcute. Il y avait dès-lors autant de centres d'action que d'origines distinctes de nerfs. Willis avait encore à ce sujet devancé Boerhaave, comme déjà nous l'avons dit; son système n'a jamais été bicn compris, et peut-être avait-il à craindre, à l'époque où il écrivit, que ses détracteurs ne le comprissent trop bien : considérant que les mammifères étaient seuls pourvus de circonvolutions, et que l'homme était placé à leur tête par la profondeur et l'étendue de ces replis, il fit de ces parties le réscrvoir des idćes; il doua la substance corticale de la faculté de les reproduire (organe de la mémoire ) ; le corps calleux, de celle de les féconder (organe de l'imagination ); les corps striés et la couche optique placés entre ces deux 
PHYSTOLOGIE ET PATHOLOGIE.

principales parties, furent le sensorium commune et l'organe les mouremens volontaires. Les idécs furent donc localisćes par cet habile médecin, mais les localisatious ne furent point spécifiées: MII. Gall et Spurzheim ont entrepris cette dernière tâche, en supposant comme démontré que les saillies ex térieures du u crâne répètent exactement les saillies des circonvolutions, et que les goûts, les penchans, l'aplitude de l'esprit et ses diverses applications, se trouvent trahis de cette manière par les enfoncemens ou les saillies des coronaux, des temporaux, des parićtaux et de l'occipital. C'est cependant celte partic, tout-à-fait hypothéthique, de leur beau travail, qui en a précisément fait la fortune.

Les actes de l'encéphale dérivant tous de la scnsibilité et des mouvemens volontaires, on devait d'abord s'occuper de localiser ces deux propriétés fondamentales dans la masse de l'encéphale, comme on avait tenté de le faire sur ses enveloppes. Willis ayant fait du cervelet l'organe de la sensibilité, il fut presque reçu par ceux qui adoptèrent ses vues, que les lobes cérébraux étaient complètement insensibles; ils furent réservés par ces divers auteurs aux mouvemens volontaires. Mais Lorry ayant trouvé que la moelle allongće était la source des mouvemens et le foyer de la sensibilité, les lobes cérébraux et le cervelet se trouvèrent en même temps tépossédés de leurs prérogatives. Puis, plus tard, on a reconnu l'influence 
que le cervelet exerçait sur les mouvemens : on a alors exagéré cette influence, comme Willis avait exagéré celle des lobes cérébraux. Les lobes cérébraux considérés primitivement comme le principe des mouvemens volontaires, sont considérés maintenant comme leur étant tout-à-fait étrangers. On a vu, de plus, que dans les idées de Reil et d'Anermann, ils devaient être le principal foyer de la sensibilité. Ainsi en moins d'un demi-siècle, les lobes cérébraux et le cervelet ont entièrement échangé leur destination.

De plus, dès que l'on s'occupa de la physiologie du cerveau, la différence des deux substances qui le composent frappa tous les esprits: de cette différence, que l'on exagéra beaucoup, on déluisit une diversité d'actions; on supposa que deux parties, dont l'une était blanche et l'autre grise, ne sauraient concourir à des actions semblables; on a déjà vu à ce sujet les hypothèses de Willis, de Wepfer, de Vieussens et de MM. Gall et Spurzheim; je passe sous silence celle de Riolan, et jarrive aux opinions de nos jours. Selon les uns, la matière grise est la partie éminemment active de l'encéphale, c'est l'organe de la sensibilité; et selon les autres, elle est le principe unique des mouvemens: on conçoit que chez ces derniers la matière blanche est la partie sensible de l'encéphale, et que pour les premiers elle est l'organe des mouvemens. Pour juger par un seul fait ces deux opinions, il n'y a qu'è considérer la moelle épinicre. chez 
tes animaux dans lesqueis la matiere blanche la constitue uniquement; l'irritation de cette moclle épinière provoque les plus vives douleurs et les convulsions les plus actives. Donc la matiere grise n'est ni l'organe unique de la sensibilité, ni le principe des mouvemens; donc aussi la matière blanche excite à-la-fois et les mouvmens et la sensibilité.

En définitive donc, la sensibilité a été attribuée exclusivement au cervelet, aux hémisphères cérćbraux et à la moelle allongée; le principe des mouvemens a tour-à-tour été placé dans les lobes cérébraux, dans la moelle allongée et dans le cervelet; et selon que l'une ou l'autre de ces proprićtés a été déplacée, on a changé la destination des parties fondamentales de l'encóphale. A quoi iient cette oscillation? Toujours à cette malhenreuse tendance qui nous porte à généraliser des résultats particuliers.

Il est évident, par exemple, que d'après les expériences physiologiques, la moelle allongée est le foyer principal de la sensibilité. Il n'esî pas moins certain que les altérations pathologiques du pont de Varole, et de la parlic de la moelle allongée qu'il embrasse sont toujours accompagnées de la perie de la sensibilité. Mais s'ensuit-il que le cervelet et les lobes cérćbraux soient insensibles? non, sans loutc : rien ne mène à colle induction, ni l'analorie, ni l'expéricnce. L'analogic la repousse, car elle: nous montre partout le sys- 
tème nerveux sensible. L'expérience la rejette également, car toutes les fois que l'on plonge un instrument à une certaine profondeur, soit dans les lobes cérébraux, soit dans le cervelet, une douleur vive se manifeste: la scnsibilité est mise en jeu. Les maladies donnent le même résultat queles expériences : des douleurs aiguës précèdent ct accompagnent les lésions matérielles de ces deux organes (1). Dans les expériences, de même que dans les cas pathologiques, le cervelet paraît plus sensible que les lobes cérébranx, et ce résultat est conforme à la distinction expérimentale des nerfs, en ccux qui sont sensibles et ceux qui sont irritables. La scnsibilité est donc répandue dans toute la masse de l'encéphale, quoiqu'il paraisse bien établi que la moelle allongée est le principal siége de cette propriété.

Lin sera-t-il de même des mouvemens volontaircs? Si l'on avait bien réfléchi aux faits d'après lesquels Willis avait fait des lobes cérébraux le centre unique de cet ordre de mouvemens; si les observations innombrables qui constatent l'existence des paralysics à la suite des lésions maté-

(1) Il est à remarquer que chez les oiseaux, les lobes cérébraus et le cervelet sout beaucoup moins sensibles que chez les manmifères; ce que Vicq- l'Azyr arait déjà observé. Remarquez anssi que c'est ioujours chez les oiseaux que l'on a fait les expériences d'ajrès lesquelles on préjuge l'insensibilité du cervelet et des lobes cérúbraux. 
PHYSIOLOGIE ET PATIOLOGIE.

rielles de ces lobes, avaient été prises en considération, assurément jamais les physiologistes n'eussent conclu de leurs expériences sur certains animaux, que ces parties étaient complètement étrangères aux mouvemens volontaires. Les résultats de l'expérience pathologique sont ici si tranchés, si multipliés, si décisifs, qu'il faut les taire pour les récuser. Car l'influence des hémisphères cérébraux sur ces mouvemens ressort de toutes parts : cette vérité intéresse de trop près la médecine pour la traiter légèrement.

Et d'abord, nous devons observer combien est compliquée la détermination du siége spécial des mouvemens volontaires dans l'encéphale. Nous avons déjà vu que la protubérance annulaire et la moelle allongée semblent les tenir exclusivement sous leur dépendance; nous avons trouvé aussi que le cervelet exerce sur leur manifestation une puissante influence; nous avons remarqué, enfin, leur discordance chez les mammifères à la suite des lésions des tubercules quadrijumeaux. Si nous avions généralisé chacun de ces résultats en particulier, nous aurions pu considérer comme l'organe des mouvemens volontaires l'une ou l'autre de ces partics, ou même toutes les trois réunies, et dis-lors les lobes cérébraux se fussent trouvés en dehors de nos explications; mais en appliquant à ces lobes la méthode qui nous a dirigés dans les autres partics, nous allons déterminer la part 
qu'ils prennent dans l'exercice de ces mouvemens.

Observons encore que les altérations de la moelle allongée et de la protubérance annulaire frappent également d'immobilité les extrémités supérieures et inférieures; que les hémisphères ducervelet paralysent plus spécialement les membres inférieurs que les supérieurs, et disons par anticipation que les lobes cérébraux influencent plus généralement et plus spécialement les membres supérieurs que les inférieurs. C'est ce que nous exposerons après avoir établi que les radiations des corps striés tiennent plus spécialement sous leur dépendance le membre inférieur, et celles de la couche optique le membre supérieur (1).

Le nommé Vandaest, tailleur d'habits, âgé de vingt-deux ans, était affecté depuis dix-huit mois d'un écoulement purulent de l'oreille droite, accompagné d'une dureté de l'ouïe du même côté, et survenu spontanément, sans qu'il se rappelât avoir reçu des coups à la tète, ou fait des chutes sur cette partie. Dans les derniers jours de mars 1811 , ce jeune homme éprouva le soir un violent frisson, suivi la nuit d'une sueur abondante, d'ure vive céphalalgie et d'efforts pour vomir, sans vomissement, quoiqu'il n'eût pris que de l'eau sucrée

(1) Voyez Annualre midico-chirurgical des hópitaux do Paris, année 1819 
dans le cours de la journée. Ces frissons et l'accès fébrile qui les suivait se renouvelèrent tous les soirs, et, soit qu'il eût ou qu'il n'eût pas mangé dans la journée, les cnvies de vomir se manifestaient pendant la période du frisson et de la chaleur. Dès le sixième ou septième accès, il éprouva, quelques instans avant son invasion, des mouvemens convulsifs dans le bras gauche; mouvemens convulsifs à la suite desquels le bras était si fatigué, que le malade ne pouvait se livrer à son travail habituel que dans le milieu de la journée.

Le 15 avril de la même année, il fut obligé de le cesser tout-à-fait, le vésicatoire et les linimens avec lesquels il avait frictionné ce membre n'ayant apporté aucun amendement à la faiblesse et à l'engourdissement qui succédaient aux mouvemens convulsifs. Les accìs cessèrent alors de se manifester ; mais le malade était faible, abattu, mélan * colique; une douleur vive et profonde de la tête ne le quittait jamais, et la suppuration de l'oreille droite coulait toujours avec la même abondance. Ses amis le voyant s'affaiblir de jour en jour, le firent porter à l'Hôtel-Dieu le 24 du même mois. Voicil'état dans lequelil se trouvait le soir de son entrée. Céphalalgie aiguē, paupières fermées, douleur vive des yeux provoquée par la lumière, vue nette, pupille ni dilatée ni contractée; le malade avait couvert sa tête avec le drap du lit pour garantir ses yeux du jour. A neuf heures du soir, convulsions violentes du bras gauche, louleur do tête plus 
vive, respiration accélérée, pouls fréqueut et serré.

Le 25 , à la visite du matin, les couvulsions du bras avaient cessé; le malade était très-affaissé, taciturne; la langue ćtaitjaune, chargée, la bouche pâtcuse; toute la nuit il avait éprouvé des nausées. On prescrivit un vomitif, des lavemens éralliens et une tisane amère : il vomit abondamment; les vomissemens ou les efforts pour vomir continuèrent toute la journée; le soir la face était profondément altérée, les yeux ternes; le bras, qui, pendant le vomissement, avait été agité par les convulsions, était dans une roideur tétanique; on ne pouvait le fléchir sans de grands efforts; le pouls était vif, petit, la respiration gênée; les yeux étaient d'une telle susceptibilité à l'action de la lumière, qu'il avait toujours la figure recouverte du drap du lit. Les facultés intellectuelles étaient parfaitement intègres.

Le 26 et le 27 , même état; légères envies de vomir, face terreuse, mouvemens convulsifs légers dans le bras, et à des intervalles éloignés ; pouls petit, très-fréquent, faiblesse-très-grande. ( Lim. végétale, Hoffm. trente gouttes, julep centi. sirop d'ipécacuanha une once, lavement de camomille avec un demi-gros de camphre. ) La nuit trèsmauvaise. Céphalalgie continuelle.

Le $2 \delta$ au nuatin, face hippccratique, pouls petit, intermittent, respiration trés-difficile. Céphalalgie vive, facultés intcllectuclles libres. (Même prescription.) A une heure de l'après-midi, perte de 
connaissance; convulsion du bras gauche, suivie d'une roideur tétanique des plus prononcées; écoulement par le nez et par la bouche d'une écume blanehe mousseuse, comme dans les accès épileptiques : à une heure et demie, le malade présente tous les signes d'une mort prochaine, et la cause de la maladic étanl inconnue, on ne change rien au traitement. Mort à deux heures aprèsmidi,

J'ai copić textuellement l'observation : car les doutes que nous avait laissés la maladie nous firent apporter une attention parliculière à l'autopsie cadavérique. L'abdomen n'offrit rien de particulier, ainsi que les organes thoraciques.

Le crâne ouvert, les membranes nous parurent dans leur état ordinaire. A la base du cerveau, et à la partie du lobe postérieur qui repose sur la face supérieure du rocher et la tente du cervelet, nous trowâmes, du côté droit, les altérations qui suivent : $1^{\circ}$. cxtéricurcment, dans l'étendue d'un pouce, la coulcur di lobe postérieur était d'un noir livide; cette partic mćlanosée était entouréc, dans l'élenduc de deux pouces environ, d'une aréle de couleur jaune pâle : toule la partic altérćc ólait beaucoup plus molle que le reste de l'hémisphòre. $2^{\circ}$. Le cerveau, incisé transversalement sur cetie partie noire livide, nous pénétrâmes dans un foyer d'environ deux pouces el

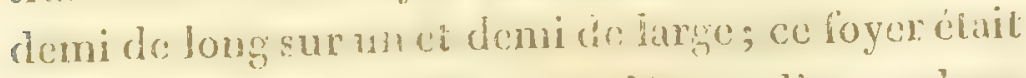
rempli par un pulrilage, verdâtie, d'une odeur 
infecte. Ce putrilage enlevé par le moyen de l'eau, les parois internes du foyer étaient tapissées d'aspérités rougeâtres, et à quelques lignes de leur épaisseur, les vaisseaux sanguins étaient singulièrement développẻs.

Le rapport de cette altération avec le rocher du còté droit nous ayant fait soupçonner que la maladie s'était peut-être propagée de l'intérieur de l'oreille ả l'encéphale, nous recherchâmes avec soin leurs connexions, mais nous n'en trouvâmes aucune d'immédiate. Toutefois les lames supérieures du rocher étaient cariées; les canaux demi - circulaires avaient disparu; la caisse était remplic par unc matière purulente jaune, analogue à celle que le malade rendait par le conduit auditif externe; la membrane du tympan était détruite à sa parlie inférieure, et c'est au travers de cette perforation que s'échappait la suppuration de l'oreille interne.

C'était la première fois que j'observais un fait si décisif; il était en effet impossible de ne pas rapporter les convulsions du bras gauche à l'irritation vive et prolongée dont la base du lobe postérieur droit avait été lc siége. Ce rapport fut aussitôt saisi par tous les élèves de la clinique, el plus spécialement par ceux qui me secondaient dans mes recherches sur les maladies organiques du cerveau : depuis, l'observation l'a si fréquem-ment confirmé, qu'il est peu de points de la pathologie micux établis que celui-li ; mais avaut le 
passer outre, je désire montrcr, dans l'intérêt de la science et de la vérité, qu'un fait à-peu-près semblable s'était offert à un médecin très - judicieux, et qu'il en avait déduit les mêmes conséquences. J'ai trouvé, il y a quatre ans, ce fait dans les oeuvres de Sandifort.

Un homme d'un tempérament mélancolique avait vécu dans un état de santé permanent jusqu'à sa quarante-deuxième année; à cette époque des contrariétés domestiques fréquemment renouvelées le firent devenir tout-à - fait mélancolique. Dans cet état, il euî recours aux soins d'un médecin pcu habile, qui lui fit prendre beaucoup de vomitifs et le soumit à un usage abondant d'eau froide; à la suite de ces moyens, et aussi après que le malade se fut plusieurs fois exposé à un air très-froid, il fut pris subitement d'une attaque d'épilepsie. Cette attaque terminće, et le malade revenu à lui-même, il éprouva subitement aussi au bras et à la main gauche une telle irritabilité et une si grande seusibilité, que par l'impression de l'air froid sur ces partics il éprouvait des mouvemens convulsifs, et seulement de ce membre supéricur, des muscles de la bouche, des joues ct de toute la tête. Dans la suite, chaque fois qu'il y avait de grandes variations atmosphériques, ou seulement les variations provenant des changemens des saisons, et même lorsqu'il éprouvait des affections tristes, aussitôt l'accès épileptique se reproduisait. 
Le malade vécut ainsi pendant quatre ans sous l'influence de cette affection.

Pendant tout cet espace de temps le malade se plaignit d'une douleur obtuse lui faisant ćprouver comme un sentiment de pression dans le còté droit de la tête, qu'il désignait en portant la main vers la région inférieure du pariétal, et au-dessous : cette douleur disparaissait quelquefois complètement, d'autres fois la durće était longue ou couite; les paroxysmes étaient plus ou moins éloignés, et plus ou moins douloureux : indépendamment du lieu de la douleur que le malade précisait, comme on l'a vu plus haut, il assurait encore que le volume n'en devaic pas dépasser celui d'un ouf de poule. La dernière année de sa vie il eut de temps à autre un flus hémorroïdal, qui ne le soulagea. point.

Pendant quatre années que dura cette maladie, le malade eut recours à plusicurs médecins; plusieurs vésicatoires furent appliqués sur le bras affecté (le gauche); lesquels étant guéris, on appliquait alternativement un séton à la nuque : la dernière année il conserva un vésicatoire au bras.

Le malade, fatigué de l'inutilité des moyens employés, désireux de recouvrer sa santé première, et ayant trouvé dans des livres de médecine l'indication de médicamens qu'il croyait propres à son état, eut recours aux eaux minérales acidules, qu'il prit : ces caux relâchèrent le ventre, firent revenir 
un peu l'appétit; mais quelque temps après il survint des romissemens de matières glaireuses.

Pendant tout ce temps, la doulcur de tête que le malade ressentait sous le parictal droit augmenta; on y appliqua un onguent balsamique dans lequel entrait le castoreum, et dont le malade s'était bien trouvé précédemment; mais cela ne produisit rien cette fois-ci, la douleur de tête s'accrut continuellement. On appliqua un cataplasme avec de la mie de pain, ce qui parut soulager un peu le malade : enfin le jour suivant il tomba dans la stupeur; on lui donna une polion antispasmodique forte; mais il mourut arant d'avoir fini cette potion.

A louverture du cadavre on trouva :

Que le foic était plus gorgé de sang que de coutume; il était un peu verdâtre à sa concavité inféricure. Les intestins avoisinant la vésicule du fiel étaient plus verdâtres que de coutume. Les viscères de l'abdomen étaient aussi plus gorgés de sang; du reste, les viscères pectoraux ne présentèrent aucune altération:

Le crâné étant cnlevé, on trouva vis-à-vis le lieu où le malade avait rapporlé la doulcur pendant la vie, la substance corlicale airsi que la médullaire voisine, beaucoup plus dures que de coutume ct vérilablement squirrheuses. Au-dessous de ce lieu induré, lequel le defunt avait souvent désigne avec le doigt, on découvrit un abce's qui aurait pu contenir un $x u f$ de poule; la cavité de cet abcés élail remplie par une ma- 
tière jaune grumeleuse, molle, muqueuse; le fond de l'abcès était occupé et environné d'une substance d'un rouge livide, dans laquelle les petits vaisseaux parurent enflammés.

Si maintenant nous rapprochons les altérations encéphaliques observées sur ce cadavre, des douleurs que le malade a éprouvées, du siége, des symptômes, et si nous les comparons, nous pourrons en déduire trois corollaires très-uliles pour la pratique : $1^{\circ}$. que la structure, la fabrique et l'action du cerveau sont décussées ou entrecroisées; de telle sorte que l'action et le sentiment de l'un des côtés du corps résident dans le côté opposé de l'encéphale; car le malade avait toujours souffert du côté droit, où l'abcès a été trouvé après la mort, et l'irritabilité, les convulsions, se sont manifestées toujours sur le bras gauche; $2^{\circ}$. que la cause des spasmes, des convulsions et de l'épilepsie, peut être placée profondément hors de la portée des moyens de l'art; $3^{\circ}$. que, d'après une multitude d'observations comparées habilemeut, on pourra désigner la partie du cerveau affectée par le membre frappé de paralysie ou de convulsions. Ainsi, si c'est le bras droit, l'altération organique sera dans l'encéphale, dans la portion qui correspond au-dessus du pariétal gauche, et vice versit. Ainsi, dans notre histoire, par exemple, cette douleur et cet abcès étaient dans l'os pariétal droit; les convulsions et l'irritabilité étaient dans le bras gauche. 
Voici d'autres faits qui justificnt ces prévisions.

Copeau (François), âgé de cinquante-neuf ans, tourneur en ivoire, avait reçu, il y avait trois ans, un coup de marteau au-dessus et en arrière de l'oreille gauche. Six mois après, il fut pris de convulsions qui dégénérèrent en épilepsie : ces convulsions débutaient toujours par le bras gauche, où il ressentait une faiblesse très-marquée plusieurs jours après l'attaque. Deux ans après la première invasion de l'épilepsie, les accès se succédaient avec rapidité; à cette époque, le bras gauche restait immobile, même pendant les convulsions; il était complètement paralysé pendant les temps ordinaires.

Le 20 mars 1818 , le malade entra à l'hôpital de la Pitié, affecté d'une pneumonie aiguë, qui résista à tous les moyens qui furent employés. Il mourm! le 25 du même mois.

Voici les symptômes qu'il nous avait présentés : la face était pâle, le regard hébêté; les réponses étaient justes, mais tardives : le bras gauche était complètement paralysé, l'avant-bras était fléchi sur le bras, les doigts fléchis dans la paume de la main ; le malade paraissait éprouver une grande douleur, quand on voulait les étendre. Le bras gauche était en ontre émacié; le pouls de ce côté élait beaucoup moins fort que celui du côté opposé; les mouvemens étaient libres dans la jambe gauche et dans tout le côté droit.

II. 
Lc poumon droit était hépatisé dans toute son étendue, et adhérent aux côtes dans son tiers inférieur.

La base du lobe postéricur de l'hémisphère droit du cerveau étalt dans un état d'induration remarquable. Le lobe incisé en cet endroit, nous rencontrâmes daus son centre un abcès qui aurait pu contenir un petil ouf de poule; la cavité de cet abcès était remplie par une matière jaune verdâtre, cntrecoupée par une multitude de petits vaisseaux. Ce foyer correspondait aux radiations postéricures de la couche optique.

Ges faits mettent hors de doute l'influence spéciale que les radiations inférieures de la couche optique exercent sur les mouvemens du bras : les suivans nous montreront le mème effet produit par les radiations moyennes et supérieures.

Berscot (Pierre-Antoine), âgé de soixante-neuf ans, entra à l'Hôtel-Dieu le $\mathrm{x}^{\text {or }}$ mars 1811 , et mourut le 20 du même mois. A son entrée, il était paralysé du bras gauche depuis trois jours. Il fut traité par les purgatifs, les sudorifiques et les vésicatoires; mais tous ces moyens furent sans aucun effet: le bras gauche resta toujours dans une immobilitć parfaite.

L'autopsie montra un épanchement considérable de sang, en caillots consistans et séparés, logé dans la convexité du lobule postérieur de lhémisphère Iroit du ccrveau, à une liggne ou 
deux en dehors et au-dessus de la voûte du ventricule latéral.

Traduisons en un autre langage les faits prócis que contiont cotte observation : paralysie du seul bras gauche; destruclion d'une grande partie des radiations optiques postérieures et moyennes du côté droit.

Genevay, tailleur, âgé de quarante-huit ans, d'un tempérament sanguin-pléthorique, élait depuis plus d'un an affecté d'une paralysie du bras gauche (peut-ètre à la suite d'une attaque d'apoplexie ).

Dans le commencement de pluviose an XII, il fut pris d'une somnolence assez prononcée; le $S$ il fut admis à l'infirmcrie de l'hospice de Bicêtre (où il était comme infirme depuis quelques années), présentant l'état suivant : face rougc, pommettes violettes, somnolence presque continuelle, pendant lacielle la bouche restait ouverte' ; la respiration se faisait avec bruit; et le ronflement survenait aussitôt que Genevay fermait les yeux : dès qu'on l'agitait un peu, il les ouvrait, répondait aux questions qu'on lui adressail, mais en témoiguant du mécontentement de ce qu'on le dérangeait; plis il s'endormait de nouvcau. Il était conslamment cn supination, le bras gauche fléchi, immobile ct appliqué sur la poitrine; le ventre était souple, les selles nulles depais deux jours, les urines faciles el fréquentes, le pouls plein et tendu. 
Ces symptômes persistèrent, la peau devint chaude, la soif vive; le râle survint et la mort eut lieu le 11 du même mois, trois jours après l'entrée du malade.

Les vaisseaux extérieurs du crâne étaient gorgés et distendus par le sang; ceux des méninges ainsi que le sinus de la dure-mère étaient dans le même état : la substance cérébrale avait sa consistance naturelle; la médullaire offrait un grand nombre de points rouges à l'incision, provenant de la dilatation des vaisseaux qui la traversent : on trouva à la partie postérieure de l'hémisphère droit une caverne pouvant contenir une petite noix, remplie par une sérosité rousse; cette caverne était logée dans la substance médullaire: au pourtour, et dans l'espace d'un pouce environ, l'encéphale était plus dur que dans le reste de son étendue et d'une couleur jaunâtre. Les quatre ventricules étaient distendus par une sérosité roussâtre; les plexus choroïdiens offraient un grand nombre de vésicules pleines d'une sérosité limpide.

Pour ne pas multiplier inutilement les observations relatives à ce point important de la physiologie et de la pathologie du cerveau, je vais en emprunter une à M. de Lassone, consignée dans l'Histoire de l'Académie royale des sciences (1).

(1) Plusieurs de mes élèves, mais sur.tout M. le docteur Lacrampe-Loustau, dans șon excellent Mémoire, m'ayant fait 
- Une femme âgée d'environ trente-deux ans, " tut de grands chagrins; l'évacuation de ses règles - fut supprimée, et il lui survint les symptômes ordinairesdans ce cas, mais sans aucun mal de tête. H. de Lassone travailla à détruire ces accidens par les moyens convenables; les symptômes disparurent et il en espérait une guérison complète, " lorsqu'à l'occasion d'un nouveau chagrin, la " malade ressentit une grande pesanteur de tête, v accompagnée de douleur; ce qui lui occasiona - quelques jours après une faiblesse dans le genre - nerveux et un tremblement presque universel. "En cet état la malade ne voulut plus prendre - de remèdes : le tremblement parut cependant "diminuer, mais l'abattement augmenta d'autant - plus; l'usage des sens et les mouvemens des - parties extérieures furent presque entièrement " abolis; le pouls et la connaissance se soute"naient du reste assez bien, et l'organe de la voix " était libre. Cette espèce de paralysie se dissipa "peu-à-peu avec la grande pesanteur de tête, mais

hommage de cette découverte publiée en 1819 dans l'Annuaire des hôpitaux, page 329, j'aurais pu taire cette observation, ainsi que celle de Sandifort, rapportée plus haut, et réserver pour moi seul l'honneur que les médecins pourront y attacher. Mais l'intérêt de la science devant passer avant tout, j'ai cru utile de faire voir que dans tous les temps et dans tous les lieux, la nature se dévoile d'une manière uniforme à ceux qui observent avec attention et sans idées préennfues. 
- Ie mal sembla affecter tour-d-tour différens

- points du cerveau, et différens organes, qui ap-

" paremment lui répondent par le commerce des

- nerfs. D'abord la malade ressentit une douleur

- profonde au sommet de la lête, et sa vue s'affai"blit; le point douloureux parut changer de lieu,

"sans qu'on pût désigner précisément l'endroit " du crâne où il répondait; la vue se rétablit, " mais les yeux n'eurent plus de mouvemens; "quelques jours aprés la vue s'obscurcit de nou- veau, et toutes les parties de l'oeil furent affec"tées; la langue tomba aussi en paralysie, et la sa"live coulait involontairement de la bouche; ces - accidens disparurent assez vite, mais la malade - se plaignit quelque temps après d'un nouveau "point douloureux à-peu-près vers le centre de * l'os occipilal, ce qui augmenta pendant quatre à - cinq jours, ct quui fut accompagné de fréquens "maux de coeur et de palpitations; tous ces ac" cidens se dissipérent, et le point douloureux se " fit ressentir plus haut derrière la tête, vers l'angle " de l'occipital; alors les bras devinrent paraly" tiques, la douleur se calma en partie et s'ap"procha de l'os pariétal du côté droit de la tête; "le bras droit recouvra le mouvenent, l'autre „ resta paralytique, et la douleur de tête ne se " dissipa point. Quelque temps après il sc forma " un autre point douloureux vers le bord anté" ricur du même pariétal, avec une espèce d'en- gourdissement lo long de l'épine du dos; la jambe 
" et le pied gauche perdirent le mouvernent et res-

" tèreut en cet état conjointement avec l'extrémité " supéricure du mème côté. Ces partics, qui * avaient perla le mouvement, n'en étaient pas n moins sensibles aux impressions du froid et de " la chaleur, et les corps aigus y causaient par * leurs piqûres des douleurs aussi vives que dans - les parties entièrement saines; enfin les douleurs " de tête augmentèrent peu-à-peu, devinrent gé" nérales, le pouls s'affaiblit, et après environ * quinze jours de langueur la malade mourul. * M. de Lassone ayant ouvert la tête du cadavre, " y trouva dans les deux endroits de la partie cor" ticale du cerveau qui répondaient aux bords an" térieur et postérieur du pariétal droit, deux tı- meurs superficielles, inégales, calleuses, d'en" viron cinq lignes de diamètre, et qui parais๖ saient s'eufoncer dans la partie médullaire, que " l'on regarde comme l'origine des nerfs. Il y avait - beaucoup d'eau dans les ventricules; le cerveau "paraissait d'une consistance plus ferme qu'il ne "l'est communément."

Ce fait, d'autant plus curieux qu'il a été receuilli à une époquc où l'action spéciale des radiations de la couche optique et du corps strié n'était point connuc, nous offre un cas dans lequel leurs effets sont accumulés sur le même sujet, et indiqués pendant la vie par des douleurs aiguës. Ainsi la douleur vive qui se manifesta d'abord vers l'angle supéricur de l'occipital, correspondait manifestcment à l'irritation des deux 
lobses postérieurs des hémisphères cérébraux; alors les deux bras, et seulement les deux bras, devinrent paralytiques : la douleur s'apaisa en partic, puis elle se rapprocha de l'angle postérieur du pariétal droit; le bras gauche devint immobile, le droit recouvra les mouvemens : cette douleur se prolongea jusque vers la parlie antèrieure du même pariétal, et aussitôt la jambe gauche devint immobilc aussi. Ne suit-on pas ici la marche de l'irritation de la partie postérieure à la partie antéricure de l'hémisphère droit, et ne voit-on pas successivement le bras, et puis la jambe du côté opposé, perdre successivement le nouvement?

On jugera mieux encore de toute l'importance de ce fait, quand on aura acquis la conviction par les observations suivantes, que les radiations antérieures et moyennes du corps strié, influencent plus spécialement le membre inférieur que le supérieur.

Lecref (Louis-Marie), âgé de soixante-huit ans, peintre en bâtimens, éprouvait des étourdissemens qui se renouvelaient aux changemens brusques des saisons : ces étourdissemens s'étaient manifestés à la suite de la suppression des hémorrhoïdes qu'il avait portées pendant dix-sept ans.

Le 2 février 1817 , ce malade étant monté sur une échelle, fut pris d'un tournoiement de tête, et tomba en même temps sur le côté gauche, de la hauteur de cinq pieds: relevé par ses camarades, sa jambe gauche était paralysée; il ne pou- 
vait se soutenir, ce qui obligea de le transporter chez lui. Le bras gauche était un peu plus faible que le droit; néanmoins le malade lui fit exécuter divers mouvemens, qu'on lui recommanda de faire pour s'assurer qu'il n'y avait pas de fracture. Il n'en fut pas de même de la jambe; son immobilité complète fit croire au chirurgien qui le visita, qu'il y avait fracture du col du fémur. On administra au malade deux purgatifs; on lui fit une saignée du bras droit. Un délire continu se manifesta le 8 férrier; le 9 au soir, Lecref fut transporté à l'hôpital de la Pitié.

Le 10, il présenta l'étal suivant: face rouge et animée, contraction des mâchoires; bouche déviée à droite: langue rouge, ventre tendu, dévoiement, délire, et pendant ce délire, agitation des derux bras et de la jambe droite; immobilité de la jambe gauche.

Le 11 et 12 , le délire diminua, mais la langue devint sèche et noire; le ventre fut de plus en plus météorisé, le dévoiement devint presque continuel; il s'y joignit une paralysie de la vessie.

Le 14 et 15 , il tomba dans une adynamie dont aucun moyen ne put le retirer. Il mourut dans la matinée du 16 .

Autopsie. Les intestins, principalement les grêles, étaient phlogosés dans presque toute leur étendue; il y avait deux petites ulcérations dans le coecum, dont toute la muqueuse était épaissie; le péritoine était enflammé, et présentait çà 
et là quelques granulations sur sa superficie. Le crâne ouvert, les vaissea ux de la pic-mère parurent très-injectés; il s'écoula une certaine quantité de sérosité au moment où le cerveau fut retiré de la boîte osseuse du crâne.

Le cerveau considéré par sa base, on aperçut un engorgement des vaisscaux de la pic-mère, correspondant aux racines du nerf olfactif droit. Le cerveau incisé dans cette partie, on pénétra à sept ou huit lignes dans la profondeur du lobe antérieur droit, dans un foyer sanguin, dont l'étendue avait un pouce d'avant en arrière : sa partie postérieure était adossée à la parie antérieure, et latérale du corps strié droit; son cenire correspondait à la partic antérieure et moyeune de la radiation antérieure du corps strié; sa base reposait sur les faisceaux des pédoncules, qui, après s'être dégagés de dessous le corps strié, vont se rendre dans la base du lobe antérieur. Le demi-centre ovale du même côté était injecté dans toute son ćtendue; le reste de l'hémisphc̀re droit était sain, ainsi que le gauche, dont les vaisseaur étaient également injectés ; le cervelet et la protubérance annulaire étaient intacts.

Une femme de soixante-dix-neuf ans (nommée Marguerite Quérat), qui séjourna dans ma division du 25 avril 1822 au 25 janvier 1825 , était paraplégique. D'abord, la cuisse et la jambe droites avaient perdu lemouvement, puis la jambe gauchc arait été affectée de la même manière : la parole était Irès- 
difficile et sur-tout extrêmement lente; l'odorat était tellement obtus, que la malade vivait habituellement dans la malpropreté la plus dégoútante. La tête était tonjours penchée sur la poitrine. Les radiations antérieures ct inférieures du corps strié des deux côtés étaient ramollies; la couleur de la partie de l'encéphale altérée élait d'un blanc sale: à gauche l'altération était plus étendue qu'à droite; les pédoncules du nerf olfactif étaient d'un gris et d'une dureté quiles rapprochait de la lame cornée; la commissure antérieure était diminuée de volume.

Il est inutile de rapporter de nouveaux faits à ce sujet, car ils se répètent tous les uns les autres; toujours les paralysies particlles da bras et de la jarnbe, dépendant d'une lésion des lobes cérébraux, coüncident avec des altérations limitées aussi, soit des radiations de la couche optique, dans le premicr cas, soit des radialions antérieures du corps strié, dans le sccond.

Il suit de là, comme conséquence immédiate, que l'altération ou la destruction de la partie moyenne du demi-centre ovale, ou l'entrelacement inféricur des radiations de la couche optique et du corps strić, sout constamment suivis d'une hómiplégic complite. Les faits qui appuient cette proposition soni si nombreux chez les anteurs, que je crois superflu d'en rapporter de nouveaux (1).

(1) Voyez it ce sujet lo premier mémnire de M. le ducteur Lacrampe-Loustan; lcs cas qu'il rapportc, recucillis à ma 
Il suit encore que, dans les hémiplégies, la jambe ou le bras sont plus ou moins affectés, selon que l'altération siége plus particulièrement ou plus profondément sur les unes ou sur les autres de ces radiations.

La femme Tenant, âgée de quarante-sept ans, était hémiplégique par suite d'une attaque d'apoplexie. Le bras avait conservé un reste de mouvement; la jambe en était totalement privée; ellc était paralysée du côté gauche. Il y avait dans l'hémisphère cérébral droit deux foyers circonscrits : l'un, et c'était le plus grand et le plus profond, dans la partie moyenne du corps strié; l'autre, plus petit, était creusé dans la partie antćrieure de la couche optique.

La femme Lanaise offrit la disposition inverse. L'hémiplégie avait commencé par le bras gauche, qui fut totalement privé de mouvement, différant en cela de la jambe, qui se mouvait encore, quoiqu'imparfaitement; la presque totalité des radiations de la couche optique étaient altérées, tandis qu'il n'y avait d'affecté que les radiations postérieures du corps strié.

La femme Grand-Pierre (Louise), à la suite d'un étourdissement qui la prit en jouant, a d'abord les doigts de la main gauche engourdis,

elinique, sont d'autant plus concluans, que d'après les symoptômes, le siége de l'altération avait été précisé avant l'ouverture des cadarres. 
et ne peut plus se livrer à ses occupations. Bientôt elle devient inquiète, criarde; elle ne peut plus mouvoir ses bras, qui sont constamment fléchis sur la poitrine; si on les étend, ils cèdent, mais se fléchissent de nouveau, comme entraînés par un mourement mécanique. La jambe gauche est entièrement immobile; la droite se meut. L'autopsie montre : $1^{\circ}$. un double ramollissement des lobules postérieurs du cerveau : or, ces lobules sont formés par les radiations postérieures de la couche optique; ce qui explique la double paralysie au bras; $2^{\circ}$. l'altération du côté droit avait envahi les radiations du corps strié; ce qui explique la paralysie de la jambe gauche; $3^{\circ}$. les radiations strićes de l'hémisphère gauche étaient intactes : de là, la persistance du mouvement de la jambe droite.

Cette action spéciale de ces deux ordres de radiations explique, selon nous, pourquoi, dans les hémiplégies, le bras est souvent plus affecté que la jambe; pourquoi, dans la guérison des paralysies, le mouvement revient plus promptement et plus complètement à la jambe qu'au bras : car les radiations de la couche optique étant plus étendues et plus disséminées que celles du corps strié, on conçoit qu'elles sont plus souvent et plus profondément atteintes que ces dernières.

Cette observation de la persistance de la paralysie des membres supérieurs est, depuis près de deux siècles, dans la science. Les uns l'ont attri- 
buée a la différence du calibre des artères des membres supérieur et inférieur; les autres, au nombre des muscles qui les meuvent; quelquesuns à la différence de longueur du col du fémur et de celui de l'humérus; et d'autres, en plus grand nombre, à l'inclinaison différente des nerís de la cuisse et du bras : on conçoit, en effet, que lorsqu'on y faisait circuler un fluide subtil, on pouvait croire que l'angle d'insertion des nerfs brachiaux sur la moelle épinière, devenait un obstacle ì son cours; tandis que la direction presque perpendiculaire des faisceaux de la queuc de cheval en favorisait, au contrairc, la marche. Mais personne, que je sache, n'avait encore songé à rechercher cette cause dans les lobes cérébraux.

Quoi qu'il en soit de cette assertion, il reste donc élabli par les faits que la moelle allongée et. la protubérance annulaire influencent également les mouvemens des deux membres; que les hémisphères du cervelet influencent plus généralement les membres inférieurs que les supérieurs; tandis qu'au contraire les lobes cérébraux tiennent plus sous leur dépendance le membre supérieur que l'inférieur.

Cetle différence d'action des radiations de la couche optique et du corps strié ne se borne pas aux mouvemens de la jambe ou du bras; elle influence encore spécialement d'autres organes, dont le trouble des fonctions intéresse au plus haut degré la pathologie. 
Ainsi les altérations des radiations de la couche optique troublent ou gènent beaucoup plus les fonctions du poumon que celles des radiations du corps strié.

De là vient qu'à la suite des hémiplégies, lorsque le mouvenent se rétablit à la jambe, et que le bras resie plus ou moins paralysé, la respiration se trouve gênée par le plus petil exercice, soit que cette gêne provienne du poumon lui-mème, soit qu'elle soit l'effet d'une diminution d'action des puissances inspiratrices, et plus spécialement du diaphragme. De là vient encore que chez ces personnes, l'acte du rire, l'éternuement, etc., sont plus gêtıés que chez les autres. Le mouvement du diaphragme est ainsi lié à celui du membre supérieur: cela explique, d'une part, l'insertion des nerfs diaphragmatiques; de l'autre, l'harmonie constante qui existe entre la fréquence de la respiration el la rapidité des mouvemens des membres.

On conçoit en effet, que siles mouvemens du diaphragme n'eussent pas été combinés avec ceux des nuembres, l'asphyxic ou la suffocation se seraient manifestées dans la course ou dans les grands exercices: c'est ce qui arrive lorsque les deux bras sont paralysés; c'est ce qui arrive lorsqu'on pralique la section des nerfs diaphragmatiques chez les mammifères: de là vient enfin le long trajet que parcourt le nerf diaphragmalique pour aller rejoindre los nerf's brachiaux, et s'insérer avec cux sur la région cervicale de la moclle épiaière. 
Les radiations de la couche optique et du corps strié exercent aussi une action spéciale sur la voix, la parole et la prononciation. M. le professeur Récamier avait, il y a plusieurs années, placé le siége de la parole dans la partie moyenne du demi-centre ovale, parce qu'il avait observé l'aphonie, à la suite des altérations organiques de cette partie: M. le docteur Bouillaud a attribué exclusivement cette propriété au lobe antérieur, parce que, dans le cas soumis à son examen, c'est à la suite des maladies du lobe antérieur que l'aphonie s'était manifestée : enfin, j'avais conclu de la lésion des radiations de la couche optique, ce que ce dernier médecin a déduit de celle du corps strié. Il résulte ainsi de ces trois ordres de faits que la voix, et sur-tout la parole, sont spécialcment influencées par le demi-centre ovale des hémisphères cérébraux. Mais ces fonctions le sont-elles au même degré et de la même manière dans toute l'étendue de ce demi-centre? $J_{\epsilon}$ crois pouvoir avancer d'après mes observations, que les radiations du corps strié influencent l'exercice de la parole d'une manière plus directe que celles de la couche optique; et qu'au contraire celles de la couche optique influencent plus la voix ou la formation des sons que celles du corps strié. D'où il résulte que dans les altérations de ces dernières radiations, l'aphonie est le résultat de la paralysie des mouvemens de la langue, et que dans celles de la couche optique, elle est l'effet 
de la paralysie du larynx : on conçoit alors que, Jors des lésions du demi-centre ovale, l'aphonie doit être le résultat de cette double action exercée et sur le larynx et sur la langue. On voit donc encore une fois que la même fonction n'est pas exclusivement assignée à tel ou tel point de la masse encéphalique, puisque déjà nous avons vu l'aphonie et le bégaiement se manifester dans les maladies de la moelle allongée et de la protubérance annulaire.

On doit surtout conclure de tout ce qui précède, que les lobes cérébraux cxercent unc action très-puissante sur les mouvemens volontaires; quc les radiations du corps strié influencent spécialement les jambes, et celles de la couche optique les bras; vérité si importante pour la pratique, que je crois utile de la confirmer par la physiologie expérimentale.

J'incisai le lobe antérieur gauche d'un chien de moyennetaille, quelques lignes au-dessus du niveau de la parlie antéricure du ventricule latéral; la patte droite postéricure fléchit seule: je fis la mêne section sur le lobe droit, et la gauche fut paralysée; alors je pus remarquer qu'eu marchant sur ses pattes de devant, l'animal trainail celles de derrière. Les pattes postérieures étaient paralysées, celles de devant ne l'étaient pas; les ratdiations antéricures du corps strié étaicut scules intéressées dans llexpérience.

Jenlevai sur un jeune chat la partic antérituro

I1. 
des deux lobes cérébraux, une ligne en avant du grand ventricule : aussitôt l'animal fléchit les jambes de derrière et se traîna sur celles de devant; placé sur le dos et maintenu dans cette position, il agitait violemment les pattes de devant, celles de derrière étaient immobiles. Les radiations de la couche optique étaient intactes, celles du corps strié étaient enlevées. J'ai varié cette expérience de différentes manières; M. le docteur Lacrampe-Loustau l'a modifiée à son tour, en se rapprochant du procédé de Saucerotte; et le résultat a toujours été le même : les extrémités postérieures ont seules été paralysées par la section des radiations anterieures du corps strié. Ces expériences reproduisent donc l'un de nos résultats pathologiques; reste à vérifier celui des radiations de la couche optique.

Le lobe cérébral droit étant mis ì découvert sur un gros chien, j'incisai sa partie postérieure au niveau de la partie moyenne de la couche optique (1); la patte antérieure gauche cessa de se mouvoir : je prolongeai l'incision sur l'hémisphère gauche et au même niveau, l'animal fléchit sur le train antéricur; en voulant marcher il tomba sur les pattes de devant, et, au moyen des pattes

(1) La bandelette blanche qui divise la couche optique en leux parties, me paraît être la ligne de démarcation de cette action spéciale; c'cst pourquoi je me suis appesanti sur ce fait dans la partie anatomique. 
de derrière restées très-agiles, il décrivait des cercles dont le centre correspondait aux pattes de devant immobiles et comme fixées sur le sol. Les radiations moyennes et postérieures de la couche optique avaient seules été divisées.

Sur un autre chien, je pratiquai une très-petite ouverture sur la partie postérieure et moyenne du coronal : je plongeai dans le lobe postérieur gauche un bistouri effilé; des mouvemens convulsifs légers se manifestèrent dans la patte droite de devant; je retirai le bistouri, et à l'aide d'un petit tube de verre jintroduisis dans la profondeur du lobe incisé quelques goustes d'acide nitrique: aussitôt les convulsions redoublèren cians la même patte : je laissai reposer le chien; une heure après, cette patte était immobile et à demi fléchie; l'animal marchait sur les trois autres. C'est le seul cas où j’aie très-bien réussi à ne léser que les radiations postérieures de la couche optique, comme cela a eu lieu dans plusieurs des cas pathologiques que jai rapportés; et chez cet animal, de même que chez nos malades, on voit que l'extrémité antérieure est uniquement affectée, d'abord de couvulsions, puis de paralysie.

L'expérience suivante, de M. le professeur Rolando, est d'antant plus curieuse à ce sujet, que chacun sait que cet habile physiologiste regarde le cervilet conmo la sonrce presque unique des mouremens volontaires. Des phénomènes fort curiens, dit-il, furcul aussi observés sur un cochon, 
chez lequel on a coupé, avec un instrument tranchant, les fibres qui vont se rendre des couches optiques aux corps cannelés. A peine fut-il opéré, que l'on observa qu'il ne remuait plus, comme auparavant, les jambes antérieures (1).

Ainsi ces expériences prouvent ce que les faits pathologiques avaient établi, savoir : que les radiations de la couche optique influencent plus spécialement les membres antérieurs, et ceux du corps strié les membres postérieurs : il est presqu'inutile d'ajouter que la jonction de ces deux ordres de radiations (ou la partie moyenne des bémisphères ) influencent tout à: la fois et ins-

(1) Les expériences de M. le docteur Loustau ont eu le même résultat. Je transcris ici un passage de son second mémoire.

"Personne n'ignore que Willis avait placé danis le corps "strié le sensorium commune, où aboutissaient toutes les sellv sations, et d'où partaient tous les mouvemens volontaires; * cette idée a été le pivot autour duquel ont été ranenés les - travaux de Pourfour-Petit, de Chopart, de Sabouraut et is de Saucerotte.

- Tous ces auteurs ont cherché à réaliser l'idéc de Willis - d'après des expériences sur les animaux vivans, et d'après - des observations pathologiques sur le cerveau de l'homme. * Saucerotle fit des expériences qui lui inontrèrent que - la partie antérieure des hémisphères cérébraux influe sur - les mouvemens de la jambe, et la partie postéricure et * supérieure de ces mêmes hémisphères sur les moure- mens du bras; ct aussitit, pour les rattacher a l'hypothèese - reçue, il supposa que tous les firisceaux médullaires: du 
tantanément les dcux membres. C'est ce que l'on produit constamment cliez les mammifères, si l'on incise profondément la partie moyenne du demicentre ovale, formé par l'entrelacement de ces radiations.

11 n'en est pas de même chez les oiseaux : jamais je n'ai pu parvenir à paralyser isolément les pattes ou les ailes, en opérant sur la partie antérieure ou postérieure de leurs lobes cérébraux, dont l'influence sur les mouvemens volontaires est beaucoup moins marquée que chez les mammifères; il en est de même chez les reptiles : j'ai remarqué seulement que chez les oiseaux, les ailes sont plus affaiblies que les pattes par l'ablation des

" cerreau se rendaient au corps sto'ie et s'entre-croisaient - dans: son centre : de là ses préçples pour l'opération du " trépan, préceptes répélés par Chopart.

$n$ Le vague el l'incertitude de ces préceptes justifient "loubli nù ils étaient tombés, et où les ont laissés tous nos * grands maîtres, les Desault, les Bichat, les Boyer, les * Richerand, et, sans aucunc exception, tous les grands chix rurgiens de nos jours!

- Pour décourrir l'action spéciale des radiations de: Ja - couche optique et du corps strié sur le mouvement. des - nembres, il fallait donc de nouveaux faits observés chez

- l'homme et de nouvelles expériences sur les animaux " vivans : ces faits et ces expériences ont été recucillis of

" exécutés de nos jours par M. Serres, et c'est en vain-

A que l'esprit de rivalité s'efforce d'en contester d'alsord la

- réalité, et puis la décourerte of la nouveauté, lorsque leur

- oertitude est démontréc. 
hémisphères cérébraux; c'est principalement chez les hirondelles que cet effet m'a paru sensible. Ainsi les expériences physiologiques, de même que les faits pathologiques, prouvent incontestablement que les hémisphères cérébraux de la classe supérieure des vertébrés concourent directement ¿̀ la production des mouvemens; la solution de continuité de leurs fibres produit la paralysie, leur irritation détermine des convulsions; les convulsions sont limitées à un membre, ou étendues à la moitié du corps, de même que les paralyșies, selon que l'irritation est circonscrite ou générale :l'application decette proposition, méconnue dans ces derniers temps (1), est sì importante pour la médecine, que nous avons dû la développer avec quelque étendue.

Les paralysies sont croisées de mème que les convulsions; c'est-à-dire que le côté droit du cerveau influénce le côfé droit du corps, et vice versa. L'action d'un hémisphère du cerveau ne s'exerce que sur le côté qui lui est opposé; jamais un hémisphère n'influe simultanément sur les deux côtés à-la-fois, soit pour déterminer une double hémiplégie, soit pour produire l'hémiplégie d'un côté et les convulsions de l'autre. On a supposé toutefois que cela pouvait avoir licu:

(1) « Mes expériences établissent que les hémisphères du - cerveau ne produisent directement aucun mourement. , (M. Flourens, pag. 275.) 
les uns ont avancé qu'il y avait des paralysies directes, produites par la lésion des hémisphères; les autres, que la même altération pouvait déterminer la paralysie croisée et les convulsions directes: enfin, ce qui approcherait du merveilleux, on a pensé que le même hémisphère pouvait aussi paralyser la jambe du côté gauche et le bras du côté d roit; on expliquail de cette manière les paralysies transverses, ou en diagonale, de Sauvages.

Ce sont surtout les paralysies directes et les convulsions directes, cö̈ncidant avec une paralysie croisée, qui ont exercé la sagacité des médecins. Il y a dans la science quelques faits et plusieurs explications. Les faits demandent à être revus; les explications, même celles qu'on a données de nos jours, ne peuvent supporter l'examen d'un physiologiste (1).

Lorsqu'il y a paralysie d'un côté et convulsion de l'autre, il existe deux affections, correspondant l'une à la paralysie, l'autre aux convulsions. André Nollet, ancien marchand de vin, âgé de soixante-onze ans, d'une forte constitution, fut frappé, dans la matinée du 31 janvier 1821 , d'une attaque d'apoplexie avec hémiplégie du côté gauche : porté le lendemain dans ma division, il nous offrit une paralysie presque complète de ce côté, et des convulsions violentes du côté droit; convul-

(1) Il n'est ici question que des explications appliquées aux altérations des hémisphères cérébraux. 
sions qui donnaient à sa physionomie un aspect hideux, à cause de l'immobilité des muscles du côté gauche de la face. L'ouverture du cadavre offrit une caverne remplie de sang, logée à la partie interne du demi-centre ovale de l'hémisphère droit; le demi-centre ovale gauche était rouge, enflammé dans toute son étendue; l'irritation était surtout prononcée au niveau de la partie moyenne du corps strié, et antérieure de la couche optique; les ablutions de la substance cérébrale ne lui faisaient rien perdre de sa rougeur.

Charles Bertin, âgé de soixante ans, d'une constitution détériorée, tomba malade le 10 décembre 1812 ; tout-à-coup il fut pris d'une difficulté d'uriner, d'une faiblesse daus les jambes et le bras droit; il n'y eut ni vertiges, ni étourdissemens précurseurs.

Le 18 décembre, jour de son entrée à l'hôpital, les mêmes symptômes persistaient, et, en outre, la face était colorée, les yeux vifs et brillans, la prononciation difficile, les idées obscurcies, le pouls dur et un peu fébrile. (Arnica avec deux gros d'acétate d'ammoniaque par pinte. )

Du 19 au 25, l'état du malade resta stationnaire; seulement les idées devinrent de jour en jour plus embarrassées, et la prononciation plus difficile. (On se borna à la tisane d'arnica.)

Le 25 au soir, le malade perd connaissance toutd-coup; la face devient rouge, les yeux étincelans; la bouche se contourne a droite; la moitie droite 
du corps est dans un état convulsif continuel, la moitié gauche tombe, au contraire, dans une immobilité absolue; le pouls est dur et tendu. (Arnica émétisé, lavement purgatif, pédiluve sinapisé.)

Le 26 , la face est décolorée, le pouls est petit, dur, tris-fréquent; une sueur visqueuse couvre la peau. (Deux larges vésicatoires aux jambes et un à la nuque. )

Le 27, prostration totale des forces, respiration un peu stertoreuse. (Vésicatoires aux cuisses.) Mort le 28 au matin.

A l'autopsie, le cerveau parut ferme au toucher; il existait dans le noyau médullaire de l'hémisphère droit, précisément en dehors et au-devant du coude que forme le ventricule latéral, un foyer contenant deux onces de sang noir; ce foyer était ouvert dans le grand ventricule; la substance cérébrale qui l'environnait était jaune et injectée dans l'espace de deux lignes. La couche optique de l'hémisphère gauche offrait aussi dans son épaisseur uı épanchement de sang noir et caillé, beaucoup moins considérable que celui de l'hémisphère droit, et ouvert comme lui dans le troisième ventricale.

Ainsi la paralysie que l'on avait remarquée du còté gauche était le résultat du grand foyer creusé dans l'hémisphère droit, et les convulsions qui existaient à droite étaient excitées par le foyer développé dans la couche oplique gauche. Tel itait aussi le cas d'une femme hémiplégique du 
côté droit depuis huit mois, et qui fut prise de convulsions du côté gauche; convulsions qui, pendant un mois, revinrent par accès simulant l'épilepsie. A l'ouverture du cadavre, on rencontra un foyer non cicatrisé dans la partie médiane du demi-centre ovale droit, et un ramollissement de deux pouces d'étendue dans la partie correspondante du demi-centre ovale gauche. Ce ramollissement était d'un gris-rougeâtre, pointillé de noir par de très-petits foyers sanguins. Les convulsions récentes du côté droit étaient évidemment l'effet de ce ramollissement, et la paralysic du côté gauche le résultat de l'ancien foyer non cicatrisé de l'hémisphère droit. Les convulsions, de même que les paralysies, sont donc croisées; lorsqu'il y a coïncidence des convulsions d'un côté, avec la paralysiè de l'autre, il y existe deux altérations spéciales, l'une correspondant à la paralysie, et l'au tre aux conrulsions. Quant aux paralysies directes, il n'en existe pas; c'est upc erreur d'observation qui les a fait admettre (1).

(1) J'ai traité ce point avec quelques détails daus l'Annuaire des hôpilaux (1819); j'y renvoie le lecteur; mais pour montrer avec quel soin doivent être recueillies les observations pour ne point s'en laisser imposer par les apparences, je vais rapporter un fait observé récemment dans ma division, et qui fit croire d'abord à l'existence d'une paralysie directe.

Dimbley (Marie), âgée de dix-neuf ans, d'une forte constitution, fut prise, le 16 janvier 1826 , d'une céphalalgic asser. 
intense, accompagnée les jours suivans de douleur vive a l'épigastre, avec nausées et romissemens de inatières bilieuses. En même temps aussi les lombes, les cuisses et les jambes étaient le siége d'une douleur conluse. Dès le 9 du même mois, cette fille avait eu ses règles, dont la durée fut de trois jours comme de coutume.

Le samedi 21 janvier, la céphalalgie, la douleur de l'épigastre, les vomissemens s'étaient dissipés; dès le matin, des boutons varioliques se manifestèrent d'abord à la face, puis sur le col et la poitrine, et ensuite sur les autres parties du corps. Le dimanche, elle entra à l'hôpital de la Pitié, et le lundi matin elle nous offrit le premier degré d'une variole semi-confluente. La variole suivit sa marche ordinaire jusqu'au jeudi 26 janvier.

Ce jour, la malade était assoupie et couchée sur le côté droit; de temps en temps elle s'agitait, et répondait brusquement aux questions qui lui étaient adressées. Si on la plaçait sur le côté gauche, elle faisait des efforts pour se remettre sur le côté droit; on s'aperçut alors que le côtć gauche était presque immobile, surtout le bras : si on lui pincait le bras gauche, elle cherchait avec la main droite à repousser celle de l'observateur. Elle accusait une douleur vire dans le côté droit de la tête. Les 27,28 et 29 , son état était le même; dans la nuit du 28 au 29 , elle tomba de son lit. La douleur de tête se fait toujours ressentir du côté droit.

Des mouvemens convulsifs se manifestent dans la nuit du 29 au 3o. Le matin, les yeux roulent dans les orbites, les mouvemens convulsifs agitent toute la face et tout le corps, principalement du côté droit. Les meoabres sont tortus, la bouche est remplic d'écume, lia lẻte est inclinée à droite; il I a suspension complète de tous les sens pendant la durée des mouremens convulsifs.

L.e 31 , les convulsions de la veille se renouvelient; le 
x février, elles sont un peu moins intenses. On remarque que dans les accès le bras droit est agité violemment et exé. cute des mouvemens précipités de flexion et d'extcnsion. Jamais la malade n'avait eu de convulsions avant la présente maladie.

Le 5 février Dimbley est mieux; la fgure s'est rapprochée de son état naiurel, la paralysie des membres gauches a presque entièrement disparu; du 6 au I4, la malade se sert du bras gauche, et prend sa tisane avec la main du même côté. Le dévoiement persiste; elle maigrit beaucoup et d'une manière rapide.

Du 15 au 26 , le dévoiement continue, la faiblesse augmente de jour en jour. Elle succombe le 27 du même mnis. Nous apprîmes alors seulement, que deux jours avant, les altaques d'épilepsie avaient reparu, ct que la nuit de la mort elle était tombée quatre fois de son lit.

Autopsie. Les membres gauches ne présentent point de. roideur, de même que la jambe droite; le bras droit est au contraire contracté, ainsi que les doights de la main du même côté.

La dure-mère ne présente rien de particulier; une.couche de pus blanchâtre liquide se remarque au-dessous de l'arachnoïle; clle occupe le centre de l'hémisphère gauche ; s'étend, d'alyant ẹ arrière et de dedans en dehors, jusqu'à un demi-pouce de la circonférence.

La substance corticale dans le lobe postéricur offre une suppuration verdâtre; la pie-mère, qui s'insinve dans les anfractuosités de cette partie de l'hémisphère, est détruite. Une section oblique de dedans en dehors étant pratiquée sur. l'hémisphère gauche, au-dessus des ventricules latéraux, montre que la suppuration occupe toute l'épaisseur du lobe postérieur. Toutcfois celte suppuration verdâtre est encore plus marquíe au centre qu'à la périphéric. La partie infé- 
rieure du lobe moyen du côté gauche présente dans la substance grise le mûme aspect verdâtre, quoique la suppuration use soit pas encore formée.

Sur le demi-centre ovale gauche, on voit encore quatre petits loyers offrint le même aspect verdatre. Trois, éloignés les uns des autres d'environ un pouce, correspondent à la couche optique et au corps strié; ils peuvent contenir un pois à cautère. Ise quatrième, situć à six lignes de l'extrémité du lobe antérieur et à sa superficie, pourrait loger une petice amande.

Dans l'hémisphère droit et dans la partie du demi-centre ovale, qui correspond au lobe mojen, existent deux petits foyers verdatres semblables pour la grandeur aux trois premiers que nous avons décrits dans l'hémisphère gauche. Ils sont à un pouce de distance l'un de l'autre, et ne s'étendent pas aú-delà de la substance blanche.

La moelle épinière était parfaitement saine. Je néglige de rappurter les altérations des atutres viscères.

Avant de commencer l'examen de cette observation, il est bon de xemarquer que ce fait avait d'autant plus fixé l'attention des ćlèves, que, peu de temps auparavant, on m'avait nié, dans l'Académic royale de Médecirie, la coincidence de l'encéphalite avec la variole conlluente. Je dois prévenir aussi qu'ayant été indisposć dans le mois de janvier, je ne vis la malade que quelques jours avant la mort; mais l'observation ayant ćté rédigéc par un de mes éléves les plus instruits, M. Martel, on peut la regarder comme parfaitement exacte. Cela étant, il faut expliquer les syunptômes par.les altéralions: ct d'abord une douleur vive se manifeste du côté droit de la tête, les nembres gauches paraissent paralysés; cela indique une irritation du côté droit du cerveau. La paralysie se dissipe assez proznptement; la malade peut se servir de la snain gauche; on trouve deux pelits foyers dans le demi- 
Quoique j'aie établi, l'un des premiers, que les lésions artificielles du corps calleux (1) n'exercent aucune influence sur les mouvemens volontaires, je pense que cette observation a besoin d'être de nouveau coufirmée chez l'homme (2). Il en

centre ovale droit. Le peu d'intensité de la paralysie coïn. cide donc avec le peu d'étendue de l'altération de l'encéphale.

Au contraire, dans les monvemens convulsifs, lé côté droit est principalement affecté; le bras droit est principalement agité, et agité de manière à fixer l'attention des malades qui avoisinent son lit. Que trouve-t-on? ce que l'on doit trouver, le lobe postérieur gauche en suppuration. Cette vive irritation de ce lobe explique donc la violence des convulsions droites, surtout du bras; de même que le peu d'intensité de la lésion du côté droit explique la faiblesse de la paralysie gauche.

Mais pourquoi la paralysie droite n'a-t-elle pas succédé à la désorganisation si profonde ile l'némisphère gauche? C'est un point encore obscur de la pathologie de l'encéphale, que je n'entreprendrai pas de traiter en ce moment. Ce que je veux faire ressortir présentement, c'est que, sans un examen approfondi, on aurait pu négliger les convulsions du bras droit, s'arrêter uniquement sur la paralysie gauche, et conclure, d'une part, l'existence d'une paralysie directe; del'autre, la coïncidence d'une seule altération produisant une paralysie directe et des convulsions croisées; ce qui, je crois, ne se présentera jamais à un observateur attentif.

(1) Annuaire des hôpitaux, troisième et quatrième expériences, pagr. 359 .

(2) Reil a rapporté un cas de lésion du corps calleux sans paralysie; mais il ne faut pas oublier que, dans les idées de ce 
estclemêmede la voûte à trois piliers: l'induction nous porte à croire que ces parties sont étrangères à l'exercice de ces mouvemens, puisque nous les voyons manquer dans des classes où ces mouvemens ont acquis leur plus haute énergie; mais, pour la solution de questions de cette importance, l'induction n'est pas preuve : il faut des faits, et des faits observés chez l'homme ( 1 ).

Avant de quitter ce qui a rapport aux paralysies, nous devons dire un mot des moyens par lesquels Ia nature en opère la guérison. Tou te paralysie coïncidant le plus fréquemment avec une solution de continuité des fibres de l'encéphale, le mouvement ne se rétablit que lorsque la réunion des fibres divisées est opérée. Il y a alors formation d'une cicatrice dans la masse de l'encéphale; j'ai prouvé ce fait à une époque où les paralysies étaient

célèbre physiologiste, la substance blanche, organe de la sensibilité, était déclarée impropre d exciter les mouvemens : or le corps calleux élant une voate entièrcment formée de matière blanche, ce fait venait merveillcusement à l'appui de sor opinion.

(1) J'ai observé trois cas de destruction presque complète de la voute; dans deux, il y avait cu une faiblesse générale de tous les membres; dans le. troisième, que j'ai publié dans le journal de M. Magendie, ce résultat élait douteux. Dans un cas semblable, publié par un de nos bons observate'urs, M. le docteur Martinet, l'intígrité des mouvemens avait survécu au ramollissement d'un hémisphère et de la voute. 
réputées incurables. Si un paralytique recouvre l'exercice des mouvemens et qu'il succombe à une autre maladie, on trouve la cicatrice au point où s'était formée la division de l'encéphale. La cicatrice est plus ou moins ferme, les lèvres de la division sont plus ou moins bien rapprochées, selon que les mouvemens sont devenus plus ou moins libres. Lescicatrices sont: ou linéaires, la guérison de la paralysie est alors complète; ou aréolaires, la guérison est alors imparfaite; les mouvemens sont plus ou moins gênés. Une paralysie étant complìtement guérie, il arrive quelquefois que, sans cause connue, et sans une nouvelle attaque d'apoplexie, la perte du mouvement reparaisse: dans ces cas, il y a in tiltration de la cicatrice; lacicatrice, de linéaire qu'elle était, devient aréolaire; les aréoles se remplissent d'une sérosité jaunâtre; les lèvres de la plaie de l'encéphale, écartécs, sont ainsi maintenues à distance. Cescas sont fréquens, surtout chez les vicillards faibles. D'aùtres fois, unc nouvelle attaque d'apoplexie, une chutc ou un coup, porté sur la tête, rompent la cicatrice; aussitôt la paralysie se reproduit. J'en ai rapporté un exemple remarquable, il y a sept ans (1). Cet effet n'a licu que dans les cicatrices récentes; les cicatrices anciennes résistent plus que les parties de l'encéphale qui les avoisincrt. On voit très-fréquemmeat des foyers sanguins rècens, creusés à côté des cicatrices,

(1) Journal universel des sciences miticales, tom. XI. 
qui ont résisté aux efforis par lesquels ont été brisées les parties de l'encéphale qui les avoisinent. On ne saurait trop admirer à ce sujet les ressources de la nature.

Quand je publiai mon mémoire sur la guérison des paralysies par la cicatrisation du cerveau, on demanda comment ce fait avait échappé si lougtemps aux recherches des observateurs. Si l'on eût consulté l'histoire de la science, la question inverse eùt été plus juste. Comment l'aurait-on découvert sous l'influence des hypothèses qui se sont disputé le domaine du système nerveux \$ De quel intérèt pouvaient être ces cicatrices, quand les mouvemens étaient attribués à l'arachnoïde, par Riolan, ot à la dure-mère, par Baglivi? Pendaut le long réve des esprits animaux, quel auteur eùt imaginé que la substance blanche pût se régénérer indépendamment de la matière grise, qui stule pouvait sécréter ces esprits? Et dernièrement cucore, quand on faisait procéder la matière blanche de li grise, quand cette dernière était une sorte de matrice où s'engendrait la première, qui cût songé à voir une cicatrice se former de toute pièce, dans le centre de la matière blanchc? Toutes ces hypothèses détournaient les observaleurs, et leur faisaient regarder comme impossible la régénération de la inatière blanche sans matière grise. Pour la découvrir, il fallait donc observer, l'esprit libre de toutes ces suppositions.

('ist une position nouvelle dans la science, que II. 
d'aroir à se demander si les hémisphères cérébraux sont les organes immédiats des sens. Cette question ressort directement néanmoins des travaux récens sur la cinquième pairc, et des faits pathologiques relatifs aux altérations du segment basilaire de l'axe cérébro-spinal du système nerveux. Il devient donc plus imporiant que jamais de chercher a préciser la part que prennent à l'exercice des sens les lobes cérébraux, pour la distinguer de celle qui paraît dévolue et à la cinquième paire et à la moelle allongúe.

Boerhaave ayant posé en principe que l'action de chaque nerf cxistait à son point d'insertion sur l'axe cérćbro-spinal, les physılogistes et les pathologistes placèrent le siége de l'odorat aux environs du champ olfactif, et celui de la vision dans la couche optique. Le sens du goût et celui de l'ouïe résidaient vers les bandes inférieures de la moelle allongée, et le toucher, sens plus général, était disséminé dans la masse des hémisphères. Voilà ce que l'on croyait, et ce qui doit être réformé ou modifié.

En attendant de nouvelles lumières, nous devons dire sur quelles bases étaient établies ces données. Sauccrotte, par ses expériences sur les animaux, semblait avoir établi sans réplique que l'odorat avait son siége dans le champ olfaclif, puisqu'il abolissait ce sens par la section de cette partie; Lover l'avait vu détruit chez l'homme par une tumeur qui comprimait les branches d'insertion 
du nerf olfactif; ct moi-même, dans plusieurs altérations organiques du champ olfactif, je croyais m'être assuré que l'olfaction était perdue dans la narine correspondante à la lésion. Mais les conclusions que nous avions déduites de ces faits et de ces expéricnces sont singulièremcnt alténuées par l'observation récente de Béclard, qui constata la persistance de l'olfaction dans une dégénérescence presque cancéreuse du champ olfactif.

L'influcnce des hémisphères cérébraux sur la vue csibien micux prouvée que cclle de l'olfaction. Coïtcr, Valsalva, Pourfour-Petit, Sauccrotte, ont constaté la perte de ce sens à la suite de l'altération ou de l'ablation des lobes cérébraux. Cet effet a été vérifić par tous les physiologistes de nos jours; mais ces expériences, en montrant l'action des hémisphères sur la vision, n'apprennent rien sur la partie qui en est spécialement le siége. La pathologie est un peu plus précise que la physiologie expérimentale.

Dans les altérations de la périphérie des lobes, à quelque profondcur qu'elles pénètrent, la vue reste intactc. Il en est de même du corps strié I a masse de la couche optique paraît être le foyer de ce sens chez l'homme; encore n'y concourt-elle pas en entier. Ainsi j'ai vu toute la surface supéricure détruitc, sans que la vision fût altérée; j’ai observé cotte destruction tantôt d'un seul côté, tantôt des deux à-la-fois, la vue restant intègre. Quand la couche optique est détruite dans sa pro- 
fondeur, la vision n'est perdue que lorsque la désorganisation pénètre au niveau du point de départ de la commissure molle : cette commissure paraît être la limite de ce sens, d'après les faits quc j'ai observés. Si les genoux sont détruits, la vue est perdue de l'oeil opposé; l'altération d'un seul ne fait que l'affaiblir.

Quelquefois les malades voient les objets doubles; quelquefois aussi ils voient les objets doubles d'un oeil et simples de l'autre : alors, la vision est singulièrement troublée. L’instinct porte constamment les malades à ne se servir que de l'œil où la vue est simple. Dans d'autres cas, la vue est d'abord double d'un oil, simple de l'autre; puis l'œil à vue simple devient double, celui à vue double devient simple. Dans quelques-unes de ces ano malies, j'ai rencontré des altérations du tubercule optique et des pédoncules cérébraux ; mais, dans tous les cas, ces altérations m'ont paru insuffisantes pour rendre compte des phénomènes observés. Si on ajoute à ces faits les cas nombreux de cécité, causés par diverses productions contre nature, qui comprimaient le nerf de la vision par des endurcissemens de son tissu, ou l'atrophie même de ce nerf, on verra que l'action immédiate des hémisphères sur la vision est mieux établie que celle de l'odorat. Toutefois, cette action n'a lieu que chez les mammifères et les oiseanx. Chez les reptiles et les poissons, les lobes cérébranx sont complètement étrangers à l'exercice: 
de ce sens (1). Quant au sens de l'ouie et du goût, je ne connais aucune observation qui puisse faire présumer une action directe cxcrcée sur eux par les lobes cérébraux.

(1) Ayant fait répéter par plusieurs de mes élèves les diverses expériences consignées dans cet ouvrage, la perte de ła vision par l'ablation des lobes cérébraux lut le phènomène qui frappa le plus leur attention; je transcris ici le passage de M. le docteur Lacrampe-Loustau, dans lequel se trouvent les probabilités anatomiques de ce fait.

"Je ne savais comment me rendre raison de la peric de la n vision par l'ablation des hémisphères cérébraux. Jc fis part - de mon embarras a M. le professeur Serres, qui ouvril $\rightarrow$ l'atlas de son anatomie comparée du cerveau, et we donna - la note suivante.

"Les cordons moyens de la moelle épinière, situés atu côté externe des pyramides, montent parallèlement ì elles jus- qu'à la partie antérieure du bulbe de la moelle allongée; n ils s'en écartent en cet cndroit, pénètrent dans l'intérieur - des tubercules quadrijumeaux, auxquels M. Serres a donné n le nom de lobes optiques dans les trois classes inférieures.

- Parvenus dans les ventricules de ces tubercules, ils se * plongent dans un renflement de matière grisâtre, et se di* visent en sortant en quatre lames, dont deux grises et deux - blanches. Les deux lames blanches, parvenues à la partie - antérieure des lobes optiques, s'adossent au faisceau des " pyramides, et vont s'épanouir avec elles dans les hémi- spheres cérćbraix. Le prolongement de ces lames n'existant - pas chez les reptiles ct les poissons, cela explique pourquoi - dans ces deux classes l'ablation des lobes céréhraux n'in" léresse en rien la vision." (Deuxième múmoire, parres 50 et 51.) 
Pour compléter les effets résultans des altérations matérielles des hémisphères cérébraux, nous devons dire un mot du trouble qui se remarque quelquefois dans la manifestation de l'intelligence.

Tantôt il y a perversion de l'intelligence par la lésion d'un seul hémisphère cérébral, tantôt cette lésion unilobaire est sans effet sur les facultés intellectuelles. C'est sur-tout lorsque l'altération est bornée, que cette intégrité de l'intelligence a été observée; et c'est aussi pour en donner l'explication, que l'on a imaginé qu'un hémisphère suppléait à l'autre, ou mieux encore qu'on ne pensait que par un seul hémisphère, comme on ne voyait que d'un oeil, qu'on n'entendait que d'une oreille. Cette manière d'expliquer l'unité de l'être sentant, au milieu de la duplicité des organes des sensations, ne mérite pas une réfutation sérieuse: c'est le dernier terme de l'abus d'une supposition de Locke.

L'action simultanće des deux hémisphères scmble mieux prouvée, daus les cas oủ la perversion de l'intelligence est perçue par le malade même: tel est celui où un seul hémisphère étant profondément affecté, le malade comprend les questions qu'cn lui adresse, médite sa réponse, et, lorsqu'il veut l'exprimer, parle tout différemment quill n'a pensè, proféredes mols, des phrases et des réponses qui ne sont ni les réponses, ni les phrases, ni les mols quil avait intention de proférer. Ta malide qui s'anerenit do cetre incohio... 
rence, s'interrompt, se reprend, rccommence et ne parvient jamais a exprimer sa pensée. Il faut avoir été témoin du combat intérieur que se livre le malade, pour concevoir ce déplorable état. Il semble, dans ces cas, que l'action des deux hémisphères est désunie; que l'hémisphère sain pense, perçoit, réfléchit, et que l'hémisphère malade exprime. L'âme se juge elle-même.

Tels sont encore les cas d'altération unilobaire, dans lesquels les malades ne peuvent proférer qu'une seule phrase, toujours la même. Ih!..La. Not... Seigneur !... répondait toujours une femme à toutes les questions : elle exprimail nettement ces mots à la suite les uns des autres; mais si, on voulait les lui faire prononcer isolément, si par exemple, on lui disait de commencer par Seigneur, elle faisait des efforts inouis sans y parvenir, etenfin après un instantelle proférait sa phrase dix ou douze fois de suile, comme par une espèce d'explosion. Tel était encore un ancien jardinier, qui prononcait avec une volubilité que pas un de nous ne pouvait égaler, Bé $B \dot{e}$ Ré... A... O... Telle est cncorc une jeune femme qui vient une ou deux fois l'an dans ma division, et dont tout le vocabulaire est renfernć rlans ces mots : ch bien! pas toujours comme ra!... Nons avons inutilement

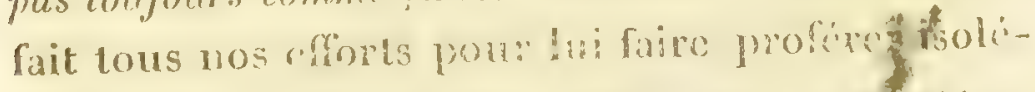

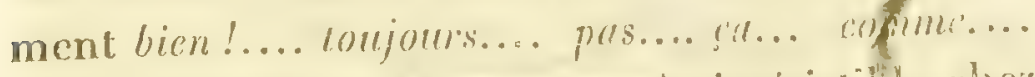
celte association de mols parait irresistilile che

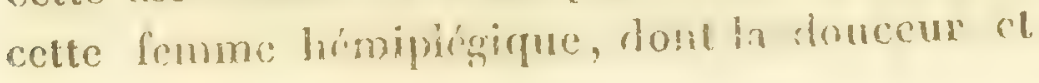


la gaîté n'ont point été altérées par cette crunlle position ( 1 ).

Constamment laltération profonde des deux lobes cérébraux trouble l'intelligence, et la trouble. en en paralysant la manifestation. Il n'y a plus de détermination réfléchie; l'œil voit, l'oreille entend, la langue et le palais goûtent; mais l'âme ne juge plus : la vie de relation est anéantie; l'homme est. complètement réduit à la vie végétative; il mange, boit et dort, ou plutôt on le fait boire et inanger, et exécuter les diverses fonctions animales, sans qu'il manifeste ni désir ni opposition. On lecouche d'un côté, il y reste; on le place de l'autre, il y reste encure : si on cherche à l'exciter fortement et qu'on y parvienne, il tient les yeux fixement ouverts; mais cette fixité du regard est le scul signe par lequel il manifeste qu'il a senti qu'il vivait encore. Ily a impression sur les sens, mais la perceplion ct la sensation sont éteintes. C'est cette absence de scnsations que l'on provoque chez les animaux, par l'ablation des lobes cérébraux, soit qu'on la pratique par le procédé de Coïter,

(1) Peut-on expliquer ces laits par l'association des mouve. mens dont Reil s'est presque seul occupé jarmi les physiologistes modernes? De la un précepte important de pratique, at qui sa'a réussi un grand zombre de fois, c'est d'apprendre i parier unx malades, quand la gućrison s'annonce; de leur enscirncele syllabaire, de les faire épeler, ot de ne leur farr. prononcer des mots entiers que lorsque toutes les combi-

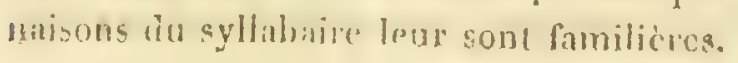


ou par celui re MM. Rolando et Flourens (1). Peut-on dire alors que les lobes cérébraux seuls veulent, se déterminent, que seuls ils percoivent, sentent? Ce qui, dans le langage de tous les physiologistes, veut dire que l'âme a perdu l'instrument de ses volontés, de ses déterminations, de ses perceptions et de ses sensations : on le croyait naguére. Mais si ce même état se produit, los lobes cérébraux étant parfaitement sains, une inconnue nouvelle est introduite dans ce problème, déjà si compliqué et si difficile. Or, les altérations profondes du segment basilaire produisent les mêmes effets que les altérations profondes des lobes cérébraux.

Ces faits prouvent incontestablement la part que prend l'encéphale dans la manifestation de lintelligence. Ils montrent des altérations des facultés intellec: 1 elles dépendantes des altérations matérielles de cet organe. En est-il de même des aliénations mentales? Les immenses recherches. de M. Ie doctcur Esquirol ont répondu négativenuent à celte question. MM. les docteurs Falret et Creerget sont d'une opinion contraire; la science attend avec intérêt le résultat de leurs travaux (2).

(1) G'est ce même élat qui a élé ubscivé dins les cas d'ossification complete, ou de pétrification de la masse de l'encéphale. T'out le monde connaît celui rapporté par le cé-lèbre Duverney.

(2) Ios procédés de l'anaromic pathologique sont si inl- 
Je n'ai eu en vue, dans ces corollaires, que de tracer une esquisse des altérations matérielles de l'axe cérébro-spinal du système nerveux, et de les rapprocher des phénomènes divers qui les accompagnent. J'ai passé avec rapidité sur des questions qui, tout-à-fait douteuses à l'époque où je commençai mes publications, ont subi les épreuves du temps, et ne sont plus aujourd'hui contestées par personne; je me suis appesanti sur d'autres, à cause des controverses dont elles sont encore l'objet, et sur-tout à raison de l'intérêt qu'elles offrent pour la pratique médicale. Dans les questions tout-à-fait nouvelles que j'ai élevées, tantôt je me suis borné à exprimer le résultat, leur développement exigeant une latitude que ne me

parfaits, qu'un grand nombre d'altérations organiques échappent encore à notre investigation. Il reste à faire pour l'anatomie pathologique ce que Bichat a exécuté pour l'histologie. Cette imperfection de cette partie fondamentale de la pathologie est attestée par les discussions qni ont suivi la publication de l'ouvrage publié par M. le docteur Petit et moi, sur la Fière entiro-mésentérique, et la loçalisation de toutes les fièvres, qui en est la conséquence immédiate. Personne ne s'est apercu de cette lacune; et ceux qui avaient le plus grand intérêt à la combler, ont apporté, au licu de faits, la métaphysique médicale du stahlianisme. En traitant, dans la pathologie du systeme nerveux, de la nature des altérations dont il est susceptible, je donnerai un apercu de ce que j'ai entrepris sur ce système seulement. L'application de ces procédés à l'étude du siége des aliénalions sera peut-être de quelque utilité. 
permettait pas le cadre de cet ouvrage; tantôt jai appuyé ces résultats par des faits, quand ces faits étaient de nature à porter avec eux la conviction. Dans tous les cas, j'en ai assez dit pour éveiller l'attention des observateurs et fournir aux bons esprits les moyens d'en déduire toutes les conséquences. Ces conséquences, je les ai depuis longtemps renfermées dans le problème suivant: Une maladie de l'axe cérébro-spinal du système nerveux étant donnée, déterminer son siége par les symptômes. C'est aux médecins et aux physiologistes à juger jusqu'à quel point j’en ai donné la solution.

Dans l'examen des fonctions des diverses parties de l'encéphale, j'ai souvent été ramené sur les hypothèses, les explications et les suppositions de toute nature, que la difficulté du sujet et les imperfections de la science ont fait naître à ses diverses époques : ce tableau des oscillations des esprits est plus nécessairc qu'on ne le pense, pour mettre un frein aux suppositions et aux hypothèses nouvelles qui reparaissent dons des ouvrages d'ailleurs recommandables, et qui le plus souvent ne sont que les hypothéses anciennes amplifiées ou mutilés : eu montrant les fincostes cficls qu'olles ont produis, ćest grantir cote paric de la science des mouvearux alangess guteile anrail i courir sous l'influcnec des nuthodes hypothéli-

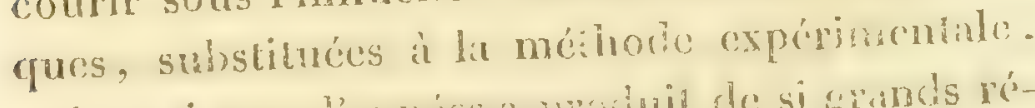

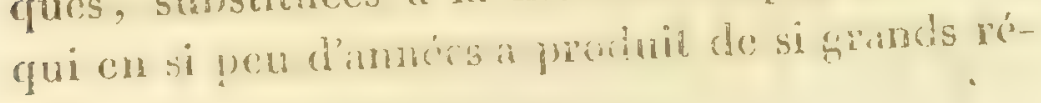
sultals. 
J'aurais bien d'autres faits à ajouter à ceux que jai rapportés; mais ils suffisent pour juger la doctrine de la locạlisation des fonctions et des maladies de l'axe cérébro-spinal. Une des erreurs de nos devanciers est d'avoir supposé qu'il n'y avait qu'une seule action, ou, en d'autres termes, qu'il y avait dans l'encéphale un point unique qui devait les régir toutes : une seconde erreur, développée dans ces derniers temps, et qui dérive de la première, est de supposer à son tour qu'une même action est exclusivement dévolue à une même partie de l'encéphale. L'une de ces suppo. sitions n'est pas plus exacte que l'autre.

11 y a unité d'action, et diversité de parties qui' concourent à la produire. Telle est la proposition la plus générale et la plus vraie qui me paraisse ressortir des faits et des expériences.

Pour que notre langage soit la représentation de nos pensées, et, autant que possible, l'expression concise des faits, je nomme excitateur le point ou la partie où paraît résider telle action. Les faits et les expériences nous permettent de distinguer les excitateurs suivans:

$1^{\circ}$. Le renflement inférieur ou la région lombaire de la moelle épinière est excitateur de l'éjaculation, de la contraction de l'utérus, de la vessie et du gros intestin.

$2^{\circ}$. La portion dorso-costale est excilateur des intustins grêles et de l'aclion des côtes.

$3^{\circ}$. La région cervicale est excitateur des agens 
inécaniques de la respiration, et plus spécialement du diaphragme.

$4^{\circ}$. Et de plus, les cordons antérieurs de la moelle épinière paraissent les excilateurs principaux des mouvemens, et les cordons postéricurs, les excitateurs principaux de la sensibilité.

$5^{\circ}$. Lc segment basilaire est un centre d'excitation. L'olive est excitateur des mouvemens du cœur; le corps restiforme, excitateur de la respiration pulmonaire. Le cordon qui sépare ces deux faisceaux est excilateur de l'estomac. Les cxcitations de conservation ou de nutrition sont ainsi concentrées dans la limite inférieure de ce segment (Organe de nutrition de Willis.)

$6^{\circ}$. La partic supérieure de ce segment est excitateur de la sensibilité et de la motilité générale. (J,orry). $\mathrm{I}_{4}$ e trapèze est excitateur des mouvemens de la face. Les faisceaux où s'insèrent les nerfs trijumeaux, l'excituleur des appareils des sens et de l'instinct.

$7^{\circ}$. Les tubercules quadrijumeaux, que Willis regardait comme les organes de conservation, sont excitateurs de l'association des mouvemens volontaires ou de l'équilibration; et de plus, les cxcitateurs du sens de la vue dans les trois classes inférieures.

$\zeta^{\circ}$. Le lobe médian du cervelet est excitateur des organes de la génération. Ses hémisphères sont c $x \rightarrow$ citateurs des mouvemens des membres, ct plus spe- 
cialement des membres pelriens. Le cervelet est excitateur du saut.

$9^{\circ}$. La couche optique est l'excitateur de la vue chez l'homme et les mammifères. Ses radiations sont excitateurs des mouvemens des membres thorachiques, et subsidiairement, excitateurs de la respiration.

10. Les radialions du corps strié sont excitateurs des membres pelviens.

11. Le champ olfactif est excitateur du sens de l'odorat.

12. Le demi-centre ovale est l'excitateur de la voix et de la parole; et de plus, les lobes cérébraux sont les excitateurs des facultés intellectuelles. Ces excitations isolées, distinctes, sont réunies, confondues, simultanées dans l'action; ce sont les instrumens divers par lesquels l'âme opère ses manifestations.

$\mathrm{Si}$, faisant maintenant un retour sur la pathologie, nous lui appliquons les données qu'elle nous a fournies, nous verrons les paralysies, les convulsions, l'épilepsie, les affections partielles des sens, peut-être même les aliénations mentales, éclairées par le double flambeau de l'anatomie pathologique et de la physiologie expérimentale; nous trouverons les viscères de l'abdomen et de la poitrine, dont l'influence sur les fonctions de l'encéphale a été si long-temps et silonguement exagérée, êtrc au contraire directement influencés dans leur ac- 
tion par l'axe cérébro-spinal du système nerveux; l'étude propre des sens s'ouvrir une route nouvelle par des acquisitions qui, quoique récentes, ont reçu presque en même temps la sanction de la physiologie et de la pathologic. Le principe d'action des organes génitaux délerminé, nons rapprochera peut-être de la connaissance de la génération, ou, du moins, nous consolera pour quelque temps de nos longs et pénibles efforts. Enlin si les longueurs et les difficultés de cette méthode expérimentale n'effraient pas les jeunes praticiens, nous verrons les suppositions, les hypothèses et les systèmes bamnis pour toujours de la médecine, el on ne traitera plus de chimérique l'espoir de voir cette belle scicnce suivre les méthodes sévères et philosophiques des sciences physiques.

IIN. 
B.

Bandelette demi-circulaire. Ce qu'elle est, II, 459. Son usage chez les reptiles ct les oiseaux, 460; ses modifications chez les mammifères, 461 .

\section{C.}

Canal vertébral. Sa formation, II, 149 .

Céphalopodes, voyez Mollusques.

Cervelet. Son état chez l'embryon des oiseaux aux diverses époques de l'incubation, I, 11-39; chez celui des reptiles aux diverses époques de formation, 68; ses dimensions chez le tétard, 8x; son état chez l'embryon de l'homme et des mammifères aux diverses époques de formation, 110 ; causes de sa formation tardive chez les embryons, 114 ; ses dimensions aux différens âges de l'homme et de l'embryon humain, 122; sa détermination chez les poissons, $199^{\ominus}$; il est toujours en rapport avec le cordon postérieur de la moelle épinière, $56_{7}$; se forme en sens inverse des hémisphères, 571 ; époques de sa formation dans les différentes classes, et de ses formes primitives, II , 542 ; de ses formes permanentes et de sa position chez les reptiles, 346 ; chez les poissons, 351 ; chez les oiseaux, 565 ; séric de ses déreloppemens chez les mammiferes, 377 ; de ses formes permanentes dans cette classe, 587 ; il ne s'accroît pas en raison du nombre des lobes dont il est composé, 599 : rapports de la matière grise et de la matière blanche qui entrent dans sa composition, dans les quatre classes de vertébrés, 402 ; sinus cérébelleux des poissons et des oiscaux, 403; corps deutelé ou rhomboïdal des mammaifères, 404: pourquoi il n'existe que dans cette classc, 405 ; rapports de développement du lobe médian et des hémisphères, 413.

Cetacés. Ils sont privés de nerfs olfactifs, I, 289 .

Champ olfactif. Son développement chez l'homme ct les mammifères, I, $17^{5}$; ses limites, 279 ; ses rapports, 280 .

Chrysochlore, voyez Taupe.

Circonvolutions. Leur formation, $\mathrm{I}, 1 / 77,178$, et II, 529; clles manquent dans les trois classes inférieures, 532 ; Jeurs xapports de développement, 559 et 560 ; elles sont tantôt symétriques, et 
tantôt non symétriques, 559-563; elles varient dans la même famille et jusque dans la même espèce, 564 ; leurs rapports avec les divisions du cervelet, 565 .

Cloison transparente. Sa formation, I, 157 ; ses rapports, II, 482 .

Commissures. Leur formation, I, 149 , et II, 450 ; se distinguent en similaires et dissimilaires, 449 .

Commissure antérieure. Elle est similaire, II , 451; ses formes et ses rapports chez les mammifères, 455 ; dans les autres classes, 453 et 458 ; elle manque chez les poissons cartilagineux et chez beaucoup d'osseux, 453 .

Commissure molle. Ses formes, son volume et ses rapports dans les quatre classes, $\cdot \mathrm{II}, 458$.

Commissure postírieurc. Elle est similaire, II, 451; manque chez les poissous et beaucoup de reptiles, ibid. ; ses formes et ses rapports dans les autres vertébrés, 452.

Cordon ou Corps restiforme. Ses rapports de position, II, 200; de développement, 201.

Corne d'Ammon des mammifères; ses rapports, II , 470.

Corps calleux. Sa formalion, I, 150 ; il n'existe que chez les mammifères, II, 480; ses rapports de développement, 481.

Corps frangé des mammifères. Ses modifications et sa position, II, 466. Corps géniculés. Époque de leur apparition chez les mammifères, I, 320 .

Corps strié. Ses dimensions chez le tétard, I, $8 \mathbf{1}$; son déreloppement chez l'embryon de l'homme et des mammiferes, 143 ; ses dimensions dans les différens âges de l'homme et de l'embryon. humain, 163; sa formation, II , 432; ses rapports de développement, 439; ce qu'on doit considérer comme le corps strié des oiseaux, 441 ; des reptiles, 446.

Couches optiques. Leur formation chez l'embryon des oiseaux, I, 18 ; clez, celui des reptiles, 67 ; leurs dimensions chez le télard, 81 ; leur développement chez l'embryon de l'homme et des mammifères, 143 ; leur dimension dans les différens âges de l'homme et de l'eubryon humain, 162 ; leurs formes primitives chez les anammifires, II, 432 ; lcurs formes permanentes et leur volume dans les trois classes in féricures, ibid.; chez les manmifères, 455 ; 


\section{pAR ORDRE ALPIABÉTIQDE.}

rapports de développement de la gerbe du faisceau, médullaire qui en diverge, 437 .

\section{D.}

Diaphragme. Il existe en rudiment chez les oiseaux, I, 496.

\section{E.}

Embryon. Se forme de la circonférence au centre, et non pas du centre à la circonférence, I, 83 et 250 ; les formes permanentes de l'encéphale des vertébrés inférieur's correspondent aux formes primitives de l'embryon des supérieurs, II, 10 ; la même chose a lieu à l'égard des invertébrés, 14.

Eminences mamillaires. Leur formation chez l'embryon humain, I, 172 ; elles n'existent que chez l'homme, II , 513 .

Encéphale. Sa formation chez l'embryon des oiseaux, I. 6 ; rapports de son poids avec celui du corps du poulet pendant l'incubation, 51 ; sa formation chez l'embryon des reptiles, 53 ; chez celui de l'homme et des mammiferes, 83 ; chez les poissons, 188 ; rapports entre l'encéphale de l'embryon des classes supérieures et celui des inférieures, 82 , et particulièrement des poissons, $187 ;$; il présente un très-grand nombre de variations chez les poissons, 185 ; différences entre celui des osseux et celui des cartilagineux, et entre les divcrses familles, 221 ; ses caractères généraux dans la classe des poissons, $\Pi$, 579 ; dans celle des reptiles, $58 \mathrm{I}$; dans celle des oiscaux, ibid.; dans celle des mammifères, 582 .

Epiphyse cérébrale (glande pinéale). Sa formation chez l'embryon des oiseaux , 1, 27; chez celui des mammilères, 158; ses rapports de développement dans les quatre classes de vertébrés, 215 ; son existence chez divers poissons, 218; sa position, II., 429; ses modifications chez les poissons, 483; chez Ies reptiles, 484; chez les oiseaux, 486 ; chez les mammifères, 489 .

\section{F.}

Fissure ammonique des nuammifères. Modifications dans les diffé? reutes faunilles de celle classe, II, 465. 
G.

Ganglions encéphaliques des invertébrés. Leur détermination, I, 402; ce qu'est l'anneau œesophagien des insectes, II, 16.

Genoux interne ct externe. Ce que sont ces tubercules, II, 435 ; leur position et leur volume, ibid.

Glande pinéale, royez Epiphyse cérébrale.

Glande pituitaire, voyez Hypophyse cérébrale.

\section{H.}

Itémisphères cérébraux. Leur état chez l'embryon des oiseaux, aux différentes époques de l'incubation, I, 6-51; chez celui des reptiles aux différentes époques de formation, 61 ; leurs dimensions chez le poulet pendant l'incubation, 35 ; chez le tétard , 81 ; leur développement chez l'embryon de l'homme et des mammifères, 86,142 ; détermination chez les poissons, 202; ils sont toujours en rapport avec le faisceau antérieur de la moelle épinière, 567 ; se forment en sens inverse du cervelet, 571 ; leurs formes générales, II, 428; leur volume et leurs formes chez les mammifères, 439,545 et 551 , et chez les poissons, 526 et 520 ; ils ont été chez ces derniers pris pour le lobe olfactif, 516 ; leur volume et leur forme chez les reptiles, 527 et 535 ; et chez les oiscaux , 539 ; nombre de leurs lobes chez les mammifères, 545 ; rapports de développement de leurs divers élémens, 577 .

Hypophyse cérébrale (glande pituitaire). Sa position, II, 429 et 492; ses modifications chez les mammifères, 492; chez les oiseaux, 494; chez les rep,tiles, $49^{5}$; chez les poissons, $49^{6}$.

\section{I.}

Insectes. Śupérieurs aux Mollusques par le degré de développement de levir système nerveux, II, 23.

Interpariétal. Son étendue est en rapport avec le rolume des tubercules quadrijumeaux, II, 333.

Lobe olfactif. Sa délermination chez les poissons osseux, I, 208: chez les carlilagineux, 24 ; rapports avec celui cles autres classes, 


\section{PAR ORDRE ALPMABÉTIQUE.}

209; sa position, II , 4030; causes de ses nombreuses variations de position, 566 ; sa saillic coincide avec la projection en avant de la face, 567 ; ses formes, son volune et sa position chez les poissons, 568 ; chez les repilies, 5 go ; chez les oiseaux, 572 ; chez les mammifères, 5,5 .

L.

Lobes optiques (tubcrcules quadrijumeaux). Leur état chez l'embryon des oisenux aux différentes époques de formation, I, 14-46; chez celui des repilies, 60 ; chez celui de l'homme et des mammifères, 86 et 132 : leur détermination chaz les poissons, 19 ! ; ils sont très-développés chez la taupe ot plusieưrs autras vertébrés privés de nerfs opticies, 351 ; sont toujours en rapport avec le faisceau moyen do la moeile épinišro, 558; grandes différences qu’ils présentent suivant les classes et les âges, II, 253; lexurs formes primiitres, 257 ; leur ciéveloppement, leux position, leurs formes et leur volume chez les reptiles, 260 ; chez les oiseaux, 265 ; chez les mammifères, $29 x$; déterminaiions données chez les oiseaux, par Trévirazus, 281 « 455 ; et par M. ảe Blainville, 290 ; oblitéxation de leur carité iatenne chez les mamanifères, 296 ; et apparition des silions trazspersazx, 297 ; rapport des tubercules quadrijumeaux enire eux chez les mammiferes, 298 ; formes et volume chez los poíssons, őoo; cavité interne, 305; tori, 307; membraue iaterae, 512; ils ont éé pris pour les hémisphères dans celte classe , 518 ; celle détermination ne peut êlre admise, 520; influence de leurs variations sur l'état général de l'encéphale des cquatre classes, 522; ils se développent en raisonz directe de la moeile épinière, 524; de plasicurs autres parlies du système nerveux, 326 ; des yeux el des norls opliques, ibid. ; cxcoptions, 329 ; rapports avec l'ctendue des interpariétaux, 333 ; rapports généraux avec les autres parties de l'encéphale, 355.

Lobule de l'hippocampe. Ses rapports de développement, I, $17^{5}$; et II, $5 / 8$.

Lobule optique. Sa position, II , 498 ; ses modifications chez les mammiferes, ibirl.; cherz les oiseaux, 505 ; chez les repliles, 507 ; chez les poissons, 508 ; il parait manquer chez divers célacés, 500. 
Lobule sphénoidal. Ses rapports de développement, I, 175 ; et II , 548,553 et 555 .

Lobule tonsillaire. Il n'exisle que chez l'homme, II, 398 .

Loi de conjugaison, I, 532; applications aux divers organes du système nerveux, 533-548.

Loi de symétrie, I, 532; application aux divers organes du système nerveux, 533-548.

\section{M.}

Mammifères. Caractères généraux de leur encéphale, II, 582 .

Membrane rayonnante des oiseaux. Elle correspond à la voûte, II , 471 ; ses modifications dan6 les différentes familles, 473 .

Métamorphoses des insectes. Les organes de la circonférence sont toujours ceux qui se inétamorphosent le plus, II, 26.

Moelle allongée. Ses rapports et sa formation chez les mammifères, I, 163 ; ses limites, II, 179; sa composition, 181; particularités qu'elle présente chez les poissons, 210 ; elle se continue avec l'axe nerveux du tronc en ligne directe chez les mammifères, les poissons et beaucoup de reptiles, 213 ; en ligne courbe chez les autres vertébrés, 214; les rapports de sa masse ne peuvent être déterminés exactement, 215 ; rapports de ses élémeus, 216.

Moelle épiniere. Son état aux diverses époques de formation chez l'embryon des oiseaux, I, 3-56; chez celui des reptiles, 57 ; chez celui de l'homme et des mammiferes, 84; ses dimensions chez le poulet pendant l'incubation, 35 ; chez le tétard, 80 ; dans les différens âges de l'homme et de l'embryon humain, 102; ses renflemens paraissent en même temps que les membres sur les côtés du corps, 99; et II, 122; son ascension dans le canal vertébral chez l'homme et les mammifères, I, 200 ; et II , 116 ; elle manque chez les invertébrés, I, 254 ; est toujours en rapport arec les hémisphères par son faisceau antérieur, 567 ; arec le cervelet par le postérieur, ibid.; avec les lobes optiques par le moyen, 568: formation de son canal, 11, 101; ses variations d'étendue dlans les quatre classes, 102-107; il angmente de capacité en raison inverse de lépaisicur de la cuuche grise, 105 ; rapports de forme de la moelle épinière arec les trous des vertebres, 109; el arec leur volume, 110 ; arec le tronc toul culicr, 121 ; avec les mem. 


\section{PAR ORDRE ALPIABÉTIQUE.}

bres, 122 ; rapports entre son ascension dans le canal vertébral, et la persistance du prolongement caudal, 116 ; rapports entre le volume et le nombre de ses renflemens et ccux des rnembres', 125 ; et des artères correspondantes, 155 ; rapports de ses modifications avec celles du système sanguin dans la métamorphose clu tétard, des batraciens et de l'embryon des mammifères, 134; elle est généralement développée en raison inverse de l'encéphale, 137 , et en raison directe des principaux nerfs crâniens, 143 ; exceptions, 145 : nombre des cordons qui la composent, 147, 150; chacun de ses segmens représente dans sa composition l'anneau. osseux qui l'environne, 149 : nombre des sillons qui existent à sa surface , 156 , 159 ; variations de ces sillons dans les quatre classes, 159 ; structure fibreuse de la moelle épinière; 162 ; elle précède l'encéphale dans sa formation, 163.

Mollusques. Le système nerveux des céphalopodes même est inférieur à celui des insectes, II, 23 .

Monstruosité. Ses causes, I, 106, 129, 478 et 504; remarques anatomiques sur diverses monstruosités, 473-491, 503-507, 575; el II, 171 ; la formation des monstres est soumise au mêrne principe que le déreloppement des êtres réguliers, I, 576 .

Mouche asile. Exception remarquable que présente dans la métamorphose son système nerveux, II, 33.

Musaraigne, vojer Taupe.

\section{N.}

Nerfs. Leur éroque de formation, T, 50 ; bien distincts chez les reptiles avant l’encéphale et la moelle épinière, 56 ; ne prennent pas leur origine dans l'encéphale pour se rendre aux organes, mais se portent des organes à l’encéphale, 345-350, 355, 409, 502 et 563.

Nerfs des invertébrés. Modifications qu'ils subissent dans la métamorphose, II , 32 ; ceux des organes des sens paraissent analogues à des rameaux du nerf trijumeau des vertébrés, I, 403 .

Nerf accessoire de Willis. Tpoque de son insertion, 1, 4fig; lieu de son insertion, 501 ; il existe chez plusicurs viscaux, 500.

Nerf acouslique ou auditif. Sa composilion chez les embryons, 3 , 421 ; son insertion el ses rapports, 422 el $4 \tilde{j} 1$; son volume cher 
les mammifères, 445; chez les oiseaux, 447; chez les reptiles, ibid.; chez les poissons, 448 ; est très-developpé chez les animaux aquatiques, 450.

Nerf de la quatrieme paire. Son insertion dans les quatre classes, $\mathrm{I}$, 344 .

Nerf de la sixieme paire. Son insertion chez les mammifères, I. 339; dans les autres classes, 343 .

Nerf de la troisieme paire. Son insertion dans les quatre classes, I, $335 \cdot 339$; son volume chez les mammilères, 339 .

Nerfs de la vision. Époque de leur insertion chez l'embryon des reptiles, des oiseaux et des mammifères, $I, 345$ : ils manquent chez la taupe et plusieurs autres vertébrés, 352 .

Nerf facial. Son insertion et ses rapports, I, 436 ; il est étranger au développement de la face, 442 ; son volume chez les mammifères, 450 ; les oiseaux et les reptiles, 452 ; les poissons, 453 .

Nerf glosso-pharyngien. Ĺpoque de son insertion, I, 469.

Nerf grand hypoglosse. Epoque de son insertion, I, $46_{9}$ : son volume dans les différentes classes, 499.

Nerf grand sympathique. Il existe chez les monstres anencéphales, I, 503 ; décroit des mammifères aux oiseaux et aux vertébrés inférieurs, $50_{7}$; il en existe quelques vesliges chez les crustacés, 508 . Nerf olfactif. On peut suivre chez les poissons osseux son implantation jusque sur les cuisses cérébrales, I, 258 ; les hémisphères cérébraux sont ordinairement plus développés chez les poissons où il est pécliculé, 262; il est toujours pédiculć chez les cartilagineux, 265 ; est tanlót pédiculé, tantôt non pédiculé chez les reptiles, 264; ses racines sont plus extéricures dans cette classe que chez les poissons osseux, 267 ; deviennent tout-ì-fait exléricures aux hémisphères chez les oiseaux, ibir.; leur disposition dans cette classe, 268 ; chez les mammifères, 272 : rapports de rolume de ce nerf chez les nammifères, 286 ; il esi très-développé chez l'embryon des mammières supérieurs, 288 ; manque chez les cétacés, 289 ; et chez les foclus monstrueux à trompe, ibid.; ne s'insère pas sur le corps strié, $290^{\circ}$; ses rapports arec la commissure antérieure, 291 ; avec le nerf trijumeau, 38z.

Nerfs opliques. Leur entrecroisement chez. les reptiles, 1, 75 et 515 , chez les poissous, 3 zo; leur jonclion chez les oiseaux, 317 ; chez 


\section{PAR ORDRE ALPHABÉTIQUE.}

les mammiferes, ${ }_{2} 24:$ ils peuvent manquer chez quelques animaux sans que la vision soit perdue, 304; manquent chez la taupe et plusieurs autres vertébrés, 350 ; chez les monstres sins yeux, 348; sont la conlinuation des lobes optiques, 307; sont formés de quatre couches chez les poissons, 308 ; leur volume dans cette classe, 306 : chez les rentiles, 311 ; cher les oiseaux; 514 ; chez les mammifères, 323 .

Nerf pneumo.gastrique. Epoque de son insertion, I, 4.69; ses variations chez les monstres, 471; son insertion dans les diliééentes classes, 491 ; son rolume, 494 .

Nerf trijumeau. Son insertion dans les rratie classes, 1,370 ; son volume proportionnel à l'étendue de la face, 377 , et en rapport arec celui de l'artère maxillaire interne, 382 ; il devient nerf oculaire chez les taupes et quelgues autres vertébrés privés cie nerf optique , 384, 388 ; nerf de l'azdition chez bearucoup de poissons, 399 ; peut-être nerf de l'olfaction chez les cétacés, $39^{8}$.

\section{O.}

Oiseaux. La longueur de lour région caudale est fixe, II, 114 ; celle de la cervicale très-variable , 115 ; caractères généraux de leur encéphale, 581.

Olfaction. L'étendue de ce sens chcz les oiseaux n'est pas proportionnelle au volume du lobe olfactif, $\mathrm{I}, 574$.

Olives. Leur formation, I, 165 ; lcurs raponorts de développement chcz les mammiferes, II , 194 ; leurs formes et leur volume dans les quatre classes, $19^{5}$; elles different beaucoup dans les trois classes inféricures de ce quielles sont chez les mammifères, $19^{8}$; modifications que subissent ieurs faisceaux chez les mammiferes, 198; dans les autres classes, 199 .

\section{P.}

Pédicule olfactif. Il n'existe pas chez les oiseaux, $T, 282$; ses rapports chez les mammiferes, 283 ; il esl creux chez les manmifíres inféricurs et chez les embryons des supérieurs, 287.

Poissons. Garactères généraux de leur encéphale, 679 .

Pont de Varole, voyez Protuberance annulaire.

Proté, voyez Taupe. 
Protubérance annulaire. Sa formation chez les mammifẻres, I, 167 ; ses rapports de développement et ses formes, II , 415-422.

Pyramides antéricures. Leur formation chez les mammiferes, I, 164; leurs rapports de position, II, 202; de développement, 203; elles sont toujours en rapport de connexion avec les hémisphères cérébraux , 567 ; leur divergence devient moins sensible des mammifères supérieurs aux inférieurs , et de ceux-ci aux autres classes, 193 ; il n’est pas exact de dire qu'elles sont développées én raison directé des hémisphères, i 192.

Pyramides postérieures. Leur formation chez les mammifères, I, 164 ; leurs rapports de position, II , 202 ; de développement , 203 ; elles sont quelquefois réunies chez les poissons par un faisceau transverse, 205 ; mais elles sont toujours directes, ibid.

\section{Q.}

Queue. Sa disparition chez le tétard, I, 79; son existence chez l'em. bryon de l'homme et de tous les mammifères, $9^{3}$; et sa disparition, 99 ; son volume est proportionnel à celui de l'artère sacrée moyenne, II, 136.

Queue-de-cheval. Sa formation, I, 103; et II , 116; il existe quelque chose d'analogue chez certains invertébrés, 119 .

\section{R.}

Rat-Taupe du Cap, voyez Taupe.

Reptiles. Caractères généraux de leur encéphale, II, 581 .

Ruban gris, II, 209; il croît dans les mêmes proportions que le trapèze, ibid. ; n'existe comme lui que chez les mammifères, 110.

\section{S.}

Septum lucidum, royez Cloison transparente.

Substance blanche et Substance grise. La blanche précède la grise, I, 257,563 et 566 ; le développement de l'une ne dépend pas de celui de l'autre, 556 ; et II, 440 ; la blanche existe seule chez quelques invertébrés, $I, 563$. 


\section{PAR ORDRE ALPHABÉTIQUE.}

T.

Taupe. Elle est, comme le rat-taupe du Cap, la chrysochlore, la musaraiggne, le protée, et plusieurs autres vertébrés, privée de nerf optique, I, 304, 352, 385, 396 ; et II, 53.

Trapèzu de la moelle allongée. Ses rapports de position, II, 206 ; de développement, 207; il n'existe que chez les mammifères, 210. Tubercules quadrijumeaux, voyez Lobes optiques.

\section{U.}

Unité de composition. Elle devient manileste, quand on étudie l'organisation intérieure des êtres, II, 5 et 12; au contraire, les différeuces frappent plus que les ressemblances; quand on ne considère que l'extérieur, ibid.; les dissemblances vont toujours en diminuant de la circonférence au centre, 8 ; pourquoi , 9 .

\section{V.}

$V$ alvule de Vieussens. Ses rapports de développement, II, 412.

$V$ entricules. Leurs variations d'étendue chez les mammifères, II, 556 ; particularités que présente dans les différentes classes le quatrieme vertricule, I, 425.

$V$ ision. Considérée chez la taupe, la chrysochlore et plusieurs atutres vertébrés privés de nerfs optiques. I, 385 et 396 ; elle ne peut s'ezercer que pax l'intcruidu des nerfs trijumeaux, II, 62 .

Vonte. Sa formation, I, 153 ; ses rapports chéz les mammilères, II, 468; sa forme, 471 ; est une commissure dissimilaire, surtout chez les oiseaux, ibid.; elle manque chez les repliles et les poissons, 479 .

\section{PARTIE PHYSIOLOGIQUE ET PATHOLOGIQUE.}

B.

Bégryement. Il est produil par l'affection de la parlic antérieure de la raoclle allongéc, II, 255; cl les altérations supérieures des 
olives, ibid.; il s'observe presçue toujours chez les hommes, et non chez les femmes, 232 .

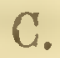

Cervelet. Son action sur les o:ganes génicaux, II, Gc:; démonstration de ceíte action par des observations patholoricrues, 602607 et 623-625, et par ces exṕricrees sü les arimaux virans, 6o8; son action sur les mo:uvements volontaires, 623 ; dímonstration cie cetie action par des observations pìnologiques, 615626 ; et par des eupériences sur les animaux vivans, 617-621 ct 626-629; ses aliérations aficcient plus les memb-es abdominaux que les tho:acigres, 630 ; résumé de ses ionctions, 717 .

Champ ófaciif. Eliets de scn aliérzion patholcgique, I, 294.

Cicatriscicion à criveau, rojez Parciysies.

Convulsions, voyez Paralysies.

Corps calleux. Il ne paraat pas aroir d"inluence sur les mouvemens volontaires, II, jo2.

Corps ou Cordon restiforme. Effets de son altération pathologique sur le pormon, Ii, 251 .

Corps strié. Son action chez ies mammiéres sur les mouremens volontaires, II, 664; et surtont su: cou: c'es membres inférieurs, ibid. et 683; observations pat'ao!ogiques, 664-C8ja ; expériences sur les animaux vivars, 689; excis ce l'aicération de ses racialions sur la voix, la parcle et la prozonciaíor, 68B; ello prociuit surtout l'aphonia par la paralysie du lut yis , 689 .

Couches optiques. Leur aclion cluez les mamaifères sur les nouvemens volontaires, II, 664; et sar-tout sar ceux des membres supérieurs, ídil. ; observaiions pathoiogiques, 664-0̂85; cxpériences sur les animaux vivans, 689; effets de l'altćration de ses radiations sur le poumon, 687; sur la voix, la parole et la prononciation, 688; cette altération produit sur-tout l'aphonic par la paralysie de la langue, ibid.

\section{D.}

Demi-centre ovale. La destruction de sa partic moyenne est toujours suivie d'une hémiplégie, II, 685. 


\section{E.}

Esprits cnimaux. Opinions des anciens physiologistes sur les esprits animaux, I, 549 ; ei $\mathrm{i}^{\prime}, 59^{5}$.

H.

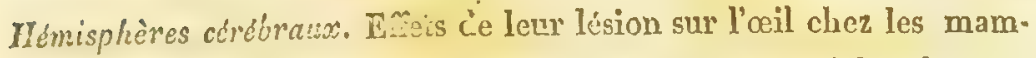
miferes, I, 531; ct II, 707 ; do leurs fonctions, 653 ; observations pathologiques, 664-683; ils ont plus d'influence sur les membres supérieurs que sur les inférieurs, 686 ; leur influence sur les mouremens volontaires est chez les oiseaux et les reptiles beaucoup moins marcuée que chez les mammifères, 693: leur action est croisée, 694; perversion de l'intelligence par leư lésion, 710-714; résumé de leurs fonctions, 718 .

\section{I.}

Instinct. Ce qu'on entend par ce mot, I, 51; les actes instinctifs paraissent sous la dépendance de la cinquième paire, II , 89 ; preuves fournies par l'esamen des invertébrés, go; et des vertébrés, 91.

Iris. Altérations produites par la lésion des tubercules quadrijumeaux, II , 649; de ses mouvemens, 651 .

\section{L.}

Lobes optiques ( Tubercules quadrijumeaux). Effets de leur altération, II, 643; observations páthologiques, 643.646, et expériences physiologiques, 647 ; leur altération chez l'homme ne détruit pas la vue, 643; dans les expériences sur les animaux vivans, leur lésion altère au contraire la vue chez les rammifères, I, 332 ; et II , 649 : et sur-tout cher. les giseaux, les reptiles et les poissons, 649 ; leur action sur la vision est toujours croisće, ibid. ; résumé de leurs fonctions, 717 .

\section{M.}

Moelle allongée. Effets de son alteration sur l'estomac, II, 252 ; sur les mouvemens et sur la parole, ibid; résumé do ses fonclious, 717. 
Moelle épinierre. Ses corcons ont paru plus imitables or plus sensibles, selon qu'ils correspondent aux branches antérieures ou aux postérieures des nerfs rachidiens, II, 221 ; observations pathologiques qui confirment cette opinion, ibid.; observation pathologique qui lui est contraire, ibid.; dans les alterations de la moelle épinière, les mouvemens ne sont lésés cque quand l'altération se rapproche de la circonférance, 222; les effets de son irritation pathologique ou de sa section sont surtout marqués chez les animaux inférieurs, ibid.; son action est directe, ibid.; et intimement liée à l'intégrité de la circulation, 223 ; elle est étrangère à la circulation chez le fœtus, 224; de son infiuence sur la respiration, ibid.; et sur les fonctions des intestins, de la vessie et des organes génitaux, 225 ; ses fonctions se développent avant celles de l'encéphale, 228; observation d'une maladie organique, 234-246 : et remarques sur cette maladie, 246 ; résumé des fonctions de la moelle épinière, 716 .

\section{N.}

Nerfs. Ils reçoivent et possèdent la faculté de sentir et de mouvoir, II , 219 ; ils ont été distingués en sensibles et irritables, 63,220 et 600 .

Nerf acoustique ou auditif. Effets de son altération pathologique sur l'audition, I, 453.

Nerf facial. Ses altérations pathologiques, I, 454 ; il peut étre considéré comme nerf respirateur de la face, ibid.

Nerf olfactif. Effets de l'altération organique de ses racines, I, 295. Nerf trijumeau. Effets de sa section sur les animaux vivans, $\Pi, 64$; et de son altération pathologique chez l'homme, $67-88$; il exerce une influence très-marquée sur l'action des sens, $9^{5}$; parait être le siége de l'instinct, ibid.

\section{0.}

Olives. Elles influent spécialement sur les mouvemens du cœur, II, 231 ; leurs altérations supérieures produisent le bégayement, 253 .

Organes génitaux. Ils ont tout à-la-fois leur principe d'action et dans le cervelet et dans la moelle épinière , II, 6og; de quelle manière agissent ces deux parties de l'axe cérébro-spinal, 609-615. 


\section{gAR ORDRE ACPHABETIQUE.}

\section{P.}

Paralysies. Leur guérison peut s'opérer par la formation d'une cica-

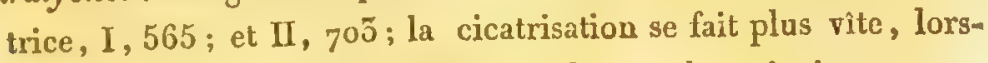
qu'elle a lieu près du demi-centre ovale, ou des principaux amas de matière blanche, I, 565; la paralysie ne peut être produite d'un côté, et les convulsions de l'autre, par la même altération, II, 640 et 694 ; lorsqu'il y a paralysie d'un côté et convulsions de l'autre, il existe deux affections, $69^{5}$; il n'y a point de paralysie directe, 698 .

Protubérance annulaire. Effets de son altération pathologique, II, $632-637$; son action est croisée, 641 .

Pyramide antérieure. Son action sur les mouvemens volontaires, II, 231 .

T.

Tubercules quadrijumeaux, royez Lobes optiques.

FIN DR LA TABLE ALPHABÉTIQUE. 

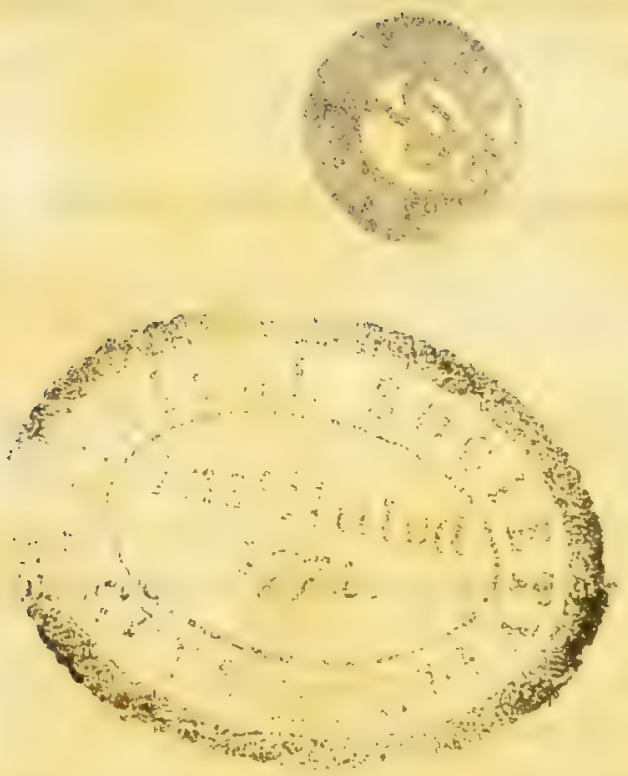





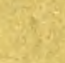


